\title{
Finding Solutions to Work-Life Conflict: Examining Models of Control Over Work-Life Interface
}

\author{
by \\ Mark Julien \\ MIR, Queen's University, Kingston, ON, Canada \\ BAH (Honours), Queen's University, Kingston, ON, Canada
}

\author{
A thesis submitted to the \\ Faculty of Graduate Studies and Research \\ in partial fulfillment \\ of the requirements for the degree of \\ Doctor of Philosophy in Management \\ Carleton University \\ Ottawa, Ontario, Canada
}

(C) Mark Julien, 2007 


$\begin{array}{ll}\begin{array}{l}\text { Library and } \\ \text { Archives Canada }\end{array} & \begin{array}{l}\text { Bibliothèque et } \\ \text { Archives Canada }\end{array} \\ \begin{array}{l}\text { Published Heritage } \\ \text { Branch }\end{array} & \begin{array}{l}\text { Direction du } \\ \text { Patrimoine de l'édition }\end{array} \\ \begin{array}{l}\text { 395 Wellington Street } \\ \text { Ottawa ON K1A ON4 }\end{array} & \begin{array}{l}\text { 395, rue Wellington } \\ \text { Ottawa ON K1A ON4 } \\ \text { Canada }\end{array}\end{array}$

Your file Votre référence ISBN: 978-0-494-33495-9 Our file Notre référence ISBN: 978-0-494-33495-9

NOTICE:

The author has granted a nonexclusive license allowing Library and Archives Canada to reproduce, publish, archive, preserve, conserve, communicate to the public by telecommunication or on the Internet, loan, distribute and sell theses worldwide, for commercial or noncommercial purposes, in microform, paper, electronic and/or any other formats.

The author retains copyright ownership and moral rights in this thesis. Neither the thesis nor substantial extracts from it may be printed or otherwise reproduced without the author's permission.
AVIS:

L'auteur a accordé une licence non exclusive permettant à la Bibliothèque et Archives Canada de reproduire, publier, archiver, sauvegarder, conserver, transmettre au public par télécommunication ou par l'Internet, prêter, distribuer et vendre des thèses partout dans le monde, à des fins commerciales ou autres, sur support microforme, papier, électronique et/ou autres formats.

L'auteur conserve la propriété du droit d'auteur et des droits moraux qui protège cette thèse. $\mathrm{Ni}$ la thèse ni des extraits substantiels de celle-ci ne doivent être imprimés ou autrement reproduits sans son autorisation.
In compliance with the Canadian

Privacy Act some supporting forms may have been removed from this thesis.

While these forms may be included in the document page count, their removal does not represent any loss of content from the thesis.
Conformément à la loi canadienne sur la protection de la vie privée, quelques formulaires secondaires ont été enlevés de cette thèse.

Bien que ces formulaires aient inclus dans la pagination, il n'y aura aucun contenu manquant.

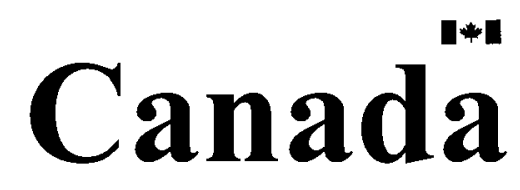




\section{Abstract}

This thesis seeks to identify workplace interventions (alternative work arrangements, perceived flexibility and supervisor support) that can be implemented at the organizational level to reduce three forms of work-life conflict (role overload, work-family interference, family-work interference). The theoretical foundation for the approach used in this thesis is Karasek's DemandControl model (Karasek, 1979; Karasek \& Theorell, 1990). The research models in this thesis adopt the basic constructs used by Karasek (i.e. demands, strain and control) as well as the two hypotheses (i.e. strain and buffering) but seek to update the conceptualization and operationalization of these constructs.

Moderated regression was used to test the hypotheses on a subsample $(n=16,366)$ of the 2001 National Work-life Conflict Study conducted by Linda Duxbury and Chris Higgins. Results indicated moderate support for Karasek's strain hypothesis as work and non-work demands were positively associated and perceived flexibility and supervisor support were negatively associated with worklife conflict. There was no support for any of the strain hypotheses involving the use of various alternative work arrangements and work-life conflict, nor for any of the buffering hypotheses (i.e., control over work-life interface will moderate the relationship between demands and work-life conflict) tested in the analyses. Results did, however, indicate that perceived flexibility was positively associated with the use of three alternative work arrangements (flextime, compressed work week and telework) and supervisor support. 
Further analysis using Chi-squared analysis and MANOVAIANOVA indicate the importance of examining both gender and job type together when exploring differences in demands (work and non-work), work-life conflict, use of alternative work arrangements, perceived flexibility and supervisor support. The findings suggest that alternative work arrangements, in and of themselves, are not helpful in reducing work-life conflict. Rather, this thesis indicates that employers wishing to help their employees reduce work-life conflict should focus instead on reducing work demands and enhancing perceived flexibility and supervisor support. 


\section{Acknowledgements}

I have been blessed with a great deal of support and encouragement from both family and friends in the last few years.

First, I would like to dedicate this thesis to my family. To my wife, Karen, whose love, on-going support and faith in me has meant more to me than words can convey. To my sons, Adam and Ben, you are the bright lights that bring me so much joy and happiness, I love the three of you so much. To my mother, Elaine, and father, Jack, who have taught me that passion, persistence and dedication will allow you to fulfill your deepest dreams. To my sister, Jo-Ann, who has shown me the importance of following your bliss and to my grandparents who have shown me the important legacy of providing unconditional love and a deep spiritual faith to children, grandchildren and greatgrandchildren.

Second, I would like to thank my supervisor, Linda Duxbury, whose tireless efforts, support and encouragement are very much appreciated. The other members of my committee (Lorraine Dyke, Judith Madill, John Zalenski and Donna Lero) deserve special mention for their helpful comments and insightful questions.

Third, I would like to thank my students and faculty from University of Regina, Carleton University and Brock University who have made teaching so enjoyable and allowed me to hone my teaching skills and find my calling. 


\section{Table of Contents}

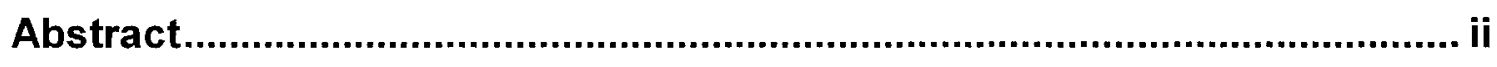

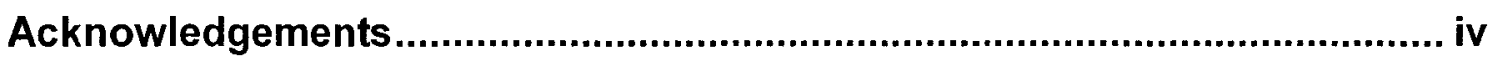

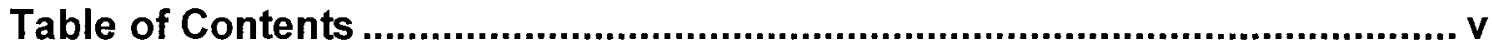

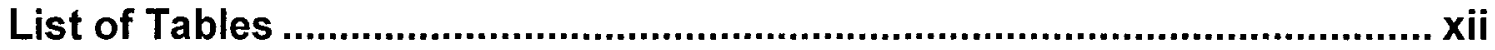

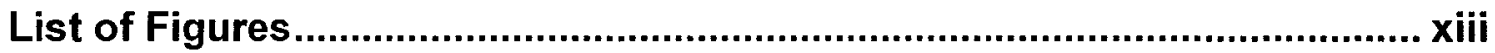

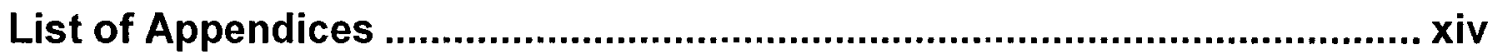

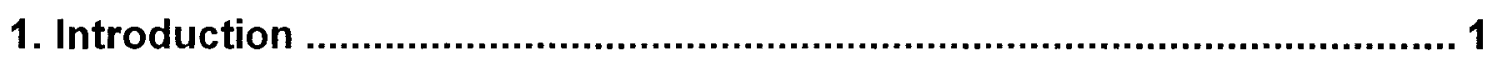

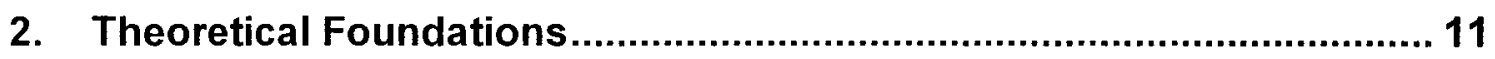

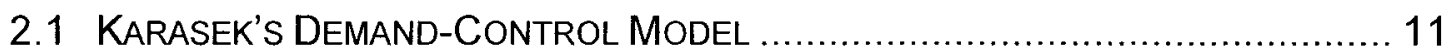

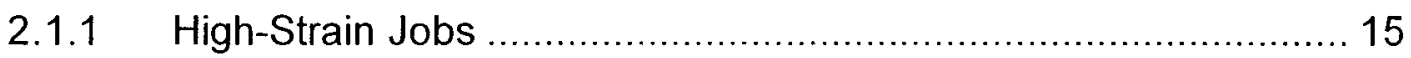

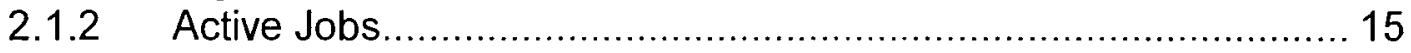

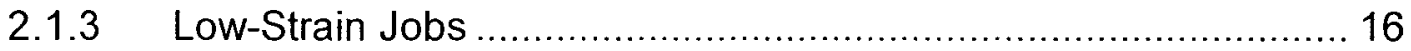

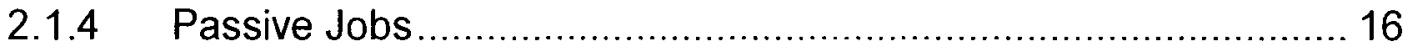

2.1.5 Implications of Karasek's Framework ........................................ 18

2.2 JOHNSON'S REFINEMENT OF KARASEK'S DEMAND-CONTROL MODEL .......... 20

2.2.1 Implications of Johnson's Framework ……............................ 23

2.3 EMPIRICAL EVIDENCE FOR KARASEK'S MODEL …................................. 24

2.3.1 How Karasek Tested His Model.............................................. 24

2.3.2 Subsequent Efforts to Test Karasek's Model ............................ 25

2.3.2.1 Difficulites in Evaluating Karasek's Model.......................... 26

2.3.3 Studies Testing Karasek's Strain Hypothesis.............................. 28

2.3.3.1 Physiological Outcomes................................................ 29

2.3.3.2 Psychological Outcomes................................................. 34

2.3.4 Studies Testing Karasek's Buffer Hypothesis ............................ 36

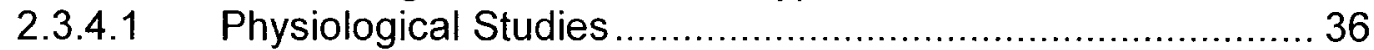

2.3.4.2 Psychological Outcomes............................................... 37

2.4 EMPIRICAL EVIDENCE FOR JOHNSON'S MODEL ......................................4 41

2.4.1 Karasek's Efforts to Test Social Support................................. 41

2.4.2 How Johnson Tested His Model ............................................. 42

2.4.3 Studies Testing Johnson's Strain Hypothesis ........................... 45

2.4.3.1 Physiological Outcomes................................................ 45

2.4.3.2 Psychological Outcomes............................................... 47

2.4.4 Studies Testing Johnson's Buffering Hypothesis .........................4 49

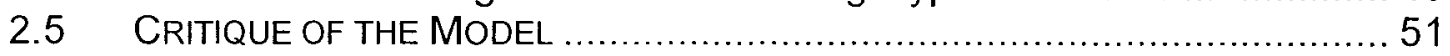

2.5.1 Criticisms of the Demands-Control Literature ........................... 51

2.5.1.1 Simplicity of the Model ................................................... 51

2.5.1.2 Operationalization of the Variables ...................................... 52

2.5.1.3 Lack of Consistency in Measuring Job Strain ...................... 53

2.5.1.4 Variability in data collection............................................ 53

2.5.1.5 Role of Socio-Economic Status ...................................... 59 


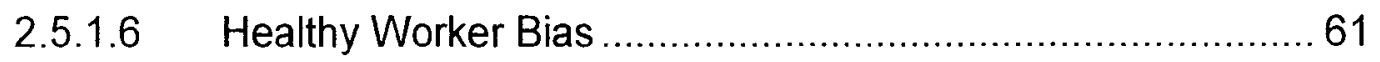

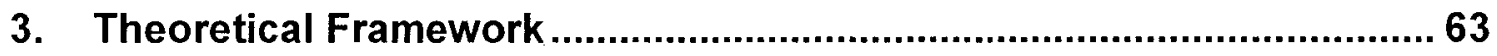

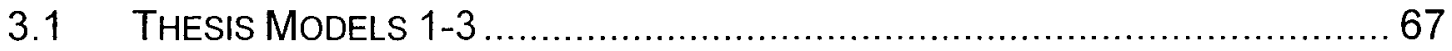

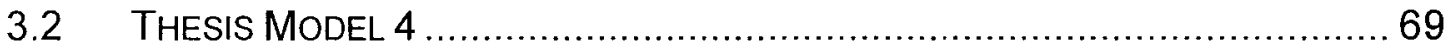

4. Work and Non-Work Demands............................................................. 71

4.1 DEFINITIONS OF WORK AND NON-WORK DEMANDS …............................. 71

4.2 RISING LEVELS OF WORK AND NON-WORK DEMANDS .............................. 72

4.3 RELATIONSHIP BETWEEN DEMANDS AND STRAIN ................................. 77

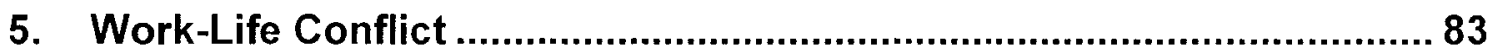

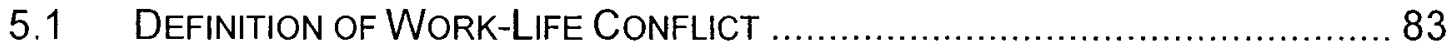

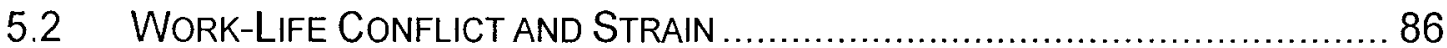

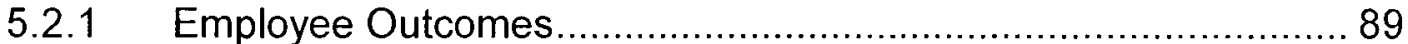

5.2.1.1 Link Between Role Overload and Employee Outcomes ....... 89

5.2.1.2 Link Between Work-Family Interference and Employee

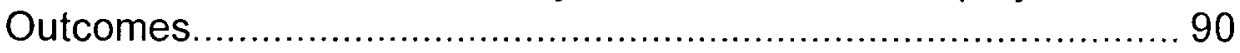

5.2.1.3 Link Between Family-Work Interference and Employee

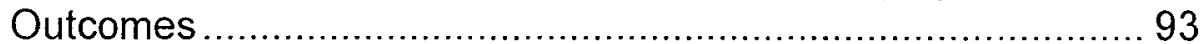

5.3 LINK BETWEen Work DEMANDS AND WoRK-LIFE ConfLICT .................... 95

5.3.1 Link Between Work Demands and Role Overload ....................... 96

5.3.2 Link Between Work Demands and Work-Family Interference....... 97

5.3.3 Link Between Work Demands and Family-Work Interference....... 99

5.4 LINK BETWEEN NON-WORK DEMANDS AND WORK-LIFE CONFLICT ............. 100

5.4.1 Link Between Non-work Demands and Role Overload ............... 100

5.4.2 Link Between Non-work Demands and Work-Family Interference

5.4.3 Link Between Non-work Demands and Family-Work Interference

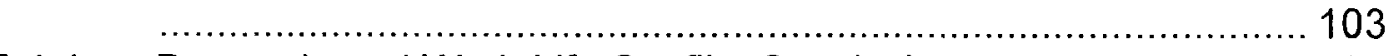

5.4.4 Demands and Work-Life Conflict Conclusion........................... 104

5.5 RISING LEVELS OF WORK-LIFE CONFLICT .............................................. 105

6. Control Over Work-Life Interface ...................................................... 110

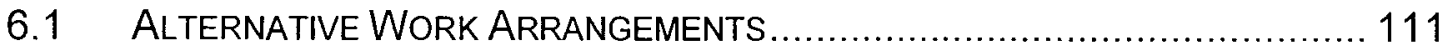

6.1.1 Compressed Work Week ..................................................... 112

6.1.1.1 Advantages and Disadvantages of Compressed Work Week

6.1.1.2 Impact of Compressed Workweek on Work-Life Conflict..... 114

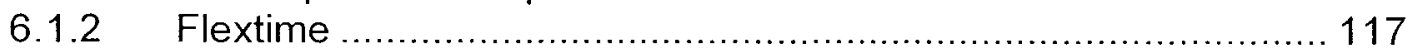

6.1.2.1 Advantages and Disadvantages of Flextime..................... 118

6.1.2.2 Impact of Flextime on Work-Life Conflict ......................... 119

6.1.3 Telecommuting ............................................................... 123

6.1.3.1 Advantages and Disadvantages of Telecommuting ........... 126

6.1.3.2 Impact of Telework on Work-Life Conflict .......................... 127

6.1.4 Shiftwork ....................................................................... 131

6.1.4.1 Advantages and Disadvantages of Shiftwork ................... 133 
6.1.4.2 Impact of Shiftwork on Work-Life Conflict ....................... 136

6.2 PERCEIVED FLEXIBILITY ........................................................ 138

6.2.1 Relationship Between Perceived Flexibility and Work-Life Conflict.. 141

6.2.2 Perceived Flexibility Moderating Demands and Work-Life Conflict .. 146

6.2.3 Relationship Between Perceived Flexibility and Alternative Work

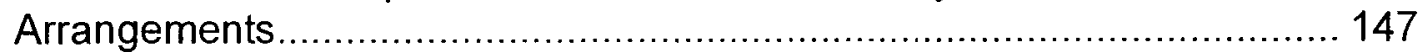

6.3 SUPERVISOR SUPPORT ........................................................... 148

6.3.1 Relationship between Supervisor Support and Work-Life Conflict

6.3.2 Supervisor Support Moderating Demands and Work-Life Conflict 151

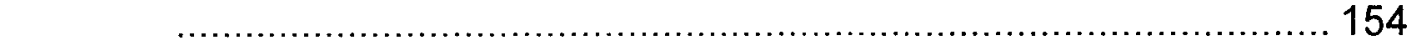

6.3.3 Relationship between Supervisor Support and Perceived Flexibility 156

6.3.4 Supervisor Support Moderating Alternative Work Arrangements and

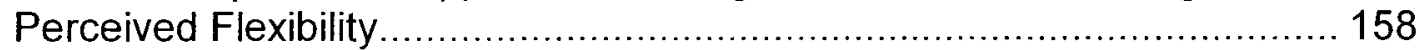

7. Impact of Gender and Job Type ........................................................... 160

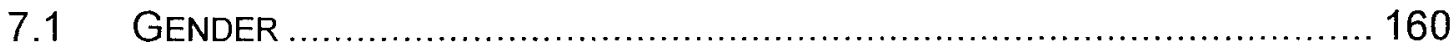

7.1.1 Gender Differences in Demands ........................................ 161

7.1.2 Gender Differences in Work-Life Conflict................................. 168

7.1.3 Gender Differences in Flextime/Perceived Flexibility ................. 175

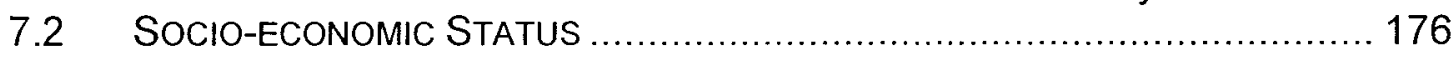

7.2.1 Job Type as a Proxy for SES .............................................. 176

7.2.2 Job Type Differences in Demands ........................................ 178

7.2.3 Job Type Differences in Work-Life Conflict .............................. 179

7.2.4 Job Type Differences in Flextime/Perceived Flexibility .............. 181

7.3 GENDER/JOB TYPE CONSIDERED TOGETHER ......................................... 183

7.3.1 Gender/Job Type Differences in Demands ............................. 184

7.3.2 Gender/Job Type Differences in Work-Life Conflict ................... 185

7.3.3 Gender/Job Type Differences in Flextime/Perceived Flexibility .. 186

8. Research Hypotheses ............................................................................ 188

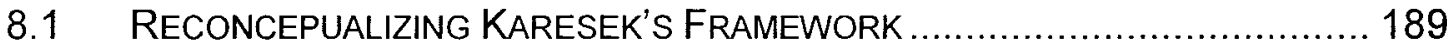

8.1.1 Model 1 Hypotheses (Role Overload) ..................................... 189

8.1.1.1 Model 1: Strain Hypotheses for Role Overload (RO) .......... 190

8.1.1.2 Model 1: Buffer Hypotheses for Role Overload.................... 191

8.1.2 Model 2 Hypotheses (Work-Family Interference) ....................... 192

8.1.2.1 Model 2: Strain Hypotheses for Work-Family Interference

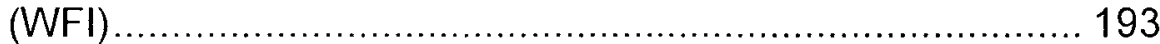

8.1.2.2 Model 2: Buffer Hypotheses for Work-Family Interference .. 193

8.1.3 Model 3 Hypotheses (Family-Work Interference) ...................... 194

8.1.3.1 Model 3: Strain Hypotheses for Family-Work Interference

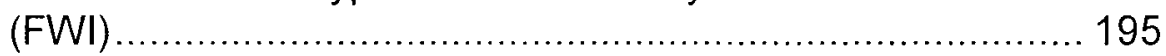

8.1.3.2 Model 3: Buffer Hypotheses for Family-Work Interference

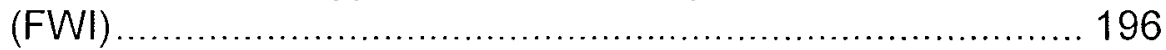

8.1.4 Justification of the Hypotheses (Models 1-3) ........................... 197 
8.1.4.1 Justification of Direct Hypotheses $(\mathrm{H} 1$ and $\mathrm{H} 2)$................. 197

8.1.4.2 Justification of Direct Hypotheses ( $\mathrm{H} 3,4,5$ and 6) ................. 198

8.1.4.3 Justification of Interaction Hypotheses $(\mathrm{H} 7-\mathrm{H} 12) \ldots \ldots \ldots \ldots . . . . .200$

8.1.5 Covariates of Models 1-3 ................................................. 201

8.2 Model Four: Hypotheses (Perceived Flexibility)........................... 203

8.2.1 Model 4: Direct Hypotheses for Perceived Flexibility............... 204

8.2.2 Model 4: Buffering Hypotheses for Perceived Flexibility ......... 204

8.2.3 Justification for Direct Hypotheses (Model 4) ........................ 204

8.2.4 Justification for Interaction Hypotheses (Model 4) .................. 205

8.2.5 Model 4 Covariates ........................................................... 206

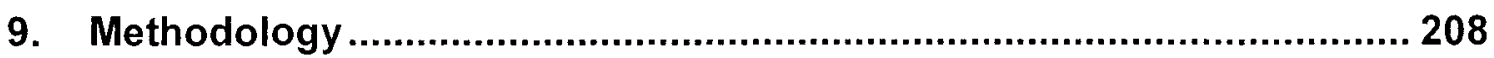

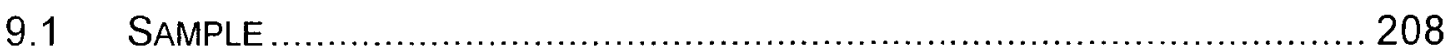

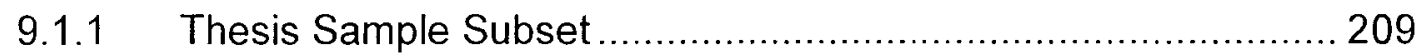

9.2 OPERATIONALIZATION OF CONSTRUCTS ………............................... 210

9.2.1 Work and Non-work demands ............................................. 210

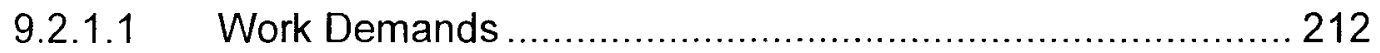

9.2.1.2 Non-Work Demands ............................................... 212

9.2.2 Measures of Strain (Work-Life Conflict) .................................. 213

9.2.3 Alternative Work Arrangements ............................................. 215

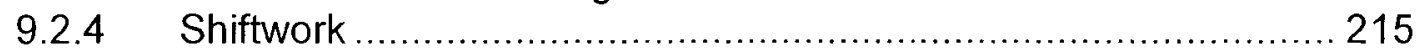

$9.2 .5 \quad$ Perceived Flexibility ........................................................ 216

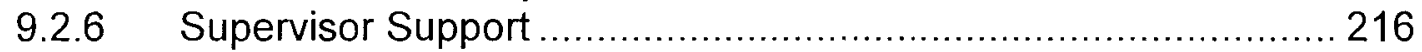

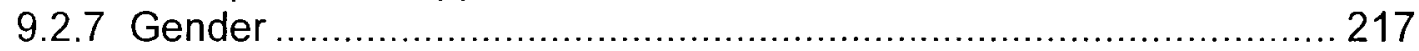

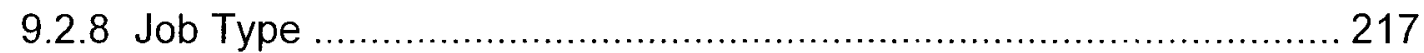

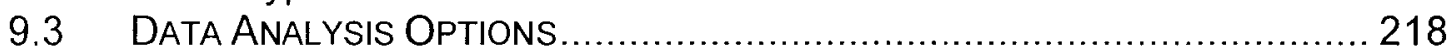

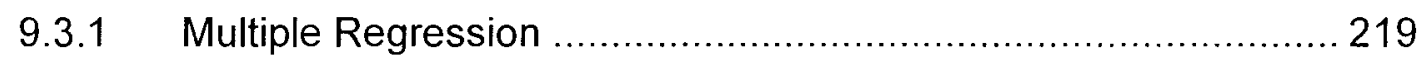

9.3.2 Structural Equation Modeling (SEM) …............................... 220

9.4 ASSUMPTIONS OF MULTIPLE REGRESSION ........................................ 222

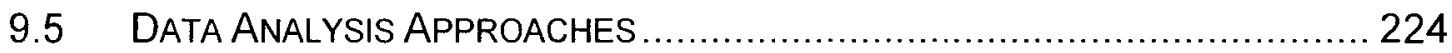

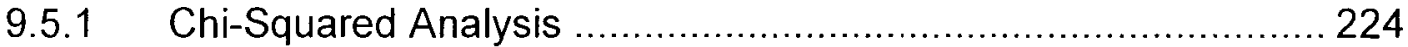

9.5.2 Analysis of Variance (MANOVA/ANOVA) ….......................... 225

9.5.3 Hierarchical Regression ....................................................... 227

9.5.3.1 Testing direct effect hypothesis ............................................ 228

9.5.3.2 Testing the moderator (i.e. interaction) hypothesis.................... 230

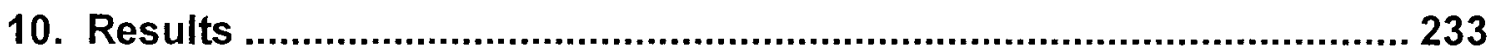

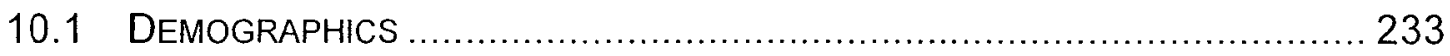

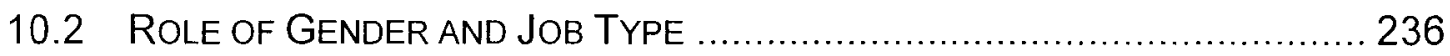

10.2.1 Examination of the Relationship Between Gender, Job Type and

Work Arrangement .................................................................. 237

10.2.2 Examination of the Relationship Between Gender, Job Type and

Work-Life Conflict.................................................................... 238

10.2.3 Examination of the Relationship Between Gender, Job Type and

Perceived Flexibility. 
10.2.4 Examination of the Relationship Between Gender, Job Type and

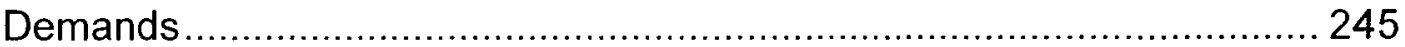

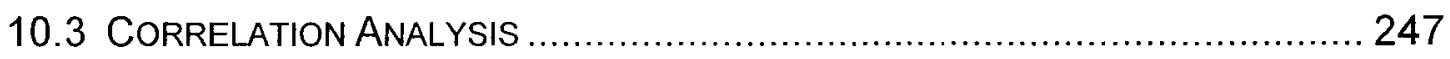

10.4 TEST OF HYPOTHESES ............................................................. 252

10.4.1 Evaluation of Relationships Between Demand, Control over Work-

Life Interface and Role Overload..................................................... 253

10.4.1.1 Relationship Between Demands and Role Overload ........... 254

10.4.1.2 Relationship Between Control Over Work-Life Interface and Role Overload........................................................................ 256

10.4.1.3 Conclusions: Role Overload Strain Hypotheses .................. 260

10.4.2 Evaluation of Factors that Moderate the Relationship Between

Demands and Role Overload ...................................................... 261

10.4.2.1 Control Over Work-Life Interface Moderates Relationship

Between Work Demands and Role Overload .................................... 265

10.4.2.2 Control Over Work-Life Interface Moderates Relationship

Between Non-work demands and Role Overload ............................... 271

10.4.2.3 Conclusions: Role Overload Buffer Hypotheses .................. 277

10.4.3 Evaluation of Relationships Between Demand, Control over Work-

Life Interface and Work-Family Interference ....................................... 277

10.4.3.1 Relationship Between Demands and Work-Family Interference

10.4.3.2 Relationship Between Control Over Work-Life Interface and Work-Family Interference ...................................................... 281

10.4.3.3 Conclusions: Work-Family Interference Strain Hypotheses. 284

10.4.4 Evaluation of Factors that Moderate the Relationship Between

Demands and Work-Family Interference ........................................... 286

10.4.4.1 Control Over Work-Life Interface Moderating Relationship

Between Work Demands and Work-Family Interference ..................... 290

10.4.4.2 Control Over Work-Life Interface Moderating Relationship

Between Non-work demands and Work-Family Interference................ 296

10.4.4.3 Conclusions: Work-Family Interference Buffer Hypotheses. 301

10.4.5 Evaluation of Relationships Between Demand, Control over Work-

Life Interface and Family-Work Interference 302

10.4.5.1 Relationship Between Demands and Family-Work Interference

10.4.5.2 Relationship Between Control Over Work-Life Interface and Family-Work Interference .................................................... 306

10.4.5.3 Conclusions: Family-Work Interference Strain Hypotheses. 309 10.4.6 Evaluation of Factors that Moderate the Relationship Between

Demands and Family-Work Interference.

10.4.6.1 Control Over Work-Life Interface Moderates Relationship

Between Work Demands and Family-Work Interference ...................... 314

10.4.6.2 Control Over Work-Life Interface Moderates Relationship

Between Non-Work Demands and Family-Work Interference ............... 320

10.4.6.3 Conclusions: Family-Work Interference Hypotheses ............. 324 
10.4.7 Evaluation of Relationships Between Work Arrangements, Supervisor Support and Perceived Flexibility

10.4.7.1 Relationship Between Alternative Work Arrangements and

Perceived Flexibility

10.4.7.2 Relationship Between Shiftwork and Perceived Flexibility ....... 327

10.4.7.3 Relationship Between Supervisor Support and Perceived

Flexibility

10.4.7.4 Conclusions: Control Over Work-Life Interface Direct

Hypotheses.

10.4.8 Evaluation of Supervisor Support Moderates the Relationship

Between Work Arrangements and Perceived Flexibility....

10.4.8.1 Conclusions: Control Over Work-Life Interface Buffer

Hypotheses.

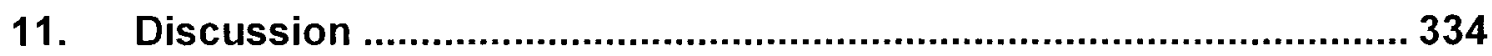

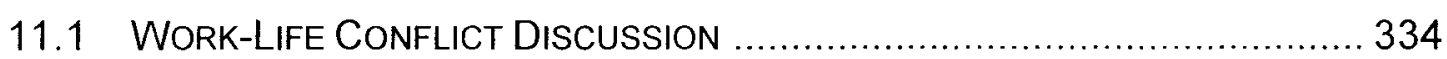

11.2 RELATIONSHIP BETWEEN GENDER AND JOB TYPE …............................ 337

11.3 RELATIONSHIP BETWEEN GENDER, JOB TYPE, DEMANDS, CONTROL OVER

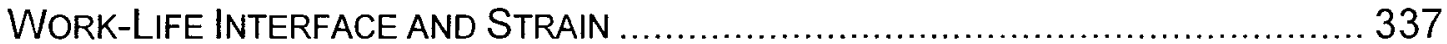

11.3.1 Relationship Between Gender, Job Type and Demands ........... 338

11.3.2 Relationship Between Gender, Job Type and Work-Life Conflict 341

11.3.3 Relationship Between Gender/Job Type and The Use of Alternative

Work Arrangements ................................................................... 345

11.3.4 Relationship Between Gender/Job Type and Control Over Work-

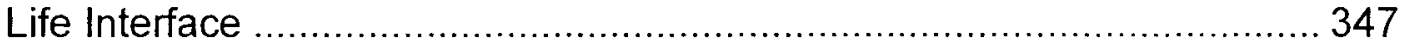

11.4 DISCUSSION OF RELATIONSHIPS BETWEEN MODELS 1-3 _.................. 352

11.4.1 Relationship Between Work Demands and WLC .................... 354

11.4.2 Relationship Between Non-Work Demands and WLC ........... 355

11.4.3 Demands Predicting Variance in Work-Life Conflict.................. 356

11.4.4 Relationship Between Control over Work-Life Interface and Strain

11.4.4.1 Relationship Between Alternative Work Arrangements and

Work-Life Conflict .............................................................. 359

11.4.4.2 Relationship Between Perceived Flexibility and Work-Life Conflict........................................................................... 361

11.4.4.3 Relationship Between Supervisor Support and Work-Life

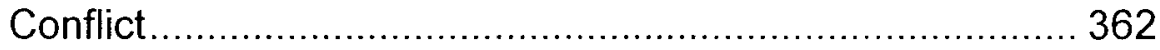

11.4.4.4 Relative Impact of Predictors and Work-Life Conflict........... 363

11.4.5 Buffering the Relationship Between Demands and Strain ......... 370

11.5 PREDICTING PERCEIVED FLEXIBLITY ............................................. 373

11.5.1 Relationship Between Control Over Work-Life Interface and

Perceived Flexibility ...................................................................... 375

11.5.2 Relative Impact of Predictors and Perceived Flexibility............. 376

11.5.3 Moderating the Relationship Between AWA and Perceived

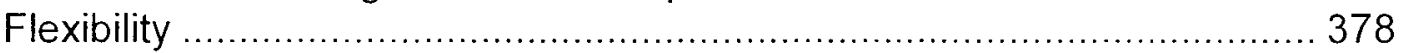

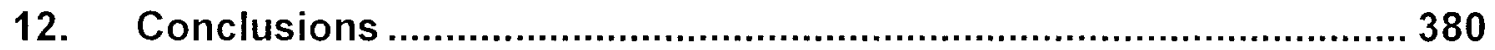




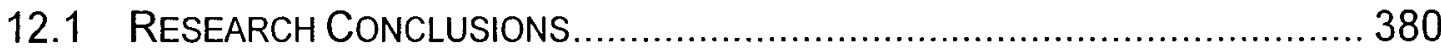

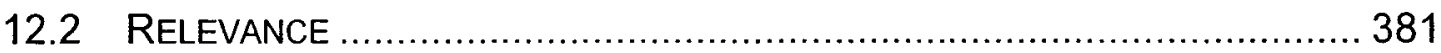

12.2.1 Theoretical/Conceptual Contributions ..................................... 381

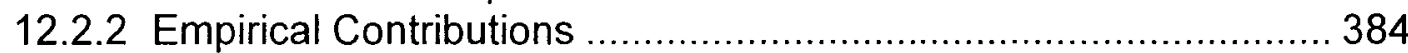

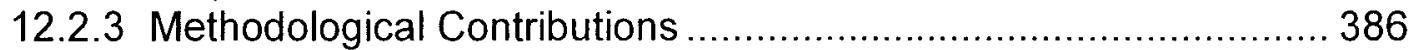

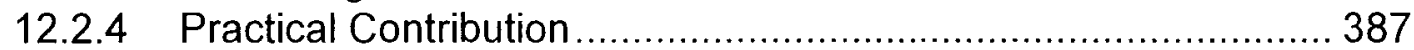

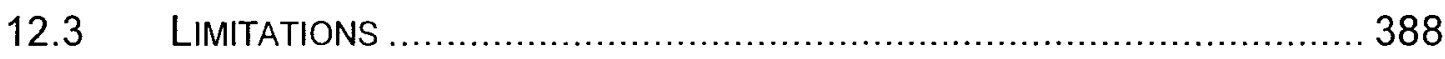

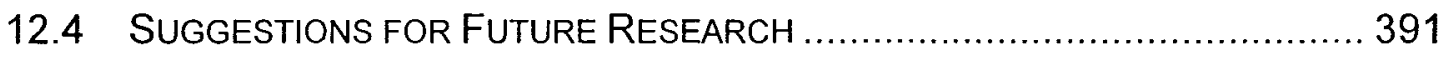

Appendix A Summary of Relevant Survey Questions ........................... 396

Appendix B Checking Assumptions of Regression ................................ 399

APPENDIX B.1 CHECKING ASSUMPTIONS OF NORMALITY-DEPENDENT VARIABLES 399 APPENDIX B.2 CHECKING ASSUMPTIONS OF LINEARITY AND HOMOSCEDASTICITY. 405

Appendix C Summary of Testing Strain and Buffer Hypotheses.......... 411

Appendix D Unstandardized and Standardized Coefficients for Models 1-

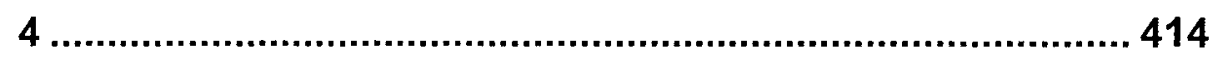

Appendix $E$ Comparison of Change in $R^{2}$ (Models 1, 2, 3 and 4) ............ 426

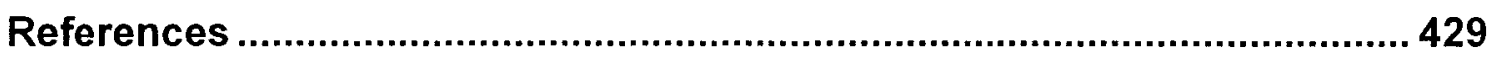




\section{List of Tables}

\begin{tabular}{|l|l|}
\hline Table & Page \\
\hline Table 1 CVD Symptoms at Low and High Social Support & 44 \\
\hline Table 2 Construct Comparison (Karasek/Thesis) & 63 \\
\hline Table 3 Summary of MANOVA/ANOVAs Used in Thesis & 226 \\
\hline Table 4 Summary of Demographic Characteristics & 234 \\
\hline Table 5 Use of Work Arrangements by Gender and Job Type & 237 \\
\hline Table 6 Results of Gender/Job Type MANOVA/ANOVA & 240 \\
\hline $\begin{array}{l}\text { Table 7 Correlation Analysis (Covariates, Independent, Dependent and } \\
\text { Moderator Variables) }\end{array}$ & 248 \\
\hline Table 8 Strain Hypotheses Results for Model 1 & 254 \\
\hline Table 9 Pratt's Coefficient: Role Overload & 256 \\
\hline Table 10 Buffer Hypotheses Results for Model 1 & 264 \\
\hline Table 11 Strain Hypotheses Results for Model 2 & 278 \\
\hline Table 12 Pratt's Coefficient: Work-Family Interference & 280 \\
\hline Table 13 Buffer Hypotheses Results for Model 2 & 289 \\
\hline Table 14 Strain Hypotheses Results for Model 3 & 303 \\
\hline Table 15 Pratt's Coefficient: Family-Work Interference & 305 \\
\hline Table 16 Buffer Hypotheses Results for Model 3 & 313 \\
\hline Table 17 Direct Hypotheses Results for Model 4: Control & 325 \\
\hline Table 18 Interaction Hypotheses Results for Model 4: Control & 330 \\
\hline Table 19 Summary of Strain Hypotheses Tested for Models 1-3 & 352 \\
\hline Table 20 Summary of Buffer Hypotheses Tested for Models 1-3 & 353 \\
\hline Table 21 Pratt's Standardized Measure for Predicting Role Overload & 365 \\
\hline $\begin{array}{l}\text { Table 22 Pratt's Standardized Measure for Predicting Work-Family } \\
\text { Interference }\end{array}$ & 365 \\
\hline Table 23 Pratt's Coefficients for Predicting Family-Work Interference & 366 \\
\hline Table 24 Summary of Direct Hypotheses Tested for Model 4 & 374 \\
\hline Table 25 Summary of Interaction Hypotheses Tested for Model 4 & 374 \\
\hline Table 26 Pratt's Coefficients for Predicting Perceived Flexibility & 376 \\
\hline
\end{tabular}




\section{List of Figures}

\begin{tabular}{|l|l|}
\hline Figure & Page \\
\hline Figure 1 Karasek's Demand-Control Model & 15 \\
\hline Figure 2 Johnson's Demand-Control-Support model & 21 \\
\hline Figure 3 General Framework: Mapping Thesis Models 1-3 to Karasek & 69 \\
\hline Figure 4 General Framework: Mapping Thesis Model 4 & 70 \\
\hline Figure 5 Comparison of Work-Life Outcomes (1991 vs. 2001) & 107 \\
\hline Figure 6 Theoretical Framework: Thesis Model 1 & 190 \\
\hline Figure 7 Theoretical Framework: Thesis Model 2 & 192 \\
\hline Figure 8 Theoretical Framework: Thesis Model 3 & 195 \\
\hline Figure 9 General Framework: Mapping Thesis Model 4 & 203 \\
\hline Figure 10 Gender/Job Type Interaction for Work-Family Interference & 242 \\
\hline Figure 11 Gender/Job Type Interaction for Perceived Flexibility & 244 \\
\hline Figure 12 Interaction of Gender/Job Type for Non-Work Demands & 246 \\
\hline Figure 13 Interaction of Work Demands x Shiftwork & 268 \\
\hline Figure 14 Interaction of Work Demands x Perceived Flexibility & 270 \\
\hline Figure 15 Interaction of Non-work demands x Supervisor Support & 276 \\
\hline Figure 16 Interaction of Work Demands x Shiftwork & 293 \\
\hline Figure 17 Interaction of Work Demands x Supervisor Support & 296 \\
\hline Figure 18 Interaction of Work Demands x Perceived Flexibility & 318 \\
\hline $\begin{array}{l}\text { Figure 19 Relationship Between Control Over Work-Life Interface, } \\
\text { Demands and Work-Life Conflict }\end{array}$ & 382 \\
\hline
\end{tabular}




\section{List of Appendices}

\begin{tabular}{|l|l|}
\hline Appendix & Page \\
\hline Appendix A Summary of Relevant Survey Questions & 396 \\
\hline Appendix B Checking Assumptions of Regression & 399 \\
\hline Appendix C Summary of Testing Strain and Buffer Hypotheses & 411 \\
\hline Appendix D Unstandardized and Standardized Coefficients for \\
Models 1-4 & 414 \\
\hline Appendix E Comparison of Change in $\mathrm{R}^{2}$ (Models 1, 2, 3 and 4) & 426 \\
\hline
\end{tabular}




\section{Introduction}

"Most workers today, regardless of gender, have family responsibilities, and most married workers, regardless of gender, have an employed spouse. But jobs are still designed as if workers have no family responsibilities" (Bailyn, Drago, and Kochan, 2001, p. 1).

The average North American worker fulfills many roles on a day-to-day basis. He/she works in paid employment outside the home, raises children, volunteers and may also look after an elderly parent. At the same time that North Americans are juggling several different roles there is a perception that the level of work demands keeps escalating, as employees are expected to do more with less. The research in both Canada and the United States would suggest that this perception is true (e.g. Bailyn, Drago, and Kochan, 2001; Duxbury and Higgins, 2002b).

For many employees, the result of trying to balance life in different domains (i.e. work and non-work) is a feeling that there just aren't enough hours in the day (i.e. role overload) and that in order to be effective in one domain, the other domain gets short-changed (interference between work and non-work domains). Role overload and role interference are both forms of work-life conflict.

This thesis seeks to identify mechanisms that can be implemented at the organizational level to reduce both of these forms of work-life conflict. The theoretical foundation for the approach used in this thesis is Karasek's DemandControl model (Karasek, 1979; Karasek and Theorell, 1990). This model placed front and center the notion that having a sense of control over one's work 
environment has a positive impact on one's health. Karasek's model is one of the most often cited and empirically tested models in the strain literature (e.g. Kristensen, 1995; Landsbergis, Schurman, Israel, Schnall, Hugentobler, Cahill, and Baker, 1993; Schnall, Landsbergis, and Baker 1994; Van der Doef and Maes, 1998, 1999).

Karasek's model centres around two different hypotheses: the strain hypothesis and the buffering hypothesis. The strain hypothesis looks at two predictors of job strain: job demands (defined by Karasek as the psychological demands employees face at work, i.e. how hard an employee works) and decision latitude (defined by Karasek as the working individual's potential control over his/her tasks and his/her conduct during the working day). ${ }^{1}$ The strain hypothesis states that there is a negative association between job demands and decision latitude. In other words, high job demands and low decision latitude are associated with high strain jobs whereas low demands and high decision latitude are associated with low strain jobs. Workers in high strain jobs are more likely than other workers to experience negative health outcomes (i.e. strains) such as coronary heart disease and depression (Karasek, 1979).

The buffer hypothesis suggests that decision latitude can moderate the relationship between work demands and strain. This hypothesis implies that the magnitude of the effect of work demands on strain depends on the level of decision latitude. In other words, high levels of decision latitude can weaken the positive association between work demands and strain.

1 Please note that Karasek used the term decision latitude when referring to his control construct. To avoid confusion, I will use the term decision latitude when referring specifically to Karasek's work but will use the term control elsewhere in this thesis. 
The research models in this thesis adopt the basic constructs used by Karasek (i.e. demands, strain and control) as well as the two hypotheses (i.e. strain and buffering) but seek to update the conceptualization and operationalization of these constructs. Karasek's conceptualizations of demands, control and strain were formulated in the 1970s and while meaningful in the context of that time period are less relevant now. Karasek's notion of demands focused exclusively on work demands and did not include non-work demands. This focus on work demands reflected the predominant paradigm at the time that the work and nonwork domains were separate worlds (Kanter, 1977) and that there was no spillover between the domains of work and personal life. In other words, when Karasek first proposed his model, the boundary between work and nonwork was perceived to be highly inflexible (Kirchmeyer, 1995).

The dramatic increase in the labour force participation of women has blurred the boundary between work and life. The magnitude of the change can be seen by considering the following data on maternal employment and family type. In 1946, 18\% of women worked in paid employment (Williams, 2004). Marshall (2005), using OECD data, reports that in $2003,68.5 \%$ of Canadian women aged 25 to 64 worked in paid employment. The result is that the vast majority of Canadian mothers work outside the home. For instance, in 2004, $73 \%$ of all women with children under 16 years of age and $67 \%$ of women with children under 6 years of age are part of the labour force (Lindsay and Almey, 2006). The percentage of dual-income families in Canada has doubled in the 
past three and a half decades rising from 33\% in 1967 to $66 \%$ in 2003 (Lindsay and Almey, 2006).

The American figures show a similar trend. The majority of American couples are dual-earners (78\% in 2002; Bond et al., 2003). Furthermore, an overwhelming number of mothers with children work: in $2005,71 \%$ of women with children under 18 were in the labor force (BLS, 2005d).

The reality for many North Americans is that they perform both workrelated duties (e.g. paid employment, unpaid overtime) as well as non-work related duties (e.g. childcare, eldercare, household chores, volunteer work) essentially putting in double or triple duty in the course of a week. Karasek's conceptualization of demands has therefore been updated in this thesis to include both work demands and non-work demands.

Karasek's notion of strain is also updated in this thesis to reflect the fact that strain is not only job-related. The demise of the traditional male breadwinner family and the ascendance of the dual-income family have introduced a new form of strain into the research lexicon: work-life conflict. The conceptualization of strain in this thesis has therefore been expanded to include a measure of strain that reflects the difficulty in juggling the responsibilities of the work and non-work domains. This notion of strain is referred to as work-life conflict and is operationalized in this thesis to include: role overload (defined as occurring when employees feel overwhelmed because they simply cannot complete all their responsibilities within the prescribed timeframe); work-family interference (defined as occurring when work responsibilities make it physically 
or psychologically harder for an employee to fulfil his or her family responsibilities) and family-work interference (defined as occurring when family responsibilities make it physically or psychologically harder for an employee to fulfil his or her work responsibilities).

Finally, this thesis has modified the conceptualization of control to accommodate the changed nature of demands and strain and now looks at control over the work-life interface. As seen in section 5.5, there has been a rise in the levels of work-life conflict among North American workers. Therefore, it is important for employers to understand how they can help employees reduce work-life conflict. The desire to reduce work-family conflict has led many companies to experiment with different ways to help employees balance work and non-work life. For today's dual earner parent, alternative work arrangements, perceived flexibility and supervisor support may increase the amount of control they have over their work-family interface. Alternative work arrangements such as flextime, compressed work week and telework have the potential to increase an employees' ability to control the timing of their workday, their work week and their workplace respectively while shiftwork may make control over the work-life interface more difficult (Johnson, 1997).

This thesis also includes two other potential forms of control over the work-life interface: perceived flexibility (defined as the employee having a sense of control over the hours worked and the location of work; Duxbury and Higgins, 2001) and supervisor support (defined as working for a supervisor who communicates effectively, respects his/her employees, supports employee 
development and gives employees the flexibility to meet non-work responsibilities) (Duxbury and Higgins, 2001).

The first three models of this thesis seek to test a new conceptualization of Karasek's model and determine if the model conceived in the 1970 s to predict job strain has relevance to our understanding of how work and non-work demands are associated with work-life conflict.

Furthermore, these models test whether or not the six forms of control over the work-life interface (i.e. flextime, compressed work week, telework, shiftwork, perceived flexibility and supervisor support) moderate the relationship between work demands and work-life conflict as well as the relationship between non-work demands and work-life conflict.

This thesis also seeks to develop a new model (model 4) that explores the relationships between the aforementioned conceptualizations of control (alternative work arrangements, perceived flexibility and supervisor support). Unfortunately, there is little literature available which looks at the relationship between alternative work arrangements, perceived flexibility and supervisor support. This thesis seeks to provide some understanding of how these three constructs are related. Firstly, Model 4 tests whether or not the four work arrangements (e.g. flextime, compressed work week, telework and shiftwork) are associated with perceived flexibility. Secondly, Model 4 also tests whether or not supervisor support is associated with perceived flexibility. Thirdly, Model 4 tests whether or not supervisor support moderates the relationship between each of the four work arrangements and perceived flexibility. 
Specifically, this thesis seeks to answer the following questions:

1a) What is the relationship between work demands and work-life conflict (role overload, work-family interference, family-work interference)?

1b) What is the relationship between non-work demands and work-life conflict (role overload, work-family interference, family-work interference)?

2) What is the relationship between the various conceptualizations of control (i.e. flextime, compressed work week, telework, shiftwork, perceived flexibility, and supervisor support) examined in this thesis and work-life conflict (role overload, work-family interference, family-work interference)?

3) To what extent do the forms of control examined in this thesis (i.e. flextime, compressed work week, telework, shiftwork, perceived flexibility, and supervisor support) moderate the relationship between work demands and worklife conflict (i.e. role overload, work-family interference, family-work interference)?

4) To what extent do the forms of control examined in this thesis (i.e. flextime, compressed work week, telework, shiftwork, perceived flexibility, and supervisor support) moderate the relationship between non-work demands and work-life conflict (i.e. role overload, work-family interference, family-work interference)?

5) What is the relationship between the various forms of control examined in this thesis (i.e. flextime, compressed work week, telework, shiftwork, and supervisor support) and perceived flexibility? 
6) To what extent does supervisor support moderate the relationship between the different work arrangements examined in this thesis (i.e. flextime, compressed work week, telework, shiftwork) and perceived flexibility?

This thesis uses data from the 2001 National Work-Life Conflict Study conducted by Linda Duxbury and Chris Higgins to answer the questions listed above. The Duxbury and Higgins study surveyed over 31,000 Canadian respondents on a broad range of questions. Hierarchical multiple regression was used to test both the strain and buffer hypotheses.

The results of this thesis are of potential interest to employees, employers, and academics. At the employee level, overload and interference have been found to be positively associated with poor physical health (e.g. Frone, Russell and Cooper, 1997; Thomas and Ganster, 1995), job dissatisfaction (Bacharach, Bamberger and Conley, 1991; Burke, 1993; Higgins, Duxbury and Irving, 1992; Kossek and Ozeki, 1998), depression or poor psychological health (Frone, Russell and Cooper, 1992a; Netemeyer et al., 1996), misuse of alcohol and drugs (Frone, Russell, and Cooper, 1993; Frone, Russell, and Cooper, 1997), and withdrawal from family interaction (MacEwen and Barling, 1994; Paden and Buehler, 1995; Repetti and Wood, 1997).

Employees are not the only stakeholder group to be negatively impacted by high levels of work-life conflict. High levels of work-life conflict can also impact employers in a number of ways. For example, work-life conflict has been found to be positively associated with intent to turnover (e.g. Anderson et al., 2002; Riley and McCloskey, 1997), job dissatisfaction (e.g. Bacharach, 
Bamberger and Conley, 1991; Kossek and Ozeki, 1998), and lower organizational commitment (e.g. Good et al., 1988; Netemeyer et al., 1996).

Society ultimately pays a price for high levels of work-life conflict. Higgins, Duxbury and Johnson (2004) in a report for the Public Health Agency of Canada (formerly Health Canada) have documented the association between high levels of work-life conflict and costs to the Canadian health care system. For example, the authors note that high levels of role overload cost the system $\$ 1.8$ billion per year in doctor's visits, 3.8 billion per year in hospital stays and $\$ 250$ million per year in visits to hospital emergency rooms. The authors then examined high levels of work-family interference and health care costs and noted that high levels of work-family interference cost the system $\$ 2.8$ billion per year.

Given the high costs of work-life conflict to employees, employers and society, it is important to understand how to reduce levels of work-life conflict. This thesis helps in this regard by evaluating the link between three organizational interventions and lower work-life conflict. This thesis will also interest academics who study work-life conflict as well as alternative work arrangements, perceived flexibility and supervisor support. Unlike many previous studies in these areas (e.g. Bailyn, 1989; Beach, 1989; Crossman and Burton, 1993) where sample size was low, this thesis uses a large data set thereby permitting generalizability of results. Testing of a reconceptualization and modernized form of Karasek's model also contributes to theoretical development in this area. Furthermore, thesis model 4 increases our understanding of how alternative work arrangements are related to perceived flexibility. 
This thesis is divided into 12 sections (including the introduction). First, the theoretical foundation is presented in section 2. Karasek's demand-control model is presented followed by the modification of the Karasek model presented by Johnson who added social support to Karasek's framework. The empirical evidence supporting or refuting both Karasek and Johnson's models is presented as well as a discussion of various methodological and theoretical issues associated with Karasek's model. The third section of the thesis presents the theoretical framework guiding this thesis. Demands (both work and non-work demands), work-life conflict (role overload, work-family interference and familywork interference) and control over work-life interface (alternative work arrangements, perceived flexibility and supervisor support) are then reviewed in sections 4,5 and 6 respectively. Factors that might confound the findings (i.e. gender and socio-economic status) are discussed in the literature review in section 7 . Section 8 outlines the hypotheses to be tested. Section 9 describes and justifies the statistical technique (i.e. hierarchical regression) used in this thesis to test the models and discusses how the constructs are operationalized. The results are presented in section 10 while section 11 discusses the key findings. Section 12 is the concluding section where the contribution of the thesis, its limitations and areas of future research are presented. 


\section{Theoretical Foundations}

"There is no use worrying about things over which you have no control, and if you have control, you can do something about them instead of worrying" (Stanley C. Allyn). (www.wisdomquotes.com/002537.html)

This section will review the theoretical foundations of this thesis and the empirical work that has emerged as a result of these theories. First, the conceptual model of Karasek and the subsequent refinements of Johnson will be discussed as well as their strain and buffer hypotheses. Second, the empirical evidence for both Karasek's and Johnson's strain and buffer hypotheses will be presented. The section concludes with a discussion of the conceptual and methodological criticisms of their work.

\subsection{Karasek's Demand-Control Model}

As noted in the introduction, the theoretical underpinnings of this thesis are based on Karasek's Demand-Control model. Before we can understand the thesis model, therefore, we must understand Karasek. This section will present Karasek's definitions of key terms (i.e. demands, control, and strain) followed by his Demand-Control Model.

Karasek's model first appeared in a seminal article in Administrative Science Quarterly in 1979. Karasek defined demands as the "psychological stressors involved in accomplishing the work load, stressors related to unexpected tasks, and stressors of job-related personal conflict" (Karasek, 1979, 
p. 291). This notion of demands centred on psychological demands at work and was characterized as how hard an employee works (Karasek, 1979). Such demands may involve meeting objectives (e.g. how many widgets an employee can make in an hour), juggling priorities (e.g. meeting conflicting deadlines), and trying to get all the work done (e.g. work overload). Other examples of demands may include interpersonal conflicts with supervisors, clients or coworkers as well as the fear of losing one's job (Karasek and Theorell, 1990).

Karasek called his control construct decision latitude which he defined as "the working individual's potential control over his tasks and his conduct during the working day" (Karasek, 1979, p. 289). He defined two subcomponents of decision latitude: task authority and skill discretion. Task authority was defined as the ability of the worker to influence decisions regarding his or her job (Theorell, 1989). Examples of such decisions include goal setting as well as deciding what and how something is achieved. Skill discretion, on the other hand, was defined relative to "personal control in the future" (Theorell, 1989, p. 50). Skill discretion includes the notion that a worker's skills are being fully utilized, that they have opportunities to learn and that they have task variety. Karasek and Theorell (1990) assumed that workers high in skill discretion develop a "sense of mastery" and are able to confidently deal with future challenges effectively.

According to Karasek (1979) the task authority and skill discretion constructs are similar to other measures in the literature (e.g. discretion and qualification scale, Gardell, 1971; intellectual discretion, Kohn and Schooler, 
1973; autonomy in task organization and variety in skill use, Hackman and Oldham, 1975). This idea was supported by Theorell (1989) who noted that task authority and skill discretion are highly correlated. This is not surprising since Karasek theorized that "a high level of skill gives the worker control over which specific skills to use to accomplish the task" (Karasek and Theorell, 1990, p. 58).

Despite the high correlation between these two factors, Karasek (1989) maintains that task authority and skill discretion are theoretically distinct constructs. Theorell (1989) cites a study conducted by Theorell, Hamsten, de Faire, Orth-Gomer, and Perski (1987) which illustrated the distinctive and separate nature of these constructs. In this study, Karasek's demand-control questionnaire was validated using six widely different occupations. The authors noted that baggage carriers rated themselves low in skill discretion but high in task authority (i.e. they felt confident that they could communicate with their supervisors when there were too many bags in one place and resolve this problem). On the other hand, symphony musicians felt they had a high level of skill discretion but low task authority to play the pieces they wished to play.

Karasek defined job strain as "an overload condition experienced by an organism's control system when it attempts to maintain integrated functioning in the face of too many environmental challenges" (Karasek and Theorell, 1990, $p$. 87). Karasek theorized that the body reacts to strain by pushing itself out of equilibrium (defined by Karasek as a rest state) and works to re-establish this equilibrium. Without proper rest or relaxation, the body pushes itself toward exhaustion. Karasek theorized that attempts to relax may be impaired if the 
subject cannot "follow its own optimal course to coordinate action - that is, if it has low decision latitude" (Karasek and Theorell, 1990, p. 88). On the other hand, individuals with a high level of decision latitude can better cope with the job demands they experience because they have more flexibility in how they cope with those job demands. This flexibility allows those with high decision latitude to effectively find solutions to these job demands that correspond to the employees' own unique skills, needs and circumstances (Wall et al., 1996). It is important to recognize that Karasek (1979) did not measure strain directly but instead relied on indirect measures such as coronary heart disease and depression.

Karasek's (1979) model allows for four possible outcomes from the association between job demands and decision latitude (control): high demands, low decision latitude (high strain jobs), high demands, high decision latitude (active jobs), low demands, high decision latitude (low strain jobs) and low demands, low decision latitude (passive jobs). According to this model, as demands increase and decision latitude decrease, job strain increases. The reverse is also hypothesized to be true. As decision latitude increases and job demands decrease, job strain decreases. The results are shown below. Details on the four proposed types of jobs (i.e. high strain, active, low strain, passive) are given following Figure 1. 
Figure 1 (Karasek's Demand-Control Model, Karasek and Theorell, 1990) Job Demands

Decision Latitude

High (control)

\begin{tabular}{|l|l|}
\multicolumn{1}{l}{ Low } & High \\
\hline Low Strain & Active \\
\hline Passive & High Strain \\
\hline
\end{tabular}

\subsubsection{High-Strain Jobs}

A high strain job is defined as an occupation where job demands are high and decision latitude (control) is low. High strain jobs are represented in the lower right hand quadrant of Figure 1. Examples of high strain jobs include assembly line workers and wait staff. It is suggested by Karasek and Theorell (1990) that it is this group where the health risks will be highest. These workers do not have the decision latitude to develop effective solutions to the high level of job demands inherent in their jobs. Interestingly, large numbers of high strain occupations such as garment stitcher, waitress, telephone operator and administrative assistants are female-dominated occupations.

\subsubsection{Active Jobs}

An active job is defined as an occupation where job demands and decision latitude (control) are both high. Active jobs are represented in the upper right hand quadrant of Figure 1. Examples of active jobs are professional jobs such as surgeons, judges, lawyers, engineers, professors and managers. These tend to be jobs carrying a high prestige factor. Karasek and Theorell (1990) also note that many of the jobs in this quadrant are male-dominated. Karasek and 
Theorell (1990) suggest that it is the high level of decision latitude (i.e. control) that allows workers in active jobs to meet the high demands of their jobs by developing creative solutions to the problems brought on by high demands.

Karasek and Theorell (1990) predict that the combination of high job demands and high control will result in only an average level of negative health outcomes.

\subsubsection{Low-Strain Jobs}

A low strain job is defined as an occupation where job demands are low and yet decision latitude (control) is high. Low-strain jobs are represented in the upper left hand quadrant of Figure 1. Examples of low-strain jobs would be repair personnel, linemen and natural scientists. Again, it is suggested that many of the jobs in the low strain quadrant are male-dominated. The high degree of decision latitude combined with low job demands leads to Karasek's prediction that this group of workers should experience lower than average levels of strain and illness because control can be exercised for each of the few demanding situations encountered on a daily basis. Karasek suggests that "these people are actually made both happier and healthier than average by work" (Karasek and Theorell, 1990, p. 36).

\subsubsection{Passive Jobs}

A passive job is defined as an occupation where both job demands and decision latitude (control) are low. Passive jobs are represented in the bottom left hand quadrant of Figure 1. Security personnel, sales clerks, billing clerks and 
custodial staff are examples of passive jobs. It is predicted by Karasek and Theorell (1990) that the long-term effects of low job demands and low decision latitude (i.e. control) is a loss of motivation and productivity due to the lack of job challenges combined with a rigid work environment.

Curiously, despite the negative picture presented of the passive job holder, Karasek and Theorell (1990) predict that employees in this group will experience only average levels of psychological strain and illness. It is interesting to note that this level of strain is the same level predicted for those holding active jobs. Karasek suggests this is because "although each stressor exposure would result in substantial residual psychological strain (just as in the high-strain circumstance), the low job demands of this work situation mean that fewer stressors are confronted" (Karasek and Theorell, 1990, p. 38).

The combination of job demands, decision-latitude (i.e. control) and job strain lead to two separate and distinct hypotheses: the strain hypothesis and the buffer hypothesis. Each of these hypotheses will be discussed in turn.

The Strain Hypothesis

$\mathrm{H}_{1}$ : The strain hypothesis states that there is a positive association between job demands and job strain such that as job demands increase, job strain increases. This hypothesis also states that there is a negative association between decision latitude and job strain such that as decision latitude decreases, job strain increases. High job demands and low decision latitude result in high strain jobs whereas low job demands and high decision latitude will lead to low strain jobs. According to this conceptualization, job demands and decision 
latitude should each be considered as independent variables while strain is the dependent variable. This additive effect hypothesizes that job demands and decision latitude each directly and independently contribute to the outcome (i.e. strain).

The Buffer Hypothesis

$\mathrm{H}_{2}$ : The buffer hypothesis contends that decision latitude can moderate the positive relationship between job demands and strain (i.e. high levels of decision latitude can buffer the negative effects of high job demands on strain). To test this hypothesis, job demands are treated as the independent variable, strain is the dependent variable and decision latitude is the moderator variable. For this hypothesis to hold, the interaction effect between job demands and decision latitude must be significant. The implication is that high levels of decision latitude can weaken the association between job demands and strain.

According to Van der Doef and Maes (1998), the practical implications of the two hypotheses differ. In the case of the buffering hypothesis, the focus is on finding ways for organizations to enhance employees' levels of decision latitude without due regard for the level of demands. In the case of the strain hypothesis, since work demands are independently associated with strain, it is inadequate to merely focus on enhancing control. One must also examine ways of reducing the level of job demands.

\subsubsection{Implications of Karasek's Framework}

Karasek's Demand-Control model dovetails with the literature on workplace stress. In this context, Karasek's notion of job demands can be 
considered a stressor. Selye (1976) defined stressors as factors in a person's environment which put physical or emotional demands on that person. The resulting association of high job demands and low decision latitude that lead to job strain are consistent with others' conceptualization of stress (i.e. negative reaction by an individual to stressors within his/her environment; Selye, 1976). For most employees, the resulting association of job demands and decision latitude occurs over a long time period (i.e. years). Therefore, the stressors Karasek refers to are considered chronic, not acute (Greenhaus and Parasuraman, 1986).

Stress theorists (e.g. Buunk et al., 1998) are also interested in what moderates the relationship between the stressor and the stress reaction (i.e. physical outcomes such as high blood pressure, psychological outcomes such as depression and behavioural outcomes such as absenteeism). Initial research examining strain in the workplace (e.g. Dohrenwend and Dohrenwend, 1974; Hinkle, 1974; Kornitzer et al., 1982; and Shekelle et al., 1979) did not however consider the impact of control. The failure of researchers to consider how control affected the relationship between job demands and strain led to erroneous assumptions about who is most likely to suffer physical symptoms as a result of high job demands. For example, early researchers initially assumed that executives who had higher work demands would experience higher levels of physiological symptoms such as heart disease. This assumption helped to foster what Landsbergis calls "the myth of executive stress" (Landsbergis, 1988, p. 219). 
In discussing the findings of the four quadrants of occupations, Karasek and Theorell (1990) suggest that it is not executives and managers with the highest level of strain and health problems in the workforce. Rather, it is those workers in low-status jobs with no decision-making freedom. In other words, this model would predict that it is "the bossed, not the bosses who experience the most stress in our society" (Karasek and Theorell, 1990, p. 44).

A key contribution of Karasek's Demand-Control model is the recognition of the important role that control can play in reducing levels of strain. This thesis explores the idea that control can be a potentially powerful moderator of the stressor-strain relationship and tests several different updated conceptualizations of control (i.e. alternative work arrangement, perceived flexibility, supportive supervisor) on the relationship between demands and work-life conflict. Further discussion of this thesis' conceptualizations of control can be found in section 3 .

Karasek's model was a catalyst for further theoretical contributions to the job strain field. It was Johnson $(1985,1986)$ and Johnson and Hall $(1988)$ who expanded upon Karasek's model and added the crucial role of social support to the discussion. These conceptualizations are discussed in the section below.

\subsection{Johnson's Refinement of Karasek's Demand-Control Model}

In response to the criticism that Karasek's model was too simple, Karasek et al. (1982) and others (e.g. Johnson, 1985, 1986) began to explore the role of social support on the Demand-Control model. Influenced by Karasek's DemandControl model, Johnson began to consider what role social support would have 
on moderating the stressor-strain relationship. The origins of Johnson's conceptualizations emerged from his work on his doctoral dissertation (Johnson, 1986) and were published in Johnson and Hall's 1988 article in American Journal of Public Health.

Johnson developed his model as follows: Instead of the $2 \times 2$ table developed by Karasek, he postulated eight possibilities with high and low levels of each of the following constructs: job demands, work control (Johnson used this term in lieu of decision latitude) and social support. Johnson termed those high in social support 'collective' and those low in social support 'isolated'.

Johnson's demand-control-support (D-C-S) model is depicted below.

Figure 2 Johnson's demand-control-support model (Johnson, 1989)

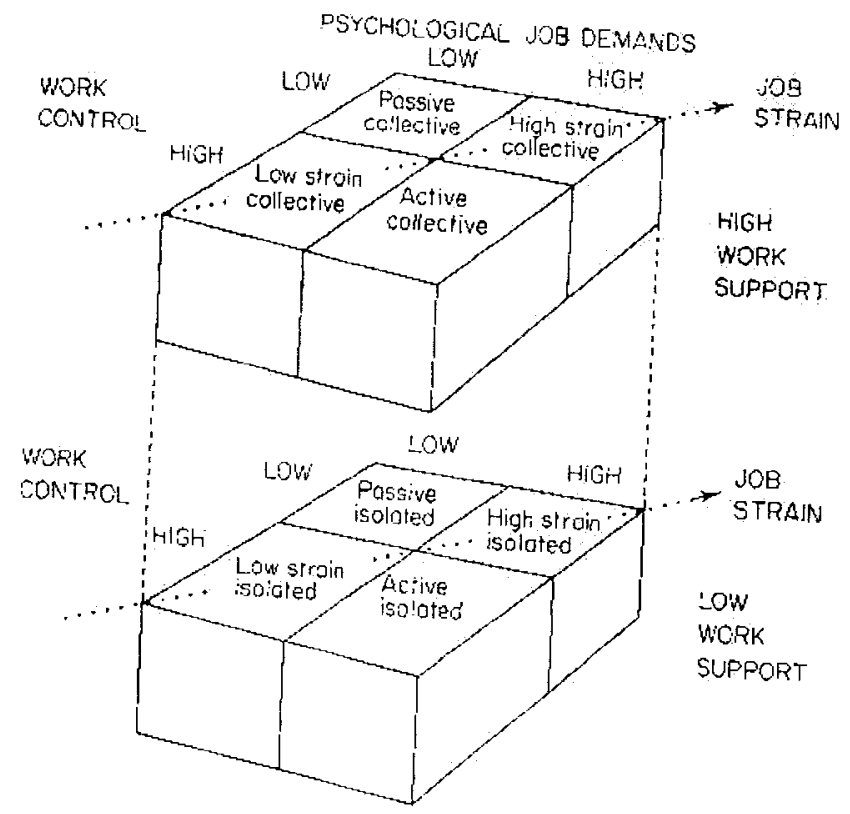


Johnson's model examines the crucial role of social support and extends Karasek's model. The combination of job demands, decision-latitude (i.e. control), social support and job strain lead Johnson to formulate hypotheses in a similar fashion to Karasek (i.e. strain hypothesis and buffer hypothesis). Consistent with our discussion of Karasek, each of Johnson's hypotheses will be presented in turn. First, we will examine the strain hypothesis followed by the buffer hypothesis.

The Strain Hypothesis

$H_{1}$ : The strain hypothesis states that there is a positive association between job demands and job strain, a negative association between work control and job strain and a negative association between social support and job strain. High job demands, low work control and low social support result in the category of high strain isolated whereas low job demands, high work control and high social support will result in the category of low strain collective. In other words, this model conceptualizes job demands, work control and social support as independent variables and strain as the dependent variable. Furthermore, the additive effect hypothesis suggests that job demands, work control and social support directly and independently contribute to the outcome (i.e. strain).

The Buffer Hypothesis

$\mathrm{H}_{2}$ : The buffer hypothesis suggests that work control and social support can both moderate the relationship between job demands and strain. High levels of work control can moderate the negative effects of high job demands on strain. Furthermore, high levels of social support can moderate the negative effects of 
high job demands on strain. This hypothesis implies that there are two interaction effects (between job demands and work control as well as between job demands and social support). This model is tested as follows. Job demands are treated as the independent variable, strain as the dependent variable while work control and social support are the moderator variables. These interactive effects imply that the magnitude of the effect of job demands on strain depends on the level of work control and the level of social support. In other words, high levels of work control and high levels of social support can weaken the positive association between job demands and strain.

\subsubsection{Implications of Johnson's Framework}

Johnson $(1985,1986)$ 's model was an improvement over Karasek's Demand-Control model because it increased awareness that social support and control can both play an important role in weakening the relationship between job demands and strain. In other words, it recognized that control, while important, is not the only influential resource to help employees cope with job demands. The social support provided by coworkers (for example, showing concern when someone is having a bad day) could also be an important resource for employees. Johnson's model supports the inclusion of social support (in the form of supervisor support) in the thesis model. The operationalization of supervisor support is found in section 3 of the thesis. 


\subsection{Empirical Evidence for Karasek's Model}

This sub-section will examine the empirical evidence to support Karasek's strain and buffer hypotheses. We will first examine Karasek's initial efforts to test these hypotheses followed by the efforts of others.

\subsubsection{How Karasek Tested His Model}

Karasek's model has been empirically tested using a variety of different demand-control questionnaires but the Job Content Questionnaire (JCQ) (Karasek, 1985) is the most frequently used instrument. Over forty research groups in seven countries have used the JCQ (Karasek and Theorell, 1990) to test Karasek's model. According to Theorell (1996), the JCQ is an expansion of Karasek's (1979) original questionnaire and contains additional scales such as social support and physical job demands. This questionnaire has been tested extensively and has been used in the United States, Europe and Japan.

Karasek empirically tested his demand-control model and published the results in Administrative Science Quarterly in 1979. The data used came from both Swedish and American national surveys. According to Karasek (1979), the Swedish data came from a random sample of the full adult population (approximately 1:1,000) aged 15 to 75 , with impressive response rates of 92 percent and 85 percent for the years 1968 and 1974 respectively. One strength of the Swedish phase of the study is its longitudinal approach (the same workers were interviewed in both 1968 and 1974). 
The American survey used in Karasek's 1979 paper was the University of Michigan Quality of Employment Survey for 1972. This survey is based on a national stratified sample of housing units and was not longitudinal. The response rate for the American sample was $76 \%$.

Karasek (1979) concluded from these data that those who worked high strain jobs (i.e. the combination of high job demands and low decision latitude were most likely to report exhaustion, job dissatisfaction and depression (compared with those working in passive, active and low strain jobs). After Karasek published his findings in 1979, he teamed up with Tores Theorell, an epidemiologist with backgrounds in both cardiovascular and psychosocial stress research. Their joint collaboration led to the research reported in Karasek, Baker, Marxer, Ahlbom, and Theorell (1981). In this research, 1,461 randomly selected Swedish working men were interviewed in 1968 about their work and about heart disease symptoms. The authors reported that men who worked in high strain jobs had an increased risk of cardio heart disease and increased cardio-vascular mortality.

\subsubsection{Subsequent Efforts to Test Karasek's Model}

Other efforts to empirically test Karasek's model followed his initial efforts. It is important to recognize that job strain is never tested directly in any of these studies. Instead, researchers measured job strain indirectly using a variety of physiological and psychological outcomes. A review of the empirical research testing Karasek's two hypotheses (i.e. strain and buffering) for these outcomes will be presented below. 
Research support for Karasek's demand/control model has certainly not been unequivocal. Many authors who have conducted reviews of the empirical evidence (Kristensen, 1995; Landsbergis et al., 1993; Schnall, Landsbergis, and Baker 1994; Van der Doef and Maes, 1998, 1999) with respect to this model suggest there is empirical support for the strain hypothesis (i.e. a negative association exists between job demands and decision latitude such that high levels of job demands and low levels of decision latitude result in high levels of job strain). It should be acknowledged, however, that some studies (e.g. Alterman et al., 1994; Reed et al., 1989) refute the strain hypothesis. The buffer hypothesis (the notion that decision latitude can actually moderate the impact of job demands on job strain) has received less support than the strain hypothesis and the research is mixed in its findings.

A review of the literature shows that the strength of the findings often depends on whether the outcome being studied is physiological (e.g. coronary heart disease, high blood pressure) or psychological (e.g. depression). The research suggests that the physiological outcomes tend to show the strongest resultant support for a negative association between job demands, decision latitude and job strain. It can be argued that the physiological studies which reject the strain hypothesis have serious methodological flaws. Support for this contention is given in subsection 2.3.3.

\subsubsection{Difficulites in Evaluating Karasek's Model}

Comparing the results obtained from the various studies testing Karasek's model is difficult for several reasons including the use of various 
operationalizations of both demands and decision latitude, the use of a variety of outcomes of job strain (i.e. physiological, psychological and behavioural) and the variety in sample characteristics (single occupation versus multi-occupation studies, average age of the group). Each of these difficulties will be discussed in further detail in the subsection below.

First, there are significant differences in how constructs such as job demands and decision latitude have been conceptualized and measured. The majority of researchers testing Karasek's demand-control model used Karasek's operationalization of job demands (e.g. Landsbergis, 1988; Schnall et al., 1990; Warr, 1990). However, other operationalizations of job demands have included workload (Fox et al., 1993) and interpersonal conflicts (Spector, 1987b). As well; the demands scale (de Jonge, and Landeweerd, 1993) contained both quantitative and qualitative aspects of demands (e.g. working under pressure of time, job complexity, working hard and strenuous work).

While many researchers (e.g. Alfredsson, Karasek and Theorell, 1982; Alterman et al., 1994; Landsbergis et al., 1994; Parkes, 1991; Parkes, Mendham and von Rabenau, 1994; Pieper, LaCroix and Karasek, 1989; Vermeulen and Mustard, 2000) have used Karasek's operationalization of decision latitude (i.e. control), other measures of decision latitude can be found in the organizational psychology literature including: self-determination (Kauppinen-Toropainen, 1981), constraints (Payne and Fletcher, 1983), discretion (Spector, 1987b), autonomy (de Jonge et al., 1999) and control (Dwyer and Ganster, 1991; Sauter et al., 1989). For example, an alternate operationalization of control is found in 
Dwyer and Ganster's (1991) 22-item control scale. Sample questions include how much control do you have over the variety of methods you use in completing your work? and how much can you control the number of times you are interrupted while you work? Other studies using Dwyer and Ganster's (1991) control scale include Fox, Dwyer and Ganster (1993) and Schaubroeck, Lam and Xie (2000). de Jonge et al. (1999) used yet another operationalization of control: the Maastricht Autonomy Questionniare (MAQ) (de Jonge, 1995) which consisted of ten items using a five point Likert scale. The MAQ asks workers to assess the level of freedom they have on a variety of tasks and the discretion they have to complete their work. Authors such as de Rijk et al. (1998) also used de Jonge's scales.

Several studies also differ with respect to what outcomes were measured (e.g. coronary heart disease versus blood pressure) as well as the characteristics of the sample group (single occupation versus multi-occupation studies, average age of the group). Despite these methodological challenges, the rest of this section will attempt to provide an overview of the empirical support for Karasek's model and address some of the empirical inconsistencies.

\subsubsection{Studies Testing Karasek's Strain Hypothesis}

This subsection will review the research testing Karasek's strain hypothesis. Physiological and psychological outcomes will each be reviewed in turn. 


\subsubsection{Physiological Outcomes}

Researchers have measured physiological outcomes such as heart disease and blood pressure when examining the impact of job strain. The physiological studies provide more convincing support for Karasek's strain hypothesis than psychological measures. In addition, the fact that a wide range of methodologies (e.g. cohort studies, case control studies and studies using disease symptom indicators) yield virtually identical results strengthens our confidence in the findings from this body of research.

A number of the studies in this area (e.g. Alfredsson and Theorell, 1983; Alfredsson, Karasek and Theorell, 1982; Alfredsson, Spetz and Theorell, 1985; Karasek et al., 1981; Karasek, Theorell, Schwartz, Schnall, Pieper and Michela, 1988; Theorell, Hamsten, deFaire, Orth-Gomer, and Perski, 1987) suggest that there is a strong link between working in high strain jobs (i.e. low decision latitude and high demand) and heart disease.

A number of key studies also support Karasek's strain hypothesis. For example, the Framingham Heart Study (La Croix, 1984) studied 328 women over a period of 10 years and found a much higher relative risk of coronary heart disease for women in high strain jobs.

Haan (1985) conducted a prospective study of 600 Finnish men and 292 Finnish women and found that high strain jobs were associated with higher risk of cardio-vascular disease and a higher rate of mortality. Alfredsson et al. (1982) examined data from 334 Swedish males with new cases of myocardial infarctions 
as well as 882 randomly selected males matched for age and also found an association between high strain jobs and heart attacks.

There have been other studies with larger samples that also support Karasek's strain hypothesis. Karasek and Gardell (1984) studied 2500 men and women who had experienced forced job changes. Those who moved to jobs with greater levels of decision latitude had lower prevalence of coronary heart disease. Karasek, Gardell and Lindell (1987) examined 2409 American male participants in the Health Examination Survey (HES) and 2432 American participants in the First National Health and Nutritional Examination Survey (NHANES I) and noted that high strain jobs were associated with an increased prevalence of heart attacks (i.e. myocardial infarctions) in both survey groups. Alfredsson et al. (1985) examined data from 958,096 employed Swedish men and women and concluded that high strain jobs were associated with higher rates of hospitalization for myocardial infarctions. In all the aforementioned studies, Karasek's operationalizations of demands and control (i.e. decision latitude) were used.

Schnall, Landsbergis, and Baker (1994) reviewed 36 empirical studies (published between 1981 and 1993) that tested Karasek's strain hypothesis. The majority of these studies found a significant positive relationship between high strain jobs and either cardiovascular disease (CVD) or cardiovascular disease risk factors such as hypertension. Reviews by Landsbergis et al. (1993) and Kristensen (1995) have come to similar conclusions about the association between high strain jobs and cardiovascular disease. 
While it is acknowledged that not all empirical studies have been supportive of Karasek's job strain model, there are often significant flaws in studies where no associations were found between job strain and a given indicator (e.g heart disease). One of the oft-cited studies in the literature purporting to refute Karasek's model is the Honolulu Heart Study (Reed et al., 1989). This 10 year longitudinal study of Japanese-American men in Honolulu concluded that no associations were found between job decision latitude, psychological work demands and cardiovascular heart disease. However, two concerns about this study suggest that their conclusions may be flawed. Karasek and Theorell (1990) note that the first concern is that the conventional high-strain occupations in manufacturing were almost absent in this population and that the high-strain population was quite small. Second, Karasek and Theorell (1990) note that a significant amount of the subjects' work experience took place back in the 1930s when Honolulu was pre-industrialized and therefore less likely to suffer from high strain occupations.

Other studies such as the General Electric study in the United States (Alterman et al., 1994) show only weak associations between high strain jobs and cardiovascular illness risk. For example, Alterman et al. (1994) reported results from the 25 year follow-up of 1683 men who participated in the Chicago Western Electric study from 1957 to 1973 . This study did show an increased risk of heart disease with reduced decision latitude, but no association between job demands (similar operationalization to Karasek) and heart disease. 
It is important, however, to note that age may play a factor in these results. Much like the Honolulu Heart Study, many participants were either relatively old already at the start of the follow-up (approaching retirement age) or retired before the end of the follow-up period. Theorell (1996) notes that participants younger than 55 years of age in general have shown a stronger association between high strain jobs (i.e. high job demands, low decision latitude) and coronary heart disease than older participants. This is most likely due to the healthy worker effect which suggests that those participants who suffer adverse health impacts from a given job often quit thus leaving behind those who have managed to adapt or effectively cope with the pressures of the job (Monk and Folkard, 1992).

Similar to the association between job strain and cardio-vascular heart disease, studies on blood pressure and job strain are mostly supportive of Karasek's job strain model. Pieper, La Croix and Karasek (1989) have summarized relationships for all the major risk factors by using five different major U.S. data bases (U.S. HES, U.S. HANES, U.S. HANES II, the Exercise Heart Study, and the Western Collaborative Group Study). In three of the databases, there was a significant association between high strain jobs (i.e. high job demands, low decision latitude) and systolic blood pressure. In all the analyses, adjustment was made for age, race, education, and body mass index. According to Theorell and Karasek (1996), the link between job strain and blood pressure is consistent across many studies despite differences such as gender and measurement technique (i.e. self-measured versus fully automated). 
For example, Theorell et al. (1988) conducted a longitudinal study on 58 men and women. On four separate occasions throughout the course of a year, the men and women measured their own blood pressure once every hour during four working days. There was an association between job strain and mean systolic blood pressure such that those in high strain jobs had higher blood pressure levels than the other types of occupations. Theorell et al. (1991) took fully automated blood pressure measurements on a group of men participating in a blood pressure screening study. High job strain was associated with higher diastolic blood pressure readings than the other strain categories. Theorell, Ahlberg-Hulten, Jodko, Sigala and de la Torre (1993) studied a group of health care personnel (all women) who took their own blood pressure once every hour over the course of their work day. High strain jobs were associated with higher levels of both diastolic and systolic blood pressure. Schnall et al.'s (1990) 87 subjects with hypertension were compared to 128 without hypertension. Selfreported job strain was significantly related to higher levels of hypertension.

A review by Van der Doef and Maes (1998) of 51 studies of Karasek's demand-control model and physical health outcomes concluded that the strain hypothesis (i.e. that there is an association between job demands and decision latitude such that those in low decision latitude, high demand jobs suffer the highest level of job strain) received support especially with respect to cardiovascular disease. Van der Doef and Maes (1998) concluded their study by noting that working in a high strain job appears to be associated with an elevated risk for cardiovascular disease. 


\subsubsection{Psychological Outcomes}

A review of the research on psychological outcomes and job strain using Karasek Demand-Control model can be found in Van Der Doef and Maes (1999). Overall there was good empirical support for Karasek's strain hypothesis and the association with negative psychological outcomes. Van Der Doef and Maes (1999) noted that 28 out of 41 studies (e.g. Braun and Hollander, 1987; Landsbergis, 1988; Wall, Jackson, Mullarkey and Parker, 1996) dealing with general psychological well-being and 18 out of 30 studies dealing with job satisfaction (e.g. Amick and Celentano, 1991; Arnetz, Andreasson, Stranberg, Eneroth, and Kallner, 1987; Cahill and Landsbergis, 1996; de Jonge and Landeweerd, 1993; Kauppinen-Toropainen, Kandolin and Mutanen, 1983; Landsbergis, 1988; Warr, 1990) were supportive of Karasek's strain hypothesis. For example, Braun and Hollander (1987) surveyed 795 German men and women and concluded that job strain was positively associated with psychological distress. Landsbergis (1988) surveyed 289 hospital and nursing home employees and found job strain was positively associated with both depression and job dissatisfaction. Wall, Jackson and Parker (1996) surveyed 1,451 manufacturing employees and found that job strain was positively associated with both depression and anxiety.

There is also some evidence to support the negative association between job satisfaction and job strain such that those in high strain jobs tend to report lower levels of job satisfaction. For example, Amick and Celentano (1991) surveyed 4,903 postal workers and found a negative association between job 
strain and job satisfaction. Another survey of postal workers found similar results. Cahill and Landsbergis (1996) surveyed 4,018 post office mail handlers and concluded that job strain was positively associated with both psychophysiological strain and job dissatisfaction. Arnetz et al. (1988) surveyed 36 surgeons and 30 general practitioners and found a negative association between job strain and work satisfaction. A study by de Jonge and Landeweerd (1993) of 1,226 health care personnel concluded that job strain was negatively associated with job satisfaction. Warr (1990) surveyed 839 men and 847 women in England and noted job strain was negatively associated with job satisfaction and positively associated with depression.

Other studies have found mixed support for Karasek's strain hypothesis. For example, Kushnir and Melamed (1991) surveyed 798 industrial workers in Israel and found an association between high strain jobs and irritability but not between high strain jobs and anxiety.

Other studies have not supported Karasek's model. For example, Carayon (1993) surveyed 122 office workers in a longitudinal survey and found no association between high strain jobs and higher levels of depression and anxiety. Parkes, Mendham and von Rabenau (1994) surveyed 145 employees in a psychiatric hospital and noted that high strain jobs were not associated with lower levels of job satisfaction. Noor (1995) surveyed 180 women (professionals and administrative assistants) in a longitudinal study and concluded that there was no support for Karasek's strain hypothesis. 
Van der Doef and Maes (1999) note that there are differences between those studies using psychological outcomes (e.g. depression) who are supportive of Karasek's strain hypothesis and those that are not. Studies supportive of Karasek tended to be larger in sample size, tended to include samples from the general population (therefore more generalizable) and tended to be crosssectional. Non-supportive studies tended to be smaller in sample size and longitudinal in design.

\subsubsection{Studies Testing Karasek's Buffer Hypothesis}

In reviewing the empirical literature involving Karasek's buffer hypothesis, and comparing it to what has been done on the strain hypothesis, two points are clear. First, there are fewer studies that tested the buffer hypothesis. Second, there is less support for the buffer hypothesis in the empirical literature. This section will review the empirical literature for both physiological and psychological outcomes.

\subsubsection{Physiological Studies}

It is acknowledged that there is limited empirical support for the buffering hypothesis when physiological outcomes are used. For example, Landsbergis (1988) surveyed 289 hospital and nursing home employees and concluded that the interaction between demands and control was statistically significant for heart disease symptoms. As well, a study by Fox, Dwyer and Ganster (1993) investigated levels of a range of physiological outcomes in a study of 136 nurses. In this study the interactive effects of subjective job demands and decision 
latitude predicted blood pressure. The authors concluded their study by suggesting that high levels of decision latitude can help to mitigate the impact of high demands in the workplace. A study by de Jonge and Landeweerd (1993) of 1,226 health care workers found a significant interaction of demand-control for health complaints.

The majority of the studies testing Karasek's buffering hypothesis using physiological outcomes do not support this hypothesis. For example, the General Electric study ( $n=1,683$ men) in the United States (Alterman et al., 1994) found no interaction between job demands and decision latitude for cardiovascular illness risk. Suadicani et al. (1993) surveyed 1,638 Danish workers and reported no evidence of an interaction between demand and control when the outcome measured was heart disease. Carayon (1993) in a longitudinal study of 122 office workers found no evidence to support the buffer hypothesis. Kushnir and Melamed (1991) surveyed 798 Israeli workers and concluded that the data supported the strain hypothesis, but not the buffering hypothesis. Similarly, work by Perrewe and Anthony (1990) ( $n=47$ blue collar workers), Sauter et al. (1989) ( $n=251$ office workers) and Spector (1987b) $(n=136$ female clerical workers) failed to find a significant interaction effect and concluded that the buffer hypothesis was not supported.

\subsubsection{Psychological Outcomes}

A review of the empirical literature dealing with psychological outcomes again finds limited support for Karasek's buffering hypothesis with only a few studies identifying significant interaction effects (i.e. Parkes, Mendham, and von 
Rabenau, 1994; Parkes and von Rabenau, 1993). Both supportive studies were the result of a survey of 145 employees in a psychiatric hospital and both studies concluded that decision latitude did buffer the relationship between demands and job satisfaction.

It should be noted that several studies found mixed support for the buffering hypothesis. While Landsbergis et al. (1992) did find that decision latitude moderated the demand-strain relationship for depression, no interactions were found for anxiety in their sample of 297 male employees. Marshall et al. (1991) surveyed 326 female social workers and did find support for Karasek's buffering hypothesis for psychological distress but not for well-being. Wall, Jackson, Mullarkey and Parker (1996) surveyed 1,451 manufacturing employees and did find an interaction effect between demands and depression, demands and anxiety and demands and job satisfaction but only for a more focused operationalization of control (i.e. the measure does not contain items on skill use and task variety as in Karasek's decision latitude instrument). This operationalization comes from Jackson et al.'s (1993) timing control and method control scales. A sample question is "to what extent can you decide how to go about getting your job done?" The authors did not find any support for Karasek's buffering hypothesis when they used Karasek's operationalization of control (i.e. decision latitude).

The majority of the empirical studies reviewed which tested Karasek's buffering hypothesis did not find significant interaction effects. The fact that these studies used a variety of outcomes increases our confidence that such an 
effect may not be prevalent. For example, Carayon (1993) failed to detect interaction effects on three outcomes (i.e. tension-anxiety, depression and daily life stress) for his sample of 122 office workers. Chay (1993) also did not find any support for Karasek's buffering hypothesis on outcomes such as well-being and job satisfaction for his sample of 117 small business owners and employees. Larger samples have also failed to find interaction effects. For example, Baker, Israel, and Schurman (1996) surveyed 1,000 employees working in manufacturing and found no support for Karasek's buffering hypothesis on depression. Cahill and Landsbergis (1996) surveyed 4,018 postal workers and did not find any interaction effects on the relationship between demands and either job dissatisfaction nor pyschophysiological strain. A survey of 1,226 health care personnel by de Jonge and Landeweerd (1993) failed to find an interaction on the relationship between demands and job satisfaction. Fletcher and Jones (1993) surveyed 985 female and 1289 male employees and found no support for Karasek's buffering hypothesis on four different outcomes including life satisfaction, anxiety, depression and job satisfaction. Other studies have also failed to find support for Karasek's buffering hypothesis including Bromet et al. (1988), Kawakami et al. (1992), Landsbergis (1988), Melamed, Kushnir and Meir (1991), Payne and Fletcher (1983), Spector (1987b) and Warr (1990).

This then leads the reader to question what differentiates the studies that are supportive of Karasek's strain and buffering hypotheses from those that are not supportive? Van Der Doef and Maes (1999) suggest three possible differences: which hypothesis is being tested (i.e. strain or buffering), whether the 
study is cross sectional or longitudinal in nature, and how job demands and decision latitude are conceptualized. Karasek's strain hypothesis has received more (but by no mean unanimous) empirical support than the buffer hypothesis. Cross-sectional studies in general are supportive of both Karasek's hypotheses (e.g. Braun and Hollander, 1987; Wall et al., 1996), while the limited number of longitudinal studies (e.g. Carayon, 1993; Kawakami et al., 1992; Muntaner et al., 1991) are not supportive of either hypothesis. Finally, studies that used a more focused and specific conceptualization and operationalization of both job demands (e.g. time pressure) and control (e.g. decision authority over pace of work) were more likely, on average, to support the buffering effect of decision latitude (e.g. Kushnir and Melamed, 1991; Kivimaki and Lindstrom, 1995; Wall et al., 1996).

In conclusion, the research shows a stronger level of support for Karasek's strain hypothesis than for Karasek's buffer hypothesis. In other words, the empirical research indicates that high strain jobs (i.e. low decision latitude, high job demands) are positively associated with a number of physiological (e.g. heart disease, blood pressure) and psychological (e.g. distress, depression) outcomes. Support for Karasek's buffering hypothesis is weaker with only a few studies supporting the notion that decision latitude can decrease the positive relationship between job demands and strain (e.g. Landsbergis, 1988; Parkes and von Rabenau, 1993). While a few studies offered mixed support for the buffering hypothesis (e.g. Landsbergis et al., 1992; Marshall et al., 1991), the 
majority of studies offered no support at all (e.g. Carayon, 1993; Cahill and Landsbergis, 1996).

\subsection{Empirical Evidence for Johnson's Model}

This section will examine the empirical evidence to support Johnson's strain and buffer hypotheses. We will first examine Karasek et al.'s (1982) efforts to test for social support. Second, Johnson's initial efforts to test both strain and buffering hypotheses will be presented followed by a summary of the efforts of others to test these hypotheses.

\subsubsection{Karasek's Efforts to Test Social Support}

Karasek, Triantis, and Chaudhry (1982) were the first researchers to take Karasek's demand-control model and extend it to measure the role of social support (predating Johnson's efforts). Karasek et al. (1982)'s sample was 1016 male workers. Data used for this study came from the U.S. national survey, the University of Michigan Quality of Employment study for 1972 (nationally stratified sample). Psychological strain measures included an eight-item scale measuring affective mood (here termed depression because of the question content), a twoitem scale measuring happiness with life in general (Karasek, 1979), and a tenitem scale measuring job-related depressed mood related to job satisfaction. The three scales are Institute for Social Research scales (Quinn and Shepard, 1974). Also included was a five-item scale measuring job dissatisfaction behaviour related to job attachment (Kalleberg, 1977), and a self-report scale of absenteeism. Finally, the survey also included four measures of supervisory support (instrumental, attentive socio-emotional, tolerant socio-emotional and 
demanding-authoritarian) and two measures of coworker support were identified (instrumental and socio-emotional).

Karasek et al. (1982) used multiple regression to test for the direct effect of social support (i.e. testing the strain hypothesis). Results indicated that social support (i.e. both coworker and supervisor support) was negatively associated with depression and absenteeism and positively associated with job and life satisfaction.

Karasek et al. (1982) used MANCOVA (rather than regression) to test for the buffering effects of social support. They did this by dividing their sample into three levels of support: high, medium and low. A buffering effect of both coworker and supervisor support was noted in just over half of the tests (18 out of 35). In other words, the relationship between job demands and job strain grew weaker with higher levels of coworker and supervisor support. Interestingly, the buffering effect was statistically significant more often when the dependent variables were depressed mood, life dissatisfaction and job dissatisfaction (17 out of 28 tests were significant) compared to the use of absenteeism as a dependent variable (only one in 7 tests were significant).

\subsubsection{How Johnson Tested His Model}

Development of Johnson's demand-control-support model and the subsequent testing of that model resulted from his doctoral dissertation (Johnson, 1986). For his doctoral dissertation, Johnson (1986) studied the interaction between job demands, work control and social support. The analysis 
was based upon 13,779 subjects ( $52 \%$ men and $48 \%$ women) responding to the annual Swedish Survey of Living Conditions. Johnson teamed up with Ellen Hall (project coordinator) and published their findings (Johnson and Hall, 1988). Johnson and Hall used Karasek's operationalization of job demands (Karasek, 1979) and a work control scale which the authors describe as a "linear composite which measured decision-making authority, task variety and personal freedom on the job" (Johnson and Hall, 1988, p. 1337). The authors noted that the operationalization of work control is similar to Karasek's measure of decision latitude. Respondents were asked to indicate for 11 items on a 3 point Likert scale $(0=$ never, $1=$ sometimes, and $2=$ often $)$ the level of control they felt they had at work. Sample questions included the amount of influence the respondent felt they had over the planning of work, how time was used at work and the freedom the respondents had to receive a phone call at work.

Two aspects of support were measured: the opportunity to interact with coworkers at work and outside of work (Johnson and Hall, 1988). The social support scale consisted of five dichotomous items. For example, respondents were asked whether they could talk to co-workers during their breaks and whether they had met with a co-worker outside of the workplace. It is interesting to note that unlike Karasek et al. (1982), Johnson does not operationalize social support to include supervisor support.

Johnson and Hall (1988) used the Cochrane Mantel-Haenszel Chi to test for associations between job demands, work control and social support. They calculated the ratio of the combined exposure to their risk factors (i.e. low work 
control, low social support) and divided by that effect if the risk factors were acting independently. Johnson and Hall (1988) found that each of the variables (i.e. high job demands, low work control and low social support) independently contributed to a higher risk of cardio-vascular disease and concluded that the data support the strain hypothesis. In other words, each of the following variables at a certain level (i.e. high job demands, low work control and low social support) independently contributed to a higher risk of cardio-vascular disease.

When the buffer hypothesis was tested, Johnson and Hall (1988) were surprised by their finding. Amongst subjects who worked in low social support occupations, it was workers with high levels of both job demands and work control that reported the highest level of cardio-vascular disease. For high social support occupations, the data revealed that the group with high job demands and low work control had the highest level of cardio-vascular disease. Table 1 summarizes Johnson and Hall (1988)'s findings.

Table 1 CVD Symptoms at Low and High Social Support (Johnson and Hall, 1988)

\begin{tabular}{|l|l|l|}
\hline Level of Strain & $\begin{array}{l}\text { Incidence of CVD } \\
\text { at low social } \\
\text { support }\end{array}$ & $\begin{array}{l}\text { Incidence of CVD } \\
\text { at high social } \\
\text { support }\end{array}$ \\
\hline $\begin{array}{l}\text { High Strain (high demands, low } \\
\text { decision latitude) }\end{array}$ & 2.17 & 1.82 \\
\hline $\begin{array}{l}\text { Active (high demands, high decision } \\
\text { latitude) }\end{array}$ & 2.55 & 1.58 \\
\hline $\begin{array}{l}\text { Low Strain (low demands, high } \\
\text { decision latitude) }\end{array}$ & 1.43 & 1.00 \\
\hline $\begin{array}{l}\text { Passive (low demands, low decision } \\
\text { latitude) }\end{array}$ & 1.95 & 1.44 \\
\hline
\end{tabular}


It is noteworthy that the highest incidence of cardiovascular disease occurred in the group that was low in social support yet high in job demands and high in work control. This, Johnson (1989) admits, was an unexpected and unpredicted finding. What this indicated to Johnson was that neither work control nor support by themselves adequately moderate the impact of job demands on strain. He went on to postulate that without support, work control is no longer an effective moderator on the demand-strain relationship (Johnson, 1989) and ultimately concluded that it is the combination of high social support and decision latitude together which reduce the impact of high job demands on cardiovascular risk.

\subsubsection{Studies Testing Johnson's Strain Hypothesis}

Other studies have built on Johnson's work. A few studies have supported Johnson's strain hypothesis (i.e. there is a negative association between social support and different outcomes such as CVD and psychological strain). Empirical evidence for Johnson's strain hypothesis using physiological outcomes are presented first. This is followed by evidence obtained using psychological outcomes.

\subsubsection{Physiological Outcomes}

Johnson, Hall and Theorell (1989) conducted a 9 year follow up study of 7,219 Swedish men and concluded that there was support for the strain hypothesis such that those in the high strain isolated category (i.e. high job demands, low decision latitude and low social support) suffered higher rates of 
both cardio-vascular mortality and morbidity than subjects in other categories. A study by de Jonge and Landeweerd (1993) of 1,226 health care workers found support for Johnson' s strain hypothesis associated with health complaints. Lerner et al. (1994) surveyed 1,319 men and women and noted that the high strain isolated group (i.e. low decision latitude, high job demands, low support) had the lowest quality of life scores. Two sample groups were studied by Parkes, Mendham and von Rabenau (1994). Study 1 focused on 145 health care workers and study 2 focused on 180 student teachers. In both studies, those in the high strain isolated group (i.e. low decision latitude, high job demands, low support) had the highest level of psychosomatic complaints.

Other studies have found mixed support for Johnson's strain hypothesis. Astrand, Hanson and Isacsson (1989) surveyed 391 male Swedish factory employees. While Astrand et al. (1989) did not find high strain isolated employees (i.e. high job demands, low decision latitude, low social support) had a statistically significant higher mortality rate than other employees, they did find that the combination of high decision latitude and high social support irrespective of demand level was associated with a lower mortality rate than other combinations.

Other studies have concluded that there is no empirical support for Johnson's strain hypothesis. For example, Theorell, Harms-Ringdahl, AhlbergHulten and Westin (1991) surveyed 136 men and 52 women in six occupations and found no support for Johnson's strain hypothesis such that no additive effects of demand, control or support were associated with back, neck and 
shoulder pain. Similarly, Amick and Celentano (1991) surveyed 4,903 postal workers and found no additive effects of demand, control and support on psychosomatic symptoms. Israel, House, Schurman, Heaney, and Mero (1989) surveyed 630 manufacturing employees and found no support for Johnson's strain hypothesis for global health symptoms. Van der Beek, Oort-Marburger and Frings-Dresen (1994) surveyed 439 truck drivers and found the strain hypothesis was not supported for either musculoskeletal complaints or general psychosomatic complaints.

\subsubsection{Psychological Outcomes}

Similar to the findings for physiological outcomes, the empirical evidence supporting Johnson's strain hypothesis and psychological outcomes is inconsistent. Overall, while there is very limited evidence to support this hypothesis, a study by Cahill and Landsbergis (1996) of 4,018 postal employees did report support for Johnson's strain hypothesis for both psychophysiological strain and job dissatisfaction.

Other studies report mixed results. Amick and Celentano (1991) surveyed 4,903 postal workers and concluded that Johnson's strain hypothesis was supported for job satisfaction when support was operationalized as supervisor support but not when support was operationalized as coworker support. Baker et al. (1996) surveyed 1,000 manufacturing employees and did not find support for Johnson's strain hypothesis when the outcome variable was depression but did find support when the outcome was negative job feelings. Fletcher and Jones (1993) in their study of 985 female and 1289 male employees presented a mixed 
set of results. For example, those employees in high strain isolated jobs (i.e. high job demands, low decision latitude, low social support) reported higher levels of anxiety and depression. However, the results were supportive of an association between high strain isolated jobs and both life satisfaction and job satisfaction but only for women. Melamed et al. (1991) in their survey of 267 female social workers found support for Johnson's strain hypothesis for burnout but not job satisfaction. Moyle (1995) in his study of 143 employees found support for Johnson's strain hypothesis for job satisfaction but not mental health.

Other studies have shown no support for Johnson's strain hypothesis. For example, Bourbonnais et al. (1996) surveyed 2,889 male and female white collar public sector employees and found no evidence to indicate that those in the high strain isolated category (i.e. high job demands, low decision latitude and low social support) reported higher levels of psychological distress. Bromet et al. (1988) surveyed 325 male power plant employees and found no evidence to support the strain hypothesis and affective disorders. Chay (1993) in their study of 117 small business owners and employees concluded that those in high strain isolated jobs were not more likely to report lower levels of well-being or job satisfaction. Clegg, Wall and Kemp (1987) surveyed 209 manufacturing employees and found no evidence that those in high strain isolated jobs (i.e. low decision latitude, high job demands, low social support) reported lower levels of mental health or job satisfaction. The survey by de Jonge and Landeweerd (1993) of 1,226 health care personnel did not find support for Johnson's strain hypothesis for the outcome of job satisfaction. Kawakami et al. (1992) in their 
study of 468 male Japanese blue collar employees found no evidence that employees in high strain jobs reported higher levels of depression. Lerner et al. (1994) in their survey of 1,319 male and female employees found no association between high strain jobs and quality of life. Other studies not showing support for Johnson's strain hypothesis include Muntaner et al. (1991) and Smulders and Nijhuis (1999).

There are two reasons for the difference in findings according to Van der Doef and Maes (1999). First, similar to the results for Karasek's demand-control model, there is a distinct difference between cross-sectional studies (which generally showed support for Johnson) and the longitudinal studies (which generally were not supportive). Second, studies where symptoms were selfreported had a much higher chance of generating supportive results in comparison to those studies where more objective measures were used (e.g. Muntaner et al., 1991).

\subsubsection{Studies Testing Johnson's Buffering Hypothesis}

Since there are very few studies testing Johnson's buffering hypothesis, for the sake of readability, empirical studies using physiological and psychological outcomes to operationalize job strain will be presented together in this section.

Most of the studies done on this topic do not support Johnson's buffering hypothesis (i.e. interaction effects are not significant). A study by de Jonge and Landeweerd (1993) of 1,226 health care workers, for example, found no support 
for Johnson buffering hypothesis on health complaints or job satisfaction. This finding was echoed by Lerner et al. (1994) in their study of 1,319 respondents. Lerner et al. (1994) found no interaction effects on the outcome of quality of life. Chay (1993) in their study of 117 small business owners and employees concluded that there were no interaction effects to support the buffering hypothesis for either well-being or job satisfaction. Lerner et al. (1994) in their survey of 1,319 male and female employees found no interaction effects for control and social support on the relationship between job demands and strain (operationalized as quality of life). Melamed et al. (1991) in their survey of 267 female social workers found no support for Johnson's buffer hypothesis for both burnout and job satisfaction. Smulders and Nijhuis (1999) surveyed 165 workers and found no evidence to support the notion that control and support moderate the relationship between job demands and strain (i.e. burnout).

That being said, there is limited evidence that does support the buffering hypothesis. Two sample groups were studied by Parkes, Mendham and von Rabenau (1994). Study 1 focused on 145 health care workers and study 2 focused on 180 student teachers. In both studies, interaction effects were detected indicating support for Johnson's buffering hypothesis for the outcome of psychosomatic complaints.

In conclusion, there is more evidence to suggest that those working high strain isolated jobs experience higher levels of negative physiological and psychological outcomes (i.e. strain hypothesis) than there is for the buffer hypothesis. While it is acknowledged that some evidence supports the strain 
hypothesis, this evidence is certainly not overwhelming. Given the various ways that researchers have measured demands, control, support and the outcomes, it is not surprising that evidence for the strain hypothesis is mixed. On the other hand, there is very little evidence to support the buffer hypothesis since the majority of studies failed to find interactions.

\subsection{Critique of the Model}

As with any model, it is inevitable that various perceived shortcomings emerge. The Karasek model is no exception. This section will summarize both the construct and methodological criticisms associated with this framework.

\subsubsection{Criticisms of the Demands-Control Literature}

A review of the literature uncovered several criticisms associated with Karasek's model and how the constructs have been operationalized and analyzed. Each criticism will be examined in turn.

\subsubsection{Simplicity of the Model}

A recurrent criticism of Karasek's demand-control model is its simplicity. This point has been raised and acknowledged in Karasek and Theorell's (1990) Healthy Work. Karasek and Theorell argue that the simplicity of the model can be regarded as a strength, not necessarily a weakness. Karasek and Theorell (1990) contend that adding a large number of variables to a given model is likely to weaken the associations for job demands or decision latitude in the model and could lead to what Karasek and Theorell term 'statistical indeterminacy' (Karasek and Theorell, 1990). 


\subsubsection{Operationalization of the Variables}

The operationalization of the variables in Karasek's model has also come under fire. The operationalization of both job demands and decision latitude have sparked debate. First, there has been a debate of how job demands are operationalized. An article by de Jonge suggests that Karasek's operationalization of job demands seems "to be a mixture of job stressors, job complexity, and lack of control" (de Jonge et al., 1999, p. 97). If correct, then this brings researchers back to the same issues that were problematic among the studies that predated Karasek's demand-control model. Karasek has positioned his model as a differentiation between job demands and decision latitude and therefore the model's validity is called into question if the two operationalizations are conceptually the same.

As well, critics have also raised some concerns about the decision latitude component. Several authors (de Jonge et al., 1999; Frese, 1989; Ganster, 1989, 1995; Kasl, 1996) have also noted that, similar to the job demands construct, the decision latitude operationalization is also a composite of quite disparate elements (e.g. skill level, skill variety, job scope and job complexity). According to these authors, skill level, skill variety, job scope and job complexity are all conceptually different from decision latitude. In fact, researchers have been more successful at finding support for both the strain and buffer hypotheses of Karasek's when they've used a more focused operationalization of control (e.g. Wall et al., 1995). 


\subsubsection{Lack of Consistency in Measuring Job Strain}

Another problem is the fact that there is no consistency in how job strain is measured. As mentioned previously, job strain has been measured in an indirect fashion by examining a variety of outcomes purported to result from job strain including physiological outcomes (e.g. heart disease) and psychological outcomes (e.g. anxiety). It becomes difficult to develop a consensus on how much empirical support exists for Karasek's model if there are so many different ways of measuring job strain. It is important to note that these criticisms do not undermine the model itself or how it has been tested.

\subsubsection{Variability in data collection}

The key observation in reviewing this literature is the various approaches to measuring job demands, decision latitude and outcomes make comparisons of the empirical studies difficult. Kristensen (1995) perhaps says it best when he notes:

"It makes little sense to compare these relative risks because of substantial differences between the studies with regard to population, assessment of job strain, control with confounders, definition of 'low strain' group, study design etc." (Kristensen, 1995, p. 19).

Much of the variability in methodology hinges on whether individuals have been asked to assess their own work situations with respect to decision latitude (i.e. self report) versus the use of expert ratings or methods which focus on the use of aggregated data based on representative workers in the occupations in the population (Theorell, 1996). 
The literature has extensively documented the advantages and disadvantages as they apply to self-reports of decision latitude as well as selfreports of various outcomes of job strain (Costa and McCrae, 1980; Karasek and Theorell, 1990; Kasl, 1998; Spector, 1992). Therefore, to avoid redundancy, we will discuss the issue of using self-reports in both contexts. It should be noted that any discussion surrounding the pros and cons of using self-reports does not only apply to Karasek's work but to any study using such an approach.

A great deal of the research testing Karasek's model (e.g. Melamed et al., 1991; Smulders and Nijhuis, 1999) has used self-report instruments to measure decision latitude and the symptoms of job strain. Respondents fill in a questionnaire asking a variety of questions about decision latitude (control) and its sub-components (task authority and skill discretion) and they are asked about the frequency and severity of a given outcome.

Advantages to using self-report questionnaires include the simplicity of administration (Karasek and Theorell, 1990), the relatively low expense, the convenience for both respondent and researcher (Kasl, 1998) and the ability to survey a large sample of respondents.

However, the literature has noted several key disadvantages of using selfreports including the possibility of negative affectivity (e.g. Costa and McCrae, 1980). Watson and Pennebaker define negative affectivity (NA) as "a broad range of aversive mood states, including anger, disgust, scorn, guilt, fearfulness, and depression" (Watson and Pennebaker, 1989, pp. 234-5). This personality trait could potentially skew the results of a given study. Studies (e.g. Costa and 
McCrae, 1980) suggest that those respondents displaying a high NA score are likely to report more stress, distress and physical discomfort over time and regardless of the situation, even in the absence of any overt or objective stressor. This ability to focus on the glass half empty is significant for self-reports of decision latitude because someone with high negative affectivity may underestimate the level of decision latitude they actually possess at work (Pennebaker and Watson, 1988).

Despite these concerns over NA, some literature downplays the influence of this trait. First, there are a wide variety of conceptualizations for NA including neuroticism, introversion, aversive mood (e.g. anger, guilt, and fearfulness), and low self-esteem (Watson and Pennebaker, 1989). Such a diversity of conceptualizations may make comparisons across studies difficult. Second, Vermeulen and Mustard (2000) note that controlling for negative affect decreases but does not eliminate the relationship between job strain and a given physiological (e.g. heart disease) or psychological (e.g. anxiety) outcome (Brief et al., 1988; Parkes, 1990). Third, several studies have investigated whether negative affectivity artificially raised the association between the stressor and the given health outcome. Chen and Spector (1991) and Decker and Borgen's (1993) results revealed that negative affectivity did not account for significant shared variance between stressors and outcomes such as anger and frustration.

Other commentators (e.g. Frese and Sabini, 1985; Frese and Zapf, 1988; Kasl, 1989, 1996; Spector, 1992; Zapf, 1989) have noted another problem with self-reporting is common method variance. Method variance is defined as, "the 
overlap in variance between two variables attributable to the type of measurement instrument used rather than due to a relationship between the underlying constructs" (Avolio et al., 1991, p. 572). Method variance could be problematic, therefore, because researchers could reach erroneous conclusions when testing Karasek's model. Researchers may conclude that the strain hypothesis is valid when in fact the variance explained was due to common method variance. However, researchers such as Evans (1985) argue that while it is true that common method variance might weaken a given interaction, it cannot create a spurious interaction (Evans, 1985).

Thomas and Ganster (1995) suggest it is important to recognize that an important part of studying decision latitude is to understand the perceptions of the respondents. In such a case, it makes sense to use self-reports. Moreover, given the sensitive nature of many surveys (e.g. opinions about supervisors, income, symptoms of depression), self-reporting can help preserve anonymity which may increase the participation rate and lessen the influence of social desirability (defined as the tendency for a respondent to choose the socially desirable response, regardless of the accuracy of their response) (Spector, 1987b).

Self-report tools could potentially become problematic because a group of respondents either under-emphasizes or over-emphasizes the influence of a given stressor depending on their familiarity with the issues of job strain (Zapf, 1989). For example, an assembly line worker is so used to his/her work situation that he/she denies some of the day-to-day hazards associated with the position 
which could lead to negative findings of job strain. On the other hand, health care professionals are more familiar with the issues around job strain which could lead to positive findings of job strain (de Jonge and Kompier, 1997).

There are two main alternatives to self-report when measuring job demands, decision latitude and the outcomes of job strain. Researchers can use either expert ratings or some type of aggregate method. Each option will be reviewed in turn with the advantages and disadvantages summarized.

Due to the difficulties associated with self-reporting, it has been suggested that expert ratings can be used instead. A subject matter expert examines the job description and observes the workplace and then scores the level of job demands and decision latitude for a given occupation. This method assumes that a neutral third party will be able to more accurately capture the true nature of the decision latitude than individuals who might be likely to exaggerate their levels of decision latitude.

However, expert observations are not without their share of problems. First, these ratings are time consuming and costly (Karasek, 1997). Second, the accuracy of the expert rating may depend on the skill level of the position under study. For instance, experts are more likely able to accurately assess a low skill job where the workers perform repetitive tasks versus knowledge worker jobs. (Semmer et al.,1996). Third, Karasek and Theorell (1990) also note that these observers may also be subject to biases such as halo effect (where an impression of a given trait or behaviour unduly influences the overall impression of the subject) and stereotyping. Fourth, even when experts spend considerable 
time in the workplace observing their subjects, they are likely to miss important events that might happen at certain times of the year (e.g. complete breakdown or machine failure; Semmer et al., 1996). Finally, an observer may mistake the behaviour of the subject for a feature of the job. For example, an observer may observe a high-strung employee acting in a frantic manner and conclude that the job is hectic (Semmer et al., 1996).

Due to the problems associated with self-report and expert techniques, some researchers (e.g. Muntaner et al., 1991) favour using an aggregated method. This aggregated method is purported to be more objective than selfreporting. With this technique, all workers with the same job title are given the average values of the responses given by workers of that particular job. This avoids an individual's subjective assessment of his/her position which can help avoid the problem of negative affectivity (Theorell, 1996).

The aggregate technique raises some serious concerns. Schnall, Landsbergis, and Baker (1994) note that due to the heterogeneity of skill levels, job demands and autonomy within a given profession (e.g. nurse, teacher) there can be large within-occupation differences in the scores. Aggregating these scores means masking the within occupation differences in scores which could underestimate true associations between decision latitude and the health outcome under study (Theorell, 1996; Kristensen, 1995). Furthermore, titles can be misleading. As Karasek (1989) notes, "even detailed systems like the US Census 3 digit occupational categories (with several hundred useful categories) 
will place all bakers into the same category whether they work in a luxury pastry shop or tend a machine at a bread-baking factory" (Karasek, 1989, p. 144).

Given the criticisms associated with self-report of physiological and psychological outcomes, commentators such as Zapf (1989) have suggested that the best way to further our understanding of the impact of decision latitude on job strain is to pursue more objective methods of collecting the data. Objective methods assess the outcome under study independently of the job incumbent (cf. Frese and Zapf, 1988, 1994; Spector, 1992). For example, measurements of a specific physiological function can be taken several times a day (e.g. blood pressure). Supporters of this approach suggest that these objective methods are more accurate and are not subject to various perceptual biases of the subjects. However, it should be pointed out that these objective methods may also be problematic.

Arguments against objective measures can be made as follows. First, Cohen et al. (1995) argue that the perception of the environmental situation of a given subject is directly relevant to their perceived stress. Therefore, you need to capture this perception through a tool such as self-report. Second, there are many pragmatic reasons for rejecting a more objective approach including the difficulty of obtaining results, the expense, the time involved and the inability to conduct objective measures on a large sample (Kasl, 1998).

\subsubsection{Role of Socio-Economic Status}

A recurring theme in the literature is the potential for socio-economic status (SES) to confound the job strain findings in Karasek's demand-control 
model. Socio-economic status can confound the findings in two ways. First, there is a great deal of research that suggests a strong positive association between lower SES and the outcomes used to quantify job strain such as coronary heart disease (Albright, Winkleby, Ragland, Fisher and Symer, 1992; Johnson and Hall, 1988; Marmot and Theorell, 1991; Popock et al., 1987). Therefore, an argument could be made that it is not the fact that those with lower SES are in low decision latitude-high demand jobs that is responsible for the higher incidents of cardiovascular disease but other nonwork-related factors such as diet and lifestyle. However, Theorell (1996) argues that work-related factors do have an impact on cardiovascular disease and the research suggests that lack of decision latitude and lack of social support are all indeed more common in those of lower SES. Theorell does not deny the influence of nonwork factors on cardiovascular disease (e.g. heredity and lifestyle) but instead argues that the influence of the workplace must also be considered.

Second, it can be argued that the low levels of decision latitude and high levels of job demands in Karasek's demand-control model are not measures of job strain per se but instead measures of socio-economic status. Karasek and Theorell refute this claim by arguing that his job strain construct is "almost orthogonal to most social class measures" (Karasek and Theorell, 1990, p. 50) concluding that social class confounding does not appear to be responsible for his research findings. In fact, blue collar jobs such as repairman, waitress, janitor can be found in three of the four quadrants (i.e. low strain, high strain and passive) of Karasek's job strain model. 
It is suggested that SES does not account for all the variance explained by the demand-control model. It is important to recognize (as do both de Jonge et al., 1999; Karasek and Theorell, 1990) that employees with lower SES are more likely to hold jobs high in demands yet low in decision latitude and are more likely to contract heart disease due to the previously mentioned risk factors. Therefore, controlling for SES allows researchers to see the strength of the relationship between high job demands and low decision latitude when the effect due to SES is factored out.

A recognition of the potentially confounding effect of SES meant that many studies involving job strain and coronary heart disease (CHD) control for SES either via education level (Alfredsson et al., 1982; Astrand et al., 1989; Falk et al., 1992; Karasek et al., 1981; Karasek et al., 1988; Theorell et al., 1987a; Theorell et al., 1991) or via occupational status (Astrand et al., 1989; Johnson and Hall, 1988; La Croix, 1984; Reed et al., 1989). Similarly, some blood pressure studies involving job strain controlled for education (e.g. Theorell, 1993) or occupational status (Harenstam et al., 1988; Light et al., 1992; Theorell et al., 1988). This thesis controls for the potentially confounding impact of socio-economic status.

\subsubsection{Healthy Worker Bias}

Researchers in the area of workplace stress must be aware of biases that can negatively affect their results. One of these biases is the ability for subjects to self-select out of a given occupation (i.e. the healthy worker effect). This scenario discussed in Karasek and Theorell (1990) could result in subjects electing to leave a high strain job. It is suggested that this could lead to the 
"underestimation of the true risk associated with job strain" (Theorell and Karasek, 1996, p. 17). According to Schnall, Landsbergis, and Baker (1994), studies suggest that selection out of high strain jobs does occur with advancing age. This lends support to the possibility of this bias occurring. For example, US data show relatively fewer occupants of high strain jobs among older age groups (Karasek et al., 1988).

Finally, it is interesting to note that the operationalization of demands, control, and strain in Karasek and Johnson's models are dated and do not reflect the realities of today's workforce. This thesis addresses the issue by updating the constructs so they are meaningful to today's employees. 


\section{Theoretical Framework}

This section provides the theoretical framework for this thesis. First, the similarities and differences between Karasek's model and the thesis models are presented. Second, the thesis models are presented. Details on each construct and the hypothesized relationships between the constructs in the models are provided in more detail in subsequent chapters.

The research model developed for this thesis uses Karasek's DemandControl Model as its theoretical underpinning. The research model has a number of features in common with Karasek's model: the basic constructs (i.e. demands, strain and control) included in the models are identical as are the two hypotheses (strain versus buffer hypotheses). However, the conceptualization and operationalization of these constructs in this research are quite different from those used by Karasek. Key differences are summarized in Table 2 below.

Table 2 Construct Comparison (Karasek/Thesis)

\begin{tabular}{|l|l|l|}
\hline Construct & Karasek & Thesis \\
\hline Demands & Work & Work and Non-Work \\
\hline Strain & Job Strain & $\begin{array}{l}\text { Work-Life conflict (role } \\
\text { overload, work-family } \\
\text { interference, family-work } \\
\text { interference) }\end{array}$ \\
\hline Control & $\begin{array}{l}\text { Decision Latitude (task } \\
\text { authority and skill } \\
\text { discretion) }\end{array}$ & $\begin{array}{l}\text { Control over work-life } \\
\text { interface (flextime, } \\
\text { compressed work week, } \\
\text { telework, shiftwork, } \\
\text { perceived flexibility and } \\
\text { supervisor support) }\end{array}$ \\
\hline Control of confounds & None & $\begin{array}{l}\text { Gender and job type } \\
\text { (surrogate measure of } \\
\text { SES) }\end{array}$ \\
\hline
\end{tabular}


Karasek's view of demands (1985) focused on workplace demands on the employee (both psychologically and physically). This conceptualization, by necessity limits the scope of study to what happens to the employee at work. Recent trends in North American society such as the increased labour force participation of women suggest that focusing only on the workplace may yield an incomplete picture. Many North Americans are doing double and triple duty as fathers, mothers, spouses, and volunteers in addition to performing paid employment and unpaid overtime. Therefore, it makes sense to examine both work and non-work demands of people rather than just demands at work (Bianchi and Raley, 2005; Frankenhaeuser, 1997; MacDermid and Harvey, 2006). The constructs used in this thesis measure both work and non-work demands. A detailed discussion on work and non-work demands can be found in section 4 of this thesis.

Karasek's notion of strain (1979) centres on job strain and his strain and buffer hypotheses focus on job-related constructs only. When Karasek's model was developed in the 1970s, most employees were men living in a traditional family unit where there was a clear division of labour associated with gender (Kanter, 1977). In other words, the men worked outside the home in paid employment and the women were responsible for most of the household and child rearing duties. This does not describe the reality of most employed Canadians today. Dual-income families are the norm today (seventy-five percent of couples with children work outside the home in paid employment; Vanier Institute of the Family, 2005). Furthermore, men are spending more time doing 
housework and helping raise their children (Bianchi and Raley, 2005; Bond et al., 1998) compared to their male counterparts in 1970s. Dual-income couples are now concerned with work-life conflict. Karasek's model needs to be updated to reflect these changes.

A conceptualization of strain that includes both work and nonwork strains should yield a more complete picture of the challenges faced by today's employee. Therefore, strain for the purposes of this research model is conceptualized as work-life conflict and operationalized to include role overload, work-family interference and family-work interference. Details on these constructs are given in section 5 .

Karasek defined his control construct (i.e. decision latitude) as "the working individual's potential control over his tasks and his conduct during the working day" (Karasek, 1979, p. 289). Karasek conceptualized his control construct to be comprised of two parts: task authority and skill discretion. Task authority was defined as the ability of the worker to influence decisions regarding his or her job (Theorell, 1989). The second component, skill discretion, focuses on the employee using his/her full set of skills and having the opportunity to learn new skills (Theorell, 1989).

As noted previously in Section 2 of this thesis, Karasek's conceptualization of control (i.e. decision latitude) has resulted in some construct and methodological concerns. A number of authors (e.g. de Jonge et al., 1999; Ganster, 1995; Kasl, 1996) have concluded that Karasek's conceptualization has confused control (i.e. decision latitude) with other important job characteristics 
that do not equate to control. This conclusion is supported by the fact that studies (e.g. Wall et al.; 1996) that measured control specifically observed the hypothesized interaction between demand and control. Studies using Karasek's broader construct of control (which included variables like task variety), on the other hand, found no such interaction.

The research model for this thesis has attempted to develop new constructs of control that have relevance for today's workforce but overcome the noted criticisms. Given the positive association between work demands and work-life conflict as well as non-work demands and work-life conflict (see sections 5.3 and 5.4 respectively), what type of control could help employees cope with work-life conflict? It is suggested that enhancing an employee's control over their work-life interface could help to reduce levels of work-life conflict. Alternative work arrangements (e.g. flextime, compressed work week and telecommuting), perceived flexibility and supervisor support are all potential sources of control to assist the employee in controlling his/her work-life interface. A detailed review of the literature on the association between work-life conflict and alternative work arrangements, perceived flexibility and supervisor support can be found in sections $6.1,6.2$ and 6.3 respectively.

Gender and socio-economic status (SES) may confound any study of the relationship between demands and strain because of their association with the key constructs of the model. Please note that more details on controlling for covariates are provided in section 7. In Karasek's model, there was no discussion of confounding variables and therefore no effort to control for these 
covariates. It is important to control for gender because there is strong evidence in the literature to suggest that men and women have different sets of work and non-work demands. Women tend to put in more hours in the non-work domain (Anthony, 2002; MacBride-King and Bachmann, 1999) while men tend to spend more time in paid employment (Fast and Frederick, 2004; Shields, 1999). There are differences too in the nature of the non-work responsibilities performed with respect to timing and control. This thesis also controls for socio-economic status by controlling job type. Using job type as a proxy for SES is a common approach in empirical studies (e.g. Fenwick and Tausig, 2001; Hill et al., 2001). As seen in section 7, managers and professionals differ from non-professionals with respect to demands and work-life conflict therefore necessitating controlling for job type.

The preceding section used Karasek's model to theoretically justify the model to be tested in this thesis. More details on the four specific models to be tested in this thesis are presented below.

\subsection{Thesis Models 1-3}

This section presents models 1-3 and provides a brief description in order to provide a framework for the reader. Once the thesis models are presented, the thesis will then turn to the relevant literature on each subject area. Thesis Models 1-3 take the basic framework of Karasek but substitute Karasek's operationalization of demands, strain and control with conceptualizations presented earlier. Consistent with the approach taken by Karasek, models 1-3 will test both strain and buffer hypotheses. These models are identical theoretically and vary only with respect to the type of work-life conflict considered 
as the outcome variable. In other words, they differ only with respect to how they operationalize strain. A different form of strain is tested in each model (i.e. model 1 tests role overload, model 2 tests work-family interference and model 3 tests family-work interference). For example, in model 1 , the strain hypotheses are tested as follows. First, it is posited that both work demands and non-work demands are positively associated with role overload (model 1 ). Second, it is hypothesized that the different forms of control included in this thesis (i.e. flextime, compressed work week, telework, perceived flexibility and supervisor support) are negatively associated with role overload (model 1). The relationship between shiftwork and role overload is also tested but in this case, it is hypothesized that shiftwork will be positively associated with role overload.

Third, the buffer hypotheses are tested. The buffer hypothesis posits that each form of control included in this thesis will moderate the relationships between work demands and role overload as well as the relationship between non-work demands and role overload. Specifically, the buffer hypothesis suggests that flextime, compressed work week, telework, perceived flexibility and supervisor support will weaken the relationship between demands (i.e. work and non-work demands) and role overload and that shiftwork will strengthen the relationship between demands and role overload.

Models 2 and 3 then follow the same pattern of testing for work-family interference and family-work interference. Please note that gender and socioeconomic status (job type) are controlled in Models 1-3. Models 1-3 are summarized in Figure 3. 
Figure 3 General Framework: Mapping Thesis Models 1-3 to Karasek

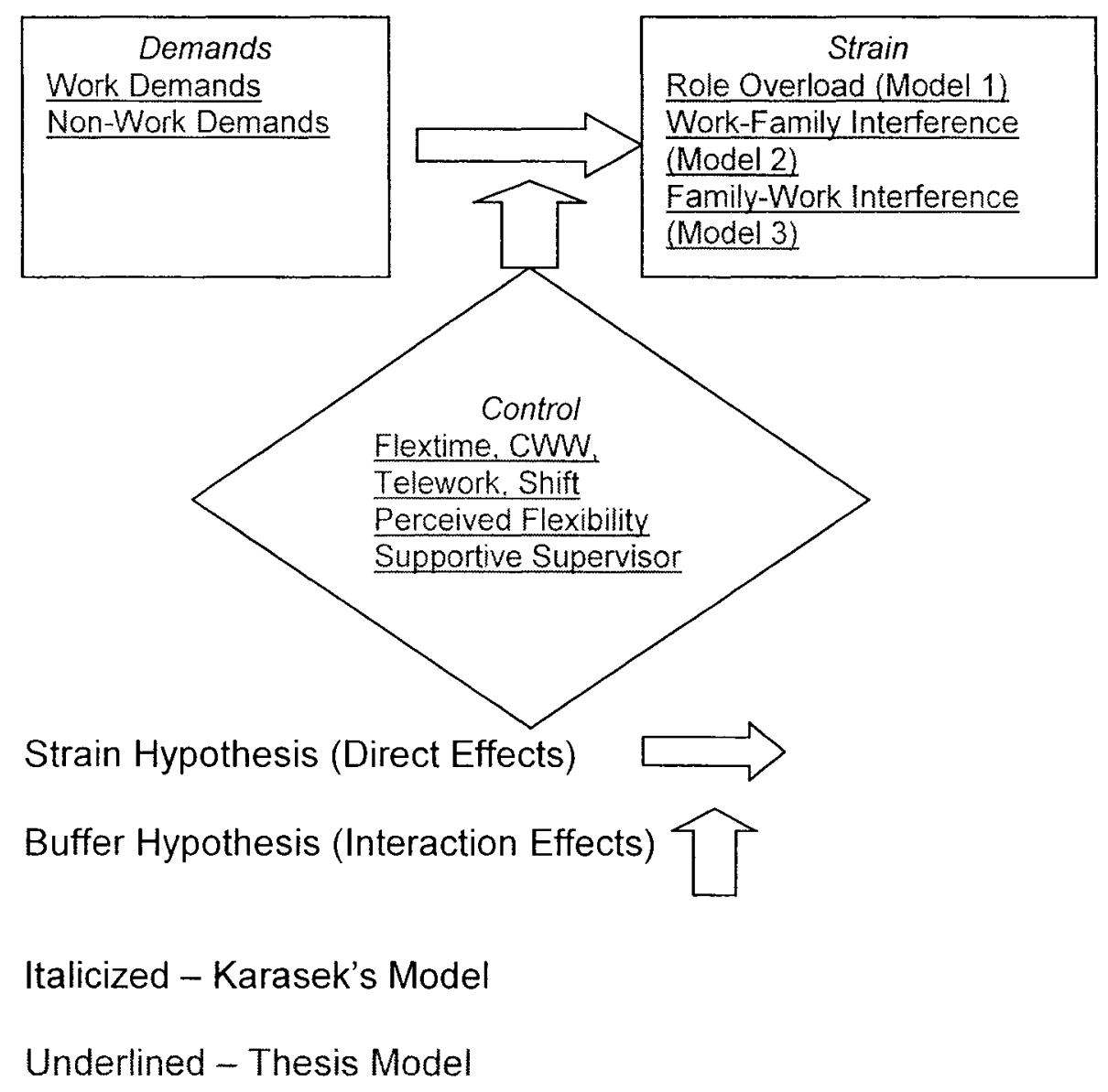

The literature supporting these associations can be found as follows:

demands (section 4), strain (i.e. work-life conflict) (section 5), control (section 6), and confounds (section 7). The specific hypotheses to be tested can be found in section 8.

\subsection{Thesis Model 4}

The fourth thesis model seeks to understand the relationship between the six control variables specified in thesis models 1-3. In this model (which is shown in Figure 4), alternative work arrangements are hypothesized to have a direct effect on perceived flexibility. Specifically, it is posited in this thesis that 
there is a positive association between perceived flexibility and each of the following three alternative work arrangements: flextime, compressed work week and telecommuting. Conversely, it is hypothesized that there is a negative association between perceived flexibility and shiftwork. Finally, we hypothesize that supervisor support can have two relationships with perceived flexibility: (1) a direct effect (i.e. the higher the supervisor support, the higher the perceived flexibility) or (2) an interaction effect (i.e. supervisor support can moderate the relationship between the work arrangement and perceived flexibility). Thesis model 4 is diagrammed below.

Figure 4 General Framework: Mapping Thesis Model 4

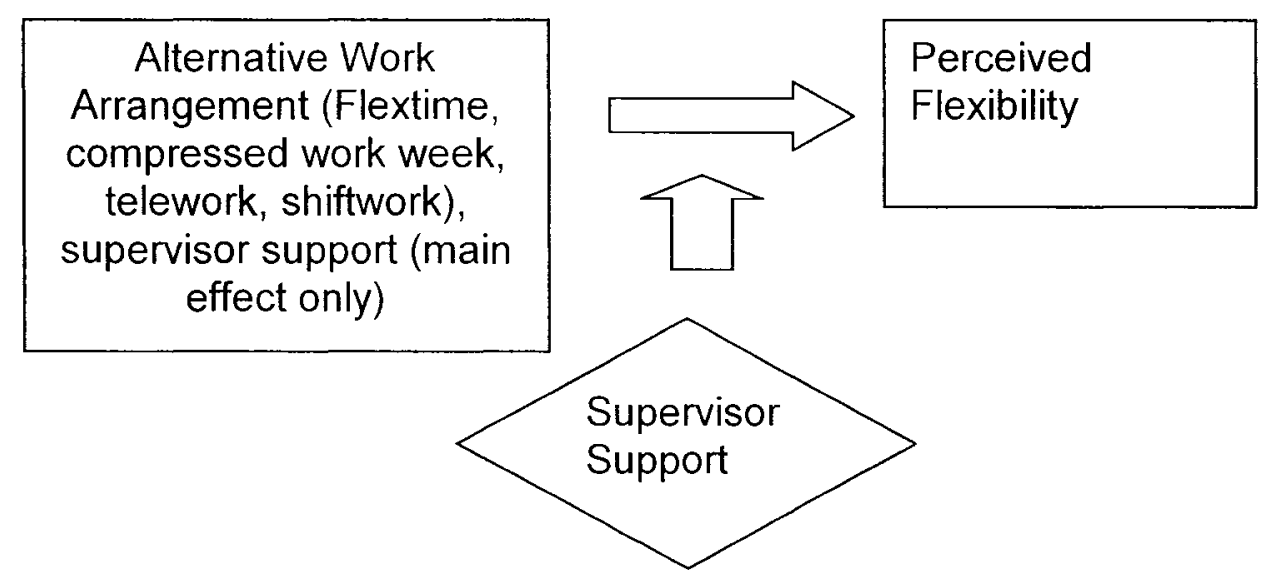

Please note that, consistent with the approach used for Thesis Model 1-3, gender and socio-economic status (job type) were controlled in all analyses testing model 4. As well, work demands and non-work demands were also controlled in these analyses in model 4 . The literature supporting these associations can be found as follows in sections 6 (control) and 7 (confounds). The specific hypotheses to be tested can be found in section 8 . 


\section{Work and Non-Work Demands}

"By working faithfully eight hours a day, you may eventually get to be boss and work twelve hours a day" Robert Frost (1875-1963), US poet (attrib). Cited in White et al., 2003, p. 175.

This section reviews the literature that justifies the conceptualization of demands in the thesis models 1-3 as time in work and non-work activities.

The definition of work and non-work demands used in this thesis is provided followed by a discussion of the ever-increasing demands employees face at work and at home. The literature justifying the association between excessive demands and strain is then reviewed.

\subsection{Definitions of Work and Non-work demands}

Karasek's notion of demands centred on work demands only. Given the fact that most Canadians fulfil many different roles (which may include employee, spouse, parent, caregiver, friend, volunteer), it can be argued that restricting the discussion of demands to include only work demands results in a limited, incomplete notion of demands. It is argued in this thesis that a more complete conceptualisation of demands would include both work and non-work demands as such a conceptualisation would yield a more complete picture of the day-today pressures faced by Canadians.

It is also important to contrast this thesis' operationalization of demands to Karasek's. Karasek (1979) operationalized demands as psychological demands that assessed how hard the respondent works. This thesis uses a different operationalization for demands where work and non-work demands are 
quantified by the number of hours a respondent spends on that activity in a given week. Work demands are defined as the time spent (i.e. number of hours per week) by employees on job-related duties (Duxbury and Higgins, 2002b). Such demands include hours spent at work, commuting to and from work; supplementary work at home, and overtime. Non-work demands are defined as the time spent by employees on non-work related activities (Duxbury and Higgins, 2002b). Non-work demands include household responsibilities, elder/care childcare, and volunteering.

The decision to use a measure of demands which quantifies the number of hours spent by the respondent in a given week is further explained in section

\subsection{1.}

\subsection{Rising Levels of Work and Non-work Demands}

Both men and women are contending with ever increasing demands in their work and personal lives. Employees in the workplace today are working longer hours than their counterparts from twenty years ago (Bond et al., 2003). Not only are employees spending more time at work, an increasing number of employees are doing supplementary work at home after their regular workday is done. A number of pieces of data can be used to support the contention that Canadians and Americans are working more hours.

In Canada, the number of Canadians working 50 or more hours per week rose from 11.3 percent in 1976 to 26 percent in 2001 (Duxbury and Higgins, 2002b). A report from Statistics Canada noted that $8 \%$ of all Canadian employees and $11.4 \%$ of Canadian male employees worked 2,400 or more hours 
in 2001 (Heisz and LaRochelle-Cote, 2006). This translates into an average of 46 hours per week. At the same time, those working between 35 and 39 hours per week dropped from $48 \%$ to $27 \%$ in the past decade (Duxbury and Higgins, 2002b). Statistics Canada also has noted a drop in those working a standard work week (working between 35 and 40 hours per week) from $47.4 \%$ in 1978 to $34.1 \%$ in 2000 (Heisz and LaRochelle-Cote, 2006).

Furthermore, the average amount of time employees spend in workrelated activities has risen from 42 hours per week to 45 hours per week in the past decade (Duxbury and Higgins, 2001). This upward trend is also reflected in a report from the OECD that states that in the period from 1970 to 2002, per capita hours worked by Canadians rose by more than 15\% (OECD, 2004).

The same trend can be seen in the United States. Bailyn et al. laments that Americans "now work the longest hours in the industrialized world" (Bailyn, Drago, and Kochan, 2001, p. 6). Figures from the American Time Use Survey report that Americans working full-time report working 9.2 hours on a typical workday (BLS, 2005a). Figures from the National Study of the Changing Workforce showed an increase in the combined hours of work (paid and unpaid) of dual-income couples climbing from 70 hours a week in 1977 to 82 hours a week in 2002 (Bond et al., 2003). Two other reports echo this finding. Jacobs and Gerson (2004) using data from the U.S. Current Population Survey $(n=50,000)$ note that the proportion of dual-income couples working very long work weeks (defined as couples working a total of 100 hours or more per week) climbed from $3.1 \%$ in 1970 to $9.3 \%$ in 2000 . The OECD report indicates the work 
hours per capita in the United States has climbed $20 \%$ in the period between 1970 and 2002 (OECD, 2004). Bianchi and Raley (2005) report that American women are working an average of 344 hours more per year in 2000 than their counterparts in the 1960s.

A significant contributing factor to the increasing numbers of hours spent working is the amount of overtime workers put in (often without pay). Using data from Statistics Canada, White (2002) noted that one out of every five (i.e. 2.3 million) Canadian workers works overtime in a given week. The average amount of overtime worked is 9 hours per week resulting in approximately 20 million hours of overtime per week (White, 2002). White (2002) goes further to mention that over half $(10,731,800)$ of those overtime hours per week are unpaid.

Results from two separate reports indicate a similar situation for our American counterparts. First, De Frank and Ivancevich (1998) report that census data indicate 60 percent of U.S. workers bring work home at night (De Frank and Ivancevich, 1998). Second, the Bureau of Labor Statistics (BLS) found that in September 2005 the average weekly overtime for employees working in manufacturing was 4.4 hours per week (BLS, 2005b).

Canadian workers are not only spending more time at work, they are more likely than ever to bring work home with them. Data from the Workplace and Employee Survey by Statistics Canada (2004) shows $14.1 \%$ of respondents performed unpaid work at home in addition to their normal work hours.

In the United States, the trend is similar. Bond et al. (1998) report that almost one-third of employees bring work home once or more often per week 
and that men and women are equally likely to bring work home. This figure represents a ten percent increase over the past twenty years.

What are the implications of all this extra work for North Americans? According to De Frank and Ivancevich (1998), it means less time to spend in other domains such as with family and friends. The reality according to De Frank and Ivancevich (1998) is that today's worker must work more hours as a result of mandatory overtime or feel compelled to work more in order to be seen as committed and to keep their jobs.

It is also important to review the trends for non-work demands. Two questions will be answered: How do the number of hours spent on work-related activities compare to the hours spent on non-work-related activities? How has the time spent in non-work activities changed over the past decade? Work by Duxbury and Higgins $(2001,2002 b)$ can be used to answer these questions. Their data indicates that Canadians are spending a significantly higher amount of time in work activities compared to non-work activities. Respondents to their survey spent an average of 17.1 hours per week in non-work-related activities (Duxbury and Higgins, 2002b). This figure is substantially lower than the average time spent in work-related activities ( 45 hours per week; reported in Duxbury and Higgins, 2001).

Duxbury and Higgins (2002b) reported that, relative to a decade earlier, respondents spent less time on non-work activities such as child care $^{2}$, home

2 Duxbury and Higgins (2002b) note that the 1991 and 2001 data are not completely comparable due to the differences in how respondents were asked to report on the amount of time spent on child care-related activities. In order to make comparisons between the 1991 and 2001 data, the authors extrapolated weekly data from the 1991 data. 
chores and leisure. Duxbury and Higgins (2002b) report a comparison of the data from 1991 and 2001 and show that both men and women reported a decrease in time spent in home chores (20\%) and time spent in leisure activities (40\%). While men reported spending $15 \%$ less time in child care, women reported a drop of $33 \%$. Given the finite nature of time, such results are hardly surprising given the concomitant increase in the number of hours the average Canadian is spending in work-related activities (Duxbury and Higgins, 2002b).

A review of the American literature on non-work-related activities indicate some patterns are similar to the Canadian experience while other patterns differ. For example, the NCSW (Bond et al., 2003) noted that both men and women reported less leisure time than respondents from twenty-five years ago (which is consistent with the Canadian data). Men in 1977 reported having 2.1 hours of leisure time per workday (compared to 1.3 hours in 2002) while women in 1977 reported having 1.6 hours of leisure time per workday (compared to just 0.9 hours in 2002) (Bond et al., 2003).

However, American men reported spending more time in child care and household activities (Bianchi and Raley, 2005; Bond et al., 1998) whereas the Canadian data shows a decrease in time spent in these domains. American women also reported that they spent more time on child care activities but less time on chores than they did twenty years ago (Bianchi and Raley, 2005; Bond et al., 1998). This differs from the Canadian experience where women are spending less time in both child care and household activities. 
In conclusion, North Americans are putting in more hours to do their job. As a result, the literature (e.g. Bond et al., 1998; Duxbury and Higgins, 2001) indicates that people are feeling overworked and overwhelmed. At the same time, when respondents have less time for themselves it means not having the time to re-energize and recuperate from these increased demands (Anderson et al., 2002; De Frank and Ivancevich, 1998; Frone, 2000; Voydanoff, 2004). What is the consequence of the increasing level of work demands (including extra hours working at home and paid/unpaid overtime)? The next section makes the case that this increase in demands can be associated with greater levels of strain.

\subsection{Relationship between demands and strain}

There is extant support in the literature for the notion that employees who consistently work long hours are more likely to suffer from a host of physical and psychological strains (Sparks et al., 1997). Working long hours has been linked to negative outcomes such as: heart disease (e.g. Uehata, 1991), physical health problems (e.g. Austin Knight, 1995), stress (e.g. Hall and Savery, 1986), fatigue (e.g. Shimonitsu and Levi, 1992), and depression (e.g. Shields, 1999). While these outcomes are not the primary focus of this thesis, establishing this link helps to position the association between demands and strain.

Once this association has been established, we then make the case in section 5 that work-life conflict can be considered a form of strain. As well in section 5, we show how increased demands have been found to be associated with increased levels of work-life conflict. 
Researchers have found evidence to indicate that working long hours is associated with heart disease and other physical health problems.

One of the earliest studies linking longer work hours with physical problems (Buell and Breslow, 1960) found a significant relationship between the number of hours worked and coronary heart disease (CHD) deaths. Using American census data (sample of 22,176 ), workers who worked more than 48 hours a week were twice as likely to die from coronary heart disease than those working a standard 40 hour work week. Two additional studies have detailed an association between working long hours and coronary heart disease but only for women (Alfredsson, Spetz and Theorell, 1985; Starrin, Larsson, Brenner, Levi, and Petterson, 1990). As noted in Sparks et al. (1997), however, both studies used small samples.

Uehata (1991) interviewed 203 Japanese families who had recently lost a loved one due to a heart attack. Uehata (1991) discovered that two-thirds of the people who died were working 60 or more hours a week and working at least 50 hours of overtime per month before the attack. Uehata (1991) argues that working excessive hours was a leading factor in promoting the attacks. Uehata (1991) offers a feasible explanation for how working excessive hours can lead to higher risk of heart disease. He notes that working long hours can result in poor lifestyle choices such as smoking, abusing alcohol, not making physical exercise a priority, and making poor nutritional choices. Over a period of years, these lifestyle choices take a toll on the human body as people gain weight and 
possibly become overweight or obese. This, in turn, substantially increases the risk of cardiovascular disease.

Austin Knight (1995) surveying employers who represent one million white-collar workers in Britain found over half the respondents reported physical health problems due to working long hours. Other researchers have found a link between working excessive overtime and deleterious physical health outcomes. For example, Hayashi et al. (1996) studied 46 male white collar Japanese workers and found that the 24-hour average blood pressure of the overtime groups (working an average of 60 hours a month of overtime) was higher than the control groups. Nakamura et al. (1998) found an association between working overtime and both obesity (using body mass index) ${ }^{3}$ and waist circumference in 248 white collar Japanese workers. Shields (1999) found that Canadian men (sample of 2,151 respondents) who worked long hours in 1994/95 had increased odds (1.4) of having excess body weight.

Researchers have also found a link between long working hours and a host of psychological outcomes including stress, distress, fatigue, exhaustion and anxiety. Several researchers have found an association between working long hours and higher levels of stress (Hall and Savery, 1986; Moss, Reid, Jackson, Lam, and Morris, 1996; Savery, 1986; Sease and Scales, 1998). Several of the studies involved large samples (e.g. Hall and Savery, 1986 studied 532 managers in Western Australia). Not only has there been a link between

3 A mathematical formula to assess relative body weight. The measure correlates highly with body fat. Calculated as weight in kilograms divided by the square of the height in meters (American Heart Association, 2005). 
excessive work hours and general levels of stress but also other psychological symptoms such as fatigue, exhaustion, and distress.

In Japan, employees work much longer hours (i.e. 200-500 hours more) than their Western counterparts (Sparks et al., 1997). Shields (1999) notes that the Japanese have named such deaths Karoshi, meaning 'death from overwork'.

Shimonitsu and Levi (1992) have found two-thirds of Japanese employees reported fatigue as a result of working long hours. Ono, Watanabe, Kaneko, Matsumoto, and Miyako (1991) in a survey of Japanese flight attendants found that long working hours were associated with high levels of fatigue. In Hong Kong, another place associated with long working hours, Lo (2003) interviewing 50 female married professionals in Hong Kong concluded that long working hours led to higher levels of exhaustion.

Several recent surveys have used large samples to show a positive association between working long hours and negative psychological outcomes. For example, a survey of 790 Ohio respondents by Roxburgh (2004) found that longer work hours (operationalized as time pressure) was positively associated with distress for both men and women. Fenwick and Tausig (2001) using data from the 1992 National Study of the Changing Workforce (sample size 2,905) found working longer hours associated with higher levels of both burnout and distress. Shields (1999) in a survey of 1,649 Canadian women found that those who worked long hours had double the odds of reporting a major depressive episode compared to those who worked standard hours. 
There were also studies that linked long working hours with both physical and psychological outcomes. Barton and Folkard (1993) found that British shiftworkers $(n=261)$ who worked more than 48 hours a week suffered a variety of mental and physical health problems such as anxiety, cardiovascular problems, digestive problems and neuroticism than those who worked fewer hours. Sparks et al. (1997) identified 21 study samples for their meta-analysis on the impact of long work hours. The authors concluded working longer hours per week was associated with higher levels of both physiological (e.g. coronary heart disease, hospitalization, mortality rate) and psychological (e.g. depression, irritability, anxiety) outcomes.

Sparks et al. (1997) points out that the majority of studies examining the relationship between long working hours and various negative health outcomes use self-report to measure hours worked and health. Sparks et al. (1997) recognize that it is possible that factors such as negative affectivity might be responsible for the reports of ill-health and not long working hours. However, Sparks et al. (1997) point to several studies (e.g. Alfredsson et al., 1985; Buell and Breslow, 1960; Starrin et al., 1990; Uehata, 1991) that used non-self-report measures and still have found working more hours associated with negative health outcomes.

While there are many studies documenting the association between working long work hours and several deleterious consequences, few studies have documented the impact of higher than average nonwork hours on psychological outcomes. The notable exceptions are Tierney, Romito and 
Messing (1990) and Hall (1992). Tierney, Romito and Messing (1990) surveyed 539 female hospital workers in Quebec and concluded that spending more hours on nonwork tasks (e.g. household chores and childcare) was associated with higher levels of exhaustion and insomnia. Hall (1992) using data from the Swedish Central Bureau of Statistics' Survey of Living Conditions (sample size was 12,772 respondents) concluded that women were more likely than men to perform the bulk of home duties (i.e. increased demand) and to report psychosomatic strain.

To conclude, the literature suggests that people are working longer hours and that these rising demands are taking a toll on their physical and mental health. It is logical to wonder about the impact of long working hours and increased demands (operationalized as time spent in various work and non-work activities) on work-life conflict. This is the subject of the next section. 


\section{Work-Life Conflict}

"We cannot build an effective organization on the foundation of broken homes and strained personal relationships" (Senge, 1990, p. 312).

This section will establish that there is a price to be paid for the frenetic pace of life many of us experience as we perform the Herculean challenges of trying to prioritize and juggle competing demands in multiple domains (as parent, spouse, worker). This price is increased work-life conflict (the strain component of models 1-3). This section has four subsections. First, we will define what is meant in this thesis by the term work-life conflict and examine the individual elements that comprise work-life conflict (i.e. role overload, work-family interference and family-work interference). Second, we will make the case that work-life conflict can be considered a form of strain and provide evidence of how this type of strain negatively impacts employees. Third, we will establish the association between both work and non-work demands and work-life conflict. In the final subsection, we present data showing how work-life conflict is rising in our society which is problematic given the link between work-life conflict and numerous negative outcomes established earlier.

\subsection{Definition of Work-Life Conflict}

Higgins, Duxbury and Lee (1994) define work-life conflict as having three key subcomponents: family-work interference, work-family interference and role overload. Family-work interference occurs when family responsibilities negatively impact one's ability to fulfill work duties (e.g. a parent is unable to attend a dinner meeting with a client because his/her child's daycare closes at 6 
PM and there is no alternate care available). Work-family interference occurs when work responsibilities negatively impact one's ability to complete family responsibilities (e.g. an overnight business trip prevents a parent from being able to attend their child's school play or sporting event). Role overload occurs when the various demands from people's different roles (e.g. employee; parent, spouse, volunteer) become overly taxing on one's time and energy. For example, feelings of exhaustion that result from trying to rush home from work, make supper, help children with homework and get them ready for bed. The essence of the work-life conflict issue is best summed up by Duxbury and Higgins when they said,

"In this sense, then, work-life conflict can be seen to have two major components: the practical aspects associated with time crunches and scheduling conflicts (i.e. an employee cannot be in two different places at the same time), and the perceptual aspect of feeling overwhelmed, overloaded or stressed by the pressures of multiple roles" (Duxbury and Higgins, 2001, p. 3).

Early research efforts (e.g. Pleck, Staines and Pleck, 1980; Staines and Pleck, 1983) examining the construct of work-family conflict or work-family interference (the common terms at the time) often saw this construct as unidimensional. However, there has been an evolution of conceptual thought on the nature of work-life conflict. Gutek et al. (1991) argued that work-family interference and family-work interference are two separate and distinct constructs; that discriminant validity between the two constructs is high, and that each domain has the potential to interfere with the other. Several studies (e.g. 
Frone et al., 1992a, 1992b, 1997b; Gutek et al., 1991; Kossek and Ozeki, 1998; Netemeyer et al., 1996) have empirically tested and supported this notion.

Frone et al. (1997b) argue that it makes sense that work-family interference and family-work interference are conceptually different because the antecedents and outcomes are often different. Frone et al. (1997b) demonstrated that work-to-family interference is primarily caused by work-related stressors and that it predicts family-related outcomes (e.g. family distress), whereas family-to-work interference is caused by family-related stressors and predicts work-related outcomes (e.g. job dissatisfaction). Hammer et al. (2002) summarizes Frone et al.'s efforts by noting that the underlying assumption is that "high levels of interference from one role to a second role makes meeting the demands of the second role more difficult" (Hammer et al., 2002, p. 85).

Research in the area of work-life conflict is also supportive of the notion that work-family interference tends to be the stronger of the two forms of interference (compared with family-work interference) and a number of studies (e.g. Burke and Greenglass, 1999; Eagle, Miles and Icenogle, 1997; Frone, 2000; Frone, Russell, and Cooper, 1992b; Frone, Yardley and Markel, 1997; Grandey and Cropazano, 1999; Gutek, Searle, and Klepa, 1991; Netemeyer et al., 1996; Williams and Alliger, 1994) have concluded that work-family interference is more likely to take place than family-work interference. Moreover, results from a recent meta-analysis by Kossek and Ozeki (1998) reported that work-family interference is more likely to negatively impact job satisfaction than family-work interference. 
Greenhaus and Parasuraman (1999) argue that given the important role work plays in our society and the fact that most people are economically dependent on their employers as their sole source of income, it makes sense that work-family interference is likely to be the dominant form of interference.

Another aspect of work-life conflict is role overload. Whereas interference can be seen a time-based component (Greenhaus and Beutell, 1985) where the demands of one domain (e.g. work) interfere with the demands of another domain (e.g. family), role overload is the strain-based component. In essence, role overload occurs when the sum total of one's activities becomes more than that person can comfortably perform (Voydanoff and Kelly, 1984). This strain can be associated with feeling exhausted and overwhelmed (Duxbury and Higgins, 2001; Wallace, 1999).

Duxbury and Higgins (2001) argue that in order to gain an accurate assessment of the level of work-life conflict experienced, one must capture both the practical (i.e. time-based) and perceptual (i.e. strain-based) components by measuring all three aspects of work-life conflict: work-family interference, familywork interference and role overload.

\subsection{Work-Life Conflict and Strain}

This section establishes the notion that work-life conflict can be considered a form of strain. First, we will discuss the theoretical foundations for such a proposition in this section and then present the empirical support establishing the negative outcomes of work-life conflict. 
In order to establish the connection between work-life conflict as a form of strain, one must begin by defining what we mean by strain and then establish certain assumptions about the nature of time and our relationship with work and non-work. Strain can be defined as, "a great or excessive pressure, demand or stress, on one's body, mind or resources" (freedictionary.com, 2006). In discussing work-life conflict, we will make two key assumptions.

First, using the scarcity hypothesis, time and energy are seen as finite, fixed resources. In other words, time spent on one activity (e.g. work) means that time is not available to spend on another activity (e.g. family). Furthermore, the energy expended in one domain means energy that is not available to be spent elsewhere. Second, consistent with the role conflict perspective, based on role theory, work and non-work responsibilities are seen to be inherently in conflict (Barnett and Gareis, 2006). Such a paradigm has been the dominant perspective in the work-family research for two decades (Barnett and Gareis, 2006; Thompson, Beauvais and Allen, 2006).

Work-life conflict has its roots in Kahn et al.'s notion of inter-role conflict (Kahn et al., 1964). Employees experience inter-role conflict when "the pressures associated with membership in one organization are in conflict with pressures stemming from membership in other groups" (Kahn et al., 1964, p. 20). Thomas and Ganster (1995) argue that work-family conflict is a "particular type of interrole conflict in which pressures from the work role are incompatible with pressures from the family role" (Thomas and Ganster, 1995, p. 7). 
In the context of work-life conflict, these pressures are the time demands that multiple stakeholders put on employees. Barnett and Gareis (2006) argue that the more roles a person assumes, the greater demands on that person's energy. However, energy spent in one domain means less energy available to be spent in other domains. The result, according to Gutek et al. (1991) is that the diminished energy leads to a reduction in role quality in the domain that has been neglected. Once that person's energy is depleted, it becomes even harder for that individual to juggle their responsibilities and the roles may begin to interfere with each other. For example, when an employee is at work or traveling on business, it is physically impossible for that employee to take care of an elderly family member or attend a school graduation. As well, as pointed out by Thompson et al., (2006), an employee might physically be present at a family even but distracted by thoughts of work. The ensuing tension and conflict in trying to balance work and non-work demands result in overload, strain, and other negative repercussions (Barnett and Gareis, 2006, Thomas and Ganster, 1995; Thompson, Beauvais and Allen, 2006).

In section 4 on Demands, it was discovered that the average North American is working longer hours in his/her job, doing supplementary work at home and working more overtime. Such an effort to work more hours will undoubtedly have an impact on workers' personal lives. Duxbury and Higgins (2001) argue that since time is a finite resource, employees have less time to spend in their private lives and most likely have a harder time balancing work and family. Both the feeling of not having enough time to do everything and the 
constant pull of trying to be in two separate places at the same time so as to balance work and family responsibilities is likely to lead to conditions of strain for employees.

Work-life conflict can also be considered a form of strain because of the number of adverse outcomes associated with this construct.

\subsubsection{Employee Outcomes}

The literature provides support for the notion that employees who experience high levels of role overload, work-family interference, and family-work interference suffer from a variety of physical, psychological, and behavioural difficulties including poor physical health, marital and family problems, life dissatisfaction, depression, exhaustion, alcohol and drug abuse. Evidence supporting this claim is reviewed below.

\subsubsection{Link Between Role Overload and Employee Outcomes}

Role overload is defined as the various demands from people's different roles (e.g. employee, parent, spouse, volunteer) becoming overly taxing on one's time and energy (Duxbury and Higgins, 2001). It is logical to assume that those experiencing high role overload will report negative physical and psychological outcomes as a result of feeling rushed, having too much to do and running low on energy.

The literature provides support for the idea that role overload (sometimes operationalized as time pressure) is associated with various negative personal outcomes. Role overload has been associated with depression (e.g. Robinson and Godbey, 1997; Roxburgh, 2004), lower levels of life satisfaction (e.g. 
Robinson and Godbey, 1997), poorer physical health (Bittman, 1998; Higgins, Duxbury and Johnson, 2004; Madsen, 2003; Robinson and Godbey, 1997), family distress (Crouter, Bumpus, Maguire and McHale, 1999; Frone, Yardley and Markel, 1997) and stress (Gerstel and Gallagher, 1993; Guelzow, Bird and Koball, 1991; Kelloway, Gottlieb, and Barham, 1999; Paden and Buehler, 1995; Robinson and Godbey, 1997; Roxburgh, 2004; Schieman, 1999; Williams and Alliger, 1994).

\subsubsection{Link Between Work-Family Interference and Employee Outcomes}

Work-family interference is defined as occurring when work responsibilities negatively impact one's ability to complete family responsibilities (Duxbury and Higgins, 2001). This notion of interference can lead to feelings of frustration over trying to juggle multiple responsibilities. This frustration in turn can result in a variety of physical and psychological outcomes as discussed below.

Relative to the other two components of work-life conflict (i.e. role overload and family-work interference) there is much more extensive empirical support for the notion that work-family interference is associated with a number of negative outcomes at the employee level including poorer physical health, psychological symptoms such as depression and behavioural outcomes such as marital difficulties, family difficulties and alcoholism.

There is ample research (e.g. Adams and Jex, 1999; Burke, 1988; Higgins, Duxbury, and Johnson, 2004; Frone, Russell and Barnes, 1996; 
Grandey and Cropanzano, 1999, Guelzow et al., 1991; Kinnunen and Mauno, 1998; Marks, 1998; Netemeyer et al., 1996; Thomas and Ganster, 1995) to suggest that higher levels of work-family interference are associated with poor physical health (measured as poor physical health and symptoms of poor physical health). Another common finding in the literature is the notion that work-family interference is negatively associated with life satisfaction (e.g. Adams et al., 1996; Aryee, 1992; Bedeian et al., 1988; Higgins, Duxbury and Irving, 1992; Kopelman et al., 1983; Netemeyer et al., 1996; Perrewe, Hochwarter, and Kiewitz, 1999; Rice et al., 1992).

Work-family interference has also been positively associated with a variety of psychological maladies including depression (Duxbury and Higgins, 1997; Frone et al., 1994; Frone et al., 1995; Frone et al., 1996; Hammer et al., 2005; Klitzman et al., 1990; MacEwen and Barling, 1994; Netemeyer et al., 1996; Thomas and Ganster, 1995; Vinokur, Pierce, and Buck, 1999), exhaustion (Boles et al., 1997; Lo, 2003), stress (Anderson et al., 2002; Duxbury and Higgins, 1997; Parasuraman et al., 1992; 1996), anxiety (Beatty, 1996, Frone, 2000; Greenglass et al., 1989), burnout (Burke, 1988; Lo, 2003), and general psychological distress (Burke and Greenglass, 1999; Grandey and Cropanzano, 1999; Marks, 1998; O'Driscoll et al., 1992; Parasuraman, Purohit, Godshalk and Buetell, 1996; Smith Major, Klein and Ehrhart, 2002; Vinokur, Pierce, and Buck, 1999).

There is also support in the empirical literature for the notion that workfamily interference is negatively associated with marital happiness (operationalized as marital adjustment and marital satisfaction in most studies) 
(Beatty, 1996; Bedeian et al., 1988; Greenhaus et al., 1987; Netemeyer et al., 1996; MacEwen and Barling, 1994; Parasuraman et al., 1989; Vinokur, Pierce, and Buck, 1999). There is also support in the literature for the propositions that work-family interference is negatively associated with both family satisfaction (e.g. Aryee et al., 1999; Bedeian et al., 1988; Carlson and Perrewe, 1999; Frone et al., 1994; Kopelman et al., 1983; Parasuraman et al., 1992; Rice et al., 1992) and quality of family life (Higgins, Duxbury and Irving, 1992) but positively associated with family distress (Frone et al., 1992a; Frone, Yardley and Markel, 1997; Grandey and Cropanzano, 1999; Vinokur, Pierce, and Buck, 1999).

Several studies by Frone and his co-authors have helped support the proposition that work-family interference is positively associated with substance abuse. Specifically, studies by this group of researchers have revealed positive relationships between work-family interference and heavy alcohol use (Frone et al., $1993 ; 1994 ; 1996 ; 1997$ a) and work-family interference and a variety of substance abuse disorders such as alcohol abuse, alcohol dependence, drug abuse and drug dependence (Frone, 2000).

A number of factors increase our confidence in these findings. First, the studies were remarkably consistent in finding the associations between higher levels of work-family conflict and the negative outcomes featured in this section (i.e. poor physical health, lower levels of life satisfaction, higher levels of depression, marital and family dissatisfaction and substance abuse) despite the fact that the researchers used a variety of measures of work-family interference (e.g. Bohen and Viveros-Long, 1981; Frone, Russell, and Cooper, 1992a; Gutek 
et al., 1991; Kopelman et al., 1983). Second, a number of the studies cited in this section (e.g. Adams and Jex, 1999; Anderson et al., 2002; Burke, 1988; Frone, 2000; Frone, Russell and Barnes, 1996; Frone, Russell and Cooper, 1993; Frone, Russell and Cooper, 1995; Greenglass et al., 1988; Hammer et al., 2005; Rice et al., 1992), involved fairly large (i.e. 450 respondents or more) samples which increases the generalizability of the findings.

\subsubsection{Link Between Family-Work Interference and Employee Outcomes}

Family-work interference occurs when family responsibilities negatively impact one's ability to fulfill work duties (Duxbury and Higgins, 2001). Similar to the argument in the previous section (i.e. work-family interference), this notion of interference will lead to feelings of frustration when juggling various tasks becomes difficult. This frustration is responsible for a variety of negative outcomes as discussed below.

There is also a significant body of evidence linking family-work interference to a variety of negative personal outcomes. For example, high family-work interference has been associated with lower levels of life satisfaction (Adams et al., 1996; Netemeyer et al., 1996; Parasuraman, Purohit, Godshalk, and Beutell, 1996; Perrewe, Hochwarter, and Kiewitz, 1999) and poorer family functioning (Bernas and Major, 2000; Kinnunen and Mauno, 1998).

As well, family-work interference has been positively associated with higher levels of stress (Anderson et al., 2002; Burke and Greenglass, 1999; Kelloway, Gottlieb and Barham, 1999; Kirchmeyer and Cohen, 1999; MacEwen 
and Barling, 1994; Marks, 1998; O'Driscoll et al., 1992; Vinokur, Pierce, and Buck, 1999; Williams and Alliger, 1994), poorer mental health (Frone, 2000; Frone, Russell and Barnes, 1996; Frone, Russell and Cooper, 1992a; Frone, Russell and Cooper, 1997; Hammer et al., 2005; Higgins, Duxbury and Johnson, 2004; Kirchmeyer and Cohen, 1999; MacEwen and Barling, 1994; Netemeyer et al., 1996; Vinokur, Pierce, and Buck, 1999), poorer physical health (Adams and Jex, 1999; Frone, Russell and Cooper, 1992a; Frone, Russell, and Barnes, 1996; Frone, Russell and Cooper; 1997; Grzywacz and Marks, 2000; Higgins, Duxbury and Johnson, 2004; Madsen, 2003; Marks, 1998; Netemeyer et al., 1996), and substance dependence disorders such as alcohol abuse, alcohol dependence, drug abuse and drug dependence (Frone, Rusell and Barnes, 1996; Frone, 2000).

Three studies featured a longitudinal design which was useful in establishing not only an association between family-work interference and a negative outcome but to establish causality. For example, Frone, Russell and Cooper (1997) found that family-to-work interference was longitudinally related to higher incidence of hypertension (i.e. blood pressure above the normal range; Heart Center, 2005), poor physical health and higher levels of depression in a sample of 267 employed parents. Hammer et al. (2005) found family-work interference was related to higher incidents of depression in both men and women in a national U.S. survey of 234 dual earner couples. Brough, O'Driscoll and Kalliath (2005) studied 398 employed men and women in New Zealand and 
noted that family-work interference was negatively associated with family satisfaction.

To summarize, there is a substantive body of literature to support the associations between work-family interference and a host of negative outcomes as well associations between family-work interference and a host of negative outcomes. The evidence to support the notion that role overload is associated with various employee negative outcomes, while not as voluminous, shows the same trends. There are both theoretical justifications and empirical evidence as to how and why work-life conflict can be considered a type of strain.

\subsection{Link Between Work Demands and Work-Life Conflict}

Thesis models 1-3 hypothesize a relationship between demands (work and nonwork) and work-life conflict (role overload, work-family interference, family-work interference). Literature supporting these relationships are reviewed below.

How exactly do work demands (i.e. committing a high number of hours to work-related activities) impact work-life conflict? The rational theory of work-life conflict (Gutek et al., 1991) observes that there are only a finite number of hours in one's day and that time spent in the work domain means less time to spend in the non-work domain. Voydanoff (2004) argues that having less time available to spend with family (spouse, children, elderly parents) makes it harder for people to meet their non-work obligations and have time to properly perform family duties and sustain healthy family relationships.

This section will examine the association between work demands and 
work-life conflict by examining the link between work demands and the three subcomponents of work-life conflict: role overload, work-to-family interference and family-work interference. Each association will be discussed in turn.

\subsubsection{Link Between Work Demands and Role Overload}

Role overload is defined as the various demands from people's different roles (e.g. employee, parent, spouse, volunteer) become overly taxing on one's time and energy. Consistent with the rational theory of work-life conflict (Gutek et al., 1991), there are only so many hours in the day. As work demands increase (defined as number of hours spent on work-related activities), less time is available for non-work activities. It is suggested that, for many people, spending more time at work would lead to a feeling of insufficient time to recharge the batteries. Lack of downtime might result in people feeling physically and emotionally drained and losing their sense of perspective by feeling rushed and feeling they don't have time for themselves. All of this could lead to perceptions of role overload. Therefore, it is reasonable to assume that as people work longer hours that such efforts could potentially result in a feeling of diminished energy and time. The following literature supports the positive association between work demands and role overload hypothesized in model 1.

A number of studies have concluded that working longer hours is positively associated with role overload. Voydanoff (2004) using data from the 1997 NSCW (1938 employed adults) found a strong positive relationship between the hours worked and strain (operationalization similar to role overload). Fu and Shaffer (2001)'s study of 267 academic, administrative and general grade 
staff at a Hong Kong university found a positive association between hours spent in paid work and role overload (operationalized as strain-based conflict). Smith Major, Klein and Ehrhart (2002) in their study of 513 employees in one Fortune 500 company, found a positive association between hours spent in paid work and role overload. Parasuraman, Purohit, Godshalk and Beutell (1996)'s study of 111 entrepreneurs noted a positive association between work-role overload and time commitment at work (measured as the number of hours spent in workrelated functions). Frone, Yardley and Markel (1997) study of 372 employed adults working for a financial services company in Canada determined that work time commitment (measured as the number of hours spent in work-related functions) was positively and statistically significantly associated with work overload.

Wallace (1997) found a positive, statistically significant association between hours worked in paid employment and role overload (operationalized as work overload). Galambos and Walters (1992) studied 96 dual-earner Canadian couples and found a statistically significant positive association between the hours worked and role overload (operationalized as role strain) for both husbands and wives.

\subsubsection{Link Between Work Demands and Work-Family Interference}

Work-family interference was defined in section 5.1 as work responsibilities negatively impacting one's ability to complete family responsibilities. The argument linking work demands and work-family 
interference is rooted in Kahn's interrole conflict and was previously discussed in section 5.2. The central premise is the assumption under the rational theory of work-life conflict that time is a fixed resource and that time spent in one domain means less time in other domains as well as physically not being able to be at non-work activities when one is at work (Gutek et al., 1991).

It is hypothesized that excessive work demands (e.g. excessive overtime, business trips out of town, last minute call-ins to work) mean less time to complete family responsibilities (e.g. family dinners, taking children to their activities, and spending time with elderly parents) due to the fact that there are a limited number of hours in a given day. Furthermore, since employees cannot be two places at once, the aforementioned work demands mean that the employee may miss out on key family events and milestones (e.g. birthday parties, anniversaries, hockey championships). The empirical literature provides additional support for the positive association between demands and work-family interference hypothesized in model 2.

The empirical literature is supportive of the notion that working excessive hours is associated with higher levels of work-family interference (e.g. Austin Knight, 1995; Carlson and Perrewe, 1999; Fenwick and Tausig, 2001; Frone, Yardley and Markel, 1997; Fu and Shaffer, 2001; Galambos and Walters, 1992; Galinsky, Bond and Hill, 2004; Gutek et al., 1991; Madsen, 2003; Smith Major, Klein and Ehrhart, 2002; Maume and Houston, 2001; Netemeyer et al., 1996; Parasuraman et al., 1996; Pleck, Staines and Lang, 1980; Shamir, 1983; Silver, 2000; Staines and Pleck, 1983; Thompson, Beauvais, and Lyness, 1999; Van 
der Hulst and Geurts, 2001; Voydanoff, 1988; Voydanoff, 2004; White et al., 2003). The majority of the aforementioned studies (e.g. Austin Knight, 1995; Fenwick and Tausig, 2001; Galinsky, Bond and Hill, 2004; Major, Klein and Ehrhart, 2002; Maume and Houston, 2001; Pleck, Staines and Lang, 1980; Silver, 2000; Staines and Pleck, 1983; Van der Hulst and Geurts, 2001; Voydanoff, 1988; Voydanoff, 2004; White et al., 2003) used large sample sizes (i.e. a minimum of 450 respondents) which contributes to our confidence in this association.

Of course, not all studies have supported the positive relationship between work hours and work-life conflict. Wallace (1999)'s study of 512 Canadian lawyers for example did not find that longer work hours were associated with higher levels of work-to-nonwork conflict for her sample of lawyers. Wallace (1999) admits that this finding was unexpected and contrary to what was hypothesized. However, Wallace (1999) suggests that lawyers tend to have some level of discretion over their work schedules which might help them meet their non-work obligations. The impact of perceived flexibility of work schedules will be more fully explored in subsection 6.5 .

\subsubsection{Link Between Work Demands and Family-Work Interference}

Conceptually, we could argue that there is a link between work demands and family-work interference. Excessive work demands and workplace pressures from supervisors to put work ahead of family have the potential to influence the employee to see family responsibilities as obstacles to 
accomplishing their work and career ambitions (Behson, 2002; Boyar et al., 2003; Voydanoff, 2005). Furthermore, North Americans are doing more supplementary work at home (Duxbury and Higgins, 2001) which could blur the boundaries about when and how the supplementary work gets done. This could increase family-work interference if employees who do unpaid overtime at home feel pressure from spouse or children to not get work done but instead to engage in family-related activities. Both scenarios could lead to a heightened sense of family-work interference.

Unfortunately, there are only a handful of studies affirming the positive association between work hours and family-work interference (e.g. Boyar et al., 2003; Duxbury, Higgins, Lee and Mills, 1992; Greenhaus and Beutell, 1985; Gutek et al., 1991; Van der Hulst and Geurts, 2001) hypothesized in model 3. This study should add to this body of evidence and increase our understanding of the relationship between work demands and family-work interference.

\subsection{Link Between Non-work Demands and Work-Life Conflict}

This section will examine the association between non-work demands and work-life conflict. It is structured in the same manner as the previous section.

\subsubsection{Link Between Non-work Demands and Role Overload}

The rationale for finding a link between non-work demands and role overload is similar to the argument presented for work demands and role overload. Rational theory would suggest that someone who is spending a large 
number of hours on non-work demands (e.g. childcare, housework, eldercare, volunteering) could experience a lower level of energy and perceive that there are not enough hours in the day to complete all they have to do at work and home. Yet, the literature presented below indicates the empirical evidence is limited and contradictory.

Fu and Shaffer (2001) did not find a statistically significant association between hours spent on household work and role overload (operationalized as strain-based family-interference) in their study of 267 employees at a Hong Kong university. On the other hand, Frone, Yardley and Markel (1997) studied 372 employed adults working for a financial services company in Canada. Family time commitment (measured as the number of hours spent in family-related responsibilities) was positively and statistically significantly associated with family role overload. This thesis hopes to shed further light on this issue by exploring the relationship between non-work demands and role overload (model one).

\subsubsection{Link Between Non-work Demands and Work-Family Interference}

Theoretically, the link between non-work demands and work-family interference is rooted in Kahn et al.'s (1964) inter-role conflict. The tension inherent between the conflicting demands of work and non-work result in interrole conflict. Those employees with higher levels of non-work demands may feel frustration that work is interfering with their ability to complete non-work tasks. This frustration will lead to higher levels of work-family interference. Whereas, 
those employees with lower levels of non-work demands may not feel the same level of frustration at the level of work-family interference.

Unfortunately, there are few studies that explore the association between non-work demands and work-family interference. The majority of the studies that do exist, however, report a positive association between non-work demands and work-family interference. Parasuraman, Purohit, Godshalk and Beutell (1996)'s study of 111 entrepreneurs noted a statistically significant positive association between time commitment to family (measured as the number of hours spent in nonwork-related functions) and work-family conflict. Yang et al. (2000) surveyed 129 American and 181 Chinese respondents. Non-work demands (operationalized as family demands) were positively and statistically significantly associated with work-family interference for both the American and Chinese groups. Carlson and Perrewe (1999) surveyed 403 respondents who worked for a state government in the American southeast and found that non-work demands and work-family interference were positively and statistically significantly associated.

There was one study where no positive relationship was found between non-work demands and work-family interference. Voydanoff's 1988 study which analyzed data from the 1977 Quality of Employment Survey (757 married men and 270 married women) did not find a statistically significant association between non-work demands and work-family conflict. All in all, however, both the theory and the empirical evidence support the hypothesized positive 
association between non-work demands and work-family interference posited in model 2.

\subsubsection{Link Between Non-work Demands and Family-Work Interference}

Kahn et al.'s (1964) inter-role conflict also helps us to explain the positive association between non-work demands and family-work interference. More hours spent in the nonwork domain means less time available for working outside the home. According to inter-role conflict theory, this can create the perception among those with high levels of non-work demands that family is interfering with work responsibilities.

An employee with a high level of non-work demands does not have the flexibility to put off completing these demands. Children need to be fed and put to bed and elderly parents need to be cared for. In this case, it is possible that the employee would be unwilling or unable to stay at work late or travel for work. In essence, the employee is experiencing a high level of interole conflict due to incompatible role demands.

Only a few studies were found which discussed the relationship between non-work demands and family-work interference. Gutek et al., 1991 surveyed two different samples: 423 psychologists and 209 senior managers and found a positive association between non-work demands and family-work interference in both samples. Kirchmeyer (1995) in her survey of 221 Canadian managers found a statistically significant positive correlation between nonwork hours and family-work interference. Frone, Yardley and Markel (1997) studied 372 
employed adults working for a financial services company in Canada. Family time commitment (measured as the number of hours spent in family-related responsibilities) was positively and statistically significantly associated with family-work interference. Fu and Shaffer (2001) surveyed 267 employees in a Hong Kong university and did not find a statistically significant association between non-work demands and time based family-interference with work. Both the theory and the empirical evidence support the hypothesized positive association between non-work demands and family-work interference posited in model 3 .

\subsubsection{Demands and Work-Life Conflict Conclusion}

In conclusion, there is a larger body of empirical literature linking work demands and work-life conflict than could be found for non-work demands and work-life conflict). Overall, there is ample literature to indicate that there are positive associations between both work demands and role overload as well as work demands and work-family interference. While there are relatively fewer studies for the work-demands-family-work interference link, the studies are nonetheless supportive.

For non-work demands and role overload, results are both very limited and rather mixed and therefore inconclusive. For both the associations between non-work demands and work-family interference and non-work demands and family-work interference, research is limited but supportive. 


\subsection{Rising Levels of Work-Life Conflict}

Before presenting the information summarizing the rise in work-life conflict in North America, it is important to take a step back and ask ourselves why should we care about rising levels of work-life conflict? Section 5.2.1 outlined the various negative employee outcomes associated with work-life conflict.

Furthermore, there is additional literature to indicate that work-life conflict has negative repercussions for the employer in the form of lower job satisfaction (e.g. Adams et al., 1996; Anderson et al., 2002; Boles et al., 2001; Frone, Yardley and Markel, 1997; Voydanoff and Kelly, 1984); lower organizational commitment (e.g. Aryee, 1992; Duxbury and Higgins, 1997; Good et al., 1988; Greenhaus and Beutell, 1985; Netemeyer et al., 1996; O'Driscoll et al., 1992; Perrewe, Ralston, and Ferdandez, 1995; Thompson, Beauvais and Lyness, 1999) and higher turnover intentions (e.g. Anderson et al., 2002; Aryee, 1992; Boyar et al., 2003; Burke, 1988; Good et al., 1988; Grandey and Cropanzano, 1999; Greenhaus, Parasuraman and Collins, 2001; Kelloway et al., 1999; Jones and Butler, 1980; Netemeyer et al., 1996; Thompson, Beauvais and Lyness, 1999). Furthermore, Higgins, Duxbury and Johnson (2004) argue that high levels of work-life conflict (in the form of role overload) cost Canada's health care system $\$ 6$ billion dollars.

Given the proposition that work-life conflict is problematic for employees, employers and society, all three stakeholders should rightly be concerned if levels of work-life conflict are on the rise. Rising work-life conflict levels could manifest themselves in rising levels of physical and psychological ailments, which in turn will negatively impact our publicly funded health care system in the 
form of more doctor's visits. Furthermore, increased levels of work-life conflict could result in increased turnover and absenteeism for employers.

How pervasive is work-life conflict in North American society? Are levels of worklife conflict improving or worsening? This section provides data to answer both of these questions and make the case that levels of work-life conflict are on the rise and that most North Americans are having tremendous difficulty "balancing the competing role of employee, parent, spouse, and eldercare giver" (Duxbury and Higgins, 2001, p. 39).

There is evidence that work-life conflict has increased in Canada and the United States. A large sample study by Duxbury and Higgins (2001) noted that relative to their 1991 results, significant increases were reported in role overload (in 2001, 59 percent reported high levels of role overload, an increase of 12 percentage points from 1991) and family-work interference (in 2001, 10\% reported high level of this interference, double the percentage from 1991). These increases (shown in Figure 5) were experienced regardless of gender, job type or parental status. The biggest increases were seen for role overload (i.e. having too much to do and too little time, feeling rushed as well as physically and mentally exhausted; Duxbury and Higgins, 2001). 
Figure 5 Comparison of Work-Life Outcomes (1991 vs. 2001) (Duxbury and Higgins, 2001).

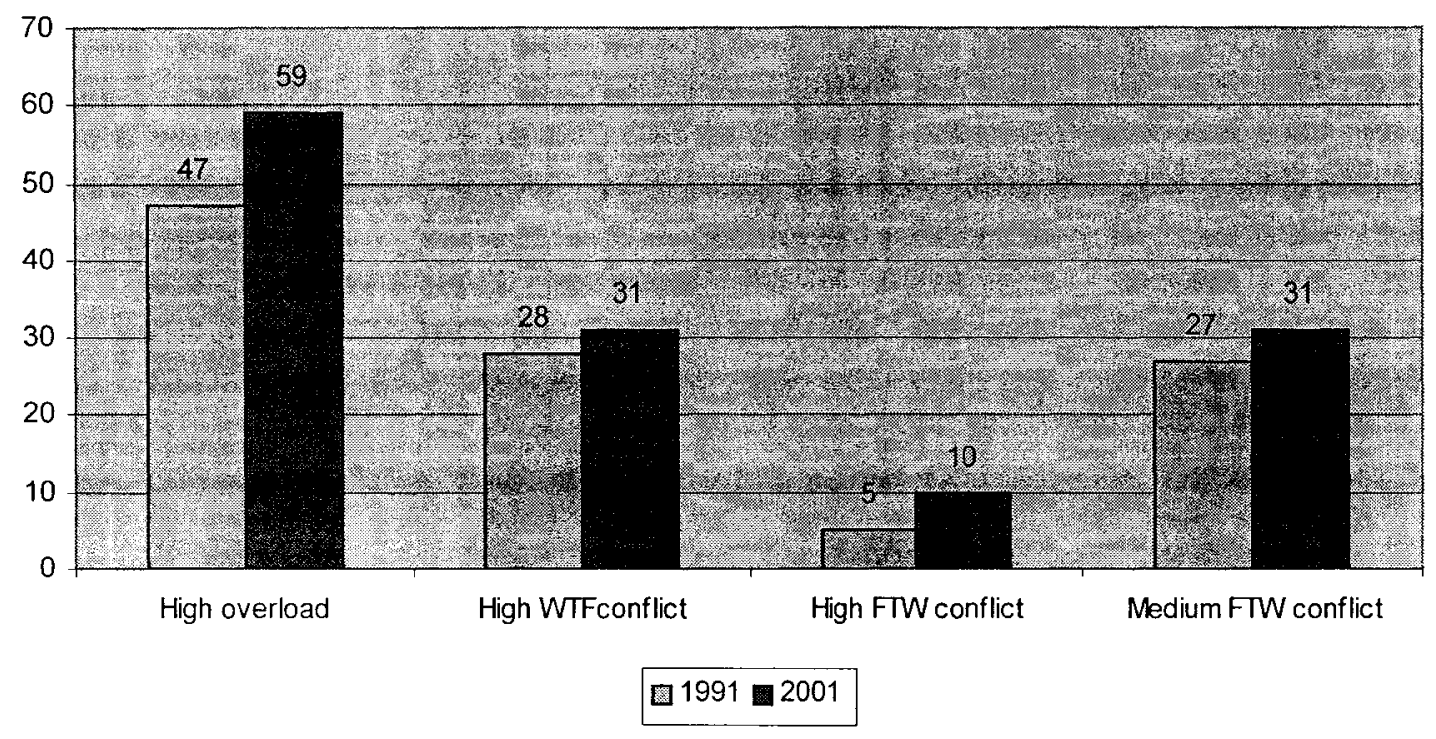

There is a similar trend in the United States with Americans reporting higher levels of role overload (operationalized as working harder and faster and feeling overworked and overwhelmed). An ever-increasing number of American workers surveyed for the NSCW (National Survey on the Changing Workforce) felt that their workplace had become a more demanding environment. For example, 68 percent of workers reported that they had to work very fast (an increase of $15 \%$ from 1977), 88 percent had to work very hard (an increase of 18 percent from 1977), and 60 percent did not have enough time to finish everything that needs to get done on the job (an increase of 20 percent from 1977; Bond et al., 1998). Galinsky, Kim and Bond (2001) commented on how hard Americans felt they were working. The results of their nationally representative sample found that: $28 \%$ of employees felt overworked often or very often in the past three months; $28 \%$ felt overwhelmed by how much work they had to do often or 
very often in the past three months and $29 \%$ felt that they did not have the time to step back and process or reflect on the work they are doing often or very often during the past three months.

When Galinsky, Kim and Bond (2001) also factored in those who feel overworked at least sometimes, there were a substantial number of respondents who felt overworked: $54 \%$ felt overworked at least sometimes in the past three months; $55 \%$ felt overwhelmed by how much work they had to do at least sometimes in the past three months and $59 \%$ felt they did not have time to step back and reflect on the work they were doing at least sometimes in the past three months. Galinsky, Kim and Bond (2001) created an overall index of feeling overworked by averaging answers to the three questions above. On a scale of 1 to 5 (with $1=$ never and $5=$ very often), respondents had an average score of 2.7 , indicating that the average employee sometimes feels overworked. In summary, the 1997 National Study of the Changing Workforce (NSCW) found that today's jobs "consume more physical and emotional energy ... and require longer hours than were required 20 years ago" (Hill et al., 2001, p. 50).

Our American counterparts are also experiencing significant levels of work-life conflict. Whereas thirty percent of respondents in the late 1970 s and early 1980 s were claiming that their job interfered with work somewhat or a great deal (e.g. Galinsky, Ruopp, and Blum, 1983; General Mills, Inc., 1981; Quinn and Staines, 1979), more recent data indicate these numbers have climbed. The most recent NSCW survey (2002) now indicates that employees with families report higher levels ( $45 \%$ experienced 'some' or 'a lot') of interference between 
their jobs and their family lives compared to respondents in 1977 (34\% experiencing 'some' or 'a lot') (Bond et al., 2003).

In conclusion, work-life conflict is a pervasive influence in North American society and the data indicates that this conflict is becoming worse for the average person. Higher levels of demands appear to have resulted in higher levels of work-life conflict. This outcome threatens to compromise quality of life and could result in poorer physical and mental health and poorer interpersonal relationships with family, friends and coworkers. What can be done to address this situation? The next section examines the literature that supports our contention that the various forms of control considered in this research (i.e. alternative work arrangements, perceived flexibility and supervisor support) could potentially reduce the incidence of work-life conflict. 


\section{Control Over Work-Life Interface}

"Most powerful is he who has himself in his own power" (Seneca)

www.quotationspage.com/quotes/seneca/31

Why do researchers need to broaden their conceptualization of control beyond what was offered by Karasek? We have established in this literature review that employees are experiencing higher levels of work (and perhaps nonwork demands) and a commensurate increase in work-life conflict. We have also established that work-life conflict has serious outcomes for employees, employers and society. In such an environment, it is important to consider what can be done to reduce work-life conflict. Specifically, this thesis examines whether or not three different organizational interventions (alternative work arrangements, perceived flexibility, supervisor support) can moderate the relationships between work demands and work-life conflict and the relationships between non-work demands and work-life conflict.

Given that our conceptualizations of both demands and strain have been expanded to recognize the influence of both work and non-work domains, it makes sense to consider the role of control in this context. Accordingly, the literature was examined with the goal of discovering organizational factors that researchers had identified as helping workers balance responsibilities in both domains. Control over work-life interface is operationalized in three ways in this thesis: as alternative work arrangements (e.g. flextime, compressed work week, telework and shiftwork), as perceived flexibility and as supervisor support. These factors were selected for inclusion in this thesis as all have been linked in the 
research literature to an increased ability to make decisions and choices that can help them better balance work and family responsibilities.

The discussion of alternative work arrangements is discussed in section 6.1. This will be followed by a discussion of perceived flexibility in section 6.2 and supervisor support in section 6.3. In each case, the theoretical underpinnings of each construct will be discussed with respect to how each construct is considered a form of control followed by empirical support for the notion that the construct in question is associated with work-life conflict.

\subsection{Alternative Work Arrangements}

Quite often a conventional Monday to Friday, 9 to 5 schedule does not permit employees the flexibility required in order to juggle work, childcare/eldercare and other personal responsibilities. For example, Nollen (1979) points out that regular work schedules can be problematic because these schedules may not be compatible with child care arrangements and may not allow sufficient time to meet the busy demands of family life. Therefore, it makes sense to look at alternatives to this conventional work schedule to see what arrangements might be conducive to a more balanced sense of work and life.

Four work arrangements are considered in this thesis: compressed work week, flextime, telework and shiftwork. It is important to recognize that some work arrangements, in theory, offer more control over timing of work (i.e. flextime, compressed work week) and location of work (telecommuting) than the regular 9 to 5 , Monday to Friday work week. Some arrangements (i.e. shiftwork) offer less. Whether this theoretical form of control translates into lower levels of work-life 
conflict will be explored in this thesis. Literature supporting such a link is reviewed below. For each sub-section, the work arrangement will be defined first. This is followed by data estimating the percentage of the workforce using this arrangement. The advantages and disadvantages of the work arrangement will be presented followed by a discussion of the impact of these work arrangements on work-life conflict.

\subsubsection{Compressed Work Week}

Compressed workweek (CWW) is defined as "reallocating employee work time by condensing the total hours in the traditional work week into fewer days" (Pierce et al., 1989, p. 52). Instead of working a typical eight hour day, five days per week schedule, an employee might instead work 10 hours a day for four days. It is possible that compressed work weeks may play a role in helping to reduce work-life conflict by giving employees an increased sense of control over their work week. It could be argued that having this sense of control plays a role in lowering work-life conflict since employees are more able to tailor their work week schedule to meet their personal obligations. For example, an employee may choose a compressed work week schedule that enables him/her to attend university classes as well as attend children's school events and sporting events.

A Hewitt Associates (1995) survey of 1050 major US employers reported that roughly $20 \%$ offer compressed work weeks ${ }^{4}$. In Canada, recent figures

4 Finding up-to-date statistics with respect to compressed work week usage has become difficult since the Bureau of Labor Statistics and National Study on the Changing Workforce have collapsed compressed work week into the broader category of flexible work arrangements. 
indicate a very low number of Canadians (5.5\%) are working a compressed work week (Statistics Canada, 2004).

\subsubsection{Advantages and Disadvantages of Compressed Work Week}

An alternative arrangement such as compressed work week involves both advantages and disadvantages for both the employer and employee. Potential employer advantages may include: improved ability to recruit new employees (Nollen and Martin, 1978b; Poor and Steele, 1973), lower absenteeism (Baltes et al., 1999; Goodale and Aagaard, 1975; Harrick et al., 1986; Latack and Foster, 1985; Nollen and Martin, 1978b; Nord and Costigan, 1973; Pierce et al., 1989; Wheeler, 1970), turnover (Hartman and Weaver, 1977; Nollen and Martin, 1978b; Wheeler, 1970), job satisfaction (Baltes et al., 1999; Hartman and Weaver, 1977; Pierce et al., 1989; Venne, 1993; Williamson, Gower and Clarke, 1994), organizational commitment (Ivancevich, 1974) and productivity (e.g. Baltes et al., 1999; Campbell, 1980; Ivancevich, 1974; Ivancevich and Lyon, 1977; Nollen and Martin, 1978b; Pierce et al., 1989; Ronen, 1984).

Potential drawbacks for employers may include inconvenience for customers (Frankel, 1998; Goodale and Aagaard, 1975; Poor and Steele, 1973), scheduling difficulties (Goodale and Aagaard, 1975; Nollen and Martin, 1978b; Poor and Steele, 1973) and the possibility that the boredom and monotony of working long hours could lead to work-related accidents (Nollen and Martin, 1978b; Perlow, 1997).

Potential employee advantages include: increase of leisure times in larger blocks (i.e. more time to recuperate from work; Goodale and Aagaard, 1975; 
Hodge and Tellier, 1975; Knauth and Rutenfranz, 1982; Nollen, 1979), ability to complete errands (e.g. doctor's visit; Pierce et al., 1989), reduced weekly commuting time (Pierce et al., 1989), and reduced work-related costs (e.g. as commuting costs for gas and car maintenance, lunch etc.; Hurrell and Colligan, 1985).

Potential employee disadvantages are similar to the employer drawbacks and include reduced leisure time on scheduled workdays (Goodale and Aagaard, 1975; Hodge and Tellier, 1975), possible fatigue from working longer hours per day (Hodge and Tellier, 1975; Ivancevich, 1974; Maklan, 1977), the higher potential for accidents due to fatigue (Perlow, 1997), and the increase of potential problems associated with prolonged exposure (i.e. 10 to 12 hours) to levels of physical and chemical hazards in the workplace (Hurrell and Colligan, 1985).

\subsubsection{Impact of Compressed Workweek on Work-Life Conflict}

What impact have compressed schedules had for outcomes such as worklife conflict? Unfortunately, very few studies have been conducted in this area. Furthermore, the results from what does exist are varied. Each study will be discussed in turn.

Four studies yielded positive findings such that, working a compressed work week was associated with either better work-life balance or more time spent with family. Cunningham (1981) surveyed 17 police officers who worked a compressed work week (13 officers served as the control group and did not work a compressed work week). The officers working a compressed work week reported more time spent with family. A second study of 50 police officers by 
Pierce and Dunham (1992) found that compressed work week was associated with less schedule related interference with personal activities. Hodge and Tellier (1975) surveyed 223 employees and concluded that compressed work week was associated with more time for family. Dunham et al. (1987) found a compressed work week schedule helped reduce work-life conflict. Dunham et al. (1987) surveyed 140 employees and concluded that those working a compressed work week (a 4/40 schedule) reported less interference with personal activities involving family and friends.

Saltzstein, Ting, and Saltzstein (2001) examined the impact of compressed work week on work-life balance for a large sample of Federal government employees $(32,103)$. The authors noted that for their whole sample, a compressed work week was associated with a "modestly positive significant relationship with work-life balance" (Saltzstein et al., 2001, p. 462). This positive relationship did not, however, hold for many subgroups of the sample. For example, there was no statistically significant association between compressed work week and work-life balance for groups such as women in dual income families with children.

The authors offered a possible explanation for this finding. Working the longer hours each day associated with compressed work weeks is not helpful for most parents. For example, a long compressed work day may mean that the parent may not see their children at all on certain days (Saltzstein, Ting and Saltzstein, 2001). For parents of children in daycare, their work schedules are 
unlikely to be compatible with most day care schedules where parents are expected to pick up their children by a specified time.

Fast and Frederick (1996) found a mixed result when examining the Canadian General Social Survey from 1992 (sample size 5,060 employed Canadians). Women using compressed work weeks actually had higher levels of perceived time stress (conceptually similar to role overload) than those working a regular 9-5 schedule. For men, there were no differences between those working a compressed schedule and a regular schedule.

What might explain the difference between these studies? Two explanations come to mind. First, the four studies (i.e. Cunningham, 1981; Dunham et al., 1987; Hodge and Tellier, 1975; Pierce and Dunham, 1992) yielding positive results used a small sample relative to the two other national studies. Second, the four studies with positive findings did not perform any between-group analysis whereas Fast and Frederick (1996) looked at gender differences and Saltzstein, Ting and Saltzstein (2001) examined both gender and parental status differences.

Unfortunately, other studies that discussed the impact of compressed work week on work-life conflict were not found despite the use of several academic databases including ABI, Psyclnfo and Social Sciences Index. Therefore, it is hoped this thesis can help make a contribution to the literature in assessing the role a compressed work week can play in helping employees cope with work-life conflict. 


\subsubsection{Flextime}

Flextime challenges the notion that all employees must start and stop their workday at the same time. Flextime is defined as providing "employees with some degrees of autonomy in the selection of the starting and ending times for

their workday" (Pierce et al., 1989, p. 21). The freedom an employee has to start and stop their workday is usually restricted by the notion of a block of core hours that all employees work (Golembiewski, Hilles, and Kagno, 1974). For example, Company $\mathrm{A}$ might have core hours between $9 \mathrm{AM}$ and $3 \mathrm{PM}$ where all employees are expected to be at work. Outside of those core hours, employees are free to craft the schedule that meets their needs with some employees starting as early as 7 AM. It is assumed that the total number of hours worked per week by the employee will not change (e.g. 7.5 hours per day) just the start and stop time.

Employees working a flextime work arrangement are able to have some control over their work day by choosing to work the hours (within certain prescribed limits in most organizations) that best suit their personal life. It is possible that this increased control over one's work day could moderate the relationship between work/non-work demands and work-life conflict such that the employee can choose the working hours that better help them meet non-work obligations. For example, two working parents using a flextime schedule could arrange their schedule in such a way that the mother is able to bring their child to school before leaving for work and the father is able to pick up their child at the end of the school day. 
In Canada, the percentage of the workforce using flextime has doubled in a ten year period. While 17 percent used flextime in 1991 (Lipsett and Reesor, 1997), this figure increased to 35.2 percent in 2001 (Statistics Canada, 2004). In the United States, the 2002 NSCW reports that forty-three percent of respondents are able to use flextime (Bond et al., 2003). This figure represents an increase of 14 percent from ten years earlier (Galinsky et al., 2004).

\subsubsection{Advantages and Disadvantages of Flextime}

The literature suggests a number of potential advantages and disadvantages of flextime for both employer and employee. Employer advantages include reduced absenteeism (Baltes et al., 1999; Christensen and Staines, 1990; Daus et al., 1998; Harvey and Luthans, 1979; Kim and Campagna, 1981; Pierce et al., 1989; Ralston, Anthony and Gufstafson, 1985), lateness (Baltes et al., 1999; Christensen and Staines, 1990; Dalton and Mesch, 1990; Daus et al., 1998; Morgan, 1977; Pierce et al., 1989) and turnover (Baltes et al., 1999; Christensen and Staines, 1990; Daus et al., 1998; Peterson, 1980; Pierce et al., 1989); improved worker productivity (Baltes et al., 1999; Christensen and Staines, 1990; Nollen and Martin, 1978a; Pierce et al., 1989; Ronen and Primps, 1980; Welch and Gordon, 1980), organizational commitment (Pierce and Newstrom, 1982; Welch and Gordon, 1980) and job satisfaction (Bohen and Viveros-Long, 1981; Christensen and Staines, 1990; Dunham et al., 1987; Kim and Campagna, 1981; Nollen, 1979; Orpin, 1981; Pierce et al., 1989; Ronen and Primps, 1980) as well as the ability to attract and retain employees (Hurrell and Colligan, 1985). 
Employer concerns include potential difficulty in scheduling meetings (Frankel, 1998; Nollen and Martin, 1978a; Welch and Gordon, 1980), potential for poor communications among staff (Nollen and Martin, 1978a; Welch and Gordon, 1980), and difficulty in scheduling the coverage of certain jobs at needed times (Christensen and Staines, 1990; Daus et al., 1998; Nollen and Martin, 1978a; Welch and Gordon, 1980).

Employees can potentially benefit from flextime by better coordinating their family responsibilities (e.g. couples with school-aged children could arrange their workday so that one spouse drives the children to school in the morning and the other spouse can pick up the children after school; Bohen and Viveros-Long, 1981; Kim and Campagna, 1981; Welch and Gordon, 1980) and many studies report employee satisfaction with their leisure time increased after employees adopted a flextime schedule (Evans, 1973; Ronen and Primps, 1980). The literature does not identify any disadvantages of flextime from the employee perspective.

\subsubsection{Impact of Flextime on Work-Life Conflict}

The findings with respect to this issue are varied. Some studies find that flextime helps reduce work-life conflict, others report mixed (e.g. flextime helps women but not men) or neutral (i.e. flextime did not make a difference) results. Still other studies find a negative impact (i.e. flextime exacerbated work-life conflict). Studies reporting each of these types of outcomes will be reviewed below. 
There is some evidence to suggest that flextime can help employees improve work-life balance. Many of the earliest efforts to study the impact of flextime on work-life conflict tended to find that flextime did make a difference (i.e. found that flextime was negatively associated with work-life conflict).

Winnett, Neale and Williams (1982) studied 71 working parents in Washington, DC and found that flextime helped alleviate some of the difficulties in coordinating work and family responsibilities. A study by Hicks and Klimoski (1981) $(n=210)$ reported that employees who had a flextime arrangement reported less work-life conflict than did employees who had conventional arrangements. Similarly, Barling and Barenburg (1984) in a study of 54 South African employed mothers found that those who had flextime arrangement reported less work-life conflict than did employed mothers who had a regular fulltime schedule. As well, the Barling and Barenburg study reported less parentspouse and parent-self role conflict as a result of flextime. A study by Walker, Fletcher and McLeod (1975) of 125 employees in two British civil service offices found that those on flextime reported greater control over work and personal life.

A more current study supported previous findings that flextime was associated with lower levels of work-life conflict. Bond et al. (2003) in the most recent National Study of the Changing Workforce (NSCW) survey of 2, 810 respondents reported that employees with access to flextime reported less workfamily interference. Employees with flextime access were more likely to report no work-family interference (32\%) and low levels of negative work-family spillover (34\%) compared to those with little access to flextime (only $19 \%$ of whom 
reported no work-family interference and $19 \%$ reported low levels of negative work-family spillover; Bond et al., 2003).

Some studies have found that flextime helped one gender but not the other. For example, Lee (1983) who surveyed 100 employees found fathers working flextime increased time spent in child socializing activities (relationship and development activities such as playing, teaching, talking). There was no change in child-related activities for women. Fast and Frederick (1996) using data from the 1992 Canadian General Social Survey (5,060 employed Canadians) stated that flextime was associated with lower levels of perceived time stress (akin to role overload conceptually) in women but not men. White et al. (2003) using two large samples $(1,915$ and 1,474$)$ in the United Kingdom concluded that flextime reduced work-family interference for women but not men.

It should be acknowledged, however, that such positive and mixed findings on flextime and work-life conflict are not universal. Secret and Sprang (2001) of 374 working parents concluded that the ability to modify work arrangements did not reduce the work-life role strain experienced by the respondents. Secret and Sprang (2001) argue that the mere existence of familyfriendly programs is not enough to resolve work-family issues.

Secret and Sprang's findings were echoed in previous work by Bohen and Viveros-Long (1981) and Shinn et al. (1989). The sample sizes were 706 for the Bohen and Viveros-Long (1981) study and 644 for the Shinn et al. (1989) study. In both those studies, the existence of formal flextime programs were of little benefit to working parents. It could be that the type of limited flexibility offered by 
flextime (i.e. the opportunity to adjust the start and stop times by an hour or two) may not be that useful to working parents compared with the ability to take time off midday to take a sick child to the doctor or to attend a parent-teacher conference (Shinn et al., 1989). Furthermore, Shinn et al. (1989) noted that respondents found that inflexible pick up and drop off times at daycares limited the usefulness of flextime. These authors concluded that "flextime at work may not benefit parents until schools, day care centers, and other institutions are also more flexible" (Shinn et al., 1989, p. 52).

In a study of 179 employees in a wide variety of family situations and workplaces, Clark (2001) examined the relationships between three aspects of work culture (flexibility of working hours, flexibility of the work itself and supportive supervision) and work/life balance. Flexibility of work times was unassociated with any of the work or personal outcomes included in the study including the ability to balance work and family. Saltzstein et al. (2001) using data from a large sample of U.S. Federal Government employees $(32,103)$ found that flexible scheduling (i.e. flextime) had no significant impact on work-life balance.

There have even been rare instances where the results indicate that those working flextime had higher levels of work-life conflict than those on a regular work schedule. For example, Dunham et al. (1987)'s survey of 102 employees concluded that those who switched to a flextime schedule reported more interference with personal activities involving friends and family. 
The reasons for the contradictory findings are speculative but there are some sensible possibilities. Zedeck and Mosier (1990) point out that while flextime may help people to better juggle their multiple priorities, the program does nothing to mitigate the level of demands at home (i.e. child care and household duties). Sharpe, Hermse and Billings (2002) observe that many of the studies in the literature which examine the impact of flextime on work-life conflict are now dated (e.g. Nollen, 1979; Walker, Fletcher and McLeod,1975) and do not reflect recent growth in flextime programs. Sparks, Faragher and Cooper (2001) speculate that the inconsistent findings of flextime and work-life conflict may lie in whether or not the employees had a choice over their schedule. Perhaps employees who are assigned their schedule may find that schedule does not meet their needs (Sparks, Faragher and Cooper 2001). Sparks et al. (2001) point to the work done by the Canadian Aging Research Network (CARNET) that supports the critical role of employee choice over scheduling. The researchers found that workers who were able to choose their worktime schedules reported greater overall well-being and reduced work interference with family life, compared with employees assigned to their work schedule. This role of perceived flexibility will be addressed more fully in Section 6.2 .

\subsubsection{Telecommuting}

Duxbury and Higgins (2002a) define telecommuting as an alternative work arrangement where an employee regularly works at home one or more complete workdays a week in lieu of working in the office. Telecommuting can give 
employees increased control over their work location. This increased control can potentially improve the employee's ability to meet his/her work and family obligations by helping the employee time when certain tasks are accomplished. For example, a working father may choose to split his workday in such a way that he works half days in the morning while his child is at a half-day preschool and then finishes his work day in the evening when the child is asleep.

In response to the question of how many people in Canada and the United States telecommute, there appears to be no easy answer. A recent article by Duxbury and Higgins (2002a) suggests telecommuters are difficult to count and estimates vary substantially from source to source because of methodological reasons such as how the researchers obtained their sample and the definition of telecommuting that was used. Duxbury and Higgins (2002a) citing Nie (1999) note that with the speed and cost of computation and connectivity changing monthly, any estimate of how many people telecommute is likely to become outdated very quickly. Given this caveat, figures on the use of telework arrangements are provided below.

Estimates of how many North American workers telecommute vary significantly depending on whether or not the estimate counts those who telecommute for only part of their workweek. Conservative estimates tend to count those telecommuters who work exclusively from home. For example, the International Telework Association and Council (sponsored by AT and T) in their nationally representative survey of American workers estimates that 6 million Americans work exclusively from home (which represents $4.3 \%$ of the total U.S 
labour force; Davis and Polonko, 2001). Data from Statistics Canada (2004) shows only $6.4 \%$ of employees were paid within normal scheduled hours to telecommute. A similar percentage of telecommuters (i.e. $6 \%$ ) was reported by Schweitzer and Duxbury (2006) when they examined the 2001 Workplace and Employee Survey (WES). Statistics Canada (2004) reports that another $2.6 \%$ of employees report doing paid overtime from home and an additional $14.1 \%$ perform unpaid overtime from home.

However, once one factors in those employees who telecommute part of their work week but who spend part of their work week working from a second location (e.g. an office), the percentage of telecommuters increases. Recent numbers from the U.S. Bureau of Labour Statistics (BLS, 2005c) report a figure of 20.7 million telecommuters and home office workers in 2004 (15\% of the U.S. labour force). Figures by BLS do include those telecommuters who do not telecommute on a full-time basis.

According to Duxbury and Higgins (2002a), it is safe to say that based on the available literature between $8 \%$ and $10 \%$ of the U.S. workforce telecommute at least one full day per week and that millions more employees telecommute on an occasional basis. This estimate is consistent with Galinsky, Bond and Hill's (2004) figures from the recent $2002 \mathrm{NSCW}$ report which noted that $2 \%$ of employees work primarily at home, while another $9 \%$ spend part of their regular work week working from home. 


\subsubsection{Advantages and Disadvantages of Telecommuting}

Potential employer advantages include increases in productivity (Caudron, 1992; DiMartino and Wirth, 1990; Hartman et al., 1992; Huws, 1992; Metzger and Von Glinow, 1988), morale (Hill et al., 1998; Riley and McCloskey, 1997), job satisfaction (Chapman et al., 1995), and decreases in absenteeism, turnover, and office overhead (Baruch, 2000; Boyd, 2000; Riley and McCloskey, 1997). Furthermore, telecommuting could be an aid in the recruitment and retention of skilled workers (Boyd, 2000; Chapman et al., 1995; McCloskey and Igbaria, 1998).

Potential employer disadvantages include loss of synergy from employee informal social interactions which often lead to new ideas and creative solutions (Baruch, 2000), difficulties with communication (Baruch, 2000; Chapman et al., 1995), potential loss of employee commitment and loyalty (Baruch, 2000; Chapman et al., 1995), and security concerns about company equipment and confidential information (Chapman et al., 1995).

Potential employee advantages include improved performance and better productivity (Riley and McCloskey, 1997), better work-life balance (Duxbury, Higgins, and Neufeld, 1998; Madsen, 2003; Riley and McCloskey, 1997), reductions in commuting time and the stresses associated with commuting (Baruch, 2000; Chapman et al., 1995) and an increased potential for living where the employee wishes instead of where the employer is located (Baruch, 2000; Chapman et al., 1995). 
Potential employee disadvantages include social isolation (Baruch, 2000; Chapman et al., 1995), detachment from social interactions (Baruch, 2000), reduced career advancement prospects (Baruch, 2000; Riley and McCloskey, 1997) and negative health consequences (e.g. repetitive strain injuries are more likely if the home environment is unsuitable; Baruch, 2000; Chapman et al., 1995).

\subsubsection{Impact of Telework on Work-Life Conflict}

There are two schools of thought on the impact of telecommuting on worklife conflict. One suggests telecommuting decreases work-life conflict while the other suggests telecommuting may actually increase work-life conflict. The reasons for each position will be discussed followed by a review of the empirical literature. How can telecommuting reduce work-life conflict? Duxbury and Higgins (2002a) provide several suggestions including increased work-time and work-location flexibility and increased control over the pacing and scheduling of work both of which should make it easier for employees to juggle personal responsibilities around their work hours. Telework may also decrease time in commuting which may give employees more time for their families and the possibility of providing emergency care for sick children (Duxbury and Higgins, 2002a).

There is some empirical support for the notion that telecommuting reduces work-life conflict. Zedeck and Mosier (1990) noted that the early telecommuting literature speculated that working at home might reduce interrole conflict thereby 
improving work-life balance. The notion that telecommuting helped employees balance work and life was supported by empirical data collected by Duxbury, Higgins, and Neufeld (1998). It should be noted that the Duxbury, Higgins, and Neufeld (1998) study was methodologically rigorous. (i.e. pre-test, post-test control group design; use of multiple data collection techniques). There were 54 teleworkers and 36 employees in the control group. Teleworkers had significantly lower levels of both work-family interference and family-work interference. Role overload did decrease for the teleworker group but was not statistically significant. The control group's levels of role overload, work-family interference and family-work interference did not change significantly (Duxbury, Higgins and Neufeld, 1998).

Madsen (2003) studied two hundred and twenty-one employees in seven corporations in the Minneapolis/St. Paul area. Individuals who worked from home at least two days per week $(n=98)$ had lower levels of both work-family conflict and family-work conflict compared to those employees who worked onsite at their employer's place of business $(n=123)$. Lapierre and Allen (2006) surveyed 230 employees and found that telework was associated with less family-work interference.

DeLay (1995) studied the impact of telecommuting on both work-family interference and family-work interference. DeLay (1995) concluded that while telecommuters $(n=59)$ had lower levels of work-family interference than nontelecommuters $(n=46)$, there were no statistically significant differences between telecommuters and non-telecommuters' levels of family-work interference. 
DeLay (1995) speculates that telecommuting "is an intervention specifically targeted toward relieving stress due to job structure, and, thus, does not address family issues" (cited in Madsen, 2003, p. 39).

The main concern expressed by those who feel telecommuting has the potential to increase work-life conflict is the notion of permeable boundaries (Shamir and Solomon, 1985). According to researchers who espouse this view, when non-telecommuters leave work there is a clear separation and distance that they can put between themselves and their office. For telecommuters, on the other hand, the "office" is always there. This omnipresent office may lead employees to work extra hours thus making it more difficult to juggle work and family schedules (Shamir and Solomon, 1985). This desire to work harder and longer could potentially lead to workaholism and burnout (Shamir and Salomon; 1985) or what Duxbury and Higgins (2002a) call the "cyberspace sweatshop". It should be noted however that neither Duxbury, Higgins and Thomas (1996) nor Baruch (2000) found any evidence supporting the link between telework and the prevalence of 'computer widows' (i.e. a phenomenon whereby people become addicted to continuing to work on their computers and practically abandon family life).

There is some empirical support for the notion that telecommuting is either neutral or deleterious in its impact on work-life conflict. For example, a study by Hill, Hawkins and Miller (1996) concluded that employees using flexplace (i.e. telecommuting) were not more successful in achieving work-life balance relative to those without the flexibility to telecommute. This conclusion was echoed in 
McCloskey (1998) who examined the impact of teleworking on work-life conflict using a sample of 89 telecommuters and 71 non-telecommuters. McCloskey concluded that there were no statistically significant differences in the levels of work-life conflict between the telecommuters and non-telecommuters. McCloskey did note, however, that the majority of her telecommuters (i.e. 67\%) only worked from home three days or less per month. Fast and Frederick (1996) using data from the 1992 Canadian General Social Survey (5,060 employed Canadians) concluded that telecommuting was not associated with lower levels of perceived time stress (similar to role overload).

Not only are there studies suggesting telecommuting is not advantageous in helping balance work and family commitments, some studies indicate that telecommuters are worse off. For example, Baruch (2000) interviewed 62 British teleworkers and found telework was associated with both higher levels of worklife conflict and the perception that family stress was now worse compared to the levels they felt before they moved to teleworking.

A larger sample survey reached a similar conclusion to Baruch (2000). Saltzstein et al. (2001) reported that for their subsample of 2,665 employees, telecommuting exerted "a significant negative influence on satisfaction with worklife balance" (Saltzstein et al., 2001, p. 463). Saltzstein et al. (2001) suggest it is possible that parents with children at home might find working at home frustrating and might feel that interruptions had an adverse impact on both their productivity and their relationships with their children (Christensen, 1988; Metzger and Von Glinow, 1988). 
What was surprising was the fact that Saltzstein et al. (2001) found that telecommuting had negative repercussions not only for parents but also for childless workers. In this regard, he notes that "it may be that working at home generates cross-pressures not just for those with children clamoring for attention, but also for those facing greater demands (and less time) for household care and maintenance (which are more difficult to ignore working at home)" (Saltzstein et al., 2001, p. 463).

In conclusion, the results are mixed with respect to whether or not telecommuting helps to reduce work-life conflict. It is suspected that differences in sample sizes, the way that the variables were operationalized, the number of days per week the respondent spent telecommuting, and whether or not certain confounding variables were controlled could explain the reason for the different conclusions about work-life conflict and telecommuting.

\subsubsection{Shiftwork}

In attempting to define shiftwork, it is important to recognize that the literature provides no universal definition of shiftwork. Johnson (1997) attributes this to the fact that shiftworkers are found in a variety of different industries and work in a variety of circumstances (Johnson, 1997). Pierce et al. (1989) define shiftwork as a pattern of working-hour arrangements whereby employees work defined blocks of time on a regular basis. It is also important to note that shifts by their very nature can vary in a number of ways: by the number of hours that constitutes a shift (e.g. 8 or 12 hour shifts), the start and stop times for these 
shifts (e.g. some evening shifts may start at 3, 4, or 5 PM), the interruptions experienced in hours worked (i.e. some employees working at a before and afterschool program work a split shift where they may work from 7 AM until 10 AM and return the same day to work from 3 PM until 6 PM) and whether the shift is permanently fixed (i.e. the employee only works days, evenings or nights) or rotating (i.e. the employee moves from one shift schedule such as evenings and switches to nights and so on; Johnson, 1997).

A recent publication by Johnson, Lero and Rooney (2001) noted that in $1995,32 \%$ of the Canadian labour force were working rotating or non-day shifts. The Monthly Labor Review reports that 15 million Americans in 1997 (almost $17 \%$ of the labour force) work non-day shifts (Beers, 2000).

Shiftwork was included in this study for two key reasons. First, the fact that shiftwork is a commonly used work arrangement means that its impact on work-life conflict needs to be assessed. Second, it provides an effective contrast to the other alternative arrangements under consideration (compressed work week, flextime and telecommuting) in this thesis. While the other work arrangements can be hypothesized to increase an employee's control over their work hours (flextime), work week (compressed work week) or work location (telecommuting), shiftwork is typically associated with lower levels of control over the timing of work (Johnson, 1997). In most workplaces, employees are assigned their shifts and have little say over when they work. For example, Johnson (1997) found that schedule control was very low (i.e. average score of less than two on a five point scale) for rotating shiftworkers in her study. The 
inability to tailor their schedule to their personal life would suggest that this group of workers will experience more difficulty balancing work and family.

\subsubsection{Advantages and Disadvantages of Shiftwork}

Similar to the other work arrangements discussed in this paper, the available research in the area enumerates a number of advantages and disadvantages of shiftwork. Advantages of shiftwork from the employer's point of view include: being able to meet customer/societal needs (e.g. call centers, emergency services; Walker, 1978), securing a greater utilization of capital invested in plant and equipment (Pierce et al., 1989; Sergean, 1971; Walker, 1978), and reducing the number of overtime hours through the introduction of an additional shift (from two shifts a day to three shifts; Pierce et al., 1989; CLMPC, 1997).

Employer disadvantages of shiftwork include: the possibility that employees who are sleep deprived as a result of working shifts could be less productive and risk serious injury (Folkard and Monk, 1979; Monk and Folkard, 1985; Pierce et al., 1989), scheduling difficulties with some employees refusing to work the less desirable shifts (Akerstedt and Torsvall, 1978; Wedderbaun, 1978 ) and turnover attributed to those who leave the company because they do not wish to continue working shifts (Pierce et al., 1989; Torsvall and Akerstedt, 1978).

According to Weiss and Liss (1989) and Pierce et al. (1989), there are many advantages for employees who work shifts including: the existence of shift 
premiums in some organizations, the possibility to work a second job (moonlighting), the appeal to students of juggling work and school schedules, and often less supervision on certain shifts (e.g. night shift). Furthermore, some people are just better suited to certain shifts because of their biological rhythms (Monk and Folkard, 1985).

Despite the aforementioned advantages of shiftwork, the literature makes note of the physical and social disadvantages of shiftwork for the employee. One key drawback to shiftwork is the interruptions to the employee's sleep schedule. Sleep disruption is a common complaint by the majority of shiftworkers (60-70 percent; Costa, 1996; Costa et al., 2000; Luna, French and Mitcha, 1997; Pierce et al., 1989; Rutenfranz et al., 1985; Tepas and Carvalhais, 1990). Monk and Folkard explain that our circadian system "set(s) the stage for sleep at some times of day and wakefulness at others" (Monk and Folkard, 1992, p. 27). When one's circadian system is misaligned, this is disruptive to an individual's ability to sleep restfully and to function when they are awake. The result is an increase in the body's production of cortisol and a rise in body temperature when the circadian system thinks it is time for the body to wake (Monk and Folkard, 1992). These physiological changes interrupt the natural sleep process and can lead the shiftworker to feel hungry before he/she has slept restfully for a full eight hours. Furthermore, when one is awake working a night shift, the circadian system can make staying alert difficult due to body temperature drops and melatonin surges (Monk and Folkard, 1992). 
A shiftworker may also be vulnerable to outside influences that result in interrupted sleep. The reality for shiftworkers is that the majority of people are awake and performing day-to-day tasks while the shiftworker attempts to sleep. Regardless of how careful shiftworkers try to block out noise from the outside world, Monk and Folkard (1992) note that many shiftworkers complain of sleep disruptions caused by traffic and other noises outside their control.

The impact of the misalignment of the circadian system and sleep disruptions can lead to many problems for shift workers. There is concern that certain monotonous tasks (e.g. driving, quality control) while sleep-deprived could lead to a higher risk of serious accidents and injury (Costa, 1996; Folkard, Akerstedt, Macdonald, Tucker and Spencer, 2000; Folkard and Monk, 1979; Monk and Folkard, 1985; Smith, Folkard and Poole, 1994). Other consequences of a circadian system out of sync are feelings of malaise and fatigue (Rutenfranz et al., 1977; Czeisler et al., 1982). An exploratory study of nuclear power plant workers by Smith and Folkard (1993) echo the findings of the previous authors. This study examined the impact of shiftwork on outcomes such as perceived health, sleep and social problems, as well as levels of alertness. Overall, the night shift proved to be the most problematic in terms of decreases in alertness, sleep duration, and sleep quality. Other studies found a strong link between working a night shift and sleep troubles (Akerstedt, 1985; Rutenfranz et al., 1985).

Several researchers (Angersbach et al., 1980; Costa, 1996; Costa et al., 2000; Rutenfranz et al., 1977; Parkes, 1999, Smith et al., 1999; Tucker, Smith, 
Macdonald, and Folkard, 2000; Vener, Szabo, and Moore, 1989) have noted that shiftworker concerns about gastro-intestinal troubles are another frequent complaint. Problems associated with this type of distress can range from more serious conditions such as ulcers to milder troubles such as change of appetite, constipation, diarrhea or indigestion. Researchers have also found a positive association between shiftwork and psychological distress (Barton, 1994; Barton, Smith, Totterdell, Spelten, and Folkard, 1993; Costa, 1996; Tucker, Barton, and Folkard, 1996; Williamson, Gower and Clarke, 1994).

\subsubsection{Impact of Shiftwork on Work-Life Conflict}

There is some intuitive appeal to the notion that employees working shiftwork would have higher levels of work-life conflict than those working a conventional 9 to 5 schedule. Shift workers are not as easily able to schedule evening and weekend activities as their friends who work a regular 9 to 5 day (Weiss and Liss, 1989). Furthermore, certain shiftworking professions such as police officers, medical staff and fire fighters are required to work holidays such as Christmas, New Year and Easter. Therefore, it is easy for the shiftworker to feel out of sync with friends and family (Fenwick and Tausig, 2001). A review of the literature as discussed below suggests, however, there is no consensus on the impact of shiftwork on work-life conflict.

Empirical studies linking shiftwork to higher levels of work-life conflict (relative to those not working shiftwork) do exist. For example, a study of 286 hotel employees (Shamir, 1983) found a strong positive association between shiftwork and work-nonwork conflict. A survey of 828 Canadian police officers by 
Burke (1988) reported that police officers working shiftwork reported greater work-family conflict than those officers working a regular schedule. Staines and Pleck (1983) examined the data from the 1977 QES (1,515 respondents) and found that working shiftwork was associated with increased levels of work-family conflict and reduced levels of marital satisfaction, marital happiness and family satisfaction. White and Keith's (1990) study of 1,668 shiftworkers also found that shift work was associated with reduced marital happiness, more reports of parental difficulties with their children and a higher chance of becoming divorced. Bohle and Tilley (1989) interviewed sixty Australian female student nurses and concluded that shiftwork was associated with lower levels of work-family conflict. Other studies linking shiftwork to higher levels of work-life conflict include: Kingston and Nock (1985); Nock and Kingston (1984); Knutsson et al., (1986); Nock and Kingston (1988); Tasto and Colligan (1978); Tepas (1985).

Two large-sized sample studies do not, however, replicate these findings. For example, Fast and Frederick (1996) examined the 1992 General Social Survey data of 5,060 employed Canadians and they concluded that shiftwork was not associated with higher levels of perceived time stress (similar to role overload). Fenwick and Tausig (2001) examined the 1992 National Study of the Changing Workforce (NSCW) in which 2,905 employed American workers were interviewed. Fenwick and Tausig examined the impact of a variety of shift arrangements on both lack of work-home balance and conflict between workhome. In only one case (weekend work only) was a shiftwork arrangement statistically significant and positively associated with conflict between work and 
home. In the other two cases (i.e. rotating shift, non-day shift), there was no statistically significant positive association between shiftwork and conflict between work and home. Furthermore, there was no statistically significant negative association between shiftwork and work-home balance for any of the shiftwork arrangements (i.e. rotating, non-day, weekend only).

In conclusion, there is some evidence to suggest an association between shiftwork and work-life conflict but the results are certainly not unequivocal. It is hoped that the findings from this thesis will help to clarify this relationship.

Finally, it should be noted that for all four work arrangements (i.e. flextime, compressed work week, telework and shiftwork), prior efforts to examine the relationship between the work arrangement and work-life conflict have focused on establishing a direct effect. Despite the use of several different academic databases (e.g. ABI, Psyclnfo and Social Sciences Index), we were unsuccessful in finding any empirical studies which examined the role of a given alternative work arrangement or shiftwork as a moderator of the relationship between demands (either work or non-work) and work-life conflict. It is hoped that this thesis will make a contribution to the literature in this area.

\subsection{Perceived Flexibility}

This section will present evidence that perceived flexibility can be considered to be a form of control over work-life interface. First, perceived flexibility will be defined. Second, we will link perceived flexibility to the idea of control. Third, we will review the literature on perceived flexibility and its relationship to work-life conflict and alternative work arrrangements. 
A review of the literature on perceived flexibility suggests a variety of names have been used to characterize the construct we are calling perceived flexibility including: schedule control (e.g. Fenwick and Tausig, 2001; Voydanoff, 1988), schedule flexibility (e.g. Smith Major et al., 2002), schedule inflexibility (e.g. Greenhaus et al., 1989), supervisor flexibility (e.g. Greenberger, Strasser, Cummings, and Dunham, 1989), and perceived job flexibility (e.g. Hill et al., 2001). Despite the diversity of terms associated with perceived flexibility, the conceptualization remains consistent. Perceived flexibility (or its equivalent moniker) is essentially the amount of control a respondent has over their work hours and their work location (Duxbury and Higgins, 2001).

Perceived flexibility can be seen as a global form of control over the workfamily interface which encompasses perceptions of both when one works and where one works. An employee with greater perceived flexibility should be better able to balance his/her work and non-work responsibilities because he/she can decide the timing of his/her work in accordance with his/her non-work obligations. This enhanced sense of control should lead to a lower level of work-life conflict.

This construct recognizes that while the availability of an alternative work arrangement should theoretically enhance control over the timing of one's work day, this arrangement by itself may not be sufficient to help the employee balance work and family commitments. For example, if the work arrangement is imposed on the employee or if the workload, work culture and/or the employee's supervisor make it difficult for an employee to take advantage of the flexibility inherent in the alternative work arrangement, then the employee is only nominally 
working such an arrangement. In this case, a particular work schedule would not be linked to an increased sense of control.

Alternately, an employee who works a fixed schedule according to the official company rules (i.e. on the books) but who has a say in where and when they work may experience a higher amount of actual control in comparison to their alternative work arrangement counterpart.

In addition to the fact that perceived flexibility has many different names, the way it has been measured has also varied in the literature. Several authors have used a one or two item measure for perceived flexibility. For example, in the NSCW studies, Bond et al. $(1998,2003)$ used one item (i.e. control over scheduling work hours). Other authors using the NSCW data (e.g. Anderson et al., 2002; Fenwick and Tausig, 2001) also report using the same item to measure perceived flexibility. Greenhaus et al. (1989) used a two-item measure for schedule inflexibility (e.g. how flexible is your work schedule). Other authors (e.g. Duxbury and Higgins, 2001; Major Smith et al., 2001; Thomas and Ganster, 1995) have used multi-item measures of perceived flexibility. As seen in section 6.2.1, regardless of the way perceived flexibility is defined and measured, the results reported are remarkably consistent.

Literature will be reviewed in this section to support the decision to treat perceived flexibility as an independent variable (models 1-3), a moderating variable (models 1-3) and a dependent variable (model 4). The literature supporting the negative association between perceived flexibility and work-life conflict will be presented first. Second, the potential for perceived flexibility to 
moderate the relationship between demands and strain will be discussed. Third, the possible use of perceived flexibility as a dependent variable will be explored.

\subsubsection{Relationship Between Perceived Flexibility and Work-Life Conflict}

Inter-role conflict (Kahn et al., 1964) positions employee's work and nonwork demands as competing demands. Perceived flexibility helps employees fulfill these two sets of role demands (i.e. work demands and non-work demands) by reducing the probability that employees will have to simultaneously perform work and non-work demands at the same time. Heightened levels of perceived flexibility will enable employees to better balance and prioritize their competing work and non-work demands thereby reducing their levels of work-life conflict (relative to their counterparts with limited or no perceived flexibility).

There is some empirical evidence to indicate that perceived flexibility is associated with lower levels of work-life conflict. Several of these studies employ large samples and use the term schedule control to denote perceived flexibility (Anderson et al., 2002; Bond et al., 1998, 2003; Fenwick and Tausig, 2001; Galinsky, Kim and Bond, 2001; Pleck, Staines and Lang, 1980; Staines and Pleck, 1983).

Data from the two most recent NSCW reports (Bond et al., 1998, 2003) indicate that perceived flexibility (operationalized as schedule control) is associated with less negative spillover from work to home. Bond et al.'s sample sizes were 2,877 (1998) and 2,810 respondents (2003). Furthermore, data from Galinsky, Kim and Bond (2001) $(n=1,003)$ suggests that perceived flexibility is 
associated with lower levels of feeling overworked. While $45 \%$ of those who say they cannot change their work schedules to be able to work their preferred hours experience high levels of feeling overworked, only $33 \%$ of those who can change their work schedules felt this way. Anderson et al. (2002) using data from the 1997 National Study of the Changing Workforce (2,248 respondents) reported that perceived flexibility was associated with less work-family conflict. Fenwick and Tausig (2001) used data from the 1992 National Study of the Changing Workforce (2,905 respondents) and concluded that perceived flexibility significantly increases work-home balance. Two other studies (Pleck, Staines and Lang, 1980; Staines and Pleck, 1983) using the 1977 QES $(1,515$ respondents) concluded that schedule control is negatively associated with workfamily conflict.

Other researchers using a different name for perceived flexibility came to similar conclusions about the association between perceived flexibility and lower levels of work-life conflict. Hill et al. (2001) used data from a 1996 IBM work and life issues survey in the United States ( $N=6451)$. Given the same workload, individuals with perceived job flexibility (in both timing and location of work) had more favourable work-life balance. This flexibility enabled employees to work longer hours before experiencing conflict between work and family. Job flexibility was found to be particularly beneficial for parents who found this flexibility useful in dealing with unpredictable family circumstances such as a child becoming ill while at school (Hill et al., 2001). 
Large sample Canadian studies also support the notion that perceived flexibility (operationalized as control at work) is associated with lower levels of work-life conflict. For example, three different studies (Work and Family Study, Work and Home Life Study and Workplace Flexibility Study) by CARNET (the Canadian Aging Research Network) surveyed large numbers of respondents in Canada and reached similar conclusions. The first study (i.e. the 1993 Work and Family Study) surveyed respondents ( $\mathrm{N}=5496)$ from eight Canadian organizations representing five employment sectors (government, education, financial services, health care and manufacturing). The second study (i.e. the 1994 Work and Home Life Study) surveyed employees ( $N=1700)$ from three Canadian organizations. The third study (i.e. the 1995 Workplace Flexibility Study) surveyed workers $(\mathrm{N}=354)$ from a large Canadian financial institution. All three studies concluded that employees who had work arrangements that they requested reported lower levels of work-family interference than employees who were hired into or assigned to their current work arrangement (Gottlieb, Kelloway and Barham, 1998).

Other studies with smaller sample sizes have also concluded that perceived flexibility is associated with lower levels of work-life conflict. For example, Greenberger et al. (1989) concluded that supervisor flexibility (operationalized similarly to perceived flexibility) was negatively associated with role strain in a sample of 169 married women with children who were employed in Southern California. Thomas and Ganster (1995) gathered data at 45 acutecare facilities from 398 health professionals who had children aged 16 years or 
younger at home. The authors concluded that perceived flexibility (operationalized as perceptions of control over areas of work and family activities) was significantly and negatively associated with work-family conflict.

Another term for perceived flexibility is schedule flexibility. Two studies by two different sets of authors reported similar findings. Galambos and Walters (1992) studied 48 dual earner couples in British Columbia and concluded that lack of schedule flexibility was positively associated with role overload (operationalized as role strain) for both husbands and wives. Smith Major et al. (2002) surveyed 513 employees in a Fortune 500 company and noted a negative, statistically significant association between schedule flexibility and work-family interference.

In section 6.1.4, it was pointed out that shiftwork is notorious for not offering employees the opportunity to choose their schedule. Certain studies have suggested that there are certain benefits associated with employees being able to choose their own shift (a form of control). For example, Voydanoff (1988) found that those who had little choice in selecting their shift had higher levels of work-life conflict than those who were able to choose their shift. Voydanoff's study is based on a subsample ( 757 working men and 270 working women) of the 1977 QES. Havlovic, Lau and Pinfield (2002) surveyed 520 nurses in British Columbia. Those nurses who worked their preferred shift reported lower levels of interference with activities with family and friends. Barton (1994) studied 587 nurses and midwives in England and Wales and concluded that those who chose to work night shifts reported fewer health, sleep, social and domestic complaints 
compared to those who did not choose to work nights. Barton, Smith, Totterdell, Spelten and Folkard (1993) surveyed 1,082 nurses and midwives (all of whom worked shiftwork) and concluded that those with higher levels of schedule control reported lower levels of social and domestic disruption than those with lower levels of schedule control.

It should be noted that some studies found mixed support for the notion that perceived flexibility is associated with lower levels of work-life conflict. Greenhaus, Parasuraman, Granrose, Rabinowitz and Beutell (1989) surveyed a matched sample of 119 men and 119 women who were dual career couples. Greenhaus et al. (1989) reported a statistically significant positive association between schedule inflexibility and work-family interference (operationalized as time-based conflict) for men but not for women. There was no statistically significant association between schedule inflexibility and strain-based conflict (i.e. role overload) for either men or women.

In conclusion, there is strong support for the notion that perceived flexibility is associated with lower levels of work-life conflict. Compared to the literature on alternative work arrangements, two conclusions can be reached for perceived flexibility. First, fewer studies have been conducted on the relationship between perceived flexibility and work-life conflict (in comparison to the number of studies on various alternative work arrangements and work-life conflict). Second, the studies on perceived flexibility and work-life conflict have produced strong support for that particular association whereas the results for alternative 
work arrangements and work-life conflict have been more mixed and less convincing.

\subsubsection{Perceived Flexibility Moderating Demands and Work-Life Conflict}

Relative to the number of studies indicating that higher levels of perceived flexibility are associated with lower levels of work-life conflict (i.e. testing a direct effect), there are only a few studies that have tested whether or not perceived flexibility moderates the relationship between demands and work-life conflict. Among these studies, the evidence supporting an interaction effect is mixed.

Early efforts testing for interactions with perceived flexibility were encouraging. For example, Staines and Pleck (1983) using the 1977 QES data $(1,515$ respondents) concluded that perceived flexibility (operationalized as schedule control) moderated the relationship between excessive weekly hours and work-family interference as well as the relationship between weekend work and work-family interference. Staines and Pleck (1986) using a subsample of the 1977 QES data (1,090 respondents) noted that perceived flexibility (operationalized as schedule control) moderated the relationship between shiftwork and work-family interference and that these buffering effects were more evident among women than men.

Later efforts at testing for interaction effects have been less successful. Thomas and Ganster (1995) computed a series of hierarchical regressions in which the interaction between perceived flexibility (operationalized as perceptions of control over areas of work and family) and work-family conflict on 
each of the strains (i.e. job satisfaction, depression, somatic complaints, cholesterol) was tested. Unfortunately, there were no statistically significant interactions. It is important to note that the thesis models are not the same as Thomas and Ganster's model since we use work-life conflict as a dependent variable in models 1-3 whereas Thomas and Ganster used work-life conflict as an independent variable.

Smith Major et al. (2002) surveyed 513 employees in a Fortune 500 company and examined the extent to which schedule flexibility buffers the relationship between long work hours and work-family interference. Again, no interaction effects were found. Both Thomas and Ganster (1995) and Smith Major et al. (2002) concluded that while perceived flexibility is associated with lower levels of work-life conflict (demonstrating a direct effect), these researchers were unable to detect a statistically significant interaction.

\subsubsection{Relationship Between Perceived Flexibility and Alternative Work Arrangements}

This thesis also considers the question of whether or not those who work an alternative work arrangement experience a heightened sense of perceived flexibility (model 4). It stands to reason that someone working an alternative work arrangement where they can choose the hours worked (flextime), days worked (compressed work week) or location (telework) should experience greater perceived flexibility than those working a conventional Monday-Friday, 9 to 5 work schedule where work day, work hours and location are fixed. 
Conversely, because shiftwork arrangements tend to be imposed on employees, those working shiftwork should report lower levels of perceived flexibility.

A review of the literature was unable to uncover any empirical literature that explores the association between alternative work arrangements and perceived flexibility (or any of its synonyms such as perceived schedule flexibility, schedule control, schedule flexibility, schedule inflexibility, supervisor flexibility and perceived job flexibility). The following databases were consulted: $A B I$, JSTOR, EBSCO and Scholar's Portal. It is hoped that model 4 of this thesis will help to contribute to our understanding of the association between alternative work arrangements and perceived flexibility. It is possible that certain alternative work arrangements are positively associated with perceived flexibility while other alternative work arrangements are not. Such a finding would have important consequences with respect to which arrangements an employer should consider.

Therefore, it is hoped that the empirical work on the association between alternative work arrangements and perceived flexibility will contribute to the literature by helping us understand the relationship between these two constructs.

\subsection{Supervisor Support}

This section will discuss the role of supervisor support as a form of control over work-life interface. First, supervisory support will be defined. Second, we will provide evidence supporting our decision to consider supervisor support as a form of control. Third, we will review the literature on supervisor support and its 
relationship to work-life conflict (models 1,2, and 3) and perceived flexibility (model 4).

Bahr (1995) notes that a variety of definitions for supervisor support appear in the literature. For example, Hughes and Galinsky (1988) define supervisor support as supervisor sensitivity (i.e. the extent to which a supervisor is viewed as supportive of the employee in the work role as well as flexible and understanding about the employee's family role). Other authors (e.g. Goff et al., 1990; Thomas and Ganster, 1995; Warren and Johnson, 1995) define supervisor support as the degree to which supervisors are willing to discuss family-related problems and be flexible when emergencies arise. While these definitions provide a good starting point, they are not a thorough depiction of what it means to be a supportive supervisor. A better, more comprehensive definition was developed by Duxbury and Higgins (2001) who defined the construct in behavioural terms as working for a supervisor who "is a good communicator, focuses on output rather than hours, demonstrates respect for employees and supports their career development" (Duxbury and Higgins, 2001, p. 55). Furthermore, Duxbury and Higgins (1997) identify supportive supervisor behaviours such as making it easier for employees to balance work and nonwork obligations by allowing schedules to be re-arranged and flexible work arrangements to be used.

Supervisor support can be considered a form of control because of the amount of influence he/she has over key facets of their employees' day. In many organizations, it is the supervisors who have the final say on whether or 
not an employee can vary his/her hours of work or use a particular alternative work arrangement such as telework. An individual's supervisor also assigns workload. It would appear, therefore, safe to assume that an individual's supervisor has the ability to impact the sense of control the employee has over his/her workday and their ability to balance work and family.

In thesis models 1-3, it is hypothesized that supervisor support can either influence work-life conflict directly (direct effect) or indirectly by moderating the relationship between demands and work-life conflict (interaction effect). In Model 4 , it is hypothesized that supervisor support can either influence perceived flexibility directly (direct effect) or indirectly by moderating the relationship between alternative work arrangements and perceived flexibility (interaction effect). The next section will provide a brief explanation as to why supervisor support is expected to perform as hypothesized in the thesis models followed by a review of the relevant literature.

First, the literature supporting the negative association between supervisor support and work-life conflict will be presented. Second, the potential for supervisor support to moderate the relationship between demands and work-life conflict will be discussed. Third, the literature supporting the positive association between supervisor support and perceived flexibility as shown in model 4 will be presented. Fourth, the literature supporting the potential for supervisor support to moderate the relationship between alternative work arrangements and perceived flexibility (also associated with model 4) will be presented. 


\subsubsection{Relationship between Supervisor Support and Work-Life Conflict}

The direct relationship between supervisor support and work-life conflict can be justified as follows. Supervisors can play a key role in helping employees reduce work-life conflict by informing employees about corporate policies (e.g. alternative work arrangements, personal leave days) designed to help employees balance work and family obligations (Galinsky and Stein, 1990). Voydanoff argues that when supervisors "respond positively to discussing and accommodating employees' family obligations, employees are likely to feel comfortable using available work-family policies" (Voydanoff, 2004, p. 403). Furthermore, Carlson and Perrewe (1999) argue that supervisors who are flexible by allowing an employee to leave work early when the employee has to respond to a family-related issue (e.g. a sick child or elderly relative) can help the employee lower his/her work-life conflict. Carlson and Perrewe (1999) also suggest that those supervisors who are open to hearing employees' familyrelated problems and providing solutions may help reduce an employee's sense of work-life conflict. Supervisors who model a sense of work-life balance in their own lives and who don't penalize those who value work-life balance are also vital in reducing an employee's work-life conflict (Duxbury and Higgins, 1997; Thomas and Ganster, 1995).

There is some evidence to support the notion that supervisor support is directly associated with lower levels of work-life conflict. Three studies supporting this notion come from large sample studies. For example, Anderson et al. (2002) examined the 1997 NSCW (2,248 respondents) and noted that supervisor support was associated with lower levels of both work-family 
interference and family-work interference. Bond et al. (2003) reported that in the latest NSCW report (sample size was 2,810 respondents), employees who rate their supervisor high in supervisor support report less interference between job and family and lower levels of negative spillover relative to those who reported low levels of supervisor support. Grzywacz and Marks (2000) used data from the National Survey of Midlife Development in the United States $(n=1,986)$ and reported that lower levels of support at work (operationalized as both coworker and supervisor support) was associated with higher levels of work-family interference (operationalized as negative work-family spillover) and higher levels of family-work interference (operationalized as negative family-work spillover).

Other studies with relatively fewer respondents than the NSCW have also supported the notion that supervisor support is associated with less work-life conflict. Secret and Sprang (2001) who studied the responses from 374 working parents concluded that parents with supportive supervisors were 2.7 times less likely to experience role strain. Thomas and Ganster (1995) with a sample of 398 health professionals concluded that supervisor support was significantly and negatively associated with work-family conflict. Frone, Yardley and Markel (1997) with their sample of 372 employed adults noted that supervisor support was negatively and statistically significantly associated with both role overload (operationalized as work overload) and work-family conflict. The association between supervisor support and family-work conflict was not statistically significant. 
Warren and Johnson (1995) studied 116 employed mothers and reported that supervisor support (i.e. supervisor flexibility and supervisor sensitivity) was negatively associated with work-family role strain such that those with supportive supervisors reported lower levels of work-family role strain. Goff, Mount and Jamison (1990) surveyed 253 respondents of a large, Midwestern United States organization and concluded that supervisor support was associated with less work-family conflict. Jones and Butler (1980) surveyed 181 married American sailors and remarked that supervisor support was negatively associated with work-family conflict (results were statistically significant).

Some studies provided mixed results. For example, Greenglass et al. (1988) concluded that supervisor support was associated with less role conflict for women (229 teachers) but not for men (327 teachers). Hughes and Galinsky (1988) came to the opposite conclusion when the studied data from the 1977 QES. Hughes and Galinsky (1988) studied a subsample of 285 working parents and noted that supervisor insensitivity to work and family role was associated with greater work-family interference for men only. Furthermore, some studies such as Greenberger et al. (1989) have found that supervisor support was not associated with role strain for any of the three groups (i.e. 80 married men, 169 married women, 72 single women) that were studied in Southern California.

It should be noted that not all studies found a positive or neutral relationship between supervisor support and lower levels of work-family conflict. Clark (2001) found that work/family balance was lower when employees had a supportive supervisor. The authors point out that it is unlikely that supportive 
supervision is responsible for decreasing the work/family balance. It is more likely that those experiencing high levels of work/family conflict are seeking out the help of supportive supervisors. However, because the study is crosssectional, causal relationships cannot be claimed.

\subsubsection{Supervisor Support Moderating Demands and Work-Life Conflict}

An argument can also be made that supervisors can also moderate the relationship between demands and work-life conflict (models 1-3). Supervisors often are responsible for delegating work to employees. Therefore, supervisors have some influence over the level of work demands experienced by employees. Therefore, supervisors can modify the workload of an employee which in turn can reduce the level of the employee's work-life conflict. The reverse situation is also true. A supervisor also has the power to increase an employee's workload and be insensitive to work-life balance issues. In this situation, a supervisor might actually strengthen that relationship between work demands and work-life conflict.

While there is some intuitive appeal to the notion that supervisor support can moderate the relationship between demands and strain, the limited empirical literature does not provide much support for this contention. For example, Carlson and Perrewe (1999) studying 314 state government workers concluded that there was empirical support for work social support (operationalized as the quality of the relationships respondents have with their supervisors, coworkers and subordinates) providing a direct effect (i.e. work social support was 
negatively associated with work-family conflict at a statistically significant level) but not a moderating effect.

Frone, Russell and Cooper (1995) studying 596 married, employed Erie County, NY respondents concluded that social support (measured using Cohen and Hoberman's 1983 Interpersonal Support Evaluation List) did not buffer the relationships between stressors such as work overload and work-family conflict and outcomes such as depression and psychological distress.

Parasuraman, Greenhaus and Granrose (1992) interviewed 119 men and 119 women who were partners in a two-career relationship and concluded that work social support did not buffer the relationships between role stressors such as role overload and work-family conflict. Ray and Miller (1994) studied 119 women (nurses and nursing assistants). They examined various forms of support including supervisor support and concluded that there were no significant buffering effects of support on the relationship between home/work stress and outcomes such as emotional exhaustion, depersonalization and reduced personal accomplishment. Lastly, Fu and Shaffer's (2001) study of 267 employees in a Hong Kong university tested for moderator effects of supervisor support on the relationship between hours spent on paid work and various forms of work-life conflict (e.g. time-based work-family interference, strain-based workfamily interference, and behavior-based work-family interference). No moderator effects of supervisor support were found.

An extensive search of the literature yielded only one study in which supervisor support moderated the relationship between demands and work-life 
conflict. A longitudinal Dutch study $(n=6883)$ found that supervisor support moderated the impact of work demands on work-family conflict (Jansen, Kant, Kristensen, and Nijhuis, 2003). Why Jansen et al. (2003) found a buffering effect and the other studies did not is a matter of speculation. One possible explanation is how work-life conflict is measured. The other researchers used multi-item, bi-directional measures for work-life conflict (i.e. both work-family interference and family-work interference were measured) whereas Jansen et al. (2003) only used a single item to measure work-family conflict (e.g. are you able to adequately combine work and family life?). A second explanation involves the type of study. The Jansen et al. (2003) study was the only longitudinal study (the other studies used a cross-sectional design).

The conclusion for supervisor support is similar to the one reached for perceived flexibility. There is ample evidence to indicate supervisor support is associated with lower levels of work-life conflict (direct effect) but no evidence to suggest supervisor support moderates the relationship between demands and strain. This thesis will however test both relationships in Models $1-3$ as it is hoped that the more rigorous methodology used in this thesis will allow us to make more definitive statements with respect to the relationship between supervisor support and work-life conflict.

\subsubsection{Relationship between Supervisor Support and Perceived Flexibility}

The direct relationship between supervisor support and perceived flexibility postulated by model 4 is based on the premise that supervisors can 
potentially enhance an employee's perceived flexibility. Supervisors, for example, often have the discretion to permit an employee to leave early or take unofficial leave in situations where a family-related emergency has resulted (Galinsky and Stein, 1990). For example, a child calls from school because he/she is sick. Such efforts by a supervisor to be sensitive to employees' nonwork obligations and permit the time to deal with non-work related problems could potentially enhance an employee's perceived flexibility.

A review of the literature did not uncover any empirical literature that explores the association between supervisor support and perceived flexibility (or any of its synonyms such as perceived schedule flexibility, schedule control, schedule flexibility, schedule inflexibility, supervisor flexibility and perceived job flexibility). The following databases were consulted: ABI, JSTOR, EBSCO and Scholar's Portal. Therefore, it is hoped that the empirical work on the association between supervisor support and perceived flexibility will contribute to the literature. Understanding the positive association between supervisor support and perceived flexibility would have important practical implications for the workplace. If we accept the premise that supervisor support is positively associated with perceived flexibility, then organizations need to help supervisors understand how enhancing an employee's perceived flexibility is helpful to the organization. Enhanced perceived flexibility is associated with lower work-life conflict which is, in turn, associated with higher levels of organizational commitment and job satisfaction and lower levels of intent to turnover. 


\subsubsection{Supervisor Support Moderating Alternative Work Arrangements and Perceived Flexibility}

An argument can also be made that supervisors can also moderate the relationship between alternative work arrangements and perceived flexibility (model 4). This hypothesis is based on the premise that supervisors may play a critical role in the use of other forms of control (i.e. alternative work arrangements, perceived flexibility) discussed in this thesis. As noted earlier, supervisors often have the final say on whether or not an employees' request to work a given alternative work arrangement will be approved. Given the assumption that a particular work arrangement may enhance an employee's sense of perceived flexibility, the supervisor can act as the "gatekeeper" in approving the employee's request. As noted in Duxbury and Haines, "until a manager has a favorable attitude towards a given work arrangement, little or nothing is going to happen" (Duxbury and Haines, 1991, p. 96). Duxbury and Haines (1991) go further to suggest that efforts to gain supervisor buy-in must focus not on the benefits to the employee but on the benefits to the organization. This view is echoed by Powell and Mainiero (1999) who note that the degree to which alternative work arrangements are actually available to the individual depends on the immediate supervisor. Supervisor support may help the employee enhance his/her flexibility through his/her support of the given alternative work arrangement. In this context, supervisor support can be seen as a moderator variable with supervisor support buffering the relationship between alternative work arrangements and perceived flexibility. 
Unfortunately, despite using numerous academic databases (i.e. ABI, JSTOR, EBSCO and Scholar's Portal), no literature was uncovered on the potential of supervisor support to moderate the relationship between alternative work arrangements and perceived flexibility. As previously noted, it is hoped the empirical work done in this thesis will contribute to the literature. Understanding whether or not supervisor support can moderate the relationship between alternative work arrangements and perceived flexibility has practical implications for the organization. If supervisor support does moderate this relationship, then organizations need to consider how to strengthen the supervisors' role so that he/she can properly support the relationship between alternative work arrangements and perceived flexibility. If we merely focus on the relationship between alternative work arrangements and perceived flexibility, we may miss out on the crucial role played by the supervisor in the success of this relationship. 


\section{Impact of Gender and Job Type}

"I have yet to hear a man ask for advice on how to combine marriage and a career" (Gloria Steinem).

(www.brainyquote.com/quotes/authors/g/gloria steinem.html)

The literature is supportive of the notion that both gender and socioeconomic status confound the association between work/non-work demands and work-life conflict. This section will justify the decision to control for both gender and socio-economic status in the thesis model. Gender will be discussed first. This will be followed by job type. The final part of the section looks at why gender and job type should be considered together.

\subsection{Gender}

This section will demonstrate how gender can be considered a covariate of the variables (i.e. demands, work-life conflict, control) included in model 1-4 in this thesis. How do societal expectations around gender inform our discussion? This question is best answered by providing a brief summary of gender role theory. This is followed by a discussion of how gender is associated with demands, work-life conflict and control over work-life interface.

Gender role socialization is the process through which "individuals acquire a gender identity as well as ways of acting, feeling, and thinking that are appropriate to the gender expectations of their society." (Robinson and Salamon, 1987, p. 124). Gender role theory suggests that men and women have a different core role that is primary to this gender identity (Barnett and Baruch, 1987). These core roles are driven by societal expectations and pressures. Societal norms support the notion that men's core role is the good provider and 
breadwinner (Barnett and Baruch, 1987; Simon, 1995; Voydanoff, 1987). Society also supports the notion that women's core role is the nurturer and caregiver of the family (Barnett and Baruch, 1987; Simon, 1995; Voydanoff, 1987).

A review of the literature indicates that there are gender differences in three of the main constructs in our models: demands, control and work-life conflict. The relationship between gender and these three constructs will now be examined.

\subsubsection{Gender Differences in Demands}

In examining the relationship between gender and demands, it is important to consider the full array of demands faced by today's workers: those associated with employment and family (i.e. non-work demands) and not total demands. The literature (e.g. Bianchi and Raley, 2005; Marini and Shelton, 1993; Pleck and Staines, 1985; Robinson and Godbey, 1997; Zick and McCullough, 1991) shows that if you look at total demands, there are no gender differences. Men do more paid work, women more unpaid work, but total time in work and family is the same. This finding supports the decision to look at work and non-work separately in thesis models 1-3.

Gender role theory would suggest that since men envision their core role to be the breadwinner and good provider, they will spend more time than women in paid employment. Furthermore, women, in their core role as nurturer will spend more time than men in household and childcare tasks. The empirical evidence provides strong support for these suppositions. 
There is strong evidence to indicate that North American men, on average, work longer hours in paid employment than women. Men in Canada work more hours on average in paid employment (Duxbury and Higgins, 2002b; Fast and Frederick, 2004; Higgins, Duxbury and Lee, 1994; Macbride-King, 1990). Furthermore, not only are the mean number of hours in paid employment higher for men, the percentage of men working a long work week is higher than the percentage of women. Shields (1999) used data from the National Population Survey conducted by Statistics Canada ( $n=2,181$ male and 1,649 female workers, aged 25 to 54 ) to show that on average men were more likely than women to work long hours in paid employment. Among those working fulltime (defined as those working a minimum of 35 hours per week), half of the men reported working 41 or more hours per week compared to $28 \%$ of women. Men working long hours averaged 55 per week while women averaged 51 hours per week. More men worked 60 hours or more per week (i.e. 32\%) than women (i.e. $19 \%)$.

This pattern also holds true in the United States. Data from the NSCW (Bond et al., 2003) shows that men work an average of 46.1 hours per week while women work an average of 39.8 hours per week. The Bureau of Labor Statistics' survey of 13,000 Americans noted a similar pattern: employed men worked almost one hour longer ( 7.9 hours) on a given day than employed women (7.1 hours) (BLS, 2006). Using data from the U.S. Current Population Survey, Jacobs and Gerson (2004) reported that employed men work an average of 43 hours per week while employed women work an average of 36 hours per week. 
Hill (2005) using data from the $1997 \mathrm{NSCW}(n=1,314)$ reported that fathers worked an average of 8.2 hours more per week in paid employment than mothers.

These data, however, only tell part of the story. According to Frankenhaeuser (1997), a person's total workload includes regular employment and overtime at work as well as housework, child care, and elder care.

Therefore, it is important to also examine the literature on non-work demands for both men and women. Deutsch (2006) suggests that gender role theory is helpful in explaining why women still perform an inordinate amount of the child care and household responsibilities (compared to their male counterparts). 'Women 'do gender' by doing housework and child care ... Men 'do gender' by refraining from doing housework and treating their paid employment as breadwinning" (Deutsch, 2006, p. 230). Deutsch (2006) argues that it is this focus on one's primary core role along with societal pressures to be the good provider (for men) or the good caregiver (for women) that drive this division of labour.

Data from both Canada and the United States support the notion that women face higher non-work demands than men. The literature suggests that Canadian and American women spend more hours per week on household chores and child care responsibilities than men. Each of these non-work responsibilities will be looked at in turn in the section below.

While Canadian men report spending 10.7 hours per week on household chores, they report that their spouses are spending almost twice that amount of 
time (19.5 hours per week; MacBride-King and Bachmann, 1999). The 2001 Canadian census confirms the discrepancy between men and women in time spent on household tasks. While $21 \%$ of Canadian women reported spending at least 30 hours doing housework in the week before the census was taken, only $8 \%$ of men made the same claim (Anthony, 2002). Silver (2000) using data from the 1998 General Social Survey (GSS) on time use reported that mothers, on average, were performing one more hour of household work per day than their spouses. The GSS interviewed almost 11,000 Canadians age 15 and over in the 10 provinces. Duxbury and Higgins (2002) ( $n=31,571$ respondents) reported that women, on average, spent 12.2 hours on household work per week while men, on average, spent 10.1 hours per week (Duxbury and Higgins, 2002).

The U.S. data reveals a similar discrepancy in the hours spent on household work. Bond et al., (2003) report that the vast majority of women in dual-earner couples took greater responsibility for cooking (77 percent) and cleaning (78 percent) compared to their partners. The recent BLS survey suggests that women are far more likely to spend time in a given day on household activities (84\%) than men (65\%) (BLS, 2006). Not only are women more likely to spend time on household activities on a given day, the hours they contribute to household responsibilities ( 2.7 hours per day) is greater than the hours spent by men (2.1 hours per day) (BLS, 2006).

A similar story unfolds when one examines the gender differences with respect to child care. Canadian Census data reveals that sixteen percent of working women reported spending 30 hours or more per week taking care of 
their children while only seven percent of working men put in these hours (Anthony, 2002). Interestingly, these percentages remained unchanged since the census in 1996. Silver (2000) has also noted that a gap exists between the amount of time that mothers spend on child-related tasks compared to their spouses. This gap persists until the child is in his/her teens. As noted in the section on the discrepancy of household duties between men and women, data for the Silver (2000) study came from the 1998 General Social Survey on time use.

Mothers working full-time spent an average of two hours more each day with their children (aged 5 and under) than fathers did (Silver, 2000). The gap does narrow as the child gets older but mothers still spent more time on average with their children aged 5 to 8 (i.e. 1.5 hours per day) and with their children aged 9 to 12 (i.e. 50 minutes per day) than their spouses. Silver (2000) notes the discrepancy between the spouses does not end until the children are 13-14 years old. More recent data from the General Social Survey of 2005 (Marshall, 2006) noted that there was still a gap between the average amount of time working mothers spent with their children in child-related activities ( 2 hours a day) and working fathers ( 1 hour a day).

Data from the 2001 census confirm that women are also overwhelmingly more likely than men to be primarily responsible for making child care arrangements and taking children to doctor's appointments (Anthony, 2002).

Similar discrepancies in the hours devoted to childcare can be seen in the U.S. data. Seventy percent of women in dual career couples with children 
reported that they take greater responsibility for routine child care compared to their partners (Bond et al., 2003). Women are also far more likely to be the ones to take time off from work to handle non-routine child-related responsibilities (e.g. staying at home with a sick child). While 31 percent of fathers in dual-earner couples with children said they had taken time off of work to help their children, $72 \%$ of mothers in dual-earner couples with children reported doing so (Bond et al., 2003). This gap is echoed in the BLS survey which reported that women were spending far more time ( 2.5 hours per day) on primary child care activities (defined as physical care, playing with children, traveling with children) than men (1.3 hours per day) (BLS, 2006). Hill (2005), using data from the 1997 NSCW $(n=1,314)$, reported that fathers spent less time (i.e. 7.1 fewer hours per week) looking after their children than mothers.

While it is fair to say that men's participation in household and child care activities has increased in the last three decades, it must be recognized that it is still far less than the hours that women devote to these tasks. For example, fathers in 2002 are spending an average of 42 minutes more time each day helping with household chores relative to their counterparts from 1977 (Bond et al, 2003). A similar finding was reported for fathers and child care. In 2002, fathers spent an hour more each day on child care than fathers in 1977 (Bond et al., 2003). While women are spending less time each day on household chores (from 3.7 hours in 1977 to 3 hours in 1992), their time spent in child-related activities remains virtually unchanged (Bond et al, 2003). Bond et al. (2003) note that while there are encouraging signs that men are taking more responsibility for 
household chores and child care, women still spend more time in both activities than men. Women spend an average of one extra hour a day on household activities and slightly less than an extra hour $(0.8)$ on childcare compared to men. This statistic is echoed in findings by Gerstel and Sarkisian (2006) who found that although men in the year 2000 averaged 9.7 hours per week on housework, this figure represented half of the hours women spent on housework. Hill (2005) using data from the $1997 \mathrm{NSCW}(n=1,314)$ reported that fathers spent less time (i.e. 6.9 fewer hours per week) on household duties than mothers. Bianchi and Raley $(2005)(n=19,013)$ reported that, in 2000 , mothers spent almost twice as many hours per week doing household tasks as fathers. Furthermore, Bianchi and Raley (2005) reported that, in 2000 , fathers were spending $2 / 3$ of the time mothers were spending with their children.

If we frame this discussion using Karasek's Demand-Control model, the following scenario emerges. The literature suggests that women are working more hours in domestic duties (i.e. higher levels of non-work demands) than men (e.g. Anthony, 2002) and the very nature of these activities is subject to less flexibility and discretion (less control; e.g. Hill et al., 2001; Mills et al., 2000; Roxburgh, 2002; 2004).

Barnett and Baruch (1987) suggest that most husbands' and fathers' household activities can be characterized as low demand and high control which places these men in Karasek's low strain quadrant. The authors then contrast this situation with the reality for many women. "Little control, relentless demands and great responsibility exposes wives and mothers to many frustrations and 
failures, and, applying Karasek's model, may account in part for the stressfulness of these roles" (Barnett and Baruch, 1987, p. 133). High demands and low control on the home front would place many women in the high strain quadrant of Karasek's model.

Thus, in conclusion, women and men seem to be operating in different realities both at work and at home. The average woman is more likely than the average man to find herself putting more time into household and child care responsibilities and tends to perform those low discretion tasks frequently (e.g. meal preparation, house cleaning). The average man (compared to the average woman) is likely to work more hours in paid employment but spend fewer hours in household tasks and perform high discretion tasks (e.g. snow shoveling, lawn mowing) periodically.

\subsubsection{Gender Differences in Work-Life Conflict}

A number of theories can be used to predict gender differences in worklife conflict. Gender role theory leads to very different predictions for men and women with respect to work-life conflict. Gender role theory predicts that men focus on their career first, are more likely to feel pressure from work demands and more likely to report higher levels of work-family interference (Gutek et al., 1991; Hill, 2005). Women, on the other hand, are more likely to feel pressure from non-work demands and are more likely to report higher levels of family-work interference (Gutek et al., 1991; Hill, 2005).

The rational theory of work-family (Gutek, Searle and Klepa, 1991) holds that more time spent in one domain can be linked to higher levels of interference 
in the other domain. Since men spend more time in paid employment than women, rational theory suggests that men should experience higher levels of work-family interference than their female counterparts. Since women spend more time in family demands (child care and household chores), women should experience higher levels of family-work interference than men.

Theoretically, then we are justified in controlling for gender in the thesis model. The research in this area is not, however, unequivocal. Rather, studies offer mixed evidence as to which gender experiences higher levels of work-life conflict.

Several studies indicate that men and women experience a similar level of work-life conflict (e.g. Eagle, Miles and Icenogle, 1997; Frone et al., 1996; Frone, 2000; Hill et al., 2001; Kinnunen and Mauno, 1998; Pleck, Staines and Lang, 1980; Shamir, 1983; Voydanoff, 1988). For example, Eagle et al. (1997) in a survey of 393 university employees found no gender differences in the level of work-life conflict. Eagle et al. argue that their results indicate that work-life conflict is equally important to both men and women and that "changes in social conceptions of gender, parenthood and work identity may be the cause" (Eagle et al., 1997, p. 180). Other non-North American studies have come to a similar conclusion that men and women share similar levels of work-life conflict. An Israeli study by Shamir (1983) of 286 hotel employees reported that felt worknonwork conflict was similar between men and women. A Finnish study of 145 married male employees and 356 Finnish married female employees (Kinnunen 
and Mauno, 1998) reported no gender differences in levels of work-family interference and family-work interference.

These findings are bolstered by large sample studies which also found no gender differences in the levels of work-life conflict. For example, both Pleck, Staines and Lang (1980) and Voydanoff (1988) using 1977 QES subsamples of 1084 and 1027 respectively concluded that men and women reported similar levels of work-family conflict. Hill et al. (2001) using data from a 1996 IBM work and life issues survey $(6,451$ respondents) reported that men and women reported similar levels of work-family balance. Two key studies by Frone et al. (1996) of employed parents (496 and 605 respondents) noted that the results failed to support their hypotheses that work-family conflict would be more strongly related to the outcomes among women and family-work conflict would be more strongly related to the outcomes among men. Four years later, Frone (2000) echoed this finding in his study of 2700 employed adults in a nationally representative sample by stating that the negative effects of work-family conflict impacted men and women equally.

For studies where gender differences have been reported, results indicate it is more common for women to report higher levels of various forms of work-life conflict (i.e. role overload, work-family interference, family-work interference) than men (Aryee et al., 1999; Crouter, 1984; Duxbury, Higgins and Mills, 1992; Duxbury and Higgins, 1998; Duxbury and Higgins, 2003; Duxbury, Higgins and Lee, 1994; Greenglass et al., 1989; Gutek et al., 1991; Hammer, Allen, and Grigsby, 1997; Hughes and Galinsky, 1988; Robinson and Godbey, 1997; 
Roxburgh, 2002; Roxburgh, 2004; Wallace, 1999; Williams and Alliger, 1994; Zukewich, 2000; Zukewich, 2003). We will compare each form of work-life conflict separately.

There is some evidence to support the claim that women experience higher levels of role overload than men. For example, Higgins, Duxbury and Lee (1992) surveyed 14,549 Canadians who worked for private sector employers. They concluded that women were significantly more likely to report high levels of role overload (30\%) than their male counterparts $(19 \%)$. More recent results by Duxbury and Higgins (2003) of 31, 571 respondents came to a similar conclusion that women were more likely to report high levels of role overload than men.

Other large sample (i.e. at least 700 respondents) Canadian and American studies (Duxbury, Higgins and Mills, 1992; Duxbury et al.,1994; Duxbury and Higgins, 1998; Higgins et al., 1994; Mattingly and Bianchi, 2003; Robinson and Godbey, 1997; Roxburgh, 2002; Roxburgh, 2004; Zukewich, 2000; Zukewich, 2003) also concluded that women suffered higher levels of role overload than men. Roxburgh $(2002,2004)$ and Mattingly and Bianchi (2003) operationalized role overload as time pressure while Zukewich $(2000,2003)$ operationalized role overload as time stress.

There are also several North American studies to show women experience higher levels of work-family interference than men (e.g. Duxbury et al., 1994; Greenglass et al., 1988; Gutek et al., 1991; Higgins, Duxbury and Lee, 1994; Higgins, Duxbury and Lee, 1994; Hill, 2005; Hughes and Galinsky, 1988). For example, Duxbury et al.'s (1994) study of 1,989 single parent and dual 
income employees with children aged 6-12 years old concluded that women had higher levels of work-family interference than men. Greenglass et al. (1988) in another Canadian study, this time involving Canadian teachers (327 men and 229 women) concluded that role conflict was higher for women than men. Two other studies by Higgins, Duxbury and Lee $(1992,1994)$ with samples of 14,549 and 3,616 respondents respectively concluded that work-family interference was stronger for women than men.

American studies have also yielded similar findings. For example, Gutek et al. (1991) using two samples of psychologists (423 respondents) and 209 senior managers concluded that for both samples, women experienced higher levels of work-family conflict than men. Hughes and Galinsky (1988) studied 285 working parents and noted that mothers with children under 18 were more likely to have greater work-family interference than fathers with children under 18 .

It is worth noting that the finding that women suffer higher levels of workfamily conflict than men are not universal. Wallace (1999) in a study of Canadian lawyers (338 respondents) stated that men experienced greater time-based conflict (akin to work-family interference) than women. In Madsen's (2003) survey of telecommuters (136 respondents), male telecommuters had higher levels of work-family conflict than female telecommuters. One might suggest that the use of a sole profession by Wallace (1999) and a specialized work arrangement by Madsen (2003) means we cannot generalize their findings. More generalizable is the finding of the latest NSCW study by Bond et al. (2003) which concluded that men reported higher levels of work-family interference than women. Bond et 
al. (2003) suggest that, by virtue of the number of hours men spend at work, it is logical to assume that they might report higher levels of work-family interference than women.

It should be noted that, relative to the number of studies examining role overload and work-family interference, fewer studies have focused specifically on the outcome of family-work interference. The limited studies available indicate women experience higher levels of family-work interference. For example, Aryee et al., (1999) studied 243 Hong Kong parents in dual-career families and concluded that men reported lower levels of family-work conflict. Higgins, Duxbury and Lee (1994) in a survey of 3,616 Canadian respondents noted that women with children under 12 experienced significantly higher levels of familywork interference than their male counterparts. Williams and Alliger (1994) reported that the spillover of distress from family-work interference was stronger for women than men in their study of 41 working parents from Albany, New York. Crouter (1984) in a study with albeit a rather small sample, (55 respondents from one manufacturing plant) concluded that women reported higher levels of familywork interference than men. Hill (2005) using data from the 1997 NSCW $(n=1,314)$ reported that fathers reported lower levels of family-work conflict than mothers. Behson (2002) in his sample of 141 employees, found that women reported higher levels of family-work conflict than men.

Why might some studies not find gender differences? Greenhaus and Parasuraman (1999) suggest three possible explanations. First, some women may choose jobs that are less demanding and therefore less likely to generate 
work-life conflict. Second, some women may decide to put their careers first and either not get married or start a family. Third, perhaps women are better at coping with the higher levels of demands that diminish their perceived level of work-life conflict.

Furthermore, when one reviews how work-family conflict was measured, one comes to an interesting observation. When work-family conflict was measured unidimensionally (e.g. Hill et al., 2001; Pleck, Staines and Lang, 1980; Voydanoff, 1988), there tended to be a finding of no gender differences on workfamily conflict. When authors used multidimensional measures of role overload and interference (e.g. Aryee et al., 1999; Duxbury et al. 1992; Duxbury et al., 1994; Higgins et al., 1994; Roxburgh, 2002, 2004; Zukewich, 2000, 2003), gender differences on various aspects of work-life conflict were reported. In conclusion, although the findings were not universal, there is fairly strong evidence to suggest that men and women do differ with respect to worklife conflict. Specifically, there is strong support to indicate that women experience higher levels of role overload than men. There is no consensus with respect to which gender experiences higher levels of work-family interference and limited evidence to suggest that women experience higher levels of familywork interference than men. Nevertheless, the possibility that gender is associated with the key outcomes (i.e. demands, work-life conflict) considered in this study supports our decision to take gender into account in our analysis. 


\subsubsection{Gender Differences in Flextime/Perceived Flexibility}

In model 4 of this thesis, we propose that alternative work arrangements (i.e. flextime, compressed work week, telework) are associated with perceived flexibility. Therefore, it makes sense for us to also examine the literature to see if there are differences between men and women in their access to various alternative work arrangements or perceptions of perceived flexibility. Using gender role theory, it could be argued that given the primary role of men as breadwinners and that society values this role, that men could be at a disadvantage when it comes to access to flextime or perceived flexibility. Furthermore, women would be allowed more leeway or flexibility to attend to nonwork obligations because society views their dominant role as one of caregiver. The very limited literature on this topic does not support this assumption.

Fast and Frederick $(1996)(n=5,060)$ reported that flextime was more available for men than for women. A similar conclusion was reached by Golden (2001) $(n=50,000)$ and Ferrer and Gagne (2006). Hammer et al. (1997) $(n=399$ dual-career couples) reported that men had greater perceived flexibility than women. Nevertheless, the fact that gender differences with respect to access to flexible work arrangement have been reported in the literature supports the decision to control for gender when testing model 4 . 


\subsection{Socio-economic Status}

The section on socio-economic status is divided into two parts: First, we will discuss why job type was chosen as a proxy for socio-economic status. Second, we will review the literature that supports our contention that socioeconomic status is associated with the main constructs in our model: demands, work-life conflict and control over work-life interface.

\subsubsection{Job Type as a Proxy for SES}

Although there is no general agreement on the definition of socioeconomic status (SES), it can be seen as a measure of an individual's or group's standing in the community (Mukherjee, 1999). Researchers are faced with choices of which measure of SES to use when they wish to control for SES. Three common proxies for SES are job type, income and education. There are several reasons why job type as a proxy for SES was chosen for this thesis.

First, job type is a common choice of proxy for SES in empirical studies For example, large sample studies $(n=6451)$ examining perceived job flexibility (in both timing and location of work) such as Hill et al. (2001) controlled for socioeconomic status by using job type. Fenwick and Tausig's (2001) study of 2,905 full-time workers also controlled for SES using job type. As well, certain small sample studies (e.g. Galambos and Walters, 1992; Wallace, 1999) controlled for SES using job type.

Second, using education as a proxy for SES can be problematic. Quite often, there can be a mismatch between the education of the job-holder and their socio-economic status. In other words, there are many well-educated Canadians 
who work low-paying jobs and vice versa. In fact, Statistics Canada reports that, in 2001, one in five workers with a university degree was overqualified (i.e. their job required only a high school diploma) (Gervais, Li and Duval, 2006). In these cases, focusing on education level alone could mean that their SES would be inaccurate. Therefore, job type is the better choice.

Third, using income as a proxy for SES can be problematic due to the regional differences in income in Canada. A summary of the weekly earnings across Canada suggests there are significant regional variations in income. For example, while Prince Edward Islanders earned an average of $\$ 562.48$ weekly, those in Ontario made $\$ 740.19$ per week (Toronto Star, August 21,2004 ).

In this thesis, job type is conceptualized into two groups: managers/professionals and non-professionals. The decision to dichotomize is supported by the fact that there are significant differences between these two groups. Duxbury and Higgins' (2001) reported that there was a strong association between income, education and job type in their dataset (please note that this thesis used this set of data to test our models). Respondents in nonprofessional positions were more likely to have a high school education or less, receive lower financial remuneration and say that, in their family, "money is tight." The men and women in managerial and professional positions, on the other hand, were more likely to have a university education, be in families that earned $\$ 100,000$ or more a year and say that, in their family, "money was not an issue." As noted in the upcoming section, there are significant differences in the level of 
demands and work-life conflict between managers/professionals and nonprofessionals.

\subsubsection{Job Type Differences in Demands}

Managers and professionals (considered to be of a higher SES than nonprofessionals) have greater work demands than non-professionals. Compared to non-professionals, managers and professionals spend more hours at work in a given week, are more likely to spend weekday and weekend nights away from home traveling for work, and spend more time working unpaid overtime and performing supplemental work at home (Duxbury and Higgins, 2001).

The notion that managers and professionals face higher levels of demands is echoed in other studies. Higgins, Duxbury and Lee (1992) noted that managers and professionals work longer hours per day and tend to do more supplementary work at home than non-professional respondents. Shields (1999) in a large sample study of employed Canadians (2151 men and 1649 women) concluded that men and women in white-collar professions work longer hours than blue-collar jobs. Duxbury and Higgins (1998) in their work-life balance study in Saskatchewan (5,426 respondents) noted that managers and professionals worked more hours per week than non-professionals.

In the United States, American managers and professionals are more likely to work a long work week (defined by the Bureau of Labor Statistics as 49 hours a week or more) than non-professionals (US Department of Labor, 2000). Hill et 
al. (2006) noted that managers and professionals worked more hours per week than non-professionals in a global survey of 300,000 IBM employees.

\subsubsection{Job Type Differences in Work-Life Conflict}

There is an interesting division in the literature on the question of which group (i.e. managers/professionals vs. non-professionals) experiences less worklife conflict. On the one hand, the empirical literature from the previous subsection suggests that managers/professionals work more hours than nonprofessionals. Please recall that in section 5.3, there is strong empirical evidence indicating that working longer hours was associated with high levels of work-life conflict. Therefore, it could be argued that managers and professionals, by virtue of the longer hours worked (relative to non-professionals), should report higher levels of work-life conflict. Another argument for managers/professionals reporting higher levels of work-life conflict can be found in the rational theory of work-family conflict (Gutek et al., 1991). Working long hours at work leaves less time for managers/professionals to fulfill their non-work obligations. Being unable/unwilling to fulfill these obligations could lead managers/professionals to feel a heightened sense of work-family interference. Lastly, there is a culture of hours (Lewis and Cooper, 1999) often found among professionals/managers where dedication to one's organization is expressed in the number of hours one puts in and a manager/professional feels he/she cannot leave until after his/her boss has left for the day. This leads to a lot of face time for these managers/professionals and putting in these hours leaves less time for non-work demands (as argued in the previous point). 
A contrasting notion in the literature suggests that those of higher SES (i.e. managers and professionals) should have an easier time balancing work and life compared to those of lower SES (i.e. non-professionals) because managers and professionals earn higher salaries than non-professionals (Duxbury and Higgins, 2001). The higher salaries earned by professionals and managers for example enable them to purchase time saving goods (e.g. maid services, ordering meals to go, live-in nannies; Duxbury and Higgins, 2001) which could reduce demands in the family domain.

Given the conflicting theoretical rationales for which job type should experience higher levels of work-life conflict, it is not surprising to find conflicting empirical findings. Roxburgh (2002) concluded that those of higher SES (measured as income) experienced greater time pressure (akin to role overload) relative to those of lower SES in her study in Ohio (734 respondents). Robinson and Godbey (1997) in their study which involved 5,358 respondents filling in detailed time diaries concluded that time pressures were more frequently reported by those considered affluent (i.e. higher SES) relative to the non-affluent (i.e. lower SES) respondents in the study. Duxbury and Higgins (1998) in their work-life balance study in Saskatchewan $(5,426$ respondents) noted that managers and professionals were more likely to report high levels of role overload as well as work-family interference than non-professionals. Two other studies (i.e. Clarkberg and Moen, 2001; Jacobs and Gerson, 1998) with sample sizes of 9108 and 50,000 respectively noted that role overload (operationalized as overwork) is higher for managers and professionals than non-professionals. 
As well, Grzywacz et al. (2002) $(n=741)$ found that managers and professionals were most likely to experience work-family spillover (compared to nonprofessionals). Hill et al. (2001) $(n=6451)$ concluded that professionals/managers reported higher levels of work-family conflict than non-professionals.

Not all studies are supportive of the notion that those of higher SES have more time pressure. For example, Voydanoff and Kelly (1984) noted that those of higher SES (operationalized as high income respondents) were less likely to report time shortage in their survey of 468 working parents in a U.S. southeastern county. Furthermore, Roxburgh (2004) concluded that higher SES (operationalized as income) did moderate the association between time pressure and depression in her study of 790 full-time Ohio workers. Roxburgh suggested that "income may provide access to resources such as household help that may assist in reducing the stressful consequences of time pressure" (Roxburgh, 2004, p. 117).

In conclusion, the literature is supportive of the notion that those of higher SES put more time into paid employment and the bulk of the studies suggest that managers/professionals experience higher levels of work-life conflict. The fact that SES is linked to work-life conflict additionally supports our decision to control for SES in this analysis.

\subsubsection{Job Type Differences in Flextime/Perceived Flexibility}

Some authors have suggested that managers/professionals theoretically have more control over the timing and place of work (Duxbury and Higgins, 2002; 
Galinsky et al., 2004; Hill et al., 2001). As such, managers/professionals should be more able to change their schedule to meet sudden changes in their personal lives (for example, having to leave work to pick up a sick child) as well as tailor their schedule to meet the day-to-day family obligations (for example, scheduling to start work early to be home when children come home from school).

There is some evidence to suggest there are two key differences between managers/professionals and non-professionals when it comes to control (as operationalized in this thesis). First, managers/professionals differ from nonprofessionals in terms of the usage of alternative work arrangements. While nonprofessionals are much more likely to work a traditional 9 to 5 workday and perform shiftwork, managers and professionals are more likely to have access to flextime work arrangements (Duxbury and Higgins, 2002). This finding is echoed by other large sample studies such as Golden (2001). Using data from the Current Population Survey $(n=50,000)$, Golden (2001) reported that managers/professionals had more access to flextime than non-professionals. Swanberg et al. (2005) using data from the $1997 \mathrm{NSCW}(\mathrm{n}=2877)$ reported that those lower in SES (i.e. lower income, less education and who were paid hourly) had less access to flextime than those higher in SES (i.e. higher income, more education, and salaried).

Hughes and Galinsky (1994) came to similar conclusions in their survey of 523 full-time employees that nonprofessionals had less access to flextime than professionals/managers. Furthermore, other studies came to similar conclusions 
(Applebaum and Golden, 2003; Bond, 2002; USDL, 2002) that nonprofessionals have less access to flextime than managers/professionals.

Second, managers and professionals are more likely to occupy occupations with high degrees of flexibility and personal control over the timing of work compared to non-professionals (Duxbury and Higgins, 2001; Hill et al., 2001). Swanberg et al. (2005) as well as Hughes and Galinsky (1994) came to the same conclusion. The fact that SES is linked to flextime/perceived flexibility once again supports our decision to control for SES in model 4.

\subsection{Gender/Job Type Considered Together}

Lindsey and Almey (2006) note that most women still work in the traditional female-dominated occupations. In 2004, over two-thirds (67\%) of employed women worked in jobs such as teaching, nursing, clerical and service positions (Lindsey and Almey, 2006). Given the phenomena of occupation compression that typically affects women, it makes sense to examine gender and job type together. This section will review the literature for differences between the following four groups (i.e. male professionals/managers, female professionals/managers, male non-professionals and female non-professionals) on the key variables of interest (i.e. demands, work-life conflict, and access to flexible work arrangements/perceived flexibility). The decision to consider gender and job type together provides further support for our decision to control for both these constructs in our analysis. 


\subsubsection{Gender/Job Type Differences in Demands}

Given the gender role theory which suggests that males are likely to focus on the breadwinning role and value working longer hours and given the recent discussion under job type which suggests that there are organizational pressures for managers/professionals to put in long hours, it should not be surprising that the literature supports the notion of male manager/professionals working longer hours than the other groups (i.e. female manager/professionals, female nonprofessionals, male non-professionals). Furthermore, Still and Williams (2006) argue that society expects mothers to be ever present for their children and this expectation clashes with what organizations want: "the ever-available worker". The implication of these two ideas was put forward by Barnett and Baruch (1987) who noted that men are less likely than women to feel guilty or frustrated at having to put in overtime or do supplementary work at home.

There is some data to support the assumption that male manager/professionals will spend a higher average number of hours in work per week and will be more likely to report working a long work week (defined as working more than 49 hours a week). Jacobs and Gerson (1998) using data from the Current Population Survey $(n=50,000)$ noted that male manager/professionals were more likely than male non-professionals, female manager/professionals and female non-professionals to work more hours per week on average and were more likely to work a minimum of 50 hours per week. This finding was echoed by the U.S. Department of Labor (U.S. Department of Labor, 2002). 


\subsubsection{Gender/Job Type Differences in Work-Life Conflict}

Given the empirical support for the positive association between hours worked and work-life conflict, it is assumed that male manager/professionals should report higher levels of work-life conflict (relative to male nonprofessionals, female manager/professionals and female non-professionals). This assumption is not supported by the literature.

The literature suggests that managerial and professional men experience lower levels of work-life conflict than women. For example, Roxburgh (2002) in her study of 734 Ohio workers reported that white collar men are substantially less time pressured (similar to role overload) than white collar women while blue collar men and women were equally time pressured. Gutek et al., 1991 surveyed two different samples: 423 psychologists and 209 senior managers and found that female professionals and managers experienced higher levels of work-family interference than their male counterparts in both samples. Maume and Houston (2001) in their sample of 1090 white-collar respondents concluded that women reported higher levels of work-family conflict. Duxbury and Higgins (2001) found that professional women experienced the highest levels of role overload and work to family interference in both 1991 and 2001 samples.

These results would suggest there is more to this story than merely the number of hours one works as otherwise, male manager/professionals should report the highest level of work-life conflict. Most likely the salience of gender role theory plays a hand in the explanation. Because of gender socialization and gender expectations, women are still expected to perform the majority of nonwork responsibilities (i.e. child care and household chores). Wallace (1999) 
notes that professional women still perform the majority of the household and child-care tasks relative to their partners.

Both Gutek et al. (1991) and Wallace (1999) speculate that professional women experience higher levels of work-life conflict because they equally value and are simultaneously emphasizing both their professional and personal roles as employee, mother and spouse. Professional men on the other hand, according to Wallace (1999) tend to value the good provider paradigm and don't mind putting in extra hours to provide for their families. These men are less likely worry about neglecting their family than their female counterparts.

\subsubsection{Gender/Job Type Differences in Flextime/Perceived Flexibility}

While there are studies which focus on the issue of whether men or women, professionals/non-professionals have greater access to flextime or report higher levels of flexibility, there are no studies which look at the impact of gender and job type together. Despite efforts to uncover such studies, academic databases such as ABI, JSTOR and Social Sciences Index produced no such studies.

The literature suggests that women and men are operating in different realities. The average woman tends to find herself occupationally segregated in a job with low levels of discretion and high demands. The average woman puts more time into household and child care responsibilities and tends to perform those tasks with less discretion that need to be performed more frequently (e.g. meal preparation, house cleaning) than the tasks performed by her male 
counterpart (e.g. snow shoveling, lawn mowing). Even within the professional ranks, women receive lower levels of pay, have less discretion and have the dual burden of trying to excel in both professional and home capacities. It is no wonder that professional women tend to report higher levels of work-life conflict than their male counterparts. It is for these reasons that gender and job type must be considered jointly and controlled for in this thesis. 


\section{Research Hypotheses}

A major objective of this thesis is to test three updated models of Karasek and Johnson's Demand-Control-Support model. While the independent and moderator variables are identical in these three models, the dependent variable differs, reflecting the fact that we are exploring the validity of the theoretical framework for three different forms of work-life conflict: role overload, workfamily interference and family-work interference. Another major objective of this thesis is to test a new model (i.e. model 4) which looks at the relationship between a number of forms of control (alternative work arrangements, supportive supervisor) and perceived flexibility.

This section is divided into two main parts: part one focuses on models 1 3 while part two deals with model 4. Part one is structured as follows. For models $1-3$, we will begin by presenting a diagram that summarizes the main theoretical relationships being examined. This is followed by a list of the direct and interaction hypotheses associated with models 1-3. A justification for these hypotheses will then be provided followed by a discussion of the covariates related to models $1-3$.

The second part of the section provides similar information for Model 4. The diagram summarizing Model 4's theoretical relationships is presented first. This is followed by a summary of the direct and interaction hypotheses associated with this model that will be tested in this theses. The hypotheses are then justified followed by a discussion of the covariates associated with model 4 . 
The literature used to support these hypotheses was presented earlier in the thesis in sections 4 (demands), 5 (strain), and 6 (control over work-life interface). A table summarizing the testing approach for the four thesis models can be found in Appendix C.

\subsection{Reconcepualizing Karesek's Framework}

As noted earlier, the first model to be tested in this thesis updates Karesek's 1979 Demand-Control model. In all cases demands are operationalized as the amount of time per week spent in work and non-work activities while control is operationalized in three ways: work arrangement, perceived flexibility, and supportive supervision. The three models differ, however, with respect to how they conceptualize the strain component of the model with model one focusing on role overload, model two on work interferes with family and model three using family interferes with work. Relevant details are given below.

\subsubsection{Model 1 Hypotheses (Role Overload)}

This section presents the theoretical framework for thesis model 1 followed by the direct and interaction hypotheses for this model. The theoretical framework for thesis model 1 is found in figure 6. 
Figure 6 Theoretical Framework: Thesis Model 1

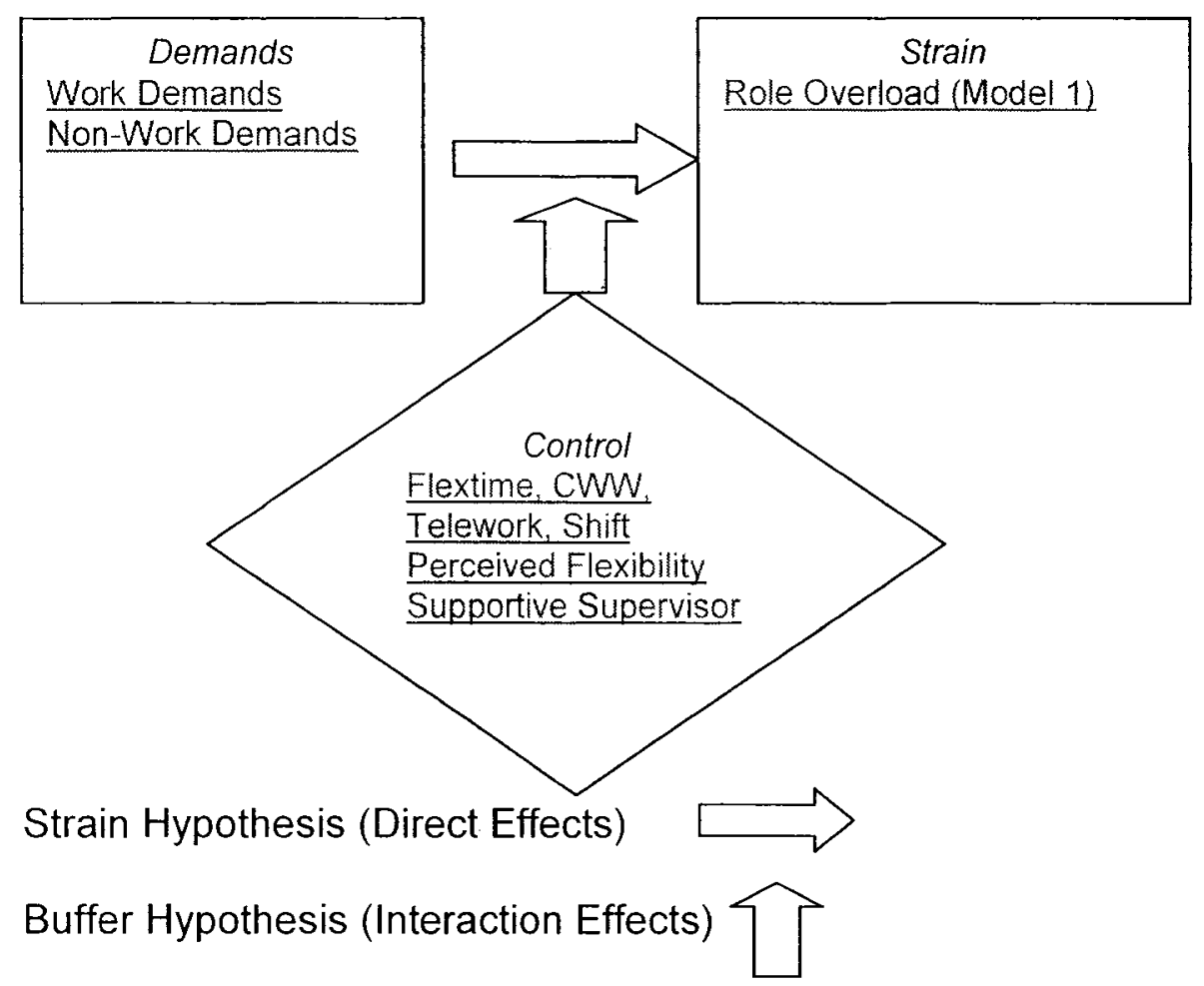

Twenty hypotheses ( 8 direct hypotheses and 12 interaction hypotheses) are tested for model one. Each set of hypotheses will be discussed in turn.

\subsubsection{Model 1: Strain Hypotheses for Role Overload (RO)}

$\mathrm{ROH} 1$ : Work demands will be positively associated with role overload. $\mathrm{ROH} 2$ : Non-work demands will be positively associated with role overload.

ROH3: The use of alternative work arrangements (AWA) will be negatively associated with role overload. Specifically employees who work:

a. flextime (versus a regular work day)

b. compressed work week (versus a regular work day)

c. telework (versus a regular work day)

will report lower levels of role overload than their counterparts who work a regular work day. 
ROH4: The use of shiftwork arrangements will be positively associated with role overload.

ROH5: Perceived flexibility will be negatively associated with role overload.

ROH6: Supervisor support will be negatively associated with role overload.

\subsubsection{Model 1: Buffer Hypotheses for Role Overload}

ROH7: Alternative work arrangements will moderate the relationship between work demands and role overload. Specifically

a. The relationship between work demands and role overload will be weaker for those who use flextime arrangements than those who work a regular work day.

b. The relationship between work demands and role overload will be weaker for those who work a compressed work week than those who work a regular work day.

c. The relationship between work demands and role overload will be weaker for those who telework than those who work a regular work day.

d. The relationship between work demands and role overload will be weaker for those who work a regular work day than those who perform shiftwork.

ROH8: Perceived flexibility will moderate the relationship between work demands and role overload. Specifically, the relationship between work demands and role overload will decrease as perceived flexibility increases.

ROH9: Supervisor support will moderate the relationship between work demands and role overload. Specifically, the relationship between work demands and role overload will decrease as supervisor support increases.

ROH10: Alternative work arrangements will moderate the relationship between non-work demands and role overload. Specifically

a. The relationship between non-work demands and role overload will be weaker for those who use flextime arrangements than those who work a regular work day.

b. The relationship between non-work demands and role overload will be weaker for those who work a compressed work week than those who work a regular work day.

c. The relationship between non-work demands and role overload will be weaker for those who telework than those who work a regular work day.

d. The relationship between non-work demands and role overload will be weaker for those who work a regular work day than those who perform shiftwork.

ROH11: Perceived flexibility will moderate the relationship between non-work demands and role overload. Specifically, the relationship between non-work demands and role overload will decrease as perceived flexibility increases. 
ROH12: Supervisor support will moderate the relationship between non-work demands and role overload. Specifically, the relationship between non-work demands and role overload will decrease as supervisor support increases.

\subsubsection{Model 2 Hypotheses (Work-Family Interference)}

This section presents the theoretical framework for thesis model 2 followed by the direct and interaction hypotheses for this model. The theoretical framework for thesis model 2 is found in figure 7.

Figure 7 Theoretical Framework: Thesis Model 2

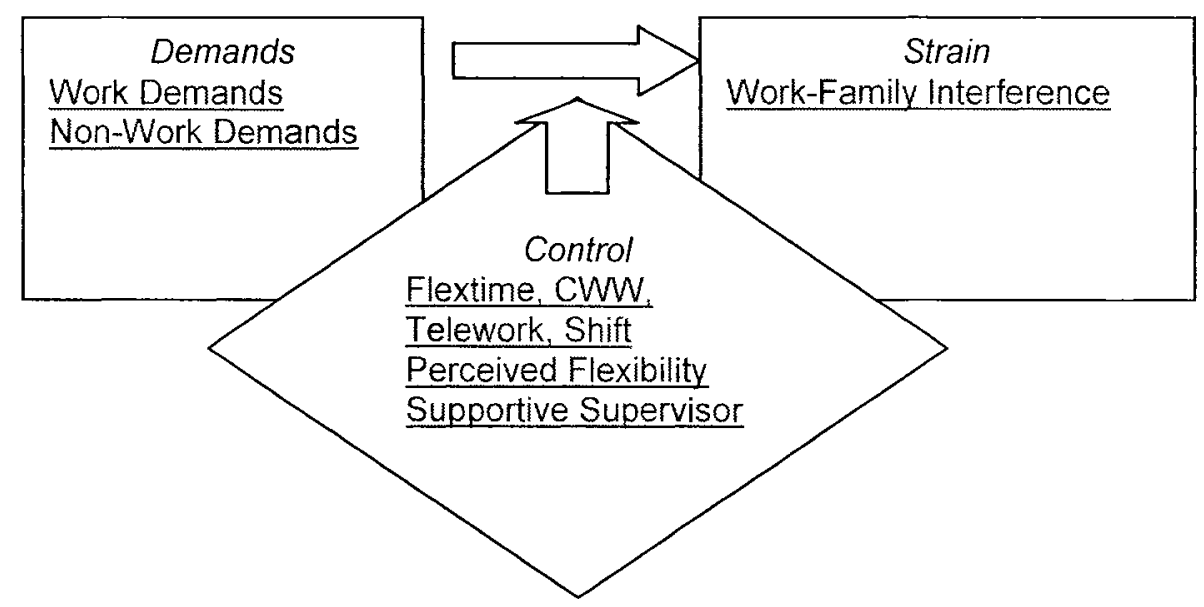

Strain Hypothesis (Direct Effects)

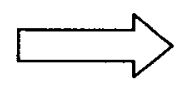

Buffer Hypothesis (Interaction Effects)

Twenty hypotheses ( 8 direct hypotheses and 12 interaction hypotheses) are tested for model two. Each set of hypotheses will be discussed in turn. 


\subsubsection{Model 2: Strain Hypotheses for Work-Family Interference (WFI)}

WFIH1: Work demands will be positively associated with work-family interference.

WFIH2: Non-work demands will be positively associated with work-family interference.

WFIH3: The use of alternative work arrangements (AWA) will be negatively associated with work-family interference. Specifically employees who work:

a. flextime (versus a regular work day)

b. compressed work week (versus a regular work day)

c. telework (versus a regular work day)

will report lower levels of work-family interference than their counterparts who work a regular work day.

WFIH4: The use of shiftwork arrangements will be positively associated with work-family interference.

WFIH5: Perceived flexibility will be negatively associated with work-family interference.

WFIH6: Supervisor support will be negatively associated with work-family interference.

\subsubsection{Model 2: Buffer Hypotheses for Work-Family Interference}

WFIH7: Alternative work arrangements will moderate the relationship between work demands and work-family interference. Specifically

a. The relationship between work demands and work-family interference will be weaker for those who use flextime arrangements than those who work a regular work day.

b. The relationship between work demands and work-family interference will be weaker for those who work a compressed work week than those who work a regular work day.

c. The relationship between work demands and work-family interference will be weaker for those who telework than those who work a regular work day.

d. The relationship between work demands and work-family interference will be weaker for those who work a regular work day than those who perform shiftwork.

WFIH8: Perceived flexibility will moderate the relationship between work demands and work-family interference. Specifically, the relationship between 
work demands and work-family interference will decrease as perceived flexibility increases.

WFIH9: Supervisor support will moderate the relationship between work demands and work-family interference. Specifically, the relationship between work demands and work-family interference will decrease as supervisor support increases.

WFIH10: Alternative work arrangements will moderate the relationship between non-work demands and work-family interference. Specifically

a. The relationship between non-work demands and work-family interference will be weaker for those who use flextime arrangements than those who work a regular work day.

b. The relationship between non-work demands and work-family interference will be weaker for those who work a compressed work week than those who work a regular work day.

c. The relationship between non-work demands and work-family interference will be weaker for those who telework than those who work a regular work day.

d. The relationship between non-work demands and work-family interference will be weaker for those who work a regular work day than those who perform shiftwork.

WFIH11: Perceived flexibility will moderate the relationship between non-work demands and work-family interference. Specifically, the relationship between non-work demands and work-family interference will decrease as perceived flexibility increases.

WFIH12: Supervisor support will moderate the relationship between non-work demands and work-family interference. Specifically, the relationship between non-work demands and work-family interference will decrease as supervisor support increases.

\subsubsection{Model 3 Hypotheses (Family-Work Interference)}

This section presents the theoretical framework for thesis model 3 followed by the direct and interaction hypotheses for this model. The theoretical framework for thesis model 3 is found in figure 8. 
Figure 8 Theoretical Framework: Thesis Model 3

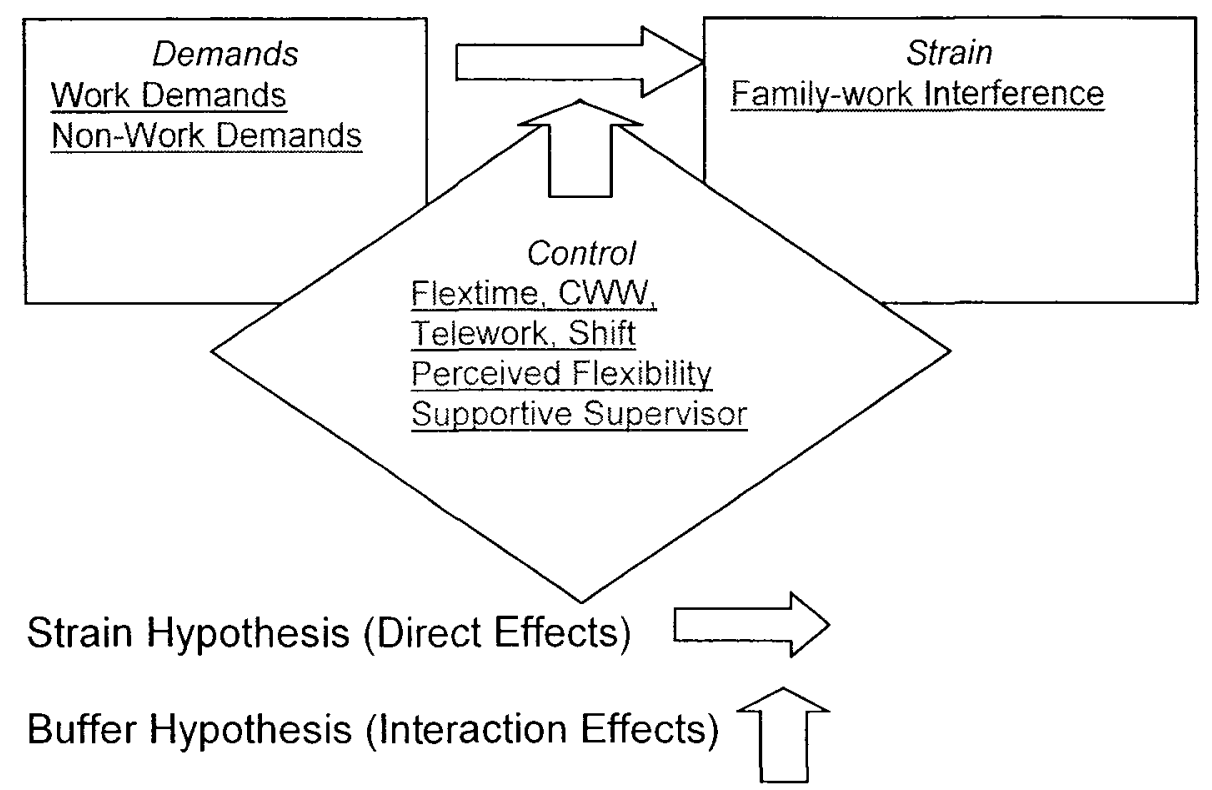

Model 3 has 20 hypotheses ( 8 direct hypotheses and 12 interaction hypotheses). Each set of hypotheses will be presented in turn.

\subsubsection{Model 3: Strain Hypotheses for Family-Work Interference (FWI)}

FWIH1: Work demands will be positively associated with family-work interference.

FWIH2: Non-work demands will be positively associated with family-work interference.

FWIH3: The use of alternative work arrangements (AWA) will be negatively associated with family-work interference. Specifically employees who work:

a. flextime (versus a regular work day)

b. compressed work week (versus a regular work day)

c. telework (versus a regular work day)

will report lower levels of family-work interference than their counterparts who work a regular work day.

FWIH4: The use of shiftwork arrangements will be positively associated with family-work interference. 
FWIH5: Perceived flexibility will be negatively associated with family-work interference.

FWIH6: Supervisor support will be negatively associated with family-work interference.

\subsubsection{Model 3: Buffer Hypotheses for Family-Work Interference (FWI)}

FWIH7: Alternative work arrangements will moderate the relationship between work demands and family-work interference. Specifically

a. The relationship between work demands and family-work interference will be weaker for those who use flextime arrangements than those who work a regular work day.

b. The relationship between work demands and family-work interference will be weaker for those who work a compressed work week than those who work a regular work day.

c. The relationship between work demands and family-work interference will be weaker for those who telework than those who work a regular work day.

d. The relationship between work demands and family-work interference will be weaker for those who work a regular work day than those who perform shiftwork.

FWIH8: Perceived flexibility will moderate the relationship between work demands and family-work interference. Specifically, the relationship between work demands and family-work interference will decrease as perceived flexibility increases.

FWIH9: Supervisor support will moderate the relationship between work demands and family-work interference. Specifically, the relationship between work demands and family-work interference will decrease as supervisor support increases.

FWIH10: Alternative work arrangements will moderate the relationship between non-work demands and family-work interference. Specifically

a. The relationship between non-work demands and family-work interference will be weaker for those who use flextime arrangements than those who work a regular work day.

b. The relationship between non-work demands and family-work interference will be weaker for those who work a compressed work week than those who work a regular work day.

c. The relationship between non-work demands and family-work interference will be weaker for those who telework than those who work a regular work day.

d. The relationship between non-work demands and family-work interference will be weaker for those who work a regular work day than those who perform shiftwork. 
FWIH11: Perceived flexibility will moderate the relationship between non-work demands and family-work interference. Specifically, the relationship between non-work demands and family-work interference will decrease as perceived flexibility increases.

FWIH12: Supervisor support will moderate the relationship between non-work demands and family-work interference. Specifically, the relationship between non-work demands and family-work interference will decrease as supervisor support increases.

\subsubsection{Justification of the Hypotheses (Models 1-3)}

This subsection justifies the relationships posited in thesis models 1-3. It is important to recognize that thesis models $1-3$ are identical with respect to the independent and moderator variables, it is only the dependent variables that differ (i.e. role overload, work-family interference, family-work interference). In order to avoid redundancy, we will present a common justification for the direct and interaction hypotheses that covers models 1,2 and 3. This justification will be presented in such a way that we differentiate the relationships between the independent variables/moderator variables and overload and the relationships between the independent variables/moderator variables and interference. Such an approach recognizes that overload and interference are conceptually different constructs (please see section 5).

\subsubsection{Justification of Direct Hypotheses ( $\mathrm{H} 1$ and $\mathrm{H} 2)$}

Karasek's strain hypothesis posited that demands were positively associated with job strain. Models 1-3 in this thesis represent an updated conceptualization of Karasek's strain hypothesis. We hypothesize that work and non-work demands will be positively associated with: role overload (model 1), 
work-family interference (model 2), and family-work interference (model 3). Empirical evidence supporting these assertions can be found in sections 5.3 and 5.4. Role overload occurs when the various demands from people's different roles (e.g. employee, parent, spouse, volunteer) become overly taxing on one's time and energy (Duxbury and Higgins, 2001). It is logical, therefore, to assume that those working longer hours (either work or non-work hours) would be more vulnerable to feeling overwhelmed and lacking in energy and be more likely to report high levels of role overload.

In the context of interference (both work-family and family-work interference), time spent in one domain means time unavailable to be spent in other domains as well as physically not being able to be two places at once (Gutek et al., 1991). The tension inherent between the conflicting demands of work and non-work has been shown to result in inter-role conflict. Therefore, higher levels of work demands and non-work demands will be associated with higher levels of interference.

\subsubsection{Justification of Direct Hypotheses ( $\mathrm{H} 3,4,5$ and 6$)$}

Karasek's strain hypothesis also posited that decision latitude (i.e. control) was negatively associated with job strain. Models $1-3$ in this thesis represent an updated conceptualization of Karasek's strain hypothesis. This thesis examines three forms of control over work-life interface (i.e. alternative work arrangements, perceived flexibility and supervisor support). Control over work-life interface should be associated with lower levels of work-life conflict. Evidence supporting 
this assertion can be found in sections 6.1.1.2 (compressed work week), 6.1.2.2 (flextime), and 6.1.3.2 (telework).

Theoretically, alternative work arrangements should result in more control over when and where demands (i.e. both work and non-work) get done. This heightened sense of control can help employees work more efficiently which in turn should lead to lower perceptions of role overload. This increased sense of control should also help reduce interference as employees are better able to schedule their work and non-work obligations and therefore better able to meet their work and non-work obligations.

Literature summarizing the association between shiftwork and work-life conflict can be found in section 6.1.4.2. This literature indicates that the relationship between shiftwork and work-life conflict should be very different than that between work-life conflict and the other alternative work arrangements examined in this thesis. Shiftwork is often assigned and therefore shiftworkers have no control over the shift they work. This inability to move their shift and this decline in perceived control experienced by shiftworkers could be associated with higher levels of feeling overwhelmed (i.e. role overload) and frustration at not being able to effectively meet work and non-work obligations (i.e. interference).

There is some literature (see section 6.2.1) which suggests that it may not be the alternative work arrangement per se that results in lower levels of work-life conflict but rather it may be the amount of control the employee perceives they have over when and where they work that is important. Whereas alternative work arrangements can be seen to theoretically give employees more control 
over the work-life interface, as noted earlier this relationship is not guaranteed (i.e. culture does not support appropriate use of flexible work arrangement). Perceived flexibility, on the other hand, is operationally defined as the actual amount of control an employee has over their work-life interface. As noted earlier the literature supports the idea that as perceived control increases, levels of work-life conflict (both overload and interference) will decline.

Supervisor support is the third conceptualization of control included in this thesis. The argument for the inclusion of supervisor support is very similar to the one made for perceived flexibility. Research in the area of supervisor support and work-life conflict (please see section 6.3.1) suggests that it may not be the alternative work arrangement per se that results in a greater sense of control but rather the actions of the employee's immediate supervisor. Supervisors can play a key role by providing information to employees on corporate policies (e.g. alternative work arrangements, and personal leave days), allowing an employee to leave early to handle a non-work emergency and modeling a sense of work-life balance. Allowing employees more control over their schedule will help the employee work more efficiently thereby reducing overload. Allowing employees to leave early to handle a personal crisis will help employees feel lower levels of interference between work and family.

\subsubsection{Justification of Interaction Hypotheses ( $\mathrm{H} 7-\mathrm{H} 12)$}

Karasek's buffering hypothesis posited that control could moderate the relationship between demands and job strain. Models $1-3$ in this thesis represent an updated conceptualization of Karasek's buffering hypothesis. 
Models 1-3 hypothesize that the various forms of control explored in this thesis (i.e. alternative work arrangements, perceived flexibility, supportive supervisor) will all moderate the association between demands (work and non-work) and work-life conflict (overload and interference). The literature supporting these hypotheses were reviewed in Sections 6.1,6.2 and 6.3. Basically these hypotheses (H7-12) all test the notion that employees with similar demands at work and at home will experience different perceptions of overload and interference depending on the amount of control they have. These hypotheses are based on the supposition that employees with a heightened sense of control should: (1) be able to work more efficiently and therefore perceive lower levels of overload than their counterparts with similar levels of demands but lower levels of control and (2) be better able to time when and where their work and non-work obligations are met, thereby reducing their perceptions of interference relative to those who report similar levels of demands but lower levels of control. Three forms of control over work-life interface are tested in these hypotheses: alternative work arrangements, perceived flexibility and supervisor support. Literature justifying our conceptualization of each of these conditions as types of control over the work-life interface was given earlier.

\subsubsection{Covariates of Models 1-3}

Covariates can affect the relationship between the independent and dependent variables such that it is unclear whether the independent variable impacted the dependent variable or whether the covariate impacted the 
dependent variable (Stevens, 1996). Appendix C provides a summary of the covariates included in each analysis. Key details are provided below.

In section 7 we established that gender and job type were associated with demands and work-life conflict. Consequently it was necessary to include gender and job type as covariates for all of the hypotheses tested in models 1, 2 and 3. Similarly, for the strain hypotheses associated with models 1-3 (i.e. H1$\mathrm{H} 6$ ), to ensure that the findings are due to the independent variable under consideration we controlled for the variables not directly associated with the given hypothesis. For example, controlling for non-work demands in $\mathrm{H} 1$ allows us to ensure the results are due to the independent variable under consideration (i.e. work demands), not sample differences with respect to non-work demands. Similarly, controlling for work demands when testing $\mathrm{H} 2$, allows us to ensure the findings are due to non-work demands not sample differences in work demands. For H3-H6, gender, job type, work demands and non-work demands were controlled so that we can ensure the findings are due to the independent variable under consideration (e.g. flextime in $\mathrm{H} 3 \mathrm{a}$ ) rather than sample differences in demands. These covariates (i.e. gender, job type, work demands, non-work demands) were also controlled for when we tested the buffer hypotheses (i.e. $\mathrm{H} 7-\mathrm{H} 12)$.

Furthermore, flextime, compressed work week, telework and shiftwork were controlled when testing hypotheses $\mathrm{H} 7 \mathrm{a}, \mathrm{b}, \mathrm{c}, \mathrm{d}$ and $\mathrm{H} 10 \mathrm{a}, \mathrm{b}, \mathrm{c}, \mathrm{d}$ respectively. Perceived flexibility was controlled when testing $\mathrm{H} 8$ and $\mathrm{H} 11$ and supervisor support were controlled when testing $\mathrm{H} 9$ and $\mathrm{H} 12$. Such an 
approach allows us to ensure that the findings are due to the interaction term under consideration and not the aforementioned covariates.

\subsection{Model Four: Hypotheses (Perceived Flexibility)}

What is not well understood in the literature is the relationship between alternative work arrangements and perceived flexibility as well as the potential for supervisor support to moderate the relationship between alternative work arrangements and perceived flexibility (see section 6.2.3 and 6.3.3). Model 4 was developed to allow us to develop both direct and interaction hypotheses and test these hypotheses. This section will present Model 4 followed by the hypotheses. This will be followed by the justification for the hypotheses and a discussion of the covariates.

Thesis model 4 is diagrammed below.

Figure 9 General Framework: Mapping Thesis Model 4

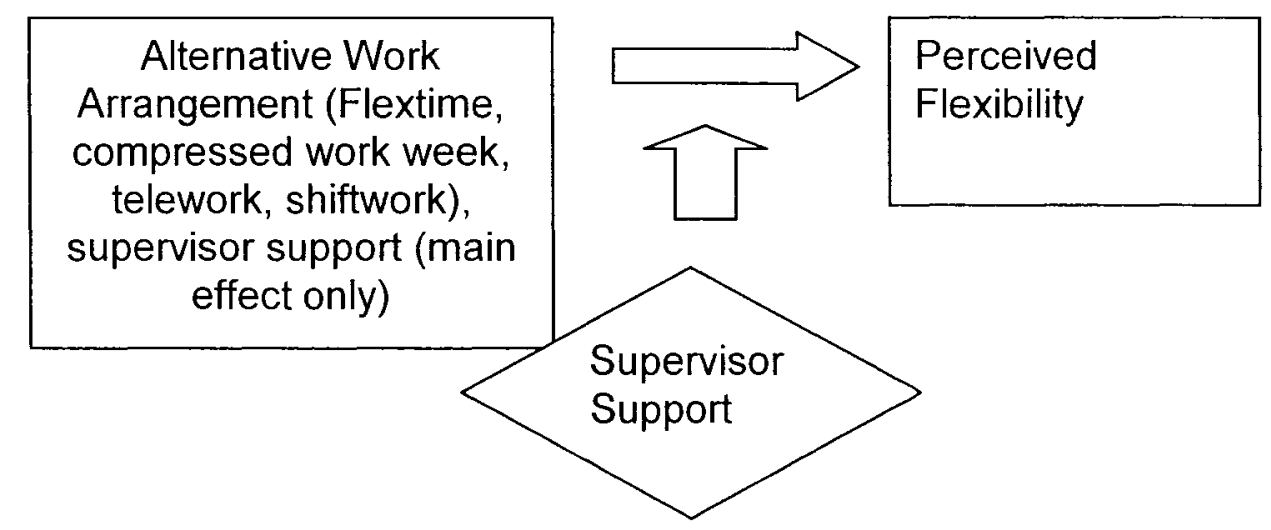

Model 4 has 9 hypotheses ( 5 direct hypotheses and 4 interaction hypotheses).

Each set of hypotheses will be presented in turn. 


\subsubsection{Model 4: Direct Hypotheses for Perceived Flexibility}

PFLEXH1: The use of alternative work arrangements (AWA) will be positively associated with perceived flexibility. Specifically employees who work:

a. flextime (versus a regular work day)

b. compressed work week (versus a regular work day)

c. telework (versus a regular work day)

will report higher levels of perceived flexibility than their counterparts who work a regular work day.

PFLEXH2: The use of shiftwork arrangements will be negatively associated with perceived flexibility.

PFLEXH3: Supervisor support will be positively associated with perceived flexibility.

\subsubsection{Model 4: Buffering Hypotheses for Perceived Flexibility}

PFLEXH4: Supervisor support will moderate the impact of alternative work arrangements on perceived flexibility. Specifically higher levels of supervisor support will strengthen the positive relationship between perceived flexibility and working:

a. flextime (versus a regular work day)

b. compressed work week (versus a regular work day)

c. telework (versus a regular work day)

PFLEXH5: Supervisor support will moderate the impact of shiftwork on perceived flexibility such that higher levels of supervisor support will weaken the negative relationship between shiftwork and perceived flexibility.

\subsubsection{Justification for Direct Hypotheses (Model 4)}

Theoretically, alternative work arrangements should increase an employee's ability to vary their work hours (flextime, compressed work week) or work location (telework). This increased ability to vary work hours/location should, in turn, increase their perception that they have increased flexibility with respect to scheduling of work. Empirical evidence to support this association can be found in section 6.2.3. 
As previously discussed, shiftwork arrangements are most often imposed upon the employee. Unlike the circumstances in PFLEXH1, in this case, shiftwork is likely to be associated with an employee feeling that he/she has less control over his/her work-life interface. This reduced lack of control is likely to manifest itself in perceptions of less perceived flexibility over the timing of one's hours. Empirical evidence to support this association can be found in section 6.2.3.

As previously discussed, a supervisor plays a central role in how the employee spends his/her day and whether an employee can informally leave the workplace early. Given this context, it can be argued that theoretically there should be a positive association between supervisor support and perceived flexibility. Empirical evidence to support this association can be found in section

\subsection{3.}

\subsubsection{Justification for Interaction Hypotheses (Model 4)}

Theoretically, it is possible to hypothesize that the behaviour of one's immediate supervisor will moderate the relationship between the employee's work arrangement and the amount of flexibility they perceive. A supervisor can influence the extent to which employees can actually vary their hours of work, days of work and location of work. Supervisors are often the ones to approve a given work arrangement and often have the discretion to allow an employee to unofficially leave early or start late on a given day. In other words, it is possible that an employee who works a regular schedule but reports to a supportive 
supervisor perceives greater flexibility than an employee who works flextime but reports to a manager who makes taking flexible hours difficult.

As previously noted, shiftwork arrangements tend to be imposed upon the employee. It is possible that having a supportive supervisor could weaken the relationship between shiftwork and perceived flexibility. Empirical evidence to support these associations can be found in section 6.3.4.

\subsubsection{Model 4 Covariates}

Covariates were also controlled for when testing the various hypotheses in Model 4 (see Appendix $C$ for a summary of what covariates were included in each analysis). For all hypotheses (PFLEX H1-5), gender, job type, work and non-work demands were controlled. In section 7 we established that gender and job type were associated with access to work arrangements and perceived flexibility. Consequently it was necessary to include gender and job type as covariates for all of the hypotheses tested in model 4. Controlling for work and non-work demands allows us to ensure the findings are due to the independent variable under consideration (e.g. flextime) and not due to the influence of work and non-work demands in PFLEX H1-3 (direct hypotheses).

Please note that gender, job type, work demands, non-work demands and supervisor support were controlled for in Hypotheses PFLEX H4 and PFLEX H5 (interaction hypotheses). Furthermore, flextime, compressed work week, telework were controlled when testing hypotheses PFLEX 4a,b, and c, respectively. Shiftwork was controlled when testing hypothesis PFLEX 5. 
Controlling for these covariates allows us to ensure the findings are due to the interaction term under consideration (e.g. flextime $x$ supervisor support) and not due to the influence of the aforementioned covariates. 


\section{Methodology}

The methodology section is divided into five sections. The first subsection will discuss the sample used in this thesis. The second subsection presents the measures used. This is followed in the third subsection by a discussion of the possible options for analyzing the data. The fourth subsection will discuss the assumptions involved with regression while the fifth section will outline the approach to analyzing the data.

\subsection{Sample}

The sample for this thesis comes from the 2001 National Work-life Conflict Study conducted by Linda Duxbury and Chris Higgins. The researchers (Duxbury and Higgins) contacted 2,978 Canadian organizations with a minimum of 500 employees each.

A total of 100 organizations (representing $3.4 \%$ of the total sample frame) participated in the Duxbury/Higgins study. Forty employers were from the private sector, 22 from the public sector and 38 from the not-for-profit sector. There were a total of 31,571 respondents (representing a $26 \%$ response rate). The lower response rate can be explained by the length and comprehensive nature of the survey and the absence of follow-up reminder notices (Duxbury and Higgins, 2001).

The use of a secondary data set for analysis has both advantages and disadvantages. Advantages may include: access to a large sample which greatly enhances the statistical power and the generalizability of the findings; access to well-validated measures; avoidance of the large expense of primary data 
collection as well as the ability to progress more quickly to the data analysis phase (Birnbaum, 2007; Dillon, Madden and Firtle, 1994; Green, Tell, and Albaum, 1993). Disadvantages may include: no control over the data collection process which limits the constructs and items that the secondary researcher may wish to explore; insufficient definition/documentation of the constructs may lead to confusion on the part of the secondary researcher about what the construct means; and the risk of using a dataset that is outdated (Birnbaum, 2007; Dillon, Madden and Firtle, 1994; Green, Tell, and Albaum, 1993).

\subsubsection{Thesis Sample Subset}

The entire sample of respondents from Duxbury and Higgins' (2001) National Work-Life Conflict Study was initially included in the analysis. This decision was based on the fact that increasing work demands, increasing nonwork demands and work-life conflict have the potential to impact all employees, regardless of gender, parental status or job type and that limiting the study of work-life conflict to subgroups such as those who are married and/or parents limits the generalizability of the findings (Boyar et al, 2003; Grzywacz and Marks, 2000). Both Boyar et al. (2003) and Grzywacz and Marks (2000) acknowledge that single childless adults often have nonwork obligations to parents, brothers/sisters and other family members.

Those respondents who worked part-time were screened out of the sample because the literature suggests that the organizational reality is quite different for part-timers (Bond et al., 2003; Higgins, Duxbury and Johnson, 2000; Werbel, 1985). White (1983) has characterized many part-time jobs as job 
ghettos with low pay, routine activities, and few promotional opportunities. These disadvantages have also been raised by Barnett (1998). A total of 4,317 respondents who work less than 35 hours per week were filtered out. This step resulted in a remaining sample of 27,254 respondents.

Those respondents who answered all categorical information (i.e. gender, job type, work arrangement, shiftwork) and who answered a minimum of one item for each construct of interest (i.e. work demands, non-work demands, role overload, work-family interference, family-work interference, perceived flexibility and supervisor support) were included for further analysis. Such an approach is consistent with other researchers (e.g. Duxbury and Higgins, 2001).

Respondents who failed to reply to at least one item per construct $(n=10,888)$ were filtered out of the dataset. This filtering step resulted in the sample of respondents being reduced from 27,254 to 16,366 .

\subsection{Operationalization of Constructs}

This section gives a brief overview of how each construct in the thesis models were operationalized in this research. The measures themselves can be found in Appendix A.

\subsubsection{Work and Non-work demands}

Respondents were asked to estimate the amount of time (in hours) spent per week in work and non-work activities. The decision to measure demands in this way is driven by the following considerations. First, measuring demands in this way is consistent with how the majority of researchers (e.g. Austin Knight, 
1995; Duxbury and Higgins, 2001; Fenwick and Tausig, 2001; Fu and Shaffer, 2001; Galinsky, Bond and Hill, 2004; Gutek et al., 1991; Silver, 2000) have measured demands when focusing on the relationship between demands and a given measure of strain (e.g. physical illness, depression, stress, work-life conflict).

Measuring demands in terms of hours is also consistent with the findings reported earlier (Section 4.3) establishing a positive association between demands (measured in terms of hours) and strain (outcomes such as depression, poor health and work-life conflict). Using hours to operationalize demands will allow also us to compare the association between both work and non-work demands and our three measures of work-life conflict.

Second, Duxbury and Higgins (2002b) argue that time is a fixed commodity and that time spent in one activity means that time is not available for other activities. Given the time commitment required of most workers (Duxbury and Higgins, 2002b), time spent at work leaves less time for other activities.

Many researchers (e.g. Duxbury and Higgins, 2002b; Frone et al., 1997; Grzywacz and Marks, 2000; Major, Klein and Ehrhart, 2002; Thompson, Beauvais and Lyness, 1999) point out that previous research suggests that time spent at work is positively associated with work-life conflict. This makes sense because more time spent at work means less time at home with the family. Insufficient time spent on family demands could negatively impact relationships with spouse and children (De Frank and Ivancevich, 1998; Gutek et al., 1991; Voydanoff, 2004). Therefore, the way this thesis measures demands is 
consistent with the notion of time as a fixed, finite resource and this measurement is consistent with the hypothesized relationships between demands and work-life conflict.

\subsubsection{Work Demands}

Work demands were quantified in this thesis by asking respondents to indicate the number of hours spent per week on the following four work-related activities: driving or commuting to and from work; in paid employment; working at home outside regular hours and educational activities (e.g. taking night courses, training for work). Total work demands per week were calculated for each respondent by summing the hours reported in each of the four work-related activities.

\subsubsection{Non-Work Demands}

Non-work demands were quantified by asking respondents to indicate the number of hours spent per week in home chores and errands, in child care or activities with children, in caring for elderly relatives or dependents and in volunteer activities. Similar to the approach used with work demands, total nonwork demands per week were calculated for each respondent by summing the hours reported in each of the four nonwork-related activities. In both cases, the higher the total number of hours spent in work and non-work activity, the higher the level of demands.

While it may have been possible to combine the work demands and nonwork demands scores into a composite total demands score, it was decided to keep the two scores separate as a global measure of demands would not permit 
the researcher to examine the impact of each demand separately. The need for such an examination can be obtained from the literature (e.g. Bianchi and Raley, 2005; Marini and Shelton, 1993; Robinson and Godbey, 1997; Zick and McCullough, 1991) that shows that while gender is not associated with total demands, it is strongly linked to the two components of total demands in that men do more paid work, women more unpaid work. Examinations that focus on total demands only could, therefore, come to erroneous conclusions with respect to the relationship between gender and demands.

In addition, focusing on total demands would not allow us to examine the relationship between work demands and our three measures of work-life conflict. By the same token, we would not be able to examine the relationship between non-work demands and these three measures of work-life conflict. There are also some theoretical consideration raised by Frone (2003) for examining the relationship between the two types of demands and work-life conflict separately. Frone (2003) argues that work-family interference and family-work interference are conceptually different and that work demands tend to be associated with work-family interference and non-work demands tend to be associated with family-work interference. The testing of such relationships would not be possible with a global measure of demands such as total demands.

\subsubsection{Measures of Strain (Work-Life Conflict)}

In this thesis, strain was operationalized as three different conceptualizations of work-life conflict: role overload, work-family interference and family-work interference. Role overload was quantified in this study using 
five items from a scale developed by Bohen and Viveros-Long (1981).

Respondents were asked to indicate using a 5 point Likert format $(1=$ strongly disagree, $3=$ neutral and $5=$ strongly agree) to what extent they agreed or disagreed with five statements associated with role overload. A sample question includes "I feel physically drained when I get home from work". Role overload was calculated for each respondent by computing an average score from the five items. Higher scores indicated greater role overload. The Cronbach's alpha for role overload for the subsample of 16,366 respondents was 0.87 . Tabachnick and Fidell (2001) suggest that Cronbach alphas in excess of 0.60 provide adequate reliability.

A modified version of the scale developed by Gutek, Searle and Kelpa (1991) was used to measure both work-to-family and family-to-work interference. Respondents were asked to indicate using a 5-item Likert scale (1 = strongly disagree, $3=$ neutral and $5=$ strongly agree) how much they agreed or disagreed with the following six questions concerning work-to-family interference and four questions about family-to-work interference. A sample question for the workfamily interference includes: "My work schedule often conflicts with my personal life". A sample question for family-work interference includes: "Making arrangements for children while I work involves a lot of effort". Work-family interference was calculated for each respondent by computing an average score from the six items. Family-work interference was calculated for each respondent by computing an average score from the four items. Higher scores for workfamily interference and family-work interference indicated higher levels of 
interference. The Cronbach's alphas for work-family interference and familywork interference were 0.91 and 0.87 respectively.

\subsubsection{Alternative Work Arrangements}

Work arrangement was operationalized by asking respondents to: "Please

FILL in the oval for the phrase that best describes how your employment is arranged". The description used for each of the following four work arrangements was as follows:

REGULAR: you work a set number of hours each week, arriving and departing at the same time each day.

FLEXTIME: you vary your arrival and departure times around a 'core' time when you should be at work.

COMPRESSED WORK WEEK: you get one working day off each week or two in return for longer hours.

WORK AT HOME: you spend part of your regular work week working at home.

In each case, the alternative work arrangement (i.e. flextime, compressed work week, telework) was assigned a value of 1 while the alternative condition (regular work week) was assigned a value of -1 . This means that the number of respondents analysed in the sample will change for the analyses associated with flextime, compressed work week and telework. For example, when testing hypotheses associated with flextime, those working compressed work week and telework are excluded from the analysis.

\subsubsection{Shiftwork}

Shiftworkers were identified by looking at the following survey question with three possible responses:

Do you work shifts? No; Yes, I work a fixed shift; Yes, I work a rotating shift. 
Shiftwork was dummy coded such that those working a regular work week were assigned a code of -1 and those working shiftwork (fixed and rotating) were both assigned a code of 1 . The coding was done in this fashion to be consistent with the hypotheses outlined in section 8 .

\subsubsection{Perceived Flexibility}

Perceived flexibility is defined as the amount of flexibility respondents perceive they have over their work hours and their work location. The measure of perceived flexibility used for this thesis was developed and tested by Duxbury and Higgins. Duxbury and Higgins first developed a four-item scale of perceived flexibility for their 1991 work-family study (Duxbury, Higgins, Lee and Mills, 1991). This measure was refined over the course of several studies and the measure of perceived flexibility used in this thesis included ten items.

Respondents were given a 5 point Likert scale (where a $1=$ very difficult, a $3=$ neither easy nor difficult, and a 5 = very easy) to use to respond to these questions. A sample question included how easy is it for you to vary your work hours? Perceived flexibility was calculated for each respondent by computing an average score from the ten items. Higher scores reflect greater perceived flexibility. The Cronbach's alpha for perceived flexibility for the subsample was 0.85 .

\subsubsection{Supervisor Support}

Supervisor support is defined as working for a supervisor who "is a good communicator, focuses on output rather than hours, demonstrates respect for employees and supports their career development" (Duxbury and Higgins, 2001, 
p. 55). The measure of supervisor support used for this thesis was developed and tested by Duxbury and Higgins between 1995 and 2001 (Duxbury and Higgins, 2001). The measure includes sixteen behaviours associated with being a supportive or a non-supportive supervisor. Six of these behaviours (e.g. makes me feel guilty about time off for personal or family reasons) are non-supportive and ten of these behaviours (e.g. listens to my concerns) are supportive. Respondents were asked to indicate using a five point Likert scale ( 1 = strongly disagree, $3=$ neutral, $5=$ strongly disagree) the extent to which they agreed or disagreed that their supervisor had engaged in each of these 16 behaviours. The six non-supportive behaviours were reverse-coded and a total supervisor support score was then calculated for each respondent by computing an average score for responses from the sixteen items. Higher scores reflect greater supervisor support. The Cronbach's alpha for supervisor support for the subsample was 0.91 .

\subsubsection{Gender}

Gender was quantified by asking respondents whether they were male or female. Males were dummy coded -1 and females coded 1 .

\subsubsection{Job Type}

Job type was quantified by asking respondents which of eight options best described the type of work they did: managerial, professional, technical, administrative, clerical, retail, production, or other. Managers/Professionals were grouped into one category (dummy coded -1 ) while all other job types were combined into a category called non-professional (coded 1). The decision to 
include the technical group of respondents into the non-professional category was driven by the finding in Duxbury and Higgins (2002b) that technical employees do not differ substantially from the non-professional employees in their sample (the reader will recall that this thesis uses the same dataset). The main differences in their sample were between managers/professionals and the other respondents (Duxbury and Higgins, 2002b).

As seen in section 7 of the thesis, there are differences in the level of demands and work-life conflict experienced by these two groups. These differences support the dichotomization of the eight categories into the two groups used in this thesis.

\subsection{Data Analysis Options}

Once the data is collected, researchers must make several key decisions about how to analyze the data. Two potential options for analyzing the data will be addressed. A review of the advantages and disadvantages of each option will be discussed followed by a justification for using the technique chosen to test the hypotheses (i.e. multiple regression).

Before outlining the data analysis options, it is useful to remember that this thesis is testing for both direct and interaction effects. Theoretically, it is possible to use either multiple regression or structural equation modeling for analyzing the data. Each technique will be defined and subsequently discussed in turn in the sections below. 


\subsubsection{Multiple Regression}

The first option for analyzing the data is multiple regression. Regression allows researchers to assess the relationship between a given dependent variable and several independent variables (Tabachnick and Fidell, 2001).

In this thesis, the research hypotheses posit a series of relationships testing both direct effects (between the independent variables and the dependent variable) and interaction effects (changes in the relationship between independent variables and the dependent variable as a function of the level of the moderator variable). Regression can be used to effectively assess the following: Are there statistically significant relationships for both direct effects and interaction effects? If so, are these relationships of practical significance? If these relationships are both statistically significant and practically significant, we can plot the interactions (Aiken and West, 1991).

There are four key advantages to using multiple regression in this thesis. First, the use of regression and its emphasis on assessing the strength of the relationships between both independent and dependent variables as well as independent variables and moderator variables is consistent with the hypotheses developed in this thesis. Second, regression is capable of handling categorical or dichotomous variables in addition to continuous variables. Third, procedures for analyzing, detecting and examining the nature of interaction effects in multiple regression have been well-established (Aiken and West, 1991; Jacquard, Turrisi, and Wan, 1990). Fourth, regression allows the researcher to control for possible confounds (gender and job type in this thesis). 
It should be noted that using multiple regression in this thesis analysis is not without drawbacks. Unlike structural equation modeling, multiple regression can only analyze one dependent variable at a time. To compensate for this fact, we tested four separate models.

\subsubsection{Structural Equation Modeling (SEM)}

The second option for data analysis is structural equation modeling.

Structural equation modeling (SEM) is a collection of statistical techniques that allow researchers to examine a set of relationships between one or more independent variables and one or more dependent variables (Ullman, 2001, 653). SEM examines these relationships by producing an estimated population covariance matrix and comparing this matrix with the sample covariance matrix (Ullman, 2001).

A key advantage to structural equation modeling is the ability to examine multiple predictive relationships involving more than one dependent measure simultaneously (Anderson and Gerbing, 1988). In the absence of testing for interaction effects, this approach is a more efficient way of testing hypotheses and examining the relationships among multiple outcomes. Another key advantage of structural equation modeling is the ability to examine latent variables (i.e. variables that are not directly measured but instead assessed indirectly based on observed indicators) in the research model.

While many authors cite the powerful ability of SEM to analyze complex relationships simultaneously (e.g. Ullman, 2001), it is acknowledged that a key disadvantage is the ensuing complexity that results from using this 
technique (Ullman, 2001). Furthermore, many authors (e.g. Brannick, 1995; Kelloway, 1995) have argued that it is pointless to use more complex statistical techniques when simpler methods are equally effective.

It is also important to remember that testing buffer hypotheses by looking for interaction effects is an integral feature of the thesis. Relative to multiple regression, the procedures for testing, detecting and describing interactions using SEM are less well-established (Cortina, Chen and Dunlop, 2001; Kline and Dunn, 2000). In fact, there is confusion among researchers using SEM about how to incorporate interaction effects into SEM (Cortina, Chen and Dunlap, 2001).

Furthermore, the procedures that have been advocated by Ping (1995), Hayduk (1996) and Kenny and Judd (1984) for testing interaction effects in SEM have been characterized as "easily prone to error" (Kline and Dunn, 2000, p. 127). Cortina et al. (2001) observe that there is a lack of user friendliness when it comes to describing the techniques for testing interactions using SEM. Cortina et al. (2001) take SEM researchers to task for not providing sufficient rationale for their techniques, for not describing their suggested techniques in detail, and for providing techniques that tend to be extremely complicated and cumbersome (Cortina et al., 2001).

These problematic issues with assessing interaction effects in SEM may help explain Cortina et al., (2001)'s observation that "the number of studies testing multiplicative effects with SEM is only slightly larger than it was in the 
wake of Kenny and Judd's (1984) seminal article on the topic" (Cortina et al., 2001, p. 325).

In conclusion, multiple regression is the most appropriate statistical analysis technique for this thesis as testing for interactions with multiple regression is well-established.

\subsection{Assumptions of Multiple Regression}

Several assumptions are associated with multiple regression including: the dependent variable is distributed normally (Stevens, 1996), there is a linear relationship between the dependent variable and the independent variables and there is homoscedasticity (i.e. constant errors of prediction across levels of the dependent measures) between predicted dependent variable scores and errors of prediction. Violations of normality and linearity assumptions could lead to an increase in the risk of Type 1 error (the probability of rejecting the null hypothesis when it is true) and decreases in power (the probability of rejecting the null hypothesis when it is false).

Normality of variables can be assessed either statistically or graphically (Tabachnick and Fidell, 2001). With large samples such as that used in this analysis, Tabachnick and Fidell (2001) recommend looking at the shape of the distribution instead of using formal inference tests. SPSSX was used to examine frequency histograms and normal probability plots to look for evidence of skewness (i.e. a skewed variable is a variable whose mean is not in the center of the distribution) and kurtosis (i.e. a distribution that is either too peaked with short, thick tails or too flat with long, thin tails) (Tabachnick and Fidell, 2001). 
Evidence of skewness and kurtosis would suggest the curve in question is not normal. No evidence of skewness or kurtosis was found for any of the dependent variables used in this thesis. Please see the histograms and probability plots in Appendix B1.

Nonlinearity and homoscedasticity was assessed by inspecting scatterplots using SPSS (Tabachnick and Fidell, 2001). Homoscedasticity occurs when the variance of residual error is constant for all values of the independent variables (Berry, 1993). Heteroscedasticity (i.e. the failure of homoscedasticity) results in a weakening of the ability to detect a linear relationship between the variables in question (Tabachnick and Fidell, 2001). Researchers can compare the standardized residuals versus the predicted values. A linear relationship among variables will result in the "standardized residuals being scattered randomly about a horizontal line" (Stevens, 1996, p. 93). An inspection of the scatterplots revealed no evidence of nonlinearity and no evidence of heteroscedasticity in any of the variables included in this thesis. The scatterplots are located in Appendix B2.

Tabachnick and Fidell (2001) also point out the problems associated with multicollinearity. Multicollinearity is defined as extremely high correlation (.90 or higher) between two independent variables (Tabachnick and Fidell, 2001). As discussed by Stevens (1996), there are three reasons why multicollinearity is problematic. First, the size of the multiple correlation $(R)$ will be limited because both predictors are trying to explain the same variance on the dependent variable. Second, multicollinearity makes it difficult to assess the relative 
importance of a certain predictor (e.g. predictor A) because another predictor (e.g. predictor B) is confounding the predictive ability of predictor A. Third, multicollinearity increases the variances of the regression coefficients thereby resulting in a more unstable prediction equation.

Fortunately, there are ways of checking for multicollinearity. A review of the correlations in section 10.2 shows no evidence of multicollinearity (Tabachnick and Fidell, 2001 suggest high correlations of 0.90 or higher are evidence of multicollinearity).

An effective strategy for reducing multicollinearity when interactions are calculated is to center the continuous independent variables and moderating variables (Aiken and West, 1991). More detail about centering the variables is provided later in this section.

\subsection{Data Analysis Approaches}

This section will outline the approaches used to analyze the data in this thesis. Chi-squared analysis, MANOVA and regression analysis were performed and each will be discussed in turn.

\subsubsection{Chi-Squared Analysis}

In order to develop a better sense of how the covariates can potentially affect the results of this thesis, we need first to examine the relationship between gender and job type. Since the gender and job type are categorical variables, we used the chi-square test of independence to examine this relationship (Tabachnick and Fidell, 2001). The null hypothesis is that there are no 
statistically significant relationship between gender and job type. If the $p$-value is less or equal to $.001^{5}$, we can reject the null hypothesis and conclude that there are statistically significant differences in the interaction between gender and job type.

This technique was also used to explore the relationship between the usage of the various work arrangements (compressed work week, flextime, telework and shiftwork) and gender and job type. The null hypothesis is that there are no statistically significant differences in the usage of various work arrangements by gender and job type. If the p-value is less than or equal to .001 , we can reject the null hypothesis and conclude that there are statistically significant differences in the interaction between gender and job type in the usage of various work arrangements.

\subsubsection{Analysis of Variance (MANOVA/ANOVA)}

Analysis of variance allows researchers to examine whether or not there are statistically significant mean differences among groups (Tabachnick and Fidell, 2001). In this thesis the relationship between gender, job type and all the continuous variables in the analysis were examined using ANOVA/MANOVA. The analysis proceeded as follows. First we examined the F-statistic associated with the gender by job type interaction effect to determine if the interaction was significant or not ( $p$ value of 0.001 or less). If yes, we interpreted the data by plotting the interaction. If the interaction effect was not significant, we interpreted the gender and job type main effects separately.

5 Please note that the rationale for the stricter alpha level is presented at the end of this section. 
The decision whether to use multivariate analysis of variance (MANOVA) or analysis of variance (ANOVA) is driven by the number of dependent variables analyzed. MANOVA is used in cases where there is more than one dependent variable and these dependent variables are either moderately or highly correlated (Duxbury and Higgins, 2005). As shown in Table 3 below, a MANOVA design was used to explore the relationship between gender, job type and work-life conflict because the three dependent variables (role overload, work-family interference and family-work interference) are moderately or highly correlated. MANOVA was also used to test the relationship between gender, job type and our two continuous measures of perceived control, perceived flexibility and supervisor support. This was done because these two variables are moderately correlated. An ANOVA design (i.e. one dependent variable) was used for work demands and non-work demands because these two variables are weakly correlated.

Table 3 Summary of MANOVA/ANOVAs Used in Thesis

\begin{tabular}{|l|l|l|}
\hline $\begin{array}{l}\text { Independent } \\
\text { variables }\end{array}$ & Dependent Variables & $\begin{array}{l}\text { Variance design } \\
\text { used }\end{array}$ \\
\hline Gender, job type & $\begin{array}{l}\text { Role overload, work-family } \\
\text { interference, family-work } \\
\text { interference }\end{array}$ & MANOVA \\
\hline Gender, job type & $\begin{array}{l}\text { Perceived flexibility, } \\
\text { supervisor support }\end{array}$ & MANOVA \\
\hline Gender, job type & Work demands & ANOVA \\
\hline Gender, job type & Non-work demands & ANOVA \\
\hline
\end{tabular}




\subsubsection{Hierarchical Regression}

A hierarchical regression approach was used to test the thesis hypotheses. Hierarchical regression allows the researcher (rather than SPSS) to choose the order in which variables are entered into the regression model based on their understanding of the theoretical literature. The advantage of hierarchical regression is that the researcher can see the unique contribution of the addition of a new independent variable or interaction term to the regression model by examining the change in $R^{2}$ (Tabachnick and Fidell, 2001). The $R^{2}$ is the "percent of variance in the dependent that is explained by the independent variables" (Duxbury and Higgins, 2005, p. 65).

This thesis tests both direct and interaction hypotheses for four different models. A table summarizing the testing approach for the four thesis models can be found in Appendix C. In summary, we are using hierarchical regression to test 8 direct hypotheses and 12 interaction hypotheses for each of models 1,2 and 3 (total of 60 regressions). Model 4 tests 5 direct and 4 interaction hypotheses (an additional 9 regressions). Such an approach was necessary in order to assess the unique contribution of the independent and moderator variables on the various dependent variables. It is acknowledged that running this many regressions increases the risk of a type 1 error (Stevens, 1996). In order to counter such a problem, Stevens (1996) recommends setting a strict alpha level in the order of 0.001 . This recommendation was followed in this thesis. 
Before undertaking the regression we followed the advice of Aiken and West (1991) and centered all continuous independent variables and moderators. Centering involves converting a continuous independent measure so that each variable has a mean of zero (Tabachnick and Fidell, 2001). Centering the variables reduces potential difficulties associated with multicollinearity by reducing the correlations between the independent variable and the interaction term as well as the correlations between the moderator variable and the interaction term (Aiken and West, 1991).

In this thesis centered scores were obtained by subtracting the sample mean from each respondent's score, resulting in transformed scores with a mean of zero (Aguinis, 2004). For example, the total sample mean for work demands is 48.85 hours per week. To center the score this mean of 48.85 was subtracted from the reported work demand score of each of the 16,366 respondents in the sample. In the case of a respondent with a reported work demands score of 70.85 this would result in a centered score of 22 .

The procedure for testing the direct and interaction (i.e. buffering) hypotheses was identical for all four thesis models. The procedures used in this thesis are outlined in the sections below.

\subsubsection{Testing direct effect hypothesis}

In order to test the direct effect (i.e. strain) hypotheses, the covariates (gender and job type) were first entered into the regression equation. Such an approach is recommended as a way of controlling for the effect of these 
covariates in the analysis (Aiken and West, 1991; Cohen and Cohen, 1983). As discussed in section 7 , the literature suggests that both socio-economic status (represented in this thesis by job type) and gender are likely to act as covariates which can affect the relationship between each independent variable (i.e. work demands and non-work demands) and the dependent variables (role overload, work-family interference, family-work interference). Work demands and/or nonwork demands were also included as covariates in a number of the analyses to ensure that the findings were due to the independent variable under consideration (please see Appendix $C$ for a detailed summary of how each hypothesis was tested). The $R^{2}$ resulting from this first regression run tells us the amount of variance in the dependent variable explained by the covariates. For example, to test $\mathrm{ROH} 1$ (i.e. work demands are positively associated with role overload), the covariates gender, job type and non-work demands were entered in the regression equation and the percentage of the variance in role overload explained by the covariates was represented by the resulting $R^{2}$.

The independent variable (varies depending on the hypothesis being tested) was entered into the regression equation in step 2. The impact of adding this independent variable on our ability to explain the variation in our dependent variable is then determined by examining the change in $R^{2}$ from step 1 to step 2 (i.e. the additional percentage of variance in the dependent variable explained by the addition of that variable of interest) The hypothesis is tested by looking at whether or not this change in $\mathrm{R}^{2}$ is statistically significant. As noted earlier, a strict alpha of less than or equal to 0.001 is required for significance in this thesis. 
The third step of our hierarchical regression analysis involves an assessment of the relative importance of the independent variables in the model being tested. Relative importance of the predictor variables (i.e. work demands, non-work demands) is obtained by calculating Pratt's coefficient and Pratt's standardized measure (Thomas et al., 1998). Pratt's coefficient is calculated by multiplying the standardized regression coefficient of the predictor variable by the correlation of the predictor variable and the dependent variable. Pratt's standardized measure is calculated by dividing Pratt's coefficient by the total variance explained. All standardized Pratt measures sum to 1 (Thomas et al., 1998).

\subsubsection{Testing the moderator (i.e. interaction) hypothesis}

Steps one and two of these analysis are identical to those described for testing of direct effect hypothesis. In this case, however, step 3, requires the addition of the interaction term into the equation. For example, to test $\mathrm{ROH} 7 \mathrm{a}$, the covariates (i.e. gender, job type, work demands, non-work demands) were entered into the regression equation. Second, the independent variable (i.e. flextime) was entered. Third, the interaction term (i.e. work demands $x$ flextime) was entered.

In order to determine whether or not the interaction term was statistically significant, we examine the change in $R^{2}$ from step 2 to step 3 using the same procedure as described in the previous section. In this case we also examine the unstandardized regression coefficient (b). Unstandarized regression coefficients 
indicate the average amount the dependent variable increases when the independent variable increases one unit associated with the interaction term (Stevens, 1996; Tabachnick and Fidell, 2001). Aguinis (2004) and Aiken and West (1991) recommend that researchers who are interpreting interaction effects should examine unstandardized, rather than standardized, regression coefficients. They justify this recommendation by noting that the interpretation of the standardized solution (i.e. the beta) "provided by computer packages is fraught with great difficulties because even if the predictors are standardized, their product in not necessarily standardized" (Aguinis, 2004, p. 38).

If the $p$-value for the interaction term is less than or equal to our alpha of 0.001 , then we can accept the moderation hypothesis. For example, we can say for $\mathrm{ROH} 7 \mathrm{a}$ that the relationship between work demands and role overload will be weaker for those who use flextime arrangements than those who work a regular work day.

Finally, in all of the above analysis it is important to examine practical as well as statistical significance. Such a step is necessary because the size of the thesis dataset $(16,366$ respondents) means that a result may be statistically significant but not practically significant. Only those results that were statistically significant were examined for practical significance.

Two key methods were used in this thesis to determine practical significance. First, we examined the relative impact of the interaction variable at various levels of a given predictor by plotting that interaction. Plotting the interaction allows researchers to understand whether the interaction effect (e.g. 
work demands $\mathrm{x}$ supervisor support) is stronger at low, medium or high values of the independent variable (e.g. work demands). An interaction term that results in a different slope at different levels of an independent variable (e.g. work demands) is deemed to be practically significant (Aguinis, 2004). Second, we examined the change in $R^{2}$ from step 2 (adding the independent variable) to step 3 (adding the interaction variable). Aguinis (2004) recommends that a change in $\mathrm{R}^{2}$ of $1 \%$ or higher is practically significant. 


\section{Results}

The results section is divided into four subsections. First, the demographics of the sample will be presented. Second, the relationship between gender, job type and the following variables, demands, work-life conflict, work arrangements, perceived flexibility, and supervisor support will be explored. Third, the correlations between the variables in this thesis are presented and discussed. Fourth, the results of the hierarchical regression will be presented for each of the four thesis models.

\subsection{Demographics}

This subsection will present detailed information on the demographic composition of the subsample analysed in this thesis. This is followed by a summary of both the means and standard deviations for all the variables analyzed in this thesis. Table 4 summarizes the demographic information in this thesis ${ }^{6}$.

6 Please note that for those cells with asterisks, the mean is presented first followed in brackets by the standard deviation. 
Table 4 Summary of Demographic Characteristics

\begin{tabular}{|l|l|l|l|}
\hline & Men & Women & Total \\
\hline Number and Percentage of Respondents & $7130(44 \%)$ & $9236(56 \%)$ & $16,366(100 \%)$ \\
\hline Average Age of Respondents (years) & 44.34 & 42.01 & 42.95 \\
\hline $\begin{array}{l}\text { Number and Percentage of Married } \\
\text { Respondents }\end{array}$ & $5194(73 \%)$ & $6971(75 \%)$ & $12165(77 \%)$ \\
\hline Number and Percentage of Parents & $5621(79 \%)$ & $6226(75 \%)$ & $11847(72.4 \%)$ \\
\hline $\begin{array}{l}\text { Education Level (Post-secondary degree or } \\
\text { higher) }\end{array}$ & $4047(57 \%)$ & $5884(64 \%)$ & $9931(61 \%)$ \\
\hline Income Range (\$40,000-\$59,999) & $2312(32 \%)$ & $3277(35 \%)$ & $5589(34 \%)$ \\
\hline $\begin{array}{l}\text { Number and Percentage of Manager/ } \\
\text { Professionals (Job Type) }\end{array}$ & $4171(58 \%)$ & $4070(44 \%)$ & $8241(50.4 \%)$ \\
\hline $\begin{array}{l}\text { Number and Percentage of Non- } \\
\text { Professionals (Job Type) }\end{array}$ & $2959(42 \%)$ & $5166(56 \%)$ & $8125(49.6 \%)$ \\
\hline $\begin{array}{l}\text { Mean and Standard Deviation for Work } \\
\text { Demands }\end{array}$ & $50.40^{\star}(9.75)$ & $47.63^{\star}(8.80)$ & $48.85(9.33)^{\star}$ \\
\hline $\begin{array}{l}\text { Mean and Standard Deviation for Non-Work } \\
\text { Demands }\end{array}$ & $18.58^{\star}(11.37)$ & $18.72^{\star}(11.74)$ & $18.66(11.58)^{\star}$ \\
\hline $\begin{array}{l}\text { Mean and Standard Deviation for Role } \\
\text { Overload }\end{array}$ & $3.45^{\star}(.88)$ & $3.69^{*}(.89)$ & $3.58(0.89)^{\star}$ \\
\hline $\begin{array}{l}\text { Mean and Standard Deviation for Work- } \\
\text { Family Interference }\end{array}$ & $2.84^{\star}(1.00)$ & $2.74^{\star}(1.02)$ & $2.78(1.02)^{\star}$ \\
\hline $\begin{array}{l}\text { Mean and Standard Deviation for Family- } \\
\text { Work Interference }\end{array}$ & $2.24^{*}(.90)$ & $2.21^{\star}(.98)$ & $2.22(0.95)^{\star}$ \\
\hline $\begin{array}{l}\text { Mean and Standard Deviation for Perceived } \\
\text { Flexibility }\end{array}$ & $3.27^{*}(.86)$ & $3.13^{\star}(.93)$ & $3.19(0.82)^{\star}$ \\
\hline $\begin{array}{l}\text { Mean and Standard Deviation for Supervisor } \\
\text { Support }\end{array}$ & $3.54^{*}(.72)$ & $3.61^{\star}(.78)$ & $3.58(0.76)^{\star}$ \\
\hline Number and Percentage working M-F 9 to 5 & $4509(63 \%)$ & $5281(57 \%)$ & $9790(60 \%)$ \\
\hline Number and Percentage working Flextime & $1933(27 \%)$ & $2057(22 \%)$ & $3990(24 \%)$ \\
\hline Number and Percentage working CWW & $974(13.7 \%)$ & $1446(15.7 \%)$ & $2420(14.7 \%)$ \\
\hline Number and Percentage working Shiftwork & $1115(15.6 \%)$ & $1300(14.1 \%)$ & $2415(14.7 \%)$ \\
\hline Number and Percentage working Telework & $81(1.1 \%)$ & $85(0.9 \%)$ & $166(1.0 \%)$ \\
\hline
\end{tabular}

A total of 16,366 respondents are included in the analysis in this thesis.

The average age of the respondents is 42.95 years of age. Over three quarters of respondents $(77 \%)$ are married and parents have an average of 1.5 children. A majority of respondents (61\%) have a college diploma or university degree, and the average respondent indicates that they are earning between $\$ 40,000$ and $\$ 59,999$ annually. The means and standard deviations for work demands, non-work demands, role overload, work-family interference, family-work

$7 \mathrm{CWW}$ is the short form for Compressed Work Week 
interference, perceived flexibility and supervisor support are summarized in Table 4.

In reviewing the means in Table 4, several points are worth noting. First, respondents on average, report heavy work demands ( 48.85 hours in work per week). This is substantively higher than the amount of time spent in non-work activities (mean of 18.66 hours per week). Second, respondents report greater levels of role overload (mean of 3.58) than any other form of work-life conflict. While respondents also report moderate levels of work-family interference (mean of 2.78), very few report family-work interference (mean of 2.22). These findings are consistent with those reported by other work-life researchers (e.g. Frone, 2003). To sum up, individuals in this sample reported moderate to high levels of role overload, moderate to low levels of work-family interference and low levels of family-work interference. Third, the majority $(60 \%)$ of respondents work a conventional Monday-Friday, 9 to 5 schedule. Almost a quarter of respondents work a flextime arrangement while an equal percentage of respondents work compressed work week and shiftwork. A very small percentage of respondents work telework.

Finally, respondents reported moderate to high levels of supervisor support (3.58) and moderate levels of perceived flexibility (3.19). The results present a picture of the average Canadian as one who works a fixed work day, is experiencing high demands at work and high levels of role overload but also receives moderately high levels of support from their supervisor. Furthermore, it would appear that having a supportive supervisor does not necessarily lead to 
increased levels of perceived flexibility as more individuals reported high levels of supervisor support than those who reported high levels of perceived flexibility.

\subsection{Role of Gender and Job Type}

The literature review indicated that both gender and job type could potentially be associated with many of the key variables of interest in this thesis (i.e. demands, work-life conflict, perceived flexibility, supervisor support, work arrangements). This section of the thesis explores the relationship between gender, job type and all the variables considered in this analysis for the research sample. This analysis was undertaken to confirm the need to include gender and job type as covariates in the regression analysis.

Table 4 provides data on the number of men and women in this sample who hold managerial/professional versus non-professional positions.

Examination of the data in this table indicates that the sample is fairly welldistributed with respect to gender and job type. Just over half (56\%) of the respondents are women, just under half are men (44\%). Similarly $50.4 \%$ of the respondents work in managerial and professional positions while $49.6 \%$ are nonprofessional.

As noted in section 9.5.1, we used the chi-square test of independence to see if gender is associated with job type (Tabachnick and Fidell, 2001). The null hypothesis is that there is no association between gender and job type. In this case, the null hypothesis is rejected $\left(X^{2}=335.28 ; d f=1 ; p\right.$-value of .000$)$ therefore there is a statistically significant association between gender and job type. Examination of the data in Table 4 shows that men are over-represented in the manager/professionals sample and under-represented in the non-professionals 
sample relative to their female counterparts. These findings are consistent with the idea of gender compression which was discussed earlier in the thesis.

\subsubsection{Examination of the Relationship Between Gender, Job Type and Work Arrangement}

Before undertaking regression analysis, we examined the relationships

between gender, job type and the use of various work arrangements of interest in this thesis (i.e. flextime, compressed work week, telework, and shiftwork).

Tabachnick and Fidell (2001) recommend performing a chi-squared analysis to test these relationships as gender, job type and work arrangement are all categorical variables. Table 5 provides a summary of the use of the arrangements by gender and job type.

Table 5 Use of Work Arrangements by Gender and Job Type ${ }^{8}$

\begin{tabular}{|l|l|l|l|}
\hline & Mgr/Prof. & Non Prof. & $\mathbf{n}=$ \\
\hline \% working flextime & & & \\
\hline Male & $1342(32 \%)$ & $591(20 \%)$ & 1933 \\
\hline Female & $1165(29 \%)$ & $892(17 \%)$ & 2057 \\
\hline$n=$ & 2507 & 1483 & 3990 \\
\hline & & & \\
\hline$\%$ working CWW & & & \\
\hline Male & $538(13 \%)$ & $436(15 \%)$ & 974 \\
\hline Female & $569(14 \%)$ & $877(17 \%)$ & 1446 \\
\hline$n=$ & 1107 & 1313 & 2420 \\
\hline & & & \\
\hline$\%$ teleworking & & & 81 \\
\hline Male & $61(1 \%)$ & $20(0.6 \%)$ & 85 \\
\hline Female & $57(1 \%)$ & $28(0.5 \%)$ & 166 \\
\hline$n=$ & 118 & 48 & \\
\hline & & & \\
\hline$\%$ working shiftwork & & & 1115 \\
\hline Male & $600(14 \%)$ & $515(17 \%)$ & 1300 \\
\hline Female & $652(16 \%)$ & $648(13 \%)$ & 2415 \\
\hline$n=$ & 1252 & 1163 & \\
\hline
\end{tabular}

\footnotetext{
${ }^{8}$ The percentage of usage was calculated by taking the number of respondents who worked that arrangement and divided by the total number of respondent for that category.
} 
The following conclusions can be drawn from the data. The use of flextime depends on gender and job type $\left(X^{2}=69.80, d f=1 ; p\right.$-value $\left.<.001\right)$. Two key differences were observed. First, managers and professionals, regardless of gender, were more likely to work flextime than their non-professional counterparts. Second, males, regardless of job type, were more likely to work flextime than females.

The use of a compressed work week also depends on gender and job type $\left(X^{2}=59.18, d f=1 ; p\right.$-value <.001). Two key differences were observed. First, non-professionals, regardless of gender, were more likely to work compressed work weeks than their manager/professional counterparts. Second, while there were no gender differences in the manager/professional sample, female non-professionals were more likely to work compressed work weeks than male non-professionals.

In the case of telework and shiftwork, we failed to reject the null hypothesis. The data does not support the idea that the use of either telework $\left(X^{2}=1.37, d f=1 ; p\right.$-value $\left.<.241\right)$ or shiftwork $\left(X^{2}=3.28, d f=1 ; p\right.$-value $\left.<.073\right)$ is associated with gender or job type.

\subsubsection{Examination of the Relationship Between Gender, Job Type and Work-Life Conflict}

Consistent with the procedure outlined in section 9.5.2, we examined the relationship between gender, job type and all the continuous variables in the analysis using ANOVAMANOVA. The analysis proceeded as follows. First we examined the F-statistic associated with the gender by job type interaction effect 
to determine if the interaction was significant or not ( $p$ value of 0.001 or less). If yes, we interpreted the data by plotting the interaction. If the interaction effect was not significant, we interpreted the gender and job type main effects separately.

The decision whether to use multivariate analysis of variance (MANOVA) or analysis of variance (ANOVA) is driven by the number of dependent variables analyzed. MANOVA is used in cases where there is more than one dependent variable and these dependent variables are either moderately or highly correlated (Duxbury and Higgins, 2005). A MANOVA design was used to explore the relationship between gender, job type and work-life conflict because the three dependent variables (role overload, work-family interference and family-work interference) are moderately or highly correlated. MANOVA was also used to test the relationship between gender, job type and our two continuous measures of perceived control: perceived flexibility and supervisor support. This was done because these two variables are moderately correlated. An ANOVA design (i.e. one dependent variable) was used in the work demands and non-work demands analysis because these two variables are weakly correlated. The results of these analyses are summarized in Table 6. 
Table 6 Results of Gender/Job Type MANOVA/ANOVA

\begin{tabular}{|c|c|c|c|}
\hline DV & Interaction & Gender Main Effect & Job Type Main Effect \\
\hline WD & Not significant & $\begin{array}{l}\text { Men report higher } \\
\text { work demands } \\
\text { than women }\end{array}$ & \begin{tabular}{ll}
\multicolumn{3}{l}{ Manager/professionals } \\
report higher work \\
demands than non- \\
professionals & \\
\end{tabular} \\
\hline NWD & $\begin{array}{l}\text { Significant } \\
\text { For women, non-professionals } \\
\text { report higher non-work demands } \\
\text { than manager/professionals } \\
\text { For men, manager/professionals } \\
\text { report higher non-work demands } \\
\text { than non-professionals }\end{array}$ & & \\
\hline $\mathrm{RO}$ & Not significant & $\begin{array}{l}\text { Women report } \\
\text { higher RO than } \\
\text { men }\end{array}$ & $\begin{array}{l}\text { Manager/professionals } \\
\text { report higher RO than non- } \\
\text { professionals }\end{array}$ \\
\hline$\overline{W F I}$ & $\begin{array}{l}\text { Significant } \\
\text { No gender difference in WFI for } \\
\text { manager/professionals. Male non- } \\
\text { professionals report higher WFI } \\
\text { than female non-professionals }\end{array}$ & & \\
\hline FWI & Not significant & Not significant & $\begin{array}{l}\text { Manager/professionals } \\
\text { report higher FWI than } \\
\text { non-professionals }\end{array}$ \\
\hline Pflex & $\begin{array}{l}\text { Significant } \\
\text { For men, manager/professionals } \\
\text { report higher levels of Pflex than } \\
\text { non-professionals } \\
\text { For women, non-professionals } \\
\text { report higher levels of Pflex than } \\
\text { manager/professionals }\end{array}$ & & \\
\hline SS & Not significant & $\begin{array}{l}\text { Women report } \\
\text { higher SS than } \\
\text { men }\end{array}$ & $\begin{array}{l}\text { Manager/professionals } \\
\text { report higher SS than non- } \\
\text { professional }\end{array}$ \\
\hline
\end{tabular}

Legend: $D V=$ dependent variable; Male Pro=Male Managers/Professionals; Male Non-Pro=Male Non-Professionals; Female Pro=Female Managers/Professionals; Female Non-Pro=Female NonProfessionals; $\mathrm{G}=\mathrm{Gender}, \mathrm{JT}=\mathrm{Job}$ Type; $\mathrm{G} \times \mathrm{JT}=$ interaction of gender and job type; $\mathrm{WD}=$ work demands; NWD=non-work demands; RO=Role Overload, WFI=Work-Family Interference; FWI=Family-Work Interference; Pflex=Perceived Flexibility; SS=Supervisor Support

While the interaction term of gender/job type for role overload is not significant $(F$-stat $=.385 ; \mathrm{df}=3,16360 ; \mathrm{p}$-value $=.535)$, both main effects are Women (regardless of job type) report higher levels of role overload (3.69) than men (3.45) $(F$-stat $=367.08 ; d f=1,16362 ; p$-value $=<.001)$. Furthermore, 
manager/professionals (regardless of gender) report higher levels of role overload (3.65) than non-professionals (3.51) (F-stat=165.41; df=1, 16362; $p$ value $=<.001)$.

The interaction term of gender/job type for work-family interference is significant $(F-$ stat $=12.70 ; d f=3,16360 ; p$-value $=<.001)$. The results of the interaction of gender/job type for work-family interference are plotted in Figure 10. From this figure, we can see that for manager/professionals, work-family interference is not associated with gender. However, male non-professionals report higher levels of work-family interference (2.97) than female nonprofessionals (2.55). 
Figure 10 Gender/Job Type Interaction for Work-Family Interference

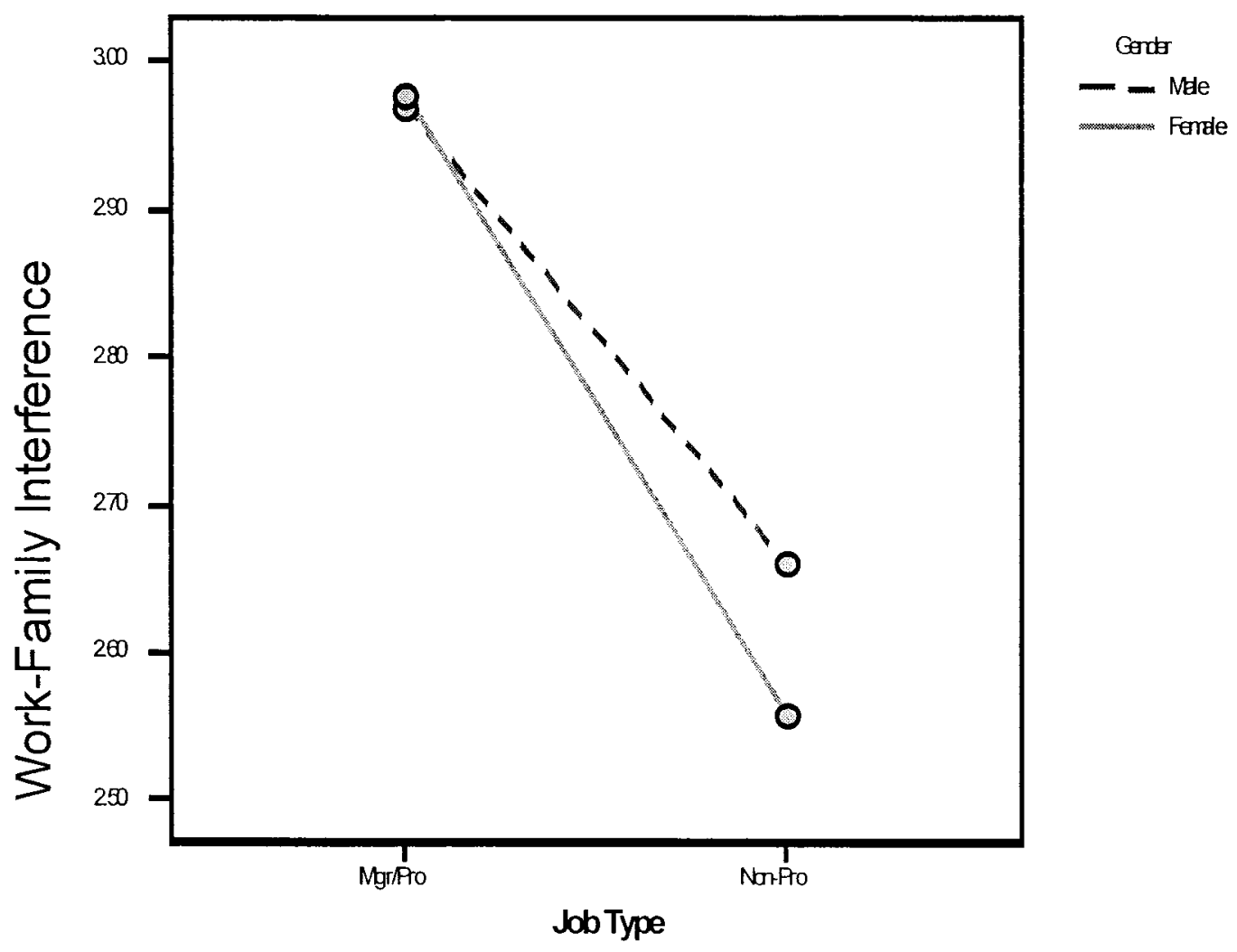


While neither the family-work interference interaction of gender/job type $(F-$ stat $=.530 ; d f=3,16360 ; p$-value $=.466)$ nor the gender main effect $(F-$ stat=.736; $d f=1,16362 ; p$-value .380 ) are significant in the analysis, the job type main effect is significant $(\mathrm{F}$-stat $=17.36 ; \mathrm{df}=1,16362 ; \mathrm{p}$-value $<.001)$. The data show that manager/professionals report higher levels of family-work interference (2.26) than non-professionals (2.19).

\subsubsection{Examination of the Relationship Between Gender, Job Type and Perceived Flexibility}

The interaction term of gender/job type for perceived flexibility is significant ( $F$-stat $=50.02 ; d f=3,16362 ; p$-value $<.001)$. The results of the interaction of gender/job type for perceived flexibility are plotted in Figure 11. For men, manager/professionals report higher levels of perceived flexibility (3.31) than non-professionals (3.23). For women, non-professionals report higher levels of perceived flexibility (3.17) than manager/professionals (3.06). 
Figure 11 Gender/Job Type Interaction for Perceived Flexibility

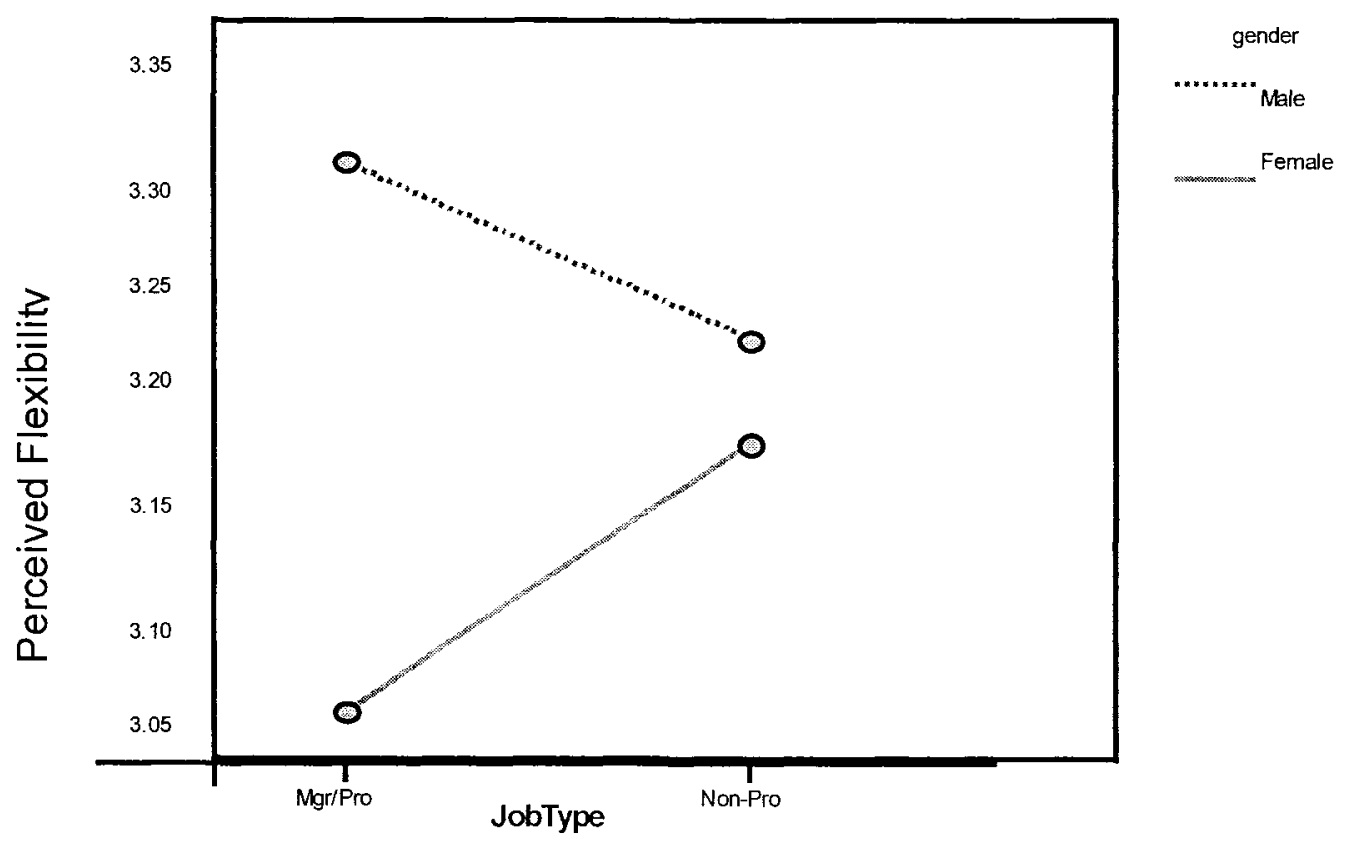


While, the interaction term of gender/job type for supervisor support is not significant $(F-$ stat $=.7 .00 ; d f=3,16362 ; p$-value $=.008)$, both main effects are. Women (regardless of job type) report higher levels of supervisor support (3.61) than men $(3.54)(F-s t a t=46.67 ; d f=1,16362 ; p$-value $=<.001)$. Managers/professionals (regardless of gender) report higher levels of supervisor support (3.61) than non-professionals (3.56) $(F-s t a t=27.71 ; d f=1,16362 ; p$-value $=<.001)$.

\subsubsection{Examination of the Relationship Between Gender, Job Type and Demands}

While the interaction term of gender/job type for work demands is not significant $(F-$ stat $=4.18 ; d f=3,16362 ; p$-value $=.041)$, both main effects are significant. Men (regardless of job type) report higher levels of work demands (50.40 hours per week) than women (47.63 hours per week) ( $F$-stat= 250.82; $\mathrm{df}=1,16362 ; p$-value $=<.001)$. Furthermore, manager/professionals $($ regardless of gender) report higher levels of work demands ( 50.80 hours per week) than non-professionals $(46.86$ hours per week $)(F$-stat $=618.15 ; \mathrm{df}=1,16362 ; p$-value $=$ $<.001)$.

The interaction term of gender/job type for non-work demands is significant $(F$-stat $=10.95 ; d f=3,16362 ; p$-value $<.001)$. The results of the interaction of gender/job type for non-work demands are plotted in Figure 12. From this figure, we can see that for women, non-professionals report higher levels of non-work demands ( 18.85 hours per week) than manager/professionals (18.57 hours per week). For men, manager/professionals report higher levels of 
non-work demands (18.97 hours per week) than non-professionals ( 18.03 hours per week).

Figure 12 Interaction of Gender/Job Type for Non-Work Demands

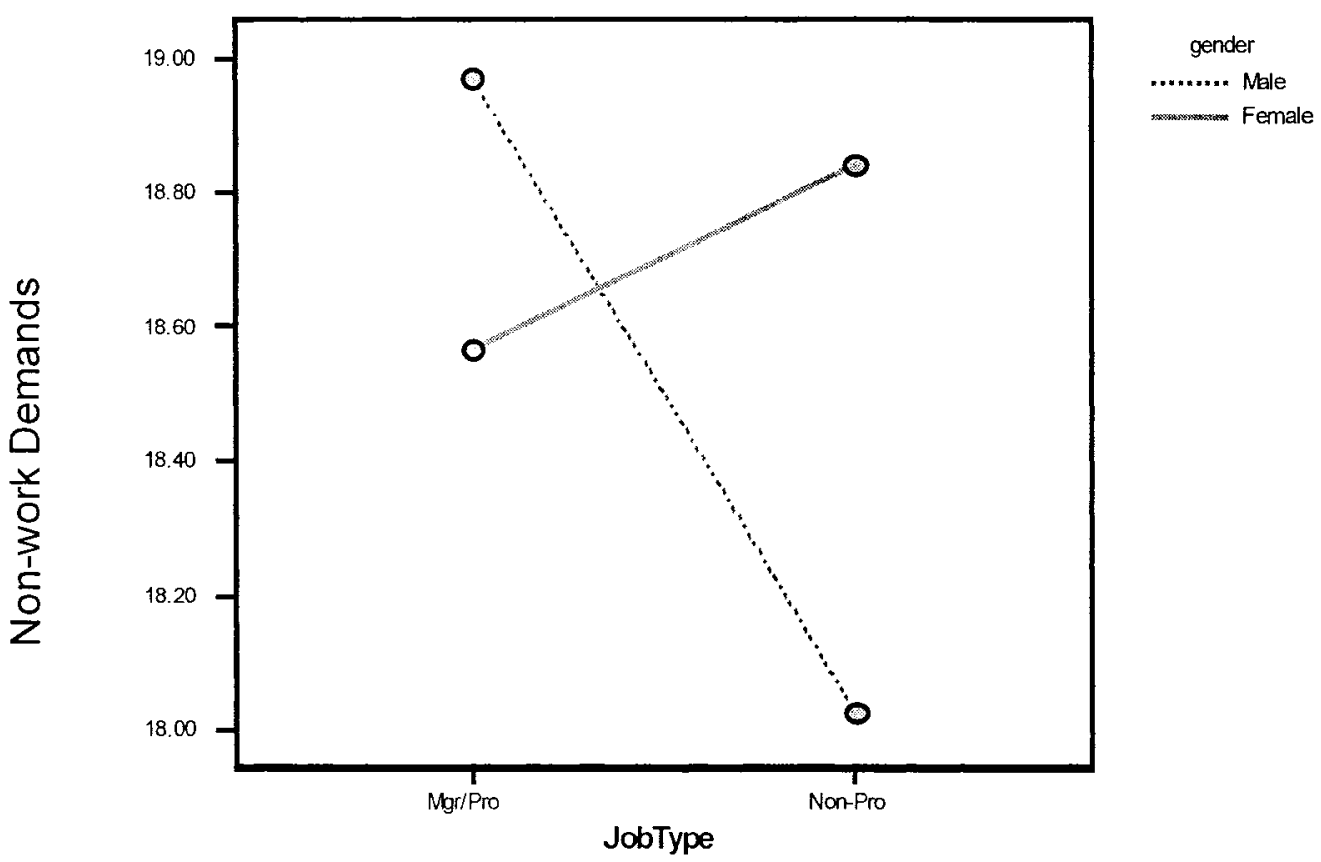


In conclusion, the above findings support our decision to control for both gender and job type in the analysis.

\subsection{Correlation Analysis}

As part of the preliminary analysis, a correlation analysis among all the study measures was performed. Doing so is a good first step to see if the relationships are in the hypothesized direction as well as an effective way to check for multicollinearity. Table 7 shows the results of this analysis. 
Table 7 Correlation Analysis (Covariates, Independent, Dependent and Moderator Variables)

\begin{tabular}{|c|c|c|c|c|c|c|c|c|c|c|c|c|c|}
\hline & 1 & 2 & 3 & 4 & 5 & 6 & 7 & 8 & 9 & 10 & 11 & 12 & 13 \\
\hline $1 G$ & $\ldots$ & & & & & & & & & & & & \\
\hline $2 \mathrm{JT}$ & $.143^{\star \star \star}$ & --- & & & & & & & & & & & \\
\hline $3 W D$ & $-.149^{\star \star \star}$ & $-.212^{\star \star \star}$ & $\cdots$ & & & & & & & & & & \\
\hline 4 NWD & .006 & -.010 & $.011^{\star \star \star}$ & --- & & & & & & & & & \\
\hline $5 \mathrm{RO}$ & $135^{\star \star \star}$ & $-.079^{\star \star \star}$ & $171^{\star \star \star}$ & $.150^{\star \star \star}$ & -- & & & & & & & & \\
\hline $6 \mathrm{WFI}$ & $-.048^{* \hbar \star}$ & $-.186^{\star \star \star}$ & $293^{* \star \star}$ & $.115^{\star \star \star}$ & $.618^{* \pi \star}$ & --- & & & & & & & \\
\hline $7 \mathrm{FWI}$ & -.012 & $-.035^{\star \star \star}$ & $.034^{\star \star \star}$ & $.235^{\star \star \star}$ & $.314^{\star \star \star}$ & $.357^{\star \star \star}$ & -- & & & & & & \\
\hline 8 Flex & $-.056^{\star \star \star}$ & $-.152^{\star \star \star}$ & $.091^{\star \star \star}$ & -.014 & -.002 & $.044^{\star \star \star}$ & $.027^{\star \star \star}$ & $--\cdot$ & & & & & \\
\hline $9 \mathrm{CWW}$ & .017 & .003 & $-.073^{\star \star \star}$ & .013 & .015 & -.020 & .013 & & -- & & & & \\
\hline 10 Tele & -.017 & $-.064^{\star \star \star}$ & $.056^{\star \star \star}$ & .017 & .015 & $.034^{\star \star}$ & $.032^{\star \star \star}$ & & & - & & & \\
\hline 11 Shift & $-.022^{\star \star}$ & -.012 & $.025^{\star \star}$ & -.014 & -.004 & $.090^{* \star *}$ & -.013 & $-.080^{* * *}$ & $-.036^{\star \star \star}$ & $-.050^{\star * \star}$ & $\cdots$ & & \\
\hline 12 Pflex & $-.081^{* \star \star}$ & .007 & $-.130^{\star \star \star}$ & $-.101^{\star \star \star}$ & $-.353^{\star \star \star}$ & $-.418^{\star \star \star}$ & $-.176^{\star \star \star}$ & $.186^{\star \star \star}$ & $.108^{\star \star \star}$ & $.118^{\star \star \star}$ & $-.223^{\star \star \star}$ & -- & \\
\hline $13 \mathrm{SS}$ & $.047^{\star \star \star}$ & $-.031^{\star \star \star *}$ & $-.052^{\star * \star}$ & $-.052^{\star \star \star}$ & $-.241^{\star \star \star}$ & $-.304^{\star \star \star}$ & $-.150^{\star \star \star}$ & $.062^{\star \star \star}$ & -.008 & .007 & $-.074^{\star \star \star}$ & $.325^{\star \star \star}$ & -- \\
\hline
\end{tabular}

${ }^{* * *}$ Correlation is significant at 0.001 level ${ }^{* \star}$ Correlation is significant at 0.01 level

(Legend: $G=$ gender; JT=job type; WD=work demands; NWD=non-work demands; RO=role overload; WFI=work-family interference;

FWl=family-work interference; Flex=flextime; CWW=compressed work week; Tele=telework; Shift=shiftwork; Pflex=perceived flexibility;

SS=supervisor support) 
This section will summarize the statistically significant correlations between the variables of interest in this thesis. Consistent with the previous practice of using a strict alpha level, the discussion of significant correlations will focus on those correlations significant to a level of 0.001 . Being male is positively associated with job type such that males are more likely to be manager/professionals. Males are also more likely to work flextime, and report higher work-family interference and perceived flexibility (i.e. positive correlation). Being female is positively associated with role overload and supervisor support.

Several key associations can be observed with respect to job type. Being a manager/professional is positively associated with work demands, role overload, work-family interference, family-work interference and supervisor support. Furthermore, managers/professionals are more likely to work flextime and telework arrangements than non-professionals. The findings are consistent with information presented in the demographic and gender/job type sections of the results.

Demands are associated with work-life conflict as follows. Work demands are positively associated with role overload, work-family interference and familywork interference. Similarly, non-work demands are positively associated with role overload, work-family interference, and family-work interference. Furthermore, work demands are positively associated with non-work demands indicating that demands in one domain do not decline just because demands in the other domain are high. Finally, it is interesting to note that both work 
demands and non-work demands are negatively associated with perceived flexibility as well as supervisor support.

All three forms of work-life conflict are inter-correlated. Role overload is positively associated with work-family interference and family-work interference and work-family interference is also associated with family-work interference. The association between role overload and work-family interference in particular is very strong (0.615) suggesting that these two forms of work-life conflict have a common cause.

Perceived flexibility is associated with all three forms of work-life conflict as well as alternative work arrangements, shiftwork and supportive supervision. Each association will be discussed in turn. Perceived flexibility is negatively associated with all three forms of work-life conflict such that higher levels of perceived flexibility are associated with lower levels of role overload, work-family interference and family-work interference. These findings are consistent with the literature presented earlier. Perceived flexibility is also positively associated with working flextime, compressed work week and telework suggesting that these three work arrangements do, in fact, enhance an employee's ability to control the work-life interface. Consistent with the extant body of literature, perceived flexibility is negatively associated with shiftwork. Finally, it is interesting to note the positive association between perceived flexibility and supportive supervision. This association is consistent with the relationships tested in Model 4. 
Finally, supportive supervision is negatively associated with all three forms of work-life conflict and shiftwork $(p<0.001)$ and positively associated with the use of flextime.

In conclusion, several key insights emerge after reviewing the correlations. First, the fact that there is no evidence of multicollinearity in the data support the undertaking of regression analysis. Second, the significant correlations between gender and job type and most of the constructs in our models reinforce the need to control for these two variables in the analysis. Third, the correlations provide support for the models in this thesis. For example, the fact that both types of demands (work demands and non-work demands) are positively associated with all three forms of work-life conflict is consistent with the six strain hypotheses found in models 1-3. It is also consistent with the literature in this area (e.g. Fenwick and Tausig, 2001; Voydanoff, 2004). Similarly, the fact that perceived flexibility and supervisor support are both negatively associated with all three types of work-life conflict further supports the strain hypotheses found in models 1-3. The correlations are also helpful in supporting our hypotheses in Model 4 that perceived flexibility will be positively associated with flextime, compressed work week, telework and supervisor support and that perceived flexibility will be negatively associated with shiftwork.

Finally, the correlations are less supportive of the strain hypotheses developed for alternative work arrangements in models 1-3. In fact, the significant positive associations between flextime and work-family interference, flextime and family-work interference, telework and work-family interference and 
telework and family-work interference run contrary to the strain hypotheses developed for models 1-3 which predict that flextime and telework should be negatively associated with the three measures of work-life conflict.

\subsection{Test of Hypotheses}

This section will present the results for the test of the hypotheses associated with each of the four thesis models. For models $1-3$, results associated with the strain hypotheses will be presented first. This will be followed by the results linked to the buffer hypotheses. For model 4 , results associated with the direct hypotheses are presented followed by the results for the interaction hypotheses ${ }^{9}$. Please recall that in order to control for Bonferroni inequality, we will only consider p-values less than or equal to 0.001 to be statistically significant.

These results include a review of the hypothesis being tested, whether or not the hypothesis was supported by the data, and a summary of the $R^{2}$, change in $R^{2}$ and $p$-value for each regression equation. To enhance the readability of this section key data such as the standardized and unstandardized regression coefficients obtained when testing the hypothesis for models $1-4$ are included in Appendix $D$ as opposed to being presented in the text. The standardized regression coefficients are used as recommended by Thomas, Hughes and Zumbo (1998) to calculate Pratt's coefficient and Pratt's standardized measure.

9 Please note that for models $1-3$, the terms strain and buffer hypotheses are used in order to be consistent with Karasek's terminology in the Demand-Control model. For model 4, the terms direct and interaction hypotheses are used because this model is not an extension of the Karasek model and the terms direct and interaction are more consistent with the intent of model 4. 
The unstandardized regression coefficients, on the other hand, are used, as recommended by Aiken and West (1991) to interpret interaction effects.

\subsubsection{Evaluation of Relationships Between Demand, Control over Work- Life Interface and Role Overload}

This section summarizes the regression results obtained when testing the 8 strain hypotheses associated with Model 1 . The results for each of these regressions are summarized in Table 8 . The actual variables included in each of these regressions are provided in Appendix C. The discussion proceeds as follows. Findings with respect to testing the strain hypotheses dealing with the relationship between demands and role overload will be presented first. This is followed by the findings obtained with the strain hypotheses pertaining to the relationship between different forms of control over the work-life interface and role overload. 
Table 8 Strain Hypotheses Results for Model 1

\begin{tabular}{|l|l|l|l|l|l|l|}
\hline $\begin{array}{l}\text { Strain Hypotheses For } \\
\text { Model 1: Role } \\
\text { Overload }\end{array}$ & $\begin{array}{l}\text { Step 1 } \mathrm{R}^{2} \\
\text { (CV) }\end{array}$ & $\begin{array}{l}\text { Step 2 } \\
\left(\text { Total R } \mathrm{R}^{2}\right)\end{array}$ & $\begin{array}{l}\text { p- } \\
\text { value } \\
\text { at step } \\
2\end{array}$ & df & F-stat & $\begin{array}{l}\Delta \text { in R } \\
\text { due to } \\
\text { IV }\end{array}$ \\
\hline $\begin{array}{l}\text { ROH1: WD positively } \\
\text { associated with RO }\end{array}$ & $5.0 \%$ & $8.1 \%$ & $<.001$ & 4,16361 & 360.78 & $3.1 \%$ \\
\hline $\begin{array}{l}\text { ROH2: NWD positively } \\
\text { associated with RO }\end{array}$ & $6.0 \%$ & $8.1 \%$ & $<.001$ & 4,16361 & 360.78 & $2.1 \%$ \\
\hline $\begin{array}{l}\text { ROH3a: Flextime } \\
\text { negatively associated } \\
\text { with RO }\end{array}$ & $8.1 \%$ & $8.2 \%$ & .031 & 5,13774 & 244.80 & $0.1 \%$ \\
\hline $\begin{array}{l}\text { ROH3b: CWW } \\
\text { negatively associated } \\
\text { with RO }\end{array}$ & $7.8 \%$ & $7.9 \%$ & .008 & 5,12204 & 209.45 & $0.1 \%$ \\
\hline $\begin{array}{l}\text { ROH3c: Telework } \\
\text { negatively associated } \\
\text { with RO }\end{array}$ & $7.9 \%$ & $7.9 \%$ & .985 & 5,9950 & 169.83 & $0 \%$ \\
\hline $\begin{array}{l}\text { ROH4: Shiftwork } \\
\text { positively associated } \\
\text { with RO }\end{array}$ & $8.1 \%$ & $8.1 \%$ & .606 & 5,16360 & 288.66 & $0 \%$ \\
\hline $\begin{array}{l}\text { ROH5: Perceived } \\
\text { flexibility negatively } \\
\text { associated with RO }\end{array}$ & $8.1 \%$ & $17.5 \%$ & $<.001$ & 5,16360 & 691.94 & $9.4 \%$ \\
\hline $\begin{array}{l}\text { ROH6: Supervisor } \\
\text { Support negatively } \\
\text { associated with RO }\end{array}$ & $8.1 \%$ & $13.6 \%$ & $<.001$ & 5,16360 & 515.41 & $5.5 \%$ \\
\hline
\end{tabular}

10.4.1.1 Relationship Between Demands and Role Overload

Two strain hypotheses postulate relationships between demands and role overload. Results associated with the testing of each of these hypotheses are presented below.

Hypothesis $\mathrm{ROH} 1$ postulates that work demands will be positively associated with role overload. This hypothesis is supported by the data. This hypothesis is tested as follows. In step 1, the three covariates (gender, job type and non-work demands) are entered into the regression equation. These 
covariates explain $5.0 \%$ of the variance in role overload. When the independent variable work demands is added to the regression equation in step 2 , an additional $3.1 \%$ of the variance is explained above that already explained by the covariates (for a total $R^{2}$ of $8.1 \%$ ). The $p$-value is $<.001$ indicating that this relationship is statistically significant. The finding that work demands are positively associated with role overload is supportive of the hypothesis.

Hypothesis $\mathrm{ROH} 2$ postulates that non-work demands will be positively associated with role overload. Again, this hypothesis is supported by the data. This hypothesis is tested as follows. In step 1, the three covariates (gender, job type and work demands) are entered into the regression. These covariates explain $6.0 \%$ of the variance in role overload. When the independent variable (non-work demands) is added into the regression equation in step 2, an additional $2.1 \%$ of the variance is explained above that already explained by the covariates (for a total $\mathrm{R}^{2}$ of $8.1 \%$ ). The $\mathrm{p}$-value is $<.001$ indicating that this relationship is statistically significant. Therefore, we can conclude that non-work demands are positively associated with role overload, a finding that is supportive of the hypothesis.

We can also assess the magnitude of variable importance of the independent variables (i.e. work demands and non-work demands) at predicting role overload by calculating Pratt's standardized measure (Thomas et al., 1998). As noted earlier, Pratt's coefficient is calculated by multiplying the standardized coefficient by the correlation of the independent variable and the dependent variable. Pratt's standardized measure is calculated by dividing Pratt's 
coefficient by the total variance explained. All standardized Pratt measures sum to 1 . The Pratt's standardized measures obtained when both work demands and non-work demands are included in the regression equation predicting role overload are shown in Table 9.

Table 9 Pratt's Coefficient: Role Overload

\begin{tabular}{|l|l|l|l|l|}
\hline $\begin{array}{l}\text { Independent } \\
\text { Variable }\end{array}$ & $\begin{array}{l}\text { Standardized } \\
\text { Coefficient }\end{array}$ & $\begin{array}{l}\text { Correlation } \\
\text { of IV and } \\
\text { DV }\end{array}$ & $\begin{array}{l}\text { Pratt's } \\
\text { Coefficient }\end{array}$ & $\begin{array}{l}\text { Standardized } \\
\text { Pratt's } \\
\text { Measure }\end{array}$ \\
\hline $\begin{array}{l}\text { Work } \\
\text { Demands }\end{array}$ & .182 & .171 & .031122 & .586964 \\
\hline $\begin{array}{l}\text { Non-work } \\
\text { demands }\end{array}$ & .146 & .150 & 0.0219 & .413036 \\
\hline $\begin{array}{l}\text { Variance } \\
\text { Explained }\end{array}$ & - & - & .0530 & 1 \\
\hline
\end{tabular}

The fact that Pratt's standardized measure is slightly higher for work demands (.586964) than for non-work demands $(.413036)$ can be interpreted to mean that work demands predict relatively more variance in role overload than do non-work demands.

\subsubsection{Relationship Between Control Over Work-Life Interface and Role Overload}

Section 3 of this thesis identified the three different forms of control over work-life interface (work arrangements, perceived flexibility and supervisor support) included in this thesis. In model one we test 6 strain hypotheses that postulate relationships between these different forms of control over the work-life interface and role overload. Specifically:

- Hypothesis $\mathrm{ROH} 3$ postulates that the use of alternative work arrangements (AWA) will be negatively associated with role overload. Specifically employees who work:

- flextime (versus a regular work day) 
- compressed work week (versus a regular work day)

- telework (versus a regular work day)

will report lower levels of role overload than their counterparts who work a regular work day.

- Hypothesis ROH4 postulates that the use of shiftwork arrangements will be positively associated with role overload.

- Hypothesis $\mathrm{ROH} 5$ postulates that perceived flexibility will be negatively associated with role overload.

- Hypothesis ROH6 postulates that supervisor support will be negatively associated with role overload.

Each of these hypotheses will be discussed in turn in the section below.

The three hypotheses exploring the relationship between the use of alternative work arrangements and role overload are not supported by the data. Results supporting this conclusion are reviewed below.

Hypothesis $\mathrm{ROH} 3 a$ (i.e. flextime negatively associated with role overload) is tested as follows. The four covariates (gender, job type, work demands and non-work demands) are entered into the regression equation in step 1 . These covariates explain $8.1 \%$ of the variance in role overload. The addition of the independent variable flextime in step 2 results in only a $0.1 \%$ increase in our understanding of the variance in role overload above that already explained by the covariates (for a total $R^{2}$ of $8.2 \%$ ). The $p$-value (.031) indicates that this relationship is not statistically significant. Therefore, we reject the hypothesis that those who work flextime will report lower levels of role overload than their counterparts who work a regular work day.

Hypothesis $\mathrm{ROH} 3 \mathrm{~b}$ (compressed work week negatively associated with role overload) is tested as follows. The four covariates (gender, job type, work 
demands and non-work demands) are entered into the regression equation in step 1 . These variables explain $7.8 \%$ of the variance in role overload. The addition of the independent variable compressed work week in step 2 results in only a $0.1 \%$ increase in our understanding of the variance in role overload above that already explained by the covariates (for a total $R^{2}$ of $7.9 \%$ ). The $p$-value $(.008)$ indicates that this relationship is not statistically significant. Therefore, we reject the hypothesis that those who work compressed work weeks will report lower levels of role overload than their counterparts who work a regular work day. Hypothesis ROH3c (telework negatively associated with role overload) is tested as follows. The four covariates (gender, job type, work demands and nonwork demands) are entered into the regression equation in step 1 . These covariates explain $7.9 \%$ of the variance in role overload. The addition of the independent variable telework in step 2 results in no additional increase in our understanding of the variance in role overload above that already explained by the covariates. The p-value (.985) indicates that this relationship is not statistically significant. Therefore, we reject the hypothesis that those who telework will report lower levels of role overload than their counterparts who work a regular work day.

Hypothesis ROH4 postulates that the use of shiftwork arrangements will be positively associated with role overload. This hypothesis is not supported by the data. This hypothesis is tested as follows. In step 1 , the four covariates (gender, job type, work demands and non-work demands) are entered into the regression equation. These covariates explain $8.1 \%$ of the variance in role 
overload. The addition of the independent variable shiftwork in step 2 results in no additional increase in our understanding of the variance in role overload above that already explained by the covariates. The p-value (.606) indicates that this relationship is not statistically significant. Therefore, we reject the hypothesis that the use of shiftwork will be positively associated with role overload.

Hypothesis ROH5 postulates that perceived flexibility will be negatively associated with role overload. This hypothesis is supported by the data. This hypothesis is tested as follows. In step 1, the four covariates (gender, job type, work demands and non-work demands) are entered into the regression equation. These covariates explain $8.1 \%$ of the variance in role overload. The addition of the independent variable perceived flexibility in step 2 results in an additional $9.4 \%$ of the variance in role overload above that already explained by the covariates (i.e. total $R^{2}$ of $17.5 \%$ ). The $p$-value $(<.001)$ indicates that this relationship is statistically significant thereby supporting our hypothesis that perceived flexibility will be negatively associated with role overload.

Hypothesis ROH6 postulates that supervisor support will be negatively associated with role overload. The data supports this hypothesis. In step 1, the four covariates (gender, job type, work demands and non-work demands) are entered into the regression. These covariates explain $8.1 \%$ of the variance in role overload. The addition of the independent variable supervisor support in step 2 results in an additional $5.5 \%$ of the variance in role overload above that already explained by the covariates (i.e. total $R^{2}$ of $13.6 \%$ ). The $p$-value $(<.001)$ indicates that this relationship is statistically significant thereby supporting the 
hypothesis that supervisor support will be negatively associated with role overload.

\subsubsection{Conclusions: Role Overload Strain Hypotheses}

In conclusion, half of the strain hypotheses for role overload are statistically significant. Both work demands and non-work demands are positively associated with role overload while perceived flexibility and supervisor support were both negatively associated with role overload (all statistically significant at a p-value of .001). Furthermore, a review of Pratt's standardized measures indicate that work demands is a stronger predictor of role overload than non-work demands.

There is mixed support for Karasek's strain hypothesis when strain is operationalized as role overload. The strain hypotheses are strongly supported when strain is operationalized as role overload and demands are operationalized as work and non-work demands. The level of support for Karasek's strain hypothesis varies depending upon how control is operationalized. When control is operationalized as either perceived flexibility or supervisor support, there is strong support for the strain hypothesis. However, when control is operationalized as a given work arrangement (i.e. flextime, compressed work week, telework, shiftwork), there is no support for the strain hypothesis.

A review of the betas for perceived flexibility $(B=-.307)$ and supervisor support $(B=-.278)$ indicate that both perceived flexibility and supervisor support are important predictors of role overload. It is important to note that the strongest associations were obtained in the regression equations where 
perceived flexibility (change in $R^{2}$ of $9.4 \%$ ) and supervisor support (change in $R^{2}$ of $5.5 \%$ ) were the independent variables used to predict role overload. The fact that both these relationships were highly significant and negative (i.e. higher control, lower role overload) when work and non-work demands were controlled for support the idea that perceived flexibility and supervisor support help employees cope with role overload. In the case of the three alternative work arrangements (i.e. flextime, compressed work week and telework) and shiftwork, no statistically significant association is found with role overload when work and non-work demands, gender and job type are controlled for. This would suggest that such work arrangements do not help employees cope with role overload.

\subsubsection{Evaluation of Factors that Moderate the Relationship Between Demands and Role Overload}

This section summarizes the regression results obtained when testing the

following 12 buffer hypothesis associated with Model 1 :

- Hypothesis $\mathrm{ROH} 7$ postulates that alternative work arrangements will moderate the relationship between work demands and role overload. Specifically:

a. The relationship between work demands and role overload will be weaker for those who use flextime arrangements than those who work a regular work day.

b. The relationship between work demands and role overload will be weaker for those who work a compressed work week than those who work a regular work day.

c. The relationship between work demands and role overload will be weaker for those who telework than those who work a regular work day.

d. The relationship between work demands and role overload will be weaker for those who work a regular work day than those who perform shiftwork.

- Hypothesis ROH8 postulates that perceived flexibility will moderate the relationship between work demands and role overload. Specifically, the relationship between work demands and role overload will decrease as perceived flexibility increases 
- Hypothesis ROH9 postulates that supervisor support will moderate the relationship between work demands and role overload. Specifically, the relationship between work demands and role overload will decrease as supervisor support increases

- Hypothesis ROH10 postulates that alternative work arrangements will moderate the relationship between non-work demands and role overload. Specifically:

a. The relationship between non-work demands and role overload will be weaker for those who use flextime arrangements than those who work a regular work day.

b. The relationship between non-work demands and role overload will be weaker for those who work a compressed work week than those who work a regular work day.

c. The relationship between non-work demands and role overload will be weaker for those who telework than those who work a regular work day.

d. The relationship between non-work demands and role overload will be weaker for those who work a regular work day than those who perform shiftwork.

- Hypothesis ROH11 postulates that perceived flexibility will moderate the relationship between non-work demands and role overload. Specifically, the relationship between non-work demands and role overload will decrease as perceived flexibility increases.

- Hypothesis $\mathrm{ROH} 12$ postulates that supervisor support will moderate the relationship between non-work demands and role overload. Specifically, the relationship between non-work demands and role overload will decrease as supervisor support increases.

The results for each of these regressions are summarized in Table 10. The actual variables included in each of these regressions are provided in Appendix C. The discussion is divided into two main parts according to the type of demand being considered: work demands (presented first) and non-work demands (presented second). Each of these two parts have an identical structure. Data showing the extent to which the various work arrangements moderate the relationship between demands and overload are presented first. 
This is followed by the presentation of data showing the extent to which the other two forms of control included in this thesis, supervisor support and perceived flexibility are able to moderate the relationship between demands and role overload. 
Table 10 Buffer Hypotheses Results for Model 1

\begin{tabular}{|l|l|l|l|l|l|l|l|l|}
\hline $\begin{array}{l}\text { Buffer Hypothesis for Model 1: } \\
\text { Role Overload }\end{array}$ & $\begin{array}{l}\text { Step 1 } \\
R^{2}(\mathrm{CV})\end{array}$ & $\begin{array}{l}\text { Step 2 R } \\
(\mathrm{IV})\end{array}$ & $\begin{array}{l}\Delta \text { in R } \\
\text { due to } \\
\mathrm{IV}\end{array}$ & $\begin{array}{l}\text { Step 3 } \\
\text { (Total) } \\
\mathrm{R}^{2}\end{array}$ & $\begin{array}{l}\Delta \text { in R } \\
\text { due to IV } \\
\text { x MV }\end{array}$ & F-stat & df & $\begin{array}{l}\text {-value } \\
\text { at Step } \\
3\end{array}$ \\
\hline $\begin{array}{l}\text { ROH7a: Flextime moderates relationship } \\
\text { between WD and RO }\end{array}$ & $8.1 \%$ & $8.2 \%$ & $0.1 \%$ & $8.2 \%$ & $0 \%$ & 204.00 & 6,13773 & .758 \\
\hline $\begin{array}{l}\text { ROH7b: CWW moderates relationship } \\
\text { between WD and RO }\end{array}$ & $7.8 \%$ & $7.9 \%$ & $0.1 \%$ & $7.9 \%$ & $0 \%$ & 174.61 & 6,12203 & .494 \\
\hline $\begin{array}{l}\text { ROH7c: Telework moderates relationship } \\
\text { between WD and RO }\end{array}$ & $7.9 \%$ & $7.9 \%$ & $0 \%$ & $7.9 \%$ & $0 \%$ & 141.51 & 6,9949 & .863 \\
\hline $\begin{array}{l}\text { ROH7d Shiftwork moderates relationship } \\
\text { between WD and RO }\end{array}$ & $8.1 \%$ & $8.1 \%$ & $0 \%$ & $8.2 \%$ & $0.1 \%$ & 244.61 & 6,16359 & .001 \\
\hline $\begin{array}{l}\text { ROH8: Perceived Flexibility moderates } \\
\text { relationship between WD and RO }\end{array}$ & $8.1 \%$ & $17.4 \%$ & $9.3 \%$ & $17.5 \%$ & $0.1 \%$ & 580.06 & 6,16359 & $<.001$ \\
\hline $\begin{array}{l}\text { ROH9: Suppervisor support moderates } \\
\text { relationship between WD and RO }\end{array}$ & $8.1 \%$ & $13.6 \%$ & $5.5 \%$ & $13.6 \%$ & $0 \%$ & 430.50 & 6,16359 & .022 \\
\hline $\begin{array}{l}\text { ROH10a: Flextime moderates relationship } \\
\text { between NWD and RO }\end{array}$ & $8.1 \%$ & $8.2 \%$ & $0.1 \%$ & $8.2 \%$ & $0 \%$ & 204.25 & 6,13773 & .229 \\
\hline $\begin{array}{l}\text { ROH10b: CWW moderates relationship } \\
\text { between NWD and RO }\end{array}$ & $7.8 \%$ & $7.9 \%$ & $0.1 \%$ & $7.9 \%$ & $0 \%$ & 174.92 & 6,12203 & .139 \\
\hline $\begin{array}{l}\text { ROH10c: Telework moderates relationship } \\
\text { between NWD and RO }\end{array}$ & $7.9 \%$ & $7.9 \%$ & $0 \%$ & $7.9 \%$ & $0 \%$ & 141.51 & 6,9949 & .871 \\
\hline $\begin{array}{l}\text { ROH10d: Shiftwork moderates relationship } \\
\text { between NWD and RO }\end{array}$ & $8.1 \%$ & $8.1 \%$ & $0 \%$ & $8.1 \%$ & $0 \%$ & 240.55 & 6,16359 & .823 \\
\hline $\begin{array}{l}\text { ROH11: Perceived flexibility moderates } \\
\text { relationship between NWD and RO }\end{array}$ & $8.1 \%$ & $17.5 \%$ & $9.4 \%$ & $17.5 \%$ & $0 \%$ & 578.40 & 6,16359 & .003 \\
\hline $\begin{array}{l}\text { ROH12: Supervisor support moderates } \\
\text { relationship between NWD and RO }\end{array}$ & $8.1 \%$ & $13.6 \%$ & $5.5 \%$ & $13.7 \%$ & $0.1 \%$ & 432.66 & 6,16359 & $<.001$ \\
\hline
\end{tabular}




\subsubsection{Control Over Work-Life Interface Moderates Relationship Between Work Demands and Role Overload}

This section presents the results of the regression analysis performed to test the ability of the various forms of control included in this analysis to moderate the relationship between work demands and role overload.

Hypothesis ROH7a states that the relationship between work demands and role overload will be weaker for those who use flextime arrangements than those who work a regular work day. This hypothesis is not supported by the data. This hypothesis is tested as follows. First the four covariates (gender, job type, work demands and non-work demands) are entered into the regression equation in step 1 . These covariates explain $8.1 \%$ of the variance in role overload. Second the independent variable, flextime, is added to the regression. This variable explains a further $0.1 \%$ of the variance in role overload above that already explained by the covariates. The interaction term, flextime by work demands, is added to the regression in step three. This term did not explain any additional variance in role overload ( $p$ value .758 ) and the beta coefficient of the interaction is zero $(.000)$. Since the interaction term is not significant we reject the hypothesis that the relationship between work demands and role overload will be weaker for those who use flextime arrangements than those who work a regular work day.

Hypothesis ROH7b states that the relationship between work demands and role overload will be weaker for those who use compressed work weeks than 
those who work a regular work day. This hypothesis is not supported by the data. This hypothesis is tested as follows. First the four covariates (gender, job type, work demands and non-work demands) are entered into the regression equation in step 1. These covariates explain $7.8 \%$ of the variance in role overload. Second, the independent variable, compressed work week, is added to the regression. This variable explains a further $0.1 \%$ of the variance in role overload above that already explained by the covariates. The interaction term, compressed work week by work demands is added to the regression in step three. This term did not explain any additional variance in role overload ( $p$ value $.494)$ and the beta coefficient of the interaction is very small $(-.001)$. Since the interaction term is not significant we reject the hypothesis that the relationship between work demands and role overload will be weaker for those who use compressed work weeks than those who work a regular work day.

Hypothesis $\mathrm{ROH} 7 \mathrm{c}$ states that the relationship between work demands and role overload will be weaker for those who telework than those who work a regular work day. This hypothesis is not supported by the data. This hypothesis is tested as follows. First the four covariates (gender, job type, work demands and non-work demands) are entered into the regression equation in step 1. These covariates explain $7.9 \%$ of the variance in role overload. Second, the independent variable, telework, is added to the regression. This variable explains no further variance in role overload ( $p$ value .929 ) above that already explained by the covariates. The interaction term, telework by work demands is added to the regression in step three. This term did not explain any additional variance in 
role overload ( $p$ value .863) and the beta coefficient of the interaction was zero $(.000)$. Since the interaction term is not significant we reject the hypothesis that the relationship between work demands and role overload will be weaker for those who telework than those who work a regular work day.

Hypothesis $\mathrm{ROH} 7 \mathrm{~d}$ states that the relationship between work demands and role overload will be weaker for those who work a regular work schedule than those who perform shiftwork. This hypothesis is supported by the data. This hypothesis is tested as follows. First the four covariates (gender, job type, work demands and non-work demands) are entered into the regression equation in step 1. These covariates explain $8.1 \%$ of the variance in role overload. Second the independent variable, shiftwork, is added to the regression. This variable does not explain any variance in role overload above that already explained by the covariates. The interaction term shiftwork by work demands is added to the regression in step three. This interaction term explains a further $0.1 \%$ of the variance in role overload above that already explained by the covariates and the independent variable and is statistically significant ( $p$ value .000 ). These findings are supportive of our hypothesis that shiftwork moderates the impact of work demands on role overload such that the relationship between work demands and role overload is weaker for those who work a regular work day than those who perform shiftwork.

As noted in section 9.5.3.2, the approach taken in this thesis is to examine statistically significant interaction terms to see if they are practically significant. This is done by plotting and interpreting the interaction and examining the 
change in $R^{2}$ that can be explained by the interaction term. Figure 13 plots the interaction of work demands and shiftwork.

Figure 13 Interaction of Work Demands x Shiftwork

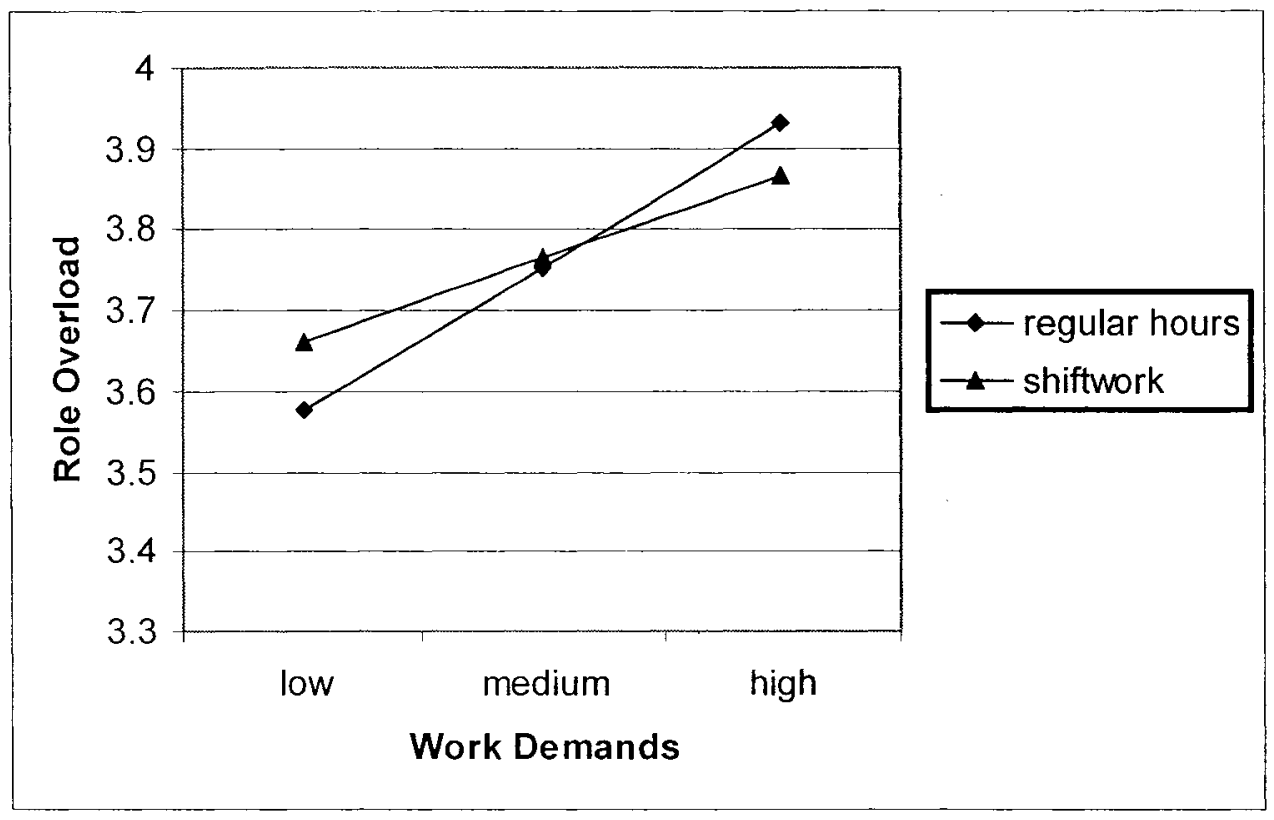

Examination of this figure indicates that the relationship between role overload and shift/regular work arrangement varies depending on the level of work demands reported by the respondent. At low levels of work demands the relationship is as hypothesized: those who work shiftwork report higher levels of role overload than those working regular hours. At high levels of work demands, on the other hand, the interaction is opposite to that hypothesized as respondents who work regular hours report higher levels of role overload than those performing shiftwork. These results could be interpreted as indicating that role overload is problematic for those who work shiftwork (regardless of level of 
work demands) but regular work arrangements are associated with higher levels of role overload when work demands rise above a critical threshold.

It is important, however, to note that the change in $R^{2}$ from step 2 (the addition of shiftwork as an independent variable) to step 3 (the addition of shiftwork by work demands) is very slight $(0.1 \%)$. These results indicate that while this result is statistically significant, it is not of practical significance.

Hypothesis ROH8 looks at the ability of perceived flexibility to moderate the relationship between work demands and role overload. This hypothesis (i.e. that the relationship between work demands and role overload will decrease as perceived flexibility increases) is supported by the data.

This hypothesis is tested as follows. First the four covariates (gender, job type, work demands and non-work demands) are entered into the regression equation in step 1 . These covariates explain $8.1 \%$ of the variance in role overload. Second the independent variable, perceived flexibility, is added to the regression. This variable explains a further $9.3 \%$ of the variance in role overload above that already explained by the covariates (for a total $R^{2}$ of $17.4 \%$ ). The interaction term perceived flexibility by work demands is added to the regression in step three. This interaction term explains a further $0.1 \%$ of the variance in role overload above that already explained by the covariates and the independent variable and is statistically significant ( $p$ value $<.001)$. These findings are supportive of our hypothesis that perceived flexibility moderates the impact of work demands on role overload such that the relationship between work demands and role overload will decrease as perceived flexibility increases. 
Consistent with the process outlined previously, we first plotted the interaction and then examined the change in $R^{2}$ from step 2 to step 3 . The results of the plotted interaction are seen in Figure 14.

Figure 14 Interaction of Work Demands x Perceived Flexibility

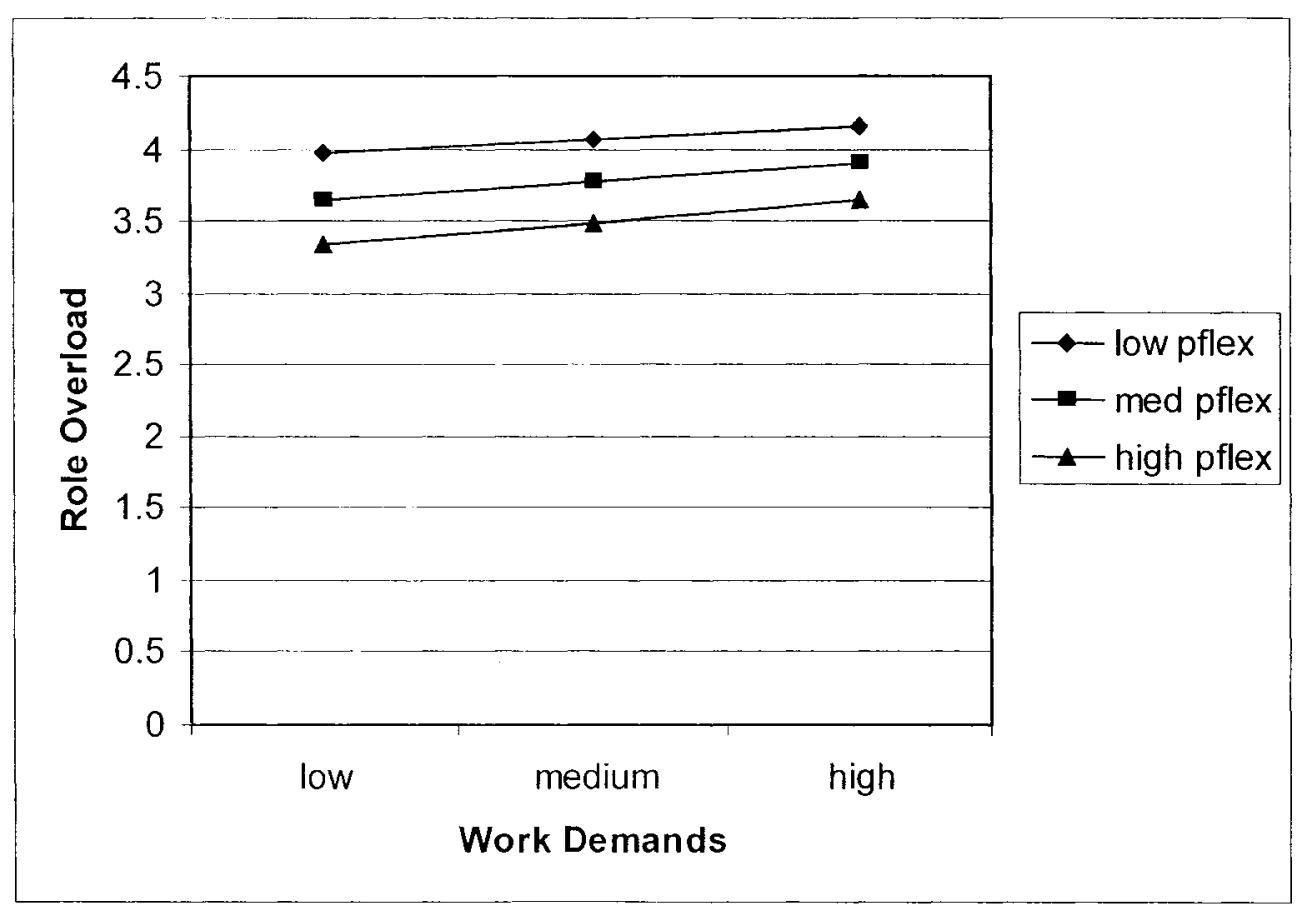

As seen in figure 14, there is very little evidence of an interaction effect for this relationship. The lines of the plot are parallel and show that those reporting high levels of perceived flexibility reported lower levels of role overload than those at medium or low levels of perceived flexibility regardless of the level of work demands. In other words, the plot of the data indicates that perceived flexibility has a direct effect on role overload. These findings are consistent with the fact that the data indicate that these results, while statistically significant, are not of practical significance (i.e. the change in $R^{2}$ was $0.1 \%$ ). 
Hypothesis ROH9 looks at the ability of supervisor support to moderate the relationship between work demands and role overload. This hypothesis (i.e. that the relationship between work demands and role overload will decrease as supervisor support increases) is not supported by the data. This hypothesis is tested as follows. First the four covariates (gender, job type, work demands and non-work demands) are entered into the regression equation in step 1. These covariates explain $8.1 \%$ of the variance in role overload. Second the independent variable, supervisor support, is added to the regression. This variable explains a further $5.5 \%$ of the variance in role overload above that already explained by the covariates (for a total $R^{2}$ of $13.6 \%$ ). The interaction term supervisor support by work demands is added to the regression in step three. This term did not explain any additional variance in role overload ( $p$ value $.022)$ and the beta coefficient of the interaction is very small (.002). Since the interaction term is not significant we reject the hypothesis that the relationship between work demands and role overload will decrease as supervisor support increases.

\subsubsection{Control Over Work-Life Interface Moderates Relationship Between Non-work demands and Role Overload}

This section presents the results of the regression analysis performed to test the ability of the various forms of control included in this analysis to moderate the relationship between non-work demands and role overload. The regression results are not supportive of the idea that alternative work arrangements 
moderate the relationship between non-work demands and role overload. Support for this contention is provided below.

Hypothesis ROH10a states that the relationship between non-work demands and role overload will be weaker for those who use flextime arrangements than those who work a regular work day. This hypothesis is not supported by the data. This hypothesis is tested as follows. First the four covariates (gender, job type, work demands and non-work demands) are entered into the regression equation in step 1 . These covariates explain $8.1 \%$ of the variance in role overload. Second the independent variable, flextime, is added to the regression. This variable explains a further $0.1 \%$ of the variance in role overload above that already explained by the covariates. The interaction term, flextime by non-work demands, is added to the regression in step three. This term did not explain any additional variance in role overload ( $p$ value .229) and the beta coefficient of the interaction is very small $(.001)$. Since the interaction term is not significant we reject the hypothesis that the relationship between nonwork demands and role overload will be weaker for those who use flextime arrangements than those who work a regular work day.

Hypothesis ROH10b states that the relationship between non-work demands and role overload will be weaker for those who use compressed work weeks than those who work a regular work day. This hypothesis is not supported by the data. This hypothesis is tested as follows. First the four covariates (gender, job type, work demands and non-work demands) are entered into the regression equation in step 1 . These covariates explain $7.8 \%$ of the variance in 
role overload. Second, the independent variable, compressed work week, is added to the regression. This variable explains a further $0.1 \%$ of the variance in role overload above that already explained by the covariates. The interaction term, compressed work week by non-work demands is added to the regression in step three. This term did not explain any additional variance in role overload ( $p$ value .139) and the beta coefficient of the interaction is very small (.001). Since the interaction term is not significant we reject the hypothesis that the relationship between non-work demands and role overload will be weaker for those who use compressed work weeks than those who work a regular work day.

Hypothesis $\mathrm{ROH} 10 \mathrm{c}$ states that the relationship between non-work demands and role overload will be weaker for those who telework than those who work a regular work day. This hypothesis is not supported by the data. This hypothesis is tested as follows. First the four covariates (gender, job type, work demands and non-work demands) are entered into the regression equation in step 1. These covariates explain $7.9 \%$ of the variance in role overload. Second, the independent variable, telework, is added to the regression. This variable explains no further variance in role overload ( $p$ value .985) above that already explained by the covariates. The interaction term, telework by non-work demands is added to the regression in step three. This term did not explain any additional variance in role overload ( $p$ value .871) and the beta coefficient of the interaction was zero $(.000)$. Since the interaction term is not significant we reject the hypothesis that the relationship between non-work demands and role 
overload will be weaker for those who telework than those who work a regular work day.

Hypothesis $\mathrm{ROH} 10 \mathrm{~d}$ states that the relationship between non-work demands and role overload will be weaker for those who work a regular work schedule than those who perform shiftwork. This hypothesis is not supported by the data. This hypothesis is tested as follows. First the four covariates (gender, job type, work demands and non-work demands) are entered into the regression equation in step 1 . These covariates explain $8.1 \%$ of the variance in role overload. Second the independent variable, shiftwork, is added to the regression. This variable does not explain any variance in role overload above that already explained by the covariates. The interaction term shiftwork by nonwork demands is added to the regression in step three. This term did not explain any additional variance in role overload ( $p$ value .823 ) and the beta coefficient of the interaction was zero $(.000)$. Since the interaction term is not significant we reject the hypothesis that the relationship between non-work demands and role overload will be weaker for those who work a regular work day than those who work shiftwork.

Hypothesis ROH11 looks at the ability of perceived flexibility to moderate the relationship between non-work demands and role overload. This hypothesis (i.e. that the relationship between non-work demands and role overload will decrease as perceived flexibility increases) is not supported by the data.

This hypothesis is tested as follows. First the four covariates (gender, job type, work demands and non-work demands) are entered into the regression 
equation in step 1. These covariates explain $8.1 \%$ of the variance in role overload. Second the independent variable, perceived flexibility, is added to the regression. This variable explains a further $9.4 \%$ of the variance in role overload above that already explained by the covariates (for a total $R^{2}$ of $17.5 \%$ ). The interaction term perceived flexibility by non-work demands is added to the regression in step three. This term did not explain any additional variance in role overload ( $p$ value .003) and the beta coefficient of the interaction is very small (.002). Since the interaction term is not significant we reject the hypothesis that that perceived flexibility moderates the impact of non-work demands on role overload such that the relationship between non-work demands and role overload will decrease as perceived flexibility increases.

Hypothesis ROH12 looks at the ability of supervisor support to moderate the relationship between non-work demands and role overload. This hypothesis (i.e. that the relationship between non-work demands and role overload will decrease as perceived flexibility increases) is supported by the data.

This hypothesis is tested as follows. First the four covariates (gender, job type, work demands and non-work demands) are entered into the regression equation in step 1 . These covariates explain $8.1 \%$ of the variance in role overload. Second the independent variable, supervisor support, is added to the regression. This variable explains a further $5.5 \%$ of the variance in role overload above that already explained by the covariates (for a total $R^{2}$ of $13.6 \%$ ). The interaction term supervisor support by non-work demands is added to the regression in step three. This interaction term explains a further $0.1 \%$ of the 
variance in role overload above that already explained by the covariates and the independent variable and is statistically significant $(p$ value $<.001)$. These findings are supportive of our hypothesis that supervisor support moderates the impact of non-work demands on role overload such that the relationship between non-work demands and role overload will decrease as supervisor support increases.

Consistent with the process outlined previously, we first plotted the interaction and then examined the change $R^{2}$ in from step 2 to step 3 . The results of the plotted interaction are seen below in Figure 15.

Figure 15 Interaction of Non-work demands x Supervisor Support

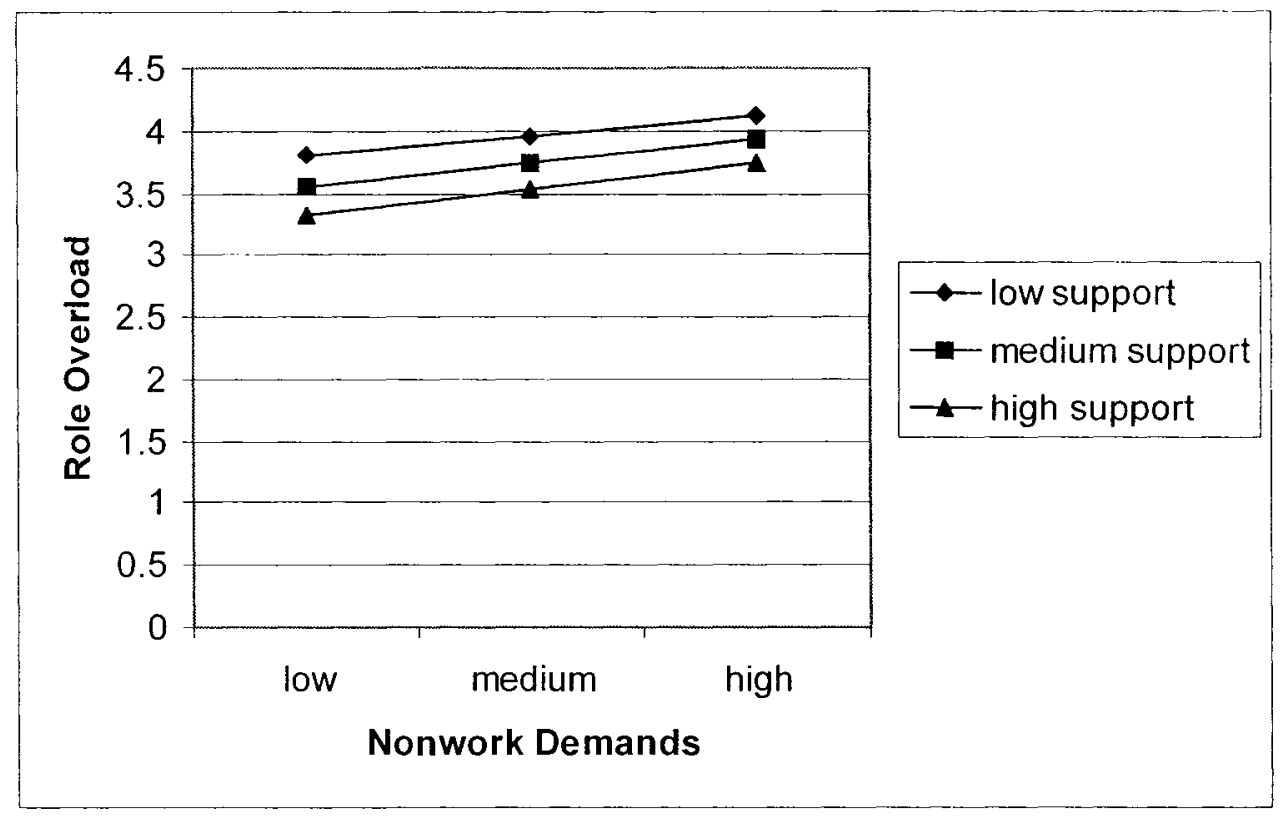

As seen in figure 15, there is very little evidence of an interaction effect for this relationship. The lines of the plot are parallel and show that those reporting high levels of supervisor support reported lower levels of role overload than those 
at medium or low levels of supervisor support regardless of the level of non-work demands. In other words, the plot of the data indicates that supervisor support has a direct effect on role overload. These findings are consistent with the fact that the data indicate that these results, while statistically significant, are not of practical significance (i.e. the change in $R^{2}$ was $0.1 \%$ ).

\subsubsection{Conclusions: Role Overload Buffer Hypotheses}

In conclusion, Karasek's buffer hypothesis is not supported when strain is operationalized as role overload, demands operationalized as work and non-work demands, and control operationalized as alternative work arrangement, perceived flexibility or supervisor support. This conclusion is based on two facts. First, nine of the twelve buffer hypotheses were not statistically significant. Second, while the buffer hypothesis was supported under specific circumstances (shiftwork by work demands, perceived flexibility by work demands, supervisor support by non-work demands), the fact that there was very little change in $R^{2}$ (i.e. only $0.1 \%$ ) and plotting the interaction term showed that the changes in role overload at various levels of the moderator and independent variable were small suggests the findings had little practical significance.

\subsubsection{Evaluation of Relationships Between Demand, Control over Work- Life Interface and Work-Family Interference}

This section summarizes the regression results obtained when testing the 8 strain hypotheses associated with Model 2. With one exception (i.e. strain is operationalized as work-family interference rather than role overload), model 2 is 
identical to model 1 . The results for each of these eight regressions are summarized in Table 11. The actual variables included in each of these regressions are provided in Appendix C. The discussion proceeds as follows.

Findings with respect to testing the strain hypotheses dealing with the relationship between demands and work-family interference will be presented first. This is followed by the findings obtained with the strain hypotheses pertaining to the relationship between different forms of control over the work-life interface and work-family interference.

Table 11 Strain Hypotheses Results for Model 2

\begin{tabular}{|l|l|l|l|l|l|l|}
\hline $\begin{array}{l}\text { Strain Hypothesis For } \\
\text { Model 2: Work-Family } \\
\text { Interference }\end{array}$ & $\begin{array}{l}\text { Step 1 } \\
R^{2} \text { (CV) }\end{array}$ & $\begin{array}{l}\text { Step 2 } \\
(\text { Total } \\
\left.\mathrm{R}^{2}\right)\end{array}$ & $\begin{array}{l}\mathrm{p} \text { - } \\
\text { value } \\
\text { at step } \\
2\end{array}$ & df & F-stat & $\begin{array}{l}\Delta \text { in } \mathrm{R}^{2} \\
\text { due to } \\
\text { IV }\end{array}$ \\
\hline $\begin{array}{l}\text { WFIH1: WD positively } \\
\text { associated with WFI }\end{array}$ & $4.8 \%$ & $11.5 \%$ & $<.001$ & 4,16361 & 481.62 & $6.7 \%$ \\
\hline $\begin{array}{l}\text { WFIH2: NWD positively } \\
\text { associated with WFI }\end{array}$ & $10.2 \%$ & $11.5 \%$ & $<.001$ & 4,16361 & 529.21 & $1.3 \%$ \\
\hline $\begin{array}{l}\text { WFIH3a: Flextime } \\
\text { negatively associated } \\
\text { with WFI }\end{array}$ & $11.9 \%$ & $11.9 \%$ & .856 & 5,13774 & 372.11 & $0 \%$ \\
\hline $\begin{array}{l}\text { WFIH3b: CWW } \\
\text { negatively associated } \\
\text { with WFI }\end{array}$ & $10.8 \%$ & $10.8 \%$ & .701 & 5,12204 & 296.56 & $0 \%$ \\
\hline $\begin{array}{l}\text { WFIH3c: Telework } \\
\text { negatively associated } \\
\text { with WFI }\end{array}$ & $11.5 \%$ & $11.5 \%$ & .345 & 5,9950 & 259.80 & $0 \%$ \\
\hline $\begin{array}{l}\text { WFIH4: Shiftwork } \\
\text { positively associated } \\
\text { with WFI }\end{array}$ & $11.5 \%$ & $12.2 \%$ & $<.001$ & 5,16360 & 452.54 & $0.7 \%$ \\
\hline $\begin{array}{l}\text { WFIH5: Perceived } \\
\text { flexibility negatively } \\
\text { associated with WFI }\end{array}$ & $11.5 \%$ & $25.7 \%$ & $<.001$ & 5,16360 & 1131.88 & $14.2 \%$ \\
\hline $\begin{array}{l}\text { WFIH6: Supervisor } \\
\text { support negatively } \\
\text { associated with WFI }\end{array}$ & $11.5 \%$ & $19.9 \%$ & $<.001$ & 5,16360 & 812.35 & $8.4 \%$ \\
\hline
\end{tabular}




\subsubsection{Relationship Between Demands and Work-Family Interference}

Two strain hypotheses postulate relationships between demands and work-family interference. Results associated with the testing of each of these hypotheses are presented below.

WFIH1 postulates that work demands will be positively associated with work-family interference. The data supports this hypothesis. This hypothesis is tested as follows. In step 1, the three covariates (gender, job type and non-work demands) are entered into the regression equation. These covariates explain $4.8 \%$ of the variance in work-family interference. When the independent variable work demands is added to the regression equation in step 2, an additional $6.7 \%$ of the variance is explained above that already explained by the covariates (for a total $R^{2}$ of $11.5 \%$ ). The $p$-value is $<.001$ indicating that this relationship is statistically significant. The finding that work demands are positively associated with work-family interference is supportive of the hypothesis.

WFIH2 postulates that non-work demands will be positively associated with work-family interference. The data supports this hypothesis. This hypothesis is tested as follows. In step 1, the three covariates (gender, job type and work demands) are entered into the regression. This step explains $10.2 \%$ of the variance in work-family interference. When the independent variable nonwork demands is added to the regression equation in step 2 , an additional $1.3 \%$ 
of the variance is explained above that already explained by the covariates (for a total $R^{2}$ of $11.5 \%$ ). The $p$-value was $<.001$ indicating that this relationship is statistically significant. The finding that non-work demands is positively associated with work-family interference is supportive of the hypothesis.

As noted earlier, we can assess the relative importance of these two independent variables (work demands and non-work demands) at predicting work-family interference by calculating Pratt's standardized measure (Thomas et al., 1998). The Pratt's standardized measure obtained when both work demands and non-work demands are included in the regression equation predicting workfamily interference are shown in Table 12.

Table 12 Pratt's Coefficient: Work-Family Interference

\begin{tabular}{|l|l|l|l|l|}
\hline $\begin{array}{l}\text { Independent } \\
\text { Variable }\end{array}$ & $\begin{array}{l}\text { Standardized } \\
\text { Coefficient }\end{array}$ & $\begin{array}{l}\text { Correlation } \\
\text { of IV and } \\
\text { DV }\end{array}$ & $\begin{array}{l}\text { Pratt's } \\
\text { Coefficient }\end{array}$ & $\begin{array}{l}\text { Pratt's } \\
\text { Standardized } \\
\text { Measure }\end{array}$ \\
\hline $\begin{array}{l}\text { Work } \\
\text { Demands }\end{array}$ & .266 & .293 & .077938 & .860357 \\
\hline $\begin{array}{l}\text { Non-work } \\
\text { demands }\end{array}$ & .110 & .115 & .01265 & .139643 \\
\hline $\begin{array}{l}\text { Variance } \\
\text { Explained }\end{array}$ & - & - & .090588 & 1 \\
\hline
\end{tabular}

The fact that Pratt's coefficient is six times higher for work demands (.860357) than non-work demands (.139643) can be interpreted to mean that work demands predict substantively more variance in work-family interference than do non-work demands. 


\subsubsection{Relationship Between Control Over Work-Life Interface and Work-Family Interference}

In model two we test 6 strain hypotheses that postulate relationships between these different forms of control over the work-life interface and workfamily interference. Specifically:

- WFIH3 postulates that the use of alternative work arrangements (AWA) will be negatively associated with work-family interference. Specifically employees who work:

a. flextime (versus a regular work day)

b. compressed work week (versus a regular work day)

c. telework (versus a regular work day)

will report lower levels of work-family interference than their counterparts who work a regular work day.

- Hypothesis WFIH4 postulates that the use of shiftwork arrangements will be positively associated with work-family interference.

- Hypothesis WFIH5 postulates that perceived flexibility will be negatively associated with work-family interference.

- Hypothesis WFIH6 postulates that supervisor support will be negatively associated with work-family interference.

Each of these hypotheses will be discussed in turn in the section below.

The three hypotheses exploring the relationship between the use of alternative work arrangements and work-family interference are not supported by the data. Results supporting this conclusion are reviewed below.

Hypothesis WFIH3a (i.e. flextime negatively associated with work-family interference) is tested as follows. The four covariates (gender, job type, work demands and non-work demands) are entered into the regression in step 1. These covariates explain $11.9 \%$ of the variance in work-family interference. The addition of the independent variable flextime results in no additional increase in our understanding of the variance in work-family interference above that already 
explained by the covariates. The p-value (.856) indicates that this relationship is not statistically significant. Therefore, we reject the hypothesis that those who work flextime will report lower levels of work-family interference than their counterparts who work a regular work day.

Hypothesis WFIH3b (compressed work week negatively associated with work-family interference) is tested as follows. The four covariates (gender, job type, work demands and non-work demands) are entered into the regression in step 1 . These covariates explain $10.8 \%$ of the variance in work-family interference. The addition of the independent variable compressed work week results in no additional increase in our understanding of the variance in workfamily interference above that already explained by the covariates. The $p$-value (.701) indicates that this relationship is not statistically significant. Therefore, we reject the hypothesis that those who work compressed work weeks will report lower levels of work-family interference than their counterparts who work a regular work day.

Hypothesis WFIH3c (telework negatively associated with work-family interference) is tested as follows. The four covariates (gender, job type, work demands and non-work demands) are entered into the regression in step 1. These covariates explain $11.5 \%$ of the variance in work-family interference. The addition of the independent variable telework results in no additional increase in our understanding of the variance in work-family interference above that already explained by the covariates. The p-value (.345) indicates that this relationship is not statistically significant. Therefore, we reject the hypothesis that those who 
telework will report lower levels of work-family interference than their counterparts who work a regular work day.

Hypothesis WFIH4 postulates that the use of shiftwork arrangements will be positively associated with work-family interference. This hypothesis is supported by the data. The hypothesis is tested as follows. In step 1, the four covariates (gender, job type, work demands and non-work demands) are entered into the regression. These covariates explain $11.5 \%$ of the variance in workfamily interference. The addition of the independent variable shiftwork results in a $0.7 \%$ increase in our understanding of the variance in work-family interference above that already explained by the covariates. The $p$-value $(<.001)$ indicates that this relationship is statistically significant thereby supporting the hypothesis that the use of shiftwork will be positively associated with work-family interference.

It should be noted, however, that in this case the change in $R^{2}$ in the amount of variance in work-family interference explained by the addition of shiftwork to the regression equation is very small (change in $R^{2}$ of $0.7 \%$ ). As noted earlier, Aguinis (2004) advises that changes in $R^{2}$ should be a minimum of $1 \%$ to be considered practically significant. Therefore, we conclude that while the findings were statistically significant, the result is not of practical significance.

Hypothesis WFIH5 postulates that perceived flexibility will be negatively associated with work-family interference. This hypothesis is supported by the data. This hypothesis is tested as follows. In step 1, the four covariates (gender, job type, work demands and non-work demands) are entered into the regression. 
These covariates explain $11.5 \%$ of the variance in work-family interference. The addition of the independent variable perceived flexibility results in an additional $14.2 \%$ of the variance in work-family interference above that already explained by the covariates (i.e. total $R^{2}$ of $\left.25.7 \%\right)$. The $p$-value $(<.001)$ indicates that this relationship is statistically significant thereby supporting our hypothesis that perceived flexibility will be negatively associated with work-family interference.

Hypothesis WFIH6 postulates that supervisor support will be negatively associated with work-family interference. The data supports this hypothesis. This hypothesis is tested as follows. In step 1, the four covariates (gender, job type, work demands and non-work demands) are entered into the regression. These covariates explain $11.5 \%$ of the variance in work-family interference. The addition of the independent variable supervisor support results in an additional $8.4 \%$ of the variance in work-family interference above that already explained by the covariates (i.e. total $R^{2}$ of $\left.19.9 \%\right)$. The $p$-value $(<.001)$ indicates that this relationship is statistically significant thereby supporting the hypothesis that supervisor support will be negatively associated with work-family interference.

\subsubsection{Conclusions: Work-Family Interference Strain Hypotheses}

In conclusion, half of the strain hypotheses for work-family interference are both statistically and practically significant. Both work demands and non-work demands are positively associated with work-family interference while perceived flexibility and supervisor support were both negatively associated with workfamily interference (all statistically significant at a p-value of .001). Also of 
importance are the Pratt's standardized measure data which indicates that work demands were approximately 6 times more important to the prediction of workfamily interference than non-work demands.

There is mixed support for Karasek's strain hypothesis when strain is operationalized as work-family interference. The strain hypotheses are strongly supported when strain is operationalized as work-family interference and demands are operationalized as work and non-work demands. The level of support for Karasek's strain hypothesis varies depending upon how control is operationalized. When control is operationalized as either perceived flexibility or supervisor support, there is strong support for the strain hypothesis. However, when control is operationalized as a given work arrangement (i.e. flextime, compressed work week, telework, shiftwork), there is very little support for the strain hypothesis. This conclusion is supported by the fact that the shiftwork hypothesis (i.e. shiftwork positively associated with work-family interference) was statistically but not practically significant. The three hypotheses for alternative work arrangements (i.e. flextime, compressed work week and telework negatively associated with work-family interference) were not statistically significant.

A review of the betas for both perceived flexibility $(B=-.431)$ and supervisor support $(B=-.391)$ indicate that both perceived flexibility and supervisor support are important predictors of work-family interference. It is important to note that the strongest associations were between perceived flexibility and work-family interference (change in $R^{2}$ of $14.2 \%$ ) and supervisor support and work-family interference (change in $R^{2}$ of $8.4 \%$ ). The fact that both these relationships were 
highly significant and negative (i.e. higher control, lower work-family interference) when work and non-work demands were controlled for support the idea that perceived flexibility and supervisor support help employees cope with work-family interference.

While the results indicate that shiftwork is positively associated with workfamily interference, the result is statistically but not practically significant. In the case of the three alternative work arrangements (i.e. flextime, compressed work week and telework), no statistically significant association is found with workfamily interference when work and non-work demands, gender and job type are controlled for. This would suggest that such work arrangements do not help employees cope with work-family interference.

\subsubsection{Evaluation of Factors that Moderate the Relationship Between Demands and Work-Family Interference}

This section summarizes the regression results obtained when testing the

following 12 buffer hypotheses associated with Model 2:

- Hypothesis WFIH7 postulates that alternative work arrangements will moderate the relationship between work demands and work-family interference. Specifically:

a. The relationship between work demands and work-family interference will be weaker for those who use flextime arrangements than those who work a regular work day.

b. The relationship between work demands and work-family interference will be weaker for those who work a compressed work week than those who work a regular work day.

c. The relationship between work demands and work-family interference will be weaker for those who telework than those who work a regular work day. d. The relationship between work demands and work-family interference will be weaker for those who work a regular work day than those who perform shiftwork. 
- Hypothesis WFIH8 postulates that perceived flexibility will moderate the relationship between work demands and work-family interference. Specifically, the relationship between work demands and work-family interference will decrease as perceived flexibility increases

- Hypothesis WFIH9 postulates that supervisor support will moderate the relationship between work demands and work-family interference. Specifically, the relationship between work demands and work-family interference will decrease as supervisor support increases

- Hypothesis WFIH10 postulates that alternative work arrangements will moderate the relationship between non-work demands and work-family interference. Specifically:

a. The relationship between non-work demands and work-family interference will be weaker for those who use flextime arrangements than those who work a regular work day.

b. The relationship between non-work demands and work-family interference will be weaker for those who work a compressed work week than those who work a regular work day.

c. The relationship between non-work demands and work-family interference will be weaker for those who telework than those who work a regular work day.

d. The relationship between non-work demands and work-family interference will be weaker for those who work a regular work day than those who perform shiftwork.

- Hypothesis WFIH11 postulates that perceived flexibility will moderate the relationship between non-work demands and work-family interference. Specifically, the relationship between non-work demands and work-family interference will decrease as perceived flexibility increases.

- Hypothesis WFIH12 postulates that supervisor support will moderate the relationship between non-work demands and work-family interference. Specifically, the relationship between non-work demands and work-family interference will decrease as supervisor support increases.

The results for each of these regressions are summarized in Table 13. The actual variables included in each of these regressions are provided in Appendix C. The discussion is divided into two main parts according to the type of demand being considered: work demands (presented first) and non-work demands (presented second). Each of these two parts have an identical 
structure. Data showing the extent to which the various work arrangements moderate the relationship between demands and work-family interference are presented first. This is followed by the presentation of data showing the extent to which the other two forms of control included in this thesis, supervisor support and perceived flexibility are able to moderate the relationship between demands and work-family interference. 
Table 13 Buffer Hypotheses Results for Model 2

\begin{tabular}{|c|c|c|c|c|c|c|c|c|}
\hline $\begin{array}{l}\text { Buffer Hypothesis for Model 2: } \\
\text { Work-Family Interference }\end{array}$ & $\begin{array}{l}\text { Step } 1 R^{2} \\
\text { (CV) }\end{array}$ & $\begin{array}{l}\text { Step } 2 \\
R^{2}(I V)\end{array}$ & $\begin{array}{l}\Delta \text { in } R^{2} \\
\text { due to } \\
\text { IV }\end{array}$ & $\begin{array}{l}\text { Step 3 } \\
\text { (Total } R^{2} \text { ) }\end{array}$ & $\begin{array}{l}\Delta \text { in } R^{2} \\
\text { due to IV } \\
\times M V\end{array}$ & F-stat & $\mathrm{df}$ & $\begin{array}{l}\text { p-value } \\
\text { at step } 3\end{array}$ \\
\hline $\begin{array}{l}\text { WFIH7a: Flextime moderates } \\
\text { relationship between WD and WFI }\end{array}$ & $11.9 \%$ & $11.9 \%$ & $0 \%$ & $11.9 \%$ & $0 \%$ & 310.10 & 6,13773 & .668 \\
\hline $\begin{array}{l}\text { WFIH7b: CWW moderates relationship } \\
\text { between WD and WFI }\end{array}$ & $10.8 \%$ & $10.8 \%$ & $0 \%$ & $10.8 \%$ & $0 \%$ & 247.38 & 6,12203 & .233 \\
\hline $\begin{array}{l}\text { WFIH7c: Telework moderates } \\
\text { relationship between WD and WFI }\end{array}$ & $11.5 \%$ & $11.5 \%$ & $0 \%$ & $11.5 \%$ & $0 \%$ & 216.48 & 6,9949 & .993 \\
\hline $\begin{array}{l}\text { WFIH7d: Shiftwork moderates } \\
\text { relationship between WD and WFI }\end{array}$ & $11.5 \%$ & $12.2 \%$ & $0.7 \%$ & $12.5 \%$ & $0.3 \%$ & 388.02 & 6,16359 & $<.001$ \\
\hline $\begin{array}{l}\text { WFIH8: Perceived flexibility moderates } \\
\text { relationship between WD and WFI }\end{array}$ & $11.5 \%$ & $25.7 \%$ & $14.2 \%$ & $25.7 \%$ & $0 \%$ & 945.09 & 6,16359 & .004 \\
\hline $\begin{array}{l}\text { WFIH9: Supervisor support moderates } \\
\text { relationship between WD and WFI }\end{array}$ & $11.5 \%$ & $19.9 \%$ & $8.4 \%$ & $20.0 \%$ & $0.1 \%$ & 679.97 & 6,16359 & $<.001$ \\
\hline $\begin{array}{l}\text { WFIH10a: Flextime moderates } \\
\text { relationship between NWD and WFI }\end{array}$ & $11.9 \%$ & $11.9 \%$ & $0 \%$ & $11.9 \%$ & $0 \%$ & 310.09 & 6,13773 & .749 \\
\hline $\begin{array}{l}\text { WFIH10b: CWW moderates } \\
\text { relationship between NWD and WFI }\end{array}$ & $10.8 \%$ & $10.8 \%$ & $0 \%$ & $10.8 \%$ & $0 \%$ & 247.15 & 6,12203 & .604 \\
\hline $\begin{array}{l}\text { WFIH10c: Telework moderates } \\
\text { relationship between NWD and WFI }\end{array}$ & $11.5 \%$ & $11.5 \%$ & $0 \%$ & $11.5 \%$ & $0 \%$ & 216.53 & 6,9949 & .607 \\
\hline $\begin{array}{l}\text { WFIH10d: Shiftwork moderates } \\
\text { relationship between NWD and WFI }\end{array}$ & $11.5 \%$ & $12.2 \%$ & $0.7 \%$ & $12.2 \%$ & $0 \%$ & 377.24 & 6,16359 & .386 \\
\hline $\begin{array}{l}\text { WFIH11: Perceived flexibility } \\
\text { moderates relationship between NWD } \\
\text { and WFI }\end{array}$ & $11.5 \%$ & $25.7 \%$ & $14.2 \%$ & $25.7 \%$ & $0 \%$ & 943.42 & 6,16359 & .296 \\
\hline $\begin{array}{l}\text { WFIH12: Supervisor support } \\
\text { moderates relationship between NWD } \\
\text { and WFI }\end{array}$ & $11.5 \%$ & $19.9 \%$ & $8.4 \%$ & $19.9 \%$ & $0 \%$ & 677.00 & 6,16359 & .526 \\
\hline
\end{tabular}




\subsubsection{Control Over Work-Life Interface Moderating Relationship Between Work Demands and Work-Family Interference}

This section presents the results of the regression analysis performed to test the ability of the various forms of control included in this analysis to moderate the relationship between work demands and work-family interference.

Hypothesis WFIH7a states that the relationship between work demands and work-family interference will be weaker for those who use flextime arrangements than those who work a regular work day. This hypothesis is not supported by the data. This hypothesis is tested as follows. First the four covariates (gender, job type, work demands and non-work demands) are entered into the regression equation in step 1 . These covariates explain $11.9 \%$ of the variance in work-family interference. Second the independent variable, flextime, is added to the regression. This variable explains no additional variance in workfamily interference above that already explained by the covariates. The interaction term, flextime by work demands, is added to the regression in step three. This term did not explain any additional variance in work-family interference ( $p$ value .668) and the beta coefficient of the interaction is zero $(.000)$. Since the interaction term is not significant we reject the hypothesis that the relationship between work demands and work-family interference will be weaker for those who use flextime arrangements than those who work a regular work day.

Hypothesis WFIH7b states that the relationship between work demands and work-family interference will be weaker for those who use compressed work 
weeks than those who work a regular work day. This hypothesis is not supported by the data. This hypothesis is tested as follows. First the four covariates (gender, job type, work demands and non-work demands) are entered into the regression equation in step 1 . These covariates explain $10.8 \%$ of the variance in work-family interference. Second, the independent variable, compressed work week, is added to the regression. This variable explains no additional variance in work-family interference above that already explained by the covariates. The interaction term, compressed work week by work demands is added to the regression in step three. This term did not explain any additional variance in work-family interference ( $p$ value .233 ) and the beta coefficient of the interaction is very small $(-.002)$. Since the interaction term is not significant we reject the hypothesis that the relationship between work demands and work-family interference will be weaker for those who use compressed work weeks than those who work a regular work day.

Hypothesis WFIH7c states that the relationship between work demands and work-family interference will be weaker for those who telework than those who work a regular work day. This hypothesis is not supported by the data. This hypothesis is tested as follows. First the four covariates (gender, job type, work demands and non-work demands) are entered into the regression equation in step 1 . These covariates explain $11.5 \%$ of the variance in work-family interference. Second, the independent variable, telework, is added to the regression. This variable explains no further variance in work-family interference ( $p$ value .345 ) above that already explained by the covariates. The interaction 
term, telework by work demands is added to the regression in step three. This term did not explain any additional variance in work-family interference ( $p$ value $.993)$ and the beta coefficient of the interaction was zero (.000). Since the interaction term is not significant we reject the hypothesis that the relationship between work demands and work-family interference will be weaker for those who telework than those who work a regular work day.

Hypothesis WFIH7d states that the relationship between work demands and work-family interference will be weaker for those who work a regular work schedule than those who perform shiftwork. This hypothesis is supported by the data. This hypothesis is tested as follows. First the four covariates (gender, job type, work demands and non-work demands) are entered into the regression equation in step 1 . These covariates explain $11.5 \%$ of the variance in workfamily interference. Second the independent variable, shiftwork, is added to the regression. This variable explains an additional $0.7 \%$ variance in work-family interference above that already explained by the covariates. The interaction term shiftwork by work demands is added to the regression in step three. This interaction term explains a further $0.3 \%$ of the variance in work-family interference above that already explained by the covariates and the independent variable and is statistically significant $(p$ value $<.001)$. These findings are supportive of our hypothesis that shiftwork moderates the impact of work demands on work-family interference such that the relationship between work demands and work-family interference is weaker for those who work a regular work day than those who perform shiftwork. 
As noted previously, the approach taken in this thesis is to examine statistically significant interaction terms to see if they are practically significant. This is done by plotting and interpreting the interaction and examining the change in $R^{2}$ that can be explained by the interaction term. Figure 16 plots the interaction of work demands and shiftwork.

\section{Figure 16 Interaction of Work Demands x Shiftwork}

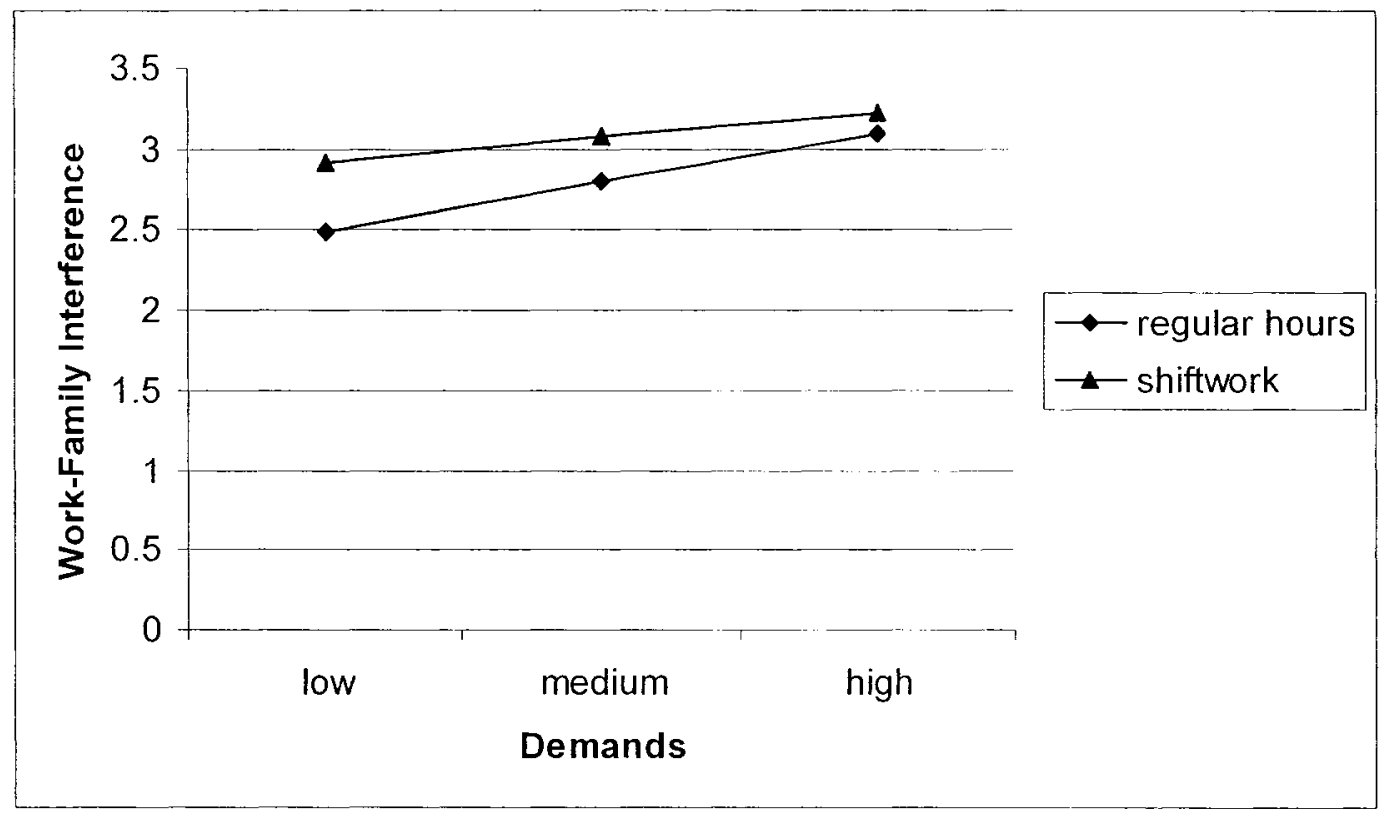

Examination of this figure indicates that the relationship between work-family interference and shift/regular work arrangement varies depending on the level of work demands reported by the individual. At low levels of work demands the relationship is as hypothesized: those who work shiftwork report higher levels of work-family interference than those working regular hours. At high levels of work demands, on the other hand, the interaction is different than the hypothesis as respondents who work regular hours and shiftworkers reported similar levels of work-family interference. This indicates that shiftwork is problematic with 
respect to work-family interference regardless of the level of work demands. It also suggests that regular work arrangements are associated with higher workfamily interference when work demands rise above a critical threshold. These findings are similar to the ones reported for role overload.

Despite this, the fact that the change in $\mathrm{R}^{2}$ was slight $(0.3 \%)$ indicates that while this result is statistically significant, it is not of practical significance.

Hypothesis WFIH8 looks at the ability of perceived flexibility to moderate the relationship between work demands and work-family interference. This hypothesis (i.e. that the relationship between work demands and work-family interference will decrease as perceived flexibility increases) is not supported by the data.

This hypothesis is tested as follows. First the four covariates (gender, job type, work demands and non-work demands) are entered into the regression equation in step 1 . These covariates explain $11.5 \%$ of the variance in workfamily interference. Second, the independent variable, perceived flexibility, is added to the regression. This variable explains a further $14.2 \%$ of the variance in work-family interference above that already explained by the covariates (for a total $R^{2}$ of $25.7 \%$ ). The interaction term perceived flexibility by work demands is added to the regression in step three. This term did not explain any additional variance in work-family interference ( $p$ value .004$)$ and the beta coefficient of the interaction is very small $(.002)$. Since the interaction term is not significant we reject the hypothesis that the relationship between work demands and workfamily interference will decrease as perceived flexibility increases. 
Hypothesis WFIH9 looks at the ability of supervisor support to moderate the relationship between work demands and work-family interference. This hypothesis (i.e. that the relationship between work demands and work-family interference will decrease as supervisor support increases) is supported by the data.

This hypothesis is tested as follows. First the four covariates (gender, job type, work demands and non-work demands) are entered into the regression equation in step 1 . These covariates explain $11.5 \%$ of the variance in workfamily interference. Second the independent variable, supervisor support, is added to the regression. This variable explains a further $8.4 \%$ of the variance in work-family interference above that already explained by the covariates (for a total $R^{2}$ of $19.9 \%$ ). The interaction term supervisor support by work demands is added to the regression in step three. This interaction term explains a further $0.1 \%$ of the variance in work-family interference above that already explained by the covariates and the independent variable and is statistically significant ( $p$ value $<.001)$. These findings are supportive of our hypothesis that supervisor support moderates the impact of work demands on work-family interference such that the relationship between work demands and work-family interference will decrease as supervisor support increases.

Consistent with the process previously outlined, we first plotted the interaction and then examined the change in $R^{2}$ from step 2 to step 3 . The results of the plotted interaction are seen in Figure 17. 
Figure 17 Interaction of Work Demands x Supervisor Support

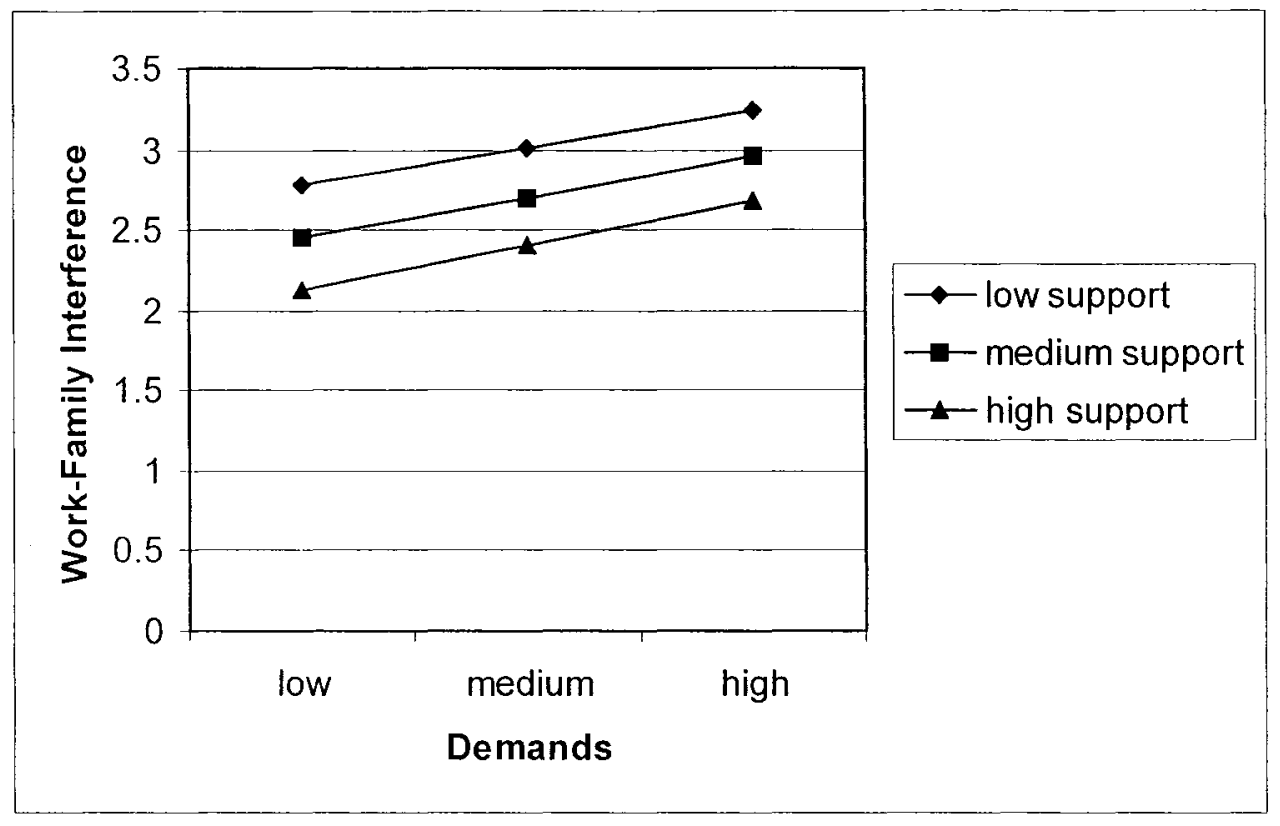

As seen in figure 17, there is very little evidence of an interaction effect for this relationship. The lines of the plot are almost parallel and show that those reporting high levels of supervisor support reported lower levels of work-family interference than those at medium or low levels of supervisor support regardless of the level of work demands. These findings are consistent with the fact that the change in $R^{2}$ was $0.1 \%$ which indicates that these results, while statistically significant, are not of practical significance.

\subsubsection{Control Over Work-Life Interface Moderating Relationship Between Non-work demands and Work-Family Interference}

This section presents the results of the regression analysis performed to test the ability of the various forms of control included in this analysis to moderate the relationship between non-work demands and work-family interference. The 
regression results are not supportive of the idea that alternative work arrangements moderate the relationship between non-work demands and workfamily interference. Support for this contention is provided below.

Hypothesis WFIH10a states that the relationship between non-work demands and work-family interference will be weaker for those who use flextime arrangements than those who work a regular work day. This hypothesis is tested as follows. First the four covariates (gender, job type, work demands and nonwork demands) are entered into the regression equation in step 1 . These covariates explain $11.9 \%$ of the variance in work-family interference. Second the independent variable, flextime, is added to the regression. This variable explains no further variance in work-family interference above that already explained by the covariates. The interaction term, flextime by non-work demands, is added to the regression in step three. This term did not explain any additional variance in work-family interference ( $p$ value .749$)$ and the beta coefficient of the interaction is zero (.000). Since the interaction term is not significant we reject the hypothesis that the relationship between non-work demands and work-family interference will be weaker for those who use flextime arrangements than those who work a regular work day.

Hypothesis WFIH10b states that the relationship between non-work demands and work-family interference will be weaker for those who use compressed work weeks than those who work a regular work day. This hypothesis is tested as follows. First the four covariates (gender, job type, work demands and non-work demands) are entered into the regression equation in 
step 1 . These covariates explain $10.8 \%$ of the variance in work-family interference. Second, the independent variable, compressed work week, is added to the regression. This variable explains no further variance in work-family interference above that already explained by the covariates. The interaction term, compressed work week by non-work demands is added to the regression in step three. This term did not explain any additional variance in work-family interference ( $p$ value .604) and the beta coefficient of the interaction is zero $(.000)$. Since the interaction term is not significant we reject the hypothesis that the relationship between non-work demands and work-family interference will be weaker for those who use compressed work weeks than those who work a regular work day.

Hypothesis WFIH10c states that the relationship between non-work demands and work-family interference will be weaker for those who telework than those who work a regular work day. This hypothesis is tested as follows. First the four covariates (gender, job type, work demands and non-work demands) are entered into the regression equation in step 1. These covariates explain $11.5 \%$ of the variance in work-family interference. Second, the independent variable, telework, is added to the regression. This variable explains no further variance in work-family interference ( $p$ value .345 ) above that already explained by the covariates. The interaction term, telework by non-work demands is added to the regression in step three. This term did not explain any additional variance in work-family interference ( $p$ value .607) and the beta coefficient of the interaction is very small (.002). Since the interaction term is not 
significant we reject the hypothesis that the relationship between non-work demands and work-family interference will be weaker for those who telework than those who work a regular work day.

Hypothesis WFIH10d states that the relationship between non-work demands and work-family interference will be weaker for those who work a regular work schedule than those who perform shiftwork. This hypothesis is tested as follows. First the four covariates (gender, job type, work demands and non-work demands) are entered into the regression equation in step 1. These covariates explain $11.5 \%$ of the variance in work-family interference. Second the independent variable, shiftwork, is added to the regression. This variable explains an additional $0.7 \%$ variance in work-family interference above that already explained by the covariates. The interaction term shiftwork by non-work demands is added to the regression in step three. This term did not explain any additional variance in work-family interference ( $p$ value .386 ) and the beta coefficient of the interaction was very small (.001). Since the interaction term is not significant we reject the hypothesis that the relationship between non-work demands and work-family interference will be weaker for those who work a regular work day than those who work shiftwork.

Hypothesis WFIH11 looks at the ability of perceived flexibility to moderate the relationship between non-work demands and work-family interference. This hypothesis (i.e. that the relationship between non-work demands and work-family interference will decrease as perceived flexibility increases) is not supported by the data. This hypothesis is tested as follows. First the four covariates (gender, 
job type, work demands and non-work demands) are entered into the regression equation in step 1 . These covariates explain $11.5 \%$ of the variance in workfamily interference. Second the independent variable, perceived flexibility, is added to the regression. This variable explains a further $14.2 \%$ of the variance in work-family interference above that already explained by the covariates (for a total $\mathrm{R}^{2}$ of $25.7 \%$ ). The interaction term perceived flexibility by non-work demands is added to the regression in step three. This term did not explain any additional variance in work-family interference ( $p$ value .296) and the beta coefficient of the interaction is very small (-.001). Since the interaction term is not significant we reject the hypothesis that perceived flexibility moderates the impact of non-work demands on work-family interference such that the relationship between non-work demands and work-family interference will decrease as perceived flexibility increases.

Hypothesis WFIH12 looks at the ability of supervisor support to moderate the relationship between non-work demands and work-family interference. This hypothesis (i.e. that the relationship between non-work demands and work-family interference will decrease as supervisor support increases) is not supported by the data. This hypothesis is tested as follows. First the four covariates (gender, job type, work demands and non-work demands) are entered into the regression equation in step 1 . These covariates explain $11.5 \%$ of the variance in workfamily interference. Second the independent variable, supervisor support, is added to the regression. This variable explains a further $8.4 \%$ of the variance in work-family interference above that already explained by the covariates (for a 
total $R^{2}$ of $19.9 \%$ ). The interaction term supervisor support by non-work demands is added to the regression in step three. This term did not explain any additional variance in work-family interference ( $p$ value .526 ) and the beta coefficient of the interaction is very small $(.001)$. Since the interaction term is not significant we reject the hypothesis that that supervisor support moderates the impact of non-work demands on work-family interference such that the relationship between non-work demands and work-family interference will decrease as supervisor support increases.

\subsubsection{Conclusions: Work-Family Interference Buffer Hypotheses}

In conclusion, Karasek's buffer hypothesis is not supported when strain is operationalized as work-family interference, demands operationalized as work and non-work demands, and control operationalized as alternative work arrangement, perceived flexibility or supervisor support. This conclusion is based on two facts. First, ten of the twelve buffer hypotheses were not statistically significant. Second, while the buffer hypothesis was supported under specific circumstances (shiftwork by work demands, supervisor support by work demands), the fact that there was very little change in $R^{2}$ (i.e. only $0.3 \%$ for the shiftwork by work demands interaction and only $0.1 \%$ for the supervisor support by work demands interaction) and plotting the interaction term showed that the changes in work-family interference at various levels of the moderator and independent variable were small suggests the findings had little practical significance. 


\subsubsection{Evaluation of Relationships Between Demand, Control over Work- Life Interface and Family-Work Interference}

This section summarizes the regression results obtained when testing the 8 strain hypotheses associated with Model 3 . With one exception, model 3 is identical to models 1 and 2. In this case, however, strain is operationalized as family-work interference rather than role overload or work-family interference. The results for each of these regressions are summarized in Table 14. The actual variables included in each of these regressions are provided in Appendix C. The discussion proceeds as follows. Findings with respect to testing the strain hypotheses dealing with the relationship between demands and familywork interference will be presented first. This is followed by the findings obtained with the strain hypotheses pertaining to the relationship between different forms of control over the work-life interface and family-work interference. 
Table 14 Strain Hypotheses Results for Model 3

\begin{tabular}{|l|l|l|l|l|l|l|}
\hline $\begin{array}{l}\text { Strain Hypothesis } \\
\text { For Model 3: } \\
\text { Family-Work } \\
\text { Interference }\end{array}$ & $\begin{array}{l}\text { Step 1 } \\
\mathrm{R}^{2} \\
\text { (CV) }\end{array}$ & $\begin{array}{l}\text { Step 2 } \\
\text { (Total } \\
\left.\mathrm{R}^{2}\right)\end{array}$ & $\begin{array}{l}\text { - } \\
\text { value } \\
\text { at step } \\
2\end{array}$ & df & F-stat & $\begin{array}{l}\Delta \text { in } \\
\mathrm{R}^{2} \\
\text { due to } \\
\text { IV }\end{array}$ \\
\hline $\begin{array}{l}\text { FWIH1: WD } \\
\text { positively } \\
\text { associated with } \\
\text { FWI }\end{array}$ & $5.6 \%$ & $5.7 \%$ & .001 & 4,16361 & 247.46 & $0.1 \%$ \\
\hline $\begin{array}{l}\text { FWIH2: NWD } \\
\text { positively } \\
\text { associated with } \\
\text { FWI }\end{array}$ & $0.2 \%$ & $5.7 \%$ & $<.001$ & 4,16361 & 247.46 & $5.5 \%$ \\
\hline $\begin{array}{l}\text { FWIH3a: Flextime } \\
\text { negatively } \\
\text { associated with } \\
\text { FWI }\end{array}$ & $5.7 \%$ & $5.7 \%$ & .002 & 5,13774 & 167.50 & $0 \%$ \\
\hline $\begin{array}{l}\text { FWIH3b: CWW } \\
\text { negatively } \\
\text { associated with } \\
\text { WFI }\end{array}$ & $5.6 \%$ & $5.6 \%$ & .136 & 5,12204 & 144.49 & $0 \%$ \\
\hline $\begin{array}{l}\text { FWIH3c: Telework } \\
\text { negatively } \\
\text { associated with } \\
\text { FWI }\end{array}$ & $5.3 \%$ & $5.4 \%$ & .014 & 5,9950 & 112.51 & $0.1 \%$ \\
\hline $\begin{array}{l}\text { FWIH4: Shiftwork } \\
\text { positively } \\
\text { associated with } \\
\text { FWI }\end{array}$ & $5.7 \%$ & $5.7 \%$ & .141 & 5,16360 & 198.41 & $0 \%$ \\
\hline $\begin{array}{l}\text { FWIH5: Perceived } \\
\text { flexibility } \\
\text { negatively } \\
\text { associated with } \\
\text { FWI }\end{array}$ & $5.7 \%$ & $8.0 \%$ & $<.001$ & 5,16360 & 285.53 & $2.3 \%$ \\
\hline $\begin{array}{l}\text { FWIH6: Supervisor } \\
\text { support negatively } \\
\text { associated with } \\
\text { FWI }\end{array}$ & $5.7 \%$ & $7.6 \%$ & $<.001$ & 5,16360 & 268.62 & $1.9 \%$ \\
\hline
\end{tabular}




\subsubsection{Relationship Between Demands and Family-Work Interference}

Two strain hypotheses postulate relationships between demands and family-work interference. Results associated with the testing of each of these hypotheses are presented below.

FWIH1 postulates that work demands will be positively associated with family-work interference. The data supported this hypothesis. The hypothesis was tested as follows. In step 1, the three covariates (gender, job type and nonwork demands) are entered into the regression. The covariates explain $5.6 \%$ of the variance in family-work interference. When the independent variable work demands is added to the regression equation in step 2 , an additional $0.1 \%$ of the variance is explained above that already explained by the covariates (for a total $R^{2}$ of $\left.5.7 \%\right)$. The $p$-value (.001) indicates that this relationship is statistically significant. The finding that work demands are positively associated with familywork interference is supportive of the hypothesis. However, the fact that we observed a change in $\mathrm{R}^{2}$ of only $0.1 \%$ when the independent variable work demands is added to the regression equation indicates that the result is statistically but not practically significant.

FWIH2 postulates that non-work demands will be positively associated with family-work interference. The data supports this hypothesis. The hypothesis is tested as follows. In step 1, the three covariates (gender, job type and work demands) are entered into the regression. These variables explain $0.2 \%$ of the variance in family-work interference. When the independent variable non-work demands is added to the regression equation in step 2 , an 
additional $5.5 \%$ of the variance is explained above that already explained by the covariates (for a total $R^{2}$ of $5.7 \%$ ). The $p$-value $(<.001)$ indicates that this relationship is statistically significant. The finding that higher levels of non-work demands is associated with higher levels of family-work interference is supportive of the hypothesis.

As noted earlier, we can assess the relative importance of these two independent variables (work demands and non-work demands) at predicting family-work interference by calculating Pratt's standardized measure (Thomas et al., 1998). The Pratt's standardized measures obtained when both work demands and non-work demands are included in the regression equation predicting family-work interference are shown in Table 15.

Table 15 Pratt's Coefficient: Family-Work Interference

\begin{tabular}{|l|l|l|l|l|}
\hline $\begin{array}{l}\text { Independent } \\
\text { Variable }\end{array}$ & $\begin{array}{l}\text { Standardized } \\
\text { Coefficient }\end{array}$ & $\begin{array}{l}\text { Correlation } \\
\text { of IV and } \\
\text { DV }\end{array}$ & $\begin{array}{l}\text { Pratt's } \\
\text { Coefficient }\end{array}$ & $\begin{array}{l}\text { Pratt's } \\
\text { Standardized } \\
\text { Measure }\end{array}$ \\
\hline $\begin{array}{l}\text { Work } \\
\text { Demands }\end{array}$ & .025 & .034 & .00085 & .015158 \\
\hline $\begin{array}{l}\text { Non-work } \\
\text { demands }\end{array}$ & .235 & .235 & .055225 & .984482 \\
\hline $\begin{array}{l}\text { Variance } \\
\text { Explained }\end{array}$ & - & - & .056075 & 1 \\
\hline
\end{tabular}

The fact that Pratt's standardized measure is sixty five times higher for non-work demands (.984482) than work demands (.015158) indicates that nonwork demands predict relatively more variance in family-work interference than do work demands. 


\subsubsection{Relationship Between Control Over Work-Life Interface and Family-Work Interference}

In model three we test 6 strain hypotheses that postulate relationships between these different forms of control over the work-life interface and familywork interference. Specifically:

- $\quad \mathrm{FWIH3}$ postulates that the use of alternative work arrangements (AWA) will be negatively associated with family-work interference. Specifically employees who work:

a. flextime (versus a regular work day)

b. compressed work week (versus a regular work day)

c. telework (versus a regular work day)

will report lower levels of family-work interference than their counterparts who work a regular work day.

- Hypothesis FWIH4 postulates that the use of shiftwork arrangements will be positively associated with family-work interference.

- Hypothesis FWIH5 postulates that perceived flexibility will be negatively associated with family-work interference.

- Hypothesis FWIH6 postulates that supervisor support will be negatively associated with family-work interference.

Each of these hypotheses will be discussed in turn in the section below.

The three hypotheses exploring the relationship between the use of alternative work arrangements and family-work interference were not supported by the data. Results supporting this conclusion are reviewed below.

Hypothesis FWIH3a (i.e. flextime negatively associated with family-work interference) is tested as follows. The four covariates (gender, job type, work demands and non-work demands) are entered into the regression in step 1. These covariates explain $5.7 \%$ of the variance in family-work interference. The addition of the independent variable flextime results in no additional increase in 
our understanding of the variance in family-work interference above that already explained by the covariates. The p-value (.002) indicates that this relationship is not statistically significant. Therefore, we reject the hypothesis that those who work flextime will report lower levels of family-work interference than their counterparts who work a regular work day.

Hypothesis FWIH3b (compressed work week negatively associated with family-work interference) is tested as follows. The four covariates (gender, job type, work demands and non-work demands) are entered into the regression in step 1 . These variables explain $5.6 \%$ of the variance in family-work interference. The addition of the independent variable compressed work week results in no additional increase in our understanding of the variance in family-work interference above that already explained by the covariates. The p-value (.136) indicates that this relationship is not statistically significant. Therefore, we reject the hypothesis that those who work compressed work weeks will report lower levels of family-work interference than their counterparts who work a regular work day.

Hypothesis FWIH3c (telework negatively associated with family-work interference) is tested as follows. The four covariates (gender, job type, work demands and non-work demands) are entered into the regression in step 1. These variables explain $5.3 \%$ of the variance in family-work interference. The addition of the independent variable telework results in a $0.1 \%$ increase in our understanding of the variance in family-work interference above that already explained by the covariates. The p-value (.014) indicates that this relationship is 
not statistically significant. Therefore, we reject the hypothesis that those who telework will report lower levels of family-work interference than their counterparts who work a regular work day.

Hypothesis FWIH4 postulates that the use of shiftwork arrangements will be positively associated with family-work interference. This hypothesis is not supported by the data. The hypothesis is tested as follows. In step 1, the four covariates (gender, job type, work demands and non-work demands) are entered into the regression. These variables explain $5.7 \%$ of the variance in family-work interference. The addition of the independent variable shiftwork results in no additional increase in our understanding of the variance in family-work interference above that already explained by the covariates. The p-value (.141) indicates that this relationship is not statistically significant. Therefore, we reject the hypothesis that the use of shiftwork will be positively associated with familywork interference.

Hypothesis FWIH5 postulates that perceived flexibility will be negatively associated with family-work interference. This hypothesis is supported by the data. The hypothesis is tested as follows. In step 1, the four covariates (gender, job type, work demands and non-work demands) are entered into the regression. These variables explain $5.7 \%$ of the variance in family-work interference. The addition of the independent variable, perceived flexibility, results in an additional $2.3 \%$ of the variance in family-work interference above that already explained by the covariates (i.e. total $R^{2}$ of $8.0 \%$ ). The p-value $(<.001)$ indicates that this 
relationship is statistically significant thereby supporting our hypothesis that perceived flexibility will be negatively associated with family-work interference. Hypothesis FWIH6 postulates that supervisor support will be negatively associated with family-work interference. The data supports this hypothesis. The hypothesis is tested as follows. In step 1, the four covariates (gender, job type, work demands and non-work demands) are entered into the regression. These variables explain $5.7 \%$ of the variance in family-work interference. The addition of the independent variable supervisor support results in an additional $1.9 \%$ of the variance in family-work interference above that already explained by the covariates (i.e. total $R^{2}$ of $7.6 \%$ ). The $p$-value $(<.001)$ indicates that this relationship is statistically significant thereby supporting the hypothesis that supervisor support will be negatively associated with family-work interference.

\subsubsection{Conclusions: Family-Work Interference Strain Hypotheses}

In conclusion, three of the eight strain hypotheses for family-work interference were both statistically and practically significant. Non-work demands was positively associated with family-work interference while perceived flexibility and supervisor support were both negatively associated with family-work interference (all statistically significant at a p-value of <.001). A review of the Pratt's standardized measures indicate that non-work demands were approximately 65 times more important to the prediction of family-work interference than work demands. In fact, the relationship between work 
demands and family-work interference while statistically significant, was not practically significant.

There is mixed support for Karasek's strain hypothesis when strain is operationalized as family-work interference. The strain hypothesis is strongly supported when strain is operationalized as family-work interference and demands are operationalized as non-work demands. However, when demand is operationalized as work demands, the results are deemed to be statistically significant but not practically significant. The level of support for Karasek's strain hypothesis varies depending upon how control is operationalized. When control is operationalized as either perceived flexibility or supervisor support, there is strong support for the strain hypothesis. However, when control is operationalized as flextime, compressed work week, telework, or shiftwork, there is no support for the strain hypothesis.

A review of the betas for both perceived flexibility $(B=-.163)$ and supervisor support $(B=-.173)$ indicate that both of these forms of control are important predictors of family-work interference. The fact that both of these relationships were highly significant and negative (i.e. higher control, lower family-work interference) when work and non-work demands were controlled for also support the idea that perceived flexibility and supervisor support help employees cope with family-work interference.

In the case of the three alternative work arrangements (i.e. flextime, compressed work week and telework) and shiftwork, no statistically significant association is found with family-work interference when work and non-work 
demands, gender and job type are controlled for. This would suggest that such work arrangements do not help employees cope with family-work interference.

\subsubsection{Evaluation of Factors that Moderate the Relationship Between Demands and Family-Work Interference}

This section summarizes the regression results obtained when testing the

following 12 buffer hypotheses associated with Model 3 :

- Hypothesis FWIH7 postulates that alternative work arrangements will moderate the relationship between work demands and family-work interference. Specifically:

a. The relationship between work demands and family-work interference will be weaker for those who use flextime arrangements than those who work a regular work day.

b. The relationship between work demands and family-work interference will be weaker for those who work a compressed work week than those who work a regular work day.

c. The relationship between work demands and family-work interference will be weaker for those who telework than those who work a regular work day. d. The relationship between work demands and family-work interference will be weaker for those who work a regular work day than those who perform shiftwork.

- Hypothesis FWIH8 postulates that perceived flexibility will moderate the relationship between work demands and family-work interference. Specifically, the relationship between work demands and family-work interference will decrease as perceived flexibility increases

- Hypothesis FWIH9 postulates that supervisor support will moderate the relationship between work demands and family-work interference. Specifically, the relationship between work demands and family-work interference will decrease as supervisor support increases

- Hypothesis FWIH10 postulates that alternative work arrangements will moderate the relationship between non-work demands and family-work interference. Specifically:

a. The relationship between non-work demands and family-work interference will be weaker for those who use flextime arrangements than those who work a regular work day.

b. The relationship between non-work demands and family-work interference will be weaker for those who work a compressed work week than those who work a regular work day. 
c. The relationship between non-work demands and family-work interference will be weaker for those who telework than those who work a regular work day.

d. The relationship between non-work demands and family-work interference will be weaker for those who work a regular work day than those who perform shiftwork.

- Hypothesis FWIH11 postulates that perceived flexibility will moderate the relationship between non-work demands and family-work interference. Specifically, the relationship between non-work demands and family-work interference will decrease as perceived flexibility increases.

- Hypothesis FWIH12 postulates that supervisor support will moderate the relationship between non-work demands and family-work interference. Specifically, the relationship between non-work demands and family-work interference will decrease as supervisor support increases.

The results for each of these regressions are summarized in Table 16. The actual variables included in each of these regressions are provided in Appendix C. The discussion is divided into two main parts according to the type of demand being considered: work demands (presented first) and non-work demands (presented second). Each of these two parts have an identical structure. Data showing the extent to which the various work arrangements moderate the relationship between demands and family-work interference are presented first. This is followed by the presentation of data showing the extent to which the other two forms of control included in this thesis, supervisor support and perceived flexibility are able to moderate the relationship between demands and family-work interference. 


\section{Table 16 Buffer Hypotheses Results for Model 3}

\begin{tabular}{|c|c|c|c|c|c|c|c|c|}
\hline $\begin{array}{l}\text { Buffer Hypothesis for Model 3: } \\
\text { Family-Work Interference }\end{array}$ & $\begin{array}{l}\text { Step } 1 \mathrm{R}^{2} \\
(\mathrm{CV})\end{array}$ & $\begin{array}{l}\text { Step } 2 \\
\mathrm{R}^{2}(\mathrm{IV})\end{array}$ & $\begin{array}{l}\Delta \text { in } \mathrm{R}^{2} \\
\text { due to } \\
\mathrm{IV}\end{array}$ & $\begin{array}{l}\text { Step } 3 \\
\left.\text { (Total } R^{2}\right)\end{array}$ & $\begin{array}{l}\triangle \text { in } R^{2} \\
\text { due to IV } \\
\times M V\end{array}$ & F-stat & $d f$ & $\begin{array}{l}\text { p-value } \\
\text { at step } 3\end{array}$ \\
\hline $\begin{array}{l}\text { FWIH7a: Flextime moderates } \\
\text { relationship between WD and FWI }\end{array}$ & $5.7 \%$ & $5.7 \%$ & $0 \%$ & $5.8 \%$ & $0.1 \%$ & 141.24 & 6,13773 & .002 \\
\hline $\begin{array}{l}\text { FWIIH7b: CWW moderates } \\
\text { relationship between WD and FWI }\end{array}$ & $5.6 \%$ & $5.6 \%$ & $0 \%$ & $5.6 \%$ & $0 \%$ & 121.53 & 6,12203 & .012 \\
\hline $\begin{array}{l}\text { FWIH7c: Telework moderates } \\
\text { relationship between WD and FWI }\end{array}$ & $5.3 \%$ & $5.4 \%$ & $0.1 \%$ & $5.4 \%$ & $0 \%$ & 94.14 & 6,9949 & .134 \\
\hline $\begin{array}{l}\text { FWIH7d: Shiftwork moderates } \\
\text { relationship between WD and FWI }\end{array}$ & $5.7 \%$ & $5.7 \%$ & $0 \%$ & $5.7 \%$ & $0 \%$ & 165.34 & 6,16359 & .883 \\
\hline $\begin{array}{l}\text { FWIH8: Perceived flexibility moderates } \\
\text { relationship between WD and FWI }\end{array}$ & $5.7 \%$ & $8.0 \%$ & $2.3 \%$ & $8.1 \%$ & $0.1 \%$ & 240.40 & 6,16359 & $<.001$ \\
\hline $\begin{array}{l}\text { FWIH9: Supervisor support moderates } \\
\text { relationship between WD and FWI }\end{array}$ & $5.7 \%$ & $7.6 \%$ & $1.9 \%$ & $7.6 \%$ & $0 \%$ & 224.29 & 6,16359 & .116 \\
\hline $\begin{array}{l}\text { FWIH10a: Flextime moderates } \\
\text { relationship between NWD and FWI }\end{array}$ & $5.7 \%$ & $5.7 \%$ & $0 \%$ & $5.8 \%$ & $0.1 \%$ & 140.53 & 6,13773 & .020 \\
\hline $\begin{array}{l}\text { FWIH10b: CWW moderates } \\
\text { relationship between NWD and FWI }\end{array}$ & $5.6 \%$ & $5.6 \%$ & $0 \%$ & $5.6 \%$ & $0 \%$ & 120.43 & 6,12203 & .701 \\
\hline $\begin{array}{l}\text { FWIH10c: Telework moderates } \\
\text { relationship between NWD and FWI }\end{array}$ & $5.3 \%$ & $5.4 \%$ & $0.1 \%$ & $5.4 \%$ & $0 \%$ & 93.93 & 6,9949 & .313 \\
\hline $\begin{array}{l}\text { FWIH10d: Shiftwork moderates } \\
\text { relationship between NWD and FWI }\end{array}$ & $5.7 \%$ & $5.7 \%$ & $0 \%$ & $5.7 \%$ & $0 \%$ & 165.93 & 6,16359 & .067 \\
\hline $\begin{array}{l}\text { FWIH11: Perceived flexibility } \\
\text { moderates relationship between NWD } \\
\text { and FWI }\end{array}$ & $5.7 \%$ & $8.0 \%$ & $2.3 \%$ & $8.0 \%$ & $0 \%$ & 237.95 & 6,16359 & .774 \\
\hline $\begin{array}{l}\text { FWIH12: Supervisor support } \\
\text { moderates relationship between NWD } \\
\text { and FWI }\end{array}$ & $5.7 \%$ & $7.6 \%$ & $1.9 \%$ & $7.6 \%$ & $0 \%$ & 223.85 & 6,16359 & .805 \\
\hline
\end{tabular}




\subsubsection{Control Over Work-Life Interface Moderates Relationship Between Work Demands and Family-Work Interference}

This section presents the results of the regression analysis performed to test the ability of the various forms of control included in this analysis to moderate the relationship between work demands and family-work interference.

Hypothesis FWIH7a states that the relationship between work demands and family-work interference will be weaker for those who use flextime arrangements than those who work a regular work day. The data did not support this hyothesis. This hypothesis is tested as follows. First the four covariates (gender, job type, work demands and non-work demands) are entered into the regression equation in step 1 . These covariates explain $5.7 \%$ of the variance in family-work interference. Second the independent variable, flextime, is added to the regression. This variable explains no additional variance in family-work interference above that already explained by the covariates. The interaction term, flextime by work demands, is added to the regression in step three. This term explains an additional $0.1 \%$ variance in family-work interference ( $p$ value $.002)$ and the beta coefficient of the interaction is very small (-.003). Since the interaction term is not significant we reject the hypothesis that the relationship between work demands and family-work interference will be weaker for those who use flextime arrangements than those who work a regular work day.

Hypothesis FWIH7b states that the relationship between work demands and family-work interference will be weaker for those who use compressed work weeks than those who work a regular work day. This hypothesis is not supported by the data. This hypothesis is tested as follows. First the four covariates 
(gender, job type, work demands and non-work demands) are entered into the regression equation in step 1 . These covariates explain $5.6 \%$ of the variance in family-work interference. Second, the independent variable, compressed work week, is added to the regression. This variable explains no additional variance in family-work interference above that already explained by the covariates. The interaction term, compressed work week by work demands is added to the regression in step three. This term did not explain any additional variance in family-work interference ( $p$ value .012) and the beta coefficient of the interaction is very small (.003). Since the interaction term is not significant we reject the hypothesis that the relationship between work demands and family-work interference will be weaker for those who use compressed work weeks than those who work a regular work day.

Hypothesis FWIH7c states that the relationship between work demands and family-work interference will be weaker for those who telework than those who work a regular work day. This hypothesis is not supported by the data. This hypothesis is tested as follows. First the four covariates (gender, job type, work demands and non-work demands) are entered into the regression equation in step 1. These covariates explain $5.3 \%$ of the variance in family-work interference. Second, the independent variable, telework, is added to the regression. This variable explains an additional $0.1 \%$ variance in family-work interference ( $p$ value .014 ) above that already explained by the covariates. The interaction term, telework by work demands is added to the regression in step three. This term did not explain any additional variance in family-work 
interference ( $p$ value .134) and the beta coefficient of the interaction is very small $(-.005)$. Since the interaction term is not significant we reject the hypothesis that the relationship between work demands and family-work interference will be weaker for those who telework than those who work a regular work day.

Hypothesis FWIH7d states that the relationship between work demands and family-work interference will be weaker for those who work a regular work schedule than those who perform shiftwork. This hypothesis is not supported by the data. This hypothesis is tested as follows. First the four covariates (gender, job type, work demands and non-work demands) are entered into the regression equation in step 1 . These covariates explain $5.7 \%$ of the variance in family-work interference. Second the independent variable, shiftwork, is added to the regression. This variable explains no additional variance in family-work interference above that already explained by the covariates. The interaction term shiftwork by work demands is added to the regression in step three. This term did not explain any additional variance in family-work interference ( $p$ value .833) and the beta coefficient of the interaction is zero $(.000)$. Since the interaction term is not significant we reject the hypothesis that the relationship between work demands and family-work interference will be weaker for those who work a regular work day than those who work shiftwork. 
Hypothesis FWIH8 looks at the ability of perceived flexibility to moderate the relationship between work demands and family-work interference. This hypothesis (i.e. that the relationship between work demands and family-work interference will decrease as perceived flexibility increases) is supported by the data. This hypothesis is tested as follows. First the four covariates (gender, job type, work demands and non-work demands) are entered into the regression equation in step 1. These covariates explain $5.7 \%$ of the variance in family-work interference. Second the independent variable, perceived flexibility, is added to the regression. This variable explains a further $2.3 \%$ of the variance in familywork interference above that already explained by the covariates (for a total $\mathrm{R}^{2}$ of $8.0 \%$ ). The interaction term perceived flexibility by work demands is added to the regression in step three. This interaction term explains a further $0.1 \%$ of the variance in family-work interference above that already explained by the covariates and the independent variable and is statistically significant ( $p$ value $<.001)$. These findings are supportive of our hypothesis that perceived flexibility moderates the impact of work demands on family-work interference such that the relationship between work demands and family-work interference will decrease as perceived flexibility increases.

Consistent with the process previously outlined, we first plotted the interaction and examined the change in $R^{2}$ from step 2 to step 3 . The results of the plotted interaction are seen in Figure 18. 
Figure 18 Interaction of Work Demands x Perceived Flexibility

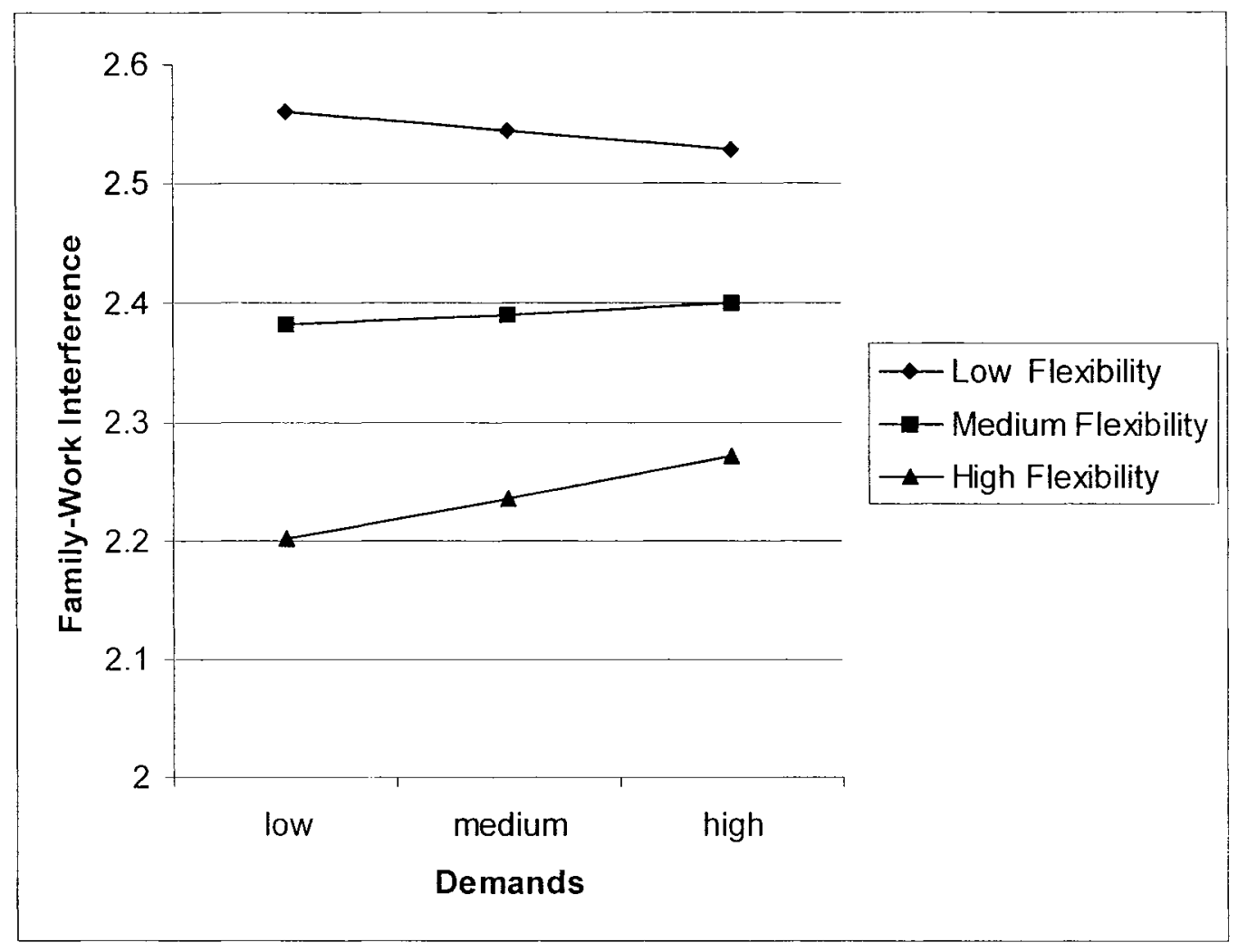

Examination of this figure indicates that the relationship between family-work interference and work demands varies slightly depending on the level of perceived flexibility reported by the individual. Specifically:

- Family-work interference is not associated with work demands at moderate levels of perceived flexibility.

- Family-work interference is positively associated with work demands when perceived flexibility is high

- Family-work interference is negatively associated with work demands when perceived flexibility is low. 
While the slopes of the lines indicate that the change is more observable at high levels of perceived flexibility than at low levels of perceived flexibility, it is also important to note that, family-work interference decreases as perceived flexibility increases, regardless of the level of work demands. These findings are consistent with the fact that the data indicate that these results, while statistically significant, are not of practical significance (i.e. the change in $R^{2}$ was $0.1 \%)$

Hypothesis FWIH9 looks at the ability of supervisor support to moderate the relationship between work demands and family-work interference. This hypothesis (i.e. that the relationship between work demands and family-work interference will decrease as supervisor support increases) is not supported by the data. This hypothesis is tested as follows. First the four covariates (gender, job type, work demands and non-work demands) are entered into the regression equation in step 1 . These covariates explain $5.7 \%$ of the variance in family-work interference. Second, the independent variable, supervisor support, is added to the regression. This variable explains a further $1.9 \%$ of the variance in familywork interference above that already explained by the covariates (for a total $R^{2}$ of $7.6 \%)$. The interaction term supervisor support by work demands is added to the regression in step three. This term did not explain any additional variance in family-work interference ( $p$ value .116) and the beta coefficient of the interaction is very small $(.002)$. Since the interaction term is not significant we reject the hypothesis that the relationship between work demands and family-work interference will decrease as supervisor support increases. 


\subsubsection{Control Over Work-Life Interface Moderates Relationship Between Non-Work Demands and Family-Work Interference}

This section presents the results of the regression analysis performed to test the ability of the various forms of control included in this analysis to moderate the relationship between non-work demands and family-work interference. The regression results are not supportive of the idea that alternative work arrangements moderate the relationship between non-work demands and familywork interference. Support for this contention is provided below.

Hypothesis FWIH10a states that the relationship between non-work demands and family-work interference will be weaker for those who use flextime arrangements than those who work a regular work day. This hypothesis is tested as follows. First the four covariates (gender, job type, work demands and nonwork demands) are entered into the regression equation in step 1. These covariates explain $5.7 \%$ of the variance in family-work interference. Second the independent variable, flextime, is added to the regression. This variable explains no additional variance in family-work interference above that already explained by the covariates. The interaction term, flextime by non-work demands, is added to the regression in step three. This term explains an additional $0.1 \%$ variance in family-work interference above that explained by the covariates and independent variable ( $p$ value .020 ) and the beta coefficient of the interaction is very small (.002). Since the interaction term is not significant we reject the hypothesis that the relationship between non-work demands and family-work interference will be 
weaker for those who use flextime arrangements than those who work a regular work day.

Hypothesis FWIH10b states that the relationship between non-work demands and family-work interference will be weaker for those who use compressed work weeks than those who work a regular work day. This hypothesis is tested as follows. First the four covariates (gender, job type, work demands and non-work demands) are entered into the regression equation in step 1 . These covariates explain $5.6 \%$ of the variance in family-work interference. Second, the independent variable, compressed work week, is added to the regression. This variable explains no additional variance in familywork interference above that already explained by the covariates. The interaction term, compressed work week by non-work demands is added to the regression in step three. This term did not explain any additional variance in family-work interference ( $p$ value .701) and the beta coefficient of the interaction is zero $(.000)$. Since the interaction term is not significant we reject the hypothesis that the relationship between non-work demands and family-work interference will be weaker for those who use compressed work weeks than those who work a regular work day.

Hypothesis FWIH10c states that the relationship between non-work demands and family-work interference will be weaker for those who telework than those who work a regular work day. This hypothesis is tested as follows. First the four covariates (gender, job type, work demands and non-work demands) are entered into the regression equation in step 1. These covariates 
explain $5.3 \%$ of the variance in family-work interference. Second, the independent variable, telework, is added to the regression. This variable explains an additional $0.1 \%$ variance in family-work interference ( $p$ value .014 ) above that already explained by the covariates. The interaction term, telework by non-work demands is added to the regression in step three. This term did not explain any additional variance in family-work interference ( $p$ value .313 ) and the beta coefficient of the interaction is very small $(-.003)$. Since the interaction term is not significant we reject the hypothesis that the relationship between non-work demands and family-work interference will be weaker for those who telework than those who work a regular work day.

Hypothesis FWIH10d states that the relationship between non-work demands and family-work interference will be weaker for those who work a regular work schedule than those who perform shiftwork. This hypothesis is tested as follows. First the four covariates (gender, job type, work demands and non-work demands) are entered into the regression equation in step 1. These covariates explain $5.7 \%$ of the variance in family-work interference. Second the independent variable, shiftwork, is added to the regression. This variable explains no additional variance in family-work interference above that already explained by the covariates. The interaction term shiftwork by non-work demands is added to the regression in step three. This term did not explain any additional variance in family-work interference ( $p$ value .067) and the beta coefficient of the interaction is very small $(-.002)$. Since the interaction term is not significant we reject the hypothesis that the relationship between non-work 
demands and family-work interference will be weaker for those who work a regular work day than those who work shiftwork.

Hypothesis FWIH11 looks at the ability of perceived flexibility to moderate the relationship between non-work demands and family-work interference. This hypothesis (i.e. that the relationship between non-work demands and family-work interference will decrease as perceived flexibility increases) is not supported by the data. This hypothesis is tested as follows. First the four covariates (gender, job type, work demands and non-work demands) are entered into the regression equation in step 1 . These covariates explain $5.7 \%$ of the variance in family-work interference. Second the independent variable, perceived flexibility, is added to the regression. This variable explains a further $2.3 \%$ of the variance in familywork interference above that already explained by the covariates (for a total $R^{2}$ of $8.0 \%$ ). The interaction term perceived flexibility by non-work demands is added to the regression in step three. This term did not explain any additional variance in family-work interference ( $p$ value .774) and the beta coefficient of the interaction is zero $(.000)$. Since the interaction term is not significant we reject the hypothesis that that perceived flexibility moderates the impact of non-work demands on family-work interference such that the relationship between nonwork demands and family-work interference will decrease as perceived flexibility increases.

Hypothesis FWIH12 looks at the ability of supervisor support to moderate the relationship between non-work demands and family-work interference. This hypothesis (i.e. that the relationship between non-work demands and family-work 
interference will decrease as supervisor support increases) is not supported by the data. This hypothesis is tested as follows. First the four covariates (gender, job type, work demands and non-work demands) are entered into the regression equation in step 1 . These covariates explain $5.7 \%$ of the variance in family-work interference. Second, the independent variable, supervisor support, is added to the regression. This variable explains a further $1.9 \%$ of the variance in familywork interference above that already explained by the covariates (for a total $R^{2}$ of 7.6\%). The interaction term supervisor support by non-work demands is added to the regression in step three. This term did not explain any additional variance in family-work interference ( $p$ value .805) and the beta coefficient of the interaction is zero $(.000)$. Since the interaction term is not significant we reject the hypothesis that the relationship between non-work demands and family-work interference will decrease as supervisor support increases.

\subsubsection{Conclusions: Family-Work Interference Hypotheses}

In conclusion, Karasek's buffer hypothesis is not supported when strain is operationalized as family-work interference, demands operationalized as work and non-work demands, and control operationalized as alternative work arrangement, perceived flexibility or supervisor support. This conclusion is based on two facts. First, eleven of the twelve buffer hypotheses were not statistically significant. Second, while a buffer hypothesis was supported under one specific circumstance (perceived flexibility by work demands), the fact that there was very little change in $R^{2}$ (i.e. only $0.1 \%$ for the perceived flexibility by work demands interaction) and plotting the interaction term showed that the 
changes in family-work interference at various levels of the moderator and independent variable were small suggests the findings had little practical significance.

\subsubsection{Evaluation of Relationships Between Work Arrangements, Supervisor Support and Perceived Flexibility}

The final model explores the relationship between the different measures of control over the work-life interface included in this thesis. This section summarizes the regression results obtained when testing the five direct hypotheses associated with Model 4. The results for each of these regressions are summarized in Table 17. The actual variables included in each of these regressions are provided in Appendix $\mathrm{C}$. Findings with respect to testing the five hypotheses posed for Model 4 are presented below.

Table 17 Direct Hypotheses Results for Model 4: Control

\begin{tabular}{|l|l|l|l|l|l|l|}
\hline $\begin{array}{l}\text { Direct Hypothesis For } \\
\text { Model 4: Perceived } \\
\text { Flexibility }\end{array}$ & $\begin{array}{l}\text { Step 1 } \\
\mathrm{R}^{2}(\mathrm{CV})\end{array}$ & $\begin{array}{l}\text { Step 2 } \\
\text { (Total } \\
\left.\mathrm{R}^{2}\right)\end{array}$ & $\begin{array}{l}\text { p-value } \\
\text { from } \\
\text { step 2 }\end{array}$ & df & F-stat & $\begin{array}{l}\Delta \text { in } \mathrm{R}^{2} \\
\text { due to } \\
\text { IV }\end{array}$ \\
\hline $\begin{array}{l}\text { PFLEXH1a: Flextime } \\
\text { positively associated } \\
\text { with perceived flexibility }\end{array}$ & $3.9 \%$ & $7.6 \%$ & $<.001$ & 5,13774 & 226.46 & $3.7 \%$ \\
\hline $\begin{array}{l}\text { PFLEX1b: CWW } \\
\text { positively associated } \\
\text { with perceived flexibility }\end{array}$ & $4.3 \%$ & $5.4 \%$ & $<.001$ & 5,12204 & 138.04 & $1.1 \%$ \\
\hline $\begin{array}{l}\text { PFLEXH1c: Telework } \\
\text { positively associated } \\
\text { with perceived flexibility }\end{array}$ & $4.1 \%$ & $5.7 \%$ & $<.001$ & 5,9950 & 120.25 & $1.6 \%$ \\
\hline $\begin{array}{l}\text { PFLEXH2: Shiftwork } \\
\text { negatively associated } \\
\text { with perceived flexibility }\end{array}$ & $3.7 \%$ & $8.7 \%$ & $<.001$ & 5,16360 & 311.85 & $5.0 \%$ \\
\hline $\begin{array}{l}\text { PFLEXH3: Supervisor } \\
\text { support positively } \\
\text { associated with } \\
\text { perceived flexibility }\end{array}$ & $3.7 \%$ & $13.8 \%$ & $<.001$ & 5,16360 & 525.91 & $10.1 \%$ \\
\hline
\end{tabular}




\subsubsection{Relationship Between Alternative Work Arrangements and Perceived Flexibility}

Three direct hypotheses postulate relationships between work arrangements and perceived flexibility.

PFLEXH1 postulates that the use of alternative work arrangements (AWA) will be positively associated with perceived flexibility. Specifically employees who work:

a. flextime (versus a regular work day)

b. compressed work week (versus a regular work day)

c. telework (versus a regular work day)

will report higher levels of perceived flexibility than their counterparts who work a regular work day.

The data supports all of these hypotheses.

To test PFLEXH1a (flextime positively associated with perceived flexibility), the four covariates (gender, job type, work demands, non-work demands) are entered into the regression in step 1. These covariates explain $3.9 \%$ of the variance in perceived flexibility. When the independent variable flextime is added into the regression equation in step 2, an additional $3.7 \%$ of the variance is explained above that already explained by the covariates (for a total $\mathrm{R}^{2}$ of $\left.7.6 \%\right)$. The $\mathrm{p}$-value $(<.001)$ indicates that this relationship is statistically significant thereby supporting the hypothesis that flextime will be positively associated with perceived flexibility.

To test PFLEXH1b (compressed work week positively associated with perceived flexibility), the four covariates (gender, job type, work demands, nonwork demands) are entered into the regression in step 1. These covariates explain $4.3 \%$ of the variance in perceived flexibility. When the independent 
variable compressed work week is added into the regression equation in step 2 , an additional $1.1 \%$ of the variance is explained above that already explained by the covariates (for a total $R^{2}$ of $5.4 \%$ ). The $p$-value $(<.001)$ indicates that this relationship is statistically significant thereby supporting the hypothesis that compressed work week will be positively associated with perceived flexibility.

To test PFLEX1c (telework positively associated with perceived flexibility), the four covariates (gender, job type, work demands, non-work demands) are entered into the regression equation in step 1. These covariates explain $4.1 \%$ of the variance in perceived flexibility. When the independent variable telework is added into the regression equation in step 2 , an additional $1.6 \%$ of the variance is explained above that already explained by the covariates (for a total $R^{2}$ of $5.7 \%)$. The $p$-value $(<.001)$ indicates that this relationship is statistically significant thereby supporting the hypothesis that telework will be positively associated with perceived flexibility.

\subsubsection{Relationship Between Shiftwork and Perceived Flexibility}

PFLEXH2 postulates that the use of shiftwork arrangements will be negatively associated with perceived flexibility. The data supports this hypothesis. To test the hypothesis, the four covariates (gender, job type, work demands, non-work demands) are entered into the regression in step 1. These covariates explain $3.7 \%$ of the variance in perceived flexibility. When the independent variable shiftwork is added into the regression equation in step 2, an additional $5.0 \%$ of the variance is explained above that already explained by the covariates (for a total $R^{2}$ of $8.7 \%$ ). The $p$-value $(<.001)$ indicates that this 
relationship is statistically significant thereby supporting the hypothesis that shiftwork will be negatively associated with perceived flexibility.

\subsubsection{Relationship Between Supervisor Support and Perceived Flexibility}

PFLEXH3 postulates that supervisor support will be positively associated with perceived flexibility. The data supports this hypothesis. To test this hypothesis, the four covariates (gender, job type, work demands and non-work demands) are entered into the regression equation in step 1. These covariates explain $3.7 \%$ of the variance in perceived flexibility. When the independent variable supervisor support is entered into the regression equation in step 2, an additional $10.1 \%$ of the variance is explained above that already explained by the covariates (for a total $R^{2}$ of $13.8 \%$ ). The p-value (<.001) indicates that this relationship is statistically significant. This finding supports the following conclusion: respondents reporting higher levels of supervisor support reported higher levels of perceived flexibility than those reporting lower levels of supervisor support thus supporting the hypothesis.

\subsubsection{Conclusions: Control Over Work-Life Interface Direct Hypotheses}

In conclusion, all five direct hypotheses are supported. All three work arrangements (i.e. flextime, compressed work week, and telework) are positively associated with perceived flexibility and employees who use any one of these three arrangements report higher levels of perceived flexibility than employees who work a regular 9 to 5 schedule. Similarly, the data show that shiftwork is 
negatively associated with perceived flexibility, which indicates that employees who perform shiftwork have lower perceived flexibility than their counterparts who work a regular 9 to 5 schedule. Finally, supervisor support is also positively associated with perceived flexibility. An evaluation of the importance of each of these forms of control to the prediction of perceived flexibility can be found in section 11.5.2.

\subsubsection{Evaluation of Supervisor Support Moderates the Relationship Between Work Arrangements and Perceived Flexibility}

This section summarizes the regression results obtained when testing the following 4 interaction hypotheses associated with Model 4:

- PFLEXH4 postulates that supervisor support will moderate the impact of alternative work arrangements on perceived flexibility. Specifically higher levels of supervisor support will strengthen the relationship between use of an alternative work arrangement and perceived flexibility for employees who work:
a. flextime (versus a regular work day)
b. compressed work week (versus a regular work day)
c. telework (versus a regular work day)

- PFLEXH5 postulates that supervisor support will moderate the impact of shiftwork on perceived flexibility such that higher levels of supervisor support will weaken the negative relationship between shiftwork and perceived flexibility.

The results for each of these regressions are summarized in Table 18.

The discussion of the findings is structured as follows. The results obtained for regressions testing if supervisor support moderates the impact of various alternative work arrangements on perceived flexibility will be presented first. This will be followed by the regression results to determine if supervisor support moderates the impact of shiftwork on perceived flexibility. 
Table 18 Interaction Hypotheses Results for Model 4: Control

\begin{tabular}{|c|c|c|c|c|c|c|c|c|}
\hline $\begin{array}{l}\text { Interaction Hypothesis for } \\
\text { Model 4: } \\
\text { Perceived Flexibility }\end{array}$ & $\begin{array}{l}\text { Step } 1 \\
R^{2} \\
(\mathrm{CV})\end{array}$ & $\begin{array}{l}\text { Step 2 } \\
R^{2} \text { (IV) }\end{array}$ & $\begin{array}{l}\Delta \text { in } R^{2} \\
\text { due to IV }\end{array}$ & $\begin{array}{l}\text { Step 3 } \\
\text { (Total } \\
R^{2} \text { ) }\end{array}$ & $\begin{array}{l}\Delta \text { in } R^{2} \\
\text { due to } \\
\text { IV } x \\
M V\end{array}$ & F-stat & $d f$ & $\begin{array}{l}\text { p-value } \\
\text { from } \\
\text { step } 3\end{array}$ \\
\hline $\begin{array}{l}\text { PFLEX4a: Supervisor } \\
\text { support moderates } \\
\text { relationship between } \\
\text { flextime and perceived } \\
\text { flexibility }\end{array}$ & $7.6 \%$ & $17.3 \%$ & $9.7 \%$ & $17.3 \%$ & $0 \%$ & 412.20 & 7,13772 & .022 \\
\hline $\begin{array}{l}\text { PFLEX4b: Supervisor } \\
\text { support moderates } \\
\text { relationship between } \\
\text { CWW and perceived } \\
\text { flexibility }\end{array}$ & $5.4 \%$ & $15.8 \%$ & $10.4 \%$ & $15.8 \%$ & $0 \%$ & 326.64 & 7,12202 & .822 \\
\hline $\begin{array}{l}\text { PFLEX4c: Supervisor } \\
\text { support moderates } \\
\text { relationship between } \\
\text { telework and perceived } \\
\text { flexibility }\end{array}$ & $5.7 \%$ & $16.3 \%$ & $10.6 \%$ & $16.3 \%$ & $0 \%$ & 275.94 & 7,9948 & .596 \\
\hline $\begin{array}{l}\text { PFLEX5: Supervisor } \\
\text { support moderates } \\
\text { relationship between } \\
\text { shiftwork and perceived } \\
\text { flexibility }\end{array}$ & $8.7 \%$ & $17.9 \%$ & $9.2 \%$ & $17.9 \%$ & $0 \%$ & 508.03 & 7,16358 & .697 \\
\hline
\end{tabular}


This section presents the results of the regression analysis performed to test the ability of supervisor support to moderate the relationship between various work arrangements and perceived flexibility. Hypothesis PFLEXH4a states that higher levels of supervisor support will strengthen the relationship between flextime and perceived flexibility. This hypothesis is not supported by the data. This hypothesis is tested as follows. First, the five covariates (gender, job type, work demands, non-work demands, flextime) are entered into the regression equation in step 1. These covariates explain $7.6 \%$ of the variance in perceived flexibility. When the independent variable supervisor support is added into the regression equation in step 2 , an additional $9.7 \%$ of the variance is explained above that already explained by the covariates for a total $R^{2}$ of $17.3 \%$ ( $p$ value $<.001)$. When the interaction term of supervisor support by flextime is added into the regression equation in step 3 , no additional variance is explained ( $p$ value was .022). Therefore, the interaction is not interpreted and we reject the hypothesis that supervisor support moderates the relationship between perceived flexibility and flextime.

Hypothesis PFLEXH4b states that higher levels of supervisor support will strengthen the relationship between compressed work week and perceived flexibility. The hypothesis is not supported by the data. This hypothesis is tested as follows. First, the five covariates (gender, job type, work demands, non-work demands, compressed work week) are entered into the regression in step 1. These covariates explain $5.4 \%$ of the variance in perceived flexibility. When the 
independent variable supervisor support is added into the regression equation in step 2 , an additional $10.4 \%$ of the variance is explained above that already explained by the covariates for a total $R^{2}$ of $15.8 \%$. ( $p$ value $<.001$ ). When the interaction term of supervisor support $x$ compressed work week is added into the regression equation, no additional variance is explained ( $p$ value was .822 ). Therefore, the interaction was not interpreted and we reject the hypothesis that supervisor support moderates the relationship perceived flexibility and compressed work week.

Hypothesis PFLEXH4c states that higher levels of supervisor support will strengthen the relationship between telework and perceived flexibility. The hypothesis is not supported by the data. This hypothesis is tested as follows. First, the five covariates (gender, job type, work demands, non-work demands, telework) are entered into the regression in step 1. These covariates explain $5.7 \%$ of the variance in perceived flexibility. When the independent variable supervisor support is added into the regression equation in step 2, an additional $10.6 \%$ of the variance is explained above that already explained by the covariates for a total $R^{2}$ of $16.3 \%$ ( $p$ value $\left.<.001\right)$. When the interaction term of supervisor support by telework is added into the regression equation, no additional variance is explained ( $p$ value .596). Therefore, the interaction is not interpreted and we reject the hypothesis that supervisor support moderates the relationship between perceived flexibility and telework.

Hypothesis PFLEXH5 states that higher levels of supervisor support will weaken the negative relationship between shiftwork and perceived flexibility. 
The hypothesis is not supported by the data. This hypothesis is tested as follows. First, the five covariates (gender, job type, work demands, non-work demands, shiftwork) are entered into the regression. These covariates explain $8.7 \%$ of the variance in perceived flexibility. When the independent variable supervisor support is added into the regression equation in step 2, an additional $9.2 \%$ of the variance is explained above that already explained by the covariates for a total $R^{2}$ of $17.9 \%$ ( $p$ value $\left.<.001\right)$. When the interaction term of supervisor support by shiftwork is added into the regression equation, no additional variance is explained ( $p$ value .697). Therefore, the interaction is not interpreted and we reject the hypothesis that supervisor support will moderate the impact of shiftwork on perceived flexibility.

\subsubsection{Conclusions: Control Over Work-Life Interface Buffer Hypotheses}

The results were not supportive for any of the four interaction hypotheses. While supervisor support has a direct effect on perceived flexibility, it did not moderate the relationship between the four work arrangements (i.e. flextime, compressed work week, telework and shiftwork) and perceived flexibility. 


\section{Discussion}

This section is structured as follows. First, we will discuss the association between the three dependent variables (i.e. role overload, work-family interference, family-work interference). Second, the relationship between the two covariates (gender and job type) will be discussed. Third, the relationship between gender/job type and the other variables of interest in this thesis will be discussed. In sections four and five the key findings with respect to thesis models 1-3 (section 4) and model 4 (section 5) will be discussed and implications of the findings presented.

\subsection{Work-Life Conflict Discussion}

This section will discuss the relationship among the three dependent variables (i.e. role overload, work-family interference and family-work interference) that comprise work-life conflict. First, it is interesting to note that the correlations between the three dependent variables are moderate to high and significant at a level of 0.001 . The fact that these three dependent variables, while conceptually distinct, are related is consistent with the literature in this area (Duxbury and Higgins, 2001; Frone, Russell and Cooper, 1992a).

The correlation between role overload and work-family interference is very high (.618) which suggests these two forms of work-life conflict may have a number of causal factors in common. A key implication of this high correlation is 
that efforts to reduce role overload may reduce work-family interference and vice versa.

The moderate correlations between role overload and family-work interference (.314) and work-family interference and family-work interference (.357) suggest that while these three variables are inter-related, family-work interference likely has a somewhat different etiology than the other two forms of strain examined in this thesis. While the differing etiologies of role overload/work-family interference and family-work interference will be discussed in more detail in sections 11.4 .3 and 11.4.4.4, two ramifications of these findings are noted here. First, these findings suggest that employers would be wise to consider a multiplicity of approaches in dealing with these three forms of work-life conflict instead of one specific approach. For researchers, these findings point to the need for additional research into the etiology of family-work interference.

Additional information on work-life conflict in Canada can be obtained by looking at the mean scores for the three dependent variables. Interestingly, respondents report higher average scores for role overload (3.58) than the other two measures of work-life conflict (mean of 2.78 for work- family interference and 2.22 for family-work interference). The literature review done for this thesis determined that there are relatively few peer-reviewed journal articles on role overload (relative to work-family interference). This is unfortunate given the fact that high levels of role overload appear to be more of a problem for Canadian employees than does role interference and that the costs associated with high levels of role overload are significant (e.g. depression, lower levels of marital and 
family satisfaction, poorer physical health). Two sets of recommendations are suggested by these findings. First, the high scores for role overload indicate there is an urgency on the part of employees, employers and society to examine ways of reducing role overload. Second, it is recommended that research be done to identify the antecedents, moderators and outcomes associated with role overload.

Also noteworthy is the fact that the mean score for work-family interference (2.78) is much higher than family-work interference (2.22). There is a good deal of empirical support for this finding. A number of studies (e.g. Eagle, Miles and Icenogle, 1997; Frone, 2000; Grandey and Cropazano, 1999) have concluded that work-family interference is more likely to take place than familywork interference.

Why should the levels of role overload and work-family interference be so much higher than family-work interference? Possible explanations can be found in the high-pressure cultures of many organizations. In many organizations, organizational downsizing has meant that the surviving employees must do the work of two or three people (leading to high levels of role overload). Furthermore, there is what Maume and Houston (2001) refer to as a "culture of overtime" in most organizations. This culture equates face time (i.e. time physically spent at work regardless of whether the time spent is productive or not) with loyalty and dedication. Maume and Houston (2001) point out that this dysfunctional practice of face time will continue as long as people are rewarded for face time with good performance evaluations, higher bonuses and 
promotions. Also, advances in technology (e.g. laptops, mobile phones) make it even easier for employees to stay connected but also make it easier to feel that the work day never really ends (Duxbury and Higgins, 2003). These pressures result in two outcomes for employees. First, employees are feeling overwhelmed and overworked by the pressures and pace of the workplace which leads to high levels of role overload. Second, employees are much more likely to focus their energies on meeting the demands of work and therefore are more likely to sacrifice family needs to meet the demands of work than vice versa (resulting in high levels of work-family interference).

\subsection{Relationship Between Gender and Job Type}

As noted earlier, job type and gender are strongly linked in the thesis sample with men being over-represented in the manager/professionals thesis sample and under-represented in the non-professionals sample relative to their female counterparts. These findings are consistent with the literature (Lindsey and Almey, 2006) and reinforce the decision to control for both gender and job type in this thesis. The large sample size allows us to look at the relationship between gender, job type and demands, control over work-life interface and strain and clear up much of the conflicting findings with respect to these issues reported in the literature.

\subsection{Relationship Between Gender, Job Type, Demands, Control Over Work-Life Interface and Strain}

This section is divided into three parts. First, we examine the relationship between gender, job type and demands (both work demands and non-work 
demands). Second, we examine the relationship between gender, job type and work-life conflict (role overload, work-family interference and family-work interference). Third, we examine the relationship between gender, job type and control over work-life interface (alternative work arrangements, perceived flexibility and supervisor support).

It is important to note that many of the studies examining gender and job type differences in work-life conflict have relatively small, nonrepresentative samples (e.g. Roxburgh, 2002). Studies examining job type differences in worklife conflict typically do not control for gender (e.g. Voydanoff and Kelly, 1984) and studies examining gender differences in work-life conflict typically do not control for job type (e.g. Frone, 2000; Hill et al., 2001). In this case, however, the large sample size (i.e. 16,366 respondents) allows us to control for gender in the analysis examining the impact of job type on demands, work-life conflict and control over work-life interface and job type in the analysis examining the impact of gender on demands, work-life conflict and control over work-life interface. As such we are able to determine key job type and gender differences in each of these constructs and clarify many of the inconsistencies in the current body of literature with respect to these relationships.

\subsubsection{Relationship Between Gender, Job Type and Demands}

The results indicate that both gender and job type are associated with work and non-work demands as follows: 
Men (50.40 hours per week) report higher levels of work demands than women (47.63), regardless of job type (i.e. interaction term not significant, main effect significant). This result is consistent with the literature (e.g. Fast and Frederick, 2004). Why should men report higher levels of work demands than women regardless of job type? Gender role theory (Barnett and Baruch, 1987; Robinson and Salamon, 1987) would suggest that because men see their primary role as being a good provider and breadwinner for their families, secure employment and advancement is more critical for men than women. As such, men should be more likely to engage in work related activities that are associated with higher job security and increased advancement such as working long hours, working paid and unpaid overtime, traveling for work and taking work home to perform in the evening. This interpretation of the data is supported by analysis done by Duxbury and Higgins (2002) who reported that the men in this sample spent more time in all of these work related activities than women, regardless of job type.

Manager/professionals (regardless of gender) report higher levels of work demands ( 50.80 hours per week) than non-professionals ( 46.86 hours per week). This result is also consistent with the literature (e.g. Shields, 1999). Why do managers and professionals regardless of gender have heavier work demands than their counterparts in non-professional positions. First, it is possible that manager/professionals work more because their jobs are more challenging and provide higher levels of intrinsic job satisfaction and personal enjoyment (Duxbury and Higgins, 2003; Van der Hulst and Geurts, 2001). Second, it may be 
that managers and professionals feel a greater obligation to work long hours because of cultural pressures on them to be available and work long hours if they want to get ahead (Lewis and Cooper, 1999; Thompson, Beauvais, and Lyness, 1999). Again, this interpretation of the data is consistent with findings reported by Duxbury and Higgins (2003) who noted that the manager/professionals in this sample were (relative to non-professionals) more likely to bring work home and travel out of town for work.

For non-work demands, the interaction of job type and gender is significant. For women, non-professionals report higher levels of non-work demands (18.85 hours per week) than manager/professionals (18.57 hours per week). For men, manager/professionals report higher levels of non-work demands (18.97 hours per week) than non-professionals (18.03 hours per week). These differences suggest that there may be shifting thinking around gender roles for manager/professionals. While non-professionals, in general, may subscribe to traditional gender roles, manager/professionals may have less entrenched views on gender roles (Falkenberg and Monachello, 1990; Rachlin, 1987). Why would there be a difference in views on gender roles? Managers/professionals are more likely to have post-secondary education (than non-professionals; Chung, 2004) and managers/professionals are more likely to be married to someone with post-secondary education (than non-professionals; Chung, 2004). It is likely that those with higher education will expect family roles (e.g. housework, child care) to be evenly shared (Rachlin, 1987). 
The finding in the literature (e.g. Anthony, 2002) that women spend more time than men in non-work demands is not supported by the data when job type is controlled for in the analysis. This is a very important finding as it casts doubt on most of the studies that report significant gender differences in non-work demands (e.g. Anthony, 2002; Hill, 2005). It also supports the need for researchers to control for both gender and job type in any analysis that looks at gender differences in work and non-work demands.

\subsubsection{Relationship Between Gender, Job Type and Work-Life Conflict}

The results of this thesis indicate that there are significant gender and job type differences in work-life conflict. Details on how gender and job type are associated with the three forms of work-life conflict examined in this thesis are discussed in detail below.

Two conclusions can be drawn from the data with respect to the relationship between gender, job type, and role overload. First, the data indicate that women (regardless of job type) report significantly higher levels of role overload (3.69) than men (3.45). This finding is consistent with the literature that notes that women report higher levels of role overload (e.g. Falkenberg and Monachello, 1990; Higgins, Duxbury and Lee, 1992; Rachlin, 1987) but somewhat surprising given the fact that there were no gender differences in work demands when job type is controlled for and the lack of a clear cut gender difference in non-work demands (i.e. depends on job type). It may be that role overload is not just a function of the amount of time an individual spends in work 
and non-work activities but is also associated with the amount of responsibility an employee has for their non-work roles. This explanation of the data is consistent with the fact that women are more likely than men to have the primary responsibility for non-work responsibilities such as child and eldercare (Anthony, 2002; Bond et al., 2003; Duxbury and Higgins, 2005).

Second, manager/professionals (regardless of gender) report higher levels of role overload (3.65) than non-professionals (3.51). This finding is consistent with the fact that, as reported earlier, managers/professionals are more likely to work longer hours and work more unpaid overtime than non-professionals. This finding also contributes to the literature in this area which has reported inconclusive findings about which job type reported higher levels of role overload when gender was not controlled for in the analysis (e.g. Roxburgh, 2002).

To draw conclusions with respect to the relationship between gender and job type and work-family interference one needs to look at both the gender and job type of the employee as the gender/job type interaction term was significant in this analysis. This analysis yields two conclusions. First, the data shows that for there are no gender differences in work-family interference for those in the manager/professional sample. Second, male non-professionals report higher levels of work-family interference (2.97) than female non-professionals (2.55).

These results help clarify the inconclusive findings in the literature exploring gender differences in work-family interference (e.g. Eagle et al., 1997). Since work-family interference depends on both gender and job type, the findings will depend upon the composition of the sample. For example, if researchers only 
looked at gender differences in work-family interference (without controlling for job type) and there are a higher number of manager/professionals in the sample, the outcome will differ (i.e. no gender differences) compared to the scenario where there are a higher number of non-professionals in the sample (i.e. gender differences).

Why do gender differences in work-family interference persist in the nonprofessional sample but not in the managerial/professional sample? First, it may be that as Duxbury and Higgins (2001) suggest organizations are more supportive of non-professional women's efforts to reduce work-family interference (relative to non-professional men) and do not penalize them when they refuse overtime and do not perform supplementary work at home. Second, according to gender role theory (Barnett and Baruch, 1987; Robinson and Salamon, 1987), female non-professionals are not expected to be the main breadwinner, while male non-professionals are held to a different standard of expectations as the familial providers and therefore male non-professionals are more susceptible to perceptions of work-family interference (than female nonprofessionals). Third, as previously mentioned in section 11.3.1, managers and professionals (regardless of gender) are expected to put work first by working longer hours. Therefore, male and female manager/professionals are likely to report similar levels of work-family interference because of these expectations and pressures from their organizations. Fourth, the expectations of work and non-work demands are beginning to converge for male and female manager/professionals. Male manager/professionals are putting more time into 
non-work demands while female manager/professionals are putting more time into work (Gerstel and Sarkisian, 2006). Therefore, this convergence of time spent on work and non-work demands could help explain the similar levels of work-family interference between male and female manager/professionals.

Finally, the fact that the gender/job type interaction term was not significant in the family-work interference analysis makes the conclusions one can draw with respect to these relationships fairly straight forward. First, the data supports the idea that manager/professionals report significantly higher levels of family-work interference (2.26) than non-professionals (2.19). Second, the data did not support differences in family-work interference between men and women. Again, these findings contribute to the research in this area as empirical studies in this area are sparse and most of the literature on the relationship between gender and job type and family-work interference is inconclusive (e.g. Roxburgh, 2002). Why would managers and professionals report higher family-work interference than non-professionals? As noted previously, the culture of hours (Lewis and Cooper, 1999) pushes manager/professional employees, regardless of their gender, to put work first (but not non-professional employees). As noted earlier in this thesis, managerial/professional employees do this by working long hours, "voluntary" overtime, and taking work home to complete in the evening. Managers/professional employees who put family before work are, therefore, acting contrary to strong organizational norms to do the reverse. It may be that in such cases, manager/professionals perceive that their family obligations are interfering with their ability to meet work obligations. 
Taken as a whole these findings have the following implications. First, researchers must control for both gender and job type when examining differences in work-life conflict. Second, the results indicate that work-life conflict affects both men and women and that organizations must not regard work-life balance as a "woman's" issue. Third, employers should consider the toll that work-life conflict is taking on managers/professionals who report higher levels of role overload, work-family interference and family-work interference. Given the resources that companies have invested in attracting and retaining, training, and motivating their human capital and given the organizational knowledge that these individuals possess, organizations need to find ways to reduce work-life conflict for all employees but especially talented managers/professionals who are more likely to be able to find meaningful work elsewhere.

Fourth, because there are gender and job type differences in work-life conflict, it is suggested that organizations cannot use a "one size fits all" approach to reducing work-life conflict. If the realities of work-life conflict are different for a male manager than a female non-professional, organizations may need to vary their approach to helping employees balance work and family and recognize that the needs of various employees and the types of solutions they need may vary by gender and job type.

\subsubsection{Relationship Between Gender/Job Type and The Use of Alternative Work Arrangements}

The results of this thesis indicate that there are significant gender and job type differences in terms of the use (and perhaps the availability) of a number of 
key alternative work arrangements (i.e. the first form of control over work-life interface). Details on how gender and job type are associated with the use of flextime, compressed work week, telework and shiftwork are discussed in detail below.

The use of flextime depends on both gender and job type. Two key differences were observed. First, managers and professionals, regardless of gender, were more likely to work flextime than their non-professional counterparts. Second, males, regardless of job type, were more likely to work flextime than females. Both findings are consistent with the limited literature on gender and job type differences in flextime use (e.g. Fast and Frederick, 1996; Golden, 2001).

The use of compressed work week depends on gender and job type. Two key differences were observed. First, non-professionals, regardless of gender, were more likely to work compressed workweeks than their manager/professional counterparts. Second, while there were no gender differences in the manager/professional sample, female non-professionals were more likely to work compressed work weeks than male non-professionals.

The differences in flextime and compressed work week point to potential concerns around who has access to these work arrangements and to the importance of fairness and equity in allocating alternative work arrangements. In fact, Duxbury and Higgins (2003) note that those employees in greatest need of flexible work arrangements (i.e. parents and those with eldercare duties) do not have access them. Duxbury and Higgins (2003) exhort employers to be more 
flexible about where and when employees work. Employers should ensure that the process for approving alternative work arrangement requests is fair and transparent and that there is mutual accountability (i.e. employees get their work done but employers are more flexible about the timing of this work). Furthermore, the process for adjusting the timing and location of work needs to be more flexible (Duxbury and Higgins, 2003).

For telework and shiftwork, the data does not support the idea that the use of either telework or shiftwork is associated with gender or job type. As for telework, the sample $(n=166)$ indicates that hardly anyone is working a formal telework arrangement on a full-time basis. People can work at home on their own time (Duxbury and Higgins, 2002) but there is a general reluctance among employers for employees to telework on the employers' time. The percentage of the sample who call themselves shiftworkers $(n=14.7 \%)$ is cause for concern given the positive association in the literature between shiftwork and work-life conflict (e.g. Burke, 1988; Shamir, 1983).

\subsubsection{Relationship Between Gender/Job Type and Control Over Work-Life Interface}

The results of this thesis indicate that there are significant gender and job type differences in these two forms of control over work-life interface (i.e perceived flexibility and supportive supervisor) examined in this thesis. Details on how gender and job type are associated with these forms of control are discussed in detail below.

To draw conclusions with respect to the relationship between gender and job type and perceived flexibility one needs to look at both the gender and job 
type of the employee as the gender/job type interaction term was significant in this analysis. This analysis yields two conclusions. First, male manager/professionals report higher levels of perceived flexibility (3.31) than male non-professionals (3.23). Second, female non-professionals report higher levels of perceived flexibility (3.17) than female manager/professionals (3.06). These findings are very interesting and lead us to ask the following questions: Why might female non-professionals report higher levels of perceived flexibility than female manager/professionals? (2) Why might male manager/professionals report higher levels of perceived flexibility than male non-professionals? Speculation with respect to both of these questions are provided below.

In response to question 1 , females are less likely than men to hold manager/professional positions. In essence, female manager/professionals can be considered a minority group (Kanter, 1993) and therefore feel their behaviour is subject to more scrutiny. Female manager/professionals feel they have to conform to the organizational norms laid out for manager/professionals (i.e. work comes first, the number of hours worked is tied to promotions) and therefore feel they must consistently demonstrate a very high level of commitment to the organization. Female manager/professionals may worry that engaging in perceived flexibility activities (e.g. varying their working hours, taking holidays when they want, taking a paid day off work when a child is sick) may signal less commitment to the organization and may adversely impact opportunities for future career advancement. Females in non-professional positions (i.e. the majority group) in the organization are less likely to feel pressured in this way 
and may be more willing to take advantage of what is offered in terms of flexibility. Furthermore, according to gender theory (Barnett and Baruch, 1987), non-professional women are seen to have primary responsibility for family and therefore can use the flexibility available from the organization without fear of career repercussions.

In response to question 2, male manager/professionals, on the other hand, may not feel the same pressure to demonstrate their commitment in the same way that female professionals do as they are in the majority and do not see taking advantage of the flexible options open to them to be a reflection of their commitment. Also these male manager/professionals are at higher levels within the organization and therefore by virtue of their position, are in a better position to vary their hours and timing of work than non-professional males.

These results differ from the literature on gender and job type differences in perceived flexibility. The literature reports managers/professionals are more likely to report perceived flexibility than non-professionals (e.g. Swanberg et al., 2005) and men are more likely to report perceived flexibility than women (e.g. Hammer et al., 1997). It should be noted that the results may differ from the literature because previous researchers have failed to control for both gender and job type in their analysis. This once again, reinforces the need for researchers to look at both gender and job type in analysis in this area.

The practical implications of the thesis results for perceived flexibility are as follows. The fact that gender and job type differences exist indicates that organizations must be mindful of equity issues around the perceptions of 
flexibility. Equity theory (Adams, 1965) suggests that those who perceive inequitable treatment can reduce their level of effort or engage in negative behaviours (e.g. leaving the organizations). Therefore, organizations must ensure that all employees have ample opportunity to enhance their perceived flexibility and that systemic organizational roadblocks to perceived flexibility (e.g. a focus on the hours one is present at work instead of one's productivity) are addressed and eliminated.

Finally, the fact that the gender/job type interaction term was not significant in the supportive manager analysis makes the conclusions one can draw with respect to these relationships fairly straight-forward. First, women (regardless of job type) report significantly higher levels of supervisor support (3.61) than men (3.54). Second, managers/professionals (regardless of gender) report higher levels of supervisor support (3.61) than non-professionals (3.56). The thesis finding that women report higher levels of supervisor support is consistent with the literature on social support (e.g. Lazarus and Folkman, 1984). The job type difference in supervisor support suggests that senior managers within organizations are more supportive than those who manage front line employees. It is difficult to determine why this is the case from these data but one can speculate that the findings could be due to the fact that micromanagement of employees decreases and trust of employees increases as one goes up the hierarchy. Alternatively, it may be that the relationship between managers and unionized employees is more adversarial than that found between non-unionized employees. 
A search for peer-reviewed literature exploring gender and job type differences in supervisor support did not turn up any research in this area. This study offers us some insight into this relationship and supports the need for further research in this area. Furthermore, given the critical role of supervisor support in reducing work-life conflict (e.g. Anderson et al., 2002), employers should examine ways to increase supervisor support for all their employees. Please note that enhancing supervisor support in organizations is discussed in greater detail in section 11.5.2.

As well, employers need to recognize that if certain groups of employees report lower levels of supervisor support (i.e. non-professionals), then employers need to focus on understanding the best practices associated with supervising non-professionals and train their supervisors on how to be more effective at supporting this group.

In conclusion, the results of this thesis present a picture of differing realities for men and women, managers/professionals and non-professionals. While men report higher levels of work demands than women, it is women who report higher levels of role overload. This suggests that researchers need to go beyond just work demands and examine other antecedents for role overload (e.g. number of hours spent on non-work demands, primary responsibility for non-work demands, the physical and psychological level of non-work demands). Manager/professionals report higher levels of work demands and all three forms of work-life conflict yet have higher levels of supervisor support than non- 
professionals. This suggests there are limits to what supervisor support can do to assist with work-life conflict if work demands are too high.

\subsection{Discussion of Relationships Between Models 1-3}

This section will discuss the implications of the findings associated with research questions 1-4. To guide the reader the results of the strain and buffer hypotheses for models $1-3$ are provided in Tables 19 and 20.

\section{Table 19 Summary of Strain Hypotheses Tested for Models $1-3^{10}$}

\begin{tabular}{|l|l|l|l|}
\hline Hypothesis & RO & WFI & FWI \\
\hline $\begin{array}{l}\text { H1 WD positively associated } \\
\text { with WLC }\end{array}$ & Supported & Supported & $\begin{array}{l}\text { Supported but } \\
\text { not substantive }\end{array}$ \\
\hline $\begin{array}{l}\text { H2 NWD positively associated } \\
\text { with WLC }\end{array}$ & Supported & Supported & Supported \\
\hline $\begin{array}{l}\text { H3a Flextime negatively } \\
\text { associated with WLC }\end{array}$ & $\begin{array}{l}\text { Not } \\
\text { Supported }\end{array}$ & Not Supported & Not Supported \\
\hline $\begin{array}{l}\text { H3b Compressed work week } \\
\text { negatively associated with } \\
\text { WLC }\end{array}$ & $\begin{array}{l}\text { Not } \\
\text { Supported }\end{array}$ & Not Supported & Not Supported \\
\hline $\begin{array}{l}\text { H3c Telework negatively } \\
\text { associated with WLC }\end{array}$ & $\begin{array}{l}\text { Not } \\
\text { Supported }\end{array}$ & Not Supported & Not Supported \\
\hline $\begin{array}{l}\text { H4 Shiftwork positively } \\
\text { associated with WLC }\end{array}$ & $\begin{array}{l}\text { Not } \\
\text { Supported }\end{array}$ & $\begin{array}{l}\text { Supported but } \\
\text { not } \\
\text { substantive }\end{array}$ & Not Supported \\
\hline $\begin{array}{l}\text { H5 Perceived flexibility } \\
\text { negatively associated with } \\
\text { WLC }\end{array}$ & Supported & Supported & Supported \\
\hline $\begin{array}{l}\text { H6 Supervisor support } \\
\text { negatively associated with } \\
\text { WLC }\end{array}$ & Supported & Supported & Supported \\
\hline
\end{tabular}

10 Please note that supported findings are both statistically and practically significant; supported but not substantive are statistically but not practically significant; not supported are neither statiscally nor practically significant 
Table 20 Summary of Buffer Hypotheses Tested for Models 1-3

\begin{tabular}{|c|c|c|c|}
\hline Hypothesis & RO & WFI & FWI \\
\hline $\begin{array}{l}\text { H7a Flextime moderates } \\
\text { relationship between work } \\
\text { demands and WLC }\end{array}$ & $\begin{array}{l}\text { Not } \\
\text { Supported }\end{array}$ & $\begin{array}{l}\text { Not } \\
\text { Supported }\end{array}$ & Not Supported \\
\hline $\begin{array}{l}\text { H7b Compressed work week } \\
\text { moderates relationship between } \\
\text { work demands and WLC }\end{array}$ & $\begin{array}{l}\text { Not } \\
\text { Supported }\end{array}$ & $\begin{array}{l}\text { Not } \\
\text { Supported }\end{array}$ & Not Supported \\
\hline $\begin{array}{l}\text { H7c Telework moderates } \\
\text { relationship between work } \\
\text { demands and WLC }\end{array}$ & $\begin{array}{l}\text { Not } \\
\text { Supported }\end{array}$ & $\begin{array}{l}\text { Not } \\
\text { Supported }\end{array}$ & Not Supported \\
\hline $\begin{array}{l}\text { H7d Shiftwork moderates } \\
\text { relationship between work } \\
\text { demands and WLC }\end{array}$ & $\begin{array}{l}\text { Supported } \\
\text { but not } \\
\text { substantive }\end{array}$ & $\begin{array}{l}\text { Supported } \\
\text { but not } \\
\text { substantive }\end{array}$ & Not Supported \\
\hline $\begin{array}{l}\text { H8 Perceived flexibility } \\
\text { moderates relationship between } \\
\text { work demands and WLC }\end{array}$ & $\begin{array}{l}\text { Supported } \\
\text { but not } \\
\text { substantive }\end{array}$ & $\begin{array}{l}\text { Not } \\
\text { Supported }\end{array}$ & $\begin{array}{l}\text { Supported but } \\
\text { not substantive }\end{array}$ \\
\hline $\begin{array}{l}\text { H9 Supervisor support } \\
\text { moderates relationship between } \\
\text { work demands and WLC }\end{array}$ & $\begin{array}{l}\text { Not } \\
\text { Supported }\end{array}$ & $\begin{array}{l}\text { Supported } \\
\text { but not } \\
\text { substantive }\end{array}$ & Not Supported \\
\hline $\begin{array}{l}\text { H10a Flextime moderates } \\
\text { relationship between non-work } \\
\text { demands and WLC }\end{array}$ & $\begin{array}{l}\text { Not } \\
\text { Supported }\end{array}$ & $\begin{array}{l}\text { Not } \\
\text { Supported }\end{array}$ & Not Supported \\
\hline $\begin{array}{l}\text { H10b Compressed work week } \\
\text { moderates relationship between } \\
\text { non-work demands and WLC }\end{array}$ & $\begin{array}{l}\text { Not } \\
\text { Supported }\end{array}$ & $\begin{array}{l}\text { Not } \\
\text { Supported }\end{array}$ & Not Supported \\
\hline $\begin{array}{l}\text { H10c Telework moderates } \\
\text { relationship between non-work } \\
\text { demands and WLC }\end{array}$ & $\begin{array}{l}\text { Not } \\
\text { Supported }\end{array}$ & $\begin{array}{l}\text { Not } \\
\text { Supported }\end{array}$ & Not Supported \\
\hline $\begin{array}{l}\text { H10d Shiftwork moderates } \\
\text { relationship between non-work } \\
\text { demands and WLC }\end{array}$ & $\begin{array}{l}\text { Not } \\
\text { Supported }\end{array}$ & $\begin{array}{l}\text { Not } \\
\text { Supported }\end{array}$ & Not Supported \\
\hline $\begin{array}{l}\text { H11 Perceived flexibility } \\
\text { moderates relationship between } \\
\text { non-work demands and WLC }\end{array}$ & $\begin{array}{l}\text { Not } \\
\text { Supported }\end{array}$ & $\begin{array}{l}\text { Not } \\
\text { Supported }\end{array}$ & Not Supported \\
\hline $\begin{array}{l}\text { H12 Supervisor support } \\
\text { moderates relationship between } \\
\text { non-work demands and WLC }\end{array}$ & $\begin{array}{l}\text { Supported } \\
\text { but not } \\
\text { substantive }\end{array}$ & $\begin{array}{l}\text { Not } \\
\text { Supported }\end{array}$ & Not Supported \\
\hline
\end{tabular}




\subsubsection{Relationship Between Work Demands and WLC}

Research Question 1a) asked: What is the relationship between work demands and work-life conflict (role overload, work-family interference, familywork interference)?

The data analysis is strongly supportive of a positive association between work demands and two forms of work-life conflict (i.e. role overload and workfamily interference) examined in this thesis. These findings are consistent with the literature on work demands and role overload (e.g.; Fu and Shaffer, 2001; Galambos and Walters, 1992; Voydanoff, 2004; Wallace, 1997) and work demands and work-family interference (e.g. Fenwick and Tausig, 2001; Galinsky, Bond and Hill, 2004; Gutek et al., 1991). They also provide partial support for Karasek's strain hypothesis that higher demands (operationalized as time spent per week in work-related activities) are associated with higher levels of strain.

It is important to recognize that work demands are not equally effective at predicting the three forms of work-life conflict considered in this analysis (controlling for gender and job type). From the data we can conclude that work demands are strongly associated with work-family interference (explaining 6.7\% of the variance in this form of work-life conflict). While work demands are also an important predictor of role overload (explaining 3.1\% of the variance in this form of work-life conflict) they are not a substantive predictor of family-work interference (predicting only $0.1 \%$ of the variance in this form of work-life conflict). This last finding is contradictory to the limited empirical literature 
(Boyar et al., 2003; Greenhaus and Beutell, 1985; Van der Hulst and Geurts, 2001) which has found a positive association between work demands and familywork interference. The discrepancy between the thesis findings and the literature may be attributed to the relatively small sample size of the studies in the literature and the fact that gender and job type were not controlled in these analyses. To avoid redundancy, the implications of the findings associated with work demands and work-life conflict will be discussed in section 11.4.3.

\subsubsection{Relationship Between Non-Work Demands and WLC}

Research Question 1b) asked: What is the relationship between non-work demands and work-life conflict (role overload, work-family interference, familywork interference)? The data analysis is supportive of positive associations between non-work demands and all three forms of work-life conflict examined in this thesis. These findings are also consistent with other literature on non-work demands and work-life conflict (e.g. Fenwick and Tausig, 2001; Fu and Shaffer, 2001; Galambos and Walters, 1992; Galinsky, Bond and Hill, 2004; Gutek et al., 1991; Voydanoff, 1988, 2004) and support Karasek's strain hypothesis that higher demands (operationalized as time spent per week in nonwork-related activities) are associated with higher levels of strain.

It is important to recognize that non-work demands are not equally effective at predicting the three forms of work-life conflict considered in this analysis (controlling for gender and job type). From the data we can conclude that non-work demands are strongly associated with family-work interference 
(explaining $5.5 \%$ of the variance in this form of work-life conflict). Non-work demands are also a predictor of both role overload (explaining $2.1 \%$ of the variance in this form of work-life conflict) and work-family interference (explaining $1.3 \%$ of the variance in family-work interference). These findings are consistent with the literature (e.g. Frone et al., 1997b) that indicates that work-family interference and family-work interference have different antecedents. Both the literature in this area and the findings from this thesis are supportive of the idea that non-work demands are an antecedent of family-work interference whereas work demands are an antecedent of work-family interference

\subsubsection{Demands Predicting Variance in Work-Life Conflict}

In response to the question of which predictor (i.e. work demands or nonwork demands) predicts more variance in the dependent variables, the answer depends on which model you examine. Key conclusions are presented below. First, the data support the idea that work demands are a better predictor of role overload than non-work demands (i.e. model 1). This conclusion is supported by the following data. First, work demands predict slightly more variance in role overload (change in $R^{2}$ of $3.1 \%$ ) than non-work demands (change in $R^{2}$ of $2.1 \%$ ). Second, the Pratt's standardized measure for work demands (.586964) was one and a half times higher than non-work demands (.413036). Theoretically it is possible that both types of demands would have the potential to leave an employee fatigued and exhausted at the end of the day. However, given the fact that the thesis data reports higher average levels of work demands ( 48.85 hours per week) than non-work demands (18.66 hours per week), it is possible that this 
is why work demands explains more variance in role overload than non-work demands. Alternately, we may wish to consider how the construct of demands was operationalized. Both work demands and non-work demands were operationalized by the number of hours respondents spent per week in domain specific activities. There are other ways of operationalizing demands (e.g. physical demands, psychological demands, and level of responsibility). Perhaps a different operationalization of demands would help us understand which form of demands (i.e. work or non-work) contributes to the prediction of role overload.

Second, the data support the idea that work demands are a better predictor of work-family interference than non-work demands (i.e. model 2). This conclusion is supported by the following data. First, work demands predict much more variance in work-family interference (change in $\mathrm{R}^{2}$ of $6.7 \%$ ) than non-work demands (change in $R^{2}$ of $1.3 \%$ ). Second, the Pratt's coefficient for work demands $(.860357)$ is six times higher than non-work demands (.139643).

Third, the data support the idea that non-work demands are a better predictor of family-work interference than work demands (i.e. model 3). Again the following data is provided to support this conclusion. First, non-work demands predict much more variance in family-work interference (change in $\mathrm{R}^{2}$ of $5.5 \%$ ) than work demands (change in $R^{2}$ of $0.1 \%$ ). Second, the Pratt's coefficient for non-work demands (.984842) was sixty-five and a half times higher than the Pratt's coefficient for work demands (.015158).

These findings are very interesting as they support the idea that role overload and work-family interference have etiologies that are similar to each 
other but quite different from that demonstrated by family-work interference. These findings result in the following implications for employers. Given that role overload and work-family interference have similar etiologies, efforts to reduce work demands are likely to result in lower levels of both role overload and workfamily interference but have little impact on family-work interference. Second, it would suggest that employers who wish to reduce family-work interference need to look at solutions other than reducing work demands and attempt to make it easier for employees to efficiently deal with non-work responsibilities. While it is acknowledged that the scope of what employers can do to reduce non-work demands is going to be limited (relative to what they can do to reduce work demands), interested employers may wish to explore the possibilities of offering on-site daycare, employee assistance programs and various concierge services.

These findings also have important implications for researchers. Relative to role overload and work-family interference, much less is known about the antecedents of family-work interference. Therefore, future research efforts should involve researching possible antecedents of family-work interference.

\subsubsection{Relationship Between Control over Work-Life Interface and Strain}

Research question 2) asks: What is the relationship between the various conceptualizations of control over work-life interface (i.e. flextime, compressed work week, telework, shiftwork, perceived flexibility, and supervisor support) examined in this thesis and work-life conflict (role overload, work-family interference, family-work interference)? 
In this case, we tested the strain hypothesis by examining the direct effect of a given alternative work arrangement, perceived flexibility or supervisor support on role overload, work-family interference or family-work interference. The results of the relationship between each form of control and work-life conflict will be discussed in turn.

\subsubsection{Relationship Between Alternative Work Arrangements and Work-Life Conflict}

This section will discuss the results of the strain hypotheses associated with the three alternative work arrangements (e.g. flextime, compressed work week and telework) and work-life conflict. The results are not supportive of any of the nine hypotheses (i.e. flextime, compressed work week, telework each negatively associated with role overload, work-family interference and familywork interference). These findings can be interpreted two ways. First, it may be that these findings refute Karasek's strain hypothesis that higher control is associated with lower levels of strain. Alternatively, it may be that alternative work arrangements, on their own, do not give employees any control over the work-life interface. The fact that both perceived flexibility and supportive managers were significantly associated with all three forms of work-life conflict (i.e. supportive of the strain hypothesis) it would seem that the second explanation of the data is more likely than the first.

Whereas the findings from this thesis are unequivocal (i.e. alternative work arrangements are not associated with lower levels of work-life conflict), the results reported in the literature have been rather inconsistent. While some 
researchers have reported a negative relationship between a given alternative work arrangement such as flextime and work-life conflict (e.g. Bond et al., 2003) others have not found a significant relationship (e.g. Secret and Sprang, 2001) or have reported a positive relationship between the alternative work arrangements and work-life conflict (e.g. Dunham et al., 2001). It is possible that the difference between the literature and the thesis findings can be attributed to studies using small sample size (e.g. Dunham et al., 2001) and not controlling for certain covariates such as gender or demands (e.g. Clark, 2001). It is important to highlight the fact that the findings from this thesis come from a large, representative sample and that covariates such as gender, demands and job type are controlled in the analysis. This increases our confidence in the findings that in fact, the use of alternative work arrangements are not associated with lower levels of work-life conflict.

The results for the shiftwork and work-life conflict hypotheses also indicate a lack of support for Karasek's strain hypothesis. Two of the three hypotheses (shiftwork positively associated with role overload; shiftwork positively associated with family-work interference) are not statistically significant. The third hypothesis (shiftwork positively associated with work-family interference) is statistically but not practically significant. These findings are different than the literature on shiftwork and work-life conflict where the majority of studies (e.g. Shamir, 1983) do report a positive relationship between shiftwork and work-life conflict. Again, when one examines our sample size and the fact that we control 
for covariates such as demands, gender and job type, this could potentially explain the difference between our results and the findings in the literature.

The fact that so few strain hypotheses are supported for the four work arrangements would imply that it takes more than a change in schedule or location to affect the three forms of work-life conflict explored in this thesis. Organizations should be aware that merely offering an alternative work arrangement may not be sufficient and that other changes can and should be offered. A review of the strain hypotheses for perceived flexibility and supervisor support will provide some guidance on what organizations can offer their employees that might be more helpful in promoting work-life balance.

\subsubsection{Relationship Between Perceived Flexibility and Work-Life Conflict}

Relative to alternative work arrangements/shiftwork, the results for perceived flexibility are much more encouraging. The data analysis was strongly supportive of a negative association between perceived flexibility and all three forms of work-life conflict examined in this thesis. The results therefore support Karasek's strain hypothesis that higher levels of control are associated with lower levels of strain when control is operationalized as perceived flexibility. These findings are consistent with other literature that support a negative association between perceived flexibility and work-life conflict (e.g. Anderson et al., 2002; Thomas and Ganster, 1995; Voydanoff, 1988).

It is important to recognize that perceived flexibility is not equally effective at predicting the three forms of work-life conflict considered in this analysis (controlling for gender, job type, work demands and non-work demands). From 
the data we can conclude that perceived flexibility explained more variance in work-family interference and had the biggest impact on this relationship (relative to the impact of perceived flexibility on either role overload or family-work interference). Perceived flexibility is negatively and strongly associated with work-family interference (explaining $14.2 \%$ of the variance in this form of work-life conflict). Perceived flexibility is also an important predictor of role overload (explaining $9.4 \%$ of the variance in this form of work-life conflict). As well, perceived flexibility is a substantive predictor of family-work interference (predicting $2.3 \%$ of the variance in this form of work-life conflict). The implications of these findings are discussed in section 11.4.4.

\subsubsection{Relationship Between Supervisor Support and Work-Life Conflict}

The data analysis is strongly supportive of a negative association between supervisor support and all three forms of work-life conflict examined in this thesis. The results therefore support Karasek's strain hypothesis that higher levels of control are associated with lower levels of strain when control is operationalized as supervisor support. These findings are consistent with other literature that support a negative association between supervisor support and work-life conflict (e.g. Anderson et al., 2002; Bond et al., 2003; Thomas and Ganster, 1995).

It is important to recognize that supervisor support is not equally effective at predicting the three forms of work-life conflict considered in this analysis (controlling for gender, job type, work demands and non-work demands). From the data we can conclude that supervisor support explained more variance in 
work-family interference and had the biggest impact on this relationship (relative to the impact of supervisor support on either role overload or family-work interference). Supervisor support is negatively and strongly associated with work-family interference (explaining $8.4 \%$ of the variance in this form of work-life conflict). Supervisor support is also an important predictor of role overload (explaining $5.5 \%$ of the variance in this form of work-life conflict). As well, supervisor support is a substantive predictor of family-work interference (predicting $1.9 \%$ of the variance in this form of work-life conflict). The implications of these findings are discussed in section 11.5.

The data indicates that supervisor support is less likely to predict familywork interference (compared with the other two forms of work-life interference). Therefore, employers may need to examine other interventions designed to help employees reduce family-work interference. Such a discussion is featured in the next section (11.4.4.4).

\subsubsection{Relative Impact of Predictors and Work-Life Conflict}

Which variable is the best predictor of each of the three forms of work-life conflict included in this thesis? Three additional regressions were performed to answer this question as the significant correlation between perceived flexibility and supervisor support (.325) indicates a potential confound between these two variables. One regression equation was run for each of the three forms of worklife conflict (i.e. role overload, work-family interference, family-work interference). The relative importance of the predictor variables (i.e. demands, control over 
work-life interface) is obtained by calculating Pratt's coefficient and Pratt's standardized measure (Thomas et al., 1998). Pratt's coefficient is calculated by multiplying the standardized regression coefficient of the predictor variable by the correlation of the predictor variable and the dependent variable. Pratt's standardized measure is calculated by dividing Pratt's coefficient by the total variance explained. All standardized Pratt measures sum to 1 (Thomas et al., 1998). Since the correlations between the predictor variables and the dependent variable and standardized regression coefficients of the predictor variables are not affected by the order in which the predictor variables are entered into the regression, all predictor variables are entered into the regression equation at once (i.e. standard multiple regression, Tabachnick and Fidell, 2001). The results can be seen in Tables 21 (role overload), 22 (work-family interference) and 23 (family-work interference). 
Table 21 Pratt's Standardized Measure for Predicting Role Overload

\begin{tabular}{|l|l|l|l|l|}
\hline Predictor & $\begin{array}{l}\text { Std. } \\
\text { Coefficient } \\
\text { of predictor }\end{array}$ & $\begin{array}{l}\text { Correlation } \\
\text { of predictor } \\
\text { and DV }\end{array}$ & $\begin{array}{l}\text { Pratt's } \\
\text { Coefficient }\end{array}$ & $\begin{array}{l}\text { Pratt's } \\
\text { Standard } \\
\text { Measure }\end{array}$ \\
\hline $\begin{array}{l}\text { Work } \\
\text { Demands }\end{array}$ & .132 & .171 & .022572 & .126374 \\
\hline $\begin{array}{l}\text { Non-work } \\
\text { demands }\end{array}$ & .108 & .150 & .016200 & .090699 \\
\hline Flextime & .040 & -.005 & -.000200 & -.001120 \\
\hline $\begin{array}{l}\text { Compressed } \\
\text { Work Week }\end{array}$ & .043 & .013 & .000559 & .003130 \\
\hline Telework & .028 & .011 & .000308 & .001724 \\
\hline Shiftwork & -.075 & -.004 & .000300 & .001680 \\
\hline $\begin{array}{l}\text { Perceived } \\
\text { Flexibility }\end{array}$ & -.291 & -.353 & .102723 & .575118 \\
\hline $\begin{array}{l}\text { Supervisor } \\
\text { Support }\end{array}$ & -.150 & -.241 & .036150 & .202394 \\
\hline $\begin{array}{l}\text { Variance } \\
\text { Explained }\end{array}$ & - & - & .178612 & 1 \\
\hline
\end{tabular}

Table 22 Pratt's Standardized Measure for Predicting Work-Family Interference

\begin{tabular}{|l|l|l|l|l|}
\hline Predictor & $\begin{array}{l}\text { Std. } \\
\text { Coefficient } \\
\text { of predictor }\end{array}$ & $\begin{array}{l}\text { Correlation } \\
\text { of predictor } \\
\text { and DV }\end{array}$ & $\begin{array}{l}\text { Pratt's } \\
\text { Coefficient }\end{array}$ & $\begin{array}{l}\text { Pratt's } \\
\text { Standardized } \\
\text { Measure }\end{array}$ \\
\hline $\begin{array}{l}\text { Work } \\
\text { Demands }\end{array}$ & .201 & .293 & .058893 & .218610 \\
\hline $\begin{array}{l}\text { Non-work } \\
\text { demands }\end{array}$ & .067 & .115 & .007705 & .028601 \\
\hline Flextime & .076 & .045 & .003420 & .012695 \\
\hline $\begin{array}{l}\text { Compressed } \\
\text { Work Week }\end{array}$ & .026 & -.029 & -.000750 & -.002800 \\
\hline Telework & .043 & .025 & .001075 & .003990 \\
\hline Shiftwork & .001 & .090 & .000090 & .000334 \\
\hline $\begin{array}{l}\text { Perceived } \\
\text { Flexibility }\end{array}$ & -.340 & -.418 & .142120 & .527549 \\
\hline $\begin{array}{l}\text { Supervisor } \\
\text { Support }\end{array}$ & -.187 & -.304 & .056848 & .211019 \\
\hline $\begin{array}{l}\text { Variance } \\
\text { Explained }\end{array}$ & - & - & .269397 & 1 \\
\hline
\end{tabular}


Table 23 Pratt's Coefficients for Predicting Family-Work Interference

\begin{tabular}{|l|l|l|l|l|}
\hline Predictor & $\begin{array}{l}\text { Std. } \\
\text { Coefficient } \\
\text { of } \\
\text { predictor }\end{array}$ & $\begin{array}{l}\text { Correlation } \\
\text { of predictor } \\
\text { and DV }\end{array}$ & $\begin{array}{l}\text { Pratt's } \\
\text { Coefficient }\end{array}$ & $\begin{array}{l}\text { Pratt's } \\
\text { Standardized } \\
\text { Measure }\end{array}$ \\
\hline $\begin{array}{l}\text { Work } \\
\text { Demands }\end{array}$ & -.003 & .034 & -.000100 & -.001090 \\
\hline $\begin{array}{l}\text { Non-work } \\
\text { demands }\end{array}$ & .215 & .235 & .050525 & .541800 \\
\hline Flextime & .052 & .022 & .001144 & .012268 \\
\hline $\begin{array}{l}\text { Compressed } \\
\text { Work Week }\end{array}$ & .021 & .004 & .000084 & .000901 \\
\hline Telework & .033 & .023 & .000759 & .008139 \\
\hline Shiftwork & -.046 & -.013 & .000598 & .006413 \\
\hline $\begin{array}{l}\text { Perceived } \\
\text { Flexibility }\end{array}$ & -.146 & -.176 & .025696 & .275549 \\
\hline $\begin{array}{l}\text { Supervisor } \\
\text { Support }\end{array}$ & -.097 & -.150 & .014550 & .156025 \\
\hline $\begin{array}{l}\text { Variance } \\
\text { Explained }\end{array}$ & - & - & .093254 & 1 \\
\hline
\end{tabular}

For role overload, there were four statistically significant and substantive predictors (all statistically significant at $<.001$ ). Perceived flexibility had the highest magnitude (Pratt's standardized measure of .575118) of variable importance (relative to all other independent variables in the model). The second most important predictor of variance in role overload was supervisor support with the second highest magnitude of variable importance (Pratt's standardized measure of .202394). The third most important predictor of variance in role overload was work demands with the third highest magnitude of variable importance (Pratt's standardized measure of .126374). The fourth most important predictor of variance in role overload was non-work demands with the fourth highest magnitude of variable importance (Pratt's standardized measure of $.090699)$. 
For work-family interference, there were four statistically significant and substantive predictors (all statistically significant at $<.001$ ). Perceived flexibility had the highest magnitude (Pratt's standardized measure of .527549) of variable importance (relative to all other independent variables in the model). The second most important predictor of variance in work-family interference was work demands with the second highest magnitude of variable importance (Pratt's standardized measure of .21861). The third most important predictor of variance in work-family interference was supervisor support with the third highest magnitude of variable importance (Pratt's standardized measure of .211019). The fourth most important predictor of variance in work-family interference was non-work demands with the fourth highest magnitude of variable importance (Pratt's standardized measure of .028601).

For family-work interference, there were three highly significant predictors (all statistically significant at $<.001$ ). Non-work demands had the highest magnitude (Pratt's standardized measure of .5418) of variable importance (relative to all other independent variables in the model) for family-work interference. The second most important predictor of variance in family-work interference was perceived flexibility with the second highest magnitude of variable importance (Pratt's standardized measure of .275549). The third most important predictor of variance in family-work interference was supervisor support with the third highest magnitude of variable importance (Pratt's standardized measure of .156025). 
The results of Tables 21 and 22 indicate that the most important predictor for both role overload and work-family interference is perceived flexibility (Pratt's standardized measure of .575118 and .527549 respectively). The second and third most important predictors for role overload was supervisor support (Pratt's standardized measure of .202394) and work demands (Pratt's standardized measure of .126374) while the second and third most important predictors for work-family interference were work demands (Pratt's standardized measure of .21861) and supervisor support (Pratt's standardized measure of .211019). The implications of these results are as follows. First, these results indicate that role overload and work-family interference have similar etiologies (as noted previously). This finding supports our earlier contention that efforts made to reduce role overload will also help reduce work-family interference. Second, in order to reduce both role overload and work-family interference, employers must emphasize enhancing perceptions of perceived flexibility among their employees. Third, employers would do well to examine the level of work demands and examine ways to reduce their employee's workloads. In essence, what is recommended is a two-pronged approach of enhancing flexibility while reducing work demands in order to reduce role overload and work-family interference.

The results for family-work interference were different than the other two forms of work-life conflict. Non-work demands explained the most variance in family-work interference than the other predictors (Pratt's standardized measure of .5418). The second most important predictor for family-work interference is 
perceived flexibility (Pratt's standardized measure of .275549 ) followed by supervisor support (Pratt's standardized measure of .156025). The implications of the results are as follows. First, family-work interference has a different etiology than either role overload and work-family interference. Employers who wish to help employees reduce family-work interference, should focus on determining how they can reducing non-work demands or help employees meet non-work demands more efficiently. This is much more difficult for employers because traditionally employers have left employees on their own to handle their non-work demands. However, recent employer interventions such as on-site daycare, concierge services and employee assistance programs have the potential to be helpful in reducing non-work demands.

Second, perceived flexibility was a significant and substantive predictor of all three forms of work-life conflict. This indicates that employers who focus their efforts on enhancing perceived flexibility. Examining the items that comprise our perceived flexibility construct can help offer concrete suggestions for enhancing perceived flexibility. It is recommended that employers allow employees:

- To vary their working hours (i.e. arrival and departure times)

- To spend some of their regular work day working at home

- To interrupt their work day for personal/family reasons and then return

- To take a paid day off work to care for a sick child or help an elderly relative

- To arrange their work schedule (i.e. shifts, overtime) to meet family/personal commitments.

As suggested in Duxbury and Higgins (2001), employers should promote enhanced flexibility by asking employees to identify what types of flexibility would be most helpful, communicate effectively with employees about what programs 
and policies are available and communicate best practices within the organization as well those practices of other organizations. Key to enhancing support for perceived flexibility is the notion that "employees must be made to feel that their career will not be jeopardized if they take advantage of supportive policies" (Duxbury and Higgins, 2001, p. 66). Enhancing perceived flexibility should help employers lower the level of work-life conflict.

Third, relative to the amount of variance explained in model 1 (role overload) or model 2 (work-family interference), less variance was explained in model 3 (family-work interference). Therefore, one concludes that Karasek's model is not as effective at explaining the relationship between demands, control and this form of strain (i.e. family-work interference) as was observed with either role overload or work-family-interference. The implication for researchers is that they should continue to pursue other theoretical models which may better explain the etiology of family-work interference. Duxbury and Higgins (2001) have identified primary responsibility for childcare as one of these antecedents. Respondents reporting high levels of family-work interference are more likely to have primary responsibility for childcare. The authors note, "these findings suggest that it is difficult to put work ahead of family if one does not have the support at home" (Duxbury and Higgins, 2001, p. 54).

\subsubsection{Buffering the Relationship Between Demands and Strain}

Consistent with Karasek's Demand-Control model, research questions 3 and 4 test whether or not control over the work-life interface can buffer 
(moderate) the relationship between demands and strain. Research Question 3 asks: To what extent do the forms of control examined in this thesis (i.e. flextime, compressed work week, telework, shiftwork, perceived flexibility, and supervisor support) moderate the relationship between work demands and work-life conflict (i.e. role overload, work-family interference, family-work interference)? Research Question 4 asks: To what extent do the forms of control examined in this thesis (i.e. flextime, compressed work week, telework, shiftwork, perceived flexibility, and supervisor support) moderate the relationship between non-work demands and work-life conflict (i.e. role overload, work-family interference, family-work interference)?

The data analysis produced unequivocal results. There is no support for Karasek's buffering hypothesis that control moderates the relationship between demands and strain. Thirteen of the eighteen buffer hypotheses are not statistically significant. The remaining five hypotheses are not substantive.

How do these results compare to the existing literature on various aspects of control over work-life interface buffering the relationship between demands and work-life conflict? First, it is important to note that no literature examining the impact of alternative work arrangements or shiftwork on the relationship between demands and work-life conflict could be identified in the literature. As such, this study adds to the extant literature in this area.

Second, the literature examining the impact of perceived flexibility on the relationship between demands and work-life conflict is inconclusive. While an early study by Staines and Pleck (1983) reported perceived flexibility had a 
buffering effect on the relationship between excessive work hours and workfamily interference, later efforts (e.g. Smith Major et al., 2002; Thomas and Ganster, 1995) reported no such buffering effect. The fact that Staines and Pleck (1983) did not control for gender, job type and demands may explain the difference in their findings and the findings in this thesis. The size of our sample and the controlling for covariates adds to the confidence in our results.

While most of the strain hypotheses are supported for thesis models 1-3, the buffering hypotheses are not supported. This has very interesting practical significance for organizations. Had the buffering hypotheses been supported, the implication for organizations would be that employees can work longer hours and not report higher levels of work-life conflict as long as they feel that they have control over their work-life interface. The fact that buffering hypotheses are not supported means that organizations should not only consider enhancing control over work-life interface but to also consider ways of reducing the number of hours employees are working if they want to decrease work-life conflict.

These findings also imply that employers need to be aware that the availability of alternative work arrangements and policies on work-family balance will do little good if their employees simply have more work to do than what is feasible to complete in a typical work week (Duxbury and Higgins, 2005

The next question then, is how can organizations increase perceptions of control over the work-life interface? How can they reduce work demands? The results from the previous section provide an answer to the first question: increase perceived flexibility and supportive management. 
The answer to the second question is more complex due to what has been referred to in the literature as the culture of hours (Lewis and Cooper, 1999). It will be difficult to reduce work hours if organizations do not recognize the link between hours at work and opportunities for advancement. Many employers confuse the amount of face time an employee puts in with his/her level of dedication (Lewis and Cooper, 1999; Thompson, Beauvais, and Lyness, 1999). A culture of overtime and facetime lead to more hours at work which, as noted earlier, result in higher levels of work-life conflict (Duxbury and Higgins, 2003; Lewis and Cooper, 1999; Thompson, Beauvais, and Lyness, 1999). It is recommended, therefore, that employers should measure productivity, not the number of hours an employee is physically at work. Changing the culture away from face time and encouraging employees to complete their work within a reasonable timeframe could potentially lead to reduced levels of work-life conflict among their employees. As well, as recommended by Duxbury and Higgins (2005), employers need to ask why their employees are putting in long hours to get the job done (e.g. unrealistic work expectations, poor planning, lack of training). Once employers understand why employees are working longer hours, they can develop the right approach to reducing these untenable workloads.

\subsection{Predicting Perceived Flexiblity}

As noted earlier, perceived flexibility appears to be one of the key mechanisms open to organizations who wish to reduce work-life conflict in their workforce. The question then become, how can we increase an employee's 
feelings of perceived flexibility? The results from the testing of Thesis Model 4 provide an answer to this question by helping us understand which variables are most strongly associated with perceived flexibility. This section will discuss the findings of thesis models 4 and is structured as follows. First, the findings for the direct effect hypotheses will be discussed. Second, relative importance of the predictors of perceived flexibility will be discussed. Third, the findings for the interaction hypotheses will be discussed. To give an overview to the reader, the results of the direct and interaction hypotheses for models 4 are provided in Table 24 and Table 25.

Table 24 Summary of Direct Hypotheses Tested for Model 4

\begin{tabular}{|l|l|}
\hline Hypothesis & Result \\
\hline Pflex 1a Flextime positively associated with perceived flexibility & Supported \\
\hline $\begin{array}{l}\text { Pflex 1b Compressed work week positively associated with } \\
\text { perceived flexibility }\end{array}$ & Supported \\
\hline Pflex 1c Telework positively associated with perceived flexibility & Supported \\
\hline Pflex 2 Shiftwork negatively associated with perceived flexibility & Supported \\
\hline $\begin{array}{l}\text { Pflex } 3 \text { Supervisor support positively associated with perceived } \\
\text { flexibility }\end{array}$ & Supported \\
\hline
\end{tabular}

Table 25 Summary of Interaction Hypotheses Tested for Model 4

\begin{tabular}{|l|l|}
\hline Hypothesis & Result \\
\hline $\begin{array}{l}\text { Pflex 4a Supervisor support moderates flextime and perceived } \\
\text { flexibility }\end{array}$ & Not supported \\
\hline $\begin{array}{l}\text { Pflex 4b Supervisor support moderates compressed work week } \\
\text { and perceived flexibility }\end{array}$ & Not supported \\
\hline $\begin{array}{l}\text { Pflex 4c Supervisor support moderates telework and perceived } \\
\text { flexibility }\end{array}$ & Not supported \\
\hline $\begin{array}{l}\text { Pflex 5 Supervisor support moderates shiftwork and perceived } \\
\text { flexibility }\end{array}$ & Not supported \\
\hline
\end{tabular}




\subsubsection{Relationship Between Control Over Work-Life Interface and Perceived Flexibility}

Research Question 5 asked: What is the relationship between the various forms of control examined in this thesis (i.e. flextime, compressed work week, telework, shiftwork, and supervisor support) and perceived flexibility?

In the case of the five direct hypotheses for Model 4, the results indicate that alternative work arrangements do make a difference when it comes to perceived flexibility. Those who work flextime, compressed work week and telework report higher levels of perceived flexibility than those working a regular Monday-Friday, 9 to 5 schedule. The results are also significant for those working shiftwork and employees who work shiftwork have lower levels of perceived flexibility than those working a regular Monday-Friday, 9 to 5 schedule. Finally, supervisor support is positively associated with perceived flexibility such that those who reported high levels of supervisor support reported high levels of perceived flexibility.

These findings have important implications for the work-life conflict literature. In essence, alternative work arrangements do help employees balance completing work and family demands but not in the way originally hypothesized. Alternative work arrangements do not directly impact any of the forms of work-life conflict studied in this thesis. Nor do they moderate the relationship between demands and work-life conflict. The implications of this 
observation and the development of a new theoretical model can be found in section 12.2.1.

\subsubsection{Relative Impact of Predictors and Perceived Flexibility}

Which variable is the best predictor of perceived flexibility? To answer this question we did one additional regression. Consistent with the approach used in 11.4.4.4, all predictors are entered into the regression at one time. The results can be seen in Table 26.

Table 26 Pratt's Coefficients for Predicting Perceived Flexibility

\begin{tabular}{|l|l|l|l|l|}
\hline Predictor & $\begin{array}{l}\text { Std. } \\
\text { Coefficient } \\
\text { of } \\
\text { predictor }\end{array}$ & $\begin{array}{l}\text { Correlation } \\
\text { of predictor } \\
\text { and DV }\end{array}$ & $\begin{array}{l}\text { Pratt's } \\
\text { Coefficient }\end{array}$ & $\begin{array}{l}\text { Pratt's } \\
\text { Standardi } \\
\text { zed } \\
\text { Measure }\end{array}$ \\
\hline Flextime & .151 & .147 & .022197 & .128485 \\
\hline $\begin{array}{l}\text { Compressed } \\
\text { Work Week }\end{array}$ & .088 & .052 & .004576 & .026488 \\
\hline Telework & .09 & .079 & .007110 & .041156 \\
\hline Shiftwork & -.187 & -.223 & .041701 & .241383 \\
\hline $\begin{array}{l}\text { Supervisor } \\
\text { Support }\end{array}$ & .299 & .325 & .097175 & .562489 \\
\hline $\begin{array}{l}\text { Variance } \\
\text { Explained }\end{array}$ & - & - & .172759 & 1 \\
\hline
\end{tabular}

For perceived flexibility, there were four statistically significant and substantive predictors (all statistically significant at $<.001$ ). Supervisor support had the highest magnitude (Pratt's standardized measure of .562489) of variable importance (relative to all other independent variables in the model). The second most important predictor of variance in perceived flexibility was shiftwork with the second highest magnitude of variable importance (Pratt's standardized measure of .241383). The third most important predictor of variance in perceived 
flexibility was flextime with the third highest magnitude of variable importance (Pratt's standardized measure of .128485). The fourth most important predictor of variance in perceived flexibility was telework with the fourth highest magnitude of variable importance (Pratt's standardized measure of .041156). The finding that supervisor support does make a difference in perceived flexibility in consistent with the literature (e.g. Thomas and Ganster, 1995). Furthermore, the size of the sample in this thesis and the controlling of covariates reinforce the credibility of these findings.

The implications for organizations are as follows. Organizations must find ways to enhance supervisor support of their employees. It is important to recognize that training initiatives could be helpful in this context. Training could raise awareness of the problems associated with work-life conflict and provide practical information on how supervisors can help to support their employees (e.g. letting them leave early in an emergency, by providing information about various alternative work arrangements, etc.). Other initiatives could focus on enhancing the supportive environment for a healthy work-life balance among employees. These initiatives could include ensuring senior management model the importance of balancing work and family, identifying best practices associated with balancing work and family, communicating those best practices and changing the reward structure so that productivity, not face time is rewarded (Duxbury and Higgins, 2003).

Furthermore, it is important for organizations to be aware that shiftwork has a negative association with perceived flexibility. Given the discussion to date 
of how perceived flexibility is associated with lower levels of role overload, workfamily interference and family-work interference, organizations would do well to be mindful of the negative effects of shiftwork and examine ways to minimize the disruption of shiftwork on employees' personal lives. It is suggested that employers consider giving employees some control over the shift they work to allow them to better meet time their non-work obligations. Furthermore, allowing employees to trade shifts and giving employees sufficient notice ahead of time about when they are scheduled to work should help to minimize the disruption to their personal lives. By the same token, it is important to recognize that while alternative work arrangements (i.e. flextime, compressed work week, telework) do have a positive association with perceived flexibility they are not the only initiative worth pursuing (i.e. supervisor support is relatively more important than alternative work arrangements in predicting perceived flexibility).

\subsubsection{Moderating the Relationship Between AWA and Perceived Flexibility}

Research question 6 asked: To what extent does supervisor support moderate the relationship between the different work arrangements examined in this thesis (i.e. flextime, compressed work week, telework, shiftwork) and perceived flexibility?

Overall, results do not support the interaction hypotheses. None of the four interaction hypotheses are statistically significant. The finding that supervisor support does not moderate the relationship between alternative work arrangements and perceived flexibility could be interpreted as the supervisor 
does not have as much discretion or influence concerning an employee's work arrangement as previously reported in the literature (e.g. Powell and Mainiero, 1999). Furthermore, an alternate explanation may be that there is a limit to how helpful a supportive supervisor is. For example, if someone is working an excessive number of hours per week, a given alternative work arrangement is unlikely to increase that individual's flexibility regardless of how supportive manager is. 


\section{Conclusions}

This part is divided into four sections. First, the research conclusions for the thesis are presented. Second, the relevance of the thesis and the contributions made are discussed. Third, the limitations of the thesis are noted. Fourth, suggestions for future research are offered.

\subsection{Research Conclusions}

In conclusion, this thesis seeks to modernize Karasek's demand-control model and update the constructs of demands, strain and control. The results of this thesis mostly support Karasek's strain hypothesis. Five of the six demand hypotheses are substantive and we conclude that work demands and non-work demands are positively associated with both role overload and work-family interference. Non-work demands are positively associated with family-work interference.

In this thesis, control over work-life interface is operationalized in three different ways: alternative work arrangements, perceived flexibility and supervisor support. Karasek's strain hypothesis (i.e. control negatively associated with strain) is partially supported depending on which aspect of control was tested. While alternative work arrangements are not associated with lower levels of work-life conflict, these arrangements are associated with higher levels of perceived flexibility. Perceived flexibility is the strongest predictor of work-life conflict (relative to alternative work arrangements and supervisor support). Supervisor support also proves to be an effective predictor of work-life 
conflict. Therefore, organizations should use two approaches in concert to reduce work-life conflict: reduce the level of work demands and increase control over work-life interface by offering alternative work arrangements in conjunction with efforts to enhance both perceived flexibility and supervisor support. In other words, alternative work arrangements on their own are insufficient interventions for organizations looking for ways to reduce work-life conflict.

This thesis reported gender and job type differences in levels of work demands and work-life conflict. Therefore, organizations must bear in mind that the realities surrounding demands and work-life conflict differ for men and women, manager/professionals and non-professionals. This fact then suggests that organizations must not strive for a one size fits all approach to reducing work-life conflict. Offering a variety of programs and policy options designed to enhance perceived flexibility and reduce work-life conflict will most likely appeal to a diverse group of employees.

\subsection{Relevance}

This thesis makes several contributions to the organizational literature from a theoretical, empirical, methodological and practical perspective. Each type of contribution will be discussed in turn.

\subsubsection{Theoretical/Conceptual Contributions}

From a theoretical and conceptual perspective, this thesis makes five contributions. First, this thesis developed an initial theoretical model to explore the relationship between various work arrangements, perceived flexibility and supervisor support. The mechanisms underlying these relationships are not very well understood in the literature. It is important to note that very few studies (a 
notable exception is Thomas and Ganster, 1995) have explicitly considered the relationship between perceived flexibility and supervisor support. For instance, no studies were found in the literature review where perceived flexibility was treated as the dependent variable.

Second, the results of this thesis (i.e. alternative work arrangements not associated with work-life conflict but alternative work arrangements positively associated with perceived flexibility) led us to develop a revised theoretical model where perceived flexibility is treated not as a dependent variable but as a mediating variable between alternative work arrangements and work-life conflict. Theoretically then the findings from this thesis support the following relationship between demands, alternative work arrangements, perceived flexibility, supportive management and work-life conflict as shown in Figure 19.

Figure 19 Relationship Between Control Over Work-Life Interface, Demands and Work-Life Conflict

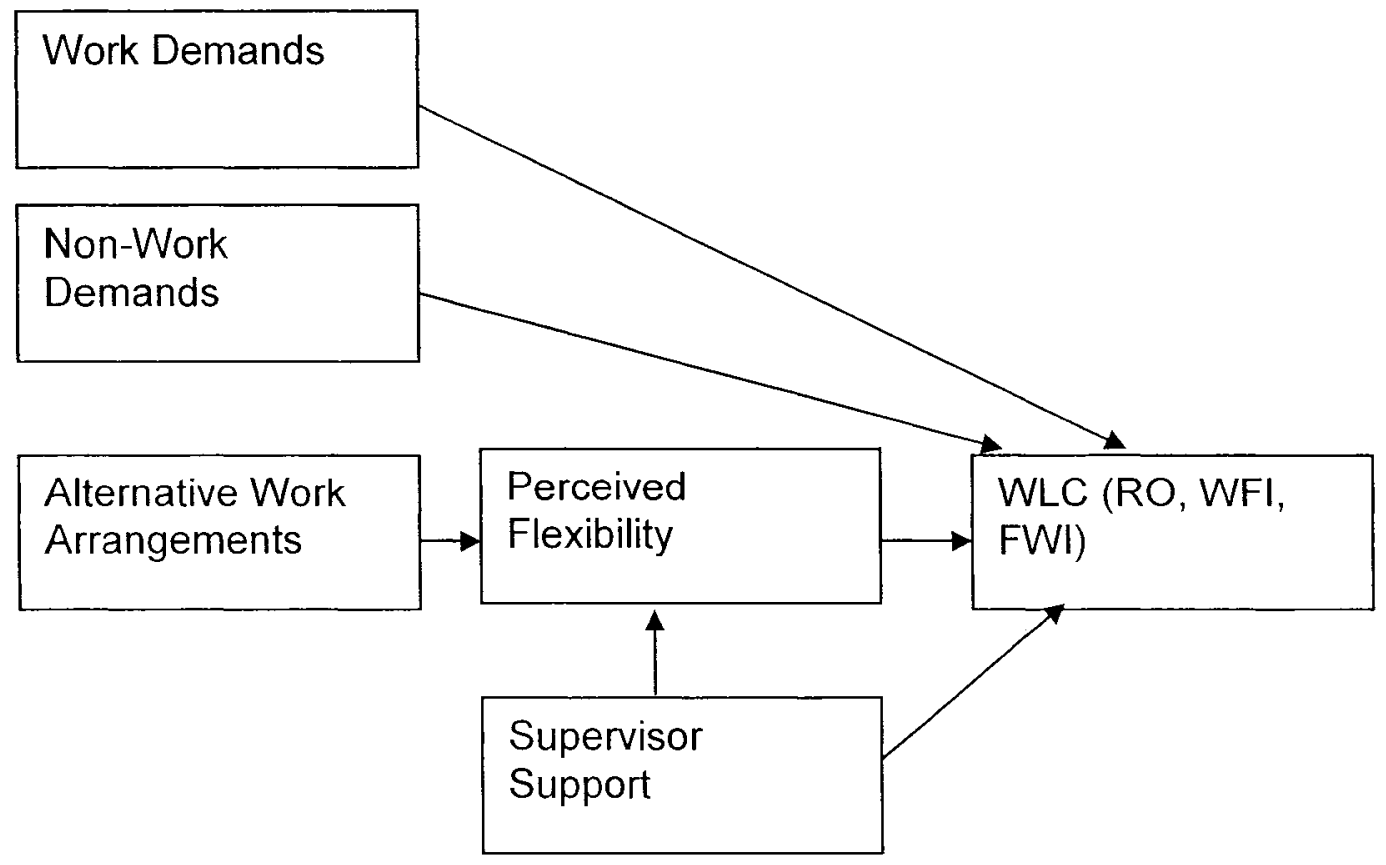


Third, learning more about the relationships between these variables (i.e. alternative work arrangements, perceived flexibility, and supervisor support) helps clear up some confusion in the literature on alternative work arrangements. Many studies have found a negative association between alternative work arrangements and work-life conflict (e.g. Bond et al., 2003). This may leave organizations and managers with the mistaken impression that all that is required to help employees balance work-life conflict is to provide an alternative work arrangement. The results of this thesis suggest that alternative work arrangements offer a good start in helping balance work and family but employers must also find ways to enhance perceived flexibility and supervisor support as well as decrease the level of work demands.

Fourth, in models 1-3, alternative work arrangements are treated as moderator variables. No other studies were found where a given alternative work arrangement moderated the relationship between demands and work-life conflict (all studies treated the work arrangement as an independent variable and examined the direct effect of the work arrangement on work-life conflict). This research provides clarity on this issue. Alternative work arrangements do not buffer the relationship between demands and work-life conflict.

Fifth, this thesis uses a well-respected theoretical model (Karasek's Demand-Control Model) to guide our research. This is significant because various authors (Baltes et al., 1999; Geurts and Demerouti, 2003; Gottlieb, Kelloway and Barham, 1998; Johnson, 1997; McCloskey and Igbaria, 1998) have 
expressed concern about the atheoretical nature of the alternative work arrangement and shiftwork literature when discussing work-life conflict.

\subsubsection{Empirical Contributions}

From an empirical perspective, this thesis makes two key contributions. First, the literature review for this thesis revealed that there is limited empirical evidence to support some of the relationships of interest in this thesis. Specifically, relative to work-family interference and family-work interference, much less empirical work has been published on role overload. In this thesis, role overload was positively associated with both work and non-work demands (statistically significant and substantive) and the mean for role overload was higher than the other two forms of work-life conflict.

Furthermore, very little empirical work has been published on the following relationships: work demands and family-work interference, non-work demands and work-family interference, non-work demands and family-work interference, compressed work week and the three forms of work-life conflict, alternative work arrangements and perceived flexibility and perceived flexibility and supervisor support. In all these cases, this thesis provided some empirical results on the aforementioned relationships. Specifically the following observations can be made with respect to each of these areas from the analysis performed in this thesis:

- There is a statistically significant positive relationship between work demands and family-work interference. This relationship is not, however, substantive. 
- There is a statistically significant and substantive positive relationship between non-work demands and work-family interference.

- There is a statistically significant and substantive positive relationship between non-work demands and family-work interference.

- Working a compressed work week makes no significant difference on any of the three forms of work-life conflict (role overload, work-family interference, family-work interference).

- There is a statistically significant and substantive positive relationship between perceived flexibility and each of the following work arrangements: flextime, compressed work week and telework.

- There is a significant and substantive positive association between perceived flexibility and supervisor support

Second, the majority of the studies examining the impact of shiftwork and alternative work arrangements on work-life conflict are dated (e.g. Bohen and Viveros-Long, 1981; Dunham et al., 1987; Hicks and Klimoski, 1981; Winnett and Neale, 1981; Shamir, 1983; Staines and Pleck, 1983). Harris (2004) points out that this means that these surveys collected data from workforces in the 1970 s. Such results are unlikely to inform us of the realities for workers, spouses, and parents of today. This thesis provides up-to-date information on key variables of interest (i.e. flextime, compressed work week, telecommuting, shiftwork, perceived flexibility and supervisor support) and work-life conflict and concluded: 
- None of the variables of interest (i.e. flextime, compressed work week, telecommuting, shiftwork, perceived flexibility and supervisor support) moderated the relationship between demands and work-life conflict.

- The work arrangements (i.e. flextime, compressed work week, telework and shiftwork) were not substantively associated with work-life conflict.

- Both perceived flexibility and supervisor support were statistically and substantively associated with work-life conflict.

\subsubsection{Methodological Contributions}

Authors (e.g. Baltes et al., 1999; Geurts and Demerouti, 2003; Gottlieb, Kelloway and Barham, 1998; Johnson, 1997; McCloskey and Igbaria, 1998) have identified several methodological concerns with previous studies in the areas of alternative work arrangements/shiftwork and work-life conflict including using homogeneous populations and small sample sizes, poorly validated measures, and not controlling for potentially significant confounding variables. Therefore, this thesis makes a contribution from a methodological perspective by using a large, nationally representative sample in its research design, using measures that have been well-established (e.g. work-life conflict, perceived flexibility, supervisor support) and have shown good reliability, and controlling for confounding variables (demands, gender and socio-economic status). 


\subsubsection{Practical Contribution}

There is a substantial body of empirical literature to indicate that there are significant negative outcomes of work-life conflict for employees, employers and society in general. For employees, high levels of work-life conflict have been associated with outcomes such as poor physical health (e.g. Burke, 1988; Thomas and Ganster, 1995), psychological problems such as depression (e.g. Frone et al., 1995; Robinson and Godbey, 1997) and substance abuse (e.g. Frone et al., 1993; Frone, 2000). For employers, work-life conflict is associated with lower job satisfaction (e.g. Adams et al., 1996; Voydanoff and Kelly, 1984); lower organizational commitment (e.g. Aryee, 1992; Netemeyer et al., 1996) and higher turnover intentions (e.g. Anderson et al., 2002; Boyar et al., 2003). For society, Higgins, Duxbury and Johnson (2004) argue that high levels of work-life conflict (in the form of role overload) cost Canada's health care system $\$ 6$ billion dollars.

Given the aforementioned costs to these stakeholders combined with the information that levels of work-life conflict have been climbing over the past decade, it is imperative that organizations try to help reduce the impact of worklife conflict. This thesis identified two interventions that can help employees cope with work-life conflict, specifically perceived flexibility and supervisor support. The results of this thesis make a compelling case for the notion that it is not enough for employers merely to offer alternative work arrangements to their employees. Alternative work arrangements are not associated with lower levels of work-life conflict and do not provide a buffering impact on the association 
between demands and strain. This thesis offers advice on what organizations can do. Organizations would do well to offer supervisors training on the impact of work-life conflict and the impact that supervisors have on employee perceived flexibility. Employers also need to ensure they are not rewarding face time and are encouraging employees to balance their work-life obligations by not working excessive overtime.

\subsection{Limitations}

As with any thesis, it is inevitable that some limitations exist. In this thesis, it is important to note five limitations. First, the research design is cross-sectional in nature. Cross-sectional designs cannot establish causality but can confirm associations. This means that a positive association between perceived flexibility and supervisor support can be demonstrated and it can be suggested that it is supervisor support that results in enhanced perceptions of flexibility. However, it is possible that those with high levels of perceived flexibility might perceive high levels of supervisor support.

Second, the fact that the data was self-reported could be a limitation. The issues associated with self-reported data were discussed in the methodological criticisms of Karasek and are threefold. One concern is the possibility that respondents might exaggerate the number of hours they have spent in various work and nonwork activities. Robinson and Godbey (1997) have shown that selfreport data of time in work activities is inflated. However, the fact that this survey was anonymous could lessen the likelihood that respondents overstated their hours worked (Thomas and Ganster, 1995). As Bond et al., (1998) noted, the issue of the number of hours reported should most likely be interpreted as a 
relative and not absolute figure. Furthermore, self-report is appropriate given the fact that the crucial measures of the study involved perceptions (e.g. work-life conflict, supervisor support, perceived flexibility).

Another concern associated with self-reports is social desirability. Social desirability is defined as the tendency for a respondent to choose the socially desirable response, regardless of the accuracy of their response (Spector, 1987b). This can be problematic because biases can have attenuating or moderating effects (Spector, 1987b). If social desirability correlates with both variables of interest, it will artifactually inflate their relationship. If social desirability correlates with only one of two variables, it will act as a suppressor and artifactually reduce the correlation between them. For example, it is more socially acceptable for men to perform housework and childcare activities. Therefore, it could be argued that men might exaggerate their contribution to the family responsibilities as a response to what is socially desirable. However, recent data from the National Study on the Changing Workforce implies that social desirability and inflation of time spent in domestic activities might not be a problem. Bond et al. (1998) reports that wives' estimates of their husbands' time spent in household activities did not differ significantly from the self-reports of these partners. Another concern with self-reports is the issue of negative affectivity (see section 2.5.1.4). It is acknowledged that this thesis did not control for negative affectivity.

Third, it could be argued that because all employees sampled worked for large organizations (i.e. at least 500 employees), the results may not be 
generalizable to those who work for smaller firms. For example, Industry Canada (2004) notes that less than $0.3 \%$ of private sector employers have more than 500 employees.

Fourth, there is the issue of nonresponse. How do those who did not respond (74\%) differ from those who did respond (26\%)? Hill et al. (2001) posit two conflicting scenarios. It is possible that those who worked the longest hours did not have time for relatively lengthy surveys and therefore chose not to respond. Therefore, those with the highest workload may be underrepresented. If so, it could be that any results may be understated. On the other hand, employees with low levels of work-life conflict who are relatively content may have chosen not to participate. If so, it is possible that the results have been overstated.

Fifth, it would have been optimal to examine all three dependent variables at once and examine the relationships between demands and strain as well as the interaction effects. While SEM would have allowed such analysis, it was not possible to perform test the moderation hypothesis with the packages that were available when the analysis was done. Future studies should use SEM to test the model in Figure 19.

Sixth, it is possible that the lack of support for the strain hypotheses associated with alternative work arrangements could be attributed to our inability to differentiate those who chose a particular work arrangement and those who had the work arrangement imposed upon them. 
Seventh, the impact of sector (i.e. public, private, not for profit) was not addressed in the thesis analysis. Duxbury and Higgins (2005) report that those respondents in the not for profit sector reported higher levels of work-family interference (relative to those working in public or private sector). As seen in the future research section, this is an area meriting further attention.

\subsection{Suggestions for Future Research}

There are several opportunities to expand on the research presented in this paper. First, future research could incorporate a longitudinal design in order to examine temporal relationships between the independent (i.e. work and nonwork demands) and dependent variables (i.e. role overload, work-family interference and family-work interference). Geurts and Demerouti (2003) argue that incorporating a longitudinal design will help us understand how issues of work-life balance change over time when major life changes occur (e.g. promotions, births, deaths, job loss). Using a longitudinal approach will help us understand "whether the work/non-work interface reflects a stable or a changing experience" (Geurts and Demerouti, 2003, p. 303).

Second, our sample was limited to employees who worked for employers with a minimum of 500 employees. This begs the question of how these results would compare for organizations with less than 500 employees (small-medium sized organizations). It would be interesting to compare the experience of those employees who work for small or medium size businesses to see if these employees experience similar levels of work-life conflict, and to see if there is more informality with respect to alternative work arrangements that might make it 
easier for these employees to gain the flexibility they need to balance work and family demands.

Third, researchers measure work-life conflict by measuring role overload (e.g. Robinson and Godbey, 1997; Shields, 1999; Voydanoff, 2004) and interference (e.g. Fu and Shaffer, 2001; Gutek et al., 1991; Madsen et al., 2003). Role overload is considered a strain-based measure of work-life conflict while interference is deemed a time-based measure of work-life conflict. However, very few studies incorporate a behaviour-based measure of work-life conflict. Guerts and Demerouti define this measure as "referring to specific patterns of role behaviour that are incompatible with expectations regarding behaviour in another role." (Geurts and Demerouti, 2003, p. 284). For example, a labour negotiator might have difficulty "turning off" his/her hard-nosed persona when dealing with his/her children or spouse. It is recommended that future research incorporate a measure of behaviour-based work-life conflict to understand how that might play a role in understanding the difficulties people having in balancing their professional and personal lives.

Fourth, while this thesis does examine supervisor support, there are a variety of other measures of social support including coworker support and spouse support. Future research could examine a measure of spouse support (Carlson and Perrewe, 1999) or coworker support (Johnson and Hall, 1988).

Fifth, this thesis did not address issues of organizational culture. It is possible that how family friendly an organization's culture is has a significant impact on an employee's work-life balance. Duxbury and Higgins (2005) 
reported that organizational culture was the most significant predictor of all three forms of work-life conflict (i.e. role overload, work-family interference and familywork interference). Future research efforts could examine the role of familyfriendly culture as a moderator of demands and work-life conflict.

Sixth, like most research in the area of work-life conflict, the individual worker has been chosen at the unit of analysis. As recognized by Bolger et al. (1990) and Barnett (1998), what happens at work affects not only the worker but his/her spouse, children and even friends and extended family and the reverse is also true. Future research could include examining the impact of the respondent's spouse's level of work and non-work demands as well as efforts to capture perceptions of work-life conflict by surveying multiple members of a household perhaps through a qualitative research design.

Seventh, the measure of demands is limited to the number of hours reported. Such a measure does not measure how physically or psychologically demanding a given role is. Future research could consider additional measures of demands in order to develop a more complete picture of how these demands impact work-life conflict.

Eighth, we can test the new model developed in this thesis and featured in figure 19 using structural equation modeling (SEM). SEM will allow us to test the relationship between the independent variables and all three dependent variables simultaneously.

Ninth, this thesis identified that family-work interference has a different etiology than either role overload or work-family interference. Furthermore, 
Karasek's model is not as helpful in understanding family-work interference (relative to role overload and work-family interference). Therefore, future research should involve exploring other theoretical models in order to identify antecedents and potential moderators of family-work interference.

Tenth, differences between sectors (i.e. private, public, not for profit) and industries were not considered in the thesis analysis. Ferrer and Gagne (2006), using the WES dataset, report that the availability of alternative work arrangements varied by industry. Future research could examine the differences in alternative work arrangement usage by industry and sector. Duxbury and Higgins (2005) report that those respondents in the not for profit sector reported higher levels of work-family interference (relative to those working in public or private sector). Therefore, future research could examine the role of industry in predicting work-life conflict.

Eleventh, part-timers were screened out of the subsample analyzed in this thesis. Future research could examine the applicability of the thesis models to part-timers and examine whether working part-time reduces work-life conflict.

Twelfth, composite measures of work and non-work demands were used when analyzing the data in this thesis. The discrete items that comprise nonwork demands such as hours spent per week in childcare or caring for elderly relatives were not analyzed individually. Research by Duxbury and Higgins (2005) indicates primary responsibility for child care was a main predictor of family-work interference. Future research could examine the relationship between these individual items of non-work demands and work-life conflict and 
examine both gender and job type differences in the level of these non-work demands. 


\title{
Appendix A Summary of Relevant Survey Questions
}

\author{
A summary of relevant questions/items from the Duxbury/Higgins \\ Balancing Work, Family and Lifestyle National Study are provided below. \\ Supervisory Support (Question 7) \\ Using a five-point likert scale ( $1=$ strongly disagree, $3=$ neutral, $5=$ strongly agree), \\ respondents answered the following sixteen questions: \\ My manager/supervisor: \\ Gives recognition when I do my job well \\ Provides constructive feedback when performance standards are not met \\ Makes it clear what is expected of me (i.e. good at communicating goals, \\ objectives, how to proceed) \\ Listens to my concerns \\ 'Puts me down" in front of colleagues or clients \\ Shares information with me \\ Is available to answer questions \\ Only talks to me when I make a mistake \\ Makes me feel guilty about time off for personl/family reasons \\ Focuses on hours of work rather than output \\ Is effective at planning the work to be done \\ Asks for input before making decisions that affect my work \\ Has unrealistic expectations about how much work can be done \\ Provides me with challenging opportunities \\ Supports my decisions (with clients, upper management) \\ Puts in long hours and expects me to do the same \\ Demands (Question 9)
}

At present, approximately how many hours per week do you spend:

Driving or commuting to and from work

In paid employment

Working at home outside regular hours

In home chores and errands

In child care or activities with your children

Caring for elderly relatives or dependents

In educational activities (i.e. taking night courses, training for work)

In volunteer activities/community work 
Perceived Flexibility (Question 13)

Using a five-point likert scale ( $1=$ very difficult $3=$ neither easy nor difficult, $5=$ very easy), respondents answered the following ten questions:

How easy or difficult is it for you:

To vary your working hours (i.e. arrival and departure times)?

To spend some of your regular work day working at home?

To take your holidays when you want?

To take time off to attend a course or conference?

To interrupt your work day for personal/family reasons and then return?

To take a paid day off work when a child is sick?

To take a paid day off work when an elderly relative needs you?

To have meals with the family?

To be home when your children get home from school?

To arrange your work schedule (i.e. shifts, overtime) to meet family/personal commitments?

Work-Life Conflict (Question 16)

Using a five-point likert scale (1=strongly disagree, $3=$ neutral, $5=$ =strongly agree), respondents answered the following fifteen questions. The author of this thesis has added the following short form to clarify which questions reflect role overload (RO), family-work interference (FWC) and work-family interference (WFC):

To what extent do you agree or disagree with the following statements:

I feel I have more to do than I can comfortably handle (RO)

I feel physically drained when I get home from work (RO)

I feel emotionally drained when I get home from work (RO)

I feel I have to rush to get everything done each day (RO)

I feel I don't have enough time for myself (RO)

Making arrangements for children while I work involves a lot of effort (FWC)

Making arrangements for elderly relatives while I work involves a lot of effort

(FWC)

My work schedule often conflicts with my personal life (WFC)

My family dislikes how often I am preoccupied with work while at home (WFC)

The demands of my job make it difficult to be relaxed at home (WFC)

My work takes time I would like to spend with family or friends (WFC)

My work makes it hard to be the kind of partner I would like to be (WFC)

My work makes it hard to be the kind of parent I would like to be (WFC)

My family/personal life often keeps me from spending the amount of time I would like on my job/career (FWC)

My family/personal life often interferes with my responsibilities at work (i.e.

getting to work on time, ability to work overtime) (FWC) 
Alternative Work Arrangements (Question 17)

Please FILL in the oval for the phrase that best describes how your employment is arranged:

REGULAR: you work a set number of hours each week, arriving and departing at the same time each day.

FLEXTIME: you vary your arrival and departure times around a "core" time when you should be at work.

COMPRESSED WORK WEEK: you get one working day off every week or two in return for longer hours.

WORK AT HOME: you spend part of your regular work week working at home.

Shiftwork (Question 18)

Do you work shifts?

NO

Yes, I work a fixed shift

Yes, I work a rotating shift

Gender (Question 46)

Are you male or female?

Job Type (Question 59)

Please FILL in the oval for the answer which best fits most of the work:

You do:

Managerial

Professional

Technical

Administrative

Clerical

Retail

Production

Other 


\section{Appendix B Checking Assumptions of Regression}

Appendix B.1 Checking Assumptions of Normality-Dependent Variables

Histogram (Role Overload)

ro

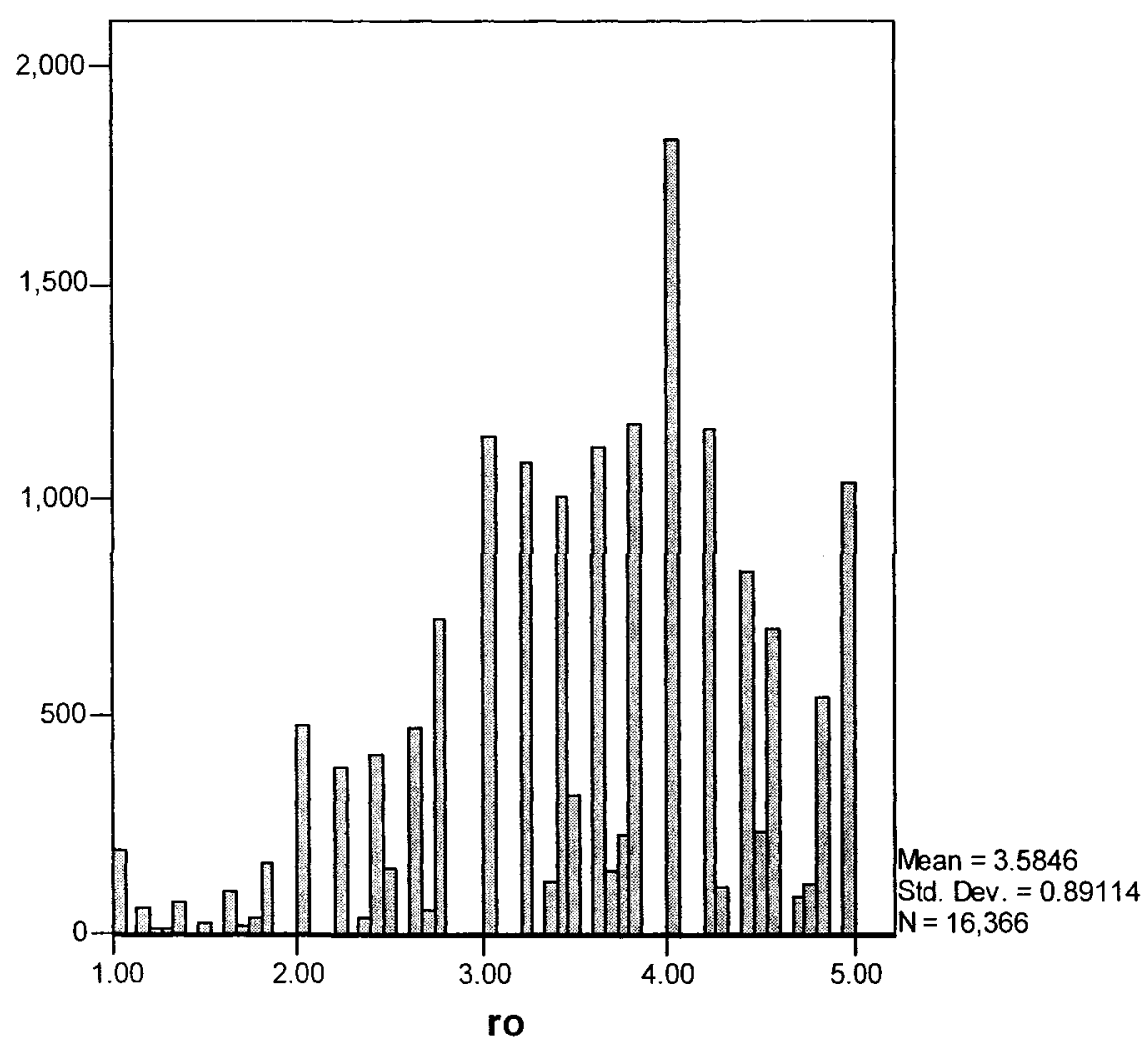


Probability Plots (Role Overload)

\section{Normal P.P Plot of ro}

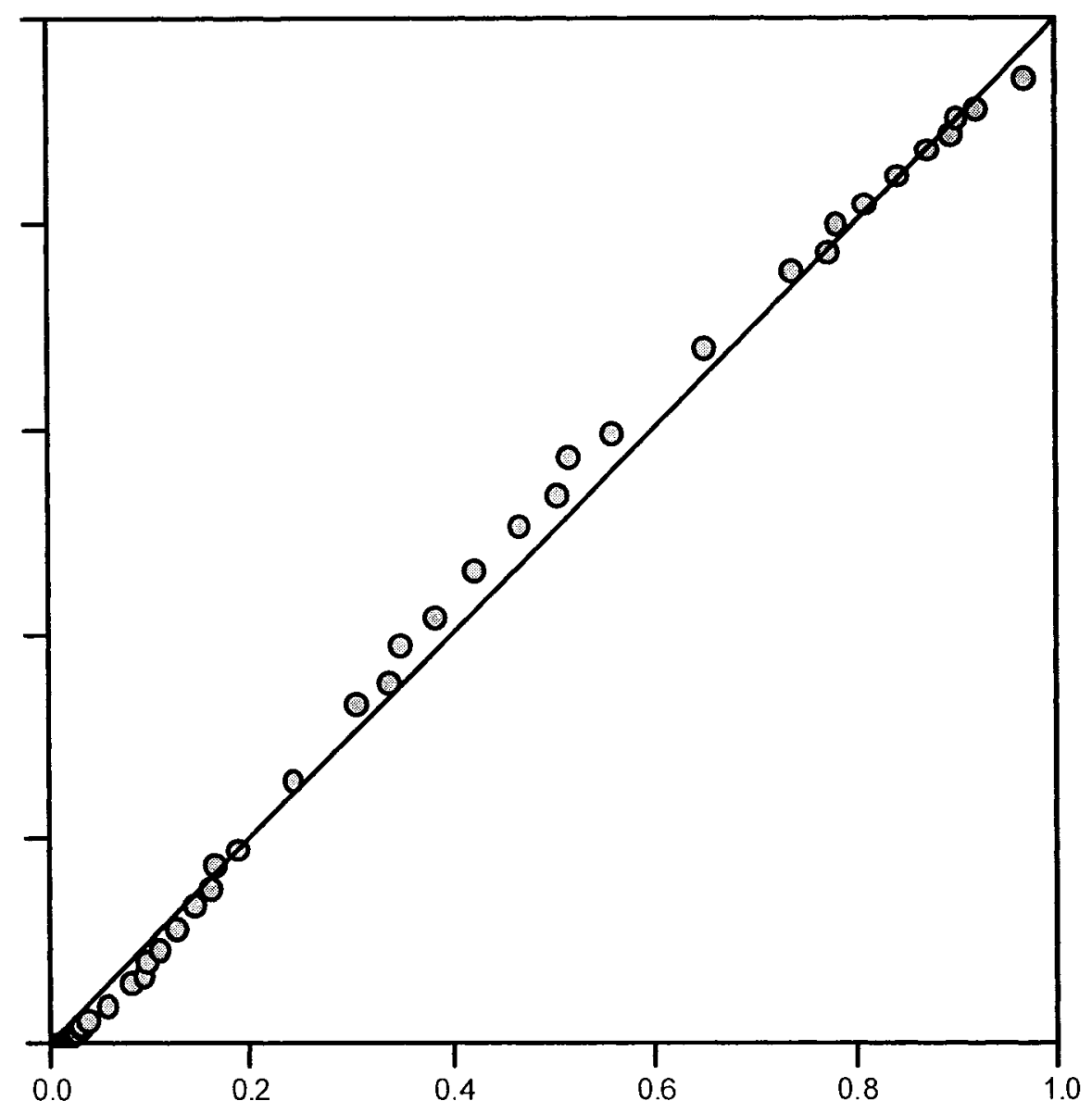

x-axis: observed y-axis: expected 
Histogram (Work-Family Interference)

wfc

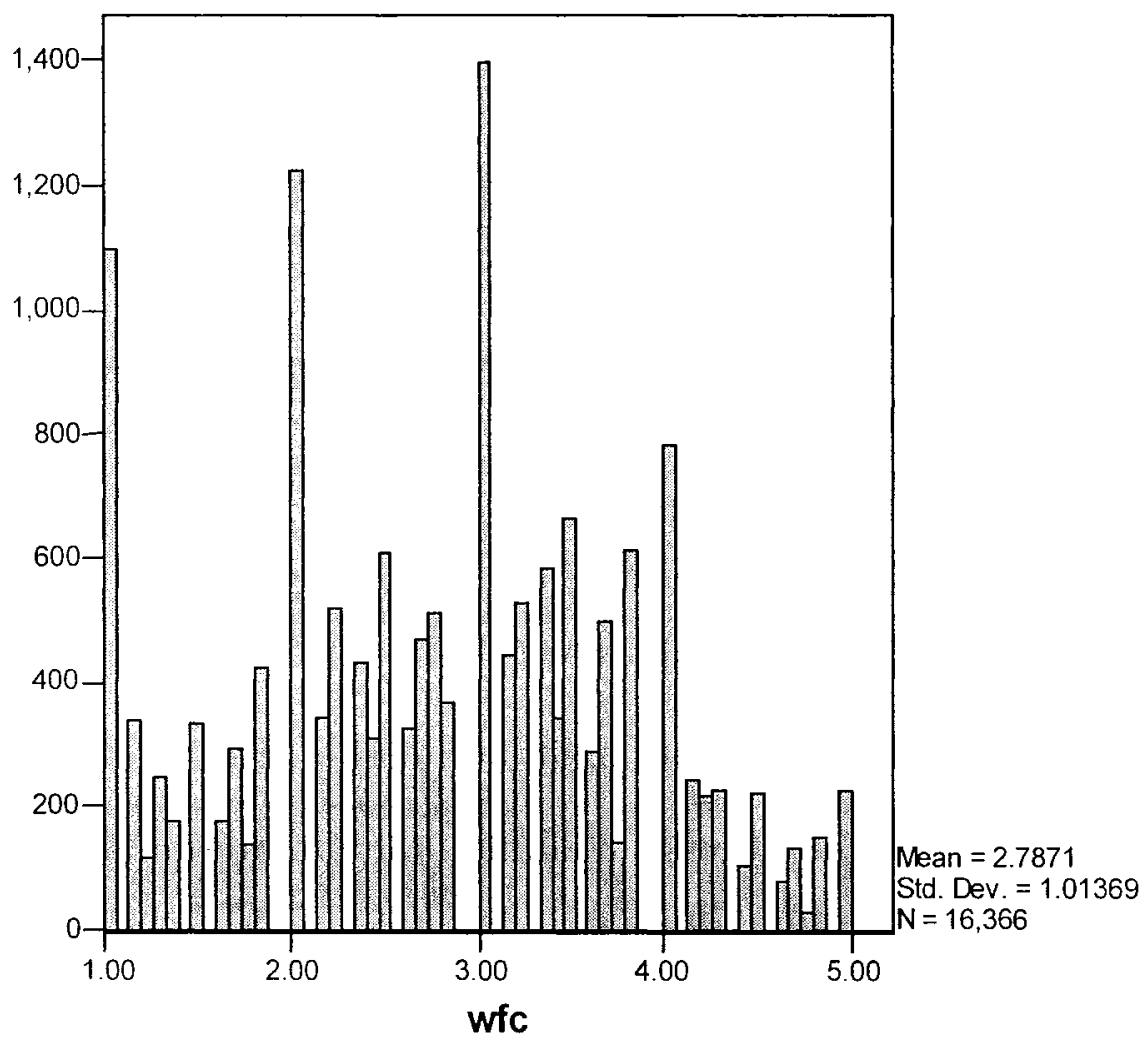


Probability Plots (Work-Family Interference)

\section{Normal P-P Plot of wfc}

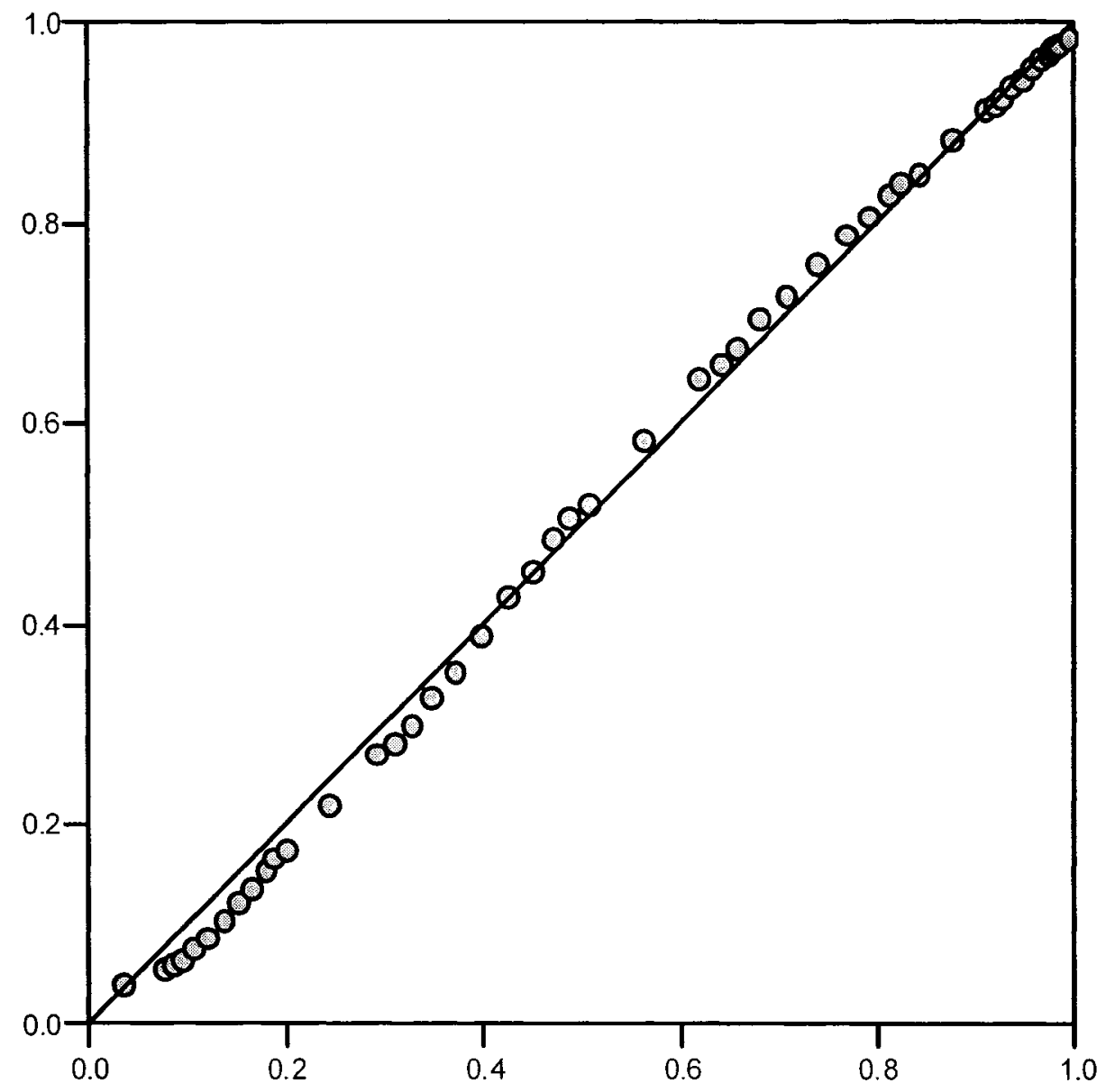

x-axis: observed y-axis: expected 
Histogram (Family-Work Interference)

fwc

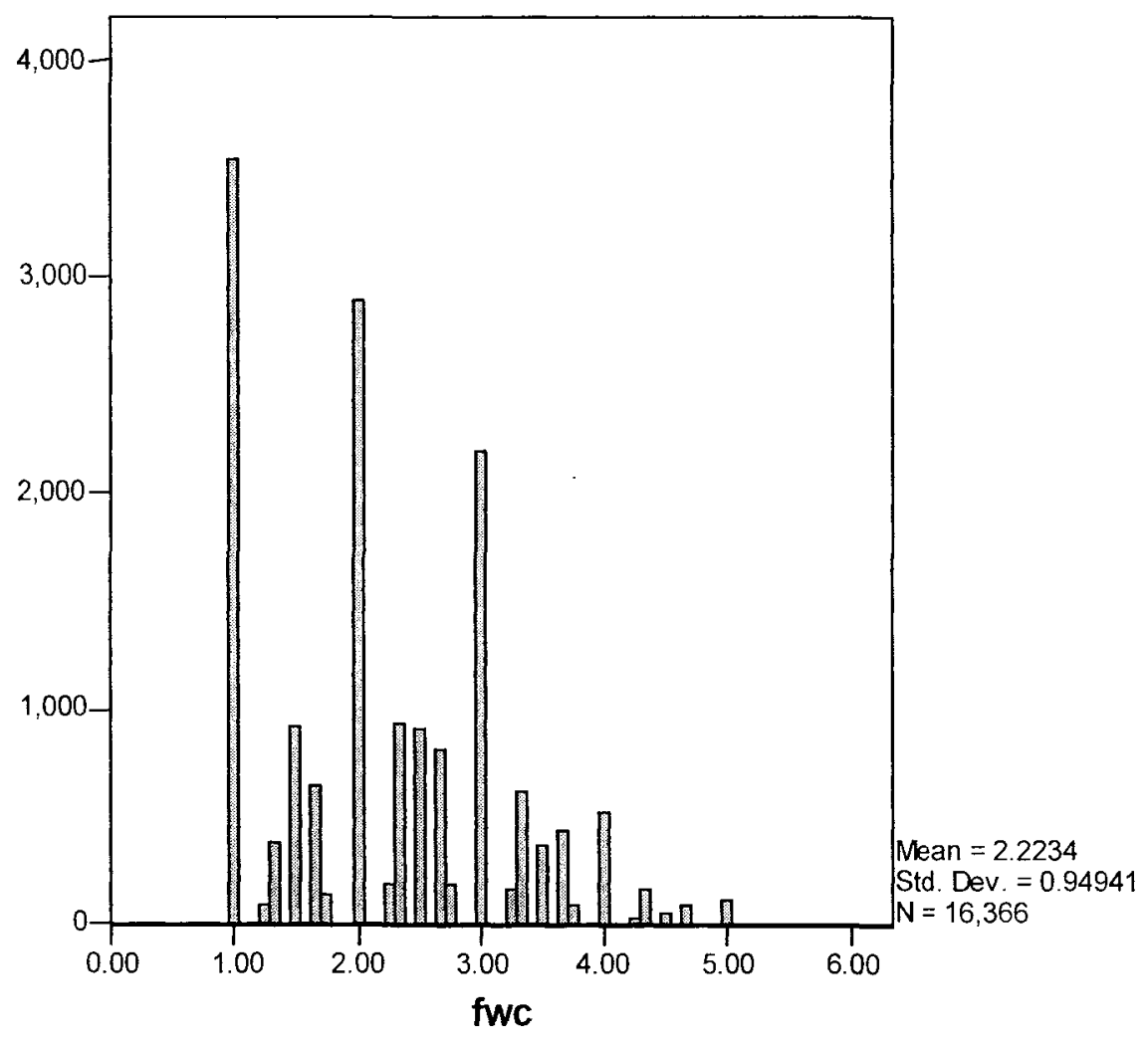


Probability Plots (Family-Work Interference)

\section{Normal P-P Plot of fwc}

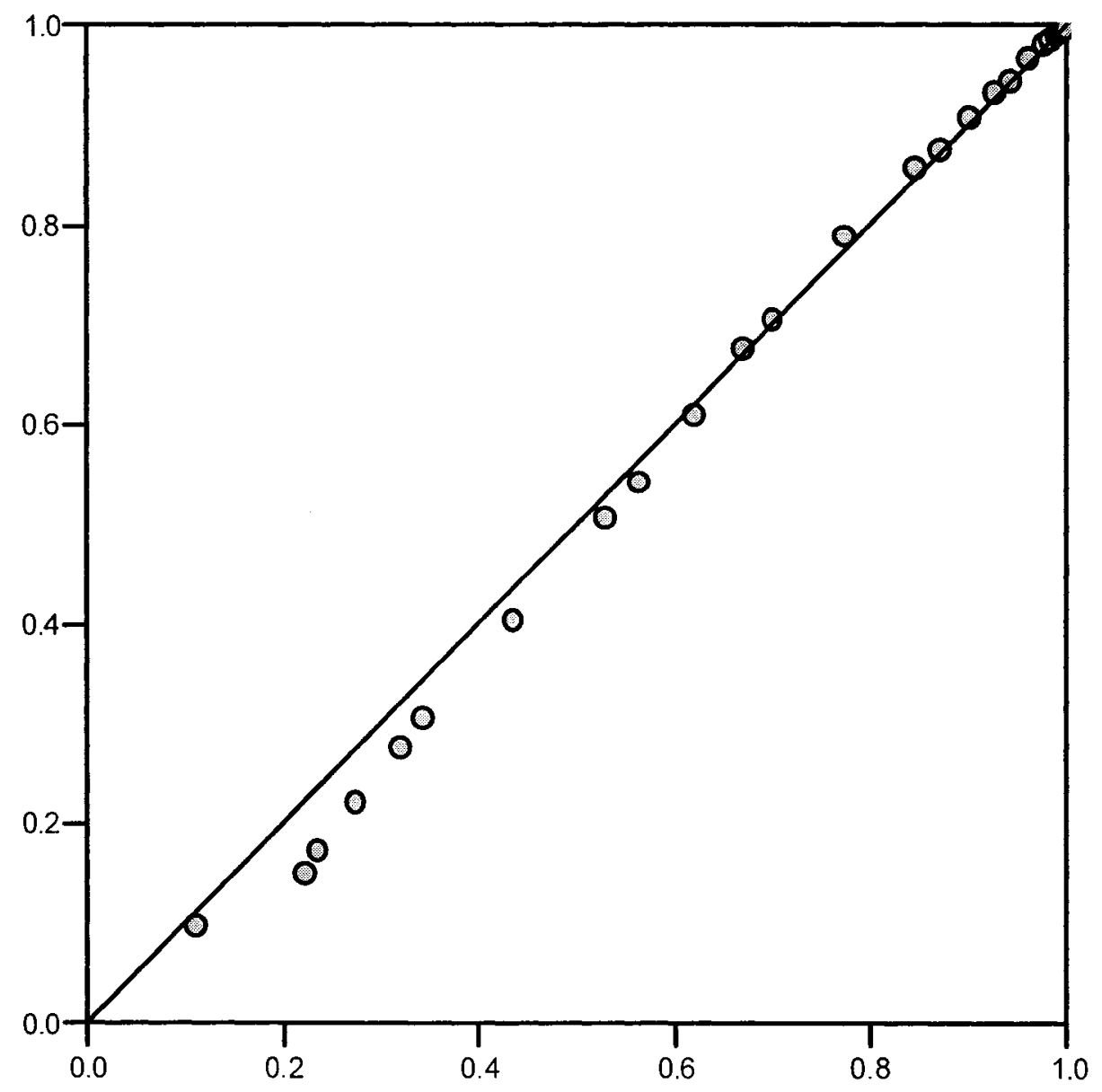

x-axis: observed y-axis: expected 
Appendix B.2 Checking Assumptions of Linearity and Homoscedasticity

Scatterplots (Work Demands and Role Overload)

\section{Scatterplot}

Dependent Variable: ro

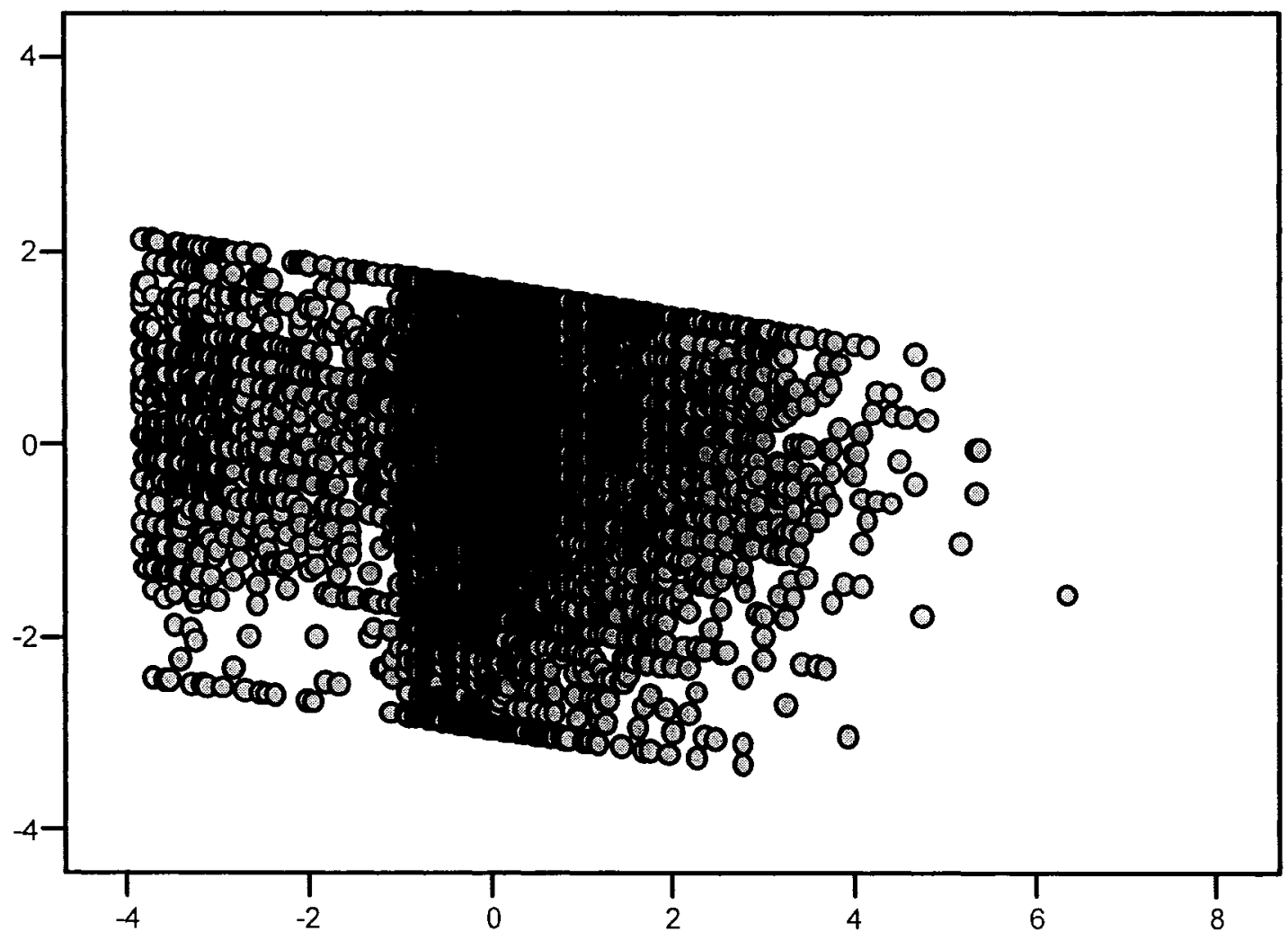

x-axis Regression Standardized Predicted Value

y-axis Regression Standardized Residual 
Scatterplots (Non-Work Demands and Role Overload)

\author{
Scatterplot
}

Dependent Variable: ro

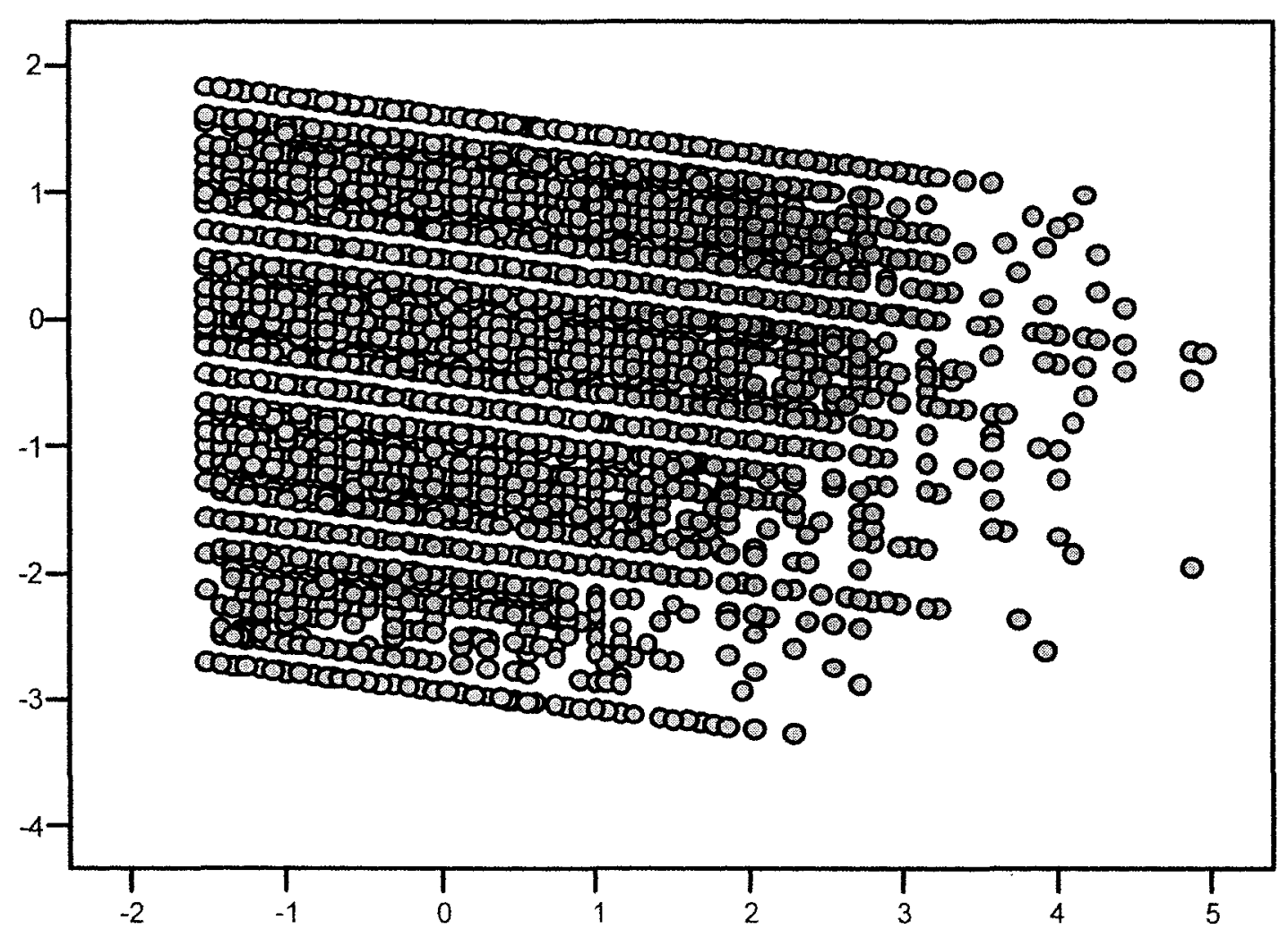

x-axis Regression Standardized Predicted Value

y-axis Regression Standardized Residual 
Scatterplots (Work Demands and Work-Family Interference)

\section{Scatterplot}

Dependent Variable: wfc

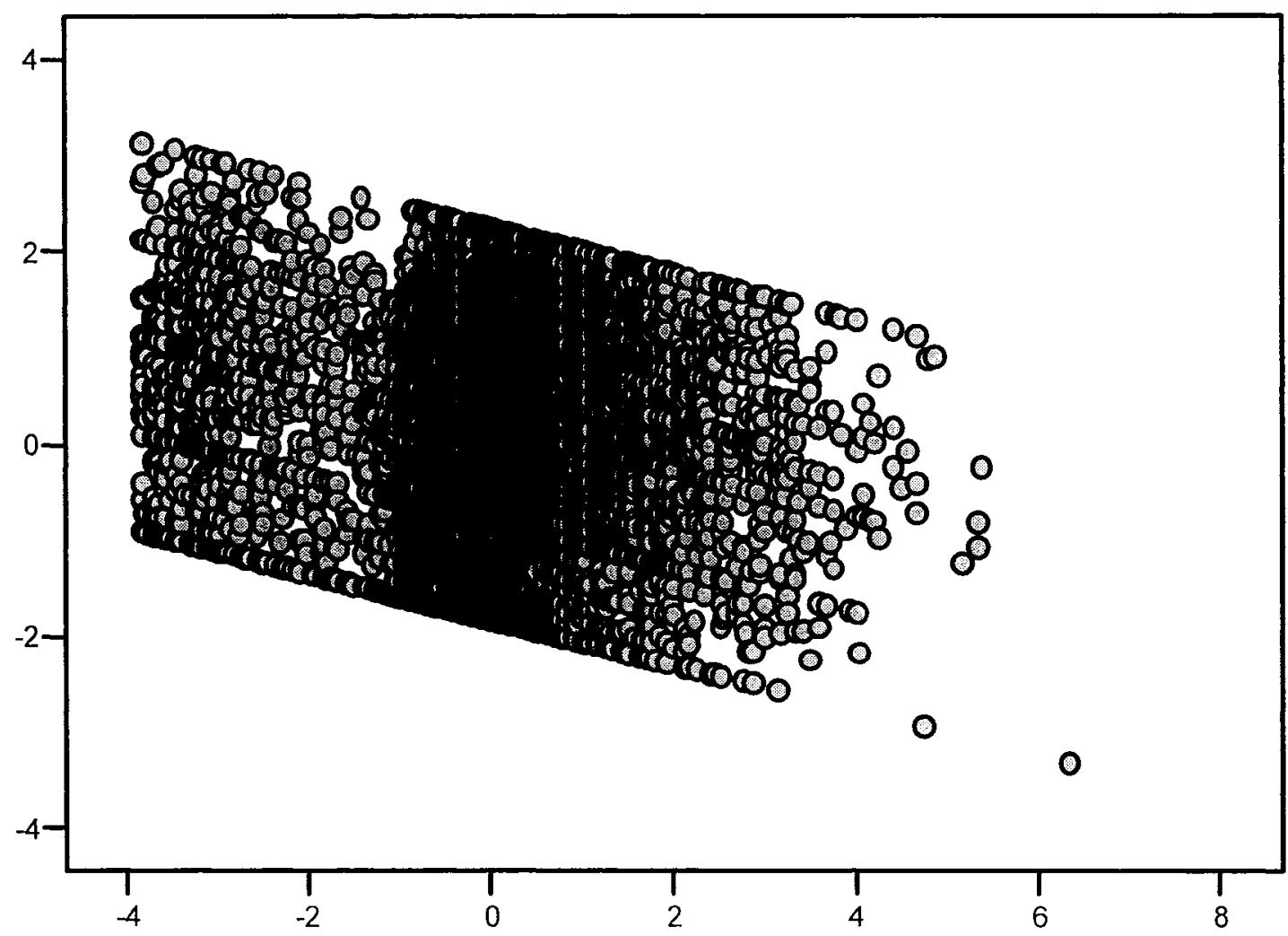

x-axis Regression Standardized Predicted Value

y-axis Regression Standardized Residual 
Scatterplots (Non-Work Demands and Work-Family Interference)

\section{Scatterplot}

\section{Dependent Variable: wfc}

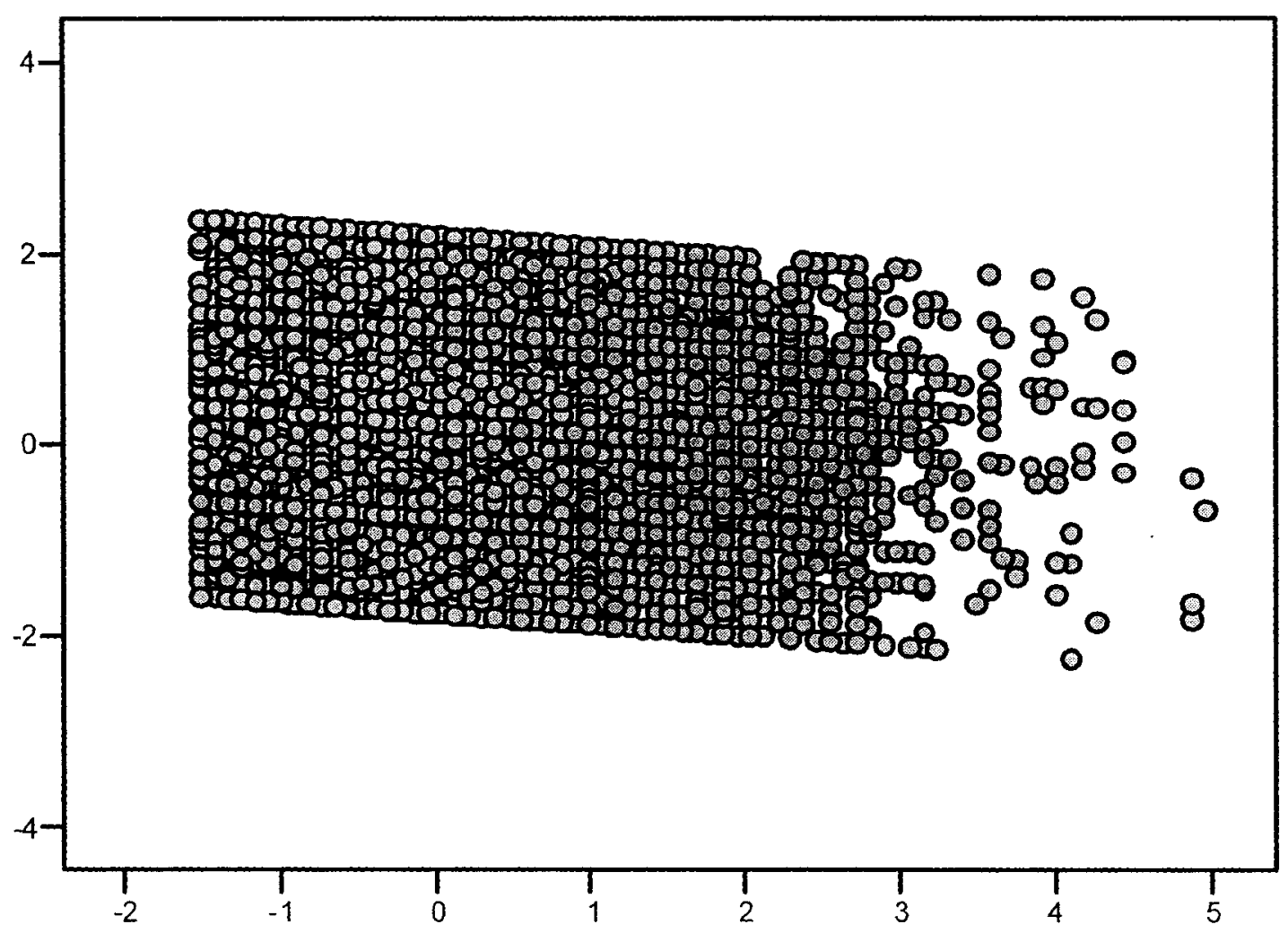

x-axis Regression Standardized Predicted Value

y-axis Regression Standardized Residual 
Scatterplots (Work Demands and Family-Work Interference)

\section{Scatterplot}

Dependent Variable: fwc

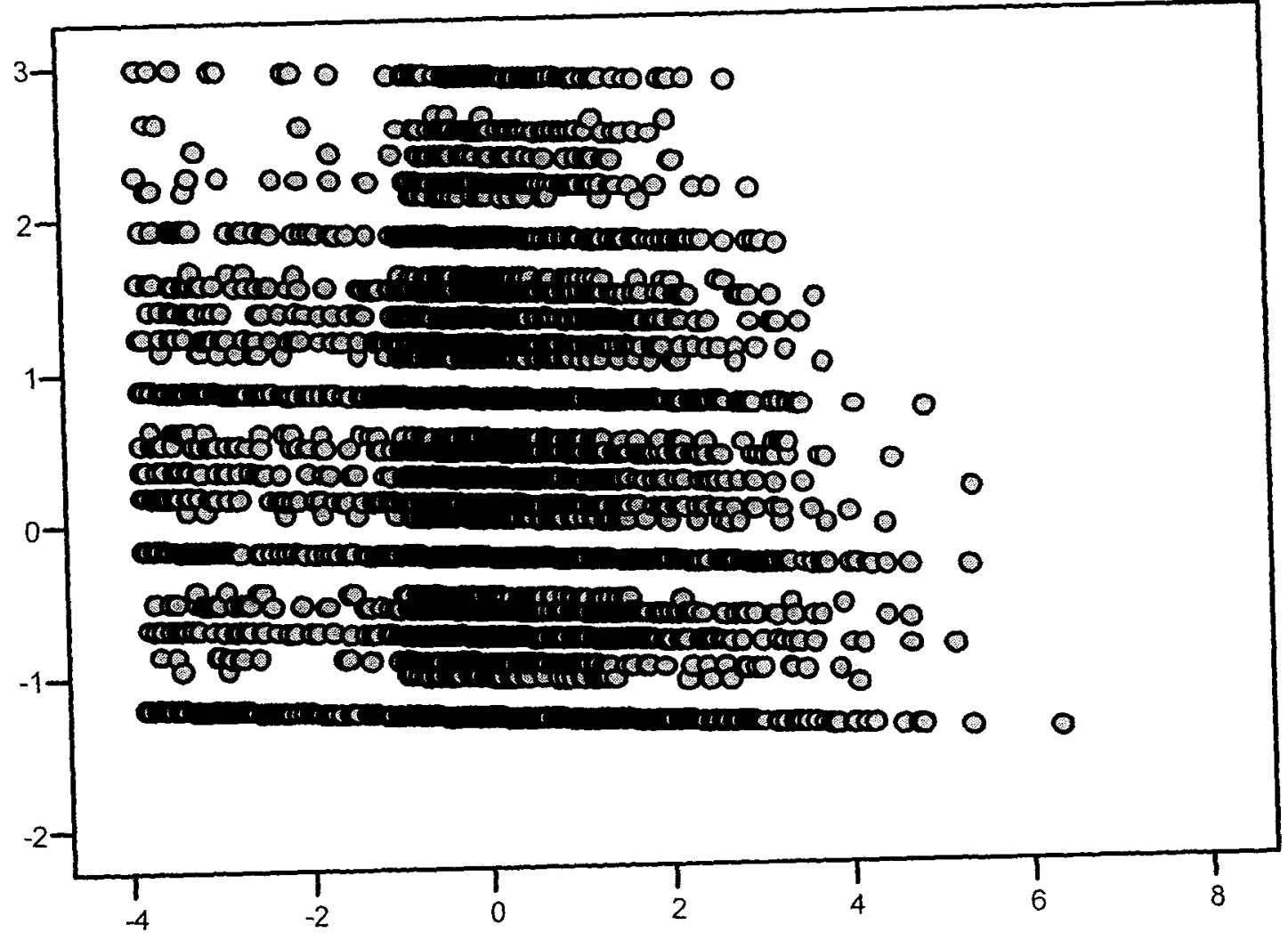

$x$-axis Regression Standardized Predicted Value

$y$-axis Regression Standardized Residual 
Scatterplots (Non-Work Demands and Family-Work Interference)

\author{
Scatterplot
}

Dependent Variable: fwc

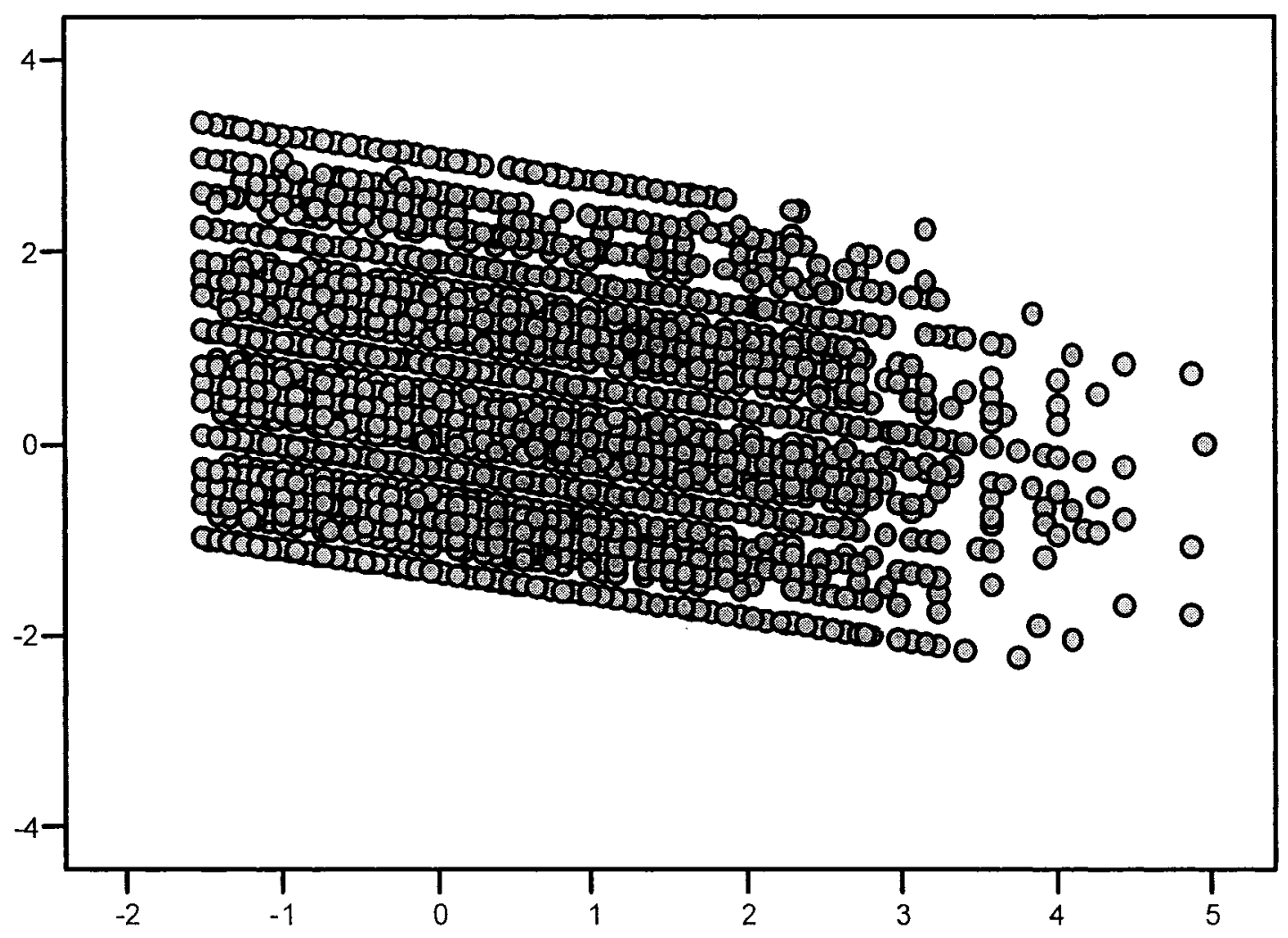

x-axis Regression Standardized Predicted Value

y-axis Regression Standardized Residual 
Appendix C Summary of Testing Strain and Buffer Hypotheses

The first table below summarizes Thesis Model 1 and the covariates, independent, moderator and dependent variables for both strain and buffer hypotheses. It was decided not re-create summary tables for Models 2 and 3 since the tables would be identical except for the dependent variables. Workfamily interference and family-work interference are the dependent variables for Models 2 and 3 respectively.

Summary of Regressions Used to Test Hypotheses Associated with Model 1

a. The Strain Hypotheses

\begin{tabular}{|l|l|l|l|}
\hline Hypothesis & Dependent Variable & $\begin{array}{l}\text { Independent } \\
\text { Variable }\end{array}$ & Co-variates \\
\hline RO1 & Role Overload & Work Demands & $\begin{array}{l}\text { Gender, Job Type, } \\
\text { Non-work Demands }\end{array}$ \\
\hline RO2 & Role Overload & $\begin{array}{l}\text { Non-work } \\
\text { Demands }\end{array}$ & $\begin{array}{l}\text { Gender, Job Type, } \\
\text { Work Demands }\end{array}$ \\
\hline RO3a & Role Overload & $\begin{array}{l}\text { Flextime (vs. } \\
\text { regular week) }\end{array}$ & $\begin{array}{l}\text { Gender, Job Type, } \\
\text { Work Demands, Non- } \\
\text { work Demands }\end{array}$ \\
\hline RO3b & Role Overload & $\begin{array}{l}\text { CWW (vs. } \\
\text { regular week) }\end{array}$ & $\begin{array}{l}\text { Gender, Job Type, } \\
\text { Work Demands, Non- } \\
\text { work Demands }\end{array}$ \\
\hline RO4 & Role Overload & $\begin{array}{l}\text { Telework (vs. } \\
\text { regular) }\end{array}$ & $\begin{array}{l}\text { Gender, Job Type, } \\
\text { Work Demands, Non- } \\
\text { work Demands }\end{array}$ \\
\hline RO5 & Role Overload & Shiftwork & $\begin{array}{l}\text { Gender, Job Type, } \\
\text { Work Demands, Non- } \\
\text { work Demands }\end{array}$ \\
\hline RO6 & Role Overload & $\begin{array}{l}\text { Supervisor } \\
\text { Support }\end{array}$ & $\begin{array}{l}\text { Gender, Job Type, } \\
\text { Work Demands, Non- } \\
\text { work Demands }\end{array}$ \\
\hline $\begin{array}{l}\text { Gender, Job Type, } \\
\text { Work Demands, Non- } \\
\text { work Demands }\end{array}$ \\
\hline
\end{tabular}


b. The Buffer Hypotheses

\begin{tabular}{|c|c|c|c|}
\hline Hypothesis & $\begin{array}{l}\text { Dependent } \\
\text { Variable }\end{array}$ & $\begin{array}{l}\text { Moderator } \\
\text { Variable }\end{array}$ & Co-variates \\
\hline RO7a & $\begin{array}{l}\text { Role } \\
\text { Overload }\end{array}$ & $\begin{array}{l}\text { Flextime } \times \text { Work } \\
\text { Demands }\end{array}$ & $\begin{array}{l}\text { Gender, Job Type, Work } \\
\text { Demands, Non-work Demands, } \\
\text { Flextime }\end{array}$ \\
\hline RO7b & $\begin{array}{l}\text { Role } \\
\text { Overload }\end{array}$ & $\begin{array}{l}\text { CWW } \times \text { Work } \\
\text { Demands }\end{array}$ & $\begin{array}{l}\text { Gender, Job Type, Work } \\
\text { Demands, Non-work Demands, } \\
\text { CWW }\end{array}$ \\
\hline RO7c & $\begin{array}{l}\text { Role } \\
\text { Overload }\end{array}$ & $\begin{array}{l}\text { Telework } x \text { Work } \\
\text { Demands }\end{array}$ & $\begin{array}{l}\text { Gender, Job Type, Work } \\
\text { Demands, Non-work Demands, } \\
\text { Telework }\end{array}$ \\
\hline RO7d & $\begin{array}{l}\text { Role } \\
\text { Overload }\end{array}$ & $\begin{array}{l}\text { Shiftwork } x \text { Work } \\
\text { Demands }\end{array}$ & $\begin{array}{l}\text { Gender, Job Type, Work } \\
\text { Demands, Non-work Demands, } \\
\text { Shiftwork }\end{array}$ \\
\hline RO8 & $\begin{array}{l}\text { Role } \\
\text { Overload }\end{array}$ & $\begin{array}{l}\text { Perceived } \\
\text { Flexibility } x \text { Work } \\
\text { Demands }\end{array}$ & $\begin{array}{l}\text { Gender, Job Type, Work } \\
\text { Demands, Non-work Demands, } \\
\text { Perceived Flexibility }\end{array}$ \\
\hline RO9 & $\begin{array}{l}\text { Role } \\
\text { Overload }\end{array}$ & $\begin{array}{l}\text { Supervisor } \\
\text { Support } x \text { Work } \\
\text { Demands }\end{array}$ & $\begin{array}{l}\text { Gender, Job Type, Work } \\
\text { Demands, Non-work Demands, } \\
\text { Supervisor Support }\end{array}$ \\
\hline RO10a & $\begin{array}{l}\text { Role } \\
\text { Overload }\end{array}$ & $\begin{array}{l}\text { Flextime } \times \text { Non- } \\
\text { work Demands }\end{array}$ & $\begin{array}{l}\text { Gender, Job Type, Work } \\
\text { Demands, Non-work Demands, } \\
\text { Flextime }\end{array}$ \\
\hline R010b & $\begin{array}{l}\text { Role } \\
\text { Overload }\end{array}$ & $\begin{array}{l}\text { CWW } \times \text { Non- } \\
\text { work Demands }\end{array}$ & $\begin{array}{l}\text { Gender, Job Type, Work } \\
\text { Demands, Non-work Demands, } \\
\text { CWW }\end{array}$ \\
\hline RO10c & $\begin{array}{l}\text { Role } \\
\text { Overload }\end{array}$ & $\begin{array}{l}\text { Telework x Non- } \\
\text { work Demands }\end{array}$ & $\begin{array}{l}\text { Gender, Job Type, Work } \\
\text { Demands, Non-work Demands, } \\
\text { Telework }\end{array}$ \\
\hline RO10d & $\begin{array}{l}\text { Role } \\
\text { Overload }\end{array}$ & $\begin{array}{l}\text { Shiftwork } \times \text { Non- } \\
\text { work Demands }\end{array}$ & $\begin{array}{l}\text { Gender, Job Type, Work } \\
\text { Demands, Non-work Demands, } \\
\text { Shiftwork }\end{array}$ \\
\hline R011 & $\begin{array}{l}\text { Role } \\
\text { Overload }\end{array}$ & $\begin{array}{l}\text { Perceived } \\
\text { Flexibility } \mathrm{x} \\
\text { Non-work } \\
\text { Demands } \\
\end{array}$ & $\begin{array}{l}\text { Gender, Job Type, Work } \\
\text { Demands, Non-work Demands, } \\
\text { Perceived Flexibility }\end{array}$ \\
\hline RO12 & $\begin{array}{l}\text { Role } \\
\text { Overload }\end{array}$ & $\begin{array}{l}\text { Supervisor } \\
\text { Support } x \\
\text { Non-work } \\
\text { Demands }\end{array}$ & $\begin{array}{l}\text { Gender, Job Type, Work } \\
\text { Demands, Non-work Demands, } \\
\text { Supervisor Support }\end{array}$ \\
\hline
\end{tabular}


Summary of Regressions Used to Test Hypotheses Associated with Model Four

a. The Direct Hypotheses

\begin{tabular}{|l|l|l|l|}
\hline Hypothesis & $\begin{array}{l}\text { Dependent } \\
\text { Variable }\end{array}$ & $\begin{array}{l}\text { Independent } \\
\text { Variable }\end{array}$ & Co-variates \\
\hline PFLEX1a & $\begin{array}{l}\text { Perceived } \\
\text { Flexibility }\end{array}$ & Flextime & $\begin{array}{l}\text { Gender, Job Type, Work } \\
\text { Demands, Non-work } \\
\text { demands }\end{array}$ \\
\hline PFLEX1b & $\begin{array}{l}\text { Perceived } \\
\text { Flexibility }\end{array}$ & CWW & $\begin{array}{l}\text { Gender, Job Type, Work } \\
\text { Demands, Non-work } \\
\text { demands }\end{array}$ \\
\hline PFLEX1c & $\begin{array}{l}\text { Perceived } \\
\text { Flexibility }\end{array}$ & Telework & $\begin{array}{l}\text { Gender, Job Type, Work } \\
\text { Demands, Non-work } \\
\text { demands }\end{array}$ \\
\hline PFLEX2 & $\begin{array}{l}\text { Perceived } \\
\text { Flexibility }\end{array}$ & Shiftwork & $\begin{array}{l}\text { Gender, Job Type, Work } \\
\text { Demands, Non-work } \\
\text { demands }\end{array}$ \\
\hline PFLEX3 & $\begin{array}{l}\text { Perceived } \\
\text { Flexibility }\end{array}$ & $\begin{array}{l}\text { Supervisor } \\
\text { Support }\end{array}$ & $\begin{array}{l}\text { Gender, Job Type, Work } \\
\text { Demands, Non-work } \\
\text { demands }\end{array}$ \\
\hline
\end{tabular}

b. The Interaction Hypotheses

\begin{tabular}{|l|l|l|l|}
\hline Hypothesis & $\begin{array}{l}\text { Dependent } \\
\text { Variable }\end{array}$ & $\begin{array}{l}\text { Moderator } \\
\text { Variable }\end{array}$ & Co-variates \\
\hline PFLEX4a & $\begin{array}{l}\text { Perceived } \\
\text { Flexibility }\end{array}$ & $\begin{array}{l}\text { Flextime } x \\
\text { Supervisor } \\
\text { Support }\end{array}$ & $\begin{array}{l}\text { Gender, Job Type, Work } \\
\text { Demands, Non-work } \\
\text { demands, Flextime, } \\
\text { Supervisor Support }\end{array}$ \\
\hline PFLEX4b & $\begin{array}{l}\text { Perceived } \\
\text { Flexibility }\end{array}$ & $\begin{array}{l}\text { CWW } x \text { Supervisor } \\
\text { Support }\end{array}$ & $\begin{array}{l}\text { Gender, Job Type, Work } \\
\text { Demands, Non-work } \\
\text { demands, CWW, } \\
\text { Supervisor Support }\end{array}$ \\
\hline PFLEX4c & $\begin{array}{l}\text { Perceived } \\
\text { Flexibility }\end{array}$ & $\begin{array}{l}\text { Telework } x \\
\text { Supervisor } \\
\text { Support }\end{array}$ & $\begin{array}{l}\text { Gender, Job Type, Work } \\
\text { Demands, Non-work } \\
\text { demands, Telework, } \\
\text { Supervisor Support }\end{array}$ \\
\hline PFLEX5 & $\begin{array}{l}\text { Perceived } \\
\text { Flexibility }\end{array}$ & $\begin{array}{l}\text { Shiftwork } x \\
\text { Supervisor } \\
\text { Support }\end{array}$ & $\begin{array}{l}\text { Gender, Job Type, Work } \\
\text { Demands, Non-work } \\
\text { demands, Shiftwork, } \\
\text { Supervisor Support }\end{array}$ \\
\hline
\end{tabular}




\section{Appendix D Unstandardized and Standardized Coefficients for Models 1-4}

Unstandardized and Standardized Coefficients for Model One (Role Overload)

Strain Hypotheses (ROH1 and ROH2)

\begin{tabular}{|l|l|l|l|}
\hline & $\begin{array}{l}\mathrm{ROH} 1 \\
\text { and } \\
\mathrm{ROH} 2\end{array}$ & $\begin{array}{l}\mathrm{ROH} 1 \text { and } \\
\mathrm{ROH} 2\end{array}$ & $\begin{array}{l}\mathrm{ROH} 1 \text { and } \\
\mathrm{ROH} 2\end{array}$ \\
\hline Variables & $\mathrm{b}$ & $\mathrm{p}$ & Beta \\
\hline Gender & .153 & $<.001$ & .170 \\
\hline Job Type & -.057 & $<.001$ & -.064 \\
\hline WD & .017 & $<.001$ & .182 \\
\hline NWD & .011 & $<.001$ & .146 \\
\hline
\end{tabular}

NOTE: $b=$ unstandardized coefficients; $p=p$ value and Beta=standardized coefficients

Strain Hypotheses (ROH3a and $\mathrm{ROH} 3 \mathrm{~b}$ )

\begin{tabular}{|l|l|l|l|l|l|l|}
\hline & ROH3a & ROH3a & ROH3a & ROH3b & ROH3b & ROH3b \\
\hline Variables & $\mathrm{b}$ & $\mathrm{p}$ & $\mathrm{Beta}$ & $\mathrm{b}$ & $\mathrm{p}$ & Beta \\
\hline Gender & .152 & $<.001$ & .169 & .152 & $<.001$ & .168 \\
\hline Job Type & -.062 & $<.001$ & -.070 & -.065 & $<.001$ & -.073 \\
\hline WD & .018 & $<.001$ & .187 & .018 & $<.001$ & .176 \\
\hline NWD & .011 & $<.001$ & .141 & .011 & $<.001$ & .143 \\
\hline Flextime & -.018 & .031 & -.018 & - & - & - \\
\hline CWW & - & - & - & .026 & .008 & .023 \\
\hline
\end{tabular}

Strain Hypotheses (ROH3c and $\mathrm{ROH} 4)$

\begin{tabular}{|l|l|l|l|l|l|l|}
\hline & ROH3c & ROH3c & ROH3c & ROH4 & ROH4 & ROH4 \\
\hline Variables & $\mathrm{b}$ & $\mathrm{p}$ & Beta & $\mathrm{b}$ & $\mathrm{p}$ & Beta \\
\hline Gender & .155 & $<.001$ & .170 & .153 & $<.001$ & .170 \\
\hline Job Type & -.068 & $<.001$ & -.075 & -.057 & $<.001$ & -.064 \\
\hline WD & .018 & $<.001$ & .181 & .017 & $<.001$ & .182 \\
\hline NWD & .010 & $<.001$ & .135 & .011 & $<.001$ & .146 \\
\hline Telework & .001 & .985 & .000 & - & - & - \\
\hline Shiftwork & - & - & - & -.005 & .606 & -.004 \\
\hline
\end{tabular}


Strain Hypotheses (ROH5 and ROH6)

\begin{tabular}{|l|l|l|l|l|l|l|}
\hline & ROH5 & ROH5 & ROH5 & ROH6 & ROH6 & ROH6 \\
\hline Variables & $\mathrm{b}$ & $\mathrm{p}$ & $\mathrm{Beta}$ & $\mathrm{b}$ & $\mathrm{p}$ & Beta \\
\hline Gender & .124 & $<.001$ & .138 & .163 & $<.001$ & .181 \\
\hline Job Type & -.059 & $<.001$ & -.067 & -.067 & $<.001$ & -.075 \\
\hline WD & .013 & $<.001$ & .136 & .016 & $<.001$ & .169 \\
\hline NWD & .009 & $<.001$ & .116 & .010 & $<.001$ & .134 \\
\hline $\begin{array}{l}\text { Perceived } \\
\text { Flexibility }\end{array}$ & -.307 & $<.001$ & -.312 & - & - & - \\
\hline SS & - & - & - & -.278 & $<.001$ & -.236 \\
\hline
\end{tabular}

Buffer Hypotheses (ROH7a and ROH7b)

\begin{tabular}{|l|l|l|l|l|l|l|}
\hline & ROH7a & ROH7a & ROH7a & ROH7b & ROH7b & ROH7b \\
\hline Variables & b & $p$ & Beta & b & p & Beta \\
\hline Gender & .152 & $<.001$ & .169 & .152 & $<.001$ & .168 \\
\hline Job Type & -.062 & $<.001$ & -.070 & -.065 & $<.001$ & -.073 \\
\hline WD & .018 & $<.001$ & .186 & .017 & $<.001$ & .171 \\
\hline NWD & .011 & $<.001$ & .141 & .011 & $<.001$ & .143 \\
\hline Flextime & -.017 & .048 & -.017 & - & - & - \\
\hline CWW & - & - & - & .026 & .007 & .023 \\
\hline $\begin{array}{l}\text { WD x } \\
\text { Flextime }\end{array}$ & .000 & .758 & .003 & - & - & - \\
\hline $\begin{array}{l}\text { WD } x \\
\text { CWW }\end{array}$ & - & - & - & -.001 & .494 & -.008 \\
\hline
\end{tabular}

Buffer Hypotheses (ROH7c and ROH7d)

\begin{tabular}{|l|l|l|l|l|l|l|}
\hline & ROH7c & ROH7c & ROH7c & ROH7d & ROH7d & ROH7d \\
\hline Variables & b & p & Beta & b & p & Beta \\
\hline Gender & .155 & $<.001$ & .170 & .153 & $<.001$ & .170 \\
\hline Job Type & -.068 & $<.001$ & -.075 & -.055 & $<.001$ & -.062 \\
\hline WD & .017 & $<.001$ & .177 & .015 & $<.001$ & .152 \\
\hline NWD & .010 & $<.001$ & .135 & .011 & $<.001$ & .147 \\
\hline Telework & .003 & .929 & .001 & - & - & - \\
\hline Shiftwork & - & - & - & .006 & .569 & .004 \\
\hline $\begin{array}{l}\text { WD } x \\
\text { Telework }\end{array}$ & .000 & .863 & -.005 & - & - & - \\
\hline $\begin{array}{l}\text { WD } x \\
\text { Shiftwork }\end{array}$ & - & - & - & -.004 & $<.001$ & -.047 \\
\hline
\end{tabular}


Buffer Hypotheses (ROH8 and ROH9)

\begin{tabular}{|l|l|l|l|l|l|l|}
\hline & ROH8 & ROH8 & ROH8 & ROH9 & ROH9 & ROH9 \\
\hline Variables & $\mathrm{b}$ & $\mathrm{p}$ & $\mathrm{Beta}$ & $\mathrm{b}$ & $\mathrm{p}$ & Beta \\
\hline Gender & .125 & $<.001$ & .139 & .163 & $<.001$ & .181 \\
\hline Job Type & -.059 & $<.001$ & -.067 & -.067 & $<.001$ & -.075 \\
\hline WD & .013 & $<.001$ & .140 & .016 & $<.001$ & .170 \\
\hline NWD & .009 & $<.001$ & .115 & .010 & $<.001$ & .134 \\
\hline $\begin{array}{l}\text { Perceived } \\
\text { Flexibility }\end{array}$ & -.313 & $<.001$ & -.318 & - & - & - \\
\hline $\begin{array}{l}\text { Supervisor } \\
\text { Support }\end{array}$ & - & - & - & -.282 & $<.001$ & -.239 \\
\hline $\begin{array}{l}\text { WD } x \\
\text { Pflex }\end{array}$ & .003 & $<.001$ & .030 & - & - & - \\
\hline WD x SS & - & - & - & .002 & .022 & .017 \\
\hline
\end{tabular}

Buffer Hypotheses (ROH10a and 10b)

\begin{tabular}{|l|l|l|l|l|l|l|}
\hline & ROH10a & ROH10a & ROH10a & ROH10b & ROH10b & ROH10b \\
\hline Variables & $\mathrm{b}$ & $\mathrm{p}$ & Beta & $\mathrm{b}$ & $\mathrm{p}$ & Beta \\
\hline Gender & .152 & $<.001$ & .169 & .152 & $<.001$ & .168 \\
\hline Job Type & -.062 & $<.001$ & -.070 & -.065 & $<.001$ & -.073 \\
\hline WD & .018 & $<.001$ & .187 & .018 & $<.001$ & .176 \\
\hline NWD & .011 & $<.001$ & .146 & .012 & $<.001$ & .152 \\
\hline Flextime & -.017 & .033 & -.018 & - & - & - \\
\hline CWW & - & - & - & .025 & .009 & .023 \\
\hline $\begin{array}{l}\text { NWD } x \\
\text { Flextime }\end{array}$ & .001 & .229 & .011 & - & - & - \\
\hline $\begin{array}{l}\text { NWD } x \\
\text { CWW }\end{array}$ & - & - & - & .001 & .139 & .016 \\
\hline
\end{tabular}

Buffer Hypotheses (ROH10c and ROH10d)

\begin{tabular}{|l|l|l|l|l|l|l|}
\hline & ROH10c & ROH10c & ROH10c & ROH10d & ROH10d & ROH10d \\
\hline Variables & $\mathrm{b}$ & $\mathrm{p}$ & $\mathrm{Beta}$ & $\mathrm{b}$ & $\mathrm{p}$ & Beta \\
\hline Gender & .155 & $<.001$ & .170 & .153 & $<.001$ & .170 \\
\hline Job Type & -.068 & $<.001$ & -.075 & -.057 & $<.001$ & -.064 \\
\hline WD & .018 & $<.001$ & .181 & .017 & $<.001$ & .182 \\
\hline NWD & .010 & $<.001$ & .129 & .011 & $<.001$ & .145 \\
\hline Telework & .001 & .967 & .000 & - & - & - \\
\hline Shiftwork & - & - & - & -.005 & .603 & -.004 \\
\hline $\begin{array}{l}\text { NWD } x \\
\text { Telework }\end{array}$ & .000 & .871 & -.006 & - & - & - \\
\hline $\begin{array}{l}\text { NWD } x \\
\text { Shiftwork }\end{array}$ & - & - & - & .000 & .823 & -.002 \\
\hline
\end{tabular}


Buffer Hypotheses (ROH11 and ROH12)

\begin{tabular}{|l|l|l|l|l|l|l|}
\hline & $\mathrm{ROH} 11$ & $\mathrm{ROH} 11$ & $\mathrm{ROH} 11$ & $\mathrm{ROH} 12$ & $\mathrm{ROH} 12$ & $\mathrm{ROH} 12$ \\
\hline Variables & $\mathrm{b}$ & $\mathrm{p}$ & $\mathrm{Beta}$ & $\mathrm{b}$ & $\mathrm{p}$ & Beta \\
\hline Gender & .125 & $<.001$ & .139 & .162 & $<.001$ & .181 \\
\hline Job Type & -.060 & $<.001$ & -.067 & -.067 & $<.001$ & -.076 \\
\hline WD & .013 & $<.001$ & .136 & .016 & $<.001$ & .169 \\
\hline NWD & .009 & $<.001$ & .117 & .010 & $<.001$ & .135 \\
\hline $\begin{array}{l}\text { Perceived } \\
\text { Flexibility }\end{array}$ & -.307 & $<.001$ & -.311 & - & - & - \\
\hline $\begin{array}{l}\text { Supervisor } \\
\text { Support }\end{array}$ & - & - & - & -.280 & $<.001$ & -.238 \\
\hline $\begin{array}{l}\text { NWD x } \\
\text { Pflex }\end{array}$ & .002 & .003 & .021 & - & - & - \\
\hline NWD x SS & - & - & - & .003 & $<.001$ & .030 \\
\hline
\end{tabular}

Unstandardized and Standardized Coefficients for Model Two (Work-Family Interference)

Strain Hypotheses (WFIH1 and WFIH2)

\begin{tabular}{|l|l|l|l|}
\hline & $\begin{array}{l}\text { WFIH1 } \\
\text { and } \\
\text { WFIH2 }\end{array}$ & $\begin{array}{l}\text { WFIH1 and } \\
\text { WFIH2 }\end{array}$ & $\begin{array}{l}\text { WFIH1 and } \\
\text { WFIH2 }\end{array}$ \\
\hline Variables & $\mathrm{b}$ & $\mathrm{p}$ & Beta \\
\hline Gender & .009 & .215 & .009 \\
\hline Job Type & -.132 & $<.001$ & -.130 \\
\hline WD & .029 & $<.001$ & .266 \\
\hline NWD & .010 & $<.001$ & .110 \\
\hline
\end{tabular}

NOTE: $b=$ unstandardized coefficients; $p=p$ value and Beta=standardized coefficients

Strain Hypotheses (WFIH3a and WFIH3b)

\begin{tabular}{|l|l|l|l|l|l|l|}
\hline & WFIH3a & WFIH3a & WFIH3a & WFIH3b & WFIH3b & WFIH3b \\
\hline Variables & $\mathrm{b}$ & $\mathrm{p}$ & Beta & $\mathrm{b}$ & $\mathrm{p}$ & Beta \\
\hline Gender & .010 & .226 & .010 & .006 & .510 & .006 \\
\hline Job Type & -.138 & $<.001$ & -.135 & -.142 & $<.001$ & -.140 \\
\hline WD & .029 & $<.001$ & .270 & .028 & $<.001$ & .250 \\
\hline NWD & .010 & $<.001$ & .108 & .010 & $<.001$ & .110 \\
\hline Flextime & .002 & .856 & .001 & - & - & - \\
\hline CWW & - & - & - & -.004 & .701 & -.003 \\
\hline
\end{tabular}


Strain Hypotheses (WFIH3c and WFIH4)

\begin{tabular}{|l|l|l|l|l|l|l|}
\hline & WFIH3c & WFIH3c & WFIH3c & WFIH4 & WFIH4 & WFIH4 \\
\hline Variables & $\mathrm{b}$ & $\mathrm{p}$ & Beta & $\mathrm{b}$ & $\mathrm{p}$ & Beta \\
\hline Gender & .009 & .381 & .008 & .011 & .149 & .011 \\
\hline Job Type & -.149 & $<.001$ & -.145 & -.131 & $<.001$ & -.130 \\
\hline WD & .029 & $<.001$ & .257 & .029 & $<.001$ & .264 \\
\hline NWD & .009 & $<.001$ & .107 & .010 & $<.001$ & .112 \\
\hline Telework & .036 & .345 & .009 & - & - & - \\
\hline Shiftwork & - & - & - & .119 & $<.001$ & 083 \\
\hline
\end{tabular}

Strain Hypotheses (WFIH5 and WFIH6)

\begin{tabular}{|l|l|l|l|l|l|l|}
\hline & WFIH5 & WFIH5 & WFIH5 & WFIH6 & WFIH6 & WFIH6 \\
\hline Variables & $\mathrm{b}$ & $\mathrm{p}$ & Beta & $\mathrm{b}$ & $\mathrm{p}$ & Beta \\
\hline Gender & -.030 & $<.001$ & -.030 & .023 & $<.001$ & .023 \\
\hline Job Type & -.136 & $<.001$ & -.134 & -.147 & $<.001$ & -.145 \\
\hline WD & .023 & $<.001$ & .210 & .027 & $<.001$ & .250 \\
\hline NWD & .006 & $<.001$ & .072 & .008 & $<.001$ & .095 \\
\hline $\begin{array}{l}\text { Perceived } \\
\text { Flexibility }\end{array}$ & -.431 & $<.001$ & -.385 & - & - & - \\
\hline SS & - & - & - & -.391 & $<.001$ & -.292 \\
\hline
\end{tabular}

Buffer Hypotheses (WFIH7a and WFIH7b)

\begin{tabular}{|l|l|l|l|l|l|l|}
\hline & WFIH7a & WFIH7a & WFIH7a & WFIH7b & WFIH7b & WFIH7b \\
\hline Variables & $\mathrm{b}$ & $\mathrm{p}$ & Beta & $\mathrm{b}$ & $\mathrm{p}$ & Beta \\
\hline Gender & .010 & .232 & .010 & .006 & .491 & .006 \\
\hline Job Type & -.138 & $<.001$ & -.136 & -.142 & $<.001$ & -.139 \\
\hline WD & .029 & $<.001$ & .271 & .027 & $<.001$ & .241 \\
\hline NWD & .010 & $<.001$ & .108 & .010 & $<.001$ & .110 \\
\hline Flextime & .000 & .958 & .000 & - & - & - \\
\hline CWW & - & - & - & -.004 & .728 & -.003 \\
\hline $\begin{array}{l}\text { WD } x \\
\text { Flextime }\end{array}$ & .000 & .668 & .004 & - & - & - \\
\hline $\begin{array}{l}\text { WD } x \\
\text { CWW }\end{array}$ & - & - & - & -.002 & .233 & .014 \\
\hline
\end{tabular}


Buffer Hypotheses (WFIH7c and WFIH7d)

\begin{tabular}{|l|l|l|l|l|l|l|}
\hline & WFIH7c & WFIH7c & WFIH7c & WFIH7d & WFIH7d & WFIH7d \\
\hline Variables & $\mathrm{b}$ & $\mathrm{p}$ & Beta & $\mathrm{b}$ & $\mathrm{p}$ & Beta \\
\hline Gender & .009 & .381 & .008 & .012 & .118 & .012 \\
\hline Job Type & -.149 & $<.001$ & -.145 & -.129 & $<.001$ & -.127 \\
\hline WD & .029 & $<.001$ & .257 & .024 & $<.001$ & .218 \\
\hline NWD & .009 & $<.001$ & .107 & .010 & $<.001$ & .112 \\
\hline Telework & .036 & .393 & .009 & - & - & - \\
\hline Shiftwork & - & - & - & .138 & $<.001$ & .096 \\
\hline $\begin{array}{l}\text { WD x } \\
\text { Telework }\end{array}$ & .000 & .993 & .000 & - & - & - \\
\hline $\begin{array}{l}\text { WD x } \\
\text { Shiftwork }\end{array}$ & & & & -.008 & $<.001$ & -.074 \\
\hline
\end{tabular}

Buffer Hypotheses (WFIH8 and WFIH9)

\begin{tabular}{|l|l|l|l|l|l|l|}
\hline & WFIH8 & WFIH8 & WFIH8 & WFIH9 & WFIH9 & WFIH9 \\
\hline Variables & $\mathrm{b}$ & $\mathrm{p}$ & Beta & $\mathrm{b}$ & $\mathrm{p}$ & Beta \\
\hline Gender & -.030 & $<.001$ & -.029 & .024 & .001 & .023 \\
\hline Job Type & -.136 & $<.001$ & -.134 & -.146 & $<.001$ & -.144 \\
\hline WD & .023 & $<.001$ & .213 & .027 & $<.001$ & .252 \\
\hline NWD & .006 & $<.001$ & .072 & .008 & $<.001$ & .095 \\
\hline $\begin{array}{l}\text { Perceived } \\
\text { Flexibility }\end{array}$ & -.436 & $<.001$ & -.389 & - & - & - \\
\hline $\begin{array}{l}\text { Supervisor } \\
\text { Support }\end{array}$ & - & - & - & -.399 & $<.001$ & -.298 \\
\hline $\begin{array}{l}\text { WD x } \\
\text { Pflex }\end{array}$ & .002 & .004 & .020 & - & - & - \\
\hline WD x SS & - & - & - & .004 & $<.001$ & .028 \\
\hline
\end{tabular}

\section{Buffer Hypotheses (WFIH10a and WFIH10b)}

\begin{tabular}{|l|l|l|l|l|l|l|}
\hline & WFIH10a & WFIH10a & WFIH10a & WFIH10b & WFIH10b & WFIH10b \\
\hline Variables & b & p & Beta & b & p & Beta \\
\hline Gender & .010 & .226 & .010 & .006 & .510 & .006 \\
\hline Job Type & -.138 & $<.001$ & -.135 & -.142 & $<.001$ & -.140 \\
\hline WD & .029 & $<.001$ & .270 & .028 & $<.001$ & .250 \\
\hline NWD & .010 & $<.001$ & .109 & .010 & $<.001$ & .113 \\
\hline Flextime & .002 & .853 & .002 & - & - & - \\
\hline CWW & - & - & - & -.004 & .690 & -.003 \\
\hline NWD x Flextime & .000 & .749 & .003 & - & - & - \\
\hline NWD x CWW & - & - & - & .000 & .664 & .005 \\
\hline
\end{tabular}


Buffer Hypotheses (WFIH10c and WFIH10d)

\begin{tabular}{|l|l|l|l|l|l|l|}
\hline & WFIH10c & WFIH10c & WFIH10c & WFIH10d & WFIH10d & WFIH10d \\
\hline Variables & b & $p$ & Beta & b & p & Beta \\
\hline Gender & .009 & .384 & .008 & .011 & .146 & .011 \\
\hline Job Type & -.149 & $<.001$ & -.145 & -.131 & $<.001$ & -.129 \\
\hline WD & .029 & $<.001$ & .257 & .029 & $<.001$ & .264 \\
\hline NWD & .011 & $<.001$ & .125 & .010 & $<.001$ & .117 \\
\hline Telework & .033 & .386 & .008 & - & - & - \\
\hline Shiftwork & - & - & - & .119 & $<.001$ & .083 \\
\hline NWD x Telework & .002 & .607 & .018 & - & - & - \\
\hline NWD $\times$ Shiftwork & - & - & - & .001 & .386 & .009 \\
\hline
\end{tabular}

Buffer Hypotheses (WFIH11 and WFIH12)

\begin{tabular}{|l|l|l|l|l|l|l|}
\hline & WFIH11 & WFIH11 & WFIH11 & WFIH12 & WFIH12 & WFIH12 \\
\hline Variables & $\mathrm{b}$ & $\mathrm{p}$ & Beta & $\mathrm{b}$ & $\mathrm{p}$ & Beta \\
\hline Gender & -.030 & $<.001$ & -.030 & .023 & .001 & .023 \\
\hline Job Type & -.136 & $<.001$ & -.134 & -.147 & $<.001$ & -.145 \\
\hline WD & .023 & $<.001$ & .210 & .027 & $<.001$ & .250 \\
\hline NWD & .006 & $<.001$ & .072 & .008 & $<.001$ & .095 \\
\hline $\begin{array}{l}\text { Perceived } \\
\text { Flexibility }\end{array}$ & -.431 & $<.001$ & -.385 & - & - & - \\
\hline $\begin{array}{l}\text { Supervisor } \\
\text { Support }\end{array}$ & - & - & - & -.392 & $<.001$ & -.292 \\
\hline $\begin{array}{l}\text { NWD x } \\
\text { Pflex }\end{array}$ & -.001 & .296 & -.007 & - & - & - \\
\hline NWD x SS & - & - & - & .001 & .526 & .004 \\
\hline
\end{tabular}

Unstandardized and Standardized Coefficients for Model Three (Family-Work Interference)

Strain Hypotheses (FWIH1 and FWIH2)

\begin{tabular}{|l|l|l|l|}
\hline & $\begin{array}{l}\text { FWIH1 } \\
\text { and } \\
\text { FWIH2 }\end{array}$ & $\begin{array}{l}\text { FWIH1 and } \\
\text { FWIH2 }\end{array}$ & $\begin{array}{l}\text { FWIH1 and } \\
\text { FWIH2 }\end{array}$ \\
\hline Variables & b & p & Beta \\
\hline Gender & -.005 & .485 & -.005 \\
\hline Job Type & -.025 & .001 & -.027 \\
\hline WD & .003 & .001 & .025 \\
\hline NWD & .019 & $<.001$ & .235 \\
\hline
\end{tabular}

NOTE: $b=$ unstandardized coefficients; $p=p$ value and Beta=standardized coefficients 
Strain Hypotheses (FWIH3a and FWIH3b)

\begin{tabular}{|l|l|l|l|l|l|l|}
\hline & FWIH3a & FWIH3a & FWIH3a & FWIH3b & FWIH3b & FWIH3b \\
\hline Variables & $\mathrm{b}$ & $\mathrm{p}$ & Beta & $\mathrm{b}$ & $\mathrm{p}$ & Beta \\
\hline Gender & -.004 & .589 & -.005 & -.001 & .925 & -.001 \\
\hline Job Type & -.022 & .006 & -.024 & -.034 & $<.001$ & -.036 \\
\hline WD & .002 & .062 & .016 & .004 & $<.001$ & .041 \\
\hline NWD & .019 & $<.001$ & .235 & .018 & $<.001$ & .227 \\
\hline Flextime & .027 & .002 & .025 & - & - & - \\
\hline CWW & - & - & - & .016 & .136 & .013 \\
\hline
\end{tabular}

Strain Hypotheses (FWIH3c and FWIH4)

\begin{tabular}{|l|l|l|l|l|l|l|}
\hline & FWIH3c & FWIH3c & FWIH3c & FWIH4 & FWIH4 & FWIH4 \\
\hline Variables & $\mathrm{b}$ & $\mathrm{p}$ & Beta & $\mathrm{b}$ & $\mathrm{p}$ & Beta \\
\hline Gender & .000 & .984 & .000 & -.005 & .469 & -.006 \\
\hline Job Type & -.031 & .001 & -.033 & -.025 & .001 & -.027 \\
\hline WD & .003 & .004 & .029 & .003 & .001 & .026 \\
\hline NWD & .018 & $<.001$ & .223 & .019 & $<.001$ & .235 \\
\hline Telework & .089 & .014 & .024 & - & - & - \\
\hline Shiftwork & - & - & - & -.015 & .141 & -.011 \\
\hline
\end{tabular}

Strain Hypotheses (FWIH5 and FWIH6)

\begin{tabular}{|l|l|l|l|l|l|l|}
\hline & FWIH5 & FWIH5 & FWIH5 & FWIH6 & FWIH6 & FWIH6 \\
\hline Variables & $\mathrm{b}$ & $\mathrm{p}$ & Beta & $\mathrm{b}$ & $\mathrm{p}$ & Beta \\
\hline Gender & -.020 & .006 & -.021 & .001 & .893 & .001 \\
\hline Job Type & -.027 & $<.001$ & -.028 & -.032 & $<.001$ & -.033 \\
\hline WD & .000 & .735 & .003 & .002 & .023 & .018 \\
\hline NWD & .018 & $<.001$ & .219 & .019 & $<.001$ & .227 \\
\hline $\begin{array}{l}\text { Perceived } \\
\text { Flexibility }\end{array}$ & -.163 & $<.001$ & -.155 & - & - & - \\
\hline $\begin{array}{l}\text { Supervisor } \\
\text { Support }\end{array}$ & - & - & - & -.173 & $<.001$ & -.138 \\
\hline
\end{tabular}

Buffer Hypotheses (FWIH7a and FWIH7b)

\begin{tabular}{|l|l|l|l|l|l|l|}
\hline & FWIH7a & FWIH7a & FWIH7a & FWIH7b & FWIH7b & FWIH7b \\
\hline Variables & $\mathrm{b}$ & $\mathrm{p}$ & Beta & $\mathrm{b}$ & $\mathrm{p}$ & Beta \\
\hline Gender & -.004 & .664 & -.004 & -.001 & .876 & -.001 \\
\hline Job Type & -.022 & .007 & -.023 & -.035 & $<.001$ & -.037 \\
\hline WD & .001 & .447 & .007 & .006 & $<.001$ & .060 \\
\hline NWD & .019 & $<.001$ & .235 & .018 & $<.001$ & .227 \\
\hline Flextime & .035 & $<.001$ & .033 & - & - & - \\
\hline CWW & - & - & - & .015 & .157 & .012 \\
\hline $\begin{array}{l}\text { WD } x \\
\text { Flextime }\end{array}$ & -.003 & .002 & -.028 & - & - & - \\
\hline $\begin{array}{l}\text { WD } x \\
\text { CWW }\end{array}$ & - & - & - & .003 & .012 & .030 \\
\hline
\end{tabular}


Buffer Hypotheses (FWIH7c and FWIH7d)

\begin{tabular}{|l|l|l|l|l|l|l|}
\hline & FWIH7c & FWIH7c & FWIH7c & FWIH7d & FWIH7d & FWIH7d \\
\hline Variables & $\mathrm{b}$ & $\mathrm{p}$ & Beta & $\mathrm{b}$ & $\mathrm{p}$ & Beta \\
\hline Gender & .000 & .998 & .000 & -.005 & .471 & -.006 \\
\hline Job Type & -.031 & .001 & -.033 & -.025 & .001 & -.026 \\
\hline WD & -.001 & .684 & -.012 & .002 & .017 & .024 \\
\hline NWD & .018 & $<.001$ & .223 & .019 & $<.001$ & .235 \\
\hline Telework & .114 & .004 & .031 & - & - & - \\
\hline Shiftwork & - & - & - & -.014 & .166 & -.011 \\
\hline $\begin{array}{l}\text { WD } x \\
\text { Telework }\end{array}$ & -.005 & .134 & -.044 & - & - & - \\
\hline $\begin{array}{l}\text { WD } x \\
\text { Shiftwork }\end{array}$ & - & - & - & .000 & .833 & -.002 \\
\hline
\end{tabular}

Buffer Hypotheses (FWIH8 and FWIH9)

\begin{tabular}{|l|l|l|l|l|l|l|}
\hline & FWIH8 & FWIH8 & FWIH8 & FWIH9 & FWIH9 & FWIH9 \\
\hline Variables & $\mathrm{b}$ & $\mathrm{p}$ & Beta & $\mathrm{b}$ & $\mathrm{p}$ & Beta \\
\hline Gender & -.020 & .006 & -.021 & .001 & .879 & .001 \\
\hline Job Type & -.026 & $<.001$ & -.028 & -.031 & $<.001$ & -.033 \\
\hline WD & .001 & .416 & .006 & .002 & .016 & .019 \\
\hline NWD & .018 & $<.001$ & .219 & .019 & $<.001$ & .227 \\
\hline $\begin{array}{l}\text { Perceived } \\
\text { Flexibility }\end{array}$ & -.169 & $<.001$ & -.161 & - & - & - \\
\hline $\begin{array}{l}\text { Supervisor } \\
\text { Support }\end{array}$ & - & - & - & -.176 & $<.001$ & -.140 \\
\hline $\begin{array}{l}\text { WD x } \\
\text { Pflex }\end{array}$ & .003 & $<.001$ & .029 & - & - & - \\
\hline WD x SS & - & - & - & .002 & .116 & .012 \\
\hline
\end{tabular}

Buffer Hypotheses (FWIH10a and FWIH10b)

\begin{tabular}{|l|l|l|l|l|l|l|}
\hline & FWIH10a & FWIH10a & FWIH10a & FWIH10b & FWIH10b & FWIH10b \\
\hline Variables & $\mathrm{b}$ & $\mathrm{p}$ & Beta & $\mathrm{b}$ & $\mathrm{p}$ & Beta \\
\hline Gender & -.004 & .597 & -.004 & -.001 & .924 & -.001 \\
\hline Job Type & .022 & .006 & -.023 & -.034 & $<.001$ & -.036 \\
\hline WD & .002 & .058 & .016 & .004 & $<.001$ & .041 \\
\hline NWD & .020 & $<.001$ & .245 & .019 & $<.001$ & .230 \\
\hline Flextime & .027 & .002 & .026 & - & - & - \\
\hline CWW & - & - & - & .015 & .140 & .013 \\
\hline $\begin{array}{l}\text { NWD } x \\
\text { Flextime }\end{array}$ & .002 & .020 & .021 & - & - & - \\
\hline $\begin{array}{l}\text { NWD } x \\
\text { CWW }\end{array}$ & - & - & - & .000 & .701 & .004 \\
\hline
\end{tabular}


Buffer Hypotheses (FWIH10c and FWIH10d)

\begin{tabular}{|l|l|l|l|l|l|l|}
\hline & FWIH10c & FWIH10c & FWIH10c & FWIH10d & FWIH10d & FWIH10d \\
\hline Variables & $\mathrm{b}$ & $\mathrm{p}$ & Beta & $\mathrm{b}$ & $\mathrm{p}$ & Beta \\
\hline Gender & .000 & .974 & .000 & -.006 & .457 & -.006 \\
\hline Job Type & -.031 & .001 & -.033 & -.026 & .001 & -.027 \\
\hline WD & .033 & .004 & .029 & .003 & .001 & .026 \\
\hline NWD & .015 & $<.001$ & .188 & .018 & $<.001$ & .222 \\
\hline Telework & .094 & .011 & .025 & - & - & - \\
\hline Shiftwork & - & - & - & -.015 & .132 & -.011 \\
\hline $\begin{array}{l}\text { NWD } x \\
\text { Telework }\end{array}$ & -.003 & .313 & -.037 & - & - & - \\
\hline $\begin{array}{l}\text { NWD } x \\
\text { Shiftwork }\end{array}$ & - & - & - & -.002 & .067 & -.019 \\
\hline
\end{tabular}

Buffer Hypotheses (FWIH11 and FWIH12)

\begin{tabular}{|l|l|l|l|l|l|l|}
\hline & FWIH11 & FWIH11 & FWIH11 & FWIH12 & FWIH12 & FWIH12 \\
\hline Variables & b & $p$ & Beta & b & p & Beta \\
\hline Gender & -.020 & .006 & -.021 & .001 & .895 & .001 \\
\hline Job Type & -.027 & $<.001$ & -.028 & -.032 & $<.001$ & -.033 \\
\hline WD & .000 & .733 & .003 & .002 & .023 & .018 \\
\hline NWD & .018 & $<.001$ & .219 & .019 & $<.001$ & .227 \\
\hline $\begin{array}{l}\text { Perceived } \\
\text { Flexibility }\end{array}$ & -.163 & $<.001$ & -.155 & - & - & - \\
\hline $\begin{array}{l}\text { Supervisor } \\
\text { Support }\end{array}$ & - & - & - & -.173 & $<.001$ & -.138 \\
\hline $\begin{array}{l}\text { NWD x } \\
\text { Pflex }\end{array}$ & .000 & .774 & -.002 & - & - & - \\
\hline NWD x SS & - & - & - & .000 & .805 & .002 \\
\hline
\end{tabular}

Unstandardized and Standardized Coefficients for Model Four (Perceived Flexibility)

Direct Hypotheses (PFLEXH1a and PFLEXH1b)

\begin{tabular}{|l|l|l|l|l|l|l|}
\hline & $\begin{array}{l}\text { PFLEXH } \\
1 \mathrm{a}\end{array}$ & PFLEX1a & $\begin{array}{l}\text { PFLEX } \\
1 \mathrm{a}\end{array}$ & $\begin{array}{l}\text { PFLEXH } \\
1 \mathrm{~b}\end{array}$ & $\begin{array}{l}\text { PFLEXH } \\
1 \mathrm{~b}\end{array}$ & $\begin{array}{l}\text { PFLEXH } \\
1 \mathrm{~b}\end{array}$ \\
\hline Variables & $\mathrm{b}$ & $\mathrm{p}$ & Beta & $\mathrm{b}$ & $\mathrm{p}$ & Beta \\
\hline Gender & -.098 & $<.001$ & -.108 & -.099 & $<.001$ & -.107 \\
\hline Job Type & .008 & .305 & .009 & .027 & .001 & .030 \\
\hline WD & -.015 & $<.001$ & .160 & -.015 & $<.001$ & -.146 \\
\hline NWD & -.007 & $<.001$ & -.094 & -.008 & $<.001$ & -.107 \\
\hline Flextime & .193 & $<.001$ & .194 & - & - & - \\
\hline CWW & - & - & - & .115 & $<.001$ & .101 \\
\hline
\end{tabular}

NOTE: $b=$ unstandardized coefficients; $p=p$ value and Beta=standardized coefficients 
Direct Hypotheses (PFLEXH1c)

\begin{tabular}{|l|l|l|l|}
\hline & $\begin{array}{l}\text { PFLEXH } \\
\text { 1c }\end{array}$ & $\begin{array}{l}\text { PFLEXH } \\
\text { 1c }\end{array}$ & $\begin{array}{l}\text { PFLEXH } \\
\text { 1c }\end{array}$ \\
\hline Variables & $\mathrm{b}$ & $\mathrm{p}$ & Beta \\
\hline Gender & -.111 & $<.001$ & -.120 \\
\hline Job Type & .023 & .013 & .025 \\
\hline WD & -.015 & $<.001$ & -.151 \\
\hline NWD & -.008 & $<.001$ & -.101 \\
\hline Telework & .455 & $<.001$ & .127 \\
\hline
\end{tabular}

Direct Hypotheses (PFLEXH2 and PFLEXH3)

\begin{tabular}{|l|l|l|l|l|l|l|}
\hline & $\begin{array}{l}\text { PFLEX } \\
\mathrm{H} 2\end{array}$ & $\begin{array}{l}\text { PFLEX } \\
\mathrm{H} 2\end{array}$ & $\begin{array}{l}\text { PFLEX } \\
\mathrm{H} 2\end{array}$ & $\begin{array}{l}\text { PFLEX } \\
\mathrm{H} 3\end{array}$ & $\begin{array}{l}\text { PFLEX } \\
\mathrm{H} 3\end{array}$ & $\begin{array}{l}\text { PFLEX } \\
\mathrm{H} 3\end{array}$ \\
\hline Variables & $\mathrm{b}$ & $\mathrm{p}$ & $\mathrm{Beta}$ & $\mathrm{b}$ & $\mathrm{p}$ & Beta \\
\hline Gender & -.096 & $<.001$ & -.105 & -.106 & $<.001$ & -.116 \\
\hline Job Type & -.010 & .133 & -.012 & .005 & .440 & .006 \\
\hline WD & -.014 & $<.001$ & -.142 & -.012 & $<.001$ & -.129 \\
\hline NWD & -.008 & $<.001$ & -.102 & -.006 & $<.001$ & -.082 \\
\hline Shiftwork & -.285 & $<.001$ & -.223 & - & - & - \\
\hline $\begin{array}{l}\text { Supervisor } \\
\text { Support }\end{array}$ & - & - & - & .382 & $<.001$ & .320 \\
\hline
\end{tabular}

Interaction Hypotheses (PFLEXH4a and PFLEXH4b)

\begin{tabular}{|l|l|l|l|l|l|l|}
\hline & $\begin{array}{l}\text { PFLEXH } \\
4 \mathrm{a}\end{array}$ & $\begin{array}{l}\text { PFLEXH } \\
4 \mathrm{a}\end{array}$ & $\begin{array}{l}\text { PFLEXH } \\
4 \mathrm{a}\end{array}$ & $\begin{array}{l}\text { PFLEXH } \\
4 \mathrm{~b}\end{array}$ & $\begin{array}{l}\text { PFLEXH } \\
4 \mathrm{~b}\end{array}$ & $\begin{array}{l}\text { PFLEXH } \\
4 \mathrm{~b}\end{array}$ \\
\hline Variables & $\mathrm{b}$ & $\mathrm{p}$ & $\mathrm{Beta}$ & $\mathrm{b}$ & $\mathrm{p}$ & Beta \\
\hline Gender & -.111 & $<.001$ & -.123 & -.115 & $<.001$ & -.124 \\
\hline Job Type & .020 & .005 & .023 & .041 & $<.001$ & .044 \\
\hline WD & -.013 & $<.001$ & -.140 & -.013 & $<.001$ & -.127 \\
\hline NWD & -.006 & $<.001$ & -.078 & -.007 & $<.001$ & -.089 \\
\hline Flextime & .175 & $<.001$ & .176 & - & - & - \\
\hline CWW & - & - & - & .120 & $<.001$ & .105 \\
\hline Telework & - & - & - & - & - & - \\
\hline SS & .361 & $<.001$ & .303 & .383 & $<.001$ & .323 \\
\hline $\begin{array}{l}\text { SS } x \\
\text { Flextime }\end{array}$ & -.024 & .022 & -.020 & - & - & - \\
\hline $\begin{array}{l}\text { SS } x \\
\text { CWW }\end{array}$ & - & - & - & -.003 & .822 & -.002 \\
\hline $\begin{array}{l}\text { SS } x \\
\text { Telework }\end{array}$ & - & - & - & - & - & - \\
\hline
\end{tabular}


Interaction Hypotheses (PFLEXH4c and PFLEXH5)

\begin{tabular}{|l|l|l|l|l|l|l|}
\hline & $\begin{array}{l}\text { PFLEXH } \\
\text { 4c }\end{array}$ & $\begin{array}{l}\text { PFLEXH } \\
4 \mathrm{c}\end{array}$ & $\begin{array}{l}\text { PFLEXH } \\
\text { 4c }\end{array}$ & PFLEXH5 & PFLEXH5 & PFLEXH5 \\
\hline Variables & $\mathrm{b}$ & $\mathrm{p}$ & Beta & $\mathrm{b}$ & $\mathrm{p}$ & Beta \\
\hline Gender & -.126 & $<.001$ & -.136 & -.108 & $<.001$ & -.119 \\
\hline Job Type & .037 & $<.001$ & .040 & .004 & .593 & .004 \\
\hline WD & -.013 & $<.001$ & -.130 & -.012 & .001 & -.125 \\
\hline NWD & -.007 & $<.001$ & -.083 & -.007 & .001 & -.086 \\
\hline Telework & .444 & $<.001$ & .124 & - & - & - \\
\hline Shiftwork & - & - & - & -.256 & $<.001$ & -.201 \\
\hline $\begin{array}{l}\text { Supervisor } \\
\text { Support }\end{array}$ & .362 & $<.001$ & .306 & .368 & $<.001$ & .307 \\
\hline $\begin{array}{l}\text { SS x } \\
\text { Telework }\end{array}$ & -.025 & .596 & -.021 & - & - & - \\
\hline $\begin{array}{l}\text { SS x } \\
\text { Shiftwork }\end{array}$ & & & & .005 & .697 & .004 \\
\hline
\end{tabular}




\section{Appendix $E$ Comparison of Change in $R^{2}$ (Models 1, 2, 3 and 4)}

\section{Strain Hypotheses (Models 1-3)}

\begin{tabular}{|c|c|c|c|c|c|c|}
\hline Hypothesis & $\mathrm{RO}$ & $\mathrm{RO}$ & WFI & WFI & FWI & FWI \\
\hline & Total $\mathrm{R}^{2}$ & $\begin{array}{l}\text { Change in } \\
R^{2} \text { by } \\
\text { adding IV }\end{array}$ & Total $\mathrm{R}^{2}$ & $\begin{array}{l}\text { Change in } R^{2} \\
\text { by adding IV }\end{array}$ & Total $\mathrm{R}^{2}$ & $\begin{array}{l}\text { Change } \\
\text { in } R^{2} \text { by } \\
\text { adding } \\
\text { IV }\end{array}$ \\
\hline $\begin{array}{l}\mathrm{H} 1(\mathrm{WD} \\
\text { positively } \\
\text { associated } \\
\text { WLC) }\end{array}$ & $8.1 \%$ & $3.1 \%$ & $11.5 \%$ & $6.7 \%$ & $5.7 \%$ & $0.1 \%$ \\
\hline $\begin{array}{l}\mathrm{H} 2 \text { (NWD } \\
\text { positively } \\
\text { associated } \\
\text { WLC) }\end{array}$ & $8.1 \%$ & $2.1 \%$ & $11.5 \%$ & $1.3 \%$ & $5.7 \%$ & $5.5 \%$ \\
\hline $\begin{array}{l}\text { H3a (Flex } \\
\text { negatively } \\
\text { associated } \\
\text { WLC) }\end{array}$ & $8.2 \%$ & $0.1 \%$ & $11.9 \%$ & $0 \%$ & $5.7 \%$ & $0 \%$ \\
\hline $\begin{array}{l}\mathrm{H} 3 \mathrm{~b} \text { (CWW } \\
\text { negatively } \\
\text { associated } \\
\text { WLC) }\end{array}$ & $7.9 \%$ & $0.1 \%$ & $10.8 \%$ & $0 \%$ & $5.6 \%$ & $0 \%$ \\
\hline $\begin{array}{l}\text { H3c } \\
\text { (Telework } \\
\text { negatively } \\
\text { associated } \\
\text { with WLC) }\end{array}$ & $7.9 \%$ & $0 \%$ & $11.5 \%$ & $0 \%$ & $5.4 \%$ & $0.1 \%$ \\
\hline $\begin{array}{l}\text { H4 } \\
\text { (Shiftwork } \\
\text { positively } \\
\text { associated } \\
\text { with WLC) }\end{array}$ & $8.1 \%$ & $0 \%$ & $12.2 \%$ & $0.7 \%$ & $5.7 \%$ & $0 \%$ \\
\hline $\begin{array}{l}\text { H5 (Pflex } \\
\text { negatively } \\
\text { associated } \\
\text { with WLC }\end{array}$ & $17.5 \%$ & $9.4 \%$ & $25.7 \%$ & $14.2 \%$ & $8.0 \%$ & $2.3 \%$ \\
\hline $\begin{array}{l}\text { H6 (SS } \\
\text { negatively } \\
\text { associated } \\
\text { with WLC) }\end{array}$ & $13.6 \%$ & $5.5 \%$ & $19.9 \%$ & $8.4 \%$ & $7.6 \%$ & $1.9 \%$ \\
\hline
\end{tabular}




\section{Buffer Hypotheses (Models 1-3)}

\begin{tabular}{|c|c|c|c|c|c|c|}
\hline Hypothesis & RO & $\mathrm{RO}$ & WFI & WFI & FWI & FWI \\
\hline & Total $\mathrm{R}^{2}$ & $\begin{array}{l}\text { Change } \\
\text { in } R^{2} \text { by } \\
\text { adding } \\
I V \times M V\end{array}$ & Total $\mathrm{R}^{2}$ & $\begin{array}{l}\text { Change } \\
\text { in } R^{2} \text { by } \\
\text { adding } \\
\mathrm{N} \times \mathrm{MV}\end{array}$ & $\begin{array}{l}\text { Total } \\
R^{2}\end{array}$ & $\begin{array}{l}\text { Change in } \\
\mathrm{R}^{2} \text { by } \\
\text { adding IV } \mathrm{x} \\
\mathrm{MV}\end{array}$ \\
\hline $\begin{array}{l}\text { H7a (Flex moderates } \\
\text { WD and WLC) }\end{array}$ & $8.2 \%$ & $0 \%$ & $11.9 \%$ & $0 \%$ & $5.8 \%$ & $0.1 \%$ \\
\hline $\begin{array}{l}\text { H7b (CWW moderates } \\
\text { WD and WLC) }\end{array}$ & $7.9 \%$ & $0 \%$ & $10.8 \%$ & $0 \%$ & $5.6 \%$ & $0 \%$ \\
\hline $\begin{array}{l}\text { H7c (Telework } \\
\text { moderates WD and } \\
\text { WLC) }\end{array}$ & $7.9 \%$ & $0 \%$ & $11.5 \%$ & $0 \%$ & $5.4 \%$ & $0 \%$ \\
\hline $\begin{array}{l}\text { H7d (Shiftwork } \\
\text { moderates WD and } \\
\text { WLC) }\end{array}$ & $8.2 \%$ & $0.1 \%$ & $12.5 \%$ & $0.3 \%$ & $5.7 \%$ & $0 \%$ \\
\hline $\begin{array}{l}\text { H8 (Pflex moderates } \\
\text { WD and WLC) }\end{array}$ & $17.5 \%$ & $0 . \overline{1 \%}$ & $25.7 \%$ & $0 \%$ & $8.1 \%$ & $0.1 \%$ \\
\hline $\begin{array}{l}\text { H9 (Supervisor support } \\
\text { moderates WD and } \\
\text { WLC) }\end{array}$ & $13.6 \%$ & $0 \%$ & $20.0 \%$ & $0.1 \%$ & $7.6 \%$ & $0 \%$ \\
\hline $\begin{array}{l}\text { H10a (Flextime } \\
\text { moderates NWD and } \\
\text { WLC) }\end{array}$ & $8.2 \%$ & $0 \%$ & $11.9 \%$ & $0 \%$ & $5.8 \%$ & $0.1 \%$ \\
\hline $\begin{array}{l}\text { H10b (CWW moderates } \\
\text { NWD and WLC) }\end{array}$ & $7.9 \%$ & $0 \%$ & $10.8 \%$ & $0 \%$ & $5.6 \%$ & $0 \%$ \\
\hline $\begin{array}{l}\text { H10c (Telework } \\
\text { moderates NWD and } \\
\text { WLC) }\end{array}$ & $7.9 \%$ & $0 \%$ & $11.5 \%$ & $0 \%$ & $5.4 \%$ & $0 \%$ \\
\hline $\begin{array}{l}\text { H10d (Shiftwork } \\
\text { moderates NWD and } \\
\text { WLC) }\end{array}$ & $8.1 \%$ & $0 \%$ & $12.2 \%$ & $0 \%$ & $5.7 \%$ & $0 \%$ \\
\hline $\begin{array}{l}\text { H11 (Pflex moderates } \\
\text { NWD and WLC) }\end{array}$ & $17.5 \%$ & $0 \%$ & $25.7 \%$ & $0 \%$ & $8.0 \%$ & $0 \%$ \\
\hline $\begin{array}{l}\text { H12 (SS moderates } \\
\text { NWD and WLC) }\end{array}$ & $13.7 \%$ & $0.1 \%$ & $19.9 \%$ & $0 \%$ & $7.6 \%$ & $0 \%$ \\
\hline
\end{tabular}


Direct Hypotheses (Model 4)

\begin{tabular}{|l|l|l|}
\hline Hypothesis & Total $\mathrm{R}^{2}$ & $\begin{array}{l}\text { Change in } \\
\mathrm{R}^{2} \text { by } \\
\text { adding IV }\end{array}$ \\
\hline $\begin{array}{l}\text { PFLEXH1a (Flextime positively associated with } \\
\text { perceived flexibility) }\end{array}$ & $7.6 \%$ & $3.7 \%$ \\
\hline $\begin{array}{l}\text { PFLEX1b (CWW positively associated with } \\
\text { perceived flexibility) }\end{array}$ & $5.4 \%$ & $1.1 \%$ \\
\hline $\begin{array}{l}\text { PFLEXH1c (Telework positively associated with } \\
\text { perceived flexibility) }\end{array}$ & $5.7 \%$ & $1.6 \%$ \\
\hline $\begin{array}{l}\text { PFLEXH2 (Shiftwork negatively associated with } \\
\text { perceived flexibility) }\end{array}$ & $8.7 \%$ & $5.0 \%$ \\
\hline $\begin{array}{l}\text { PFLEXH3 (Supervisor support positively } \\
\text { associated with perceived flexibility) }\end{array}$ & $13.8 \%$ & $10.1 \%$ \\
\hline
\end{tabular}

Interaction Hypotheses (Model 4)

\begin{tabular}{|l|l|l|}
\hline Hypothesis & Total $\mathrm{R}^{2}$ & $\begin{array}{l}\text { Change in } \\
\mathrm{R}^{2} \text { by adding } \\
\mathrm{IV} \times \mathrm{MV}\end{array}$ \\
\hline $\begin{array}{l}\text { PFLEX4a (Supervisor support moderates flextime and } \\
\text { perceived flexibility) }\end{array}$ & $17.3 \%$ & $0 \%$ \\
\hline $\begin{array}{l}\text { PFLEX4b (Supervisor support moderates CWW and } \\
\text { perceived flexibility) }\end{array}$ & $15.8 \%$ & $0 \%$ \\
\hline $\begin{array}{l}\text { PFLEX4c (Supervisor support moderates telework and } \\
\text { perceived flexibility) }\end{array}$ & $16.3 \%$ & $0 \%$ \\
\hline $\begin{array}{l}\text { PFLEX5 (Supervisor support moderates shiftwork and } \\
\text { perceived flexibility) }\end{array}$ & $17.9 \%$ & $0 \%$ \\
\hline
\end{tabular}




\section{References}

Abroms, L. \& Goldscheider, F. (2002). More work for mother: How spouses, cohabiting partners and relatives affect the hours mothers work. Journal of Family and Economic Issues, 23, 147-166.

Adams, G. \& Jex, S. (1999). Relationships between time management, control, work-family conflict and strain. Journal of Occupational Health Psychology, 4, 72-77.

Adams, G., King, L. \& King, D. (1996). Relationships of job and family involvement, family social support, and work-family conflict with job and life satisfaction. Journal of Applied Psychology, 81, 411-420.

Adams, J. (1965). Inequity in social exchanges. In Berkowitz, L. (Ed.) Advances in Experimental Social Psychology. New York: Academic Press.

Aguinis, H. (1995). Statistical power problems with moderated multiple regression in management research. Journal of Management, 21(6), $1141-1158$.

Aguinis, H. (2004). Regression analysis for categorical moderators. New York: The Guilford Press.

Aguinis, H. \& Stone-Romero, E. (1997). Methodological artifacts in moderated multiple regression and their effects on statistical power. Journal of Applied Psychology, 82(1), 192-206.

Aiken, L. S. \& West, S. G. (1991). Multiple Regression: Testing and Interpreting Interactions. Newbury Park, CA: Sage Publications.

Akerstedt, T. (1985). Shifted sleep hours. Annual Clinical Research, 17, 273279 .

Akerstedt, T. \& Torsvall, L. (1978). Experimental changes in shift schedules Their effects on well-being, Ergonomics, 21, 849-856.

Albright, C. L., Winkleby, M. A., Ragland, D. R., Fisher, J. \& Syme, S. L. (1992). Job strain and prevalence of hypertension in a biracial population of urban bus drivers. American Journal of Public Health, 82, 984-989.

Aldous, J., Mulligan, G. \& Bjarnason, T. (1998). Fathering over time: What makes the difference? Journal of Marriage and the Family, 60, 809-820. 
Alfredsson, L., Karasek, R. \& Theorell, T. (1982). Myocardial infarction risk and psychosocial environment: An analysis of the male Swedish working force. Social Science Medicine, 3, 463-467.

Alfredsson, L., Spetz, C. \& Theorell, T. (1985). Type of occupation and nearfuture hospitalization for myocardial infarction and some other diagnoses. International Journal of Epidemiology, 14, 378-388.

Alfredsson, L. \& Theorell, T. (1983). Job characteristics of occupations and myocardial infarction risk: Effects of possible confounding factors. Social Science Medicine, 17, 1497-1503.

Allen, T. (2001). Family-supportive work environments: The role of organizational perceptions. Journal of Vocational Behaviour, 58, 414435.

Allen, T., Herst, D., Bruck, C. \& Sutton, M. (2000). Consequences associated with work-to-family conflict: A review and agenda for future research. Journal of Occupational Health Psychology, 5, 278-308.

Allensbach, H. (1975). Flexible Working Hours. International Labor Office: Geneva.

Alterman, T., Shekelle, R., Vernon, S., \& Burau, K. (1994). Decision latitude, psychologic demand, job strain, and coronary heart disease in the Western Electric study. American Journal of Epidemiology, 139, 620627.

American Heart Association (2005). Glossary. http://www.americanheart.org/presenter.jhtml?identifier=1158.

Amick, B. \& Celentano, D. (1991). Structural determinants of the psychosocial environment: introducing technology in the work stress framework. Ergonomics, 34, 625-646.

Amick, B., Kawachi, I., Coakley, E., Lerner, D., Levine, S. \& Colditz, G. (1998). Relationship of job strain and iso-strain to health status in a cohort of women in the United States. Scandinavian Journal of Work Environment and Health, 24, 54-61.

Anderson, S., Coffey, B. \& Byerly, R. (2002). Formal organizational initiatives and informal workplace practices: Links to work-family conflict and jobrelated outcomes. Journal of Management, 28, 787-810. 
Anderson, J. \& Gerbing, D. (1988). Structural equation modeling in practice: A review and recommended two-step approach. Psychological Bulletin, $103,411-423$.

Aneshensel, C. (1986). Marital and employment role-strain, social support, and depression among adult women. In Hobfoll, S. (Ed.) Stress, Social Support and Women. Washington: Hemisphere Publishing Corp.

Angersbach, D., Knauth, P., Loskant, H., Karvonen, M., Undeutsch, K. \& Rutenfranz, J. (1980). A retrospective cohort study comparing complaints and diseases in day and shift workers. International Archives of Occupational and Environmental Health, 45, 127-140.

Anonymous Source (2004): http://www.utexas.edu/cc/stat/tutorials/amos Structural Equation Modeling using AMOS: An introduction.

Anthony, L. (2002). Canadian women still doing the majority of housework, childcare: 2001 census. www.broadband.rogers.com/news/national/pf story.jsp?cid=n021104A.

Anonymous (2001). Work-life conflict: a three-part problem. The Worklife Report, 13(4), 3-5.

Applebaum, E. \& Golden, L. (2002). The standard workday or the highway. Washington, DC: Center for Designing Work Wisely.

Arnetz, B., Andreasson, S., Strandberg, M., Eneroth, P., \& Kallner, A. (1987). The psychosocial work environment of physicians: Stress, health risks and work satisfaction. Lakartidningen, 84, 816-824.

Aryee, S. (1992). Antecedents and outcomes of work-family conflict among married professional women: evidence from Singapore. Human Relations, 45, 813-837.

Aryee, S., Luk, V., Leung, A. \& Lo, S. (1999). Role stressors, interrole conflict, and well-being: The moderating influence of spousal support and coping behaviors among employed parents in Hong Kong. Journal of Vocational Behavior, 54, 259-278.

Aryee, S., Srinivas, E. \& Tan, H. (2005). Rhythms of life: Antecedents and outcomes of work-family balance in employed parents. Journal of Applied Psychology, 90(1), 132-146.

Astrand, N, Hanson, R, Isacsson,S. (1989). Job demands, job decision latitude, job support, and social network factors as predictors of mortality in a 
Swedish pulp and paper company. British Journal of Industrial Medicine, $46,334-40$.

Atkinson, W. (1985). Homework. Personnel Journal, 64, 104-109.

Austin Knight (1995). Long hours culture. London: Austin Knight.

Averill, J. (1973). Personal control over aversive stimuli and its relationship to stress. Psychological Bulletin, 80, 286-303.

Avolio, B., Yammarino, F. \& Bass, B. (1991). Identifying common methods variance with data collected from a single source: An unresolved sticky issue. Journal of Management, 17(3), 571-587.

BLS (Bureau of Labor Statistics) (2005a). American Time Use Survey: 2004 results announced by $B L S$.

http://www.bls.gov/news.release/pdf/atus.pdf.

BLS (Bureau of Labor Statistics) (2005b). Employment Situation Summary. (http://www.bls.gov/news.release/empsit.nr0.htm).

BLS (Bureau of Labor Statistics) (2005c). Work at home in 2004.

(http://www.bls.gov/news.release/pdf/homey.pdf).

BLS (Bureau of Labor Statistics) (2005d). http://www.bls.gov/cps/labor2005/chart6-3.pdf.

BLS (Bureau of Labor Statistics) (2006). American Time Use Survey: 2005 results announced by BLS. http://www.bls.gov.tus/.

Bacharach, S., Bamberger, P. \& Conley, S. (1991). Work-home conflict among nurses and engineers: Mediating the impact of role stress on burnout and satisfaction at work. Journal of Organizational Behavior, 12, 39-53.

Bachler, C. (1995). Workers take leave of job stress. Personnel Journal, 74, 3846.

Bailey, D. and Kurland, N. (2000). A Review and New Directions for Telework Research: Study Telework Not Teleworkers, Working Paper, Stanford University.

Bailyn, L. (1989). Towards the perfect workplace. Communications of the ACM $32,460-472$. 
Bailyn, L., Drago, R. \& Kochan, T. (2001). Integrating work and family life: A holistic approach. A report of the Sloan Work-Family Policy Network. Cambridge, MA: MIT Sloan School of Management.

Baker, E., Israel, B. \& Schurman, S. (1996). Role of control and support in occupational stress: an integrated model. Social Science and Medicine, 43, 1145-1159.

Baltes, B., Briggs, T., Huff, J., Wright \& Neuman, G. (1999). Flexible and compressed workweek schedules: A meta-analysis of their effects on work-related criteria. Journal of Applied Psychology, 84, 496-513.

Barclay, D. (1986). The impact of the organization context on conflict in organizational buying: A systems view. Ph.d. thesis. University of Michigan.

Barham, L., Gottlieb, B. \& Kelloway, K. (1998). Variables affecting managers' willingness to grant alternative work arrangements. The Journal of Social Psychology, 138, 291-302.

Barling, J. \& Barenburg, A. (1984). Some personal consequences of 'flexitime' work schedules. Journal of Social Psychology, 123, 137-138.

Barling, J. \& Kelloway, K. (1996). Job insecurity and health: The moderating role of workplace control. Stress Medicine, 12, 253-259.

Barnes, K. (1994). Tips for managing telecommuters. HR Focus, November, 910.

Barnett, R. (1994). Home-to-work spillover revisited: A study of full-time employed women in dual-earner couples. Journal of Marriage and the Family, 56, 647-656.

Barnett, R. (1997). Gender, job stress and illness. In J. Stellman (Ed.) Encyclopedia of Occupational Health and Safety. Geneva: International Labour Office.

Barnett, R. (1998). Toward a review and reconceptualization of the work/family literature. Genetic, Social and General Psychology Monographs, 124, 125-182.

Barnett, R. (1999). A new work-life model for the twenty-first century. Annals, AAPSS, 562, 143-158. 
Barnett, R. \& Baruch, G. (1987). Social roles, gender and psychological distress. In Barnett, R., Biener, L. \& Baruch, G. (Eds.) Gender and Stress. New York: The Free Press.

Barnett, R., Biener, L. \& Baruch, G. (Eds.) (1987). Gender and Stress. New York: The Free Press.

Barnett, R. \& Brennan, R. (1995). The relationship between job experiences and psychological distress: A structural equation approach. Journal of Organizational Behavior, 16, 259-276.

Barnett, R. \& Brennan, R. (1997). Change in job conditions, change in psychological distress and gender: a longitudinal study of dual-earner couples. Journal of Organizational Behavior, 18, 253-274.

Barnett, R. \& Gareis, K. (2006). Role theory perspectives on work and family In Pitt-Catsouphes, M., Kossek, E. \& Sweet, S. (Eds.). The Work and Family Handbook: Multidisciplinary Perspectives and Approaches. Mahwah, NJ: Lawrence Erlbaum \& Associates, Publishers.

Barnett, R., Gareis, K. \& Brennan, B. (1999). Fit as a mediator of the relationship between work hours and burnout. Journal of Occupational Health Psychology, 4, 307-317.

Barnett, R., Marshall, N. \& Pleck, J. (1992). Men's multiple roles and their relationship to men's psychological distress. Journal of Marriage and the Family, 54, 358-367.

Barnett, R., Marshall, N., Raudenbush, S. \& Brennan, T. (1993). Gender and the relationship between job experiences and psychological distress: $A$ study of dual-earner couples. Journal of Personality and Social Psychology, 64, 794-806.

Barnett, R. \& Rivers, C. (1996). She works/he works: How two income families are happier, healthier, and better off. New York: Harper Collins.

Baron, R. \& Kenny, D. (1986). The moderator-mediator variable distinction in social psychological research: Conceptual, strategic and statistical considerations. Journal of Personality and Social Psychology, 51, 11731182 .

Bartley, S., Blanton, P. \& Gilliard, J. (2005). Husbands and wives in dual-earner marriages: Decision-making, gender role attitudes, division of household labor, and equity. Marriage and Family Review, 37(4), 69-93. 
Bartolome, F. \& Evans, P. (1980). Must success cost so much? Harvard Business Review, 61, 67-74.

Barton, J. (1994). Choosing to work at night: A moderating influence on individual tolerance to shift work. Journal of Applied Psychology, 79, 449-454.

Barton, J. \& Folkard, S. (1991). The response of day and night nurses to their work schedules. Journal of Occupational Psychology, 64, 207-218.

Barton, J. \& Folkard, S. (1993). Advancing versus delaying shift systems. Ergonomics, 36, 59-64.

Barton, J., Smith, L., Totterdell, P., Spelten, E. \& Folkard, S. (1993). Does individual choice determine shift system acceptability. Ergonomics, 36, 93-99.

Baruch, Y. (2000). Teleworking: benefits and pitfalls as perceived by professionals and managers. New Technology, Work and Environment, $15,34-49$.

Baruch, Y. \& Nicholson, N. (1997). Home, Sweet work: requirements for effective home-working. Journal of General Management, 23, 2, 15-30.

Bateman, T. \& Crant, J. (1993). The proactive component of organizational behavior: A measure and correlates. Journal of Organizational Behavior, 14, 103-118.

Batt, R. \& Valcour, P. (2000). Workplace flexibility, work-family integration, and employee turnover. Paper given at the conference "Work and Family: Expanding the Horizons". March 3-4, 2000, in San Francisco.

Bazerman, M. (1982). Impact of personal control on performance: Is added control always beneficial? Journal of Applied Psychology, 67, 472-479.

Beach, B. (1989). Integrating work and family life: The homeworking family. Albany, NY: State University of New York Press.

Beatty, C. (1996). The stress of managerial and professional women: Is the price too high? Journal of Organizational Behavior, 17, 233-251.

Beatty, S. (2001). Extreme Job stress: Survivors' Tales. Wall Street Journal. Vol. 237, B1.

Beauvais, L. \& Kowalski, K. (1993). Predicting work/family conflict and participation in family-supportive work behaviors: A competing test of two 
theories. Paper presented at the National Academy of Management meetings, Atlanta, GA.

Bedeian, A. G., Burke, B.G. \& Moffett, R.G. (1988). Outcomes of work-family conflict among married male and female professionals. Journal of Management, 14, 475-491.

Beehr, T. (1988). Psychological Stress in the Workplace. New York: Routledge.

Beehr, T. \& Franz, T. (1986). The current debate about the meaning of job stress. Journal of Organizational Behavior Management, 8, 5-18.

Beers, T. (2000). Flexible schedules and shift work: replacing the ' 9 -to-5' workday? Monthly Labor Review, June, 33-40.

Behson, S. (2002). Coping with family-to-work conflict: The role of informal work accommodations to family. Journal of Occupational Health Psychology, 7(4), 324-341.

Bellavia, G. \& Frone, M. (2005). Work-family conflict. In Barling, J., Kelloway, K. \& Frone, M. (Eds.) Handbook of work stress. Thousand Oaks, CA: Sage.

Bentler, P. \& Chou, C. (1987). Practical issues in structural modeling. Sociological Methods \& Research, 16, 78-117.

Berkman, L. \& Syme, S. (1979). Social networks, host resistance and mortality: A nine-year study of Alameda county residents. American Journal of Epidemiology, 109, 186-204.

Berardo, D., Shehan, C. \& Leslie, G. (1987). A residue of tradition: Jobs, careers, and spouses' time in housework. Journal of Marriage and the Family, 49, 381-390.

Bernas, K. \& Major, D. (2000). Contributors to stress resistance: testing a model of women's work-family conflict. Psychology of Women Quarterly, 24, $170-178$.

Berry, W. (1993). Understanding regression assumptions: Series: Quantitative Applications in the Social Sciences, No. 92. Thousand Oaks, CA: Sage Publications.

Beutell, N. \& Wittig-Berman, U. (1999). Predictors of work-family conflict and satisfaction with family, job, career and life. Psychological Reports, 85, 893-903. 
Bhagat, R., Lindholm, H., McQuaid, S., \& Segovis, J. (1985). Total life stress: A multi-method validation of the construct and its effect on organizationally valued outcomes and withdrawal behaviors. Journal of Applied Psychology, 70, 202-214.

Bhar, J. (1995). Developing a measure of supervisor support. MMS. Thesis. Carleton University.

Bianchi, S., Casper, L. \& King, R. (Eds.) (2005). Work, family, health and wellbeing. Mahwah, NJ: Lawrence Erlbaum Associates, Publishers.

Bianchi, S. \& Raley, S. (2005). Time allocation in families. In Bianchi, S., Casper, L. \& King, R. (Eds.) (2005). Work, family, health and well-being. Mahwah, NJ: Lawrence Erlbaum Associates, Publishers.

Bierman, A. (2007). Interactive textbook on clinical symptom research. http://symptomresearch.nih.gov/chapter 20/sec 2/cabs2pg1.htm.

Bird, C. (1999). Gender, household labor, and psychological distress: The impact of the amount and division of housework. Journal of Health and Social Behavior, 40, 32-45.

Bittman, M. (1998). The land of the lost weekend? Trends in free time among working age Australians, 1974-1992. Discussion paper no. 83, Australia Social Policy Research Centre, University of New South Wales.

Bohen, H. \& Viveros-Long, A. (1981). Balancing Jobs and Family life: Do Flexible Work Schedules Help? Philadelphia: Temple University Press.

Bohle, P. \& Tilley, A. (1989). The impact of night work on psychological wellbeing. Ergonomics, 32, 1089-1099.

Boles, J., Howard, G. \& Donofrio, H. (2001). An investigation into the interrelationships of work-family conflict, family-work conflict and work satisfaction. Journal of Managerial Issues, 13, 376-390.

Boles, J., Johnston, M. \& Hair, J. (1997). Role stress, work-family conflict and emotional exhaustion: Inter-relationships and effects on some workrelated consequences. Journal of Personal Selling \& Sales Management, $1,17-28$.

Bolger, N., DeLongis, A., Kessler, R. \& Wethington, E. (1989). The contagion of stress across multiple roles. Journal of Marriage and the Family, 51, 175-183. 
Bond, J. (2002). The impact of job and workplace factors on low-wage and income employees. Unpublished manuscript, Families and Work Institute.

Bond, J., Galinsky, E. \& Hill, J. (2004). When work works: Flexibility, a critical ingredient in creating an effective workplace. New York: Families and Work Institute.

Bond, J., Galinsky, E. \& Swanberg, J. (1998). The 1997 National Study of the Changing Workforce. New York: Families and Work Institute.

Bond, J., Thompson, C., Galinsky, E., \& Prottas, D. (2003). Highlights of the National Study of the Changing Workforce. New York: Families and Work Institute.

Bourbonnais, R., Brisson, C., Moisan, J. \& Vezina, M. (1996). Job strain and psychological distress in white-collar workers. Scandinavian Journal of Work, Environment and Health, 22, 139-145.

Bourbonnais, R., Comeau, R., \& Vezina, M. (1999). Job strain and evolution of mental health among nurses. Journal of Occupational Health Psychology, 4, 95-107.

Bowman, G. \& Stern, M. (1995). Adjustment to occupational stress: The relationship of perceived control to effectiveness of coping strategies. Journal of Counseling Psychology, 42, 294-303.

Boyar, S., Maertz, C., Pearson, A. \& Keough, S. (2003). Work-family conflict: A model of linkages between work and family domain variables and turnover intentions. Journal of Managerial Issues, 15, 175-190.

Boyd, P. (2000). Six Organizational Benefits of Telecommuting, http://pw2.netcom.com/-pboyd/orgbens.html.

Bozeman, D., Perrewe, P., Hochwarter, W. \& Brymer, R. (2001). Organizational politics, perceived control and work outcomes: Boundary conditions on the effects of politics. Journal of Applied Social Psychology, 31, 486-503.

Brannick, M. (1995). Critical comments on applying covariance structure modeling. Journal of Organizational Behavior, 16, 201-213.

Brass, D. (1985). Technology and the structuring of jobs: Employee satisfaction, performance and influence. Organizational Behaviour Human Decision Processes, 55, 216-240. 
Braun, S. \& Hollander, R. (1987). A study of job stress among women and men in the Federal Republic of Germany. Health Education Research, 2, 4551.

Braun, S. \& Hollander, R. (1988). Work and depression among women in the Federal Republic of Germany. Women and Health, 14, 5-24.

Breaugh, J. (1983). The 12 hour work day: Differing employee reactions. Personnel Psychology, 36, 277-288.

Breaugh, J. (1985). The measurement of work autonomy. Human Relations, 38, $551-570$.

Brief, A., Burke, M., George, J., Robinson, B. \& Webster, J. (1988). Should negative affectivity remain an unmeasured variable in the study of job stress? Journal of Applied Psychology, 78, 193-198.

Bromet, E., Dew, M., Parkinson, M. \& Schulberg, H. (1988). Predictive effects of occupational and marital stress on the mental health of a male workforce. Journal of Organizational Behavior, 9, 1-13.

Brown, J. (1988). Understanding Research in Second Language Learning: A Teacher's Guide to Statistics and Research Design. Cambridge, UK: Cambridge University Press.

Brough, P., O'Driscoll, M. \& Kalliath, T. (2005). The ability of 'family friendly' organizational resources to predict work-family conflict and job and family satisfaction. Stress and Health, 21, 223-234.

Bryson, R, Bryson, J. \& Johnson, M. (1978). Family size, satisfaction and productivity in dual-career couples. Psychology of Women Quarterly, 3, 167-177.

Buell, P. \& Breslow, L. (1960). Mortality from chronic heart disease in California men who work long hours. Journal of Chronic Diseases. 11, 615-626.

Buffardi, L, Smith, J., O'Brien, A., Erdwins, C. (1999). The impact of dependentcare responsibility and gender on work attitudes. Journal of Occupational Health Psychology, 4, 356-367.

Bullers, S. (1994). Women's roles and health: The mediating effect of perceived control. Women and Health, 22, 11-30.

Bumpus, M., Crouter, A. \& McHale, S. (1999). Work demands of dual-earner couples: Implications for parents' knowledge about children's daily lives in middle childhood. Journal of Marriage and the Family, 61, 465-475. 
Burden, D. \& Googins, B. (1987). Boston University balancing job and homelife study. Boston: Boston University School of Social Work.

Burke, M., Brief, A., \& George, J. (1993). The role of negative affectivity in understanding relations between self-reports of stressors and strains: $A$ comment on the applied psychology literature. Journal of Applied Psychology, 78, 402-412.

Burke, R. (1986a). Occupational and life stress and the family: conceptual frameworks and research findings. International Review of Applied Psychology, 35, 347-369.

Burke, R. (1986b). The present and future status of stress research. Journal of Organizational Behavior Management, 8, 249-267.

Burke, R. (1988). Some antecedents and consequences of work-family conflict. In Goldsmith, E. (Ed.). Work and family: Theory, research and applications. Newbury Park, CA: Select Press.

Burke, R. (1993). Toward an understanding of psychological burnout among police officers. Journal of Social Behavior and Psychology, 8, 425-438.

Burke,R. (1994). Stressful events, work/family conflict, coping, psychological burnout and wellbeing among police officers. Psychological Reports, 75, 787-800.

Burke, R. \& Greenglass, E. (1987). Work and family. In Cooper, C. \& Robertson, I. (Eds.) International Review of Industrial and Organizational Psychology. Chichester, UK: Wiley.

Burke, R. \& Greenglass, E. (1999). Work-family conflict, spouse support and nursing staff well-being during organizational restructuring. Journal of Occupational Health Psychology, 4, 327-336.

Burley, K. (1994). Gender differences and similarities in coping responses to anticipated work-family conflict. Psychological Reports, 74, 115-123.

Buunk, B., de Jonge, J., Ybema, J. \& de Wolff, C. (1998). Psychosocial aspects of occupational stress. In Drenth et al. (Eds). Handbook of Work and Organizational Psychology: Work Psychology. East Sussex, UK: Psychology Press.

Byrne, B. (2001). Strutural Equation Modeling with AMOS: Basic Concepts, Applications and Programming. Mahwah, NJ: Lawrence Erlbaum Associates. 
Cahill, J. \& Landsbergis, P. (1996). Job strain among post office mailhandlers. International Journal of Health Services, 26, 731-750.

Calvasina, E. \& Boxx, R. (1975). Efficiency of workers on the four day work week. Academy of Management Journal, 18, 604-610.

Campbell, L. (1980). Can new shift schedules motivate? Hydrocarbon Processing, April, 249-256.

Canadian Press Newswire (1996). Fewer workers had jobs in June but they made slightly more money, August 29.

Carayon, P. (1993). A longitudinal test of Karasek's job strain model among office workers. Work and Stress, 7, 299-314.

Carlson, D. \& Perrewe, P. (1999). The role of social support in the stressor-strain relationship: An examination of work-family conflict. Journal of Management, 25, 513-540.

CARNET: The Canadian Aging Research Network (1993). The work and family survey findings. Guelph, ON: University of Guelph, Psychology Department.

CARNET: The Canadian Aging Research Network (1994). The work and homelife survey findings. Guelph, ON: University of Guelph, Psychology Department.

CARNET: The Canadian Aging Research Network (1995). Flexible work arrangements: A user's guide. Guelph, ON: University of Guelph, Psychology Department.

Caudron, S. (1992). Working at home pays off. Personnel Journal, 71, 40-49.

Caudron, S. (1998). Job stress is in job design. Workforce, 77, 21-23.

Cervinka, R. (1993). Night shift dose and stress at work. Ergonomics, 36, 155160.

Chapman, A., Sheehy, N., Heywood, S., Dooley, B. \& Collins, S. (1995). The organizational implications of teleworking. In Cooper et al. (Eds.). International Review of Industrial and Organizational Psychology. v. 10. New York: John Wiley \& Sons Ltd. 
Chay, Y. (1993). Social support, individual differences and well-being: a study of small business entrepreneurs and employees. Journal of Occupational and Organizational Psychology, 66, 285-302.

Chen, P. Y. \& Spector, P. E. (1991). Negative affectivity as the underlying cause of correlations between stressors and strains. Journal of Applied Psychology, 76, 398-407.

Chesney, M., Sevelius, G., Black, G, Ward, M., Swan, G. \& Rosenman, R. (1981). Work environment, Type A behavior, and coronary heart disease risks. Journal of Occupational Medicine, 23, 551-555.

Christensen, K. (1988). Women and Home-Based Work: The Unspoken Contract. New York: Holt, Rinehart and Winston.

Christensen, K. \& Staines, G. (1990). Flextime: A viable solution to work/family conflict. Journal of Family Issues, 11, 455-476.

Christensen, P. (1997). Toward a comprehensive work/life strategy. In Parasuraman, S. \& Greenhaus, J. (Eds.) Integrating work and family: challenges and choices for a changing world. Westport, CT: Quorum Books.

Chung, L. (2004). Low-paid workers: How many live in low income families? Perspectives on Labour and Income, Statistics Canada, Catalogue no. 75-001-XIE. 5-14.

CLMPC (1997). Changing Times, New Ways of Working: Alternative Working Arrangements and Changes in Working Time. Ottawa: Canadian Labour Market and Productivity Centre.

Clark, S. (2001). Work cultures and work/family balance. Journal of Vocational Behaviour, 58, 348-365.

Clark, S. (2002). Employees' sense of community, sense of control, and work/family conflict in Native American organizations. Journal of Vocational Behavior, 61, 92-108.

Clarkberg, M. \& Moen, P. (2001). Understanding the time-squeeze: Married couples' preferred and actual work-hour strategies. American Behavioral Scientist, 44(7), 1115-1135.

Clay, R. (1997). Working mothers: Happy or haggard? In Reich, J., Bulatao, E., VandenBos, G. \& Farberman, R. (Eds.). Close up on psychology: Supplemental readings from the APA Monitor. Washington, DC: American Psychological Association. 
Cleary, P. \& Mechanic, D. (1983). Sex differences in psychological distress among married people. Journal of Health and Social Behavior, 24, 111 121.

Clegg, C. \& Wall, T. (1990). The relationship between simplified jobs and mental health: A replication study. Journal of Occupational Psychology, 63, 289296.

Clegg, C., Wall, T. \& Kemp, N. (1987). Women on the assembly line: A comparison of main and interactive explanations of job satisfaction, absence and mental health. Journal of Occupational Psychology, 60, 273-287.

Coffey, C., Skipper, J. Jr., \& Jung, F. (1988). Nurses and shiftwork: Effects on job performance and job-related stress. Journal of Advanced Nursing, 13, 245-254.

Cohany, S. (November,1998). Workers in alternative employment arrangements: a second look. Monthly Labor Review, 3-21.

Cohen, J. (1978). Partialled products are interactions; partialled powers are curve components. Psychological Bulletins, 85, 858-866.

Cohen, J. (1988). Statistical power analysis for the behavioral sciences. Hillsdale, $\mathrm{NJ}$ : Lawrence Erlbaum Associates.

Cohen, J. \& Cohen, P. (1983). Applied multiple regression/correlation analysis for the social sciences. Hillsdale, NJ: Erlbaum.

Cohen, S., Kamarck, T., \& Mermelstein, R. (1983). A global measure of perceived stress. Journal of Health and Social Behavior, 24, 385-396.

Cohen, S., Kessler, R. \& Gordon, L. (1995). Strategies for measuring stress in studies of psychiatric and physical disorders. In S. Cohen, R. Kessler \& L. Gordon (Eds.) Measuring stress (pp. 3-26). New York: Oxford University Press.

Cohen, S. \& Wills, T. (1985). Stress, social support, and the buffering hypothesis. Psychological Bulletin, 98, 310-357.

Cooper, C. (1996). Working hours and health. Work and Stress, 10(1), 1-4.

Cooper, C., Dewe, P., \& O'Driscoll, M. (2001). Organizational stress: A review and critique of theory, research, and applications. Thousand Oaks, CA: Sage Publications. 
Cooper, C. \& Smith, M. (1985). Introduction: Blue collar workers are 'at risk'. In Cooper, C. \& Smith, M. Job stress and blue collar work. New York: John Wiley and Sons Ltd

Cooper, C. \& Smith, M. (1997). Managerial Style. In J. Stellman (Ed.) Encyclopedia of Occupational Health and Safety. Geneva: International Labour Office.

Cooper, D. \& Schindler, P. (1998). Business Research Methods. Boston: Irwin: McGraw-Hill.

Corneil, W. (1997). Social Support. In J. Stellman (Ed.) Encyclopedia of Occupational Health and Safety. Geneva: International Labour Office.

Cortina, J., Chen, G. \& Dunlap, W. (2001). Testing interaction effects in LISREL: Examination and illustration of available procedures. Organizational Research Methods, 4(4), 324-360.

Costa, G. (1996). The impact of shift and night work on health. Applied Ergonomics, 27, 9-16.

Costa, G., Folkard, S., \& Harrington, J. (2000). Shift work and extended hours of work. In P. Baxter, P. Adams, T. Aw, Cockcroft, A. \& Harrington, J. (Eds.) Hunter's diseases of occupations ( $9^{\text {th }}$ ed., pp. 581-589). London: Arnold.

Costa, P. \& McCrae, R. (1980). Influence of extraversion and neuroticism on subjective well-being: Happy and unhappy people. Joumal of Personality and Social Psychology, 38, 668-678.

Coverman, S. (1989). Role overload, role conflict, and stress: Addressing consequences of multiple role demands. Social Forces, 67(4), 965-982.

Crossman, G. \& Burton, P. (1993). Telework stereotypes: A case study. Journal of Information Science, 19, 349-362.

Crouter, A. (1984). Spillover from family to work: The neglected side of the workfamily interface. Human Relations, 37, 425-442.

Crouter, A., Bumpus, M., Maguire, M. \& McHale, S. (1999). Linking parents' work pressure and adolescents' well-being: Insights into dynamics in dual-earner families. Developmental Psychology, 35, 1453-1461.

Crouter, A., Helms-Erikson, H., Updegraff, K. \& McHale, S. (1999). Conditions underlying parent's knowledge about children's daily lives in middle 
childhood: Between and within family comparisons. Child Development, 70, 246-259.

Crouter, A, Perry-Jenkins, M., Huston, T., \& Crawford, D. (1989). The influence of work-induced psychological states on behavior at home. Basic and Applied Social Psychology, 10, 273-292.

Crum, R. M., Mutaner, C., Eaton, W. \& Anthony, J. (1995). Occupational stress and the risk of alcohol abuse and dependence. Alcohol and Clinical Experimental Research, 19, 647-655.

Cummings, T., Molloy, E. \& Glen, R. (1977). A methodological critique of fiftyeight selected work experiments. Human Relations, 30, 675-708.

Cunningham, B. (1981). Exploring the impact of a ten-hour compressed shift schedule. Journal of Occupational Behavior, 2, 217-222.

Cunningham, J. (1989). A compressed shift schedule: Dealing with some of the problems of shift-work. Journal of Organizational Behavior, 10, 231-245.

Cunningham, M. (2005). Gender in cohabitation and Marriage: The influence of gender ideology on housework allocation over the life course. Journal of Family Issues, 26 (8), 1037-1061.

Cyber Dialogue (2000) Small Business/Home Office Trend Report. U.S. Small Business Advisory Service, Washington. 11.

Czeisler, C., Moore-Ede, M. \& Coleman, R. (1982). Rotating shiftwork schedules that disrupt sleep are improved by applying circadian principles. Science, $217,460-463$.

Dalton, D. \& Mesch, D. (1990). The impact of flexible scheduling on employee attendance and turnover. Administrative Science Quarterly, 35, 370-387.

Daniels, K. \& Guppy, A. (1994). Occupational stress, social support, job control, and psychological well-being. Human Relations, 47, 1523-1544.

Dannhauser, C. (1999). Who's in the Home Office? American Demographics, July, URL: http://www.demographics.com/publications/ad/00_adad/9907-ad009797.html

Daus, C., Sanders, D. \& Campbell, D. (1998). Consequences of alternative work schedules. In Cooper, C. \& Robertson, I. International Review of Industrial and Organizational Psychology. Vol. 13. New York: John Wiley \& Sons Ltd. 
Davidson, M. \& Fielden, S. (1999). Stress and the Working Woman. In Powell, G. (Ed). Handbook of Gender and Work. Thousand Oaks, CA: Sage Publications, Inc.

Davis, D. \& Polonko, K. (2001). Website: www.workingfromanywhere.org.

Davis-Blake, A. \& Pfeffer, J. (1989). Just a mirage: The search for dispositional effects in organizational research. Academy of Management Review, 14, $385-400$.

Deci, E. \& Ryan, R. (1987). The support of autonomy and the control of behavior. Journal of Personality and Social Psychology, 53, 1024-1037.

Decker, P. \& Borgen, F. (1993). Dimensions of work appraisal: Stress, strain, coping, job satisfaction and negative affectivity. Journal of Counseling Psychology, 40, 470-478.

De Frank, R. \& Ivancevich, J. (1998). Stress on the job: An executive update. Academy of Management Executive, 12, 55-67.

De Jonge, J. (1995). Job autonomy, well-being and health: A study among Dutch health care workers (PhD thesis). Maastricht: Datawyse.

De Jonge, J. \& Kompier, M. (1997). A critical examination of the demand-controlsupport model from a work psychological perspective. International Journal of Stress Management, 4, 235-258.

De Jonge, J. \& Landeweerd, J. (1993). Test of the job demand-control approach among health care workers: a secondary analysis. Gedrag en Organisatie, 21, 79-92.

De Jonge, J., van Breukelen, G., Landeweed, J. \& Nijhuis, F. (1999). Comparing group and individual level assessments of job characteristics in testing the job demand-control model: A multilevel approach. Human Relations, $52,95-122$.

De Lange, A., Toon, T., Kompier, M., Houtman, I. \& Bongers, P. (2003). The very best of the millennium: Longitudinal research and the demandcontrol (support) model. Journal of Occupational Health Psychology, 8(4), 282-305.

De Lay, N. (1995). The effects of telecommuting and gender on work-family conflict and satisfaction. Unpublished doctoral dissertation, Illinois Institute of Technology, Chicago. 
Demerouti, E, Bakker, A. \& Bulters, A. (2004). The loss spiral of work pressure, work-home interference, and exhaustion: Reciprocal relations in a threewave study. Journal of Vocational Behavior, 64, 131-149.

De Rijk, A., Le Blanc, P., Schaufeli, W. \& de Jonge, J. (1998). Active coping and need for control as moderators of the job demand-control model: Effects on burnout. Journal of Occupational and Organizational Psychology, 71, 1-18.

Deutsch, F. (2006) Experimental social psychology and the study of work and family. In M. Pitt-Catsouphes, E. Kossek, \& S. Sweet (Eds.). The work and family handbook: Multi-disciplinary perspectives and approaches. Mahwah, NJ: Lawrence Erlbaum Associates, Publishers.

Diamantopoulos, A. (1994). Modeling with LISREL: A guide for the uninitiated. Journal of Marketing Management, 10, 105-136.

Dillon, W., Madden, T.. and Firtle, N. (1994) Marketing Research in a Research Environment, New York: Irwin.

Di Martino, V. \& Wirth, L. (1990). Telework: A New way of working and living. International Labor Review, 129, 529-554.

DiNatale, M. (March 2001). Characteristics of and preferences for alterantive work arrangements, 1999. Monthly Labor Review, 28-49.

Dirkx, J. (1993). Adaptation to permanent night work: the number of consecutive work nights and motivated choice. Ergonomics, 36, 29-36.

Dohrenwend, B. \& Dohrenwend, B. (Eds.). (1974). Stressful events: Their nature and effects. New York: Wiley.

Dolan, S., Ameringen, M. \& Arsenault, A. (1992). Personality, social support, and workers' stress. Industrial Relations, 47, 125-139.

Dollard, M., Winefield, H., Winefield, A. \& de Jonge, J. (2000). Psychosocial job strain and productivity in human service workers: A test of the demandcontrol-support model. Journal of Occupational and Organizational Psychology, 73, 501-510.

Dooley, B, Byrne, M., Chapman, A., Oborne, D., Heywood, S., Sheehy, N. \& Collins, S. (1994). The teleworking experience. Basingstoke: Taylor \& Francis.

Dunahoo, C., Geller, P. \& Hobfoll, S. (1996). Women's coping: Communal versus individualistic orientation. In Schabracq, M., Winnubst, J. \& Cooper, C. 
(Eds.) (1996) Handbook of Work and Health Psychology. New York: John Wiley \& Sons Ltd.

Dunham, R. (1977) Shiftwork: A review and theoretical analysis. Academy of Management Review, 2, 624-634.

Dunham, R., Pierce, J. \& Castaneda, M. (1987). Alternative work schedules: Two field quasi-experiments. Personnel Psychology, 40, 215-242.

Dunlap, W. \& Kemery, E. (1988). Effects of predictor intercorrelations and reliabilities on moderated multiple regression. Organizational Behavior and Human Decision Processes, 41, 248-258.

Duxbury, L. \& Haines, G. (1991). Predicting alternative work arrangements from salient attitudes: A study of decision makers in the public sector. Journal of Business Research, 23, 83-97.

Duxbury, L. \& Higgins, C. (1991). Gender differences in work-family conflict. Journal of Applied Psychology, 76(1), 60-74.

Duxbury, L. \& Higgins, C. (1997). Supportive managers: What are they? Why do they matter? The HRM Research Quarterly, 1 (4), 1-4.

Duxbury, L. \& Higgins, C. (1998). Work-life balance in Saskatchewan: Realities and challenges.

Duxbury, L. \& Higgins, C. (2001). Work-life balance in the new millennium: Where are we? Where do we need to go? Work Network: Canadian Policy Research Networks, Inc.

Duxbury, L. \& Higgins, C. (2002a). Telework: A primer for the millennium. In Cooper, C. (Ed.) The New World of Work. Sage: London.

Duxbury, L. \& Higgins, C. (2002b). The National Work-Life Conflict Study: Report One. Healthy Communities Division, Health Canada.

Duxbury, L. \& Higgins, C. (2003). Work-Life Conflict in Canada in the New Millenium: A Status Report. Healthy Communities Division, Health Canada.

Duxbury, L. \& Higgins, C. (2005). Who is at risk? Predictors of work-life conflict: Report 4. Healthy Communities Division, Public Health Agency of Canada.

Duxbury, L., Higgins, C. \& Irving, R. (1989). Gender Differences in Work-Family Stress. 89-14. 
Duxbury, L., Higgins, C. \& Johnson, K. (1999). An examination of the implications and costs of work-life conflict in Canada. Ottawa: Health Canada.

Duxbury, L., Higgins, C. \& Lee, C. (1994). Work-Family Conflict: A Comparison by Gender, Family Type, and Perceived Control. Journal of Family Issues, 15, 449-466.

Duxbury, L., Higgins, C., Lee, C. \& Mills, S. (1991). Balancing Work and Family: A study of the Canadian Federal Public Sector.

Duxbury, L., Higgins, C. \& Mills, S. (1992). After-hours telecommuting and workfamily conflict: A comparative analysis. Information Systems Research, 3(2), 173-190.

Duxbury, L., Higgins, C. and Neufeld, D. (1998) Telework and the Balance Between Work and Family: Is Telework Part of the Problem or Part of the Solution? In M. Igbaria and M. Tan (Eds) The Virtual Workplace. Idea Group Publishing, Hershey, PA, pp.218-255.

Duxbury, L., Higgins, C. \& Thomas, D. (1996). Work and family environments and the adoption of computer-supported supplemental work-at-home. Journal of Vocational Behavior, 49, 1-23.

Dwyer, D. \& Ganster, D. (1991). The effects of job demands and control on employee attendance and satisfaction. Journal of Organizational Behavior, 12, 595-608.

Eagle, B., Miles, E. \& Icenogle, M. (1997). Interrole conflicts and the permeability of work and family domains: Are there gender differences? Journal of Vocational Behavior, 50, 168-184.

Eby, L, Casper, W., Lockwood, A., Bordeaux, C. \& Brinley, A. (2005). Work and family research in IO/OB: Content analysis and review of the literature (1980-2002). Journal of Vocational Behavior, 66, 124-197.

Edwards, J. \& Rothbard, N. (2000). Mechanisms linking work and family: Clarifying the relationship between work and family constructs. Academy of Management Review, 25, 178-199.

Eisenberger, R, Huntington, R., Hutchison, S. \& Sowa, D. (1986). Perceived organizational support. Journal of Applied Psychology, 71, 500-507.

Elloy, D. \& Smith, C. (2003). Patterns of stress, work-family conflict, role conflict, role ambiguity and overload among dual-career and single-career couples: An Australian study. Cross Cultural Management, 10(1), 55-66. 
Elloy, D. \& Smith, C. (2004). Antecedents of work-family conflict among dualcareer couples: An Australian study. Cross Cultural Management, 11(4), $17-27$.

Emmons, C., Biernat, M., Tiedje, L., Lang, E. \& Wortman, C. (1990). Stress, support and coping among women professionals with preschool children. In Eckenrode, J. \& Gore, S. (Eds.). Stress Between Work and Family. New York: Plenum.

Etzion (1984). Moderation effect of social support on the stress-burnout relationship. Journal of Applied Psychology, 69, 615-622.

Evans, M. (1973). Notes on the impact of flexitime in a large insurance company: Reactions of nonsupervisory employees. Occupational Psychology, 43, 237-240.

Evans, M. G. (1985). A Monte Carlo study of the effects of correlated method variance in moderated multiple regression analysis. Organizational Behavior and Human Decision Processes, 36, 305-323.

Falk, A., Hanson, B., Isacsson, S, Ostergren, P. (1992). Job strain and mortality in elderly men: social network, support and influence as buffers. American Journal of Public Health, 82, 1136-1139.

Falkenberg, L. \& Monachello, M. (1990). Dual-career and dual-income families: Do they have different needs? Journal of Business Ethics, 9, 339-351.

Fast, J. \& Frederick, J. (Winter 1996). Working arrangements and time stress. Canadian Social Trends, 14-19. Statistics Canada. Catalogue 11-008XPE.

Fast, J. \& Frederick, J. (2004). The time of our lives: Juggling work and leisure over the life cycle. Statistics Canada. Catalogue 89-584-MIE.

Feldman, D. \& Gainey, T. (1997). Patterns of telecommuting and their consequences: Framing the research agenda. Human Resource Management Review, 7, 369-388.

Fenwick, R. \& Tausig, M. (2001). Family and health outcomes of shift work and schedule control. American Behavioral Scientist, 44, 1179-1188.

Ferber, M., O'Farrell, \& Allen, L. (1991). Work and Family: Policies for a Changing Workforce. Washington, DC: National Academy Press. 
Ferrer, A. \& Gagne, L. (2006). The use of family friendly workplace practices in Canada. IRPP Working Paper Series, no. 2006-002. Institute for Research on Public Policy.

Fields, C. (1974). Variable work hours - the experience. Personnel Journal, 53, 675-678.

Fisher, S. (1985). Control and blue collar work. In Cooper, C. \& Smith, M. Job stress and blue collar work. New York: John Wiley and Sons Ltd.

Fletcher, B. (1988). The epidemiology of occupational stress. In Cooper et al. (Eds.) Causes, Coping and Consequences of Stress at Work. New York: John Wiley \& Sons Ltd.

Fletcher, B. \& Jones, F. (1993). A refutation of Karasek's demand-discretion model of occupational stress with a range of dependent measures. Journal of Organizational Behaviour, 14, 319-330.

Folkard, S., Akerstedt, T., Macdonald, I., Tucker, P. \& Spencer, M. (2000). Refinement of the three-process model of alertness to account for trends in accident risk. In S. Hornberger, P. Knauth, G. Costa, \& S. Folkard (Eds.) Shiftwork in the $21^{\text {st }}$ Century: Challenge for research and practice (pp. 49-54). Frankfurt am Main: Peter Lang.

Folkard, S. \& Monk, T. (1979). Shiftwork and performance. Human Factors, 21, 483-492.

Forester, T. (1988). The myth of the electronic cottage. Futures (June), 227-240.

Fornell, C. (1982) (Ed.). A Second Generation of Multivariate Analysis. New York: Praeger.

Foster, L, Latack,J. \& Reindl, L. (1979). Effects and promises of the shortened work week. Academy of Management Proceedings,226-230.

Fouad, N. \& Tinsley, H. (1997). Work-family balance. Journal of Vocational Behavior, 50, 141-144.

Fox, M. \& Dwyer, D. (1999). An investigation of the effects of time and involvement in the relationship between stressors and work-family conflict. Journal of Occupational Health Psychology, 4, 164-174.

Fox, M., Dwyer, D. \& Ganster, D. (1993). Effects of stressful job demands and control on physiological an attitudinal outcomes in a hospital setting. Academy of Management Journal, 36, 289-318. 
Frankel, M. (1998). Creating the family friendly workplace: Barriers and solutions. In S. Klarreich (Ed.). Handbook of Organizational Health Psychology: Programs to Make the Workplace Healthier. Madison, WI: Psychosocial Press.

Frankenhaeuser, M. (1979). Psychobiological aspects of life stress. In S. Levine \& Ursin, H. (Eds.) Coping and Health. New York: Plenum Press.

Frankenhaeuser, M. (1993a). Current issues in psychobiological stress research. In M. Vartiainen (Ed.) European views in psychology - Keynote lectures. Helsinki: Acta Psychologica Fennica XIII.

Frankenhaeuser, M. (1993b). The measurement of the total workload of men and women. In L. Levi (Ed.)A Healthier work environment - Basic concepts and methods of measurements. Geneva: WHO.

Frankenhaeuser, M. (1997). Workload. In J. Stellman (Ed.) Encyclopedia of Occupational Health and Safety. Geneva: International Labour Office.

Frankenhaeuser, M. \& Gardell, B. (1976). Underload and overload in working life: Outline of a multidisciplinary work. Journal of Human Stress, 2, 34-46.

Frankenhaeuser, M., Lundberg, U. \& Chesney, M. (1991) (Eds.) Women, Work and Health: Stress and Opportunities. New York: Plenum Press.

Fredriksen, K. \& Scharlach, A. (1999). Employee family care responsibilities. Family Relations, 48, 189-196.

Freedictionary.com (2006) Definition of strain. http://www.thefreedictionary.com/strain.

French, J. (1974). Person role fit. In McLean, A. (Ed.) Occupational Stress. Spingfield, III: Thomas.

Frese, M. (1989). Theoretical models of control and health. In Sauter, S., Hurrell, J. \& Cooper, C. (1989) Job control and worker health. New York: John Wiley and Sons Ltd.

Frese, M. (1999). Social support as a moderator of the relationship between work stressors and psychological dysfunctioning: A longitudinal study with objective measures. Journal of Occupational Health Psychology, 4 (3), 179-192.

Frese, M. \& Sabini, J. (1985). Goal Directed Behaviour: The concept of Action in Psychology. Hillsdale, NJ: Lawrence Erlbaum Associates. 
Frese, M. \& Semmer, N. (1986). Shiftwork, stress, and psychosomatic complaints: a comparison between workers in different shiftwork schedules, non-shiftworkers and former shiftworkers. Ergonomics, 29, 99-114.

Frese, M. \& Zapf, D. (1988). Methodological issues in the study of work stress: Objective vs. subjective measurement of work stress and the question of longitudinal studies. In Cooper, C. et al. (Eds). Causes, Coping and Consequences of Stress at Work. New York: John Wiley \& Sons Ltd.

Frese, M. \& Zapf. D. (1994). Action as the core of work psychology: A German approach. In H. C. Triandis, M. D. Dunnette and L. M. Hough (Eds.) Handbook of industrial and organizational psychology (Vol. 4). Palo Alto, CA: Consulting Psychologists Press. Pp. 271-340.

Fried, Y. (1988). The future of physiological assessments in work situations. In Cooper, C. et al. (Eds). Causes, Coping and Consequences of Stress at Work. New York: John Wiley \& Sons Ltd.

Fried, Y. \& Tiegs, R. (1993). The main effect model versus buffering model of shop steward social support: A study of rank-and-file auto workers in the U.S.A. Journal of Organizational Behavior, 14, 481-493.

Friedman, D. (1991). Linking Work-Family Issues to the Bottom Line. New York: The Conference Board.

Friedman, D. \& Galinsky, E. (1992). Work and family issues: a legitimate business concern. In Zedeck, S. (Ed.) Work, families and organizations. San Francisco: Jossey-Bass, Inc.

Friedman, D. \& Johnson, A. (1997). Moving from programs to culture change: the next stage for the corporate work-family agenda. In Parasuraman, S. \& Greenhaus, J. (Eds.). Integrating work and family: challenges and choices for a changing world. Westport, CT: Quorum Books.

Frone, M. (2000). Work-family conflict and employee psychiatric disorders: The national comorbidity survey. Journal of Applied Psychology, 85, 888-895.

Frone, M. (2003). Work-Family Balance. In J. Quick, \& L. Tetrick (Eds.) Handbook of Occupational Health Psychology. Washington: American Psychological Association.

Frone, M., Barnes, G. \& Farrell, M. (1994). Relationship of work-family conflict to substance use among employed mothers: The role of negative affect. Journal of Marriage and the Family, 56, 1019-1030. 
Frone, M., Russell, M. \& Barnes, G. (1996b). Work-family conflict, gender and health-related outcomes: A study of employed parents in two community samples. Journal of Occupational Health Psychology, 1, 57-69.

Frone, M., Russell, M., \& Cooper, L. (1992a). Antecedents and Outcomes of Work-Family Conflict: Testing a Model of the Work-Family Interface. Journal of Applied Psychology, 77, 65-78.

Frone, M., Russell, M., \& Cooper, L. (1992b). Prevalence of work-family conflict: Are work and family boundaries asymmetrically permeable. Journal of Organizational Behavior, 13, 723-729.

Frone, M., Russell, M., \& Cooper, L. (1993). Relationship of work-family conflict, gender, and alcohol expectancies to alcohol use/abuse. Journal of Organizational Behavior, 14, 545-558.

Frone, M., Russell, M. \& Cooper, M. (1995). Relationship of work and family stressors to psychological distress: The independent moderating influence of social support, mastery, active coping and self-focused attention. In Crandall, R. \& Perrewe, P. Occupational stress: A handbook. Washington: Taylor and Francis.

Frone, M., Russell, M. \& Cooper, L. (1997). Relation of work-family conflict to health outcomes: A four-year longitudinal study of employed parents. Journal of Occupational and Organizational Psychology, 70, 325-335.

Frone, M. \& Yardley, J. (1996). Workplace family-supportive programmes: Predictors of employed parents' importance ratings. Journal of Occupational and Organizational Psychology, 69, 351-366.

Frone, M., Yardley, J., \& Markel, K. (1997). Developing and testing an integrative model of the work-family interface. Journal of Vocational Behavior, 50, 145-167.

Fu, C. \& Shaffer, M. (2001). The tug of work and family: Direct and indirect domain-specific determinants of work-family conflict. Personnel Review, $30,502-522$.

Galambos, N. \& Walters, B. (1992). Work hours, schedule inflexibility, and stress in dual-earner spouses. Canadian Journal of Behavioural Science, 24(3), 290-302.

Galinsky, E. \& Bond, J. (1998). Business Work-Life Study. New York: Families and Work Institute. 
Galinsky, E., Bond, J. \& Friedman, D. (1993). The changing workforce: Highlights of the national study. New York: Families and Work Institute.

Galinsky, E., Bond, J. \& Hill, J. (2004). When work works: A status report on workplace flexibility. New York: Families and Work Institute.

Galinsky, E., \& Johnson, A. (1998). Reframing the business case for work-life initiatives. New York: Families and Work Institute.

Galinsky, E., Kim, S. \& Bond, J. (2001). Feeling overworked: When work becomes too much. Families and Work Institute.

Galinsky, E, Ruopp, R. \& Blum, K (1983). The Corporate Work and Family Life Study. Unpublished data.

Galinsky, E. \& Stein, P. (1990). The impact of human resource policies on employees: Balancing work/family life. Journal of Family Issues, 11, 368383.

Galt, V. (November 10, 2004). Heavy workloads hit health care: study. Globe and Mail, p. B1.

Ganster, D. (1989). Worker control and well-being: A review of research in the workplace. In Sauter, S., Hurrell, J. \& Cooper, C. (Eds.) Job control and worker health. New York: John Wiley and Sons Ltd.

Ganster, D. C. (1995). Interventions for building healthy organizations: Suggestions from the stress research literature. In L. R. Murphy, J.J. Hurrell Jr., S. L. Sauter \& C. P. Keita (Eds.) Job stress interventions. Washington: APA.

Ganster, D. (1997). Autonomy and Control. In J. Stellman (Ed.) Encyclopedia of Occupational Health and Safety. Geneva: International Labour Office.

Ganster, D. \& Fusilier, M. (1989). Control in the workplace. In Cooper et al. (Eds.) International Review of Industrial and Organizational Psychology. New York: John Wiley \& Sons Ltd.

Ganster, D. \& Schaubroeck, J. (1991). Work stress and employee health. Journal of Management, 17, 235-271.

Ganster, D. \& Schaubroeck, J. (1995). The moderating effect of self-esteem on the work stress-employee health relationship. In Crandall, R. \& Perrewe, $P$. Occupational stress: A handbook. Washington: Taylor and Francis. 
Gardell, B. (1971). Technology, alienation, and mental health. Stockholm: Personaladministrativa Radet.

Gardner, A. \& Dagnall, B. (1977). The effects of twelve-hour shifts working on absence attributed to sickness. British Journal of Industrial Medicine, 34, 148-150.

General Mills, Inc. (1981). General Mills American Family Report, Families at work: Strengths and strains. Minneapolis, MN: Louis Haldi \& Associates.

Gerstel, N. \& Gallagher, S. (1993). Kinkeeping and distress: Gender, recipients of care, and work-family conflict. Journal of Marriage and the Family, 55, 598-608.

Gerstel, N. \& Sarkisian, N. (2006). Sociological perspectives on families and work: The import of gender, class and race. In M. Pitt-Catsouphes, E. Kossek, \& S. Sweet (Eds.). The work and family handbook: Multidisciplinary perspectives and approaches. Mahwah, NJ: Lawrence Erlbaum Associates, Publishers.

Gervais, G., Li, C. \& Duval, A. (2006). The dynamics of overqualification: Canada's underemployed university graduates. Analysis in Brief, no. 39. Statistics Canada. Catalogue No. 11-621-MIE2006039.

Geurts, S. \& Demerouti, E. (2003). Work/non-work interface: A review of theories and findings. In Schabracq, M., Winnubst, J. \& Cooper, C. (Eds.) The Handbook of Work and Health Psychology. Chichester, UK: John Wiley \& Sons.

Glass, J. \& Camarigg, V. (1992). Gender, parenthood, and job-family compatibility. The American Journal of Sociology, 98(1), 131-151.

Glass, J. \& Fujimoto, T. (1994). Housework, paid work, and depression among husbands and wives. Journal of Health and Social Behavior, 35, 179191.

Goff, S., Mount, M, \& Jamison, R. (1990). Employer supported child care, workfamily conflict and absenteeism: A field study. Personnel Psychology, 43, 793-809.

Goldberger, L. \& Breznitz, S. (Eds.) (1993). Handbook of Stress: Theoretical and Clinical Aspects. New York: The Free Press.

Golden, L. (2001). Flexible work schedules: Which workers get them? American Behavioral Scientist, 44(7), 1157-1178. 
Goldsmith, E. \& Walters, T. (1989). Selected bibliography on work and family. In Goldsmith, E. (Ed.) Work and family: Theory, research and applications. Newbury Park, CA: Select Press.

Golembiewski, R., Hilles, R. \& Kagno, M. (1974). A longitudinal study of flextime effects: Some consequences of an OD structural intervention. Journal of Behavioral Science, 4, 503-532.

Gonyea \& Googins (1992) Linking the worlds of work and family: beyond the productivity trap. Human Resource Management, 31 (3), 209-226.

Good, L, Sisler, G. \& Gentry, J. (1988). Antecedents of turnover intentions among retail management personnel. Journal of Retailing, 64, 295-314.

Goodale, J. \& Aagaard, A. (1975). Factors relating to varying reactions to the 4day workweek. Journal of Applied Psychology, 60, 33-38.

Googins, B. (1997). Shared responsibility for managing work and family relationships: A community perspective. In Parasuraman, S. \& Greenhaus, J. (Eds.) Integrating work and family: challenges and choices for a changing world. Westport, CT: Quorum Books.

Gore, S. (1986). Perspectives on social support and research on stress moderating processes. Journal of Organizational Behavior Management, 8, 85-101.

Gottlieb, B, Kelloway, K \& Barham, E. (1998). Flexible work arrangements: Managing the work-family boundary. New York: Wiley.

Gove, W. (1978). Sex differences in mental illness among adult men and women: An evaluation of four questions raised regarding the evidence on the higher rates of women. Social Science and Medicine, 12B, 187-198.

Gove, W. \& Hughes, M. (1979). Possible causes of the apparent sex differences in physical health: An empirical investigation. American Sociological Review, 44, 126-146.

Government of Canada (2002). 2002 Public Service Employee Survey. Catalogue No. BT49-4/19-2002.

Gowing, M. (1997). Staffing Issues. In J. Stellman (Ed.) Encyclopedia of Occupational Health and Safety. Geneva: International Labour Office.

Grandey, A. \& Cropanzano, R. (1999). The conservation of resources model applied to work-family conflict and strain. Journal of Vocational Behavior, $54,350-370$. 
Green, P.E. Tull, D.S. and Albaum G (1993) Research methods for marketing decisions, New York: Prentice Hall.

Greenberger, D. \& Strasser, S. (1986). Development and application of a model of personal control in organizations. Academy of Management Review, $11,164-177$.

Greenberger, D., Strasser, S., Cummings, L. \& Dunham, R. (1989). The impact of personal control on performance and satisfaction. Organizational Behavior and Human Decision Processes, 43, 29-51.

Greenberger, E, Goldberg, W., Hamill, S., O'Neil, R. \& Payne, C. (1989). Contributions of a supportive work environment to parents' well-being and orientation to work. American Journal of Community Psychology, 17, 755-783.

Greenglass, E., Pantony, K. \& Burke, R. (1989). A gender-role perspective on role conflict, work stress and social support. In Goldsmith, E. (Ed.). Work and family: Theory, research and applications. Newbury Park, CA: Select Press.

Greenhaus, J. (1989). The intersection of work and family roles: individual, interpersonal, and organizational issues. In Goldsmith, E. (Ed.) Work and family: Theory, research and applications. Newbury Park, CA: Select Press.

Greenhaus, J., Bedeian, A. \& Mossholder, K. (1987). Work experiences, job performance, and feelings of personal and family well-being. Journal of Vocational Behavior, 31, 200-215.

Greenhaus, J. \& Beutell, N. (1985). Sources of Conflict Between Work and Family Roles. Academy of Management Review, 19, 76-88.

Greenhaus, J. \& Parasuraman, S. (1986). A work-nonwork interactive perspective of stress and its consequences. Journal of Organizational Behavior Management, 8, 37-60.

Greenhaus, J. \& Parasuraman, S. (1999). Research on work, family and gender: Current status and future directions. In Powell, G. (Ed). Handbook of Gender and Work. Thousand Oaks, CA: Sage Publications, Inc.

Greenhaus, J., Parasuraman, S. \& Collins, K. (2001). Career involvement and family involvement as moderators of relationships between work-family conflict and withdrawal from a profession. Journal of Occupational Health and Psychology, 6 (2), 91-100. 
Greenhaus, J., Parasuraman, S., Granrose, C., Rabinowitz, S. \& Beutell, N. (1989). Sources of work-family conflict among two-career couples. Journal of Vocational Behavior, 34, 133-153.

Greenhaus, J. \& Powell, G. (2003). When work and family collide: Deciding between competing role demands. Organizational Behavior and Human Decision Processes, 90, 291-303.

Greenlund, K., Liu, K., Knox, S., McCreath, H., Dyer, A. \& Gardin, J. (1995). Psychosocial work characteristics and cardiovascular disease risk factors in young adults: The CARDIA study. Social Science Medicine, $41,717-723$.

Grimm, L. \& Yarnold, P. (Eds). (2000). Reading and Understanding More Multivariate Statistics. Washington: American Psychological Association.

Grzywacz, J., Almeida, D., \& McDonald, D. (2002). Work-family spillover and daily reports of work and family stress in the adult labor force. Family Relations, 51, 28-36.

Grzywacz, J. \& Marks, N. (2000). Reconceptualizing the work-family interface: An ecological perspective on the correlates of positive and negative spillover between work and family. Journal of Occupational Health Psychology, 5, 111-126.

Guelzow, M., Bird, G. \& Koball, E. (1991). An exploratory path analysis of the stress process for dual-career men and women. Journal of Marriage and the Family, 53, 151-164.

Gutek, B., Searle, S. \& Klepa, L. (1991). Rational versus gender role expectations for work-family conflict. Journal of Applied Psychology, 76, 560-568.

Guttentag, M. \& Salasin, S. (1976). Women, men and mental health. In L.A. Carter, A. F. Scott, \& W. Martyna (Eds.) Women and men: Changing roles, relationships, and perceptions. New York: Aspen Institute for Humanistic Studies.

Haan, M. (1985). Job strain and cardiovascular disease: A ten-year perspective study. Paper presented at the eighteenth annual meeting of the Society for Epidemiologic Research. Chapel Hill, NC. American Journal of Epidemiology, 122, 532. (Abstract).

Hackman, J. \& Lawler, E. (1971). Employee reactions to job characteristics. Journal of Applied Psychology, 55, 259-286. 
Hackman, J. \& Oldham, G. (1975). Development of the Job Diagnostic Survey. Journal of Applied Psychology, 60, 159-170.

Hall, D. (1990). Promoting work/family balance: An Organization-Change Approach. Organizational Dynamics, 18, 4-18.

Hall, D. \& Richter, J. (1988). Balancing work life and home life: What can organizations do to help? The Academy of Management Executive, 2(3), 213-223.

Hall, E. (1989). Gender, work control, and stress: A theoretical discussion and an empirical test. International Journal of Health Services, 19, 725-745.

Hall, E. (1992). Double exposure: The combined impact of the home and work environments on mental strain and physical illness. International Journal of Health Services, 22, 239-260.

Hall, K. \& Savery, L. (1986). Tight rein, more stress. Harvard Business Review, January-February, 160-164.

Hamilton, C. (1987). Telecommuting. Personnel Journal, 66, 91-101.

Hammar, N., Alfredsson, L. \& Johnson, J. (1998). Job strain, social support at work, and incidence of myocardial infarction. Occupational Environmental Medicine, 55, 548-553.

Hammer, L., Allen, E. \& Grigsby, T. (1997). Work-family conflict in dual-earner couples: Within individual and crossover effects of work and family. Journal of Vocational Behavior, 50, 185-203.

Hammer, L. \& Barbera, K. (1997). Toward an integration of alternative work. Human Resource Planning, 20(2), 28-36.

Hammer, L., Colton, C., Caubet, S. \& Brockwood, K. (2002). The unbalanced life: Work and family conflict. In Thomas, J. \& Hersen, M. (Eds.) Handbook of Mental Health in the Workplace. Thousand Oaks, CA: Sage Publications.

Hammer, L., Cullen, J., Neal, M., Sinclair, R., \& Shafiro, M. (2005). The longitudinal effects of work-family conflict and positive spillover on depressive symptoms among dual-earner couples. Journal of Occupational Health Psychology, 10 (2), 138-154. 
Harenstam, A. \& Theorell, T. (1988). Work conditions and urinary excretion of catecholamines: A study of prison staff in Sweden. Scandinavian Journal of Work, Environment and Health, 14, 257-264.

Harrick, E., Vanek, G. \& Michlitsch, F. (1986). Alternate work schedules, productivity, leave usage and employee attitudes: A field study. Public Personnel Management, 15(2), 159-169.

Harris, H. (2004). Work-life issues and the adjustment of women international managers. Journal of Management Development, 23(9), 818-832.

Hartman, R., \& Weaver, K. (1977). Four factors influencing conversion to a fourday work week, Human Resource Management, 16, 24-27.

Hartman, R., Stoner, C. \& Arora, R. (1992). Developing successful organizational telecommuting arrangements: worker perceptions and managerial perceptions. SAM Advanced Management Journal, Summer, 35-42.

Harvey, B. \& Luthans, F. (1979). Flexitime: An empirical analysis of its real meaning and impact, MSU Business Topics, 27, 31-36.

Havlovic, S., Lau, D. \& Pinfield, L. (2002). Repercussions of work schedule congruence among full-time, part-time and contingent nurses. Health Care Management Review, 27(4), 30-41.

Hayashi, T., Kobayashi, Y., Yamaoka, K. \& Yano, E. (1996). Effect of overtime work on 24-hour ambulatory blood pressure. Journal of Occupational and Environmental Medicine, 38(10), 1007-1011.

Hayduk, L. (1996). LISREL: Issues, debates and strategies. Baltimore, MD: John Hopkins University Press.

Haynes, S., Feinleib, M. \& Kannel, W. (1980). The relationship of psychosocial factors to coronary heart disease in the Framingham study. Part III: Eight year incidence of coronary heart disease. American Journal of Epidemiology, 3, 37-58.

Haynes, S., LaCroix, A., \& Lippin, T. (1987). The effect of high job demands and low control on the health of employed women. In Quick et al. (Eds.) Work Stress. New York: Praeger Publishers.

Heaney, C, House, J., Israel, B. \& Mero, R. (1995b). The relationship of organizational and social coping resources to employee coping behaviour: a longitudinal analysis. Work and Stress, 9, 416-431. 
Heaney, C., Price, R. \& Rafferty, J. (1995a). Increasing coping resources at work: A field experiment to increase social support, improve work team functioning, and enhance employee mental health. Journal of Organizational Behavior, 16, 335-352.

Heart Center (2005). Glossary of Cardiology Terms. http://www.heartcenter.com/glossary.html.

Heck, R., Walker, R. \& Furry, M. (1996). The Workers at Work at Home, HomeBased Employment and Family Life. In R. Heck, A. Owen and B. Rowe (Eds.) National Academy Press. Washington, D.C. Pg. 41-74.

Heisz, A. \& LaRochelle-Cote, S. (2006). Work hours instability in Canada. Analytical Studies Branch Research Paper Series. Statistics Canada: Ottawa. Catalogue no. 11F0019MIE-no. 278.

Hendrick, C. \& Clark, M. (1990). Research methods in personality and social psychology. Review of Personality and Social Psychology, volume 11. Newbury Park, CA: Sage Publications.

Hendrix, W., Ovalle, N. \& Troxler, R. (1985). Behavioral and physiological consequences of stress and its antecedent factors. Journal of Applied Psychology, 70, 188-201.

Hendrix, W., Spencer, B. \& Gibson, G. (1994). Organizational and extraorganizational factors affecting stress, employee well-being, and absenteeism for males and females. Journal of Business and Psychology, 9, 103-128.

Hennon, C., Loker, S. \& Walker, R. (Eds.) (2000). Gender and Home-Based Employment. Westport, CT: Auburn House.

Hetrick, R. (2000). Analyzing the recent upward surge in overtime hours. Monthly Labor Review, February, 30-33.

Hewitt Associates (1995). Work and Family Benefits Provided by Major U.S. Employers in 1995. Lincolnshire, IL: Hewitt Associates.

Hicks, W. \& Klimoski, R. (1981). The impact of flexitime on employee attitudes. Academy of Management Journal, 24, 333-341.

Higgins, C. \& Duxbury, L. (1992). Work-family conflict: A comparison of dualcareer and traditional-career men. Journal of Organizational Behavior, 13(4), 389-411. 
Higgins, C., Duxbury, L. \& Irving, R. (1992). Work-Family Conflict in the DualCareer Family. Organizational Behavior and Human Decision Processes, 51, 51-75.

Higgins, C., Duxbury, L. \& Johnson, K. (2000). Part-time work for women: Does it really help balance work and family? Human Resource Management, 39 (1), 17-32.

Higgins, C., Duxbury, L. \& Johnson, K. (2004). Exploring the link between worklife conflict and demands on Canada's health care system: Report 3. Healthy Communities Division. Public Health Agency of Canada.

Higgins, C., Duxbury, L. \& Lee, C. (1992). Balancing Work and Family: A study of Canadian Private Sector Employees. London: Western Business School.

Higgins, C., Duxbury, L. \& Lee, C. (1994) Impact of Life-Cycle Stage and Gender on the Ability to Balance Work and Family Responsibilities. Family Relations, 43, 144-150.

Hill, E. (2005). Work-family facilitation and conflict, working fathers and mothers, work-family stressors and support. Journal of Family Issues, 26(6), 793819.

Hill, E., Campbell, A. \& Koblenz, M. (1997, March). The art of employee surveys: Using surveys for organizational change. Paper presented at the 1997 Conference Board Work and Family Conference, New York, NY.

Hill, E., Hawkins, A., Ferris, M. \& Weitzman, M. (2001). Finding an extra day a week: The positive influence of perceived job flexibility on work and family life balance. Family Relations, 50, 49-58.

Hill, E., Hawkins, A. \& Miller, B. (1996). Work and Family in the Virtual Office: Perceived influences of mobile telework. Family Relations, 45, 293-301.

Hill, E., Jackson, A. \& Martinengo, G. (2006). Twenty years of work and family at International Business Machines Corporation. American Behavioral Scientist, 49 (9), 1165-1183.

Hill, E., Miller, B., Weiner, S. \& Colihan, J. (1998). Influences of the virtual office on aspects of work and work/life balance. Personnel Psychology, 51, 667-683.

Himle, D., Jayaratne, S. \& Thyness, P. (1989). The buffering effects of four types of supervisory support on work stress. Administration in Social Work, 13, 19-34 
Hinkle, L. (1974). The effect of exposure to culture change, social change and changes in interpersonal relationships on health. In Dohrenwend, $B$. \& Dohrenwend, B. (Eds.) Stressful events: Their nature and effects. New York: Wiley.

Hipple, S. (1998). Contingent work: results from the second survey. Monthly Labor Review, November, 22-35.

Hipple, S. (2001). Contingent work in the late-1990s. Monthly Labor Review, March, 3-27.

Hipple, S. \& Stewart, J. (1996). Earnings and benefits of contingent and noncontingent workers. Monthly Labor Review, October, 22-30.

Hobfoll, S. (1986). Social support: research, theory, and applications from research on women. In Hobfoll, S. (Ed.) Stress, social support and women. Washington: Hemisphere Publishing Corp.

Hodge, B. \& Tellier, R. (1975). Employee reactions to the four-day week, California Management Review, 18, 25-30.

Holme, I., Helgeland, A., Hjermann, I., Leren, P. \& Lund-Larsen, P. G. (1980). Four-year mortality by some economic indictors: The Oslo study. Journal of Epidemiology and Community Health, 34, 48-52.

Holt, R. (1992). Occupational stress. In Goldberger, L. \& Breznitz, S. (Eds) Handbook of Stress. New York: Free Press.

House, J. (1981). Work stress and social support. Reading, MA: AddisonWesley.

Huck, S., Cormier, W. \& Bounds, W. (1974). Reading statistics and research. New York: Harper \& Row Publishers.

Hughes, D. \& Galinsky, E. (1988). Balancing work and family lives: Research and corporate applications. In Gottfried, A. \& Gottfried, A. (Eds.) Maternal employment and children's development. New York: Plenum.

Hughes, D. \& Galinsky, E. (1994). Work experiences and marital interactions: elaborating the complexity of work. Journal of Organizational Behavior, 15(5), 423-438.

Huitema, B. (1980). The analysis of covariance and alternatives. New York: John Wiley \& Sons. 
Hurrell, J. \& Colligan, M. (1985). Alternative work schedules: flextime and the compressed work week. In Cooper, C. \& Smith, M. Job stress and blue collar work. New York: John Wiley and Sons Ltd.

Huws, U. (1993). Teleworking in Britain. The Employment Dept., Research Series, 18.

Industry Canada (2004). Key small business statistics (April 2004): How many businesses are there in Canada? http://strategis.ic.gc.ca/epic/internet/insbrp-rppe.nsf/en/rd00861e.html

Israel, B., House, J., Schurman, S., Heaney, C. \& Mero, R. (1989). The relation of personal resources, participation, influence, interpersonal relationships and coping strategies to occupational stress, job strains and health: a multivariate analysis. Work and Stress, 3, 163-194.

Ivancevich, J. (1974). Effects of the shorter workweek on selected satisfaction and performance measures. Journal of Applied Psychology, 59, 717721.

Ivancevich, J. \& Ganster, D. (1986a). Stress: Theory, research and suggestions. Journal of Organizational Behavior Management, 8, 1-4.

Ivancevich, J. \& Lyon, H. (1977). The shortened workweek: A field experiment. Journal of Applied Psychology, 62, 34-37.

Ivancevich, J. \& Matteson, M. (1980). Stress at work: A managerial perspective. Glenview, IL: Scott, Foresman \& Co.

Ivancevich, J. \& Matteson, M. (1986b). Organizational level stress management interventions: A review and recommendations. Journal of Organizational Behavior Management, 8, 229-248.

Jackson, P. (1992). Specifying the buffering hypothesis: Support, strain and depression. Social Psychology Quarterly, 55(4), 363-378.

Jackson, P., Wall, T., Martin, R. \& Davids, K. (1993). New measures of job control, cognitive demand and production responsibility. Journal of Applied Psychology, 78,753-762.

Jackson, S. \& Maslach, C. (1982). After-effects of job-related stress: Families as victims. Journal of Occupational Behavior, 3, 63-77.

Jacobs, J. (1999). The sex segregation of occupations: Prospects for the $21^{\text {st }}$ century. In Powell, G. (Ed). Handbook of Gender and Work. Thousand Oaks, CA: Sage Publications, Inc. 
Jacobs, J. \& Gerson, K. (1998). Who are the overworked Americans? Review of Social Economy, 56, 442-459.

Jacobs, J. \& Gerson, K. (2004). The time divide: Work, family and gender inequality. Cambridge, MA: Harvard University Press.

Jacquard, J., Turrisi, R. \& Wan, C. (1990). Interaction Effects in Multiple Regression. London: Sage Publications.

Jamal, M. \& Baba, V. (1992). Shiftwork and department-type related to job stress, work attitudes and behavioral intentions: A study of nurses. Journal of Organizational Behavior, 13, 449-464.

Jamal, M. \& Jamal, S. (1982). Work and nonwork experiences of employees on fixed and rotating shifts: An empirical assessment. Journal of Vocational Behavior, 20, 282-293.

James, L. R. \& Jones, A. P. (1980). Perceived job characteristics and job satisfaction: An examination of reciprocal causation. Personnel Psychology, 33, 97-135.

James, L. R. \& Tetrick, L. E. (1986). Confirmatory analytic tests of three causal models relating job perceptions to job satisfaction. Journal of Applied Psychology, 71, 77-82.

Jansen, N., Kant, I., Kristensen, T., \& Nijhuis, F. (2003). Antecedents and consequences of work-family conflict: A prospective cohort study. Journal of Occupational and Environmental Medicine, 45, 479-491.

Johnson, J. (1985). The effects of control and social support on work-related strain and adverse health outcomes. Research report 39, Department of Psychology, University of Stockholm, Stockholm.

Johnson, J. (1986). The impact of the workplace social support, job demands, and work control under cardiovascular disease in Sweden. Ph.D. diss., Johns Hopkins University. Distributed by Department of Psychology, University of Stockholm, Report no. 1-86.

Johnson, J.V. (1989). Control, collectivity and the psychosocial work environment. In Sauter, S., Hurrell, J. \& Cooper, C. (Eds.) Job control and worker health. New York: John Wiley and Sons Ltd.

Johnson, J. \& Hall, E. (1988). Job strain, workplace social support, and cardiovascular disease: a cross sectional study of a random sample of 
the Swedish working population. American Journal of Public Health, 78, 1336-1342.

Johnson, J., Hall, E., Ford, D., Mead, L., Levine, D., Wang, N., \& Klag, M. (1995). The psychosocial work environment of physicians. Journal of Occupational and Environmental Medicine, 37, 1151-1159.

Johnson, J., Hall, E. \& Theorell, T. (1989). Combined effects of job strain and social isolation on cardio-vascular disease morbidity and mortality in a random sample of the Swedish male working population. Scandinavian Journal of Work, Environment and Health, 15, 271-279.

Johnson, J. V., Stewart, W. Fredlund, P., Hall, E.M., \& Theorell, T. (1990). Psychosocial job exposure matrix: An occupationally aggregated attribution system for work environment exposure characteristics. Stress Research Reports, 221: National Institute for Psychosocial Factors and Health, Stockholm.

Johnson, K. (1997). A work and family perspective on the effects of shiftwork. Master's Dissertation. Carleton University.

Johnson, K., Lero, D. \& Rooney, S. (2001). Work-Life Compendium 2001: 150 Canadian Statistics on Work, Family and Well-Being. Centre for Families, Work and Well-Being. Guelph, ON: University of Guelph.

Jones, A. \& Butler, M. (1980). A role transition approach to the stress of organizationally induced family role disruption. Journal of Marriage and the Family, 42, 367-376.

Jones, F. \& Fletcher, B. (1996). Job control and health. In M. Schabracq et al., Handbook of Work and Health Psychology. New York: John Wiley \& Sons Ltd.

Jones, F., Burke, R. \& Westman, M. (2006) (Eds). Work-life balance: A psychological perspective. New York: Psychology Press.

Joure, S., Leon, J., Simpson, D., Holley, C., \& Frye, R. (1989, March). Stress: The pressure cooker at work. The Personnel Administrator, 34, 92-95.

Judy, R. \& D'Amico, C. (1997). Workforce 2020: Work and workers in the $21^{\text {st }}$ Century. Indianapolis, IN: Hudson Institute.

Kahn, R. (1991). The forms of women's work. In Frankenhaeuser, M., Lundberg, $U$. and Chesney, $M$ (Eds.) Women, work and health, stress and opportunities. New York: Plenum. 
Kahn, R., \& Byosiere, P. (1992). Stress in organizations. In Dunnette, M. \& Hough, L. (Eds.) Handbook of industrial and organizational psychology. Volume 3 Palo Alto, CA: Consulting Psychologists Press, Inc.

Kahn, R., Wolfe, D., Quinn, R., Snoek, J. \& Rosenthal, R. (1964). Organizational stress: Studies in role conflict and ambiguity. New York: Wiley.

Kalleberg, A. (1977). Work values and job rewards: A theory of job satisfaction. American Sociological Review, 42, 124-143.

Kanner, A., Kafry, D. \& Pines, A. (1978). Conspicuous in its absence: The lack of positive conditions as a source of stress. Journal of Human Stress, 4 , 33-39.

Kanter, R. (1977). Work and family in the United States: A critical review and agenda for research and policy. New York: Russell Sage Foundation.

Kanter, R. (1993). Men and Women of the Corporation. New York: Basic Books.

Karasek, R. (1979). Job demands, job decision latitude, and mental strain: Implications for job redesign. Administrative Science Quarterly, 24, 285309.

Karasek, R. (1985). Job content questionnaire. Department of Industrial and Systems Engineering, University of Southern California, Los Angeles.

Karasek, R. (1989). Control in the workplace and its health-related aspects. In Sauter, S., Hurrell, J. \& Cooper, C. (Eds.) Job control and worker health. New York: John Wiley and Sons Ltd.

Karasek, R. (1990). Lower health risk with increased job control among white collar workers. Journal of Organizational Behavior, 11, 171-185.

Karasek, R. (1997). Demand/Control Model: A social, emotional and physiological approach to stress risk and active behaviour development. In J. Stellman (Ed.) Encyclopedia of Occupational Health and Safety. Geneva: International Labour Office

Karasek, R., Baker, D., Marxer, F., Ahlbom, A. \& Theorell, T. (1981). Job decision latitude, job demands, and cardiovascular disease: A prospective study of Swedish men. American Journal of Public Health, 71, 694-705.

Karasek, R. \& Gardell, B. (1984). Managing job stress. Unpublished manuscript. New York: Columbia University, Department of Industrial Engineering and Operations Research. 
Karasek, R., Gardell, B., \& Lindell, J. (1987). Work and non-work correlates of illness and behaviour in male and female Swedish white-collar workers. Journal of Occupational Behaviour, 8, 187-207.

Karasek, R., Theorell, T., Schwartz, J., Schnall, P., Pieper, C. \& Michela, J. (1988). Job characteristics in relation to the prevalence of myocardial infarction in the U.S. HES and HANES. American Journal of Public Health, 78, 910-918.

Karasek, R. \& Theorell, T. (1990). Healthy work: Stress, productivity, and the reconstruction of working life. New York: Basic Books.

Karasek, R., Triantis, K. \& Chaudhry, S. (1982). Co-worker and supervisor support as moderators of associations between task characteristics and mental strain. Journal of Occupational Behaviour, 3, 181-200.

Kasl, S. (1979). Epidemiological contributions to the study of work stress. In Cooper, C. \& Payne, R. (Eds.) Stress at work. New York: Wiley.

Kasl, S. (1989). An epidemiological perspective on the role of control in health. In Sauter, S., Hurrell, J. \& Cooper, C. Job Control and Worker Health. New York: John Wiley \& Sons Ltd.

Kasl, S. (1996). The influence of the work environment on cardiovascular health: A historical, conceptual, and methodological perspective. Journal of Occupational Health Psychology, 1, 42-56.

Kasl, S. (1998). Measuring job stressors and studying the health impact of the work environment: An epidemiologic commentary. Journal of Occupational Health Psychology, 3, 390-491.

Kaufmann \& Beehr (1986). Interaction between job stessors and social support: some counterintuitive results. Journal of Applied Psychology, 71, 522-526.

Kauppinen-Toropainen, K. (1981). Job demands and job content: Effects on job dissatisfaction and stress. Department of Psychology, Institute of Occupational Health, Helsinki.

Kauppinen-Toropainen, K., Kandolin, I. \& Mutanen, P. (1983). Job dissatisfaction and work-related exhaustion in male and female work. Journal of Occupational Behavior, 4, 193-207.

Kawakami, N., Haratani, T. \& Araki, S. (1992). Effects of perceived job stress on depressive symptoms in blue-collar workers of an electrical factory in 
Japan. Scandinavian Journal of Work, Environment and Health, 18, 195200.

Keita, G. (1997). Ethnicity. In J. Stellman (Ed.) Encyclopedia of Occupational Health and Safety. Geneva: International Labour Office.

Kelloway, K. (1995). Structural equation modeling in perspective. Journal of Organizational Behavior, 16, 215-224.

Kelloway, K. (1998). Using LISREL for Structural Equation Modeling: A Researcher's Guide. Thousand Oaks, CA: Sage Publications.

Kelloway, K., Gottlieb, B. \& Barham, L. (1999). The source, nature and direction of work-family conflict: A longitudinal investigation. Journal of Occupational Health Psychology, 4, 337-346.

Kemery, E, Bedeian, A., Mossholder, K. \& Touliatos, J. (1985). Outcomes of role stress: A multisample constructive replication. Academy of Management Journal, 28, 363-375.

Kenny, D. \& Judd, C. (1984). Estimating the nonlinear and interactive effects of latent variables. Psychological Bulletin, 96, 201-210.

Kim, J. \& Campagna, A. (1981). Effects of flextime on employee attendance and performance: A field experiment. Academy of Management Journal, 24, 729-741.

Kingston, P. (1990). Illusions and ignorance about the family responsive workplace. Journal of Family Issues, 11, 438-454.

Kingston, P. \& Nock, S. (1985). Consequences of the family day. Journal of Marriage and the Family, 47, 619-629.

Kingston, P. \& Nock, S. (1987). Time together among dual-earner couples. American Sociological Review, 52, 391-400.

Kinicki, A., McKee, F., \& Wade, K. (1996). Annual Review: 1991-1995:

Occupational Health. Journal of Vocational Behavior, 49, 190-220.

Kinnunen, U. \& Mauno, S. (1998). Antecedents and outcomes of work-family conflict among employed women and men in Finland. Human Relations, $51,157-177$

Kirchmeyer, C. (1995). Managing the work-nonwork boundary: An assessment of organizational responses. Human Relations, 48, 515-536. 
Kirchmeyer, C. (2000). Work-life initiatives: Greed or benevolence regarding workers' time? In Cooper, C. \& Rousseau, D. Trends in Organizational Behavior, Volume 7. New York: John Wiley \& Sons, Ltd.

Kirchmeyer, C. \& Cohen, A. (1999). Different strategies for managing the work/non-work interface: A test for unique pathways to outcomes. Work and Stress, 13, 59-73.

Kivimaki, M. \& Lindstrom, K. (1995). Effects of private self-consciousness and control on the occupational stress-strain relationship. Stress Medicine, 11, 7-16.

Kline, R. (1998). Principles and practice of structural equation modeling. New York: The Guildford Press.

Kline, T. \& Dunn, B. (2000). Analysis of interaction terms in structural equation models: A non-technical demonstration using the deviation score approach. Canadian Journal of Behavioural Science, 32 (2), 127-132.

Klitzman, S., House, J., Israel, B. \& Mero, R. (1990). Work stress, nonwork stress, and health. Journal of Behavioral Medicine, 13, 221-243.

Knauth, P. (1993). The design of shift systems. Ergonomics, 36, 15-28.

Knauth, P. (1997). Hours of work. In J. Stellman (Ed.) Encyclopedia of Occupational Health and Safety. Geneva: International Labour Office.

Knauth, P. \& Rutenfranz, J. (1982). Development of criteria for the design of shiftwork systems. In Kogi, K., Miura, T., \& Saito, H. (Eds.). Shiftwork: Its practice and improvement. Journal of Human Ergology, 2 (Supplement), 337-367.

Knuttson, A., Akerstedt, T., \& Orth-Gomer, K. (1986). Increased risk of ischaemic heart disease in shift workers. Lancet, 12, 89-92.

Kohn, M. \& Schooler, C. (1973). Occupational experience and psychological functioning: An assessment of reciprocal effects. American Sociological Review, 38, 97-118.

Kohn, M. \& Schooler, C. (1982). Job conditions and personality: A longitudinal assessment of their reciprocal effects. American Journal of Sociology, $87,1257-1286$.

Kohn, M. \& Schooler, C. (1983). Work and personality: An inquiry into the impact of social stratification. Norwood, NJ: Ablex Publishing. 
Kopelman, R., Greenhaus, J. \& Connolly, T. (1983). A model of work, family, and interrole conflict: A construct validation study. Organizational Behavior and Human Performance, 32, 198-215.

Korabik, K., Lero, D. \& Ayman, R. (2003). A multi-level approach to cross cultural work-family research: A micro and macro perspective. Internal Journal of Cross Cultural Management, 3, 289-303.

Kornitzer, M., Kittel, F., Dramaix, M. \& de Backer, G. (1982). Job stress and coronary heart disease. Advanced Cardiology, 19, 56-61.

Kossek, E., Noe, R. \& DeMarr, B. (1999). Work-family role synthesis: individual and organizational determinants. The International Journal of Conflict Management, 10, 102-129.

Kossek, E. \& Ozeki, C. (1998). Work-family conflict, policies and the job-life satisfaction relationship: A review and directions for organizational behavior-human resources research. Journal of Applied Psychology, 83, 139-149.

Kossek, E. \& Ozeki, C. (1999). Bridging the work-family policy and productivity gap. Community, Work and Family, 2, 7-32.

Kraft, P. (1984). Computer programming: Do males and females do the same work? Paper presented at the Office of Technology Assessment Symposium on the Impacts of Office Automation and Computer Mediated Work on the Quality of Worklife, Washington, DC.

Kranzler, G. \& Moursund, J. (1999). Statistics for the Terrified. Upper Saddle River, NJ: Prentice Hall.

Krausz, M. \& Freibach, N. (1983). Effects of flexible working time for employed women upon satisfaction, strains and absenteeism. Journal of Occupational Psychology, 56, 155-159.

Kristensen, T. (1991). Sickness absence and work strain among Danish slaughterhouse workers: An analysis of absence from work regarded as coping behaviour. Social Science Medicine, 32, 15-27.

Kristensen, T. (1995). The demand-control-support model: Methodological challenges for future research. Stress Medicine, 11, 17-26.

Kropf, M. (1997). A research perspective on work-family issues. In Parasuraman, S. \& Greenhaus, J. (Eds.) Integrating work and family: challenges and choices for a changing world. Westport, CT: Quorum Books. 
Kushnir, T. \& Melamed, S. (1991). Work-load, perceived control and psychological distress in type A/B industrial workers. Journal of Organizational Behavior, 12, 155-168.

La Croix, A. (1984). Occupational exposure to high demand/low control work and coronary heart disease in the Framingham cohort. Ph.D. diss., Department of Epidemiology, University of North Carolina.

La Croix, A. \& Haynes, S. (1987). Occupational exposure to high demand/low control work and coronary heart disease incidence in the Framingham cohort. Society for Epidemiological Research: Abstracts, 481.

Laflamme et al. (1998). Job strain and ambulatory blood pressure among female white-collar workers. Scandinavian Journal of Work, Environment and Health, 24, 334-343.

Landauer, J. (1997). Bottom-line benefits of work/life programs. HR Focus, July, 3-4.

Landsbergis, P. (1988). Occupational stress among health care workers: A test of the demands-control model. Journal of Organizational Behavior, 9 , 217-239.

Landsbergis, P., Schnall, P., Deitz, D., \& Pickering, T. (1992). The patterning of psychological attributes and distress by 'job strain' and social support in a sample of working men. Journal of Behavioral Medicine, 15, 379-405.

Landsbergis, P., Schurman, S., Israel, B., Schnall, P., Hugentobler, M. Cahill, J. \& Baker, D. (1993). Job stress and heart disease: Evidence and strategies for prevention. New Solution,: Summer, 42-58.

Landsbergis, P., Schnall, P., Warren, K., Pickering, T. \& Schwartz, J. (1994). Association between ambulatory blood pressure and alternative formulations of job strain. Scandinavian Journal of Work, Environment and Health, 20, 349-363.

Langan-Fox, J. (1998). Women's careers and occupational stress. In Cooper, C. \& Robertson, I. International Review of Industrial and Organizational Psychology. Volume 13. New York: John Wiley and Sons Ltd.

Lapierre, L. \& Allen, T. (2006). Work-supportive family, family-supportive supervision, use of organizational benefits, and problem-focused coping: Implications for work-family conflict and employee well-being. Journal of Occupational Health Psychology, 11(2), 169-181. 
LaRocco, J., House, J. \& French, J. (1980). Social support, occupational stress and health. Journal of Health and Social Behaviour, 21, 202-218.

Latack, J. \& Foster, L. (1985). Implementation of compressed work schedules: Participation and job redesign as critical factors for employee acceptance. Personnel Psychology, 38, 75-92.

Lazarus, R, DeLongis, A., Folkman, S. \& Gruen, R. (1985). Stress and adaptational outcomes: The problem of confounded measures. American Psychologist, 40, 770-779.

Lazarus, R. \& Folkman, S. (1984). Stress, appraisal and coping. New York: Springer Publishing Company.

Lee, C., Ashford, S., \& Bobko, P. (1990). Interactive effects of 'Type A' behavior and perceived control on worker performance, job satisfaction and somatic complaints. Academy of Management Journal, 33, 870-881.

Lee, R. (1983). Flexitime and conjugal roles. Journal of Occupational Behavior, 4, 297-315.

Lee, R. (1984). The study of flextime. Department of Management Studies, Loughborough University of Technology.

Lerner, D., Levine, S., Malspeis, S. \& D'Agostino, R. (1994). Job strain and health-related quality of life in a national sample. American Journal of Public Health, 84, 1580-1585.

Lero, D. \& Johnson, K. (1994). 110 Canadian Statistics on Work \& Family. Ottawa: The Canadian Advisory Council on the Status of Women.

Levi, L., Sauter, S. \& Shimomitsu, T. (1999). Work-related stress --- It's time to act. Journal of Occupational Health Psychology, 4, 394-396.

Levine, J. \& Pittinsky, T. (1997). Working fathers: New strategies for balancing work and family. Reading, MA: Addison-Wesley.

Lewis, S. \& Cooper, C. (1999). The work-family research agenda in changing contexts. Journal of Occupational Health Psychology, 4, 382-393.

Light, K., Turner, J. \& Hinderliter, A. (1992). Job strain and ambulatory work blood pressure in healthy young men and women. Hypertension, 20, 214-218.

Lilly, T, Catsouphes-Pitt, M. \& Googins, B. (1997). Work-Family Research: An Annotated Bibliography. Westport, CT: Greenwood Press. 
Lindsay, C. \& Almey, M. (2006). Paid and unpaid work In Statistics Canada (2006). Women in Canada: A Gender Based Statistical Report. Ottawa: Catalogue no. 89-503-XIE. 103-132.

Lipsett, B., \& Reesor, M. (1997) 'Flexible work arrangements'. Applied Research Bulletin, Human Resource Development Canada.

Lo, S. (2003). Perceptions of work-family conflict among married female professionals in Hong Kong. Personnel Review, 32, 376-390.

Lohmoller, J. (1982). An overview of the latent variables path analysis. Paper presented at the annual meeting of the American Educational Research Association, New York.

Long, S. (Ed.) (1988). Common Problems/Proper Solutions: Avoiding Error in Quantitative Research. Newbury Park, CA: Sage Publications.

Loscocco, K. \& Spitze, G. (1990). Working conditions, social support, and the well-being of female and male factory workers. Journal of Health and Social Behavior, 31, 313-327.

Lott, B. (1987). Women's lives: Themes and variations in gender learning. Monterey, CA: Brooks/Cole.

Lowe, G. (2000). The Quality of Work: A people Centred Agenda. Oxford University Press, Toronto.

Lowe, G. S. \& Northcott, H. C. (1988). The impact of working conditions, social roles, and personal characteristics on gender differences in distress. Work and Occupations, 15, 55-77.

Lowe, G. \& Schellenberg, G. (1999). Changing employment relationships: Implications for workers, employers and public policy. Canadian Policy Research Networks, Ottawa.

Luna, T., French, J. \& Mitcha, J. (1997). A study of USAF air traffic controller shiftwork: sleep, fatigue, activity, and mood analyses. Aviation Space and Environmental Medicine, 68, 18-23.

Lundberg et al. (1999). Stress and workload in men and women in high-ranking positions. Journal of Occupational Health Psychology, 4, 142-151.

MacBride-King, J. (1990). Work and Family: Employment Challenges of the '90s. Report, 59-90. 
MacBride-King, J. \& Bachmann, K. (1999). Is work-life balance still an issue for Canadians and their employers? You bet it is! The Conference Board of Canada: Ottawa.

MacDermid, S. \& Harvey, A. (2006). The work-family conflict construct: Methodological implications. In Pitt-Catsouphes, M., Kossek, E. \& Sweet, S. (Eds.) (2006). The work and family handbook: Multidisciplinary perspectives and approaches. Mahwah, NJ: Lawrence Erlbaum Associates, Publishers.

MacDermid, S., Hertzog, J., Kensinger, K. \& Zipp, J. (Summer 2001). The Role of Organizational Size and Industry in Job Quality and Work-family relationships. Journal of Family and Economic Issues, 22(2), 191-216.

MacDermid, S., Litchfield, L. \& Pitt-Catsouphes, M. (1999). Organizational Size and Work-family issues. ANNALS, AAPSS, 562, 111-126.

MacEwen, K. \& Barling, J. (1994). Daily consequences of work interference with family and family interference with work. Work and Stress, 8, 244-254.

Madsen, S. (2003). The effects of home-based teleworking on work-family conflict. Human Resource Development Quarterly, 14, 35-58.

Major, D. \& Germano, L. (2006). The changing nature of work and its impact on the work-home interface. In F. Jones, R. Burke, \& M. Westman (Eds.) Work-Life Balance: A Psychological Perspective. New York: Psychology Press.

Maklan, D. (1977). The four-day workweek: Blue-collar adjustment to a nonconventional arrangement of work and leisure time. Praeger, New York.

Marchese, M., Bassham, G. \& Ryan, J. (2002). Work-family conflict: A virtue ethics analysis. Journal of Business Ethics, 40 (2), 145-154.

Marini, M. \& Shelton, B. (1993). Measuring household work: Recent experience in the United States. Social Science Research, 22, 361-382.

Marks, N (1998). Does it hurt to care? Caregiving, work-family conflict, and midlife well-being. Journal of Marriage and the Family, 60, 951-966.

Marmot, M. G., Adelstein, A. M., Robinson, N. \& Rose, G. A. (1978). Changing social-class distribution of heart disease. British Medical Journal, 2, 1109-1112.

Marmot, M \& Syme, L. (1976). Acculturation and coronary heart disease in Japanese-Americans. American Journal of Epidemiology, 104, 225-247. 
Marmot, M. \& Theorell, T. (1991). Social class and cardiovascular disease: The contribution of work. In J. Johnson and G. Johansson (Eds.) The Psychosocial work environment: Work organization, democratization and health. Amityville, NY: Baywood, Publ., 33-48.

Marshall, K. (2005). How Canada compares in the G8. Statistics Canada.

Perspectives on Labour and Income, 17(3). Catalogue no. 75-001-XPE.

Marshall, K. (2006). Converging gender roles. Statistics Canada. Perspectives on Labour and Income, 7(7), Catalogue no. 75-001-XIE.

Marshall, N., Barnett, R., Baruch, G. \& Pleck, J. (1991). More than a job: women and stress in caregiving occupations. Current Research on Occupations and Professions, 6, 61-81.

Maruyama, G. (1998). Basics of structural equation modeling. Thousand Oaks, CA: Sage Publications.

Maruyama, S. \& Morimoto, K. (1996). Effects of long workhours on life-style, stress and quality of life among intermediate Japanese managers. Scandinavian Journal of Work, Environment and Health, 22, 353-359.

Mattingly, M. \& Bianchi, S. (2003). Gender differences in the quantity and quality of free time: The U.S. experience. Social Forces, 81(3), 999-1030.

Maume, D. \& Houston, P. (2001). Job segregation and gender differences in work-family spillover among white-collar workers. Journal of Family and Economic Issues, 22, 171-189.

Maurice, M. (1975). Shift work. Geneva, International Labor Office.

Maxwell, S. \& Delaney, H. (1993). Bivariate median splits and spurious statistical significance. Psychological Bulletin, 113, 181-190.

McClelland, G. \& Judd, C. (1993). Statistical difficulties of detecting interactions and moderator effects. Psychological Bulletin, 114, 376-390.

McCloskey, D (1998). The impact of telecommuting on the work outcomes of professionals. Unpublished doctoral dissertation, Drexel University, Philadelphia.

McCloskey, D. and M. Igbaria (1998). A Review of the Empirical Research on 
Telecommuting and Directions for Future Research. In $M$. Igbaria and $M$. Tan (Eds.) The Virtual Workplace. Idea Group Publishing, Hershey, PA, pp. 338-358.

McCright, P. (1988). The job control and job demand hypothesis in a laboratory setting. Ph.D. diss., Stanford University.

McGuire, J. \& Liro, J. (1987). Absenteeism and flexible work schedules. Public Personnel Management, 16, 47-59.

McLaney, M. \& Hurrell, J. (1988). Control, stress and job satisfaction in Canadian nurses. Work and Stress, 2, 217-224.

McNaughton, M. (1999). Work and Family—Bibliography. Vancouver: BC Council for Families.

McShane, S. (1998). Organizational Behaviour. Toronto: McGraw-Hill Ryerson.

Medalie, J., Kahn, H., Neufeld, H., Riss, E., \& Gouldborn, M. (1973). Five-year myocardial infarction incidence. Journal of Chronic Disease, 26, 329341.

Mehlman, M. (1988). Social aspects of teleworking: Facts, hopes, fears, ideas. In Korte, W., Robinson, s. \& Steinle, W. (Eds.). Telework: Present situation and future development of a new form of work organization. Amsterdam: North-Holland.

Meisner, M. (1971). The long arm of the job. Industrial Relations Journal, October, 239-260.

Melamed, S., Kushnir, T. \& Meir, E. (1991). Attenuating the impact of job demands: additive and interactive effects of personal control and social support. Journal of Vocational Behavior, 39, 40-53.

Mele, D. (1989). Organization of work in the company and family rights of the employees. Journal of Business Ethics, 8, 647-655.

Menon, S. (1999). Psychological empowerment: Definition, measurement, and validation. Canadian Journal of Behavioural Science, 31, 161-164.

Metzger, R. \& Von Glinow, M (1988). Off-site workers: At home and abroad. California Management Review, 30 (3), 101-111.

Miles, R. (1976). Role requirements as sources of organizational stress. Journal of Applied Psychology, 61, 172-179. 
Milkie, M. \& Peltola, P. (1999). Playing all the roles: Gender and the work-family balancing act. Journal of Marriage and the Family, 61, 476-490.

Miller, A. (1988). Stress on the job. Newsweek, April 25, 40-45.

Miller, J., Schooler, C., Kohn, M. L. \& Miller, K. A. (1983). Women and work: The psychological effects of occupational conditioning. American Journal of Sociology, 85, 56-64.

Mills, R, Duncan, K. \& Amyot, J. (2000). Home-Based employment and workfamily conflict: A Canadian study. In Hennon, C., Loker, S. \& Walker, R. (Eds.) Gender and Home-Based Employment. Westport, CT: Auburn House.

Moen, P. \& Dempster-McClain, D. (1987). Employed parents: Role strain, work time, and preferences for working less. Journal of Marriage and the Family, 49, 579-590.

Monk, T. \& Folkard, S. (1985). Shiftwork and performance. In S. Folkard and T. Monk (Eds.) Hours of Work - Temporal Factors in Work Scheduling. New York: John Wiley \& Sons, pp. 239-252.

Monk, T. \& Folkard, S. (1992). Making Shift Work Tolerable. London: Taylor \& Francis.

Morgan, F. (1977). Your (flex) time may come. Personnel Journal, 56, 82-85.

Moss, P., Reid, N., Jackson, S., Lam, E., \& Morris, D. (1996). The working hours, work patterns, stress levels and views of house officers - A study of a general surgical department. Part one of a Report to the West Midlands Regional Task Force (Doctors in Training), Coventry University, February, 1996.

Mott, P., Mann, F., McLoughlin, Q. \& Warwick, D. (1965). Shiftwork: The social, Psychological and Physical Consequences. Ann Arbor, MI: The University of Michigan Press.

Mowrer, O \& Viek, P. (1948). An experimental analogue of fear from a sense of helplessness. Journal of Abnormal and Social Psychology, 43, 193-200.

Moyle, P. (1995). The role of negative affectivity in the stress process: tests of alternative models. Journal of Organizational Behavior, 16, 647-668.

Muhonen, T. \& Torkelson, E. (2003). The demand-control-support model and health among women and men in similar occupations. Journal of Behavioral Medicine, 26(6), 601-613. 
Muntaner, C. \& Eaton, W. (1997). Mental illness. In J. Stellman (Ed.) Encyclopedia of Occupational Health and Safety. Geneva: International Labour Office.

Muntaner, C. \& O'Campo, P. (1993). A critical appraisal of the demand/control model of the psychosocial environment: epistemological, social, behavioral and class considerations. Social Science and Medicine, 36, 1509-1517.

Muntaner, C., Tien, A., Eaton, W. \& Garrison (1991). Occupational characteristics and the occurence of psychotic disorders. Social Psychiatry and Psychiatric Epidemiology, 26, 273-280.

Mukherjee, D. (1999). Socio-economic status and school system enrolments. http://www.aeufederal.org.au/Publications/DMukherjeepaper.pdf

Murphy, L. (1988). Workplace interventions for stress reduction. In C. Cooper, et al. (Eds.) Causes, Coping and Consequences of Stress at Work. New York: John Wiley \& Sons Ltd.

Murphy, L, Hurrell, J. \& Quick, J. (1992). Work and well-being: Where do we go from here? In J.C. Quick, L.R. Murphy \& J.J. Hurrell (Eds.) Stress and Well-Being at Work. Washington, DC: The American Psychological Association, pp. 331-347.

Murphy, S. (2000). Mental health and the workplace: A multidisciplinary examination of the individual and organizational antecedents and outcomes of stress, anxiety and depressed mood. Ph.d. Dissertation. Carleton University.

Nakamura, K., Shimai, S., Kikuchi, S., Takahashi, H., Tanaka, M., Nakano, S., Motohashi, Y., Nakadaira, H. \& Yamamoto, M. (1998). Increases in body mass index and waist circumference as outcomes of working overtime. Occupational Medicine, 48, 169-173.

Narayanan, V. \& Nath, R. (1982). A Field test of some attitudinal and behavioral consequences of flextime. Journal of Applied Psychology, 67, 214-218.

Nathanson, C. (1975). Illness and the feminine role: A theoretical review. Social Science and Medicine, 9, 57-62.

Near, Rice and Hunt (1980). The relationship between work and non-work domains. Academy of Management Review, 5, 415-429. 
Netemeyer, R., Boles, J. \& McMurrian, R. (1996). Development and validation of work-family conflict and family-work conflict scales. Journal of Applied Psychology, 81, 400-410.

Newton, T. \& Keenan, A. (1990). The moderating effect of the Type A behavior pattern and locus of control upon the relationship between change in job demands and change in psychological strain. Human Relations, 43, 1229-1255

Nie, N. (1999). Tracking our Techno Future, American Demographics, July, URL:http://www.demographics.com/publications/ad/00_ad-ad/9906ad990601.html

Niedhammer, I., Goldberg, M., Leclerc, A., Bugel, I. \& David, S. (1998).

Psychosocial factors at work and subsequent depressive symptoms in the Gazel cohort. Scandinavian Journal of Work Environment and Health, 24, 197-205.

Nishiyama, K. \& Johnson, J. (1997). Karoshi --- Death from overwork: Occupational health consequences of Japanese production management. International Journal of Health Services, 27(4), 625-641.

Nock, S. \& Kingston, P. (1984). The family work day. Journal of Marriage and the Family, 46, 333-343.

Nock, S. \& Kingston, P. (1988). Time with children: The impact of couples' worktime commitments. Social Forces, 67, 59-85.

Nollen, S.(1979). Highlights of the literature, 7: New patterns of work. Scarsdale, NY: Work in America Institute.

Nollen, S. (1982). New work schedules in practice: Managing time in a changing society. New York: Van Nostrand Reinhold Company.

Nollen, S. (1999). Flexible work arrangements: An overview of developments in the United States In Zeytinoglu, I. (Ed.) (1999). Changing work relationships in industrialized economies. Amsterdam: John Benjamins Publishing Company.

Nollen, S. \& Martin, V. (1978a). Alternative work schedules, Part l: Flextime. New York: AMACOM, a division of American Management Associations.

Nollen, S. \& Martin, V. (1978b). Alternative work schedules, Part 2 (Permanent part-time employment and Part 3 (The Compressed work week. New York: AMACOM, a division of American Management Associations. 
Nomaguchi, K., Milkie, M. \& Bianchi, S. (2005). Time strains and psychological well-being: Do dual-earner mothers and fathers differ? Journal of Family Issues, 26(6), 756-792.

Noor, N. (1995). Work and family roles in relation to women's well-being: a longitudinal study. British Journal of Social Psychology, 34, 87-106.

Nord, W. \& Costigan, R. (1973). Worker adjustment to the four-day week: A longitudinal study. Journal of Applied Psychology, 58, 60-66.

Northrup, H., Wilson, J. \& Rose, K. (1979). The twelve-hour shift in the petroleum and chemical industries. Industrial and Labor Relations Review, 32, 312321.

O'Driscoll, M. \& Beehr, T. (2000). Moderating effects of perceived control and need for clarity on the relationship between role stressors and employee affective reactions. Journal of Social Psychology, 140, 151-159.

O'Driscoll, M., llgen, D. \& Hildreth, K. (1992). Time devoted to job and off-job activities, interrole conflict, and affective experiences. Journal of Applied Psychology, 77, 272-279.

Oginska, H., Pokorski, J. \& Oginski, A. (1993). Gender, ageing, and shiftwork intolerance. Ergonomics, 35, 161-168.

O'Grady, K. E. (1982). Measures of explained variance: Cautions and limitations. Psychological Bulletin, 92, 766-777.

Ohman, A. \& Bohlin, G. (1989). The role of controllability in cardiovascular activation and cardiovascular disease: help or hindrance? In Steptoe, $A$. \& Appels, A. (Eds.) Stress, Personal Control and Health. BrusselsLuxembourg: John Wiley \& Sons Ltd.

Olson, M. (1982). New information technology and organizational culture. MIS Quarterly, Special Issue, 71-92.

Olson, M. (1989). Organizational barriers to professional telework. In Boris, E. \& Daniels, C. (Eds.). Historical and contemporary perspectives on paid labor at home. Urbana, IL: University of Illinois Press.

Olson, M. \& Primps, S. (1984). Working at Home with Computers: Work and Nonwork Issues. Journal of Social Issues, 40, 97-112.

Olsson, K., Kandolin, I. \& Toropainen, K. (1990). Stress and coping strategies of three shiftworkers. Le Travail Humain, 53, 175-188. 
Ono, Y., Watanabe, S., Kaneko, S., Matsumoto, K. \& Miyako, M. (1991). Working hours and fatigue of Japanese flight attendants. Journal of Human Ergology, 20, 155-164.

Organization for Economic Co-operation and Development (OECD) (2004). Clocking in and clocking out: Recent trends in working hours policy brief. Retrieved from: http://www.oecd.org/dataoecd/42/49/33821328.pdf.

Orpin, C. (1981). Effects of flexible working hours on employee satisfaction and performance: A field experiment. Journal of Applied Psychology, 66(1), 113-115.

Orth-Gomer, K. (1997). Social support: An interactive stress model. In J. Stellman (Ed.) Encyclopedia of Occupational Health and Safety. Geneva: International Labour Office.

Paden, S. \& Beuhler, C. (1995). Coping with the dual-income lifestyle. Journal of Marriage and the Family, 57, 101-110.

Parasuraman, S., Greenhaus, J. \& Granrose, C. (1992). Role stressors, social support, and well-being among two-career couples. Journal of Organizational Behavior, 13, 339-356.

Parasuraman, S. \& Greenhaus, J. (1997). The changing world of work and family. In Parasuraman, S. \& Greenhaus, J. (Eds.) Integrating work and family: challenges and choices for a changing world. Westport, CT: Quorum Books.

Parasuraman, S., Purohit, Y., Godshalk, V. \& Beutell, N. (1996). Work and family variables, entrepreneurial career success, and psychological well-being. Journal of Vocational Behavior, 48, 275-300.

Parker, L. (1993). When to fix it and when to leave: Relationships among perceived control, self-efficacy, dissent and exit. Journal of Applied Psychology, 78, 949-959.

Parker, L. \& Allen, T. (2001). Work/family benefits: Variables related to employee's fairness perceptions. Journal of Vocational Behaviour, 58 , 453-468.

Parker, S. \& Sprigg, C. (1999). Minimizing strain and maximizing learning: The role of job demands, job control and proactive personality. Journal of Applied Psychology, 84, 925-939.

Parkes, K. (1984). Locus of control, cognitive appraisal, and coping in stressful episodes. Journal of Personality and Social Psychology, 46, 655-668. 
Parkes, K. (1986). Coping in stressful episodes: The role of individual differences, environmental factors, and situational characteristics. Journal of Personality and Social Psychology, 51, 1277-1292.

Parkes, K. (1989). Personal control in an occupational context. In A. Steptoe, A. \& A. Appels (Eds.) Stress Personal Control, and Health. Brussels: John Wiley \& Sons Ltd.

Parkes, K. (1990). Coping, negative affectivity, and the work environment: additive and interactive predictors of mental health. Journal of Applied Psychology, 75, 399-409.

Parkes, K. (1991). Locus of control as moderator: An explanation for additive versus interactive findings in the demand-discretion model of work stress? British Journal of Psychology, 82, 291-312.

Parkes, K. (1994). Personality and coping as moderators of work stress processes: models, methods and measures. Work and Stress, 8, 110129.

Parkes, K. (1999). Shiftwork, job type and the work environment as joint predictors of health-related outcomes. Journal of Occupational Health Psychology, 4(3), 256-268.

Parkes, K., Mendham, C. \& von Rabenau, C. (1994). Social support and the demand-discretion model of job stress: tests of additive and interactive effects in two samples. Journal of Vocational Behavior, 44, 91-113.

Parkes, K. \& von Rabenau, C. (1993). Work characteristics and wellbeing among psychiatric health care staff. Journal of Community and Applied Social Psychology, 3, 243-260.

Payne, R. \& Fletcher, B. (1983). Job demands, supports and constraints as predictors of psychological strain among school teachers. Journal of Vocational Behaviour, 22, 136-147.

Pearlson, K. \& Saunders, C. (2001). There's no place like home: Managing telecommuting paradoxes. Academy of Management Executive, 15, 117128.

Pedhazur, E. (1997). Multiple regression in behavioral research: Explanation and prediction. Fort Worth, TX: Harcourt Brace College Publishers.

Pelfrene, E., Vlerick, P., Kittel, F., Mak, R., Kornitzer, M. \& De Backer, G. (2002). Psychosocial work environment and psychological well-being: 
assessment of the buffering effects in the job demand-control (-support) model in BELSTRESS. Stress and Health, 18, 43-56.

Pennebaker, J. \& Watson, D. (1988). Self-reports and physiological measures in the workplace. In Hurrell et al. (Eds.) Occupational stress. New York: Taylor and Francis.

Perlow, L. (1995). Putting the work back into work/family. Group and Organization Management, 20, 227-239.

Perlow, L. (1997). Finding time: how corporations, individuals, and families can benefit from new work practices. Ithaca, NY: Industrial Labor Relations Press, Cornell University.

Perrewe, P. \& Anthony, W. (1990). Stress in a steel pipe mill: The impact of job demands, personal control and employee age on somatic complaints. Journal of Social Behavior and Personality, 5, 77-90.

Perrewe, P., Hochwarter, W. \& Kiewitz, C. (1999). Value attainment: An explanation for the negative effects of work-family conflict on job and life satisfaction. Journal of Occupational Health Psychology, 4, 318-326.

Perrewe, P., Ralston, D. \& Ferdandez, D. (1995). A model depicting the relations among perceived stressors, role conflict and organizational commitment: A comparative analysis of Hong Kong and the United States. Asia Pacific Journal of Management, 12, 1-17.

Perry-Jenkins, M., Repetti, R. \& Crouter, A. (2000). Work and Family in the 1990s. Journal of Marriage and the Family, 62, 981-998.

Peterson, D. (1980). Flexitime in the United States: The lessons of experiences, Personnel, 57, 21-31.

Peterson, M. (1985). Attitudinal differences among work shifts: What do they reflect? Academy of Management Journal, 28, 723-732.

Peterson, M., Smith, P., Akande, A., Ayestaran, S., Bochner, S., Callan, V., Cho, N., Correia Jesuino, J., D’Amorim, M., Francois, P., Hofmann, K., Koopman, P., Leung, K., Lim, T., Mortazavi, S., Munene, J., Radford, M., Ropo, A., Savage, G., Setiadi, B., Sinha, T., Sorenson, R., \& Viedge, C. (1995). Role conflict, ambiguity, and overload: A 21-nation study. Academy of Management Journal, 38(2), 429-452.

Phelan, J., Schwartz, J., Bromet, E., Dew, M., Parkinson, D., Schulberg, H., Dunn, L., Blane, H. \& Curtis, C. (1991). Work stress, family stress, and depression in professional and managerial employees. Psychological 
Medicine, 21, 999-1012.

Pieper, C., LaCroix, A., \& Karasek, R. (1989). The relation of psychosocial dimensions of work with coronary heart disease risk factors: A metaanalysis of five United States data bases. American Journal of Epidemiology, 129, 483-494.

Pierce, J. \& Dunham, R. (1992). The 12-hour work day: A 48-hour, eight-day week. Academy of Management Journal, 35, 1086-1098.

Pierce, J. \& Newstrom, J. (1982). Employee responses to flexible work schedules: An interorganization, intersystem comparison. Journal of Management, 8, 9-25.

Pierce, J. \& Newstrom, J. (1983). The design of flexible work schedules and employee responses: Relationships and process. Journal of Occupational Behavior, 4, 247-262.

Pierce, J., Newstrom, J., Dunham, R. \& Barber, A. (1989). Alternative Work Schedules. Boston: Allyn and Bacon, Inc.

Ping, R. (1995). A parsimonious estimating technique for interaction and quadratic latent variables. Journal of Marketing Research, 32, 336-347.

Pinneau, S. (1975). Effect of social support on psychological and physiological strain, Doctoral Dissertation, University of Michigan.

Pitt-Catsouphes, M. \& Googins, B. (1999). Preface. Annals, AAPSS, 562, 8-15.

Pitt-Catsouphes, M., Kossek, E. \& Sweet, S. (Eds.) (2006). The work and family handbook: Multi-disciplinary perspectives and approaches. Mahwah, NJ: Lawrence Erlbaum Associates, Publishers.

Pleck, J. (1978). The work-family role system. Social Problems, 24, 417-427.

Pleck, J. (1985). Working wives, working husbands. Beverly Hills: Sage.

Pleck, J. (1997). Paternal involvement: Levels, sources and consequences. In Lamb, M. (Ed.) The Role of the Father in Child Development. New York: Wiley.

Pleck, J. \& Staines, G. (1985). Work schedules and family life in two-earner couples. Journal of Family Issues, 6, 61-82. 
Pleck, J., Staines, G. \& Lang, L. (March 1980). Conflicts between work and family life. Monthly Labor Review, 29-31.

Poelmans, S. (Ed.) (2005). Work and family: An international research perspective. Mahwah, NJ: Lawrence Erlbaum Associates, Publishers.

Poelmans, S., Allen, T., Spector, P., O'Driscoll, M., Cooper, C. \& Sanchez, J. (2003). A cross-national comparative study of work/family demands and resources. International Journal of Cross-Cultural Management, 3(3), 275-287.

Poelmans, S., O'Driscoll, M., \& Beham, B. (2005). An overview of international research on the work-family interface. In S. Poelmans (Ed.). Work and Family: An International Research Perspective. Mahwah, NJ: Lawrence Erlbaum Associates, Publishers.

Polivka, A. (1996a). Contingent and alternative work arrangements, defined. Monthly Labor Review, October, 3-9.

Polivka, A. (1996b). A profile of contingent workers. Monthly Labor Review, October, 10-21.

Polivka, A. (1996c). Into contingent and alternative employment: by choice? Monthly Labor Review, October, 55-74.

Poor, R. \& Steele, J. (1973). Work and leisure: The reactions of people at fourday firms. 4 Days, 40 Hours. New York: Mentor.

Popock, S., Shaper, A., Cook, D., Phillips, A. \& Walker, M. (1987). Social class differences in ischaemic heart disease in British men. Lancet II, 197-201.

Portello, J. \& Long, B. (2001). Appraisals and coping with workplace interpersonal stress: A model for women managers. Journal of Counseling Psychology, 48, 144-156.

Powell, G. (1997). The sex difference in employee inclinations regarding workfamily programs: Why does it exist, should we care, and what should be done about it (if anything). In Parasuraman, S. \& Greenhaus, J. (Eds.) Integrating work and family: challenges and choices for a changing world. Westport, CT: Quorum Books.

Powell, G. \& Mainiero, L. (1999). Managerial decision making regarding alternative work arrangements. Journal of Occupational and Organizational Psychology, 72, 41-56. 
Presser, H. (1995). Job, family, and gender: Determinants of nonstandard work schedules among employed Americans in 1991. Demography, 32(4), 577-598.

Presser, H. (1999). Toward a 24-hour economy. Science, 284, 1778-1779.

Pugliesi, K. (1995). Work and well-being: Gender differences in the psychological consequences of employment. Journal of Health and Social Behavior, 36, 57-71.

Quick, J. (1999). Editorial. Journal of Occupational Health Psychology, 4, 299300.

Quinn, R. \& Shepard, L. (1974). The 1972-73 Quality of Employment Survey, Survey Research Center, Ann Arbor, University of Michigan.

Quinn, R. \& Staines, G. (1979). The 1977 Quality of Employment Survey: Descriptive statistics with comparison data from the 1969-70 and 197273 surveys. Ann Arbor, MI: Institute for Social Research.

Raabe, P. (1990). The organizational effects of workplace family policies. Journal of Family Issues, 11, 477-491.

Rachlin, V. (1987). Fair vs. equal role relations in dual-career and dual-earner families: Implications for family interventions. Family Relations, 36, 187192.

Rahim, A. \& Psenicka, C. (1996). A structural equations model of stress, locus of control, social support, psychiatric symptoms and propensity to leave a job. The Journal of Social Psychology, 136, 69-84.

Ralston, D. (1990). How flexitime eases work/family tensions. Personnel, August, 45-48.

Ralston, D., Anthony, W. \& Gustafson, D. (1985). Employees may love flextime, but what does it do to the organization's productivity? Journal of Applied Psychology, 70, 272-279.

Ramsower, R. (1985). Telecommuting: The Organizational and Behavioral Effects of Working at Home. Ann Arbor, MI: UMI Research Press.

Ray, E., \& Miller, K. (1994). Social support, home/work stress, and burnout: who can help? Journal of Applied Behavioral Science, 30(3), 357-373. 
Reed, D., La Croix, A., Karasek, R., Miller, D. \& McLean, C. (1989). Occupational strain and the incidence of coronary heart disease. American Journal of Epidemiology, 129, 495-502.

Regression Overview (2005).

http://www2.chass.ncsu.edu/garson/pa765/regress.htm

Repetti, R. (1987). Individual and common components of the social environment at work and psychological well-being. Journal of Personality and Social Psychology, 52(4), 710-720.

Repetti, R. \& Wood, J. (1997). Effects of daily stress at work on mothers' interactions with preschoolers. Journal of Family Psychology, 11, 90108.

Rice, R., Frone, M., \& McFarlin, D. (1992). Work-nonwork conflict and the perceived quality of life. Journal of Organizational Behavior, 13, 155-168.

Riche, M. (2006). Demographic implications for work-family research In PittCatsouphes, M., Kossek, E. \& Sweet, S. (Eds.) (2006). The work and family handbook: Multi-disciplinary perspectives and approaches. Mahwah, NJ: Lawrence Erlbaum Associates, Publishers. 125-140.

Riley, F. \& McCloskey, D. (1997). Telecommuting as a response to helping people balance work and family. In S. Parasuraman \& J. Greenhaus (Eds.) Integrating work and family: challenges and choices for a changing world. Westport, CT: Quorum Books.

Ritti, R. (1971). Job enrichment in engineering organizations. In j. Maher (Ed.) New perspectives in job enrichment. New York: Van Nostrand.

Rizzo, J., House, F., \& Lirtzman, S. (1970). Role conflict and ambiguity in complex organizations. Administrative Science Quarterly, 15, 150-163.

Robbins, S. \& Langton, N. (2001). Organizational Behaviour: Concepts, Controversies, Applications. Toronto: Prentice-Hall.

Robinson, B. \& Salamon, E. (1987). Gender role socialization: A review of the literature. In E. Salamon \& B. Robinson (Eds.). Gender roles: Doing what comes naturally? Toronto: Methuen.

Robinson, J. \& Godbey, G. (1997). Time for Life: The Surprising Ways Americans Use their Time. University Park: The Pennsylvania State University Press. 
Robinson, J. \& Godbey, G. (1998). Trend, gender and status differences in Americans' perceived stress. Society and Leisure. 21, 473-489.

Robinson, J. \& Milkie, M. (1998). Back to the basics: Trends in and role determinants of women's attitudes toward housework. Journal of Marriage and the Family, 60, 205-218.

Rodgers, C. (1992). The flexible workplace: what have we learned? Human Resource Magazine, 31, 183-199.

Roehling, P., Roehling, M. \& Moen, P. (2001). The relationship between work-life policies and practices and employee loyalty: A life course perspective. Journal of Family and Economic Issues, 22, 141-170.

Ronen, S. (1981). Flexible Working Hours: An Innovation in the Quality of Work Life. New York: McGraw-Hill Book Company.

Ronen, S. (1984). Alternative Work Schedules: Selecting, Implementing and Evaluating. Homewood, IL: Dow-Jones, Irwin.

Ronen, S. \& Primps, S. (1980). The impact of flextime on performance and attitudes in 25 public agencies. Public Personnel Management, 9, 201207.

Rose, R., Hurst, M., \& Herd, A. (1979). Cardiovascular and endocrine responses to work and the risk of psychiatric symptoms among air traffic controllers. In J. Barnett (ed.) Stress and Mental Disorder. New York: Raven Press.

Rosenfield, S. (1980). Sex differences in depression: Do women always have higher rates? Journal of Health and Social Behavior, 21, 33-42.

Rosenfield, S. (1989). The effects of women's employment: Personal control and sex differences in mental health. Journal of Health and Social Behavior, $30,77-91$.

Rothstein, D. (1996). Entry into and consequences of nonstandard work arrangements. Monthly Labor Review, October, 76-83.

Rotondo, D., Carlson, D. \& Kincaid, J. (2003). Coping with multiple dimensions of work-family conflict. Personnel Review, 32, 275-296.

Rousseau, D. (1978). Characteristics of departments, positions and individuals: Contexts for attitudes and behaviours. Administrative Science Quarterly, $23,521-540$ 
Rowland, K., Ferris, G., Fried, Y. \& Simon, C. (1988). An assessment of the physiological measurement of work stress. In Hurrell et al. (Eds.) Occupational stress. New York: Francis and Taylor.

Roxburgh, S. (1996). Gender differences in work and well-being: effects of exposure and vulnerability. Journal of Health and Social Behavior, 37, 265-277.

Roxburgh, S. (2002). Racing through life: The distribution of time pressures by roles and role resources among full-time workers. Journal of Family and Economic Issues, 23, 121-145.

Roxburgh, S. (2004). There just aren't enough hours in the day: The mental health consequences of time pressure. Journal of Health and Social Behavior, 45, 115-131.

Rutenfranz, J., Colquhoun, W., Knauth, P. \& Ghata, J. (1977). Biomedical and psychosocial aspects of shiftwork: A review. Scandinavian Journal of Work, Environment and Health, 3, 165-182.

Rutenfranz, J., Haider, M, \& Koller, M. (1985). Occupational health measures for nightworkers and shiftworkers. In S. Folkard and T. Monk (Eds.) Hours of Work - Temporal Factors in Work Scheduling. New York: John Wiley \& Sons. pp. 199-210.

Rwamporo, R., Mock, S. \& Schafft, K. (2002). Work-family demands and stress: A mixed methods examination of gender differences and mediating variables. BLCC Working Paper \#02-01. Cornell Employment and Family Career Institute.

Salkind, N. (2000). Statistics for People Who Think They Hate Statistics. Thousand Oaks, CA: Sage Publications, Inc.

Salamon, E. \& Robinson, B. (1987) (Eds.). Gender roles: Doing what comes naturally? Toronto: Methuen.

Saltzstein, A., Ting, Y., \& Saltzstein, G. (2001). Work-family balance and job satisfaction: The impact of family-friendly policies on attitudes of federal government employees. Public Administration Review, 61, 452-466.

Sapsford, R. \& Jupp, V. (Eds.) (1996). Data collection and analysis. London: Sage Publications.

Sarason, I., \& Johnson, J. (1979). Life stress, organizational stress, and job satisfaction. Psychological Reports, 44, 75-79. 
Sauter, S., Hurrell, J. \& Cooper, C. (1989). Job Control and Worker Health. New York: John Wiley and Sons Ltd.

Sauter, S., Hurrell, J., Murphy, L. \& Levi, L. (1997). Psychosocial and Organizational Factors. In J. Stellman (Ed.) Encyclopedia of Occupational Health and Safety. Geneva: International Labour Office.

Sauve, R. (1999). Trends in Canadian family incomes, expenditures, savings and debt. Vanier Institute of the Family, Ottawa (http://www.vifamily.ca/pubs/sauve.htm)

Sauve, R. (2004). Profiling Canada's Families III. Vanier Institute of the Family: Ottawa, ON. (http://www.vifamily.ca/library/facts/facts.html).

Savery, L. (1986). Stress and the employee. Leadership and Organizational Development, 7(2), 17-20.

Sayer, L., Bianchi, S. \& Robinson, J. (2004). Are parents investing less in children? Trends in mothers' and fathers' time with children. The American Journal of Sociology, 110, 1-43.

Scandura, T. \& Lankau, M. (1997). Relationships of gender, family responsibility and flexible work hours to organizational commitment and job satisfaction. Journal of Organizational Behavior, 18, 377-391.

Schabracq, M. \& Cooper, C. (1997). Flexibility of labor, well-being and stress. International Journal of Stress Management, 4, 259-274.

Schabracq, M., Winnubst, J. \& Cooper, C. (Eds.) (1996). Handbook of Work and Health Psychology. New York: John Wiley \& Sons Ltd.

Schat, A. \& Kelloway, E.K. (2000). Effects of perceived control on the outcomes of workplace aggression and violence. Journal of Occupational Health Psychology, 5, 386-402.

Schaubroeck, J., Cotton, J. \& Jennings, K (1989). Antecedents and consequences of role stress: A covariance structure analysis. Journal of Organizational Behavior, 2 (1), 35-58.

Schaubroeck, J. \& Fink, L. (1998). Facilitating and inhibiting effects of job control and social support on stress outcomes and role behavior: a contingency model. Journal of Organizational Behavior, 19, 167-195.

Schaubroeck, J. \& Kuehn, K. (1992). Research design in industrial and organizational psychology. In Cooper et al. (Eds.). International Review 
of Industrial and Organizational Psychology, v. 7. New York: John Wiley \& Sons Ltd.

Schaubroeck, J, Lam, S. \& Xie, J. (2000). Collective efficacy versus self-efficacy in coping responses to stressors and control: A cross-cultural study. Journal of Applied Psychology, 85, 512-525.

Schaubroeck, J. \& Merritt, D. (1997). Divergent effects of job control on coping with work stressors: The key role of self-efficacy. Academy of Management Journal, 40, 738-754.

Schein, V (1993). The work/family interface: Challenging the 'corporate convenient'. Women in Management Review, 8, 22-27.

Schein, V., Maurer, E. \& Novak, J. (1977). Impact of flexible working hours on productivity. Journal of Applied Psychology, 62, 463-465.

Schieman, S. (1999). Age and anger. Journal of Health and Social Behavior, 40, 273-289.

Schnall, P., Landsbergis, P. \& Baker, D. (1994). Job strain and cardiovascular disease. Annual Review of Public Health, 15, 381-411.

Schnall, P., Pieper, C., Schwartz, J., Karasek, R., Schlussel, Y., Devereux, R., Ganau, A., Alderman, M., Warren, K. \& Pickering, T. (1990). The relationship between 'job strain', workplace, diastolic blood pressure, and left ventricular mass index. Journal of the American Medical Association, 263, 1929-1935.

Schroeder, L., Sjoquist, D. \& Stephan, P. (1986). Understanding regression analysis: An introductory guide. London: Sage Publications.

Schumacker, R. \& Lomax, R. (1996). A beginner's guide to structural equation modeling. Mahwah, NJ: Lawrence Erlbaum Associates.

Schweitzer, L. \& Duxbury, L. (2006). Benchmarking the use of telework arrangements in Canada. Canadian Journal of Administrative Sciences, 23(2), 105-117.

Sease, R. \& Scales, J. (1998). Work now - pay later? The impact of long work hours on health and family life. (Technical paper No. 17) Colchester, England: Institute for Social and Economic Research.

Secret, M. (2000). Identifying the family, job, and workplace characteristics of employees who use work-family benefits. Family Relations, 49, 217-225. 
Secret, M. \& Sprang, G. (2001). The effects of family-friendly workplace environments on work-family stress of employed parents. Journal of Social Service Research, 28, 21-45.

Sekaran, U. (1983). Factors influencing the quality of life in dual career families. Journal of Occupational Psychology, 56, 161-174.

Selye, H. (1974). Stress without distress. Philadelphia: JB Lipincott.

Selye, H. (1976). The stress of life. New York: McGraw-Hill.

Semmer, N., Zapf, D., \& Greif, S. (1996). 'Shared job strain': A new approach for assessing the validity of job stress measurements. Journal of Occupational and Organizational Psychology, 69, 293-310.

Senge, P. (1990). The Fifth Discipline. New York: Doubleday.

Sergean, R. (1971). Managing Shiftwork. London: Gower Press.

Shaffer, M. \& Harrison, D. (1998). Expatriates' psychological withdrawal from international assignments: work, nonwork and family influences. Personnel Psychology, 51, 87-118.

Shamir, B. (1983). Some antecedents of work-nonwork conflict. Journal of Vocational Behavior, 23, 98-111.

Shamir, B. (1992). Home: The perfect workplace? In Zedeck, S. (Ed.) Work, Families and Organizations. San Francisco: Jossey-Bass, Inc.

Shamir, B. and Salomon, I. (1985). Work at Home and the Quality of Working Life. Academy of Management Review, 10, 455-463.

Sharpe, D., Hermsen, J., \& Billings, J. (2002). Factors associated with having flextime: A focus on married workers. Journal of Family and Economic Issues, 23, 51-72.

Shekelle, B., Schneider, A., Lin, S., Raynor, W., Garron, D., Ostfeld, A., \& Paul, O. (1979). Work tension and risk of coronary heart disease (CHD). CVD Epidemiol. Newsletter no. 26. American Heart Association, National Center, 7320 Greenville Avenue, Dallas, Texas.

Shellenbarger, S. (1991). More job seekers put family needs first. The Wall Street Journal, November $15^{\text {th }}$, pp. B1, B6. 
Shellenbarger, S. (1992). Lessons from the workplace: How corporate policies and attitudes lag behind workers' changing needs. Human Resource Management, 31, 156-169.

Shellenbarger, S. (2000). Finding Ways to Keep a Partner's Job Stress from hitting home. Wall Street Journal, v. 236, B1.

Sheridan, A. \& Conway, L. (2001). Workplace flexibility: reconciling the needs of employers and employees. Women in Management Review, 16, 5-12.

Shields, M. (1999). Long working hours and health. Health Reports, 11(2), 3348.

Shimonitsu, T. \& Levi, L. (1992). Recent working life change in Japan. European Journal of Public Health, 2, 76-96.

Shinn, M., Wong, N., Simko, P. \& Ortiz-Torres, B. (1989). Promoting the wellbeing of working parents: Coping, social support and flexible job schedules. American Journal of Community Psychology, 17, 31-55.

Shore, R. (1998). Ahead of the Curve. New York: Families and Work Institute.

Siegel, P., Post, C., Brockner, J., Fishman, A. \& Garden, C. (2005). The moderating influence of procedural fairness on the relationship between work-life conflict and organizational commitment. Journal of Applied Psychology, 90(1), 13-24.

Siltanen, J., Jarman, J. and Blackburn, R. (1995). Gender Inequality in the Labour Market, Occupational Concentration and Segregation. Geneva: International Labour Office.

Silver, C. (2000). Being there: the time dual-earner couples spend with their children. Canadian Social Trends, 57, 26-29.

Simon, R. (1992). Parental role strains, salience of parental identity and gender differences in psychological distress. Journal of Health and Social Behavior, 33, 25-35.

Simon, R. (1995). Gender, multiple roles, role meaning and mental health. Journal of Health and Social Behavior, 36(2), 182-194.

Skinner, D. (1980). Dual-career family stress and coping: A literature review. Family Relations, 29, 473-480.

Skinner, E. (1996). A Guide to constructs of control. Journal of Personality and Social Psychology, 71, 549-570. 
Smith, C., Robie, C., Folkard, S., Barton, J., Macdonald, I, Smith, L., Spelten, E., Totterdell, P. \& Costa, G. (1999). A process model of shiftwork and health. Journal of Occupational Health Psychology, 4(3), 207-218.

Smith, C., Tisak, J., Hahn, S., \& Schmieder, R. (1997). The measurement of job control. Journal of Organizational Behavior, 18, 225-237.

Smith, L. \& Folkard, S. (1993). The impact of shiftwork on personnel at a nuclear power plant: an exploratory survey study. Work and Stress, 7, 341-350.

Smith, L., Folkard, S. \& Poole, C. (1994). Increased injuries on night shift. The Lancet, 244, 1137-1139.

Smith Major, V., Klein, K. \& Erhart, M (2002). Work time, work interference with family, and psychological distress. Journal of Applied Psychology, 87, 427-436.

Smock, P. \& Noonan, M. (2005). Gender, work and family well-being in the United States. In S. Bianchi, L. Casper, and R. King (Eds.) (2005). Work, family, health and well-being. Mahwah, NJ: Lawrence Erlbaum Associates, Publishers.

Smulders, P. \& Nijhuis, F. (1999). The job demands-job control model and absence behaviour: results of a 3 year longitudinal study. Work and Stress, 13, 115-131.

Smyrnios, K., Romano, C., Tanewski, G., Karosky, P., Millen, R. \& Yilmaz, M. (2003). Work-family conflict: A study of American and Australian family businesses. Family Business Review, 16, 35-51.

Sparks, K. \& Cooper, C. (1999). Occupational differences in the work-strain relationship: Towards the use of situation-specific models. Journal of Occupational and Organizational Psychology, 72, 219-229.

Sparks, K., Cooper, C., Fried, Y., \& Shirom, A. (1997). The effects of hours of work on health: A meta-analytic review. Journal of Occupational and Organizational Psychology, 70, 391-409.

Sparks, K., Faragher, B. \& Cooper, C. (2001). Well-being and occupational health in the $21^{\text {st }}$ century workplace. Journal of Occupational and Organizational Psychology, 74, 489-509.

Spector, P. (1986). Perceived control by employees: a meta-analysis of studies concerning autonomy and participation at work. Human Relations, 39, $1005-1016$. 
Spector, P. (1987a). Method variance as an artifact in self-reported affect and perceptions at work: Myth or significance problem? Journal of Applied Psychology, 72, 438-443.

Spector, P. (1987b). Interactive effects of perceived control and job stressors on affective reactions and health outcomes for clerical workers. Work and Stress, 1, 155-162.

Spector, P. (1992). A consideration of the validity and meaning of self-report measures of job conditions. In Cooper, C. et al. (Eds) International Review of Industrial and Organizational Psychology. v. 7. New York: John Wiley \& Sons Ltd.

Spector, P., Dwyer, D. \& Jex, S. (1988). Relation of job stressors to affective, health, and performance outcomes: A comparison of multiple data sources. Journal of Applied Psychology, 73, 11-19.

Spector, P. \& O'Connell, B. (1994). The contribution of personality traits, negative affectivity, locus of control and Type $A$ to the subsequent reports of job stressors and job strains. Journal of Occupational and Organizational Psychology, 67, 1-11.

Spurgeon, A., Harrington, M. \& Cooper, C. (1997). Health and safety problems associated with long working hours: a review of the current position. Occupational Environmental Medicine, 54, 367-375.

Staines, G. \& Pleck, J. (1983). The impact of work schedules on the family. Institute for Social Research. University of Michigan.

Staines, G. \& Pleck, J. (1984). Nonstandard work schedules and family life. Journal of Applied Psychology, 69(3), 515-523.

Staines, G. \& Pleck, J. (1986) Work schedule flexibility and family life. Journal of Occupational Behavior, 7, 147-223.

Standen, P., Daniels, K. \& Lamond, D. (1999). The home as a workplace: Workfamily interaction and psychological well-being in telework. Journal of Occupational Health Psychology, 4, 368-381.

Stansfeld, S., North, F., White, I. \& Marmot, M. (1995). Work characteristics and psychiatric disorder in civil servants in London. Journal of Epidemiology and Community Health, 49, 48-53.

Starrels, M. (1992). The evolution of workplace family policy research. Journal of Family Issues, 13, 259-278. 
Starrin, B., Larsson, G., Brenner, S., Levi, L. \& Petterson, I. (1990). Structural changes, ill-health, and mortality in Sweden, 1963-1983: A macroaggregated study. International Journal of Health Services, 20 (1), $27-42$.

Statistics Canada (1999). Overview of the Time Use of Canadians in 1998. Catalogue no. 12F0080XIE.

Statistics Canada (2000). Women in Canada 2000: A Gender Based Statistical Report. Ottawa: Catalogue no. 89-503-XPE.

Statistics Canada (2001). Workplace and Employee Survey.

Statistics Canada (2002). Women in Canada: Work Chapter Updates. Catalogue \#89F0133X1E.

Statistics Canada (2004). Workplace and Employee Survey Compendium. Catalogue \#71-585-XIE.

Statistics Canada (2006). Women in Canada: A Gender Based Statistical Report. Ottawa: Catalogue no. 89-503-XIE.

Staub, E, Tursky, B. \& Schwartz, G. (1971). Self-control and predictability: their effects on reactions to averse stimulation. Journal of Personality and Social Psychology, 18, 157-162.

Staw, B., Bell, N. \& Clausen, J. (1986). The dispositional approach to job attitudes: A lifetime longitudinal test". Administrative Science Quarterly, 31, 56-77.

Staw, B. \& Ross, J. (1985). Stability in the midst of change: A dispositional approach to job attitudes. Journal of Applied Psychology, 70, 469-480.

Steptoe, A. (1989). The significance of personal control in health and disease. In A. Steptoe and A. Appels (Eds.) Stress, Personal Control and Health. Brussels-Luxembourg: John Wiley \& Sons Ltd.

Steptoe, A. \& Appels, A. (1989) (Eds.) Stress, Personal Control and Health. Brussels-Luxembourg: John Wiley \& Sons Ltd.

Steptoe, A. \& Pollard, T. (1997). Selected acute physiological outcomes. In J. Stellman (Ed.) Encyclopedia of Occupational Health and Safety. Geneva: International Labour Office. 
Stevens, J. (1996). Applied Multivariate Statistics for the Social Sciences. Mahwah, NJ: Lawrence Erlbaum Associates.

Still, M. \& Williams, J. (2006). A legal perspective on family issues at work. $\ln M$. Pitt-Catsouphes, E. Kossek, \& S. Sweet (Eds.) The Work and Family Handbook: Multi-Disciplinary Perspectives, Methods and Approaches. Mahwah, NJ: Lawrence Erlbaum Associates, Publishers.

Stryker, S. (1987). Identity theory: Developments and extensions. In K. Yardley and T. Honess (Eds.) Self and Identity: Psychosocial Perspectives. New York: Wiley.

Suadicani, P., Hein, H. \& Gyntelberg, F. (1993). Are social inequalities as associated with the risk of ischaemic heart disease as a result of psychosocial working conditions? Atherosclerosis, 101, 165-175.

Swanberg, J., Pitt-Catsouphes, M., Drescher-Burke, K. (2005). A question of justice: Disparities in employees' access to flexible schedule arrangements, Journal of Family Issues, 26(6), 866-895.

Swanson, R. \& Holton, E. (1997). Human Resource Development Research Handbook. San Francisco: Berrett-Koehler Publishers.

Swanson, N. (2000). Working women and stress. Journal of the American Medical Women's Association, 55, 76-79.

Swart, J. (1978). A flexible approach to working hours. New York: AMACOM, a division of American Management Associations.

Tabachnick, B. \& Fidell, L. (2001). Using Multivariate Statistics. Boston: Allyn \& Bacon.

Tasto, D. \& Colligan, M. (1978). Health Consequences of Shiftwork. Project URU-4426, Technical Report, Menlo Park, CA: Stanford Research Institute.

Tausig, M. \& Fenwick, R. (1992). Gender differences in the causes of workers' stress. Paper presented at the International Conference on Social Stress, Venice, Italy, June.

Tausig, M. \& Fenwick, R. (2001). Unbinding Time: Alternative work schedules and work-life balance. Journal of Family and Economic Issues, 22, 101111.

Templar, A., Armstrong-Stassen, M., Devine, K. \& Solomon, N. (1999). Telework and teleworkers In Zeytinoglu, I. (Ed.) (1999). Changing work 
relationships in industrialized economies. Amsterdam: John Benjamins Publishing Company.

Tepas, D. (1985). Flexitime, compressed workweeks and other alternative work schedules. In S. Folkard and T. Monk (Eds.) Hours of work - temporal factors in work scheduling. New York: John Wiley \& Sons.

Tepas, D. \& Carvalhais, A. (1990). Sleep patterns of shiftworkers. Occupational Medicine: State of Art Review, 5, 199-208.

Theorell, T. (1989). Personal control at work and health: a review of epidemiological studies in Sweden. In A. Steptoe and A. Appels (Eds.) Stress, Personal Control and Health. Brussels-Luxembourg: John Wiley \& Sons Ltd.

Theorell, T. (1993). Medical and physiological aspects of job interventions. In Cooper, C. et al. (Eds). International Review of Industrial and Organizational Psychology. v. 8. New York: John Wiley \& Sons Ltd.

Theorell, T. (1996). Flexibility at work in relation to employee health. In M. Schabracq, J. Winnubst and C. Cooper (Eds.) Handbook of Work and Health Psychology. New York: John Wiley \& Sons Ltd.

Theorell, T., Ahlberg-Hulten, G., Berggren, T., Perski, A., Sigala, F., Svensson, J. \& Wallin, B.M. (1987). Work environment, personal habits and heart disease risk. Stress Research Reports No. 195, National Institute for Psychosocial Factors and Health and the Department of Stress Research, Karolinska Institute, Sweden.

Theorell, T., Ahlberg-Hulten, G., Jodko, M., Sigala, F. \& de la Torre, B. (1993). Influence of job strain and emotion on blood pressure levels in female hospital personnel during work hours. Scandinavian Journal of Work, Environment \& Health, 19, 264-269.

Theorell, T., de Faire, U., Johnson, J., Hall, E., \& Perski, A. (1991). Job strain and ambulatory blood pressure profiles. Scandinavian Journal of Work, Environment \& Health, 17, 380-385.

Theorell, T. \& Floderus-Myrhed, B. (1977). 'Workload' and risk of myocardial infarction: A prospective psychosocial analysis. International Journal of Epidemiology, 6, 17-21.

Theorell, T., Hamsten, A., de Faire, U., Orth-Gomer, K. \& Perski, A. (1987). Psychosocial work conditions before myocardial infarction in young men. International Journal of Cardiology, 15, 33-46. 
Theorell, T., Harms-Ringdahl, K., Ahlberg-Hulten, G. \& Westin, B. (1991). Psychosocial job factors and symptoms from the locomotor system - a multicausal analysis. Scandinavian Journal of Rehabilitation Medicine, $23,165-173$.

Theorell, T. \& Karasek, R. (1996). Current issues relating to psychosocial job strain and cardiovascular disease research. Journal of Occupational Health Psychology, 1, 9-26.

Theorell, T. \& Johnson, J. (1997). Cardiovascular diseases. In J. Stellman (Ed.) Encyclopedia of Occupational Health and Safety. Geneva: International Labour Office.

Theorell, T., Perski, A., Akerstedt, T., Sigala, F., Ahlberg-Hulten, G., Svensson, J. \& Eneroth, P. (1988). Changes in job strain in relation to changes in psychological states: A longitudinal study. Scandinavian Journal of Work, Environment and Health, 14, 189-196.

Thoits, P. A. (1992). Identity structures and psychological well-being: Gender and marital status comparisons. Social Psychology Quarterly, 55, 236-256.

Thomas, D., Hughes, E. \& Zumbo, B. (1998). On variable importance in linear regression. Social Indicators Research, 45, 253-275.

Thomas, L. \& Ganster, D. (1995). Impact of family-supportive work variables on work-family conflict and strain: A control perspective. Journal of Applied Psychology, 80, 6-15.

Thompson, C., Beauvais, L. \& Allen, T. (2006). Work and family from an industrial/organizational psychology perspective. In Pitt-Catsouphes, $M$., Kossek, E. \& Sweet, S. (Eds.) (2006). The work and family handbook: Multi-disciplinary perspectives and approaches. Mahwah, NJ: Lawrence Erlbaum Associates, Publishers.

Thompson, C., Beauvais, L. \& Lyness, K. (1999). When work-family benefits are not enough: The influence of work-family culture on benefit utilization, organizational attachment and work-family conflict. Journal of Vocational Behavior, 54, 392-415.

Thompson, J. \& Bunderson, S. (2001). Work-nonwork conflict and the phenomenology of time: Beyond the balance metaphor. Work and Occupations, 28 (1), 17-39.

Thompson, S. (1981). Will it hurt less if I can control it? A complex answer to a simple question. Psychological Bulletin, 90, 89-101. 
Tierney, D., Romito, P. \& Messing, K. (1990). She ate not the bread of idleness: Exhaustion is related to domestic and salaried working conditions among 539 Quebec hospital workers. Women and Health, 16(1), 21-42.

Tolbert, P., Valcour, M. \& Marler, J. (2002). Work schedules, work-life balance and perceived success in work life and in family life. BLCC Working Paper \#02-09. Cornell Employment and Family Careers Institute.

Toronto Star (August 21, 2004). Pay Packet, p. C10.

Torsvall, L. \& Akerstedt, T. (1978). Summary of a longitudinal study of shift work effects on well-being, Reports from the laboratory for clinical stress research, 926, 1-6.

Trent, J., Smith, A. \& Wood, D. (1994). Telecommuting: stress and social support. Psychological Reports, 74, 1312-1314.

Tucker, P., Barton, J., \& Folkard, S. (1996). Comparison of eight and twelve hour shifts: Impacts on health, well-being and alertness during the shift. Occupational Environmental Medicine, 53, 767-772.

Tucker, P. Smith, L., Macdonald, I. \& Folkard, S. (2000). Effects of direction of rotation inn continuous and discontinuous eight hour shift systems. Occupational Environmental Medicine, 57, 678-684.

Uehata, T. (1991). Long working hours and occupational stress-related cardiovascular attacks among middle-aged worked in Japan. Journal of Human Ergology, 20, 147-153.

Ullman, J. (2001). Structural Equation Modeling. In Tabachnick, B. \& Fidell, L. Using Multivariate Statistics. Boston: Allyn \& Bacon.

Underwood, P. (2000). Social support: The promise and the reality in Rice, V. (Ed.). Handbook of stress, coping and health: Implications for nursing research, theory and practice. Thousand Oaks, CA: Sage Publications, Inc.

United States Department of Labor (2000). Are managers and professionals really working more, Issues in Labor Statistics. Bureau of Labor Statistics: Washington.

United States Department of Labor. (2001). Report on the American Workforce.

United States Department of Labor (2002). Workers on flexible and shift schedules in 2001 (USDL 02-225). Washington, DC. 
Vacha-Haase, T. \& Thompson, B. (2004). How to estimate and interpret various effect sizes. Journal of Counseling Psychology, 51(4), 473-481.

Van der Beek, A., Oort-Marburger, D. \& Frings-Dresen, M. (1994). The relations between work demands and health complaints in lorry drivers. International Archives of Occupational and Environmental Health, 66, 179-184.

Van der Doef, M. \& Maes, S. (1998). The job demand-control (support) model and physical health outcomes: A review of strain and buffer hypotheses. Psychological Health, 13 (5), 909-936.

Van der Doef, M. \& Maes, S. (1999). The job demand-control (support) model and psychological well-being: a review of 20 years of empirical research. Work and Stress, 13, 87-114.

Van der Doef, M., Maes, S. \& Diekstra, R. (2000). An examination of the job demand-control-support model with various occupational strain indicators. Anxiety, Stress and Coping, 13, 165-185.

Van der Hulst, M. \& Geurts, S. (2001). Associations between overtime and psychological health in high and low reward jobs. Work and Stress, 15, 227-240.

van Dijkhuizen, N. (1980). From Stressors to Strains. Lisse: Swets and Zeitlinger.

van Dixhoorn, J. (1997). Stress at the workplace: Health and productivity - ISMA5. International Journal of Stress Management, 4, 233.

Vanier Institute of the Family (2005). http://www.vifamily.ca/library/work/kitchen digest.html.

Vener, K., Szabo, S. \& Moore, J. (1989). The effect of shift work on gastrointestinal (GI) function. A review. Chronobiologia, 16, 421-439.

Venkatesh, A. \& Vitalari, N. (1992). An emerging distributed work arrangement: An investigation of computer-based supplemental work at home. Management Science, 38(12), 1687-1706.

Venne, R. (1993). Alternative worktime arrangements: The compressed workweek. Unpublished doctoral dissertation, University of Toronto, Toronto, Ontario, Canada.

Verhaegen, P., Cober, R., De Smedt, M., Dirkx, J., Kerstens, J., Rijvers, D. \& Van Daele, P. (1987). The adaptation of night nurses to different work schedules. Ergonomics, 30, 1301-1309. 
Vermeulen, M. \& Mustard, C. (2000). Gender differences in job strain, social support at work, and psychological distress. Journal of Occupational Health Psychology, 5, 428-440.

Villa, J., Howell, J., Dorfman, P. \& Daniel, D. (2003). Problems with detecting moderators in leadership research using moderated multiple regression. The Leadership Quarterly, 14, 3-23.

Vinokur, A., Pierce, P. \& Buck, C. (1999). Work-family conflicts of women in the Air Force: Their influence on mental health and functioning. Journal of Organizational Behavior, 20, 865-878.

Viswesvaran, C., Sanchez, J. \& Fisher, J. (1999). The role of social support in the process of work stress: A meta-analysis. Journal of Vocational Behavior, 54, 314-334.

Voydanoff, P. (1984) (Ed). Work and family: Changing roles of men and women. Palo Alto, CA: Mayfield Publishing Company.

Voydanoff, P. (1987). Work and family life. Thousand Oaks, CA: Sage Publications.

Voydanoff, P. (1988). Work role characteristics, family structure demands, and work/family conflict. Journal of Marriage and the Family, 50, 749-761.

Voydanoff, P. (2004). The effects of work demands and resources on work-tofamily conflict and facilitation. Journal of Marriage and Family, 66, 398412.

Voydanoff, P. (2005). Work demands and work-to-family and family-to-work conflict: Direct and indirect relationships. Journal of Family Issues, 26, 707-726.

Voydanoff, P. \& Kelly, R. (1984). Determinants of work-related family problems among employed parents. Journal of Marriage and the Family, 46, 881892.

Wahlgreen, E. (2002). When job stress becomes disabling. Business Weekonline, 7/1/2002.

Waismel-Manor, R., Moen, P. \& Sweet, S. (2002). Managing and thriving: What factors predict dual-earner middle class couples feeling highly successful in their jobs, families and balancing both? BLCC Working Paper \#02-20. Cornell Employment and Families Careers Institute. 
Waldron, I., Weiss, C., \& Hughes, M. (1998). Interacting effects of multiple roles on women's health. Journal of Health and Social Behavior, 39, 216-236.

Walker, J., Fletcher, C., \& McLeod, D. (1975). Flexible working hours in two British government offices. Public Personnel Management,4, 216-222.

Walker, J. (1978). Human Aspects of Shiftwork. London: Institute of Personnel Management.

Wall, T., Jackson, P. \& Mullarkey, S. (1995). Further evidence of some new measures of job control, cognitive demand and production responsibility. Journal of Organizational Behavior, 16, 431-455.

Wall, T., Jackson, P., Mullarkey, S. \& Parker, S. (1996). The demands-control model of job strain: A more specific test. Journal of Occupational and Organizational Psychology, 69, 153-166.

Wallace, J. (1997). It's about time: A study of hours worked and work spillover among law firm lawyers. Journal of Vocational Behavior, 50, 227-248.

Wallace, J. (1999). Work-to-nonwork conflict among married male and female lawyers. Journal of Organizational Behavior, 20, 797-816.

Wallace, M., Levens, M. \& Singer, G. (1988). Blue collar stress. In Cooper, C. et al. (Eds). Causes, Coping and Consequences of Stress at Work. New York: John Wiley \& Sons Ltd.

Warr, P. (1987). Work, Unemployment and Mental Health. Oxford: Clarendon Press.

Warr, P. (1990). Decision latitude, job demands, and employee wellbeing. Work and Stress, 4, 285-294.

Warren, J. \& Johnson, P. (1995). The impact of workplace support on workfamily role strain. Family Relations, 44, 163-169.

Watkins, K. (1995). Changing managers' defensive reasoning about work/family conflicts. The Journal of Management Development, 14, 77-88.

Watson, D. \& Pennebaker, J. (1989). Health complaints, stress, and distress: Exploring the central role of negative affectivity. Psychological Review, 96, 234-254.

Watson, D., Pennebaker, J. \& Folger, R. (1986). Beyond negative affectivity: Measuring stress and satisfaction in the workplace. Journal of Organizational Behavior Management, 8, 141-157. 
Wedderbaun, A. (1978). Some suggestions for increasing the usefulness of psychological and sociological studies of shift work, Ergonomics, 21, 827-833.

Weingarten, K. (1978). The employment pattern of professional couples and their distribution of involvement in the family. Psychology of Women Quarterly, 3, 43-52.

Weiss, M. \& Liss, M. (1989). Night shift work: Job and family concerns. In E. Goldsmith (Ed.) Work and family: Theory, research and applications. Newbury Park, CA: Select Press.

Weissman, M. \& Klerman, G. (1977) Sex differences in the epidemiology of depression. Archives of General Psychiatry, 34, 98-111.

Welch, J. \& Gordon, D. (1980). Assessing the impact of flexitime on productivity, Business Horizons, 23, 61-65.

Werbel, J. (1985). The impact of primary life involvements on turnover: A comparison of part-time and full-time employees. Journal of Occupational Behavior, 6, 251-258.

Westman, M. \& Piotrkowski, C. (1999). Introduction to the special issue: workfamily research in occupational health psychology. Journal of Occupational Health Psychology, 4, 301-306.

Wheeler, K. (1970). Small business eyes the four-day week, Harvard Business Review, 48, 142-147.

White, J. (1983). Women and part-time work. Ottawa: Canadian Advisory Council on the Status of Women.

White, J. (2002). A new look at shorter hours of work in the communications, energy and paperworkers' union. Just Labour, 1, 41-49.

White, M., Hill, S., McGovern, P., Mills, C. \& Smeaton, D. (2003). 'Highperformance' management practices, working hours, and work-life balance. British Journal of Industrial Relations, 41, 175-195.

White, L. \& Keith, B. (1990). The effect of shiftwork on the quality and stability of marital relations. Journal of Marriage and the Family, 52, 453-462.

Whitfield, K. \& Strauss, G. (Eds.) (1998). Researching the World of Work: Strategies and Methods in Studying Industrial Relations. Ithaca, NY: ILR Press. 
Williams, C. (2004). The sandwich generation. Statistics Canada. Catalogue no. 75-001-XPE.

Williams, D. \& House, J. (1985). Social support and stress reduction. In C. Cooper and M. Smith (Eds.) Job stress and blue collar work. New York: John Wiley and Sons Ltd.

Williams, K. \& Alliger, G. (1994). Role stressors, mood spillover, and perceptions of work-family conflict in employed parents. Academy of Management Journal, 37, 837-868.

Williamson, A., Gower, C. \& Clarke, B. (1994). Changing the hours of shiftwork: A comparison of 8- and 12-hour shift rosters in a group of computer operators. Ergonomics, 37(2), 287-298.

Willmott, B. (2003). Stress standards to put pressure on employers. Personnel Today, January, 14.

Wilson, M., Dejoy, D., Vandenburg, R., Richardson, H. \& McGrath, A. (2004). Work characteristics and employee health and well-being: Test of a model of healthy organization. Journal of Occupational and Organizational Psychology, 77, 565-588.

Winnett, R. \& Neale, M. (1980). Modifying settings as a strategy for permanent, preventive behavior change: flexible work schedules and family life as a case in point. In P. Karoly and J. Steffen (Eds.) Improving the long-term effects of psychotherapy. New York: Gardner Press.

Winnett, R. \& Neale, M. (1981). Flexible work schedules and family time allocation: assessment of a system change on individual behavior using self-report logs. Journal of Applied Behavior Analysis, 14, 39-46.

Winnett, R., Neale, M. \& Williams, K. (1982). The effects of flexible work schedules on urban families with young children: Quasi-experimental, ecological studies. American Journal of Community Psychology, 10, 4964.

Winnubst, J. \& Schabracq, M. (1996). Social support, stress and organization: Towards optimal matching. In M. Schabracq, J. Winnubst, and C. Cooper (Eds.) Handbook of Work and Health Psychology. New York: John Wiley \& Sons Ltd.

Wolf, W. \& Fligstein, N. (1979a). Sex and authority in the workplace: Causes of sexual inequality. American Sociology Review, 44, 235-252. 
Wolf, W. \& Fligstein, N. (1979b). Sexual stratification: Differences in power in the work setting. Social Forces, 58, 94-107.

Wortman, C., Biernat, M., \& Lang, E. (1991). Coping with Role Overload. In M. Frankenhaeuser, U. Lundberg, and M. Chesney (Eds.) Women, Work and Health: Stress and Opportunities. New York: Plenum Press.

Wright, E., Costello, C., Hachen, D. \& Sprague, J. (1982). The American class structure. American Sociological Review, 47, 709-726.

Yang, C. \& Carayon, P. (1995). Effect of job demands and social support on worker stress: a study of VDT users. Behaviour \& Information Technology, 14 (1), 32-40.

Yang, N., Chen, C., Choi, J. \& Zou, Y. (2000). Sources of work-family conflict: A Sino-U.S. comparison of the effects of work and family demands. Academy of Management Journal, 43 (1), 113-123.

Yogev, S. (1981). Do professional women have egalitarian marital relationships? Journal of Marriage and the Family, 43, 865-871.

Zapf, D. (1989). Self and expert observation in psychological job analysis: Methodological problems in the measurement of stressors at work. Gottingen, Germany: Hogrefe.

Zapf, D., Dormann, C., \& Frese, M. (1996). Longitudinal studies in organizational stress research: A review of the literature with reference to methodological issues. Journal of Occupational Health Psychology, 1 , 145-169.

Zappert, L. \& Weinstein, M. (1985). Sex differences in the impact of work on physical and psychological health. American Journal of Psychiatry, 142, 1174-1178.

Zedeck, S. (1992). Introduction: Exploring the domain of work and family concerns. In S. Zedeck (Ed.) Work, families and organizations. San Francisco: Jossey-Bass, Inc.

Zedeck, S. \& Mosier, K. (1990). Work in the family and employing organization. American Psychologist, 45, 240-251.

Zeytinoglu, I. (1999). Flexible work arrangements: An overview of developments in Canada. In Zeytinoglu, I. (Ed.) (1999). Changing work relationships in industrialized economies. Amsterdam: John Benjamins Publishing Company. 
Zick, C. \& McCullough, J. (1991). Trends in married couples' time use: Evidence from 1977-78 and 1987-88. Sex Roles, 24, 459-487.

Zohar, D. (1999). When things go wrong: The effects of daily work hassles on effort, exertion and negative mood. Journal of Occupational and Organizational Psychology, 72, 265-283.

Zuckerman, M., Knee, R., Kieffer, S., Rawsthorne, L. \& Bruce, L. (1996). Beliefs in realistic and unrealistic control: Assessment and implications. Journal of Personality, 64, 435-464.

Zukewich, N. (2000). Paid and unpaid work. In Women in Canada 2000: A gender-based statistical report. Statistics Canada: Housing, Family and Social Statistics Division. Catalogue No. 89-503-XPE.

Zukewich, N. (2003). Work, parenthood and the experience of time scarcity. Statistics Canada. Catalogue no. 89-584-MIE-no. 1. 